
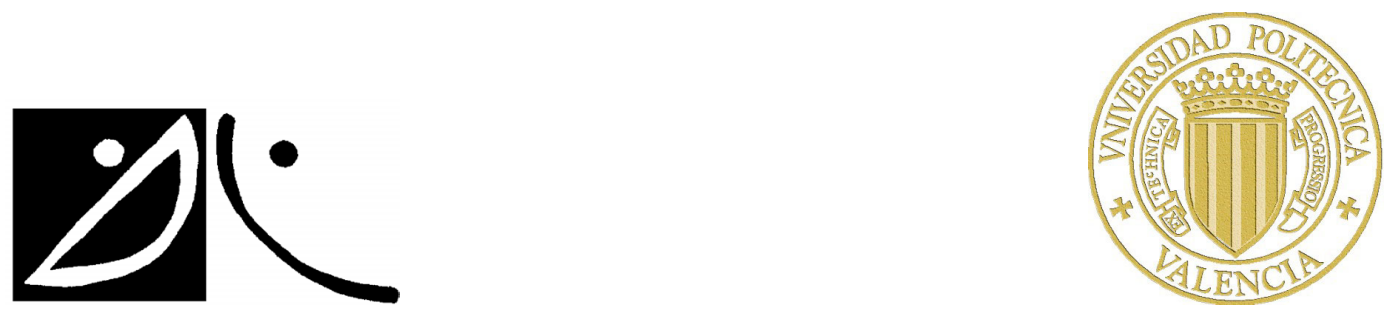

\title{
ANÁLISIS DE PROBLEMAS ARBITRARIOS DE DISPERSIÓN ELECTROMAGNÉTICA MEDIANTE MÉTODOS HÍBRIDOS
}

\author{
Héctor Esteban González \\ Director: Dr. Vicente E. Boria Esbert
}

Tesis Doctoral presentada en la Universidad Politécnica de Valencia para la obtención del título de Doctor Ingeniero de Telecomunicación

Valencia, Mayo 2002 

"Ay mi pescadito, no llores ya más, ay mi pescadito deja de llorar ..."

Capitanes Intrépidos

A mi hijo o hija que está en camino 



\section{Agradecimientos}

Resulta difícil escribir un capítulo de agradecimientos, ya que siempre temes olvidarte de alguien o no hacer justicia a la ayuda que te han prestado. Por otra parte existe la tentación de extenderse demasiado y resultar tedioso. Así que intentaré ser breve y si me olvido de alguien, por favor que no se sienta ofendido.

Mi primer agradecimiento es para Vicente Boria, porque desde que comencé el proyecto fin de carrera me hizo sentir siempre que formábamos parte de un mismo equipo. Estoy convencido de que ha sido el mejor director que yo podría haber tenido.

No me puedo olvidar de todos los miembros (profesores y becarios) del Grupo de Aplicaciones de las Microondas de Valencia y Cuenca, y de los compañeros de la Facultad de Físicas de la Universidad de Valencia. Especialmente agradezco a Santiago Cogollos el haber sido un estupendo compañero todo este tiempo, desde la época en que sólo éramos tres.

También quiero agradecer a mi mujer, mis padres y mi hermano la infinita paciencia que han tenido conmigo durante estos años.

Y por último, agradezco al lector el aprecio que hace de mi trabajo al leer esta tesis. Espero sinceramente que le resulte de utilidad.

Valencia

Héctor Esteban González

26 de Julio de 2002 



\section{Resumen}

El objetivo general de la tesis es el desarrollo de una única herramienta que permita analizar una muy amplia gama de problemas de dispersión electromagnética múltiple, con presencia de objetos dispersores de geometría arbitraria tanto metálicos como dieléctricos (con o sin pérdidas).

Para cumplir con este objetivo se utiliza en la tesis la función de transferencia o matriz de dispersión generalizada de un objeto dispersor. Esta matriz caracteriza electromagnéticamente al dispersor de forma completa ante cualquier incidencia relacionando los espectros cilíndricos de los campos incidente y dispersado por el objeto, resultando posteriormente mucho más fácil la resolución del acoplo entre varios objetos dispersores una vez que la matriz de dispersión de todos ellos ha sido obtenida.

Al comenzar la tesis se disponía de los programas necesarios para obtener la función de transferencia de objetos sencillos (tiras y cilindros 2-D), y se contaba con un método de resolución del acoplo entre múltiples dispersores que obtenía la solución final tras un proceso iterativo. Aunque este método proporcionaba buenos resultados para problemas de dispersión en espacio abierto, presentaba varias limitaciones que hacían imposible su uso para el análisis de estructuras muy cerradas, como los son la mayoría de dispositivos en guía de onda.

El primer trabajo de la tesis consiste en implementar técnicas que permiten analizar nuevos objetos como son cilindros multicapa con pérdidas eléctricas y magnéticas (solución analítica), objetos dieléctricos inhomogéneos de sección arbitraria, arcos circulares metálicos y objetos cuya geometría se puede expresar como combinaciones de objetos metálicos sencillos (tiras y cilindros). A continuación se desarrolla un nuevo método de análisis del acoplo electromagnético entre múltiples dispersores que supera las limitaciones del método anterior. Con el nuevo método es posible analizar prácticamente cualquier problema de dispersión múltiple 2-D independientemente de la posición de los objetos, incluidos muchos dispositivos reales inductivos en guía rectangular.

Una vez conseguido un método de análisis de problemas múltiples 2-D eficiente y capaz de analizar prácticamente cualquier estructura, se aplica al análisis de dispositivos inductivos en guía rectangular. Para ello se extraen los parámetros circuitales de la estructura guiada a partir de la matriz de dispersión en espacio abierto del problema 2-D equivalente. En primer lugar se desarrolla una técnica para la obtención de la matriz de admitancias generalizadas (MAG) cuyos resultados son satisfactorios si se analiza un único dispositivo, pero insuficientes si se desea caracterizar varios dispositivos en guía y enlazar sus matrices de parámetros circuitales para obtener la respuesta global de la red. Para superar esta limitación 
se desarrolla otra técnica que permite construir la matriz de dispersión generalizada (MDG) con unos resultados excelentes y se analizan con éxito una gran variedad de dispositivos en guía rectangular. Los resultados son validados mediante comparación con otros publicados en la bibliografía técnica relacionada. Esta técnica se aplica con éxito al análisis de filtros y diplexores inductivos. Además se analiza el efecto que produce la presencia de esquinas redondeadas debidas a defectos en la fabricación en dichos dispositivos, y se valida con éxito la precisión del método comparando sus resultados con medidas de la respuesta de un diplexor real con esquinas redondeadas debidas a defectos de fabricación. Como consecuencia se apunta la posibilidad de que la técnica desarrollada permita en un futuro el diseño automatizado de estos dispositivos teniendo en cuenta los defectos de mecanizado.

Además de aplicar la nueva técnica al análisis de filtros y diplexores con esquinas redondeadas, se aplica con éxito a la determinación precisa de las propiedades dieléctricas de materiales, aunque éstos sean líquidos, consiguiendo superar limitaciones (poder analizar sólo materiales con bajas pérdidas y en un margen de frecuencia estrecho) de métodos anteriores.

Finalmente, en la tesis se apuntan aplicaciones inmediatas del trabajo que aún quedan por explotar, como por ejemplo el análisis de cristales fotónicos, el diseño de dispositivos de comunicaciones teniendo en cuenta defectos de fabricación, el diseño de nuevos dispositivos tales como filtros con postes dieléctricos, etc. Y como líneas futuras de investigación relacionadas con esta nueva herramienta se destaca la extensión del método, primero al análisis de problemas capacitivos (p. ej. saltos de sección capacitivos, y estructuras en plano E en general), y posteriormente al análisis de estructuras completamente tridimensionales (mediante el uso de espectros esféricos de campo), tales como tornillos de sintonía o iris elípticos en guías de ondas, excitación mediante cable coaxial de guías y/o cavidades, antenas o cubiertas vegetales, todas ellas de gran interés práctico. 


\section{Resum}

L'objectiu general de la tesi és el desenvolupament d'una única eina que permitisca analitzar una amplia gama de problemes de dispersió electromagnètica múltiple, amb la presència d'objectes dispersors de geometria arbitraria tant metàlics com dielèctrics (amb i sense pèrdues).

Per a acomplir amb aquest objectiu s'utilitza a la tesi la funció de transferència o matriu de dispersió generalitzada d'un objecte dispersor. Aquesta matriu caracteritza electromagnèticament al dispersor de forma completa front a qualsevol incidència relacionant els espectres cilíndrics de camps incident i dispersat per l'objecte, resultant posteriorment molt més fàcil la resolució de l'acoblament entre varis objectes dispersors una volta que la matriu de dispersió de tots ells ha sigut calculada.

Al començament de la tesi es disposava dels programes necesaris per a obtindre la funció de transferència d'objectes senzills (tires i cilindres 2-D), i es contava amb un mètode de resolució de l'acoblament entre múltiples dispersors que arribava a la solució final després d'un procés iteratiu. Encara que aquest mètode proporcionava bons resultats per a problemes de dispersió en espai obert, presentava varies limitacions que feien impossible la seua utilització per a l'anàlisi d'estructures molt tancades, com ho són la majoria de dispositius en guia d'ona.

El primer treball de la tesi és implementar tècniques que permitisquen analitzar nous objectes com són cilindres multicapa amb pèrdues eléctriques i magnétiques (solució analítica), objectes dieléctrics inhomogenis de secció arbitrària, arcs circulars matàlics i objectes amb geometria que pot ésser expressada com a combinacions d'objectes metàlics senzills (tires i cilindres). A continuació es desenvolupa un nou mètode d'anàlisi de l'acoblament electromagnètic entre múltiples dispersors que supera les limitacions del mètode anterior. Amb el nou mètode és possible analitzar pràcticament qualsevol problema de dispersió múltiple 2-D independentment de la posisició dels objectes, inclosos molts dispositius reals inductius en guia rectangular.

Una volta aconseguit un mètode d'anàlisi de problemes múltiples 2-D eficient i capaç d'analitzar pràcticament qualsevol estructura, s'aplica a l'anàlisi de dispositius inductius en guia rectangular. Amb aquest objectiu s'extrauen els paràmetres circuitals de la estructura guiada a partir de la matriu de dispersió en espai obert del problema 2-D equivalent. En primer lloc es desenvolupa una tècnica per a la obtenció de la matriu d'admitàncies generalitzades (MAG) els resultats de la qual són satisfactoris si es analitza un únic dispositiu, però insuficients si es desitja caracteritzar varis dispositius en guia i enllaçar les seues matrius de paràmetres circuitals per a obtindre la resposta global de la xarxa. Per a superar aquesta 
limitació es desenvolupa una altra tècnica que permiteix construir la matriu de dispersió generalitzada (MDG) amb uns resultats excel-lents i s'analitzen amb èxit una gran varietat de dispositius en guia rectangular. Els resultats són validats mitjançant la comparación amb altres publicats en la bibliografia tècnica ralacionada. Aquesta tècnica s'aplica amb èxit a l'anàlisi de filtres i diplexors inductius. A més s'analitza l'efecte que produeix la presència de cantons redondejats a causa de defectes en la fabricació dels esmentats dispositius, i es valida amb èxit la precisió del mètode comparant els seus resultats amb mesures de la resposta d'un diplexor real amb cantons redondejats degut a defectes de fabricació. Com a conseqüència s'apunta la possibilitat de que la tècnica desenvolupada permitisca en un futur el diseny automatitzat d'aquests dispositius considerant els defectes de mecanitzat.

A més d'aplicar la nova tècnica a l'anàlisi de filtres i diplexors amb cantons redondejats, s'aplica amb èxit a la determinació precisa de les propietats dielèctriques de materials, encara que aquests siguen líquids, aconseguint superar limitacions (analitzar només materials amb baixes pèrdues $i$ en una marge de freqüència estret) de métodes anteriors.

Finalment, en la tesi s'apunten aplicacions inmediates del treball que encara queden per explotar, com per exemple l'anàlisi de cristals fotònics, el diseny de dispositius de comunicacions considerant defectes de fabricació, el diseny de nous dispositus tals com filtres amb objectes dielèctrics, etc. I com a línies futures d'investigació relacionades amb aquesta nova ferramenta es destaca la extensió del mètode, primer a l'anàlisi de problemes capacitius (per exemple salts de secció capacitius i estructures en plànol $\mathrm{H}$ en general), i posteriorment a l'anàlisi d'estructures completament tridimensionals (mitjançant l'ús d'espectres esfèrics de camp), tals com caragols metàlics de sintonia o iris elíptics en guies d'ones, excitació mitjançant cable coaxial de guies i/o cavitats, antenes o cobertes vegetals, totes elles de gran interés práctic. 


\section{Abstract}

The general goal of this work is the development of a technique that enables the analysis of a wide range of electromagnetic scattering problems, with the presence of metallic and/or dielectric scatterers with arbitrary geometry (with or without losses).

In order to achieve this goal the transfer function or generalized scattering matrix of a scattering object is used. This matrix fully characterizes the electromagnetic behaviour of a scatterer relating the cylindric spectra of the incident and scattered fields, thus being easier to solve the electromagnetic coupling among multiple objects once the scattering matrix of all the individual objects has been computed.

At the beginning of this work a software was available for computing the transfer function of simple objects (2-D metallic strips and cylinders) and also for solving the electromagnetic coupling among multiple objects using an iterative method. Although this method could solve successfully scattering problems in open space, due to some limitations it could not analyze clumped structures, like the ones that have to be solved to analyze most waveguide devices.

The first task in this thesis consists on implementing techniques for the analysis of new 2D objects such as multilayered circular cylinders with electric or magnetic losses (analytical solution), inhomogeneous dielectric objects with arbitrary geometry, metallic circular arcs and objects whose geometry can be expressed as a combination on simple metallic objects (strips and cylinders). Next a new method is developed for the analysis of the electromagnetic coupling among multiple objects that overcomes the limitations of the former method. This new method can be used to analyze almost any 2-D multiple scattering problem, regardless of the position of the individual objects, including many real rectangular waveguide devices.

Once a new and efficient method has been developed for the analysis of almost any 2$\mathrm{D}$ multiple scattering problem, the method is applied to the analysis of inductive devices in rectangular waveguide. For this purpose the circuital parameters of the guided structure must be computed from the scattering matrix of the equivalent 2-D open space problem. In the first place a new technique for the obtention of the Generalized Admitances Matrix (GAM) is implemented. The results of this technique are satisfactory if the circuital parameters of only one device are needed. However, the circuital parameters provided by this technique are not accurate enough for the characterization of multiple cascaded devices whose Generalized Admitances Matrices must be linked in order to obtain the matrix of the whole network. This limitation is overcome by developing another technique which allows the construction of the Generalized Scattering Matrix (GSM) providing excellent results. A great variety of guided devices are successfully analyzed with this technique, and the results are validated by com- 
parison with results from the technical literature. This technique is applied to the analysis of guided inductive filters and diplexers. Moreover, the effect produced by the presence of rounded corners due to fabrication tolerances is analyzed, and the accuracy of this technique is successfully validated by comparing its results with measurements of the response of a real diplexer with rounded corners du to fabrication tolerances. As a consequence of this result, the possibility that this technique allows in the future the automated design of guided devices with fabrication tolerances is pointed.

The technique for computing the GSM is also successfully applied to the precise determination of the dielectric properties of materials, even if they are liquid materials, overcoming the limitations (only low losses and a narrow frequency range) of previous methods.

Finally, immediate applications of this work are pointed, such as the analysis of bandgap materials, the design of communication devices with fabrication tolerances, the design of new devices such as filters with inductive dielectric posts, etc. And some future research lines related to the tools developed in this work ar also outlined, such as the extension of the method, first to the analysis of capacitive structures (i.e. capacitive steps, and structures in the E plane in general), and next to the analysis of completely tridimensional structures (using spherical field spectra), such as tuning screws or elliptical irises in waveguides, excitation of waveguides and/or cavities with coaxial cable, antennas or vegetation covers, all of them of great practical interest. 


\section{Índice}

$\begin{array}{lll}\text { Agradecimientos } & \text { V }\end{array}$

$\begin{array}{lll}\text { Resumen } & \text { VII }\end{array}$

Resum IX IX I

$\begin{array}{lll}\text { Abstract } & \text { XI }\end{array}$

1. Introducción 1

1.1. Motivación ........................ . . 1

1.2. Revisión bibliográfica . . . . . . . . . . . . . . . 2

1.2.1. Análisis de objetos dispersores con geometría arbitraria . . . . . . . 2

1.2.2. Problemas de dispersión múltiple . . . . . . . . . . . . . . 3

1.2.3. Dispositivos en guía rectangular . . . . . . . . . . . . . 3

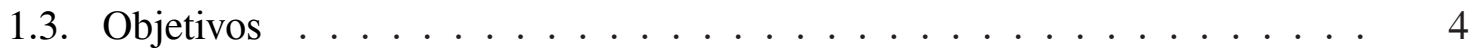

1.4. Estructura de la tesis . . . . . . . . . . . . . . 5

2. Análisis de problemas de dispersión bidimensionales 9

2.1. Introducción . . . . . . . . . . . . . . . . . 9

2.2. Dispersión de objetos aislados . . . . . . . . . . . . . . . . . 10

2.2.1. Introducción. Función de transferencia . . . . . . . . . . . 10

2.2.2. Funciones de transferencia TM y TE . . . . . . . . . . . . . . . 13

2.2.3. Matriz de corriente . . . . . . . . . . . . . . . . 18

2.2.4. Matriz de espectro . . . . . . . . . . . . . . . . . . 19

2.2.5. Objetos dispersores analizados . . . . . . . . . . . 20

2.3. Dispersión de múltiples objetos . . . . . . . . . . . . . . . 25

2.3.1. Introducción . . . . . . . . . . . . . . . 25

2.3.2. Nuevo método de análisis de problemas de dispersión múltiple . . . 28

2.3.3. Resultados . . . . . . . . . . . . . . . 37

2.4. Conclusiones . . . . . . . . . . . . . . . . . 43

3. Análisis de problemas inductivos en guías rectangulares 45

3.1. Introducción . . . . . . . . . . . . . . . . . . . 45

3.1.1. Descripción del problema . . . . . . . . . . . 45 
3.1.2. Métodos de análisis existentes . . . . . . . . . . . . . 46

3.1.3. Aplicación del método de análisis de problemas 2-D . . . . . . . . 47

3.2. Matrices generalizadas de parámetros circuitales en guías de onda . . . . . 50

3.2.1. Amplitudes de tensión y corriente modales . . . . . . . . . . 50

3.2.2. Matriz de admitancias generalizada (MAG) . . . . . . . . . . . . . . 52

3.2.3. Matriz de dispersión generalizada (MDG) . . . . . . . . . . . 53

3.3. Obtención de la MAG utilizando cortocircuitos . . . . . . . . . . . 57

3.3.1. Metodología . . . . . . . . . . . . . . 57

3.3.2. Excitación del modo $T E_{n 0}^{(j)} \ldots \ldots \ldots \ldots$. . . . . . . . . . . . . . . 59

3.3.3. Cálculo de los campos transversales . . . . . . . . . . . 62

3.3.4. Resultados . . . . . . . . . . . . . . . 76

3.4. Obtención de la MDG mediante acoplo de modos . . . . . . . . . . 85

3.4.1. Introducción . . . . . . . . . . . . . . . . 85

3.4.2. Acoplo de modos . . . . . . . . . . . . . . . . . . . . 85

3.5. Resultados . . . . . . . . . . . . . . . . . . 105

3.5.1. Obstáculos inductivos . . . . . . . . . . . . . 106

3.5.2. Discontinuidades inductivas . . . . . . . . . . . . . . 121

3.5.3. Componentes y dispositivos en plano H . . . . . . . . . . . . 133

3.6. Conclusiones . . . . . . . . . . . . . . . . . 138

4. Aplicaciones 139

4.1. Determinación de las propiedades dieléctricas de materiales . . . . . . . . . 140

4.1.1. Introducción . . . . . . . . . . . . . . . . . . . 140

4.1.2. Medida de los parámetros de dispersión . . . . . . . . . . . . . . . 141

4.1.3. Proceso de optimización . . . . . . . . . . . . . . . . . . 143

4.1.4. Función de error . . . . . . . . . . . . . . . . . . . . 144

4.1.5. Algoritmo de optimización . . . . . . . . . . . . . . . . . . . 148

4.1.6. Resultados . . . . . . . . . . . . . . . . . 150

4.2. Análisis de filtros inductivos con esquinas redondeadas . . . . . . . . . . 157

4.2.1. Introducción . . . . . . . . . . . . . . . . 157

4.2.2. Principales métodos de fabricación . . . . . . . . . . . . 157

4.2.3. Análisis de filtros inductivos con efectos de mecanizado en plano $\mathrm{H} \quad 161$

4.3. Análisis de diplexores . . . . . . . . . . . . . . . . . . . 165

4.3.1. Introducción . . . . . . . . . . . . . . . 165

4.3.2. Diferentes geometrías de diplexores . . . . . . . . . . . 167

4.3.3. Análisis de un diplexor del satélite Hispasat 1-C (Alcatel Espacio) . 169

4.3.4. Simulación . . . . . . . . . . . . . . . . . . . . . . . . . . . . . . . . . . . . . .

4.4. Conclusiones . . . . . . . . . . . . . . . . . . 185

$\begin{array}{ll}\text { 5. Conclusiones } & 187\end{array}$ 
A. Espectros plano y cilíndrico de campo. Transformaciones 191

A.1. Espectro cilíndrico . . . . . . . . . . . . . . . . . . . . . . . . 191

A.2. Espectro de ondas planas . . . . . . . . . . . . . . . . . . . . . . 192

A.3. Equivalencia entre espectro plano y cilíndrico . . . . . . . . . . . . . . . . 195

A.4. Espectro cilíndrico de una onda incidente plana . . . . . . . . . . . . 195

B. Matrices de Traslación de espectros 197

B.1. Introducción . . . . . . . . . . . . . . . . . . . . . 197

B.2. Traslación de espectro emergente a incidente . . . . . . . . . . . . . . . 197

B.3. Traslación de espectro incidente a incidente . . . . . . . . . . . . . 206

C. Número de modos cilíndricos para el acoplo con modos guiados 215

D. Sistemas de coordenadas local y global en una guía rectangular 225

E. Método de descenso del simplex $\quad 231$

F. MDG conjunta de una conexión de dos redes 239

F.1. Conexión de dos accesos cualesquiera de dos redes . . . . . . . . . . . . 239

F.2. Cambio de accesos . . . . . . . . . . . . . . . . . 250

G. Publicaciones $\quad \mathbf{2 5 5}$

G.1. Artículos en revistas de ámbito internacional . . . . . . . . . . . . . . 255

G.2. Congresos . . . . . . . . . . . . . . . . . . . 256

G.2.1. Congresos nacionales . . . . . . . . . . . . . . . 256

G.2.2. Congresos internacionales . . . . . . . . . . . . 257 


\section{Capítulo 1}

\section{Introducción}

\subsection{Motivación}

El objetivo general de la tesis es el desarrollo de una única herramienta que permita analizar una muy amplia gama de problemas de dispersión electromagnética múltiple, con presencia de objetos dispersores de geometría arbitraria tanto metálicos como dieléctricos (con o sin pérdidas). Se pretende analizar problemas bidimensionales de dispersión en espacio abierto (p.ej. antenas formadas por múltiples reflectores) y problemas reales en guías de onda rectangulares.

El origen de la tesis está en la línea de investigaciones iniciadas en el Departamento de Comunicaciones de la Universidad Politécnica de Valencia por varios profesores, entre ellos el director de la presente tesis, Vicente Boria [1,2], en la cual me integré durante la realización del proyecto final de carrera. Esta tesis es una continuación de dichas investigaciones, de manera que se toma como punto de partida un método híbrido (en parte numérico y en parte espectral) que permite el análisis de problemas múltiples bidimensionales en espacio abierto [3]. Dicho método hace uso de la función de transferencia o matriz de dispersión generalizada para caracterizar de forma individual a cada uno de los objetos dispersores.

A continuación se realiza una revisión bibliográfica en la que se detallan los principales métodos existentes para analizar objetos dispersores aislados, problemas de dispersión múltiple, y dispositivos en guía de ondas. La revisión bibliográfica evidenciará el enorme interés científico y práctico de los problemas que se van a analizar en la tesis, además de establecer un contexto en el que se justificará la elección del método híbrido descrito en [3] como punto de partida para el análisis general de problemas de geometría arbitraria. Más aún, la revisión de los métodos existentes y el conocimiento aproximado de sus prestaciones facilitará una correcta valoración posterior de los resultados que se obtengan con el nuevo método. Se terminará esta introducción detallando los objetivos de la tesis y anticipando al lector de forma resumida el contenido de los diferentes capítulos de la tesis. 


\subsection{Revisión bibliográfica}

\subsubsection{Análisis de objetos dispersores con geometría arbitraria}

Antes de discutir las investigaciones realizadas hasta la fecha sobre análisis de problemas de dispersión múltiple conviene recordar los métodos más utilizados para el análisis de dispersores individuales, pues casi todos los métodos de análisis múltiple se basan en un análisis previo de los dispersores individualmente.

La caracterización electromagnética de objetos dispersores aislados ha sido objeto de muchos estudios. Cuando la geometría de los mismos es canónica, y se puede describir de forma sencilla mediante algún sistema de coordenadas, se puede caracterizar su comportamiento electromagnético de forma analítica [4]. Si la geometría del objeto no se puede describir de forma sencilla, y si el objeto es pequeño eléctricamente, se han utilizado tradicionalmente métodos numéricos, p.ej. el método de los momentos ("Moment Method"), el método unimomento ("Unimoment Method"), o el método de las condiciones de contorno ("Boundary Contour method") [5]. Sin embargo, si el objeto dispersor es grande eléctricamente, los métodos anteriores son ineficientes y en su lugar se han utilizado tradicionalmente técnicas de alta frecuencia menos exactas como óptica física ("Physical Optics"), óptica geomética ("Geometrical Optics"), PTD, o GTD [4]. La principal desventaja tanto de los métodos numéricos como de los de alta frecuencia es su dependencia de la geometría del objeto dispersor y de la excitación. Con el objeto de evitar esta limitación están recibiendo cada vez más atención los métodos de matrices generalizadas para la caracterización de problemas de dispersión en espacio abierto [6]. En esta línea se comenzó a trabajar en el Departamento de Comunicaciones de la U.P. de Valencia, desarrollándose un método que utiliza matrices de dispersión generalizadas que relacionan espectralmente la excitación y la respuesta de los objetos dispersores [7]. Dicho método utiliza formulaciones espectrales solamente para analizar objetos con geometría canónica, y la formulación espectral se combina con el método numérico de los momentos para analizar algunos objetos con geometría no canónica tales como tiras, cilindros y reflectores metálicos. El uso de la matriz de dispersión generalizada supone una gran ventaja, pues una vez que ésta ha sido calculada, se ha conseguido una total independencia respecto de la geometría del objeto, y la dispersión ante cualquier incidencia puede obtenerse de forma inmediata. Además, para la solución de problemas de dispersión múltiple, la caracterización previa de los objetos individuales mediante matrices de dispersión permite un análisis flexible de estructuras arbitrarias ante cualquier excitación, todo ello independientemente de la geometría de los objetos individuales. Este procedimiento fue ya utilizado en el Departamento de Comunicaciones para el análisis de antenas con múltiples reflectores [1], de modo que la presencia de varios reflectores puede analizarse mediante el producto matricial de las matrices de dispersión de los reflectores siguiendo el avance de las ondas electromagnéticas.

Uno de los objetivos de la tesis será el de ampliar el método de análisis desarrollado en la U.P.V. ([7]), puesto que dicho método tan sólo es capaz de analizar algunos objetos metálicos 2-D, tales como cilindros, tiras y reflectores parabólicos e hiperbólicos. El objetivo es conseguir un método capaz de analizar prácticamente cualquier objeto 2-D de sección 
transversal arbitraria tanto metálico como dieléctrico.

Sin embargo, para un análisis más completo de un problema de dispersión múltiple, es necesario tener en cuenta el acoplo entre distintos objetos.

\subsubsection{Problemas de dispersión múltiple}

El problema de la dispersión múltiple ha sido ampliamente estudiado, de manera que se han propuesto varias formas de abordar este problema en la bibliografía técnica $[6,8]$. En [6] se propuso un método gráfico muy intuitivo y exacto pero cuya dificultad aumenta enormemente conforme crece el número de objetos dispersores. Un método iterativo que gradualmente se aproxima a la solución final siguiendo el progreso físico de las interacciones entre objetos fue propuesto en [8], pero dicho método presentaba problemas de convergencia cuando los objetos dispersores eran de gran tamaño o estaban muy próximos entre sí. Además requería de cálculos costosos para comprobar la convergencia que reducían su eficiencia. Como alternativa a estos y otros métodos de resolución de acoplo entre múltiples objetos, en el Departamento de Comunicaciones de la U.P.V. se decidió desarrollar un nuevo método iterativo que explota el concepto de las matrices de dispersión [3]. El método resuelve primero el acoplo entre dos objetos, calculando para cada uno una matriz de dispersión que proporciona la respuesta de cada objeto a cualquier incidencia en presencia del otro objeto. Posteriormente se resuelve el acoplo entre los dos primeros objetos y un tercero, y así sucesivamente hasta completar el problema de dispersión múltiple, proporcionando matrices de dispersión conjuntas que dan la respuesta de cada objeto una vez resuelta la interacción múltiple. La ventaja de este método es que no aumenta su complejidad al aumentar el número de objetos, ya que cada nuevo objeto supone sólo una iteración adicional, y resulta eficiente y exacto para dispersores de todos los tamaños. No obstante, este método, aunque exacto, eficiente y válido para analizar un amplio rango de problemas, presenta diversas limitaciones si los objetos que forman el problema múltiple están muy cerca entre sí, formando agrupaciones muy cerradas. Un objetivo de la tesis será por tanto superar estas limitaciones implementando un nuevo método de análisis del acoplo electromagnético entre objetos.

\subsubsection{Dispositivos en guía rectangular}

La caracterización electromagnética de discontinuidades y obstáculos de geometría arbitraria en una guía de ondas es otro campo de gran interés en la literatura técnica [9, 10], debido a su aplicación práctica en el diseño de muchos dispositivos de microondas, tales como filtros pasa banda [11, 12] y de banda eliminada [13], o circuladores [14]. De nuevo, si la geometría del obstáculo se ajusta a un sistema de coordenadas, se pueden obtener soluciones analíticas. Sin embargo es cada vez mayor el interés por estructuras no canónicas que permitan modelar mejor las guías reales (p. ej. guías con esquinas redondeadas [9]), codos irregulares $[10,14,15]$, o postes de geometría irregular tales como postes cuadrados [16], o múltiples postes [13]. No obstante la mayoría de estos métodos o son métodos numéricos costosos desde el punto de vista computacional, o han sido desarrollados para resolver determinados problemas muy concretos y pocos ofrecen la posibilidad de analizar un amplio 
rango de problemas de geometría arbitraria de forma razonablemente eficiente.

Para poder analizar eficientemente estructuras arbitrarias en guías de onda se han desarrollado recientemente algunos métodos. Entre ellos destaca un método que utiliza el acoplo modal ("Mode-Matching") [14] para poder analizar codos y estructuras de múltiples accesos de geometría arbitraria. No obstante no permite el análisis de obstáculos. Otra contribución importante es un método analítico que utiliza modos cilíndricos y que es capaz de analizar un amplio rango de problemas [10]. Permite al análisis de ventanas de acoplo y de obstáculos metálicos cilíndricos. Sin embargo no analiza obstáculos de geometría no cilíndrica. Para poder analizar con una sola herramienta todo tipo de estructuras inductivas arbitrarias en una guía rectangular se aplicará el método de análisis de problemas múltiples mejorado descrito con anterioridad. Dicho método debe ser altamente eficiente para el análisis de estas estructuras, ya que se puede abordar el problema como si se tratara de un problema bidimensional en espacio abierto, debido a que los campos son invariantes en una dimensión siempre que la guía reciba sólo excitaciones de los modos $T E_{m 0}^{z}$ y los obstáculos sean inductivos. Otro objetivo de la tesis es por tanto analizar el segmento irregular de la guía que contiene los obstáculos y/o discontinuidades como si se tratara de un problema en espacio abierto. Para ello se debe segmentar la guía en múltiples dispersores de pequeño tamaño, obtener la matriz de dispersión de cada objeto por separado, y luego resolver un problema de dispersión múltiple 2-D. Finalmente, se debe desarrollar un procedimiento que obtenga los parámetros circuitales de la estructura guiada a partir de las matrices de dispersión de modos cilíndricos.

\subsection{Objetivos}

Como ya se ha indicado con anterioridad, el objetivo principal de la tesis es el desarrollo de un método de análisis de problemas de dispersión múltiple con geometría arbitraria en entornos abiertos y en sistemas guiados. En la sección de revisión bibliográfica se han ido apuntando los objetivos secundarios que se deben cumplir para lograr el objetivo global de la tesis:

- Ampliar el método de análisis de dispersores 2-D ([7]) para que sea capaz de analizar una mayor variedad de objetos. Concretamente, los objetos que se desea analizar son:

Cilindros multicapa . Para analizar estos objetos se utilizará la formulación iterativa espectral de [17], ya que se trata de un método analítico y por tanto muy eficiente, que además permite introducir pérdidas eléctricas o magnéticas en cualquiera de las capas que forman el cilindro.

Objetos inhomogéneos 2-D de sección transversal arbitraria En este caso se utilizará el método descrito en [18]. Al tratarse de un método numérico resulta mucho menos eficiente que el método descrito en [17], pero por contra se puede analizar con él cualquier objeto dieléctrico 2-D de sección arbitraria.

Combinaciones de objetos metálicos sencillos Se trata de analizar combinaciones de los objetos metálicos sencillos que el método de [7] ya es capaz de analizar (tiras, 
cilindros y reflectores metálicos). De esta forma, por ejemplo, se pueden analizar objetos metálicos 2-D de sección transversal compuesta por varias tiras con la geometría que se desee.

- Desarrollar un nuevo método de análisis de problemas de dispersión múltiple que supere las limitaciones que presenta el método descrito en [3] cuando se analiza el acoplo de objetos en entornos muy cerrados (objetos muy próximos entre sí).

- Desarrollar un procedimiento que permita obtener los parámetros circuitales de una estructura guiada (matriz de admitancias generalizadas o matriz de dispersión generalizada) a partir de las matrices de dispersión de modos cilíndricos que caracteriza a los objetos del problema múltiple 2-D.

- Verificar la eficacia y eficiencia del método resultante analizando problemas guiados clásicos tales como postes metálicos [12], iris y aperturas [19], transiciones [20], postes dieléctricos con pérdidas [11], postes multicapa [21], múltiples postes dieléctricos [22], postes cuadrados [16], codos [23], codos circulares [24], estructuras de accesos múltiples [16], etc.

- Analizar dispositivos complejos en guía rectangular de interés en el sector de las comunicaciones espaciales y móviles, tales como filtros de cavidades inductivas y diplexores. También se desea analizar cómo influye en la respuesta de dichos dispositivos la presencia de pequeños errores debidos a la técnica de fabricación, ya que cuando se fabrican estos dispositivos aparecen esquinas redondeadas, debido al grosor de la fresa utilizada normalmente en dicho proceso, que pueden hacer que la respuesta del dispositivo no se ajuste a la que se obtendría con un simulador convencional que considera que dichas esquinas son rectas.

- Validar el análisis de algún dispositivo complejo con medidas de la respuesta de un prototipo realizadas en el laboratorio.

\subsection{Estructura de la tesis}

La tesis se ha estructurado en capítulos de la siguiente manera:

Capítulo 2 En este capítulo se introduce el concepto de función de transferencia o matriz de dispersión generalizada de un objeto dispersor. Dicha función de transferencia caracteriza totalmente el comportamiento electromagnético ante cualquier incidencia. Se describe la formulación utilizada para construir la función de transferencia y se enumeran los objetos para los que ha sido implementado el cálculo de su función de transferencia.

Finalmente, en este capítulo se reformula el método de análisis de problemas de dispersión múltiple 2-D descrito en [3], de modo que en lugar de resolver el acoplo iterativamente se hace de una sola vez evitando las mencionadas limitaciones del método 
anterior. Se muestran algunos resultados que demuestran que el nuevo método es capaz de analizar con éxito estructuras que no se podían analizar con el método anterior, tales como antenas de tipo bocina o estructuras inductivas en una guía rectangular (postes, codos, cavidades, etc.).

Capítulo 3 En este capítulo se desarrollan dos técnicas diferentes para la extracción de parámetros circuitales de una estructura guiada a partir de la matriz de dispersión de modos cilíndricos del problema 2-D equivalente. El primer método consigue construir la matriz de admitancias generalizadas (MAG) colocando cortocircuitos en cada uno de los puertos de acceso de la estructura. Dicho método no proporciona resultados enteramente satisfactorios, así que se aborda el problema con otra perspectiva y se consiguen extraer los parámetros de dispersión mediante una técnica de acoplo de modos. Para las dos técnicas mencionadas se analizan dispositivos inductivos sencillos en guía rectangular, y se comparan los parámetros circuitales obtenidos con resultados de la bibliografía técnica relacionada.

Capítulo 4 En este capítulo se aplica el método desarrollado en los capítulos anteriores a diversas actividades de gran interés práctico, como son la determinación de las propiedades dieléctricas de los materiales y el análisis eficaz y eficiente de filtros y diplexores inductivos en guía rectangular con defectos de mecanizado.

Capítulo 5 En este último capítulo se extraen la conclusiones finales de la tesis y se apuntan posibles líneas de investigación futuras.

Además de los capítulos mencionados, se han incluido los siguientes apéndices:

Apéndices A y B En estos apéndices se explica cómo es el espectro cilíndrico de campo electromagnético, utilizado continuamente a lo largo de la tesis, y cómo se pueden realizar cambios en el origen de coordenadas al que está referido dicho espectro.

Apéndice C En este apéndice se realiza un estudio exhaustivo de la cantidad de modos del espectro cilíndrico de campo que son necesarios para reconstruir correctamente el campo de los modos de una guía rectangular a lo largo de una circunferencia. Esto es necesario para realizar el acoplo de modos entre modos cilíndricos de espacio abierto y modos guiados.

Apéndice D Aquí se realizan los cálculos matemáticos necesarios para relacionar los sistemas de coordenadas en que están expresados los campos correspondientes a modos guiados y a modos cilíndricos de espacio abierto, y poder forzar la continuidad de dichos campos.

Apéndice $\mathbf{E}$ En este apéndice se explica el método de descenso del simplex, que es un método de búsqueda del mínimo de una función $N$-dimensional. Este método de minimización es necesario para estimar la permitividad de materiales dieléctricos en el interior de una guía rectangular. 
Apéndice F Aquí se desarrolla la formulación necesaria para enlazar las matrices de dispersión generalizadas (MDG) de dos redes de múltiples accesos conectadas en serie (un acceso cualquiera de la primera red conectado a otro acceso cualquiera de la segunda).

Apéndice G Aquí se reflejan las publicaciones científicas a que ha dado lugar el trabajo de la tesis, con el objeto de refrendar el interés científico de la misma. 


\section{Capítulo 2}

\section{Análisis de problemas de dispersión bidimensionales}

\subsection{Introducción}

En este capítulo se desarrolla un método general de resolución de problemas de dispersión múltiple. Este tipo de problemas son de gran interés práctico, pues a la hora de caracterizar electromagnéticamente una gran variedad de sistemas reales, éstos se pueden modelar como un conjunto de múltiples objetos dispersores. Así, por ejemplo, la caracterización electromagnética de cubiertas vegetales (bosques, plantaciones de arroz, trigo, etc.), de gran interés en teledetección, se puede reducir al análisis de un conjunto de múltiples cilindros finitos sobre un plano reflector [25] (ver figura 2.1).

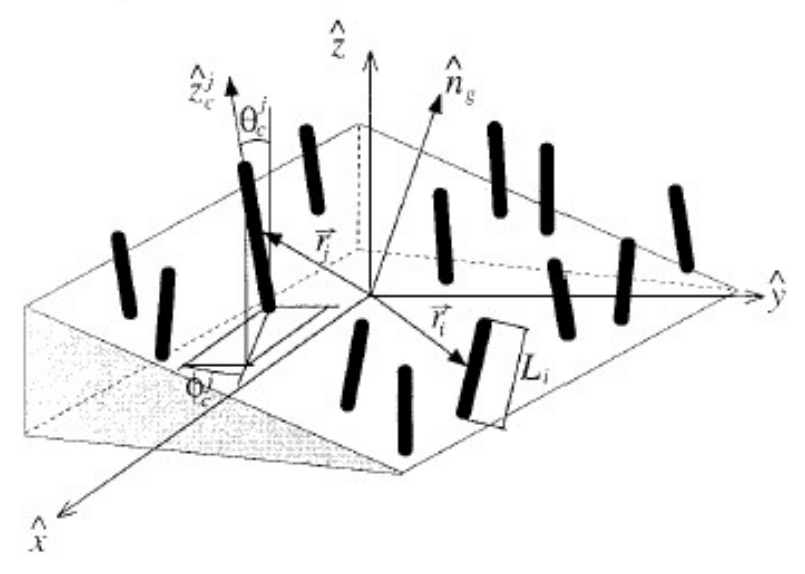

Figura 2.1: Modelo simplificado de un bosque para su caracterización electromagnética

Otros problemas reales cuya caracterización electromagnética se puede reducir a la resolución de un problema de dispersión múltiple son las antenas formadas por múltiples reflectores [1], las agrupaciones de antenas [26], o las estructuras periódicas bidimensionales 
denominadas cristales fotónicos [27]. Resulta evidente, por tanto, la utilidad de un método general de resolución de problemas de dispersión múltiple.

En la literatura técnica podemos encontrar una gran variedad de métodos que resuelven el acoplo electromagnético entre múltiples dispersores haciendo uso de matrices que caracterizan el comportamiento electromagnético de cada objeto de forma individual $[1,6,8,3]$ . A estas matrices se las denomina matrices de dispersión [6] o también funciones de transferencia [3] por su similitud con las funciones de transferencia utilizadas en la teoría de los sistemas lineales [28]. En este capítulo se desarrolla un nuevo método de análisis de problemas de dispersión múltiple que también hace uso de las funciones de transferencia individuales de cada objeto. Como se comprobará a lo largo del capítulo, el nuevo método supera las limitaciones de métodos anteriores.

En la sección 2.2 se desarrolla el concepto de función de transferencia de un objeto dispersor, y en la sección 2.3 se detalla el nuevo método de análisis de problemas de dispersión múltiple.

\subsection{Dispersión de objetos aislados}

\subsubsection{Introducción. Función de transferencia}

A continuación se describe la caracterización de un objeto dispersor mediante técnicas espectrales, utilizando lo que denominaremos función de transferencia.

El concepto de la función de transferencia se utiliza en general para sistemas electrónicos lineales invariantes en el tiempo (sistemas LTI), de modo que la función de transferencia es la respuesta en frecuencia de dicho sistema a un impulso temporal [28]. Esta función de transferencia caracteriza al sistema LTI de forma que, dada una señal cualquiera de entrada de espectro $X(f)$, se puede obtener el espectro de la señal de salida $Y(f)$ inmediatamente multiplicando dicho espectro de entrada por la función de transferencia $H(f)$ :

$$
Y(f)=H(f) \cdot X(f)
$$

Este procedimiento supone una gran ventaja en el análisis de los sistemas electrónicos lineales, puesto que una vez determinada la respuesta al impulso se conoce la respuesta del sistema a cualquier señal con sólo realizar una multiplicación en el dominio espectral.

El concepto de función de transferencia para sistemas electrónicos lineales se puede aplicar también a problemas electromagnéticos, ya que las ecuaciones de Maxwell son lineales e invariantes en el tiempo. En el caso de problemas electromagnéticos el sistema que se analizará será un objeto dispersor, la señal de entrada será una onda electromagnética incidente y la señal de salida será la onda dispersada por dicho objeto como respuesta a la onda incidente.

En los sistemas electrónicos las señales de entrada y salida $(x(t)$ e $y(t))$ presentan una variación temporal, y para utilizar la función de transferencia se transforman respectivamente al dominio de la frecuencia $(X(f)$ e $Y(f)$ ). En problemas electromagnéticos, las señales de 
entrada y salida son campos electromagnéticos cuya intensidad depende de la posición espacial $(\vec{E}(\rho, \phi, z)$ y $\vec{H}(\rho, \phi, z))$. De igual forma que a las señales con dependencia temporal les corresponde un espectro dependiente de la frecuencia temporal, a los campos electromagnéticos, dependientes del espacio, les corresponde un espectro dependiente de la frecuencia espacial (ver apéndice A). Los espectros de campo más frecuentes son los resultantes de expresar el campo como suma de ondas planas, cilíndricas, o esféricas [29].

El paralelismo entre el planteamiento para sistemas electrónicos y para problemas electromagnéticos se ilustra en la figura 2.2, donde $E I$ es el espectro del campo incidente, $E D$ es el espectro de campo dispersado y $D$ es la función de transferencia que caracteriza al objeto dispersor.

\section{SISTEMAS ELECTRÓNICOS}

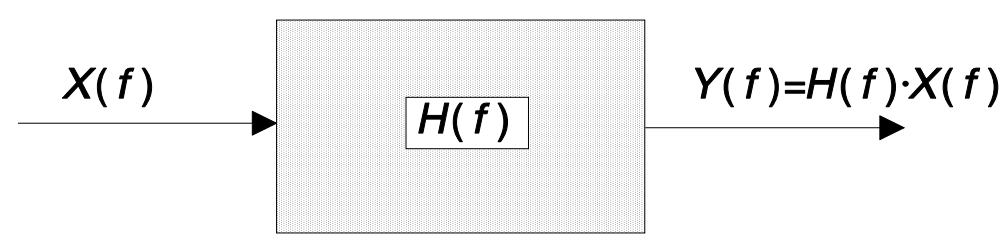

\section{PROBLEMAS ELECTROMAGNÉTICOS}

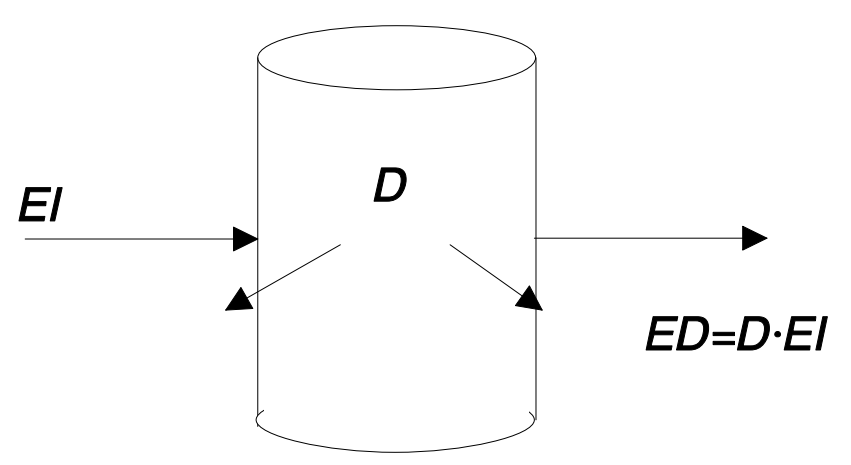

Figura 2.2: Función de transferencia en sistemas electrónicos y en problemas electromagnéticos

La función de transferencia en problemas electromagnéticos es en realidad una matriz o un operador que permite obtener el espectro del campo dispersado por el objeto en cuestión a partir del espectro del campo incidente. Por ello también se denominará matriz de caracterización. La ventaja de este procedimiento es que una vez construida la función de transferencia o matriz de caracterización de un objeto se puede prescindir de su geometría ya que su respuesta ante distintas incidencias puede ser rápidamente calculada como el producto matricial de la función de transferencia por el espectro de cada una de las ondas incidentes. Por tanto, para distintos espectros incidentes resulta inmediato el conocimiento de los cor- 
respondientes espectros dispersados una vez se ha obtenido la función de transferencia que caracteriza al objeto en cuestión.

Esta ventaja permite además analizar el acoplamiento electromagnético que se produce entre varios dispersores cuando sobre ellos incide un cierto campo eléctrico. De esta manera se puede estudiar el efecto de las interacciones que se producen entre diversos objetos sin necesidad de recurrir a la geometría de los mismos.

Dado que se pretende analizar problemas de dispersión bidimensionales, la geometría de los objetos que se va a caracterizar es invariante en una dimensión. En este caso resulta ventajoso utilizar el sistema de coordenadas cilíndricas y espectros de ondas cilíndricas, de modo que la geometría sea invariante a lo largo del eje $z$. Ello reduce considerablemente el problema, pues los espectros de campo son unidimensionales, y la función de transferencia es una matriz bidimensional. Para el caso de objetos cuya geometría no sea invariante en ninguna dimensión la función de transferencia puede obtenerse de forma similar al caso bidimensional, utilizando coordenadas esféricas en lugar de cilíndricas, y expresando los campos en función de sus espectros esféricos.

Para un análisis más sencillo de un objeto dispersor, el campo de excitación o incidencia se separa en dos polarizaciones: la $T M^{z}$ y la $T E^{z}$. En el caso $T M^{z}$ el campo eléctrico incidente está polarizado según $\hat{z}$. Este campo incidente produce sólo campo eléctrico dispersado según $\hat{z}$, debido a la invarianza de la geometría, y la relación entre espectros de campo eléctrico dispersado e incidente queda determinada mediante la función de transferencia TM del dispersor. El caso complementario es aquél donde la incidencia es $T E^{z}$, en el que el campo eléctrico incidente está contenido en el plano definido por los vectores unitarios $\hat{x} \mathrm{e}$ $\hat{y}$ (o plano $X Y$ ), y por tanto el campo magnético incidente está polarizado según $\hat{z}$, al igual que el campo magnético dispersado. La función de transferencia TE relaciona en este caso los espectros de campo magnético dispersado e incidente.

La caracterización de un dispersor se divide en dos fases. En un primer paso se calcula la matriz de corriente. Esta matriz relaciona la densidad de corriente inducida sobre la superficie o en el volumen del dispersor con el espectro de la señal incidente. Una vez construida la matriz de corriente, se construye en un segundo paso la matriz de espectro. Esta matriz relaciona el espectro de campo dispersado con la densidad de corriente. La función de transferencia, que relaciona espectro de campo dispersado con espectro de campo incidente, se obtiene por tanto multiplicando la matriz de corriente por la matriz de espectro.

El espacio se divide en tres regiones a efectos de cálculo del campo dispersado, como se muestra en la figura 2.3. El campo dispersado se puede reconstruir a partir de su espectro cilíndrico tan sólo en la zona libre de fuentes, es decir, en la zona externa a la circunferencia que contiene al objeto (región $R 3$ ). En la zona interna a dicha circunferencia pero externa al objeto (región $R 2$ ), el campo dispersado se obtiene por integración del campo dispersado por la corriente inducida sobre la superficie o en el volumen de dicho dispersor. En la región interna al objeto (región $R 1$ ), el campo será nulo si se trata de un objeto perfectamente conductor, y en caso contrario el campo se obtendrá a partir de la corriente inducida al igual que en la región $R 2$. Para obtener el espectro del campo dispersado se multiplicará la función de transferencia por el espectro de campo incidente, y para obtener la corriente inducida sobre el objeto se multiplicará la matriz de corriente por el espectro de campo incidente. 


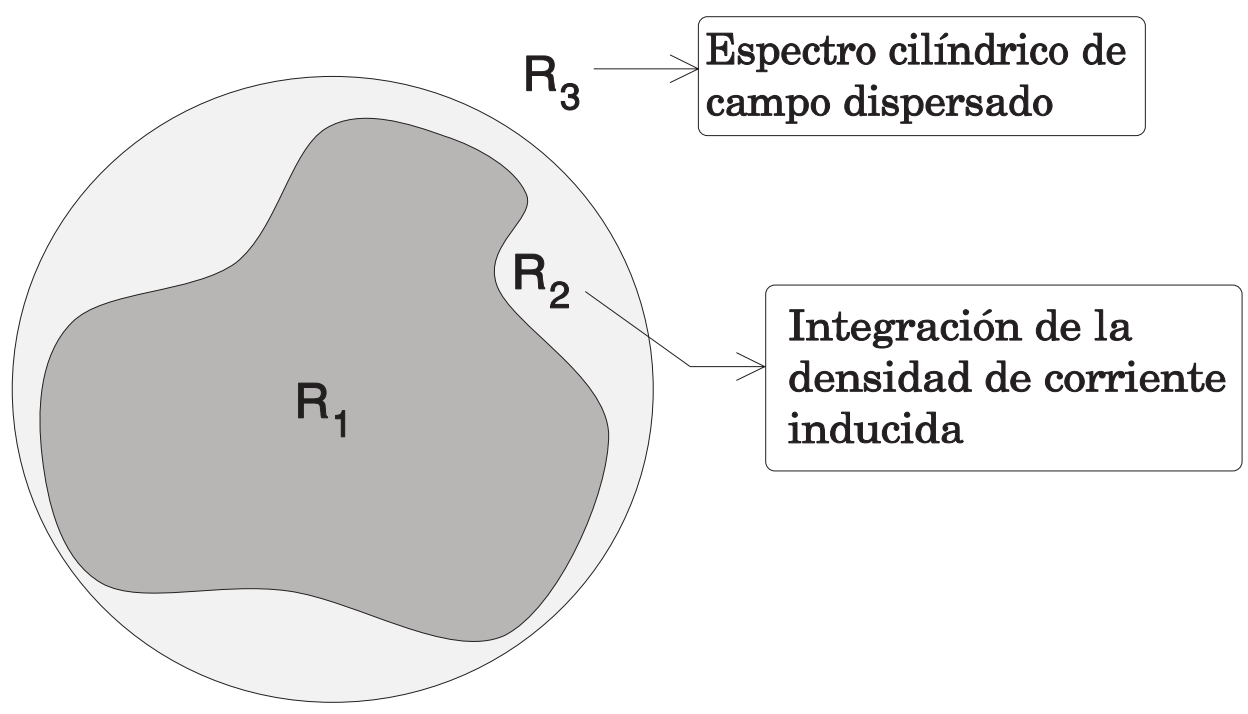

Figura 2.3: Regiones en el cálculo del campo dispersado

\subsubsection{Funciones de transferencia TM y TE}

Para caracterizar el comportamiento electromagnético de un objeto dispersor, se separa la onda de excitación en las polarizaciones $T M^{z}$ y $T E^{z}$, como ya se ha explicado anteriormente. La respuesta del objeto a cada una de estas polarizaciones la proporcionan las funciones de transferencia TM y TE, respectivamente.

El sistema de referencia que se utilizará será un sistema de coordenadas cilíndricas $(\rho, \phi)$, y el objeto dispersor se alineará a lo largo del eje $Z$, como se aprecia en la figura 2.4. Los dispersores que se van a caracterizar serán infinitos en una dimensión, como ya se ha dicho, siendo su geometría invariante en el eje $Z$. Por lo tanto el problema que se analiza será invariante en la coordenada $\hat{z}$, bidimensional, y se utilizarán tan sólo las coordenadas $\rho$ y $\phi$. Este sistema de coordenadas $(\rho, \phi)$ es local al objeto, y por tanto su origen se sitúa en algún punto interior al mismo.

Los campos incidentes $T M^{z}\left(E_{z}^{i}\right)$ y $T E^{z}\left(H_{z}^{i}\right)$ se expresarán como sumas de modos cilíndricos con valor finito en el origen (funciones de Bessel), de la forma:

$$
\begin{aligned}
& E_{z}^{i}=\sum_{p=-N_{i}}^{N_{i}} i_{p}^{\mathrm{TM}} J_{p}(k \rho) e^{j p \phi} \\
& H_{z}^{i}=\sum_{p=-N_{i}}^{N_{i}} i_{p}^{\mathrm{TE}} J_{p}(k \rho) e^{j p \phi}
\end{aligned}
$$

donde $p$ indica el orden del modo cilíndrico incidente $J_{p}(k \rho) e^{j p \phi}$ y los coeficientes $i_{p}^{\mathrm{TM}}$ e $i_{p}^{\mathrm{TE}}$ son, respectivamente, los espectros cilíndricos $T M$ y $T E$ de campo incidente. Los 


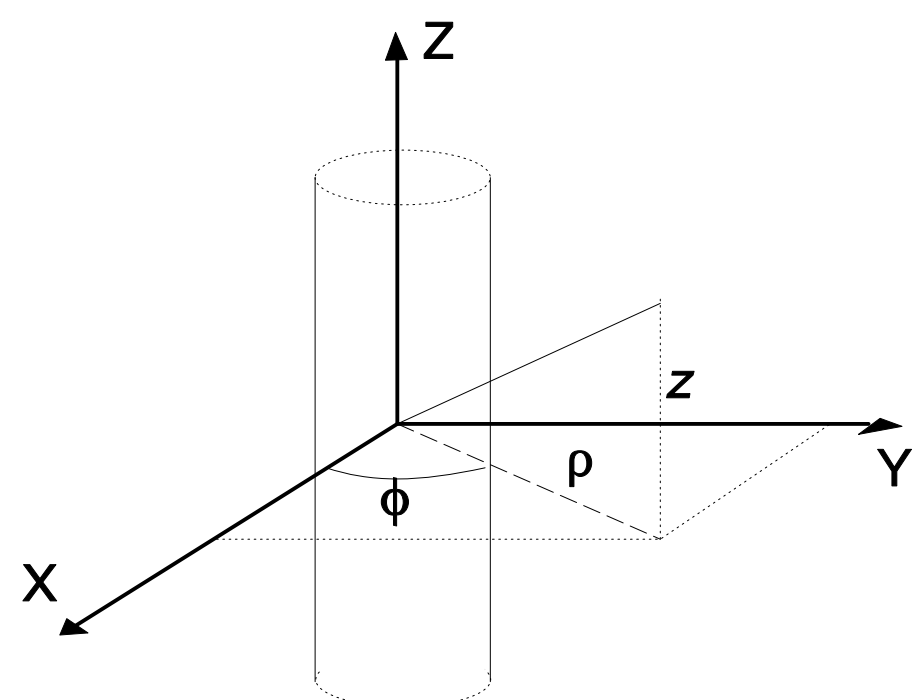

Figura 2.4: Sistema de coordenadas utilizado en la caracterización de dispersores bidimensionales

campos incidentes se deberían expresan como sumas infinitas de modos incidentes, pero a efectos prácticos estas series se truncan pues tan sólo es necesario considerar el número de modos suficiente para reconstruir de forma satisfactoria el campo en toda la superficie del objeto. Este número de modos depende principalmente de la frecuencia espacial del campo que queramos expresar como suma de modos cilíndricos, y de la región del espacio donde queramos que se reconstruya correctamente el campo. Cuanto mayor sea la frecuencia espacial, y mayor la región del espacio donde queramos reconstruir el campo, mayor será el número de modos.

Los campos incidentes se caracterizarán a efectos de cálculo por medio de vectores columna de $2 N_{i}+1$ elementos que contengan los coeficientes $i_{p}^{\mathrm{TM}}$ e $i_{p}^{\mathrm{TE}}$ de sus espectros correspondientes, presentando el siguiente aspecto:

$$
E I^{\mathrm{TM}}=\left[\begin{array}{c}
i_{-N_{i}}^{\mathrm{TM}} \\
i_{-N_{i}+1}^{\mathrm{TM}} \\
\vdots \\
i_{N_{i}-1}^{\mathrm{TM}} \\
i_{N_{i}}^{\mathrm{TM}}
\end{array}\right]_{2 N_{i}+1 \times 1}, E I^{\mathrm{TE}}=\left[\begin{array}{c}
i_{-N_{i}}^{\mathrm{TE}} \\
i_{-N_{i}+1}^{\mathrm{TE}} \\
\vdots \\
i_{N_{i}-1}^{\mathrm{TE}} \\
i_{N_{i}}^{\mathrm{TE}}
\end{array}\right]_{2 N_{i}+1 \times 1}
$$

Los campos $T M^{z}$ y $T E^{z}$ que disperse el objeto se expresarán como sumas de modos cilíndricos emergentes. Por lo tanto aparecerán funciones de Hankel de segunda especie $H_{n}^{(2)}(k \rho)$, singulares en el origen, y que se propagan hacia el infinito, dando lugar a las siguientes expresiones para los campos dispersados: 


$$
\begin{aligned}
& E_{z}^{d}=\sum_{q=-N_{d}}^{N_{d}} c_{q}^{\mathrm{TM}} H_{q}^{(2)}(k \rho) e^{j q \phi} \\
& H_{z}^{d}=\sum_{q=-N_{d}}^{N_{d}} c_{q}^{\mathrm{TE}} H_{q}^{(2)}(k \rho) e^{j q \phi}
\end{aligned}
$$

donde los coeficientes $c_{q}^{\mathrm{TM}}$ y $c_{q}^{\mathrm{TE}}$ definen respectivamente los espectros de las ondas dispersadas, mientras que $N_{d}$ es el orden del modo superior a partir de cuál se trunca la serie. Según el criterio enunciado en [4], [29] y [6], $N_{d}$ debe cumplir que:

$$
N_{d}>k a
$$

donde $k$ es el número de onda $k=2 \pi / \lambda$, y $a$ es la distancia desde el origen del sistema local de coordenadas hasta el punto de la superficie del objeto más lejano. Este criterio nos puede orientar a la hora de seleccionar el valor de $N_{i}$, el orden del máximo modo incidente, pues en la práctica $N_{i}$ debe ser también mayor que $k a$, pero no se conocen estudios concluyentes en cuanto al valor concreto que debe tomar $N_{i}$. En la práctica se ha experimentado que si $N_{i}>k R$ se consigue reconstruir correctamente una onda plana en un círculo de radio $R$ en torno al origen. No obstante, si el patrón de campo que se pretende reconstruir es más complejo (mayor variación espacial) que el de una onda plana, es necesario utilizar un mayor número de modos (en el apéndice $\mathrm{C}$ se hace un estudio exhaustivo sobre el número de modos cilíndricos incidentes necesarios para una aplicación concreta: el acoplo de modos cilíndricos y modos guiados de una guía rectangular).

Conviene pues eligir el centro del sistema local de coordenadas de forma que $a$, la distancia desde el origen al punto más lejano de la superficie del objeto, sea mínima. De esta forma se minimizarán $N_{i}$ y $N_{d}$, y por tanto serán necesarios menos modos para reconstruir los campos incidente y dispersado, y las funciones de transferencia tendrán un tamaño menor, con lo que se asegura no estar realizando más cálculos de los necesarios ni estar haciendo a su vez más lento el método.

De igual forma que para los espectros de los campos incidentes, los espectros de los campos dispersados se expresarán mediante vectores columna de $2 N_{d}+1$ elementos de la forma:

$$
E D^{\mathrm{TM}}=\left[\begin{array}{c}
c_{-N_{d}}^{\mathrm{TM}} \\
c_{-N_{d}+1}^{\mathrm{TM}} \\
\vdots \\
c_{N_{d}-1}^{\mathrm{TM}} \\
c_{N_{d}}^{\mathrm{TM}}
\end{array}\right]_{2 N_{d}+1 \times 1}, E D^{\mathrm{TE}}=\left[\begin{array}{c}
c_{-N_{d}}^{\mathrm{TE}} \\
c_{-N_{d}+1}^{\mathrm{TE}} \\
\vdots \\
c_{N_{d}-1}^{\mathrm{TE}} \\
c_{N_{d}}^{\mathrm{TE}}
\end{array}\right]_{2 N_{d}+1 \times 1}
$$


Para caracterizar al objeto dispersor se calculará la respuesta del objeto a cada modo incidente $J_{p}(k \rho) e^{j p \phi}$ posible tanto para el caso $T M^{z}$ como para el caso $T E^{z}$. Esta respuesta se calculará utilizando algún método numérico para geometrías arbitrarias, y se puede obtener de forma analítica para objetos con geometría canónica en coordenadas cilíndricas (cilindro metálico o dieléctrico). La respuesta del dispersor a cada modo $J_{p}(k \rho) e^{j p \phi} T M^{z}$ y $T E^{z}$ vendrá definida mediante espectros emergentes de la forma:

$$
\begin{aligned}
& E_{z}^{i}=J_{p}(k \rho) e^{j p \phi} \Longrightarrow E_{z}^{d}=\sum_{q=-N_{d}}^{N_{d}} d_{q p}^{\mathrm{TM}} H_{q}^{(2)}(k \rho) e^{j q \phi} \\
& H_{z}^{i}=J_{p}(k \rho) e^{j p \phi} \Longrightarrow H_{z}^{d}=\sum_{q=-N_{d}}^{N_{d}} d_{q p}^{\mathrm{TE}} H_{q}^{(2)}(k \rho) e^{j q \phi}
\end{aligned}
$$

Los coeficientes $d_{q p}^{\mathrm{TM}}$ y $d_{q p}^{\mathrm{TE}}$ indican cómo contribuye el modo $p$-ésimo del espectro incidente $T M^{z}$ o $T E^{z}$ al modo $q$-ésimo del espectro dispersado $T M^{z}$ o $T E^{z}$.

La respuesta global del objeto ante una incidencia definida por los coeficientes espectrales $i_{p}^{\mathrm{TM}} \mathrm{o} i_{p}^{\mathrm{TE}}$, puede expresarse en función de los coeficientes $d_{q p}^{\mathrm{TM}} \mathrm{o} d_{q p}^{\mathrm{TE}}$ de la forma:

$$
\begin{aligned}
E_{z}^{i} & =\sum_{p=-N_{i}}^{N_{i}} i_{p}^{\mathrm{TM}} J_{p}(k \rho) e^{j p \phi} \\
& \Longrightarrow E_{z}^{d}=\sum_{p=-N_{i}}^{N_{i}} i_{p}^{\mathrm{TM}}\left(\sum_{q=-N_{d}}^{N_{d}} d_{q p}^{\mathrm{TM}} H_{q}^{(2)}(k \rho) e^{j q \phi}\right) \\
H_{z}^{i} & =\sum_{p=-N_{i}}^{N_{i}} i_{p}^{\mathrm{TE}} J_{p}(k \rho) e^{j p \phi} \\
& \Longrightarrow H_{z}^{d}=\sum_{p=-N_{i}}^{N_{i}} i_{p}^{\mathrm{TE}}\left(\sum_{q=-N_{d}}^{N_{d}} d_{q p}^{\mathrm{TE}} H_{q}^{(2)}(k \rho) e^{j q \phi}\right)
\end{aligned}
$$

Cambiando los sumatorios de orden se obtiene la expresión definitiva para los campos dispersados por el objeto:

$$
\begin{aligned}
E_{z}^{d} & =\sum_{q=-N_{d}}^{N_{d}}\left(\sum_{p=-N_{i}}^{N_{i}} i_{p}^{\mathrm{TM}} d_{q p}^{\mathrm{TM}}\right) H_{q}^{(2)}(k \rho) e^{j q \phi} \\
H_{z}^{d} & =\sum_{q=-N_{d}}^{N_{d}}\left(\sum_{p=-N_{i}}^{N_{i}} i_{p}^{\mathrm{TE}} d_{q p}^{\mathrm{TE}}\right) H_{q}^{(2)}(k \rho) e^{j q \phi}
\end{aligned}
$$


Comparando con (2.4) se pueden expresar los coeficientes de los espectros dispersados como:

$$
\begin{gathered}
c_{q}^{\mathrm{TM}}=\sum_{p=-N_{i}}^{N_{i}} i_{p}^{\mathrm{TM}} d_{q p}^{\mathrm{TM}} \\
c_{q}^{\mathrm{TE}}=\sum_{p=-N_{i}}^{N_{i}} i_{p}^{\mathrm{TE}} d_{q p}^{\mathrm{TE}}
\end{gathered}
$$

Estas relaciones entre espectros de campo dispersado e incidente a partir del conocimiento de los coeficientes $d_{q p}^{\mathrm{TM}}$ y $d_{q p}^{\mathrm{TE}}$ pueden expresarse de forma matricial mediante las siguientes relaciones:

$$
\begin{aligned}
& E D^{\mathrm{TM}}=D^{\mathrm{TM}} \cdot E I^{\mathrm{TM}} \\
& E D^{\mathrm{TE}}=D^{\mathrm{TE}} \cdot E I^{\mathrm{TE}}
\end{aligned}
$$

donde $E D^{\mathrm{TM}}, E D^{\mathrm{TE}}$ y $E I^{\mathrm{TM}}, E I^{\mathrm{TE}}$ son, respectivamente, los vectores columna de los espectros $T M^{z}$ y $T E^{z}$ de campo dispersado e incidente descritas en (2.3) y (2.6), y $D^{\mathrm{TM}}$, $D^{\mathrm{TE}}$ son las funciones de transferencia o matrices de caracterización TM y TE del objeto. Estas matrices se construyen a partir de los coeficientes $d_{q p}^{\mathrm{TM}}$ y $d_{q p}^{\mathrm{TE}}$ de la siguiente forma:

$$
\begin{aligned}
D^{\mathrm{TM}}= & {\left[\begin{array}{cccc}
d_{-N_{d}-N_{i}}^{\mathrm{TM}} & d_{-N_{d}-N_{i}+1}^{\mathrm{TM}} & \cdots & d_{-N_{d} N_{i}}^{\mathrm{TM}} \\
d_{-N_{d}+1-N_{i}}^{\mathrm{TM}} & d_{-N_{d}+1-N_{i}+1}^{\mathrm{TM}} & \cdots & d_{-N_{d}+1 N_{i}}^{\mathrm{TM}} \\
\vdots & \vdots & \ddots & \vdots \\
d_{N_{d}-N_{i}}^{\mathrm{TM}} & d_{N_{d}-N_{i}+1}^{\mathrm{TM}} & \cdots & d_{N_{d} N_{i}}^{\mathrm{TM}}
\end{array}\right]_{2 N_{d}+1 \times 2 N_{i}+1} } \\
D^{\mathrm{TE}}= & {\left[\begin{array}{cccc}
d_{-N_{d}-N_{i}}^{\mathrm{TE}} & d_{-N_{d}-N_{i}+1}^{\mathrm{TE}} & \cdots & d_{-N_{d} N_{i}}^{\mathrm{TE}} \\
d_{-N_{d}+1-N_{i}}^{\mathrm{TE}} & d_{-N_{d}+1-N_{i}+1}^{\mathrm{TE}} & \cdots & d_{-N_{d}+1 N_{i}}^{\mathrm{TE}} \\
\vdots & \vdots & \ddots & \vdots \\
d_{N_{d}-N_{i}}^{\mathrm{TE}} & d_{N_{d}-N_{i}+1}^{\mathrm{TE}} & \cdots & d_{N_{d} N_{i}}^{\mathrm{TE}}
\end{array}\right]_{2 N_{d}+1 \times 2 N_{i}+1} }
\end{aligned}
$$

Cada columna de la matriz $D^{\mathrm{TM}}$ o $D^{\mathrm{TE}}$ es la respuesta del objeto a un modo incidente espectral distinto. Y por lo tanto el espectro dispersado será la suma de las respuestas a cada modo ponderadas por los coeficientes $i_{p}^{\mathrm{TM}} \mathrm{o} i_{p}^{\mathrm{TE}}$ del espectro incidente, lo que se consigue multiplicando matricialmente la función de transferencia TM o TE por el correspondiente espectro incidente $E I^{\mathrm{TM}}$ o $E I^{\mathrm{TE}}$. 
Una vez obtenidas las funciones de transferencia, se puede calcular inmediatamente la respuesta del objeto a cualquier onda incidente, sin necesidad de conocer cómo es realmente el objeto, lo que da idea de lo ventajoso que resulta esta técnica.

La dimensión de la matriz TM o TE de caracterización del dispersor aumenta a medida que las dimensiones eléctricas del objeto crecen, y por lo tanto cuando éste sea muy grande será conveniente fraccionar dicho objeto en varios elementos más pequeños y aplicar técnicas de realimentación múltiple o acoplo electromagnético.

En el caso de objetos dispersores con geometría canónica en coordenadas cilíndricas es posible obtener la función de transferencia y la matriz de corriente de forma analítica. Este es el caso del cilindro metálico infinito [4] y del cilindro dieléctrico multicapa infinito [17]. En el caso de objetos con geometría no canónica se debe utilizar algún método numérico, como el Método de los Momentos [30].

\subsubsection{Matriz de corriente}

En el cálculo de la función de transferencia se pueden distinguir dos pasos. En el primero se construye la matriz de corriente, que relaciona la distribución de corrientes inducidas en el dispersor con el espectro de campo incidente. En un segundo paso se construye la matriz de espectro que relaciona el espectro del campo dispersado con la distribución de corriente, de forma que la función de transferencia se obtiene como el producto de la matriz de espectro por la matriz de corrientes.

En el caso de objetos con geometría no canónica, para construir la matriz de corriente se seguirán los siguientes pasos:

1. Cálculo de la corriente $J\left(\overrightarrow{\rho^{\prime}}\right)$ inducida en el objeto como respuesta al $p$-ésimo modo incidente $E_{z}^{i}=J_{p}(k \rho) e^{j p \phi}$. Esta corriente inducida se puede calcular utilizando algún método numérico como el método de los momentos.

2. Discretización de la distribución de corriente. En el método de los momentos la corriente se expande como suma de $N_{s}$ funciones base, de la forma:

$$
J\left(\overrightarrow{\rho^{\prime}}\right)=\sum_{n=1}^{N_{s}} I_{n} f_{n}\left(\overrightarrow{\rho^{\prime}}\right)
$$

de modo que basta con conocer los pesos $I_{n}$ de dicha serie. Llamaremos $\left[I_{n p}\right]$ al vector que recoge los pesos de la mencionada serie ante la incidencia del modo $p$-ésimo. Cada elemento $I_{n p}$ de dicho vector es la amplitud de la $n$-ésima función base de corriente como respuesta al $p$-ésimo modo incidente. El vector $\left[I_{n p}\right]$ se almacena como la columna $p$-ésima de la matriz de corriente.

3. Este proceso se repite para cada modo incidente, es decir, variando $p$ desde $-N_{i}$ hasta $N_{i}$, hasta completar la matriz de corriente.

El tamaño de la matriz de corriente será de $N_{s} \times 2 N_{i}+1$. Cada elemento de la matriz de corrientes $I_{n p}$ indicará la contribución del $p$-ésimo modo incidente a la amplitud de la 
$n$-ésima función de corriente sobre el objeto, de modo que la relación entre la distribución de corriente inducida en el objeto y el espectro de campo incidente es:

$$
C O=D I \cdot E I
$$

donde $C O$ es un vector columna que contiene las amplitudes de corriente, $E I$ es el espectro de campo incidente, y $D I$ es la matriz de corrientes, de la forma:

$$
D I=\left[\begin{array}{cccc}
I_{1-N_{i}} & I_{1-N_{i}+1} & \cdots & I_{1 N_{i}} \\
I_{2-N_{i}} & I_{2-N_{i}+1} & \cdots & I_{2 N_{i}} \\
\vdots & \vdots & \ddots & \vdots \\
I_{N_{s}-N_{i}} & I_{N_{s}-N_{i}+1} & \cdots & I_{N_{s} N_{i}}
\end{array}\right]_{N_{s} \times 2 N_{i}+1}
$$

\subsubsection{Matriz de espectro}

La matriz de espectro $D E$ permitirá obtener el espectro dispersado $E D$ a partir de la corriente inducida $C O$ como:

$$
E D=D E \cdot C O
$$

Para construir la matriz de espectro se seguirán los siguientes pasos:

1. Cálculo del espectro de campo eléctrico dispersado por el $n$-ésimo elemento de corriente $f_{n}$ sobre el dispersor de amplitud unidad. A los coeficientes del mencionado espectro se les denominará $S_{q n}$, de modo que el campo dispersado por el $n$-ésimo elemento de corriente será:

$$
E_{n}^{s}=\sum_{q=-N_{d}}^{N_{d}} S_{q n} H_{q}^{(2)}(k \rho) e^{j q \phi}
$$

Cada coeficiente $S_{q n}$ indica la contribución del $n$-ésimo elemento de corriente al $q$ ésimo modo del espectro de campo dispersado. Estos coeficientes pueden obtenerse utilizando la Transformada de Fourier o el Teorema de Adición de las funciones de Bessel (ver [31]).

2. Los coeficientes $S_{q n}$ con $q$ variando desde $-N_{d}$ a $N_{d}$ se almacenan en la $n$-ésima columna de la matriz de espectro.

3. Este proceso se repite para cada elemento de corriente, es decir, variando $n$ desde 1 hasta $N_{s}$, hasta completar la matriz de espectro.

El tamaño de la matriz de espectro será de $2 N_{d}+1 \times N_{s}$.

Como ya se ha indicado, la relación entre el espectro de campo dispersado y la distribución de corrientes inducidas en la superficie del objeto es:

$$
E D=D E \cdot C O
$$


donde $E D$ es el espectro de campo dispersado, $C O$ es un vector columna que contiene las amplitudes de los $N_{s}$ elementos de corriente, y $D E$ es la matriz de espectro, de la forma:

$$
D E=\left[\begin{array}{cccc}
S_{-N_{d} 1} & S_{-N_{d} 2} & \cdots & S_{-N_{d} N_{s}} \\
S_{-N_{d}+11} & S_{-N_{d}+12} & \cdots & S_{-N_{d}+1 N_{s}} \\
\vdots & \vdots & \ddots & \vdots \\
S_{N_{d} 1} & S_{N_{d} 2} & \cdots & S_{N_{d} N_{s}}
\end{array}\right]_{2 N_{d}+1 \times N_{s}}
$$

La relación entre espectro dispersado e incidente será el producto de la matriz de espectro por la matriz de corriente:

$$
E D=D E \cdot C O=D E \cdot D I \cdot E I=D \cdot E I
$$

Por lo tanto la función de transferencia $D$ será el producto de la matriz de espectro $D E$ por la matriz de corriente $D I$.

$$
D=D E \cdot D I
$$

\subsubsection{Objetos dispersores analizados}

Para las aplicaciones que se han desarrollado en el presente trabajo únicamente ha sido necesario considerar la polarización $T M^{z}$, de modo que tan sólo se han calculado funciones de transferencia TM.

Al comenzar la tesis ya se disponía de los programas informáticos necesarios para obtener la función de transferencia TM de los siguientes objetos [7, 31]:

Cilindro metálico infinito La función de transferencia de este objeto se puede obtener de forma analítica, forzando la condición de contorno del campo eléctrico en la superficie del cilindro (campo total en la superficie metálica nulo) [4]:

$$
d_{q p}^{T M}=\left\{\begin{array}{lll}
-\frac{J_{p}(k a)}{H_{p}^{(2)}(k a)} & \text { si } & q=p \\
0 & \text { si } & q \neq p
\end{array}\right.
$$

donde $a$ es el radio del cilindro. En la figura 2.5.a se muestra la amplitud de campo eléctrico alrededor de un cilindro de radio $0,5 \lambda$ ante la incidencia de una onda plana.

Tira metálica infinita Como se trata de un objeto con geometría no canónica, su función de transferencia TM se obtiene utilizando un método numérico, concretamente el Método de los Momentos, con funciones base pulso e impulsos como funciones de test (Point Matching) (ver detalles en [31]). En la figura 2.5.b se muestra la amplitud de campo eléctrico alrededor de una tira de longitud $\lambda$ ante la incidencia de una onda plana. 


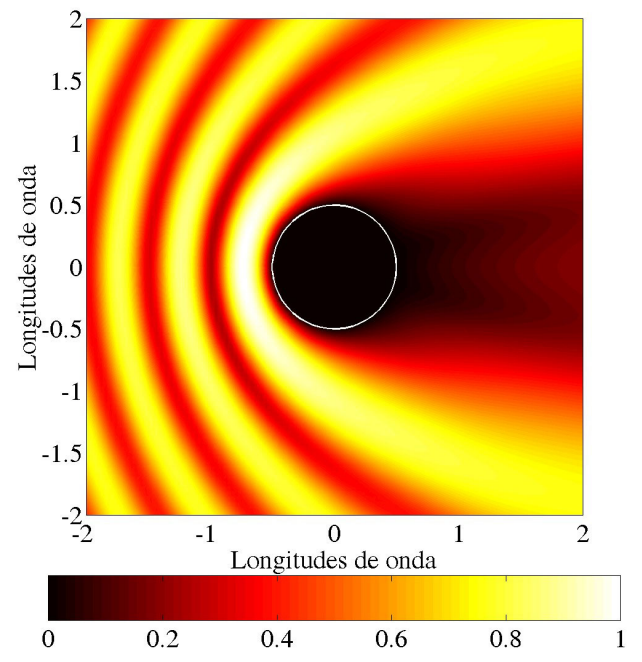

(a) Cilindro metálico. Radio $0,5 \lambda$

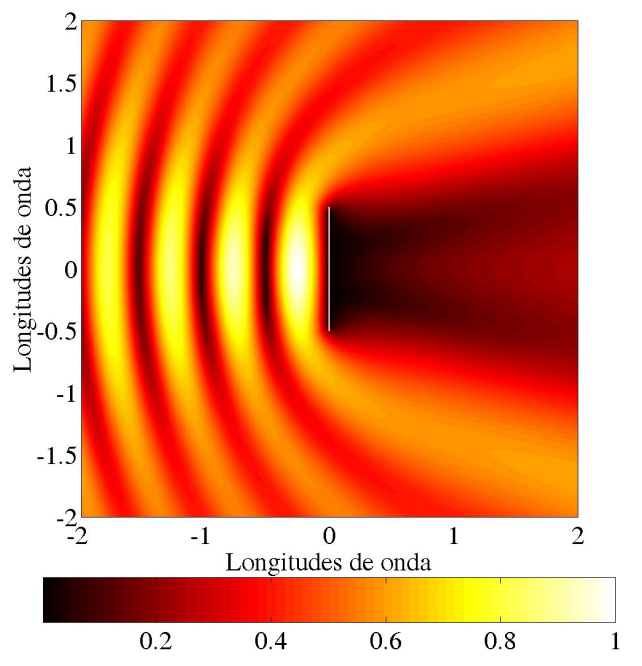

(b) Tira metálica. Longitud $\lambda$

Figura 2.5: Amplitud de campo eléctrico ante la incidencia de una onda plana

Como primer trabajo de tesis, y con el fin de poder analizar una mayor variedad de problemas de dispersión, se implementaron los programas necesarios para calcular las funciones de transferencia TM de los siguientes objetos:

Cilindro dieléctrico y/o magnético homogéneo infinito $\mathrm{Al}$ igual que con el cilindro metálico, la solución en este caso es analítica. Para resolver este problema es preciso forzar la continuidad de campo eléctrico y magnético en la superficie del cilindro, suponiendo que en el interior del mismo existe un campo $E_{z}^{c}$ que podemos expresar como suma de modos cilíndricos finitos en el origen de coordenadas:

$$
E_{z}^{c}=\sum_{n=-N_{c}}^{N_{c}} s_{n}^{T M} J_{n}\left(k_{c} \rho\right) e^{j n \phi}
$$

donde $k_{c}$ y $s_{n}^{T M}$ son respectivamente el número de onda y el espectro cilíndrico de campo $T M^{z}$ dentro del cilindro.

Tras forzar continuidad de campos eléctrico y magnético se obtiene que los elementos 
de la función de transferencia TM son:

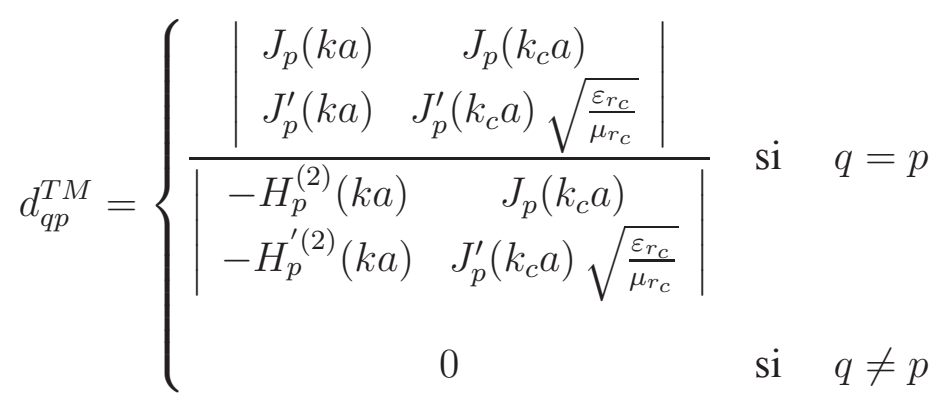

donde $a$ es el radio del cilindro, y $\varepsilon_{r_{c}}$ y $\mu_{r_{c}}$ son la permitividad y permeabilidad relativas dentro del cilindro.

Por otro lado, el espectro de campo $T M^{z}$ dentro del cilindro se relaciona con el espectro de campo incidente según la siguiente relación:

$$
s_{n}^{T M}=\frac{\left|\begin{array}{cc}
-H_{p}^{(2)}(k a) & J_{p}\left(k_{c} a\right) \\
-H_{p}^{\prime(2)}(k a) & J_{p}^{\prime}\left(k_{c} a\right) \sqrt{\frac{\varepsilon_{r_{c}}}{\mu_{r_{c}}}}
\end{array}\right|}{\left|\begin{array}{lc}
-H_{p}^{(2)}(k a) & J_{p}\left(k_{c} a\right) \\
-H_{p}^{\prime(2)}(k a) & J_{p}^{\prime}\left(k_{c} a\right) \sqrt{\frac{\varepsilon_{r_{c}}}{\mu_{r_{c}}}}
\end{array}\right|} i_{n}^{T M}
$$

Cilindro dieléctrico y/o magnético multicapa infinito En el caso de cilindros formados por varias capas de diversos materiales cilíndricos concéntricos (por ejemplo un líquido dentro de una pipeta), la solución es también analítica, debiendo obtener el campo dentro de cada capa de forma iterativa forzando la continuidad de contorno en cada separación entre dos capas (ver [17]).

Arco circular metálico El análisis de este objeto resultará necesario más adelante para poder obtener los parámetros circuitales de un codo circular en plano $\mathrm{H}$ en una guía de ondas rectangular. Como se trata de un objeto con geometría no canónica, al igual que la tira, su función de transferencia TM también se ha obtenido utilizando el Método de los Momentos, con funciones base pulso e impulsos como funciones de test (Point Matching).

Objetos dieléctricos inhomogéneos 2-D de sección transversal arbitraria Este tipo de objetos ha sido analizado utilizando el método descrito en [18]. Al tratarse de un método numérico resulta mucho menos eficiente que el método descrito en [17], pero por contra se puede analizar con él cualquier objeto dieléctrico 2-D.

Objetos compuestos de varias tiras Se han implementado los programas necesarios para poder analizar mediante el método de los momentos objetos dispersores 2-D cuya geometría pueda ser compuesta uniendo varias tiras o cilindros metálicos, de modo que el usuario especifica la geometría del dispersor indicando el número de tiras o cilindros, 
y la posición, longitud y orientación de cada una de los sub-objetos. Posteriormente se analizan todos los objetos mediante el Método de los Momentos como si se tratara de un único objeto. Esto resulta especialmente útil para caracterizar conjuntamente tiras que se sitúan tan próximas que no es posible analizarlas como dispersores aislados y posteriormente analizar el acoplo entre las mismas, a causa de su extrema proximidad, tal y como se explicará más adelante.

En las figuras 2.6 y 2.7 se muestra el campo eléctrico alrededor de los diferentes tipos de objetos 2-D que se han analizado, ante la incidencia de una onda plana.

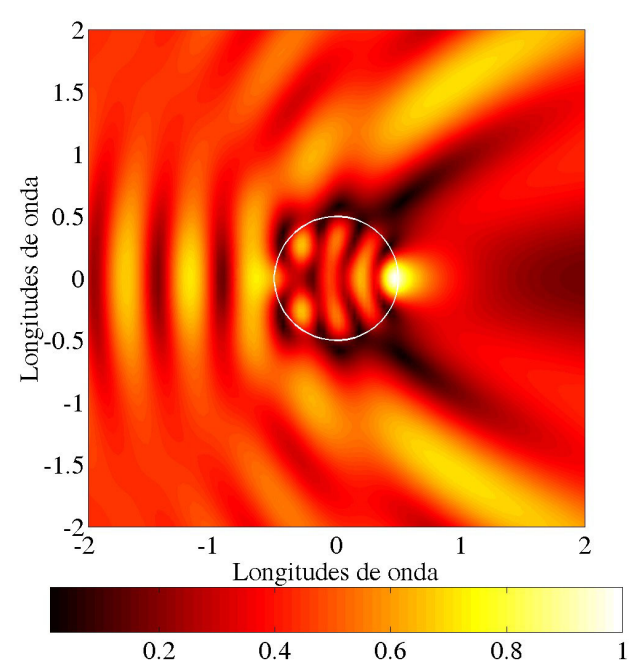

(a) Cilindro dieléctrico. Radio $0,5 \lambda, \varepsilon_{r}=4$

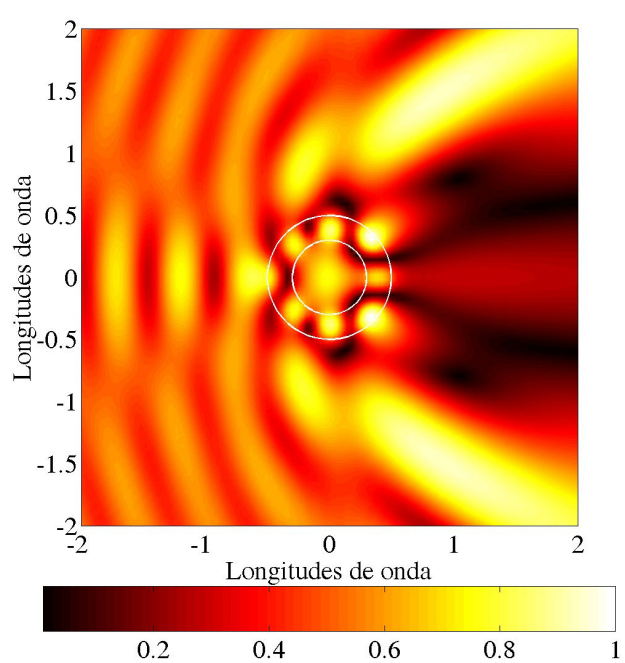

(b) Cilindro dieléctrico multicapa. Radios $0,3 \lambda$ y $0,5 \lambda$. $\varepsilon_{r}=1$ y 4

Figura 2.6: Amplitud de campo eléctrico ante la incidencia de una onda plana 


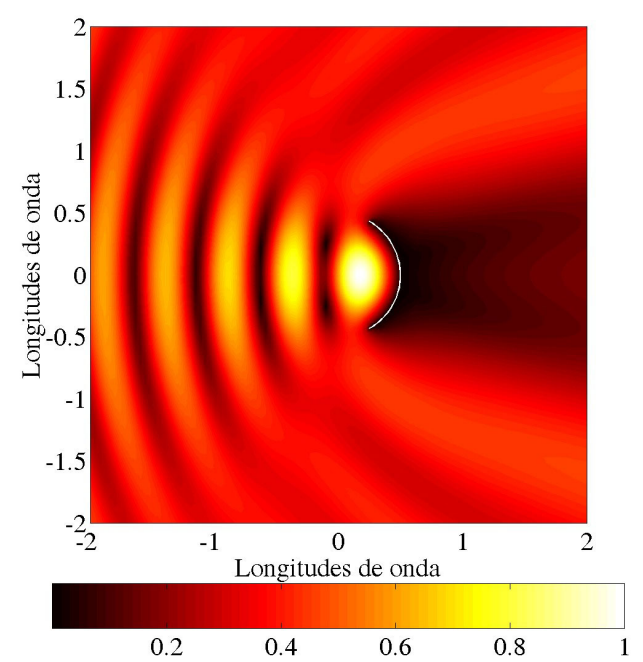

(a) Arco metálico. Radio $0,5 \lambda$

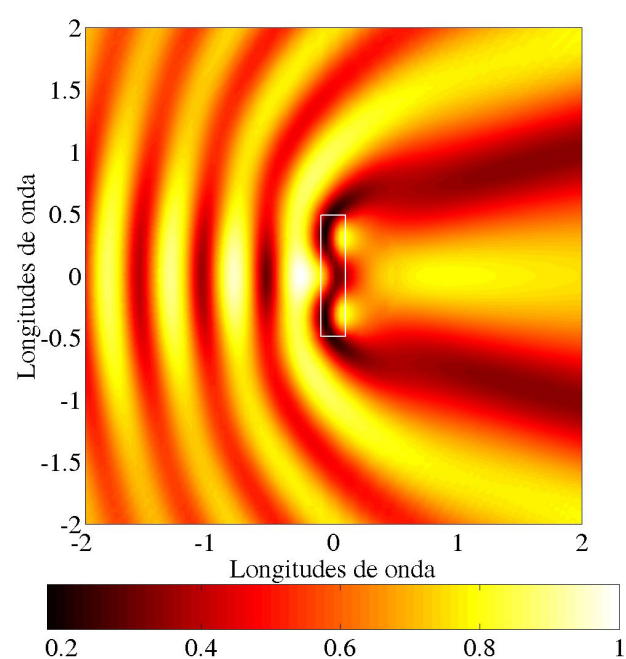

(b) Poste rectangular dieléctrico. Lados $0,2 \lambda$ y $0,98 \lambda, \varepsilon_{r}=4$

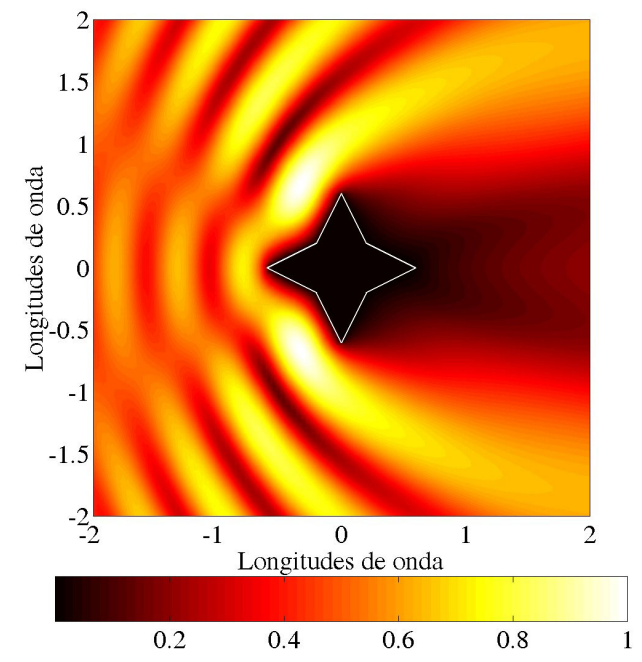

(c) Estrella compuesta por varias tiras

Figura 2.7: Amplitud de campo eléctrico ante la incidencia de una onda plana 
Además de la implementación de los programas necesarios para analizar nuevos objetos, se ha mejorado el cálculo de los elementos de la diagonal principal de la matriz de coeficientes resultante de aplicar el método de los momentos al cálculo de la corriente inducida en la superficie de un objeto metálico 2-D. Esta mejora ha supuesto una reducción del coste computacional a la cuarta parte sin pérdida de precisión en los resultados [32].

\subsection{Dispersión de múltiples objetos}

\subsubsection{Introducción}

En esta sección se analizan problemas de dispersión en los que existen múltiples objetos dispersores. En ese caso, el campo que produzca un objeto dispersor no será el mismo que cuando estaba aislado. Esto se debe a que recibirá campo procedente no sólo de la excitación exterior, sino también del resto de objetos que se hallan en su entorno, y que son a su vez excitados por la fuente externa y por el campo procedente del resto de objetos. El objetivo de esta sección será, por tanto, resolver el acoplo electromagnético que se produce entre los objetos dispersores, y obtener el campo que conjuntamente dispersan todos los objetos como respuesta a cualquier excitación externa, después de haberse producido todas las interacciones entre ellos.

Para resolver el acoplo entre múltiples objetos dispersores utilizaremos la función de transferencia de cada uno de los objetos, que como se ha visto anteriormente los caracteriza de forma individual. La simplicidad que supone caracterizar los objetos individuales mediante sus funciones de transferencia ha sido aprovechada en numerosas ocasiones para generar métodos que resuelven el acoplo entre varios dispersores [1, 6, 8]. Todos estos métodos presentan alguna limitación importante. El método propuesto en [1] tan sólo considera la primera interacción entre los objetos, resolviendo el problema de forma sencilla pero claramente incompleta y por tanto inexacta. En [6] se presenta un método que obtiene una solución completa, pero requiere para plantear el sistema de ecuaciones de la realización de unos diagramas que cada vez resultan más complejos, y por tanto inabordables conforme el número de objetos dispersores aumenta. Finalmente, el método presentado en [8] es un método que sigue el camino físico de las ondas, y va construyendo la solución de forma iterativa añadiendo a cada paso una nueva interacción entre los objetos. Las desventajas de este método son lo costoso de la comprobación de la convergencia del método (grado de cumplimiento de alguna condición de contorno en la superficie de un objeto), y la divergencia y por tanto imposibilidad de obtener solución en casos en los que los objetos dispersores se encuentran muy próximos entre sí o cuando existen a la vez objetos muy grandes y muy pequeños en el mismo problema.

En [3] se propone un método que supera las limitaciones de los métodos anteriores y resuelve el problema de dispersión múltiple de forma satisfactoria para problemas en espacio abierto. El procedimiento seguido por dicho método consiste en analizar en primer lugar el acoplo entre dos objetos, y caracterizarlos de forma conjunta mediante unas nuevas funciones de transferencia o matrices de caracterización conjuntas. En el siguiente paso los dos primeros objetos se tratan como si fueran uno sólo, y se resuelve el acoplo del conjunto for- 
mado por los dos primeros objetos con un tercer objeto. Y así, en sucesivas iteraciones (tantas como objetos), se consigue finalmente caracterizar de forma conjunta a todos los objetos mediante unas matrices de caracterización conjuntas que proporcionan el campo dispersado por los objetos ante cualquier posible incidencia.

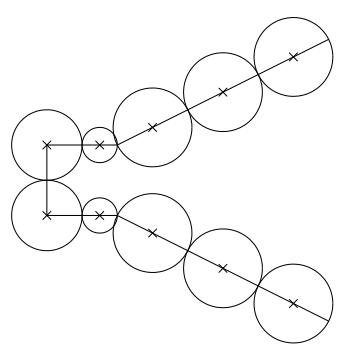

Figura 2.8: Segmentación de una bocina 2-D en un problema de dispersión múltiple

Este método se ha aplicado con éxito al análisis de problemas de dispersión múltiple tales como antenas 2-D formadas por múltiples reflectores, y en general a cualquier problema en espacio abierto aunque los objetos estén próximos entre sí o existan simultáneamente objetos muy grandes y muy pequeños. No obstante, al intentar aplicar este método al análisis de problemas en los que los objetos forman geometrías cerradas (por ejemplo al analizar una bocina 2-D como la de la figura 2.8), se descubrió que existían unas limitaciones que hacían que los resultados proporcionados por el método fueran inexactos en algunas circunstancias. En concreto, el método propuesto en [3] debe cumplir las siguientes tres normas para que la solución sea correcta al analizar la interacción entre dos grupos de objetos [33]:

1. Ningún objeto debe estar centrado dentro de la mínima circunferencia que contiene a los objetos del otro grupo (ver figura 2.9.a, objeto $C_{t}^{2}$ ). En ese caso el campo dispersado por dicho objeto no se reconstruirá correctamente en todo el contorno de todos los objetos del otro grupo.

2. Ningún objeto debe poseer parte alguna de su contorno dentro de la mínima circunferencia que contiene a ningún objeto del otro grupo (ver figura 2.9.b objetos $C_{t}^{2}$ y $C_{t}^{1}$ ). De ser así el campo dispersado por el objeto $C_{t}^{1}$ no se reconstruirá correctamente en parte de la superficie del objeto $C_{t}^{2}$.

3. El número de modos cilíndricos con los que se reconstruye el campo incidente a un grupo no debe ser excesivamente elevado. De ser así, las matrices de traslación de espectros presentarán elementos casi singulares y los sistemas matriciales que se deban resolver estarán mal condicionados. 


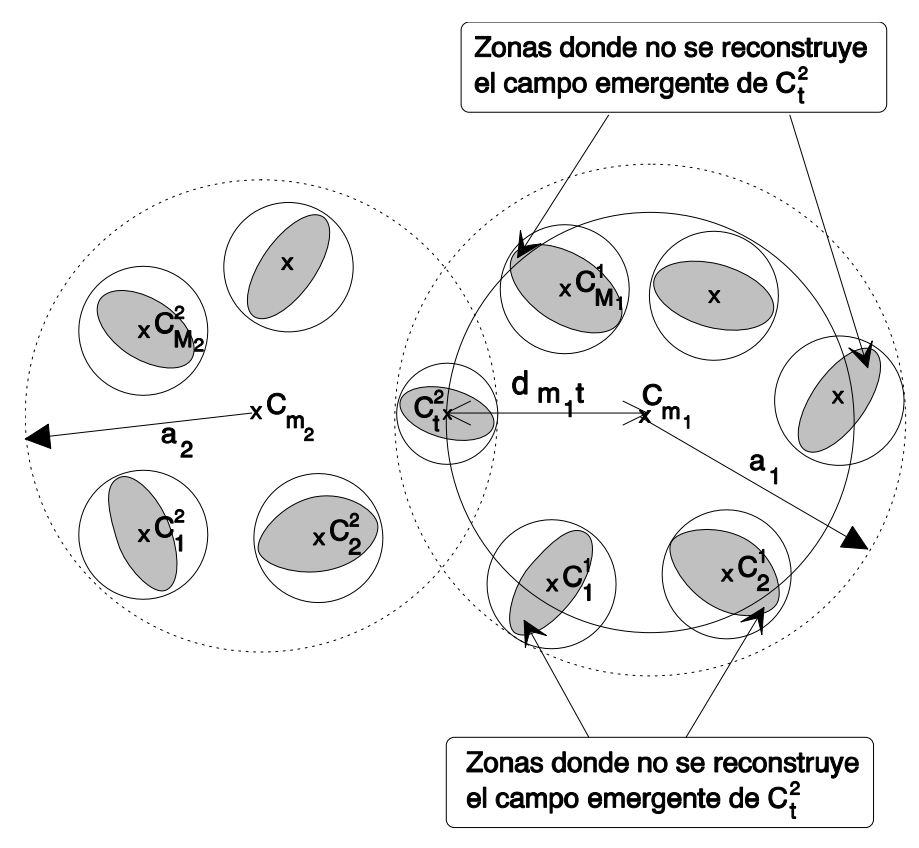

(a)

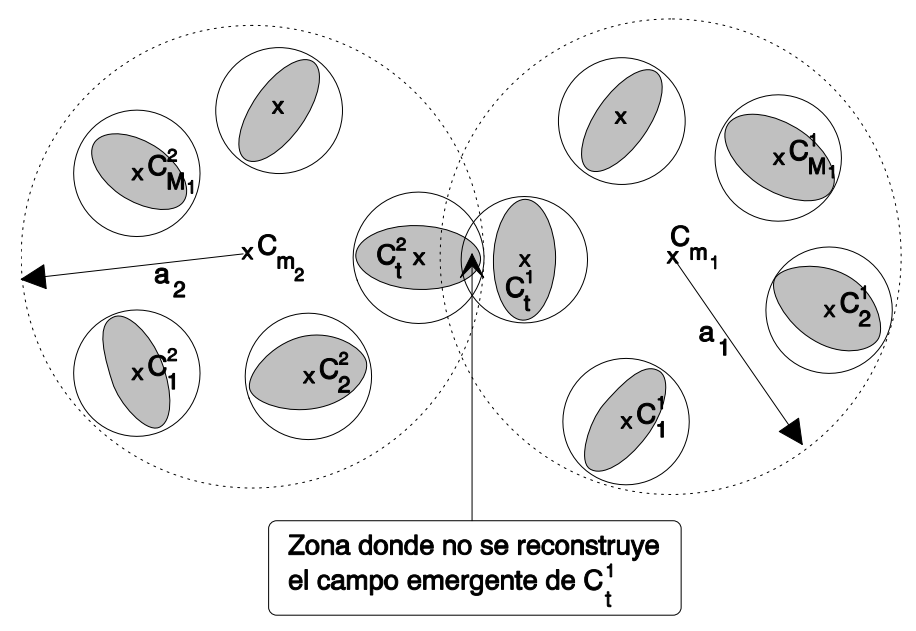

(b)

Figura 2.9: Esquema de dos situaciones en las que le método propuesto en [3] falla

Al intentar aplicar el método de [3] a problemas muy cerrados como el de la figura 2.8 , resulta imposible no incumplir alguna de las tres reglas citadas anteriormente. En consecuencia, el método resulta útil para el análisis de problemas en espacio abierto, pero es insuficiente para analizar problemas cerrados como son estructuras en guía de ondas, que son los problemas que se proponen analizar en la tesis. Por este motivo se desarrolló una 
nueva técnica de resolución de problemas de dispersión múltiple, que evita las limitaciones del método iterativo anterior descrito en [3].

\subsubsection{Nuevo método de análisis de problemas de dispersión múltiple}

Consideremos un problema general de dispersión múltiple como el que se ilustra en la figura 2.10. Deseamos obtener la respuesta electromagnética conjunta de $N$ objetos dispersores, compuestos de un material metálico o dieléctrico, cuya geometría es invariante en una dimensión (eje $\hat{z}$ ) y con una sección transversal (plano $X Y$ ) arbitraria. Cada objeto $i$ $(i \in[1,2, \ldots, N])$ estará contenido en una circunferencia de radio $r_{i}$, y vendrá caracterizado de forma individual por una función de transferencia, o matriz de caracterización, $D_{i}$. De esta forma, la matriz $D_{i}$ nos proporciona el espectro del campo dispersado por el objeto $i$ como respuesta a un espectro cilíndrico arbitrario incidente a $C_{i}$. La matriz $D_{i}$ toma un espectro cilíndrico incidente a $C_{i}$ de $2 N_{i}^{i n}+1$ modos, y devuelve un espectro cilíndrico emergente de $C_{i}$ de $2 N_{i}^{d i}+1$ modos. Por tanto $D_{i}$ es una matriz de tamaño $\left(2 N_{i}^{i n}+1\right) \times\left(2 N_{i}^{d i}+1\right)$. Tanto $N_{i}^{i n}$ como $N_{i}^{d i}$ será un número suficientemente grande como para que no se pierda precisión en los resultados. Como mínimo, ambos deben ser mayores que $K r_{i}$, donde $K$ es el número de onda y $r_{i}$ es el radio de la circunferencia que contiene al objeto $i$.

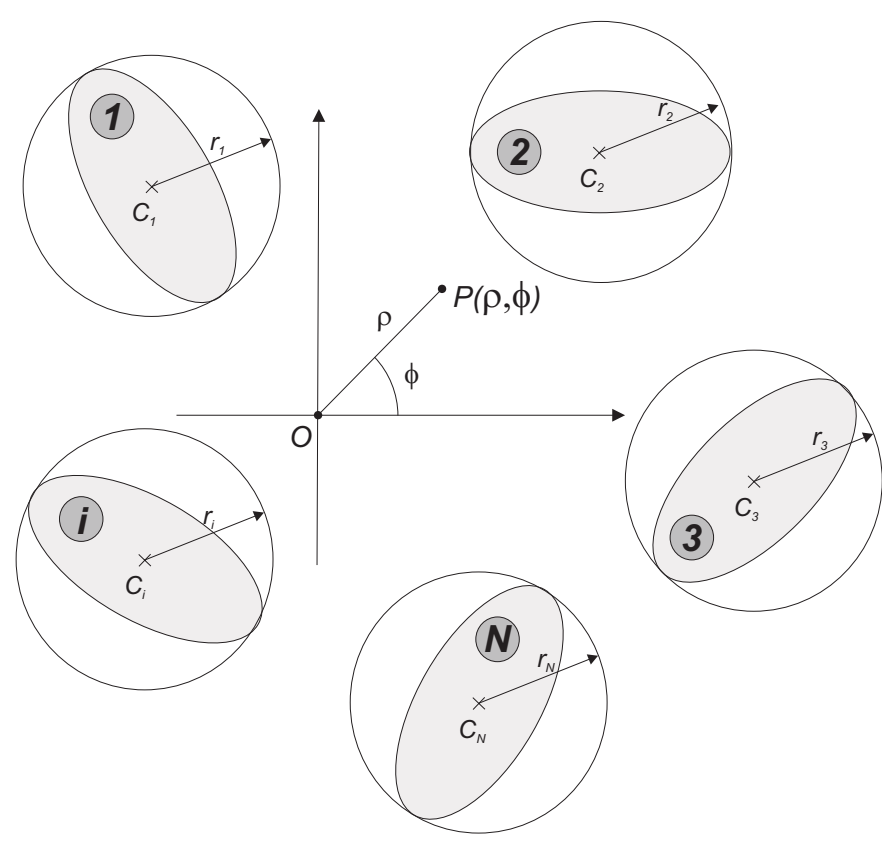

Figura 2.10: Esquema general de un problema de dispersión múltiple

El conjunto formado por los $N$ objetos será iluminado por una fuente de radiación externa caracterizada por el espectro cilíndrico de campo eléctrico, al que llamaremos EI. Dicho espectro de campo incidente estará centrado en el origen de coordenadas global a los $N$ objetos, el punto $O$ (ver figura 2.10), y será un espectro de $2 N I+1$ modos, siendo $N I$ un 
número entero suficientemente grande. Por tanto $E I$ es un vector de $2 N I+1$ elementos. Utilizaremos coordenadas cilíndricas, ya que la geometría es invariante en una dimensión, de modo que un punto cualquiera $P$ vendrá caracterizado por sus coordenadas $\rho$ y $\phi$ (ver figura 2.10).

Al iluminar la fuente externa a los $N$ objetos, éstos producirán una onda dispersada cada uno. A su vez, estas ondas incidirán sobre cada uno de los demás objetos, produciéndose como respuesta una nueva onda dispersada en cada objeto. $\mathrm{Y}$ así irían sucediéndose las interacciones entre los $N$ objetos hasta el infinito. En el régimen permanente, cuando pase el tiempo necesario para ello, todas las interacciones significativas entre los $N$ objetos ya han tenido lugar y la iluminación que recibe cada objeto $i$ (suma de las infinitas contribuciones que recibe desde el exterior y desde los $N-1$ objetos restantes) es constante y estable.

Llamaremos a $E_{i}^{\text {in }}$ al espectro cilíndrico del campo eléctrico incidente al objeto $i$ en régimen permanente. Se tratará de un vector columna de $2 N_{i}^{i n}+1$ elementos. $E_{i}^{i n}$ será la suma del campo procedente de la fuente externa y de las contribuciones procedentes de los $N-1$ objetos restantes. Si logramos conocer el valor de $E_{i}^{i n}$ para $i=[1,2, \ldots, N]$, habremos resuelto el problema, y podremos conocer el campo dispersado por cada uno de los $N$ objetos, teniendo en cuenta ya el acoplo electromagnético entre ellos. Ese campo, al que llamaremos $E_{i}^{d i}$, será:

$$
E_{i}^{d i}=D_{i} * E_{i}^{i n} \quad i \in[1,2, \ldots, N]
$$

Nuestro problema se reduce, por tanto, a conocer el valor del campo $E_{i}^{\text {in }}$ incidente a cada objeto en régimen permanente. Para hallar esas $N$ incógnitas, intentaremos relacionarlas entre ellas. Concretamente, intentaremos relacionar el valor de $E_{i}^{\text {in }}$ (campo total incidente al objeto $i$ ) con el resto de incógnitas (campo incidente al resto de objetos). En la figura 2.11 se muestran los campos incidentes a todos los objetos excepto al objeto $i$.

Cada uno de los campos $E_{k}^{i n}$, con $k \in[1, \ldots, N]$ y $k \neq i$, incide sobre el objeto $k$. Como consecuencia, dicho objeto produce un campo dispersado cuyo espectro será:

$$
D_{k} \cdot E_{k}^{i n}
$$

donde $D_{k}$ es la función de transferencia o matriz de caracterización del objeto $k$. El espectro $D_{k} \cdot E_{k}^{i n}$ está centrado en el punto $C_{k}$ (ver figura 2.12).

El campo dispersado por el objeto $k$ iluminará al objeto $i$. Y el espectro de campo incidente en $i$ debido a $k$ lo podremos obtener haciendo una transformación de espectro cilíndrico emergente de $C_{k}$ a espectro cilíndrico incidente en $C_{i}$. Esta transformación se puede hacer de forma matricial mediante la matriz de traslación de espectros $T_{i k}$ [31] (ver el apéndice B). Esta matriz será de tamaño $\left(2 N_{i}^{i n}+1\right) \times\left(2 N_{k}^{d i}+1\right)$. De forma que el campo incidente en $C_{i}$ debido al objeto $k$ será:

$$
T_{i k} \cdot D_{k} \cdot E_{k}^{i n}
$$

tal y como se muestra en la figura 2.13 . 

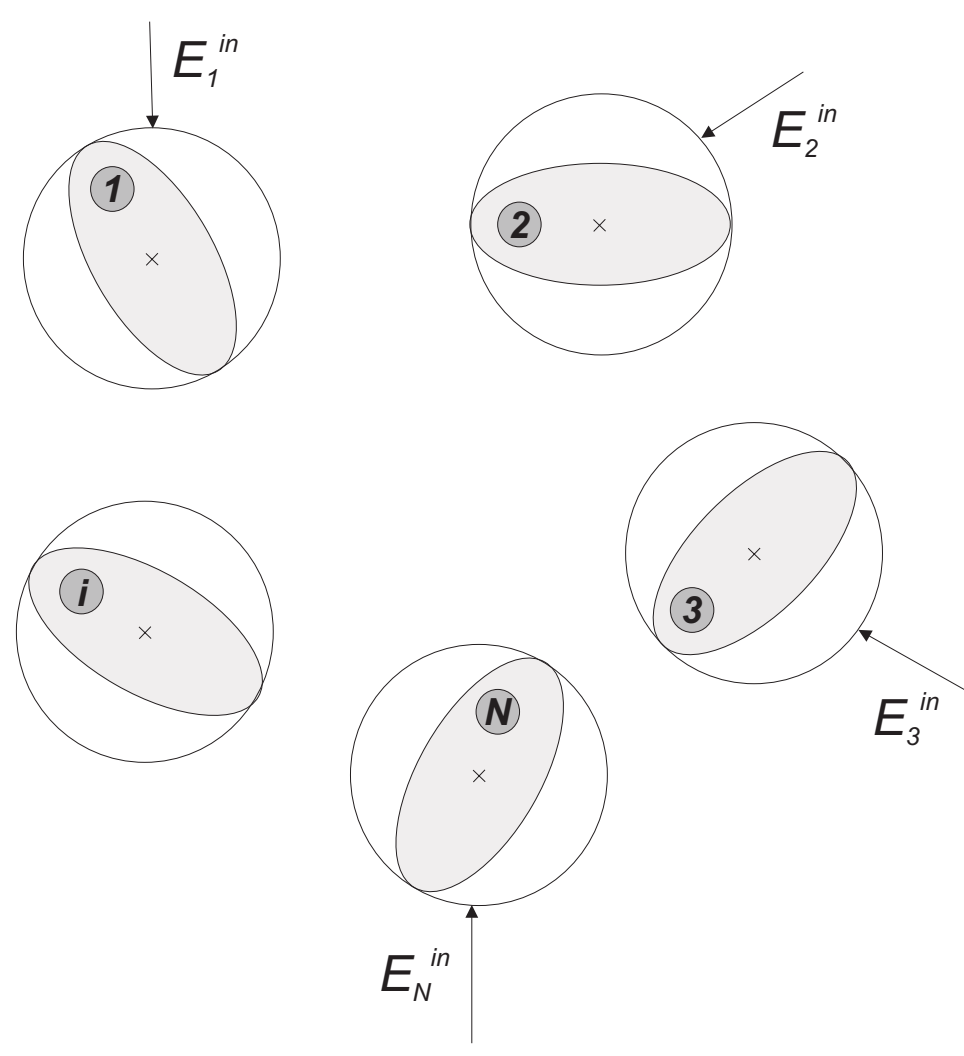

Figura 2.11: Esquema de dispersión múltiple con los campos incidentes a cada objeto

El campo total incidente en el objeto $i\left(E_{i}^{i n}\right)$ será el que le llega de los $N-1$ objetos restantes más el que le llega desde la fuente externa que ilumina al conjunto de dispersores $\left(T_{i 0} \cdot E I\right)$. Por tanto:

$$
E_{i}^{i n}=\sum_{\substack{k=1 \\ k \neq i}}^{N} T_{i k} \cdot D_{k} \cdot E_{k}^{i n}+T_{i 0} \cdot E I
$$

donde $T_{i 0}$ es una matriz diferente según la excitación sea cercana o lejana:

Excitación lejana En el caso en el que la excitación sea lejana (por ejemplo, una onda plana), el campo de excitación lo expresaremos como una suma de modos cilíndricos incidentes en un punto $C_{\text {exc }}$. Para minimizar el número de modos necesarios para expresar correctamente el campo de excitación, conviene que $C_{\text {exc }}$ esté situado en el centro de la menor circunferencia que contiene a todos los objetos. La matriz $T_{i 0}$ será en este caso una matriz de traslación de espectro incidente en $C_{\text {exc }}$ a espectro incidente en $C_{i}$ (ver apéndice $\mathrm{B}$ ).

Excitación cercana La excitación será cercana cuando ésta surja de algún punto $C_{\text {exc }}$ cercano a los objetos. En este caso conviene expresar el campo de excitación como una 


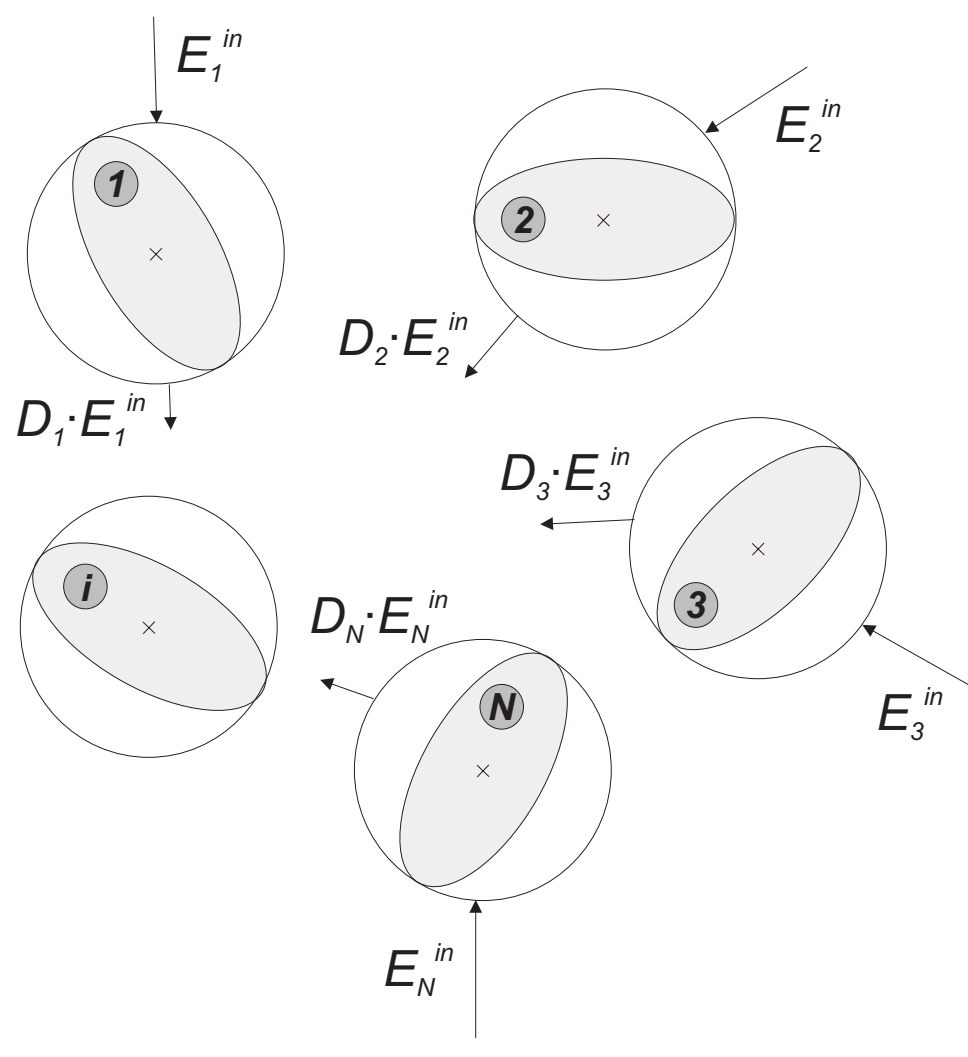

Figura 2.12: Esquema de dispersión múltiple con los campos dispersados por cada objeto

suma de modos cilíndricos emergentes (ver apéndice A) desde el punto $C_{\text {exc }}$. Y por tanto en este caso la matriz $T_{i 0}$ será una matriz de traslación de espectro emergente de $C_{\text {exc }}$ a espectro incidente en $C_{i}$.

La matriz $T_{i 0}$ será de tamaño $\left(2 N_{i}^{i n}+1\right) \times\left(2 N_{\text {exc }}+1\right)$, donde $N_{\text {exc }}$ es el número de modos del espectro cilíndrico del campo de excitación.

En la ecuación 2.26 hemos conseguido relacionar el campo incidente al objeto $i$ con el campo incidente al resto de objetos. Disponemos ya de 1 ecuación con $N$ incógnitas. Si relacionamos de igual forma el campo incidente a cada objeto con los campos incidentes a los demás objetos, obtendremos las $N$ ecuaciones de $N$ incógnitas siguientes:

$$
E_{i}^{i n}=\sum_{\substack{k=1 \\ k \neq i}}^{N} T_{i k} \cdot D_{k} \cdot E_{k}^{i n}+T_{i 0} \cdot E I \quad i=1,2, \ldots, N
$$

Pondremos los términos independientes a un lado de la ecuación, y las incógnitas al otro:

$$
E_{i}^{i n}-\sum_{\substack{k=1 \\ k \neq i}}^{N} T_{i k} \cdot D_{k} \cdot E_{k}^{i n}=T_{i 0} \cdot E I \quad i=1,2, \ldots, N
$$




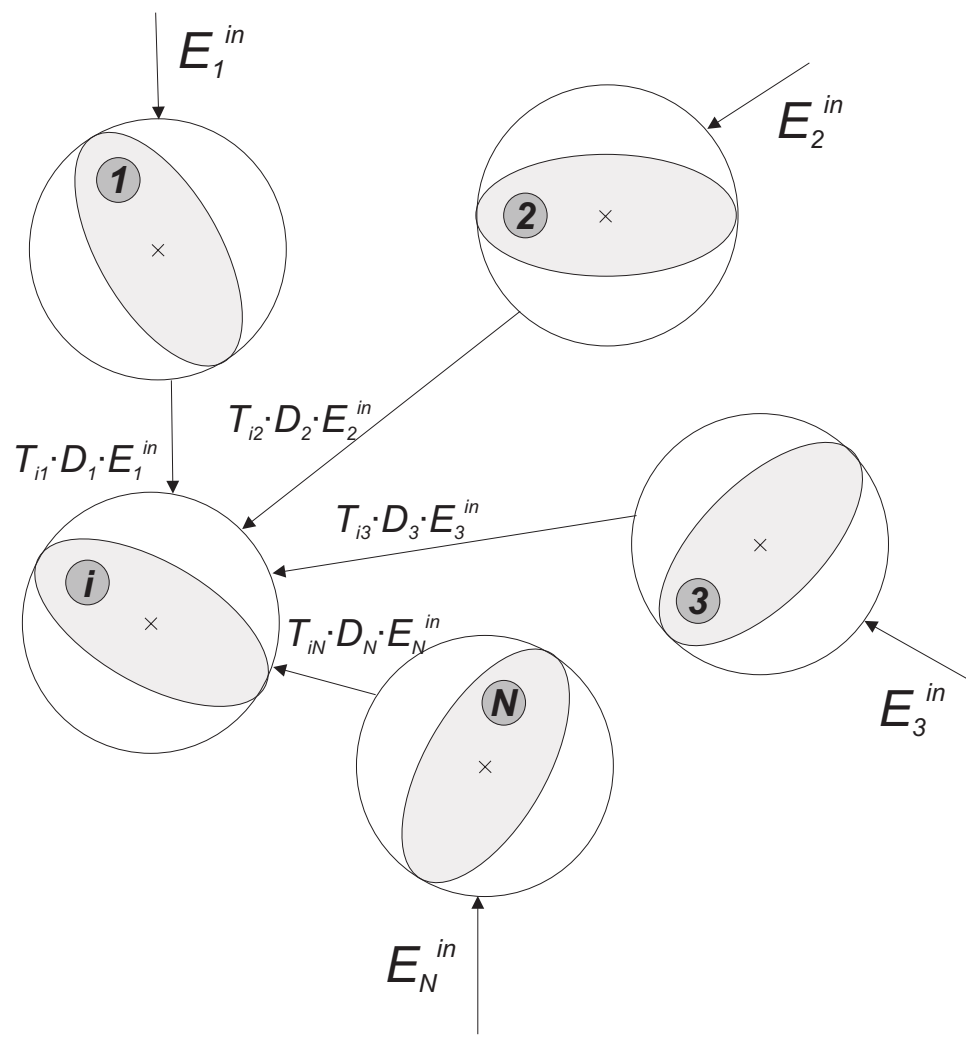

Figura 2.13: Esquema de dispersión múltiple con los campos incidentes al objeto $i$

Definiremos unos coeficientes $A_{i k}$, que serán los coeficientes de la incógnita $k$-ésima $\left(E_{k}^{i n}\right)$ en la ecuación $i$-ésima. De esta forma podemos reescribir las ecuaciones anteriores de la siguiente forma:

$$
\sum_{k=1}^{N} A_{i k} \cdot E_{k}^{i n}=T_{i 0} \cdot E I \quad i=1,2, \ldots, N
$$

donde cada coeficiente $A_{i k}$ es una matriz de tamaño $\left(2 N_{i}^{i n}+1\right) \times\left(2 N_{k}^{i n}+1\right)$ de la siguiente forma,

$$
A_{i k}=\left\{\begin{array}{cc}
I & \text { si } i=k \\
-T_{i k} \cdot D_{k} & \text { si } i \neq k
\end{array}\right.
$$

donde $I$ es una matriz identidad de tamaño $\left(2 N_{i}^{i n}+1\right) \times\left(2 N_{i}^{i n}+1\right)$.

Las $N$ ecuaciones de (2.29) se pueden escribir de forma matricial de la siguiente forma:

$$
A \cdot E=B \cdot E I
$$


donde,

$$
\begin{aligned}
& A= {\left[\begin{array}{cccc}
A_{11} & A_{12} & \cdots & A_{1 N} \\
A_{21} & A_{22} & \cdots & A_{2 N} \\
\vdots & \vdots & \ddots & \vdots \\
A_{N 1} & A_{N 2} & \cdots & A_{N N}
\end{array}\right] } \\
& E=\left[\begin{array}{c}
E_{1}^{i n} \\
E_{2}^{i n} \\
\vdots \\
E_{N}^{i n}
\end{array}\right] \\
& B=\left[\begin{array}{c}
T_{10} \\
T_{20} \\
\vdots \\
T_{N 0}
\end{array}\right]
\end{aligned}
$$

Los tamaños de la matrices $A, E, B$ y $E I$ son los siguientes:

$$
\begin{aligned}
\text { Tamaño de } A & =\sum_{k=1}^{N}\left(2 N_{k}^{i n}+1\right) \times \sum_{k=1}^{N}\left(2 N_{k}^{i n}+1\right) \\
\text { Tamaño de } E & =\sum_{k=1}^{N}\left(2 N_{k}^{i n}+1\right) \times 1 \\
\text { Tamaño de } B & =\sum_{k=1}^{N}\left(2 N_{k}^{i n}+1\right) \times(2 N I+1) \\
\text { Tamaño de } E I & =(2 N I+1) \times 1
\end{aligned}
$$

Por tanto la solución al acoplo electromagnético entre los $N$ objetos dispersores se reduce finalmente a la solución del sistema de ecuaciones lineales definido en (2.31):

$$
E=A^{(-1)} \cdot B \cdot E I
$$

Resuelto el sistema de ecuaciones ya conocemos el campo incidente a cada objeto (tenido en cuenta el acoplo entre dispersores). Por tanto, como se indicaba en (2.23), el campo $E_{k}^{d i}$ que dispersará cada objeto $k$ como respuesta a una incidencia externa $E I$ será:

$$
E_{k}^{d i}=D_{k} \cdot E_{k}^{i n}
$$

Si deseamos conocer la respuesta de los $N$ objetos ante cualquier incidencia, debemos proceder de igual forma a como procedíamos a la hora de caracterizar objetos de forma aislada: debemos obtener la respuesta de cada objeto a cada posible modo incidente.

El vector $E$ es un vector que contiene $\operatorname{los} N$ espectros $E_{k}^{i n}(k=1,2, \ldots, N)$ incidentes a los $N$ objetos cuando la excitación externa posee un espectro $E I$. Si queremos conocer la 
respuesta de los múltiples dispersores a cada uno de los $2 N I+1$ posibles modos cilíndricos incidentes, deberemos calcular el valor del vector $E$ para $2 N I+1$ excitaciones diferentes. Esto se puede hacer en un sólo paso si tomamos como espectro de campo incidente $E I$ no un vector de tamaño $2 N I+1$ con un espectro cilíndrico concreto, sino una matriz identidad $I$ de tamaño $(2 N I+1) \times(2 N I+1)$. Si hacemos esto, la columna $p$-ésima de la matriz $E I$ será un vector cuyos elementos son todos nulos excepto el elemento $p$-ésimo. Lo que supone que esa columna es el espectro cilíndrico correspondiente a un incidencia del modo cilíndrico de orden $p$ :

$$
J_{p}(K \rho) e^{j p \phi}
$$

Por tanto, siendo EI una matriz identidad, el producto

$$
E^{\prime}=A^{(-1)} \cdot B \cdot E I=A^{(-1)} \cdot B \cdot I=A^{(-1)} \cdot B
$$

proporciona una matriz $E^{\prime}$ de tamaño

$$
\sum_{k=1}^{k=N}\left(2 N_{k}^{i n}+1\right) \times(2 N I+1)
$$

que almacena en la $p$-ésima columna los espectros de los campos incidentes a los $N$ objetos cuando la incidencia externa es la de un modo cilíndrico de orden $p$.

La matriz $E^{\prime}$ está formada por $N$ submatrices de la siguiente forma:

$$
E^{\prime}=\left[\begin{array}{c}
E_{1}^{\prime} \\
E_{2}^{\prime} \\
\vdots \\
E_{N}^{\prime}
\end{array}\right]
$$

donde cada matriz $E_{k}^{\prime}(k=1,2, \ldots, N)$ es una matriz de tamaño $\left(2 N_{k}^{i n}+1\right) \times(2 N I+1)$, que almacena en cada columna $p$ el espectro de campo incidente al objeto $k$ ante la incidencia externa del modo cilíndrico de orden $p$. Si multiplicamos $E_{k}^{\prime}$ por la matriz de caracterización o función de transferencia del objeto $k$, obtendremos una matriz, a la que llamaremos $D_{k}^{\prime}$, de tamaño $\left(2 N_{k}^{d i}+1\right) \times(2 N I+1)$ :

$$
D_{k}^{\prime}=D_{k} \cdot E_{k}^{\prime}
$$

La matriz $D_{k}^{\prime}$ almacena en cada columna $p$ el espectro de campo dispersado por el objeto $k$ ante la incidencia externa del modo cilíndrico de orden $p$, ya teniendo en cuenta el acoplo con el resto de objetos. Por tanto, a esta matriz la llamaremos función de transferencia conjunta, ya que caracteriza al objeto $N$ ante cualquier posible incidencia externa teniendo en cuenta el acoplo con el resto de objetos, de igual forma que la matriz $D_{k}$ es la función de transferencia del objeto aislado.

Obteniendo las $N$ funciones de transferencia conjunta $\left(D_{k}^{\prime}\right.$, con $\left.k=1,2, \ldots, N\right)$, habremos resuelto el problema de dispersión múltiple, y podremos conocer de forma inmediata 
y muy sencilla la respuesta de los $N$ objetos dispersores ante cualquier incidencia externa. Si $\left[i_{p}\right]$ es el espectro del campo externo incidente sobre los $N$ objetos, podemos obtener el espectro del campo dispersado por cada objeto $k$ con sólo multiplicar por la correspondiente función de transferencia conjunta:

$$
\left[c_{q}^{k}\right]=D_{k}^{\prime} \cdot\left[i_{p}\right]
$$

De manera que el campo $\vec{E}_{k}(\rho, \phi)$ dispersado por el objeto $k$ ante la incidencia $\left[i_{p}\right]$ es:

$$
\vec{E}_{k}(\rho, \phi)=\sum_{q=-N_{k}^{d i}}^{N_{k}^{d i}} c_{q}^{k} H_{q}^{(2)}\left(K \rho_{k}\right) e^{j q \phi_{k}} \hat{z}
$$

donde $\rho_{k}$ y $\phi_{k}$ son las coordenadas del punto $C=(\rho, \phi)$ vistas desde el punto $C_{k}$, centro del objeto $k$ (ver figura 2.14), y se pueden obtener de la siguiente forma:

$$
\begin{aligned}
\vec{v}_{k} & =\overrightarrow{C_{k} C} \\
\rho_{k} & =\left\|\vec{v}_{k}\right\|=\sqrt{v_{k_{x}}^{2}+v_{k_{y}}^{2}} \\
\phi_{k} & =\angle \overrightarrow{v_{k}}=\arctan \frac{v_{k_{y}}}{v_{k_{x}}}
\end{aligned}
$$

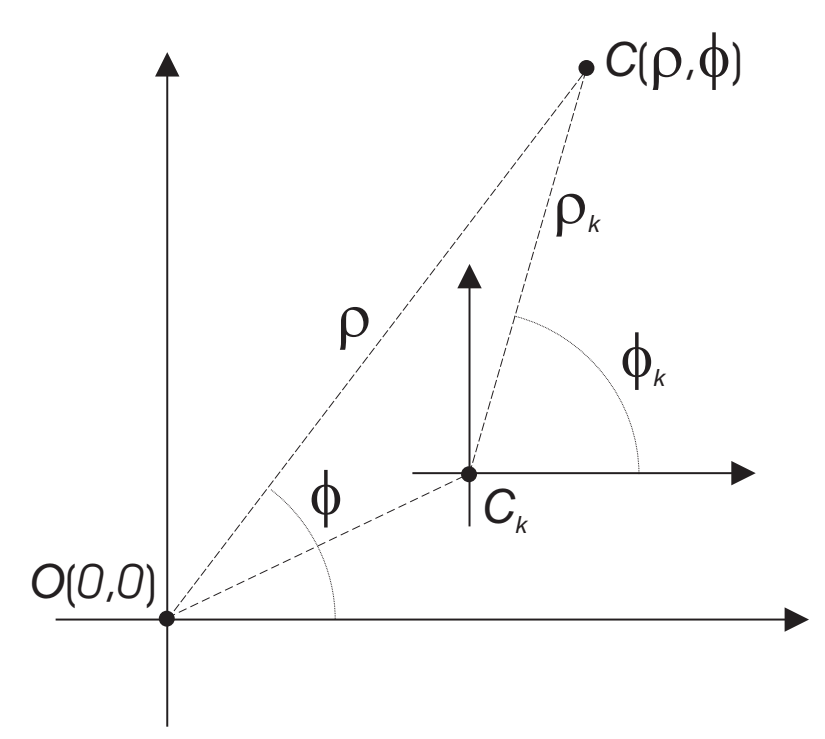

Figura 2.14: Sistemas de coordenadas global y local al objeto $k$

\section{Limitación del método}

El método de solución de acoplo entre objetos que se acaba de presentar permite el análisis de problemas de dispersión múltiple con cualquier número de objetos, y con la presencia 
de objetos de cualquier tamaño y geometría, siempre que estén previamente caracterizados de forma aislada mediante su función de transferencia o matriz de caracterización.

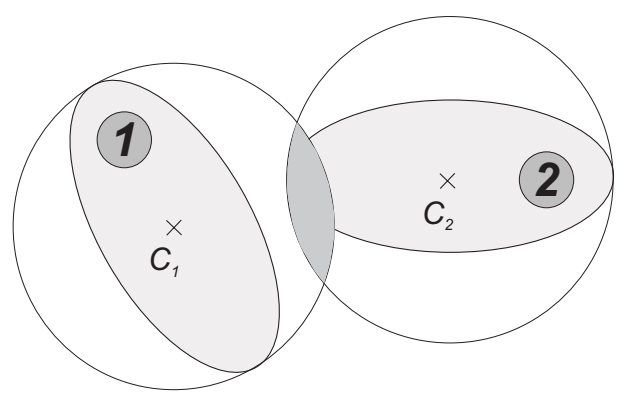

Figura 2.15: Limitación del método de acoplo múltiple. El campo no se reconstruye correctamente en la zona oscurecida

No obstante, si un objeto lo caracterizamos mediante su función de transferencia, podemos conocer el campo que este objeto produce como respuesta a cualquier incidencia; pero sólo podemos conocer este campo fuera de la circunferencia que lo circunscribe. Por tanto, no podemos situar dos objetos tan cerca que se introduzca un objeto dentro de la circunferencia que contiene a otro (ver figura 2.16).

Otra situación crítica se produce cuando las circunferencias que contienen a dos objetos se intersectan, pero ninguno de los dos objetos entra dentro de la circunferencia que contiene al otro. Esta situación se puede producir por ejemplo el analizar una estructura compleja como la de la figura 2.16.a (una unión en T en una guía rectangular). En dicha figura se muestra cómo se ha fragmentado la estructura en un conjunto de tiras metálicas (problema de dispersión múltiple), de modo que existen dos parejas de objetos (objetos 1 y 2, y objetos 3 y 4) cuyas circunferencias se intersectan sin que ningún objeto entre dentro de la circunferencia de otro. En esta situación el campo dispersado por el objeto 1 se puede reconstruir correctamente en toda la zona de interés como suma de modos emergentes de $C_{1}$ (centro del objeto 1). Por tanto en este caso no existe ninguna limitación de tipo teórico. No obstante, es necesario expresar el campo emergente de $C_{1}$ como suma de modos incidentes a $C_{2}$ (centro del objeto 2) para conocer mediante la función de transferencia del objeto 2 su respuesta ante el campo dispersado por el objeto 1 . Y la matriz de traslación de espectro de campo emergente de $C_{1}$ a campo incidente a $C_{2}$ será una matriz mal condicionada ya que el número de modos del espectro de campo incidente a $C_{2}$ se elige lo suficientemente grande como para reconstruir correctamente el campo en toda la circunferencia que contiene al objeto 2 . Y dentro de esa circunferencia hay una zona (parte de la circunferencia que contiene al objeto 1), donde el campo no se podía expresar mediante espectro de campo emergente de $C_{1}$. En definitiva, la inversión de las matrices de traslación de espectros entre objetos cuyas circunferencias se intersectan puede dar lugar a errores que se acumularán en el caso de haber varios objetos en esta situación.

Con el objetivo de evitar esta situación, en la figura 2.16.b se segmenta de forma que no se incumple esta segunda limitación del método, y de esta forma garantizamos la corrección 
de los resultados.

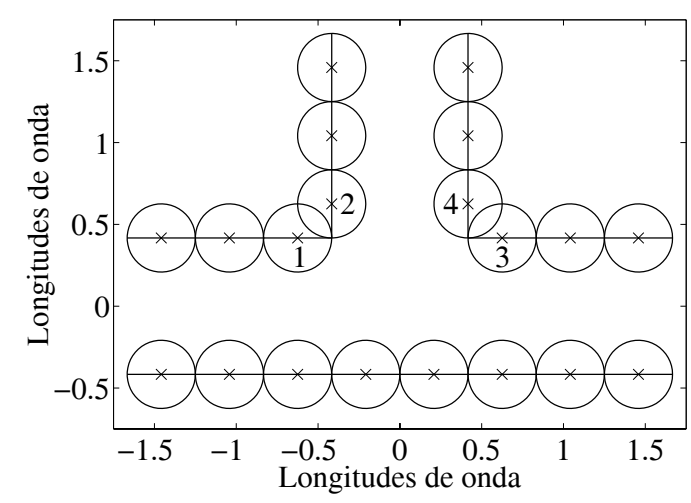

(a) Incorrecto

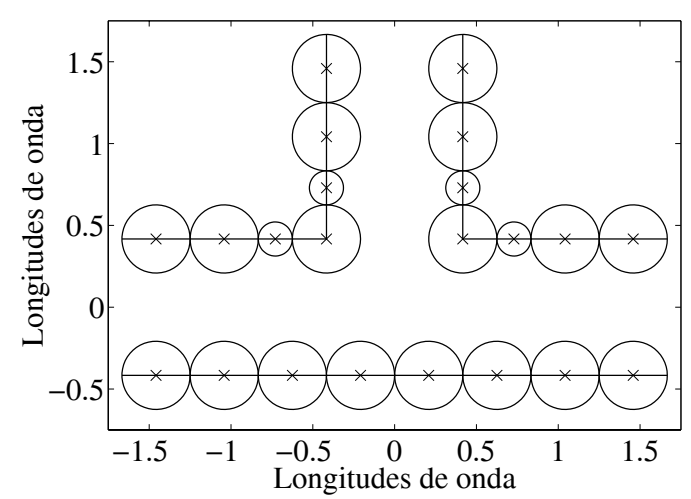

(b) Correcto

Figura 2.16: Dos situaciones similares en las que se fragmenta una estructura en múltiples dispersores de forma correcta e incorrecta

Las limitaciones que se han explicado son las únicas limitaciones del método. Por lo demás, es un método general y válido para el análisis de cualquier problema de dispersión múltiple.

\subsubsection{Resultados}

La primera estructura que se va analizar para comprobar el comportamiento del método es una antena 2-D de tipo bocina alimentada por una guía rectangular.

En la figura 2.17 se muestra la estructura analizada, y cómo ésta ha sido fragmentada en un problema de dispersión múltiple formado por varias tiras metálicas 2-D. Cada circunferencia encierra una tira metálica infinita.

La estructura se excita por una fuente puntual situada a una distancia $\lambda / 4$ de la pared posterior de la guía que alimenta a la antena. La longitud de la boca de la bocina es de $D=4 \lambda$.

En la figura 2.18 se muestran la amplitud y la fase del campo eléctrico en la zona de campo cercano de la bocina. El tiempo de cálculo invertido en la resolución de problema de dispersión y en el cálculo del campo en la rejilla que se representa en la figura en un Pentium III a $1.7 \mathrm{GHz}$ es de 44 segundos. 


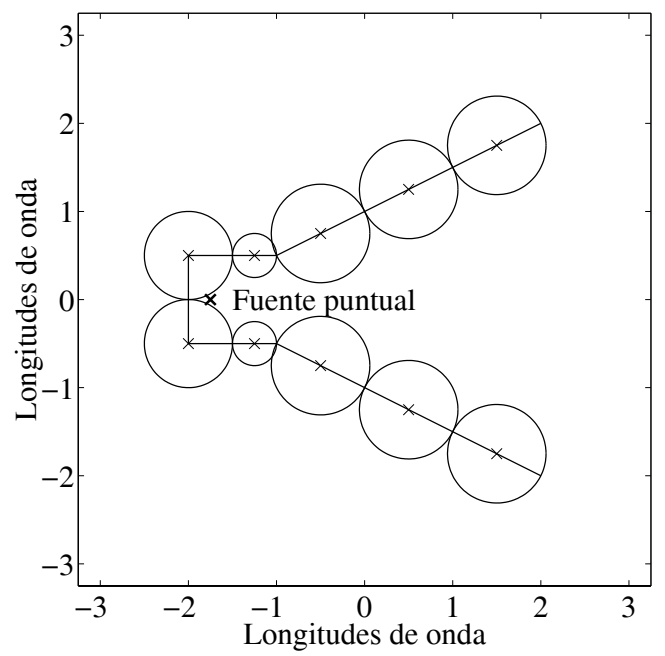

Figura 2.17: Problema de dispersión múltiple en el que se ha fragmentado una antena de tipo bocina $(D=4 \lambda)$ para su análisis

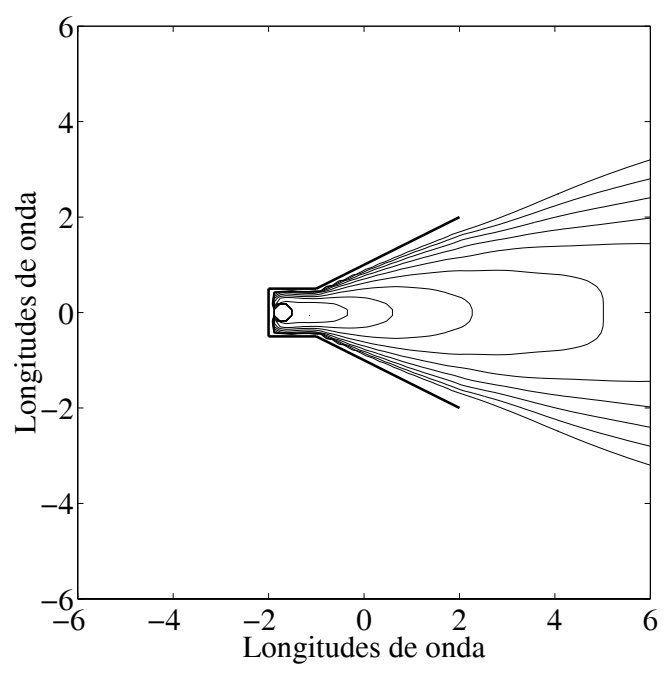

(a) Amplitud de campo eléctrico (dB)

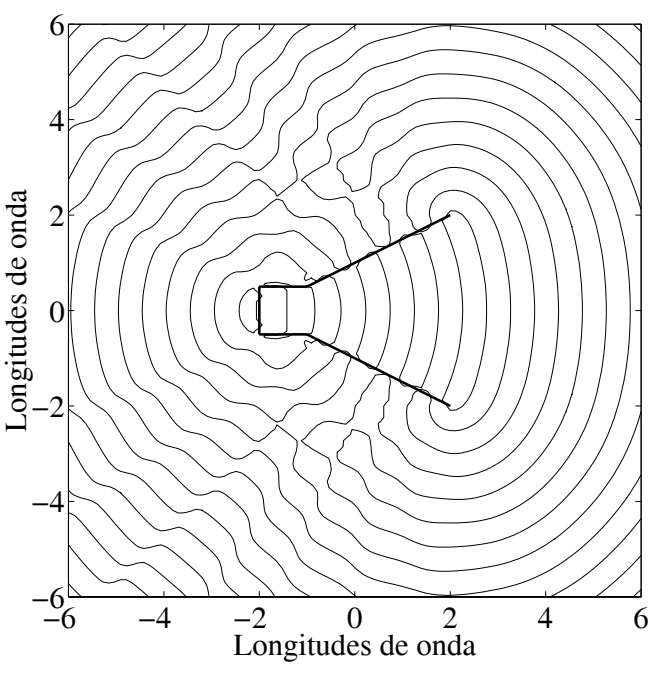

(b) Fase de campo eléctrico

Figura 2.18: Amplitud y fase del campo eléctrico en la zona de campo cercano de una bocina $2-\mathrm{D}(D=4 \lambda)$ 
Si se intenta analizar la misma bocina con el método iterativo de [3], es imposible encontrar una fragmentación en tiras 2-D que no incumpla alguna de las reglas expuestas en la página 26. A pesar de incumplir dichas reglas, se ha analizado la bocina con el método de [3], y el resultado se muestra en la figura 2.19. Al comparar con los resultados de la figura 2.18 se puede apreciar claramente la incorrección en los resultados. Se puede concluir por tanto, que el nuevo método de resolución de problemas de dispersión múltiple supera las limitaciones del método anterior.

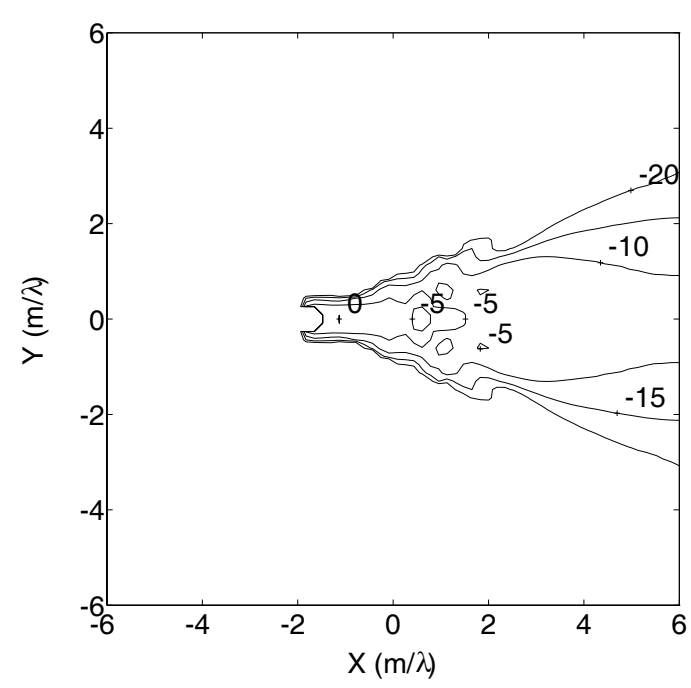

(a) Amplitud de campo eléctrico (dB)

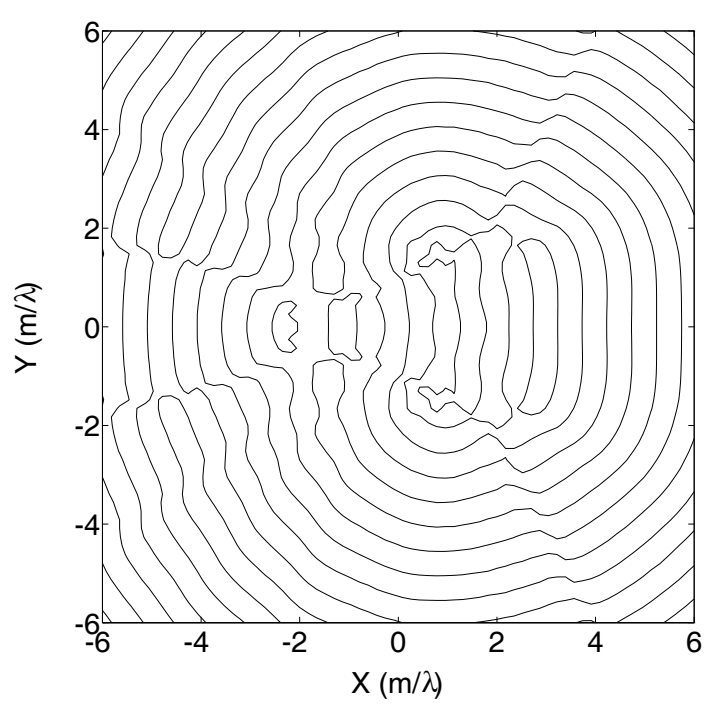

(b) Fase de campo eléctrico

Figura 2.19: Amplitud y fase del campo eléctrico en la zona de campo cercano de una bocina $2-\mathrm{D}(D=4 \lambda)$ resuelto con el método descrito en [3]

Vista la capacidad del nuevo método para el análisis de una bocina 2-D, comprobaremos ahora su capacidad para resolver problemas de mayor complejidad como es la agrupación de dos bocinas alimentadas por una guía rectangular. La boca de las bocinas es de $D=4 \lambda$, y la separación entre las dos bocinas es de $d=4 \lambda$. En la figura 2.20.a se muestra la segmentación de la estructura en tiras metálicas 2-D. Para alimentar a las dos bocinas la guía de ondas que transporta la señal se divide en dos brazos mediante una unión en T. Con el objeto de minimizar las reflexiones y maximizar la transferencia de potencia desde la guía a las dos bocinas, se han introducido unos rebajes en la estructura, tal y como se muestra en la vista en detalle de la figura 2.20.b. Nótese que los codos incluidos en la red de alimentación son circulares, lo que refuerza el carácter general del método desarrollado, pues es capaz de analizar simultáneamente estructuras con geometrías muy diversas. En las figuras 2.20.c y 2.20.d se muestran la amplitud y la fase de la agrupación de dos bocinas en campo cercano. 


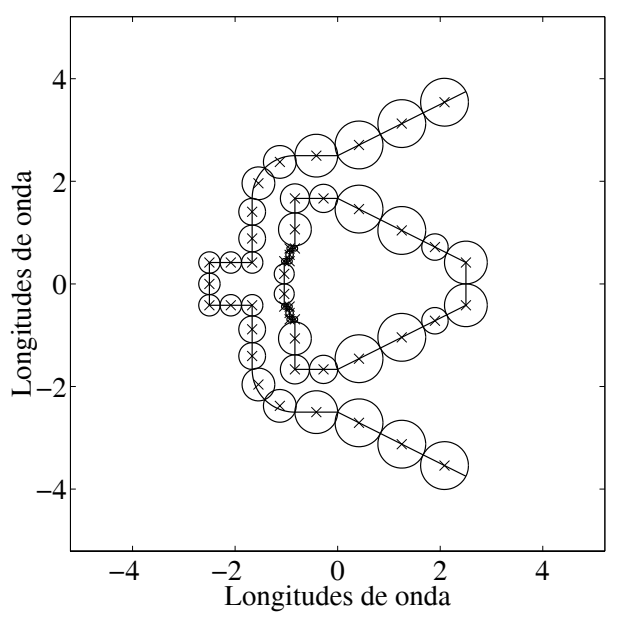

(a) Segmentación en un problema de dispersión múltiple. Vista general

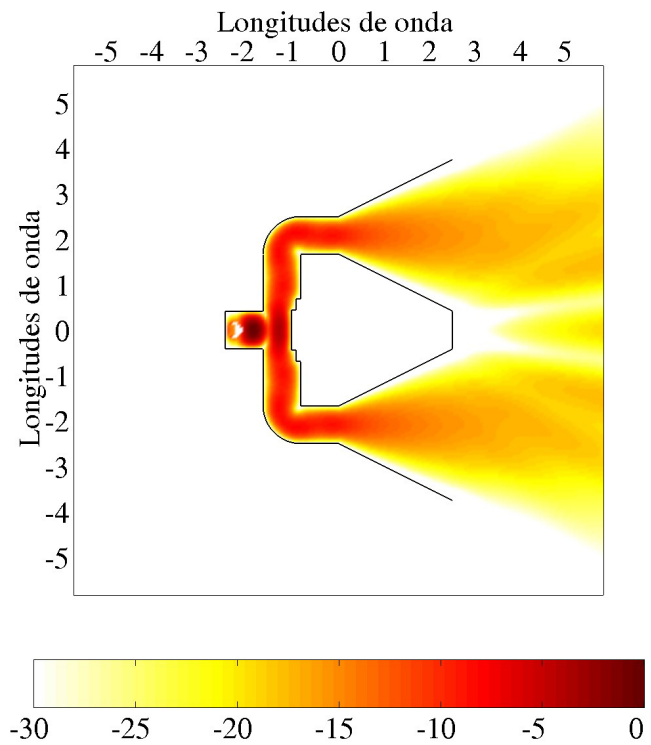

(c) Amplitud de campo eléctrico (dB)

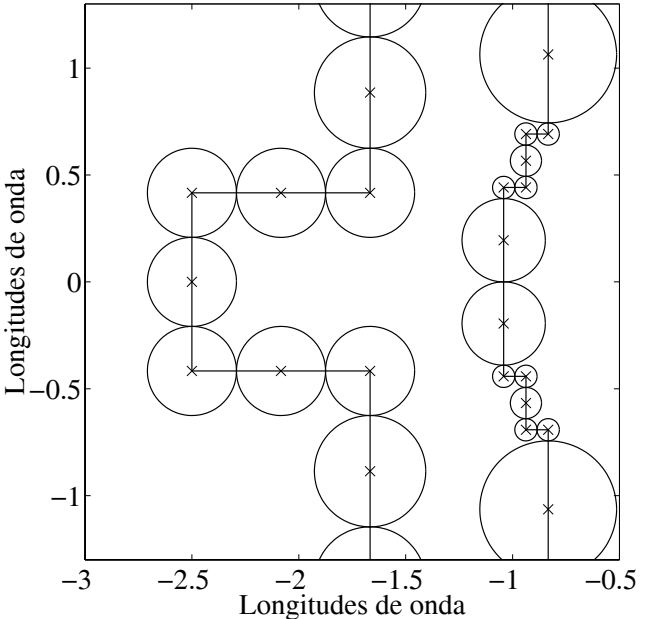

(b) Segmentación. Detalle de la unión T con entrantes

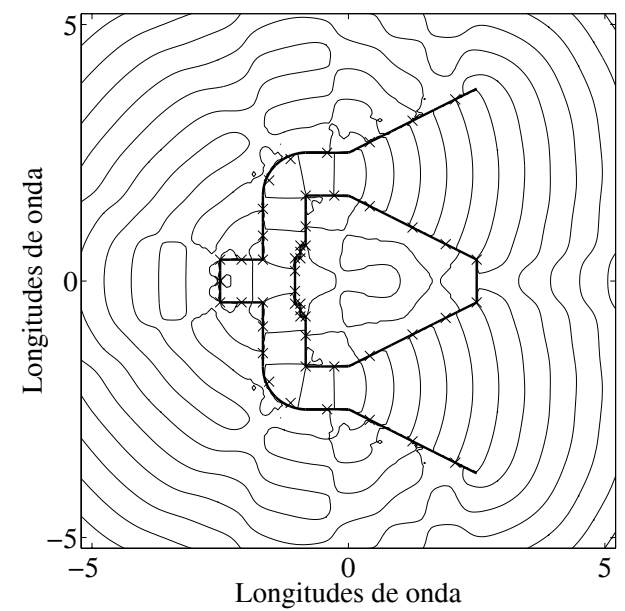

(d) Fase de campo eléctrico

Figura 2.20: Agrupación de dos bocinas 2-D alimentadas con una guía rectangular $(D=4 \lambda$ y $d=4 \lambda)$ 


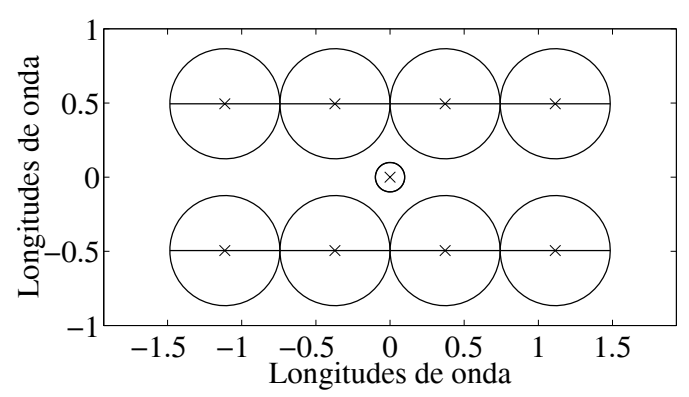

(a) Segmentación en tiras 2-D
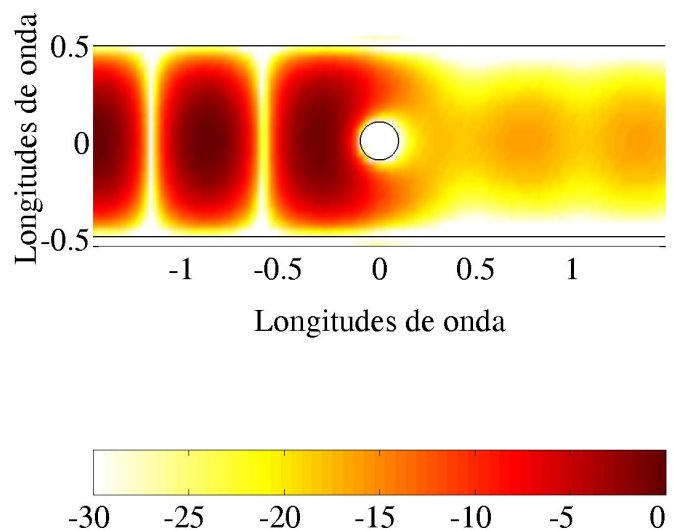

(b) Amplitud de campo eléctrico (dB). Modo $T E_{10}$
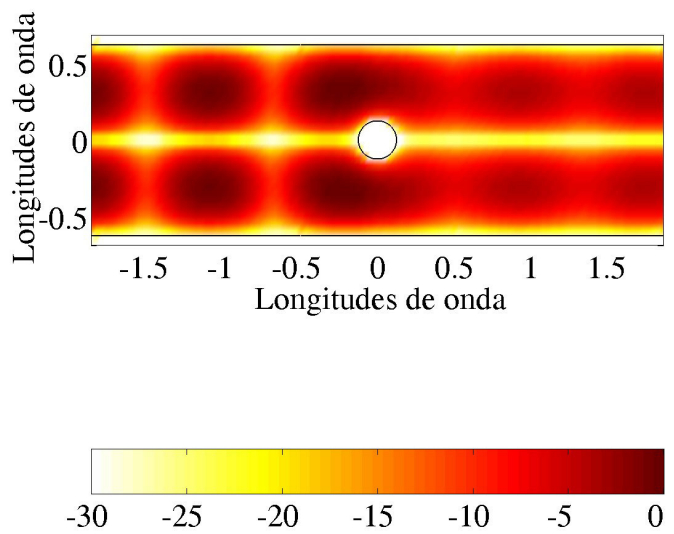

(c) Amplitud de campo eléctrico (dB). Modo $T E_{20}$

Figura 2.21: Poste metálico circular en plano $\mathrm{H}$ en el interior de una guía rectangular de anchura $a$. El diámetro del poste es $d=0,2 a$, y la longitud de onda es $\lambda=1,01 a$

En la figura 2.21 se analiza un poste circular metálico en el interior de una guía rectangular de radio $a$. Como se explica en el capítulo 3, el nuevo método de análisis de problemas de dispersión múltiple 2-D se puede aplicar a la resolución de problemas reales en plano $\mathrm{H}$ en guía rectangular, ya que en estos casos tanto la excitación (modos $T E_{m 0}$ ) como la estructura (en plano $\mathrm{H}$ ) son invariantes en una dimensión, y el problema se puede abordar como si se tratara de una estructura bidimensional. En el ejemplo de la figura 2.21 se ha considerado la existencia de un poste circular metálico de diámetro $d=0,2 a$, y la longitud de onda de trabajo es $\lambda=1,01 a$. Se ha representado la propagación del modo fundamental $T E_{10}$ 
por dentro de la guía (figura 2.21.b), y la del modo superior (figura 2.21.c). Para excitar la estructura con los modos de la guía de onda rectangular es necesario realizar un acoplo de modos de la guía rectangular a modos cilíndricos. Todo lo relativo a este acoplo de modos se explica exhaustivamente en el capítulo 3.

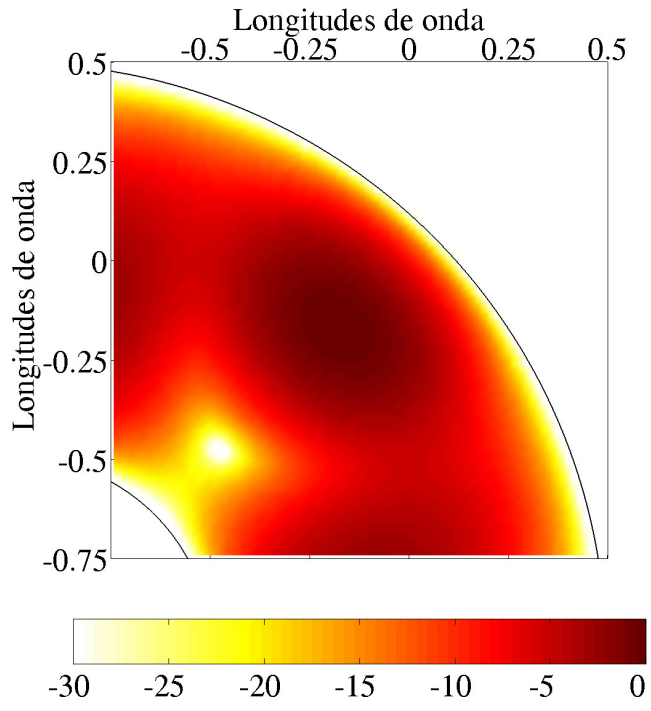

(a) Codo circular

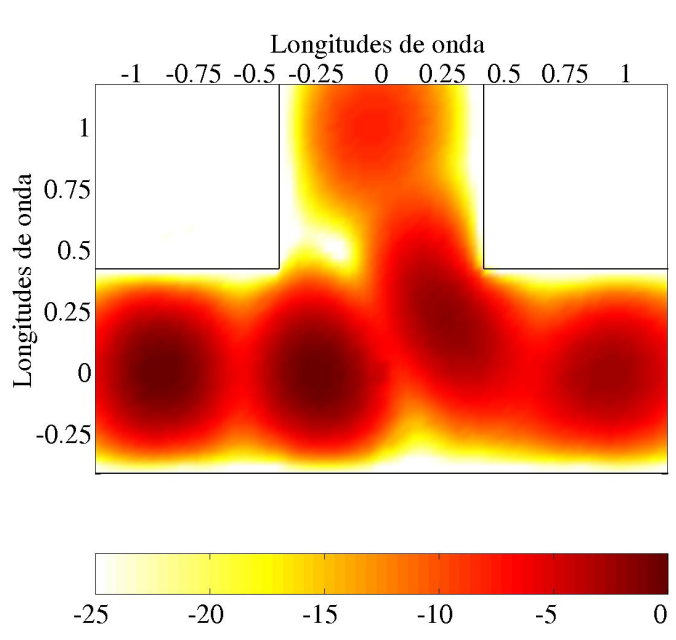

(b) Union en $\mathrm{T}$

Figura 2.22: Codo circular y unión en $\mathrm{T}$ en plano $\mathrm{H}$ en una guía rectangular excitados por el modo fundamental

En la figura 2.22 se muestran dos ejemplos más de análisis de estructuras en plano $\mathrm{H}$ en guía de onda rectangular. Se trata de un codo circular y una unión en T excitadas por el modo fundamental. El codo circular se representa en la figura 2.22.a, donde el ancho de la guía es $a$, la longitud de onda es $\lambda=1,01 a$, y los radios interior y exterior del codo son, respectivamente, $R_{1}=0,5 a$ y $R_{2}=1,05 a$. La unión en $\mathrm{T}$ se representa en la figura 2.22.b. Todos los accesos de la unión son de la misma anchura $a$, y la longitud de onda de trabajo es $\lambda=1,2 a$.

Finalmente se ha analizado la estructura de la figura 2.23.a. Se trata de una cavidad en plano $\mathrm{H}$ en una guía rectangular con un poste dieléctrico en el centro de la misma. Los accesos de la cavidad son de anchura $a_{1}=17 \mathrm{~mm}$, la anchura de la cavidad es de $a_{2}=$ $22,86 \mathrm{~mm}$, el diámetro del poste es $d=5 \mathrm{~mm}$, y su permitividad relativa es $\varepsilon_{r}=2,17$. La frecuencia de excitación es $f=28,84 \mathrm{GHz}$. 


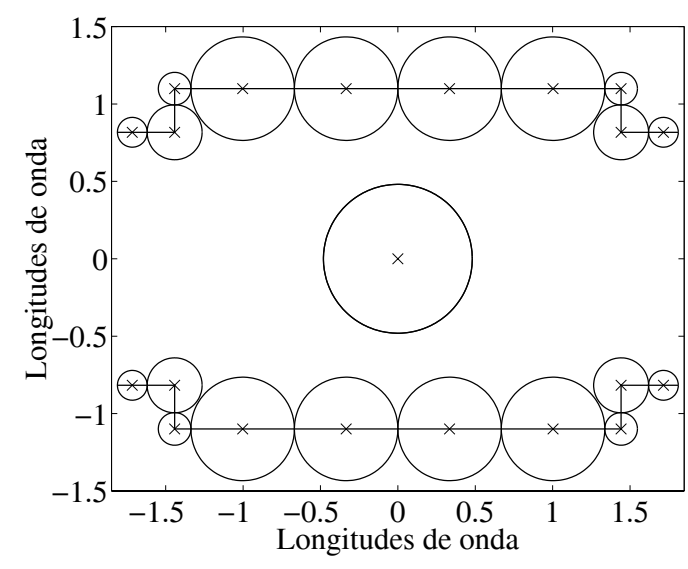

(a) Segmentación en tiras 2-D

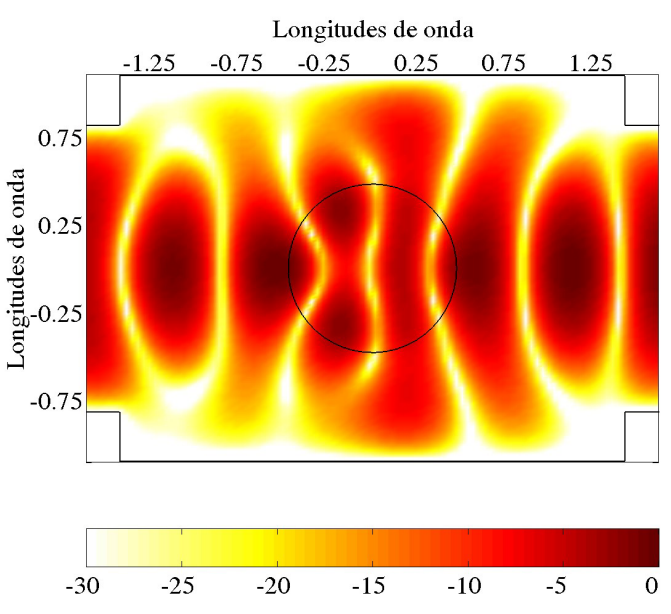

(b) Amplitud de campo eléctrico (dB)

Figura 2.23: Poste dieléctrico $\left(d=5 \mathrm{~mm}, \varepsilon_{r}=2,17\right)$ en el interior de una cavidad de anchura $a_{2}=22,86 \mathrm{~mm}$, accedida por dos guías rectangulares de anchura $a_{1}=17 \mathrm{~mm}$. $f=28.84 \mathrm{GHz}$

\subsection{Conclusiones}

En este capítulo se ha abordado con éxito el análisis de problemas de dispersión múltiple 2-D con geometría arbitraria. En primer lugar se ha introducido el concepto de función de transferencia para la caracterización electromagnética de dispersores individuales. Posteriormente, y utilizando las mencionadas funciones de transferencia, se ha desarrollado un nuevo método de resolución del acoplo electromagnético entre múltiples dispersores. Este nuevo método supera las limitaciones de métodos anteriores que también hacen uso de las funciones de transferencia. La única limitación del nuevo método, que viene impuesta por las características de los espectros de campo cilíndricos que se han utilizado, es que ningún objeto puede estar dentro de la circunferencia que circunscribe a otro. Los resultados obtenidos con el método son satisfactorios, y demuestran que el método puede analizar estructuras arbitrarias bidimensionales, y en combinación con la técnica de acoplo de modos que se explicará en el capítulo 3, puede analizar también estructuras arbitrarias en plano H en guía de onda rectangular. 


\section{Capítulo 3}

\section{Análisis de problemas inductivos en guías rectangulares}

\subsection{Introducción}

\subsubsection{Descripción del problema}

En este capítulo se describe un nuevo método que permite el análisis de problemas inductivos con cualquier geometría en una guía rectangular. Se entiende por problemas inductivos aquellos en los que la geometría de la guía es invariante en altura, es decir, en la dimensión $\hat{y}$ (ver figura 3.1). Por ejemplo, la existencia de un poste metálico que atraviese completamente la guía de arriba a abajo es un problema inductivo. El término inductivo [20] se aplica a este tipo de problemas porque en el modelo circuital de este tipo de obstáculos o discontinuidades aparece una inductancia, mientras que cuando la discontinuidad o el obstáculo se encuentran a lo largo del eje $\hat{x}$ en el circuito equivalente aparece una capacitancia, y se habla entonces de problemas capacitivos.

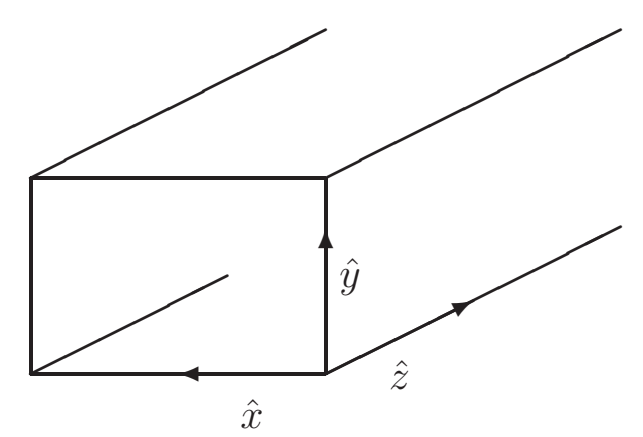

Figura 3.1: Sistema de coordenadas en una guía rectangular

Los dispositivos con geometría inductiva son de una gran aplicabilidad, y han sido tradi- 
Análisis de problemas inductivos en guías rectangulares

cionalmente un elemento clave en el diseño de muchos dispositivos de microondas, tales como filtros paso banda [11, 12] y de banda eliminada [13], filtros de postes acoplados $[34,13]$, adaptadores [35], desfasadores [35], circuladores [14, 36], cavidades con esquinas redondeadas [9], codos biselados [15, 14, 10], o diplexores y multiplexores [37]. El análisis correcto y el diseño de estos dispositivos es de gran importancia para muchas aplicaciones tales como comunicación de satélite e inalámbricas [38]. Por lo tanto, el análisis de discontinuidades y obstáculos inductivos, presentes en muchos dispositivos reales de microondas, ha recibido recientemente una considerable atención en las publicaciones técnicas de este sector $[9,10]$.

\subsubsection{Métodos de análisis existentes}

Los métodos existentes para el análisis de los dispositivos de microondas que se han mencionado con anterioridad pueden clasificarse en tres grandes grupos:

- métodos analíticos,

- métodos numéricos,

- y métodos híbridos.

Algunos ejemplos de métodos analíticos, también denominados modales, son el método de la Matriz de Admitancias Generalizada (Generalized Admittance Matrix o GAM) [39], y el método de la Matriz de Dispersión Generalizada (Generalized Sacttering Matrix o MDG) [40]. Estos métodos proporcionan resultados muy exactos y de una forma eficiente desde el punto de vista computacional. No obstante, presentan la desventaja de que son sólo aplicables a unos pocos problemas canónicos con geometrías regulares.

Una alternativa a los métodos analíticos son los métodos numéricos o de discretización espacial, tales como el método de Elementos Finitos (Finite Element o FE) [35, 41] o el método de diferencias finitas en el dominio temporal (Finite Difference Time Domain o FDTD) [42]. Estos métodos son capaces de analizar problemas con geometrías arbitrarias, pero su desventaja es el elevado consumo de memoria y de tiempo de CPU.

Como alternativa tanto a los métodos analíticos como a los numéricos, nacieron métodos que intentaban sacar partido de las ventajas de estos dos tipos de métodos: la eficiencia de los métodos modales y la flexibilidad de los métodos numéricos. Este es el objetivo de los llamados métodos híbridos, los cuales combinan las técnicas numéricas y analíticas para analizar de forma eficiente una gran variedad de problemas. En los últimos años se han desarrollado y documentado un gran número de técnicas híbridas han sido desarrolladas y documentadas en los últimos años, alcanzándose con ellas una flexibilidad cada vez mayor. Por ejemplo, un poste metálico o dieléctrico en una guía rectangular se analiza en [12, 43, 21] con una combinación del método de los momentos y acoplo modal (Mode-Matching). En [44] se presenta un método híbrido más flexible que es capaz de analizar múltiples obstáculos dieléctricos o metálicos en una guía rectangular. Sin embargo, este método necesita pasar de parámetros de admitancia a parámetros de dispersión, lo que puede producir en algunos casos singularidades. Además, no es capaz de analizar discontinuidades. Un método de acoplo modal 
con condición de contorno (Boundary Contour Mode-Matching o BCMM) se ha publicado recientemente en [14]. Este método es capaz de analizar discontinuidades con geometría arbitraria en dispositivos multipuerto, incluyendo la presencia de un poste circular metálico. Más recientemente, se ha publicado un nuevo método BCMM [45] que analiza guías rectangulares con múltiples postes de geometría arbitraria. Sin embargo, no analiza simultáneamente la presencia de múltiples puertos y/o de discontinuidades. El método BCMM de [14] ha sido ampliado por otros autores [10] hasta convertirlo en un método puramente analítico (evitando las integraciones numéricas del método anterior). Con esto han conseguido una mayor eficiencia, pero, sin embargo, el resultado ha sido de una menor flexibilidad, ya que se pueden analizar discontinuidades en dispositivos multipuerto como antes, pero ya no se puede incorporar la existencia de un poste metálico.

\subsubsection{Aplicación del método de análisis de problemas 2-D}

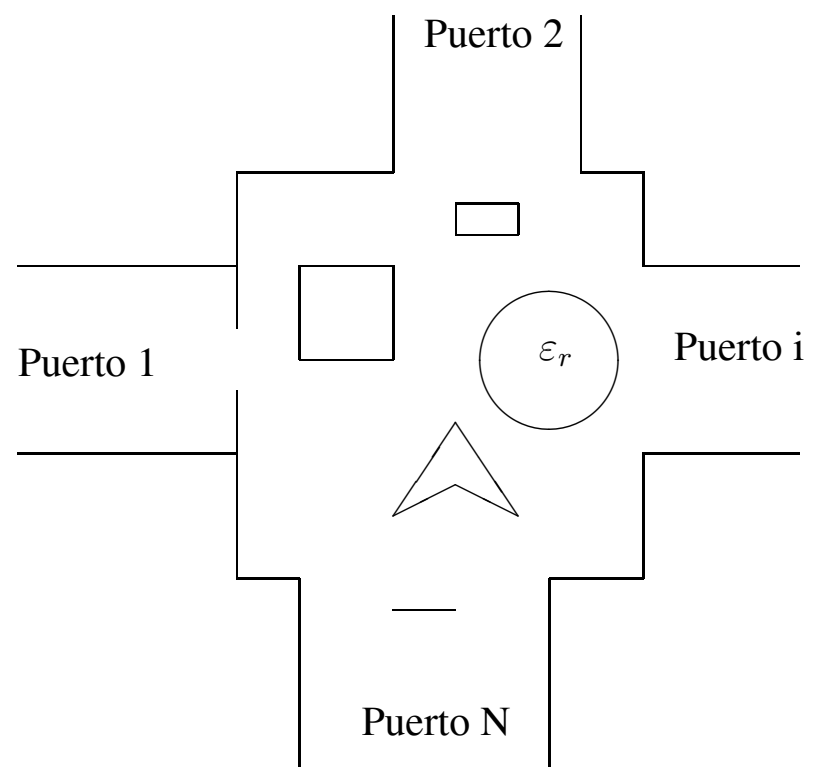

Figura 3.2: Problema bajo análisis. Geometría inductiva arbitraria en guía rectangular con múltiples objetos y discontinuidades

En este capítulo se describe cómo se ha aplicado con éxito el método de análisis de problemas de dispersión bidimensionales del capítulo 2 al análisis de problemas inductivos con geometría arbitraria en guía rectangular. Con este método se puede analizar cualquier problema inductivo en una guía rectangular. Así, se puede analizar un problema tan general como el mostrado en la figura 3.2, donde se muestra un segmento de guía rectangular al cual acceden múltiples puertos y en el que existen múltiples objetos inductivos con geometrías arbitrarias, metálicos y dieléctricos con pérdidas ( $\varepsilon_{r}$ complejo), así como discontinuidades inductivas en varios de los puertos de acceso (iris y ventanas metálicas).

El hecho de que podamos aplicar el método de resolución de problemas 2-D al análisis de una estructura real (geometría inductiva en guía rectangular) se debe a que cuando una guía 
rectangular con geometría inductiva es atacado por modos del tipo $T E_{m 0}$ (entre los cuales se halla el modo fundamental de la guía, el $T E_{10}$ ), tan sólo se excitan modos del mismo tipo. Como los campos correspondientes a los modos $T E_{m 0}$ son invariantes a lo largo del eje $\hat{y}$, y como la geometría de la guía también es invariante a lo largo del eje $\hat{y}$ en el caso de problemas inductivos, se puede abordar el estudio de este tipo de problemas como si se tratara de problemas bidimensionales.

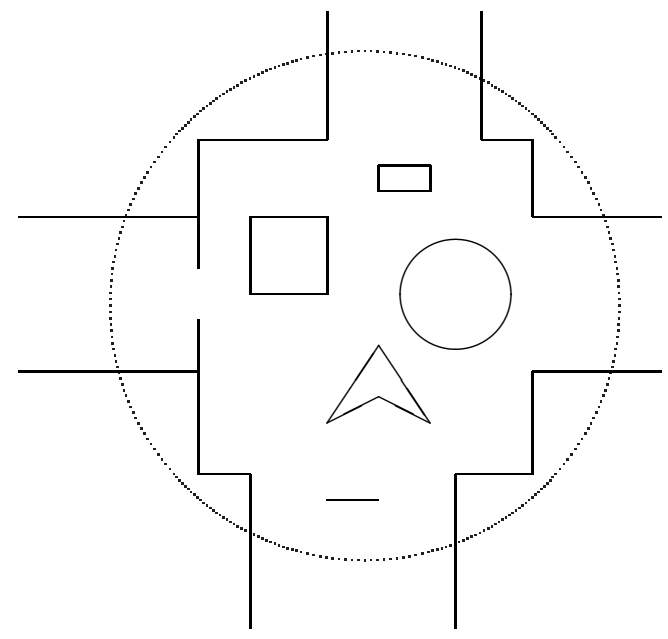

(a) Problema real en guía rectangular

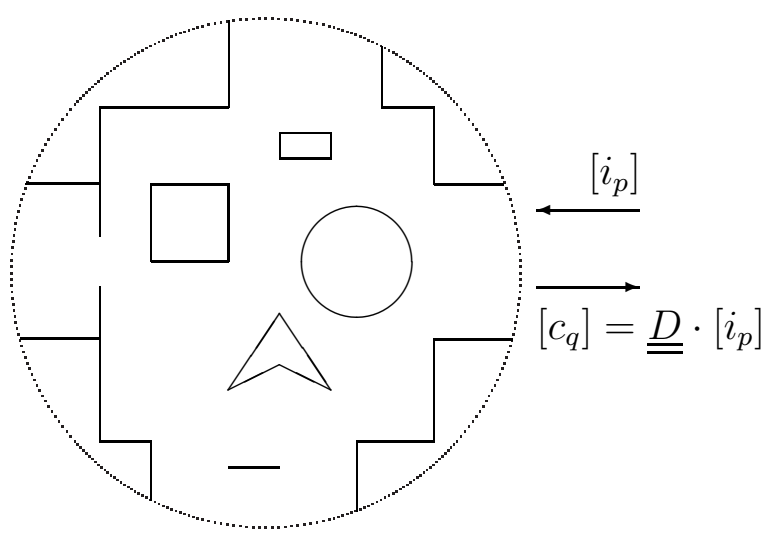

(b) Problema equivalente 2-D en espacio abierto

Figura 3.3: Esquema ilustrativo del segmento irregular de la guía rectangular que es analizado como si se tratara de un problema de dispersión bidimensional en espacio abierto

Para ello se seguirá el procedimiento siguiente:

1. En primer lugar se debe encerrar la parte de la estructura guiada con geometría no canónica en una circunferencia (ver figura 3.3.a). En dicha circunferencia se insertarán $N$ accesos de guía rectangular con geometría canónica.

2. A continuación se considerará lo que queda dentro de dicha circunferencia como un problema de dispersión múltiple 2-D en espacio abierto. En la figura 3.3.b se muestra lo que quedaría dentro de la circunferencia, que en el problema 2-D equivalente consistiría en un problema de dispersión múltiple formado por los siguientes dispersores individuales:

- Cuatro postes 2-D (uno con sección cuadrada, otro con sección circular, otro con sección rectangular, y otro con sección en forma de estrella).

- Varias tiras metálicas 2-D en las que se han segmentado las paredes de la estructura guiada y la ventana inductiva del puerto 1 .

- Una tira 2-D para modelar el iris inductivo del puerto $N$. 
3. El siguiente paso es obtener la matriz de dispersión $\underline{\underline{D}}$ que relaciona el espectro cilíndrico de campo dispersado con el de campo incidente. Para ello se utilizará alguno de los métodos de análisis descritos en el capítulo anterior.

4. Finalmente, se debe obtener una matriz de parámetros circuitales para la estructura guiada. Aunque la matriz $\underline{\underline{D}}$ contiene toda la información sobre el comportamiento electromagnético de la estructura guiada, es necesario expresar esta información en forma de parámetros circuitales. De esta forma se puede enlazar dicha estructura de forma sencilla con otros bloques, caracterizados también mediante parámetros circuitales, utilizando teoría simple de circuitos.

Cuando deseamos caracterizar dispositivos complejos mediante parámetros circuitales, las matrices de dichos parámetros que sólo tienen en cuenta interacciones asociadas al modo fundamental, resultan insuficientes. En el diseño de los actuales componentes de microondas, debido a su complejidad, resulta imprescindible que cada bloque de dichos componentes sea caracterizado teniendo en cuenta las interacciones entre modos de orden superior. Es por tanto necesario recurrir a matrices de parámetros circuitales generalizadas, las cuales relacionan amplitudes no sólo de los modos fundamentales en cada puerto de acceso, sino también de modos de orden superior, tantos como sean necesarios para una correcta caracterización de la estructura.

Se han desarrollado dos métodos diferentes para obtener matrices generalizadas de parámetros circuitales :

- Método para la obtención de matrices de admitancias utilizando cortocircuitos. Una vez calculada la matriz de admitancias se puede obtener la matriz de dispersión mediante una sencilla transformación. Este método se describe con detalle en la sección 3.3.

- Método para la obtención de la matriz de dispersión realizando un acoplo de modos cilíndricos a modos guiados en los $N$ puertos de acceso a la estructura guiada. Este otro método se describe en la sección 3.4. 


\subsection{Matrices generalizadas de parámetros circuitales en guías de onda}

En esta sección se describirá cómo son la matriz de admitancias generalizada (MAG) y la matriz de dispersión generalizada (MDG) en el caso de una estructura guiada. En sucesivas secciones se detalla cómo se obtienen la MAG y la MDG a partir de la matriz de dispersión $\underline{\underline{D}}$ calculada mediante el método híbrido 2-D.

\subsubsection{Amplitudes de tensión y corriente modales}

En una guía de ondas, los campos eléctrico y magnético los podemos expresar como una combinación lineal de las infinitas soluciones de la ecuación de onda. Estas soluciones se clasifican en tres familias de soluciones o modos de propagación: modos TEM, TE y TM. En el caso que nos ocupa (guía rectangular con estructura inductiva), tan sólo se propagarán por la guía soluciones del tipo TE (onda Transversal Eléctrica), ya que la excitación será siempre el modo fundamental $\left(T E_{10}\right)$, y las estructuras que analizaremos tendrán una geometría invariante en la dirección de eje $y$ (ver figura 3.1). Esto último garantiza que sólo se excitarán en las estructuras bajo análisis modos $T E_{m o}$. De modo que los campos eléctrico y magnético transversales en el puerto de acceso $i$ los podremos expresar como una combinación lineal de dichas soluciones $T E_{m 0}$ [20]:

$$
\begin{aligned}
& \vec{E}_{t}^{(i)}\left(x_{i}, y_{i}, z_{i}\right)=\sum_{m=1}^{\infty} V_{m}^{(i)}\left(z_{i}\right) \vec{e}_{m}^{(i)}\left(x_{i}, y_{i}\right) \simeq \sum_{m=1}^{M_{i}} V_{m}^{(i)}\left(z_{i}\right) \vec{e}_{m}^{(i)}\left(x_{i}, y_{i}\right) \\
& \vec{H}_{t}^{(i)}\left(x_{i}, y_{i}, z_{i}\right)=\sum_{m=1}^{\infty} I_{m}^{(i)}\left(z_{i}\right) \vec{h}_{m}^{(i)}\left(x_{i}, y_{i}\right) \simeq \sum_{m=1}^{M_{i}} I_{m}^{(i)}\left(z_{i}\right) \vec{h}_{m}^{(i)}\left(x_{i}, y_{i}\right)
\end{aligned}
$$

donde

- $\left(x_{i}, y_{i}, z_{i}\right)$ : son las coordenadas locales del acceso $i$, definidas según se indica en la figura 3.1.

- $M_{i}$ : Número de modos en cada puerto del acceso $i$ que se considerarán para el correcto análisis de la estructura.

- $\vec{e}_{m}^{(i)}$ y $\vec{h}_{m}^{(i)}$ : funciones vectoriales normalizadas, soluciones de la ecuación de onda para campo eléctrico y magnético en el puerto de acceso $i$ de la estructura guiada, con $m \in[1,2, \cdots, \infty[$. Estas funciones vectoriales se considerarán normalizadas según [20].

- $V_{m}^{(i)}\left(z_{i}\right), I_{m}^{(i)}\left(z_{i}\right)$ : Funciones de tensión e intensidad de corriente que describen la variación de las amplitudes (valor eficaz) de campo eléctrico y magnético para el $m$ ésimo modo en el acceso $i$ a lo largo de la dirección de propagación. 


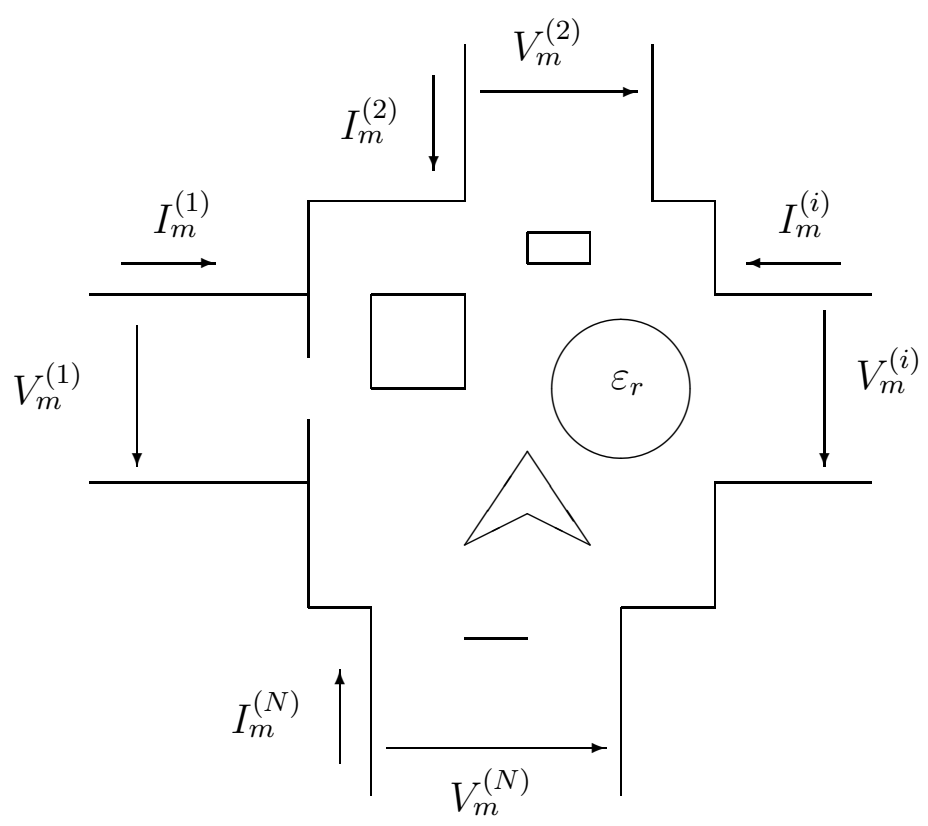

Figura 3.4: Tensiones y corrientes modales para el modo $T E_{m 0}$ en la estructura de la figura 3.2

En la figura 3.4 se han representado las amplitudes modales de tensión y corriente sobre la estructura general de la figura 3.2.

En el caso de guías de onda rectangulares, y para modos $T E_{m 0}$ :

$$
\begin{aligned}
\vec{e}_{m}^{(i)}\left(x_{i}, y_{i}\right) & =-\hat{y}_{i} \frac{\sqrt{2}}{\sqrt{a_{i} b_{i}}} \sin \left(\frac{m \pi}{a_{i}} x_{i}\right) \\
\vec{h}_{m}^{(i)}\left(x_{i}, y_{i}\right) & =\hat{x}_{i} \frac{\sqrt{2}}{\sqrt{a_{i} b_{i}}} \sin \left(\frac{m \pi}{a_{i}} x_{i}\right)
\end{aligned}
$$

donde $a_{i}$ y $b_{i}$ son la anchura y altura del acceso $i$, y los vectores unitarios $\hat{x}_{i}$ e $\hat{y}_{i}$ son los definidos en la figura 3.1. Estas funciones vectoriales normalizadas cumplen las propiedades de ortogonalidad y unitariedad:

$$
\iint_{S} \vec{e}_{m}^{(i)} \cdot \vec{e}_{n}^{(i)} \cdot \mathrm{d} S= \begin{cases}1 & m=n \\ 0 & m \neq n\end{cases}
$$

donde $S$ es la sección transversal de la guía de ondas.

Las tensiones y corrientes de cada modo se pueden obtener proyectando los campos eléctrico y magnético transversales sobre las funciones vectoriales de la siguiente forma:

$$
\begin{aligned}
V_{m}^{(i)}\left(z_{i}\right) & =\iint_{S} \vec{E}_{t}^{(i)} \cdot \vec{e}_{m}^{(i)} \mathrm{d} S \\
I_{m}^{(i)}\left(z_{i}\right) & =\iint_{S} \vec{H}_{t}^{(i)} \cdot \vec{h}_{m}^{(i)} \mathrm{d} S
\end{aligned}
$$




\subsubsection{Matriz de admitancias generalizada (MAG)}

La matriz de admitancias generalizada relaciona las amplitudes modales de corrientes y tensiones de la siguiente forma:

$$
I_{m}^{(i)}=\sum_{j=1}^{N} \sum_{n=1}^{M_{j}} Y_{m n}^{(i, j)} V_{n}^{(j)} \quad i \in[1, \cdots, N], m \in\left[1, \cdots, M_{i}\right]
$$

siendo $M_{i}$ el número de modos que se consideran necesarios para expresar correctamente el campo en el acceso $i$. Nótese que en la expresión anterior se expande la corriente modal del $m$-ésimo modo en el acceso $i$ como combinación lineal de las tensiones modales de todos los modos de cada uno de los accesos. Los pesos de esa suma son los coeficientes $Y_{m n}^{(i, j)}$, que son los que constituyen la matriz de admitancias generalizada.

En una red de $N$ accesos y con $M_{i}$ modos en cada uno de dichos accesos $(i \in[1, \cdots, N])$, existen $M$ corrientes y tensiones modales, siendo $M$ :

$$
M=\sum_{i=1}^{N} M_{i}
$$

Por lo tanto (3.8) representa $M$ ecuaciones con $M$ incógnitas. Este sistema de ecuaciones se puede expresar de forma matricial de la siguiente forma:

$$
\left[\begin{array}{c}
I^{(1)} \\
I^{(2)} \\
\ldots \\
I^{(N)}
\end{array}\right]=\left[\begin{array}{cccc}
Y^{(1,1)} & Y^{(1,2)} & \ldots & Y^{(1, N)} \\
Y^{(2,1)} & Y^{(2,2)} & \ldots & Y^{(2, N)} \\
\ldots \ldots \ldots \ldots \ldots \ldots \ldots \ldots \ldots \ldots \\
Y^{(N, 1)} & Y^{(N, 2)} & \ldots & Y^{(N, N)}
\end{array}\right] \cdot\left[\begin{array}{c}
V^{(1)} \\
V^{(2)} \\
\ldots \\
V^{(N)}
\end{array}\right]=\underline{\underline{Y}} \cdot\left[\begin{array}{c}
V^{(1)} \\
V^{(2)} \\
\ldots \\
V^{(N)}
\end{array}\right]
$$

donde cada elemento $I^{(i)}, V^{(i)}$ e $Y^{(i, j)}$ es un sub-bloque matricial con el siguiente contenido:

$$
\begin{aligned}
I^{(i)}= & {\left[\begin{array}{llll}
I_{1}^{(i)} & I_{2}^{(i)} & \ldots & I_{M_{i}}^{(i)}
\end{array}\right]^{T} } \\
V^{(i)}= & {\left[\begin{array}{llll}
V_{1}^{(i)} & V_{2}^{(i)} & \ldots & V_{M_{i}}^{(i)}
\end{array}\right]^{T} } \\
Y^{(i, j)}= & {\left[\begin{array}{llll}
Y_{11}^{(i, j)} & Y_{12}^{(i, j)} & \ldots & Y_{1 M_{j}}^{(i, j)} \\
Y_{21}^{(i, j)} & Y_{22}^{(i, j)} & \ldots & Y_{2 M_{j}}^{(i, j)} \\
\ldots \ldots & \ldots \ldots & \ldots & \ldots \ldots \\
Y_{M_{i} 1}^{(i, j)} & Y_{M_{i} 2}^{(i, j)} & \ldots & Y_{M_{i} M_{j}}^{(i, j)}
\end{array}\right] }
\end{aligned}
$$

Cada sub-bloque $I^{(i)}$ o $V^{(i)}$ representa todas las amplitudes modales de corriente o tensión del acceso $i$. Por otro lado, cada sub-bloque $Y^{(i, j)}$ contiene las contribuciones de las amplitudes de tensión de los modos del acceso $j$ sobre amplitudes de corriente de los modos del acceso $i$.

La matriz $\underline{\underline{Y}}$ de la ecuación (3.10) es la matriz de admitancias generalizada (MAG). 


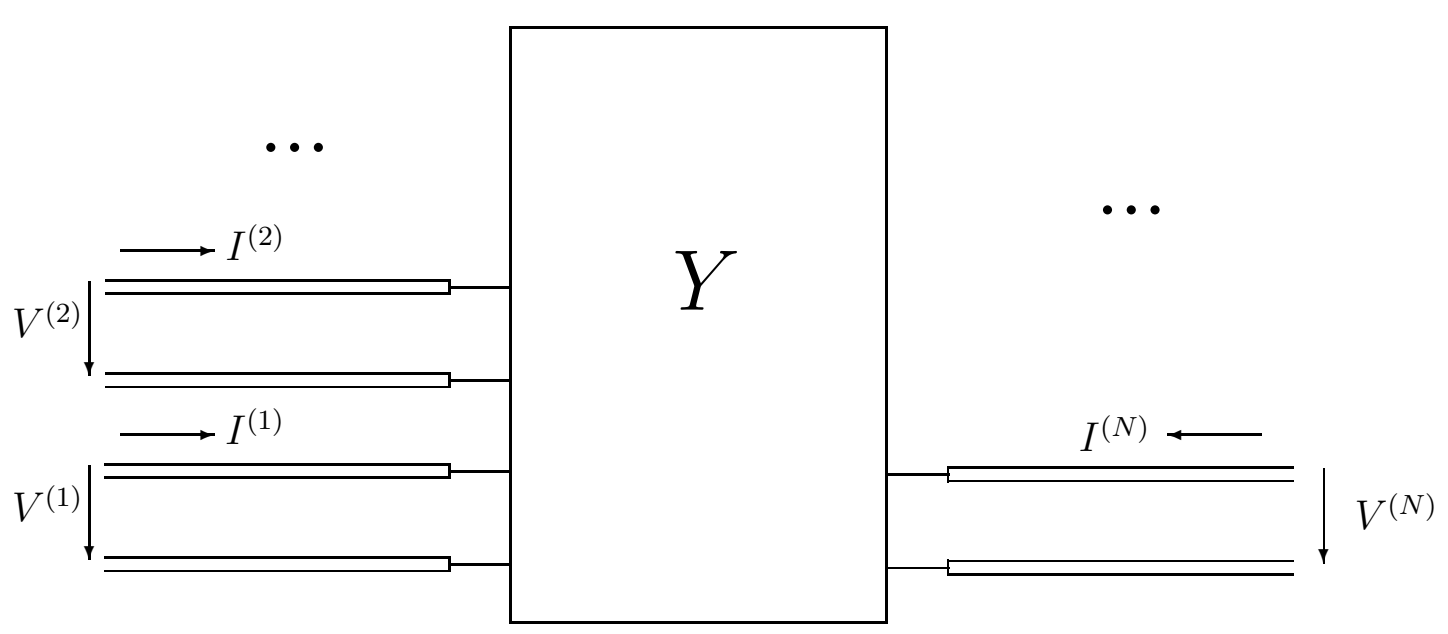

Figura 3.5: Circuito equivalente al de la figura 3.4 utilizando la matriz de admitancias generalizadas

En la figura 3.5 se muestra un circuito equivalente al de la figura 3.4. Como se puede apreciar la MAG caracteriza el comportamiento de la red hacia fuera de sus puertos de acceso al proporcionar la relación entre tensiones y corrientes modales, de modo que se puede trabajar con la estructura de la figura 3.4 de forma sencilla, sin especificar cómo es dicha estructura por dentro.

\subsubsection{Matriz de dispersión generalizada (MDG)}

Si bien la MAG relacionaba amplitudes de tensiones y corrientes modales, la matriz de dispersión generalizada (MDG), relaciona amplitudes de ondas progresivas y regresivas.

Por cada modo de propagación puede existir una onda progresiva y otra regresiva, es decir, la amplitud de tensión y corriente en cada punto del acceso $i$ se puede expresar como la suma de una onda progresiva y otra regresiva:

$$
\begin{aligned}
V_{m}^{(i)}\left(z_{i}\right) & =V_{m}^{+(i)} e^{-\gamma_{m}^{(i)} z_{i}}+V_{m}^{-(i)} e^{\gamma_{m}^{(i)} z_{i}} \\
I_{m}^{(i)}\left(z_{i}\right) & =I_{m}^{+(i)} e^{-\gamma_{m}^{(i)} z_{i}}+I_{m}^{-(i)} e^{\gamma_{m}^{(i)} z_{i}} \\
& =\frac{V_{m}^{+(i)}}{Z_{0 m}^{(i)}} e^{-\gamma_{m}^{(i)} z_{i}}-\frac{V_{m}^{-(i)}}{Z_{0 m}^{(i)}} e^{\gamma_{m}^{(i)} z_{i}}
\end{aligned}
$$

donde $\gamma_{m}^{(i)}$ es la constante de propagación del $m$-ésimo modo de propagación en el acceso $i, V_{m}^{+(i)}, V_{m}^{-(i)}, I_{m}^{+(i)}$ y $I_{m}^{-(i)}$ son las amplitudes de las $m$-ésimas ondas modales de tensión y corriente (progresiva y regresiva) en el acceso $i$, y $Z_{0 m}^{(i)}$ es la impedancia característica del $m$-ésimo modo en el acceso $i$.

Sustituyendo (3.14) y (3.15) en (3.1) y (3.2): 


$$
\begin{aligned}
\vec{E}_{t}^{(i)} & =\sum_{m=1}^{M_{i}} V_{m}^{+(i)} \vec{e}_{m}^{(i)} e^{-\gamma_{m}^{(i)} z_{i}}+\sum_{m=1}^{M_{i}} V_{m}^{-(i)} \vec{e}_{m}^{(i)} e^{\gamma_{m}^{(i)} z_{i}} \\
\vec{H}_{t}^{(i)} & =\sum_{m=1}^{M_{i}} I_{m}^{+(i)} \vec{h}_{m}^{(i)} e^{-\gamma_{m}^{(i)} z_{i}}+\sum_{m=1}^{M_{i}} I_{m}^{-(i)} \vec{h}_{m}^{(i)} e^{\gamma_{m}^{(i)} z_{i}} \\
& =\sum_{m=1}^{M_{i}} \frac{V_{m}^{+(i)}}{Z_{0 m}^{(i)}} \vec{h}_{m}^{(i)} e^{-\gamma_{m}^{(i)} z_{i}}-\sum_{m=1}^{M_{i}} \frac{V_{m}^{-(i)}}{Z_{0 m}^{(i)}} \vec{h}_{m}^{(i)} e^{\gamma_{m}^{(i)} z_{i}}
\end{aligned}
$$

Definiremos unas amplitudes normalizadas para las $m$-ésimas ondas progresiva y regresiva de la siguiente forma:

$$
\begin{aligned}
a_{m}^{(i)} & =\frac{V_{m}^{+(i)}}{\sqrt{Z_{0 m}^{(i)}}} \\
b_{m}^{(i)} & =\frac{V_{m}^{-(i)}}{\sqrt{Z_{0 m}^{(i)}}}
\end{aligned}
$$

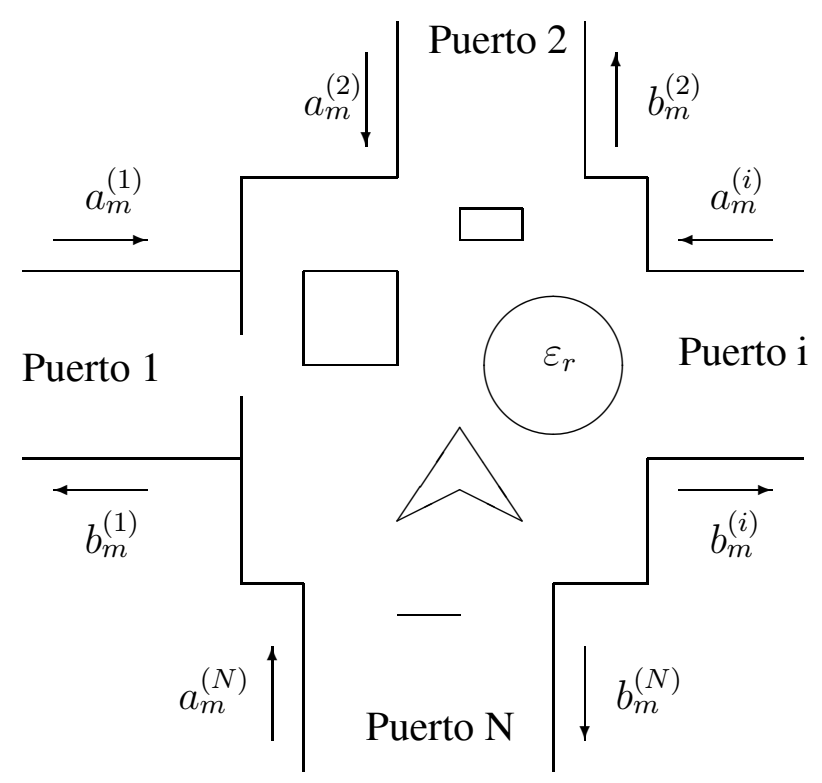

Figura 3.6: Amplitudes de las ondas progresiva y regresiva normalizadas en cada puerto de acceso de la figura 3.2 para el modo $T E_{m 0}$

En la figura 3.6 se han representado las amplitudes normalizadas de las ondas progresiva y regresiva en cada acceso correspondientes al modo $T E_{m 0}$.

Utilizando las relaciones (3.18) y (3.19), expresamos finalmente los campos transversales en función de las amplitudes modales normalizadas que aparecen en la MDG: 


$$
\begin{aligned}
\vec{E}_{t}^{(i)} & =\sum_{m=1}^{M_{i}} a_{m}^{(i)} \sqrt{Z_{0 m}^{(i)}} \vec{e}_{m}^{(i)} e^{-\gamma_{m}^{(i)} z_{i}}+\sum_{m=1}^{M_{i}} b_{m}^{(i)} \sqrt{Z_{0 m}^{(i)}} \vec{e}_{m}^{(i)} e^{\gamma_{m}^{(i)} z_{i}} \\
\vec{H}_{t}^{(i)} & =\sum_{m=1}^{M_{i}} \frac{a_{m}^{(i)}}{\sqrt{Z_{0 m}^{(i)}}} \vec{h}_{m}^{(i)} e^{-\gamma_{m}^{(i)} z_{i}}-\sum_{m=1}^{M_{i}} \frac{b_{m}^{(i)}}{\sqrt{Z_{0 m}^{(i)}}} \vec{h}_{m}^{(i)} e^{\gamma_{m}^{(i)} z_{i}}
\end{aligned}
$$

Definiendo unas nuevas funciones vectoriales normalizadas para cada acceso $i$ :

$$
\begin{aligned}
\vec{e}_{m}^{(i) \prime^{\prime}} & =\sqrt{Z_{0 m}^{(i)}} \vec{e}_{m}^{(i)} \\
\vec{h}_{m}^{(i)^{\prime}} & =\sqrt{Z_{0 m}^{(i)}} \vec{h}_{m}^{(i)}
\end{aligned}
$$

Las expresiones (3.20) y (3.21) se simplifican pues de la siguiente forma:

$$
\begin{aligned}
\vec{E}_{t}^{(i)} & =\sum_{m=1}^{M_{i}} a_{m}^{(i)} \vec{e}_{m}^{(i)^{\prime}} e^{-\gamma_{m}^{(i)} z_{i}}+\sum_{m=1}^{M_{i}} b_{m}^{(i)} \vec{e}_{m}^{(i)^{\prime}} e^{\gamma_{m}^{(i)} z_{i}} \\
\vec{H}_{t}^{(i)} & =\sum_{m=1}^{M_{i}} a_{m}^{(i)} Y_{0 m}^{(i)} \vec{h}_{m}^{(i)^{\prime}} e^{-\gamma_{m}^{(i)} z_{i}}-\sum_{m=1}^{M_{i}} b_{m}^{(i)} Y_{0 m}^{(i)} \vec{h}_{m}^{(i)^{\prime}} e^{\gamma_{m}^{(i)} z_{i}}
\end{aligned}
$$

donde $Y_{0 m}^{(i)}=1 / Z_{0 m}^{(i)}$.

La matriz de dispersión generalizada de una red de $N$ accesos de guíaondas proporciona una relación entre las amplitudes normalizadas de las ondas modales progresivas y regresivas en cada puerto de acceso de la red de la siguiente forma:

$$
b_{m}^{(i)}=\sum_{j=1}^{M} \sum_{n=1}^{M_{j}} S_{m n}^{(i, j)} a_{n}^{(j)} \quad i \in[1, \cdots, N], m \in\left[1, \cdots, M_{i}\right]
$$

siendo $M_{i}$ el número de modos que se consideran necesarios para expresar correctamente el campo en el acceso $i$. En la expresión anterior se expande la amplitud normalizada del $m$-ésimo modo reflejado o regresivo en el acceso $i$ como combinación lineal de las amplitudes normalizadas de todos los modos incidentes o progresivos de cada uno de los accesos. Los pesos de esa suma son los coeficientes $S_{m n}^{(i, j)}$, que son los que constituyen la matriz de dispersión generalizada.

En una red de $N$ accesos y con $M_{i}$ modos en cada uno de dichos accesos $(i \in[1, \cdots, N])$, existen $M$ ondas progresivas y regresivas, siendo $M=\sum_{i=1}^{N} M_{i}$. 
Por lo tanto (3.26) representa $M$ ecuaciones con $M$ incógnitas. Este sistema de ecuaciones se puede escribir en forma matricial del siguiente modo:

$$
\left[\begin{array}{c}
b^{(1)} \\
b^{(2)} \\
\ldots \\
b^{(N)}
\end{array}\right]=\left[\begin{array}{cccc}
S^{(1,1)} & S^{(1,2)} & \ldots & S^{(1, N)} \\
S^{(2,1)} & S^{(2,2)} & \ldots & S^{(2, N)} \\
\ldots \ldots \ldots \ldots \ldots \ldots & \ldots \ldots & \ldots \ldots \\
S^{(N, 1)} & S^{(N, 2)} & \ldots & S^{(N, N)}
\end{array}\right] \cdot\left[\begin{array}{c}
a^{(1)} \\
a^{(2)} \\
\ldots \\
a^{(N)}
\end{array}\right]=\underline{\underline{S}} \cdot\left[\begin{array}{c}
a^{(1)} \\
a^{(2)} \\
\ldots \\
a^{(N)}
\end{array}\right]
$$

donde cada elemento $b^{(i)}, a^{(i)}$ y $S^{(i, j)}$ es un sub-bloque con el siguiente contenido:

$$
\begin{aligned}
a^{(i)}= & {\left[\begin{array}{llll}
a_{1}^{(i)} & a_{2}^{(i)} & \ldots & a_{M_{i}}^{(i)}
\end{array}\right]^{T} } \\
b^{(i)}= & {\left[\begin{array}{llll}
b_{1}^{(i)} & b_{2}^{(i)} & \ldots & b_{M_{i}}^{(i)}
\end{array}\right]^{T} } \\
S^{(i, j)}= & {\left[\begin{array}{llll}
S_{11}^{(i, j)} & S_{12}^{(i, j)} & \ldots & S_{1 M_{j}}^{(i, j)} \\
S_{21}^{(i, j)} & S_{22}^{(i, j)} & \ldots & S_{2 M_{j}}^{(i, j)} \\
\ldots \ldots & \ldots \ldots & \ldots & \ldots \ldots \\
S_{M_{i} 1}^{(i, j)} & S_{M_{i} 2}^{(i, j)} & \ldots & S_{M_{i} M_{j}}^{(i, j)}
\end{array}\right] }
\end{aligned}
$$

Cada sub-bloque $b^{(i)}$ o $a^{(i)}$ representa todas las amplitudes normalizadas de ondas incidentes o reflejadas del acceso $i$. Por otro lado, cada sub-bloque $S^{(i, j)}$ contiene las contribuciones de las ondas incidentes de los modos del acceso $j$ sobre las ondas reflejadas de los modos del acceso $i$.

La matriz $\underline{\underline{S}}$ de la ecuación (3.27) es la matriz de dispersión generalizada (MDG).

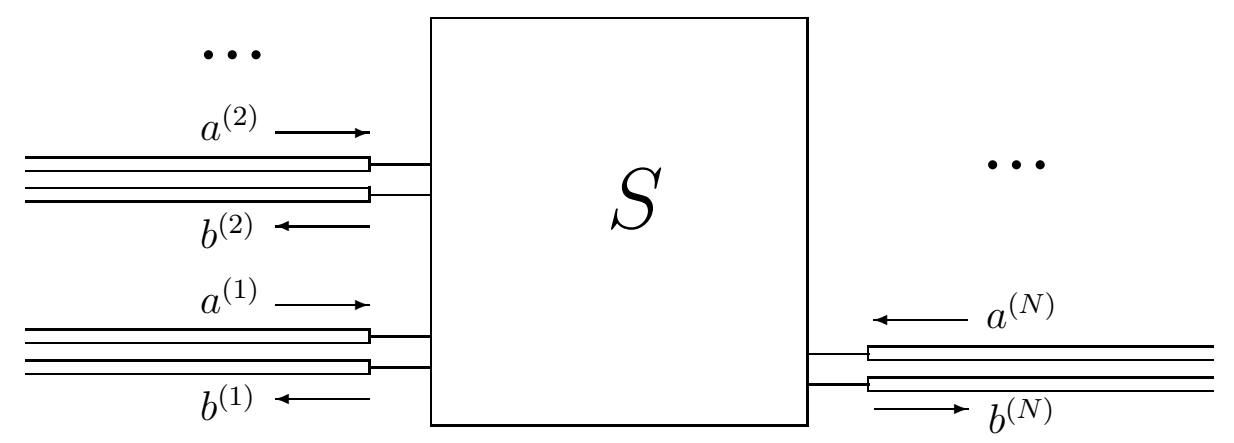

Figura 3.7: Circuito equivalente al de la figura 3.6 utilizando parámetros de dispersión

En la figura 3.7 se muestra un circuito equivalente al de la figura 3.6. La MDG, al igual que la MAG, caracteriza el comportamiento de la red hacia fuera de sus puertos de acceso. 


\subsection{Obtención de la MAG utilizando cortocircuitos}

\subsubsection{Metodología}

De acuerdo con la definición de los parámetros de admitancia dada por (3.8), se puede deducir la siguiente expresión para dichos parámetros:

$$
Y_{m n}^{(i, j)}=\frac{I_{m}^{(i)}}{V_{n}^{(j)}}, \quad \operatorname{con} \begin{cases}V_{p}^{(k)}=0 & k \neq j, \quad \forall p \\ V_{p}^{(j)}=0 & p \neq n\end{cases}
$$

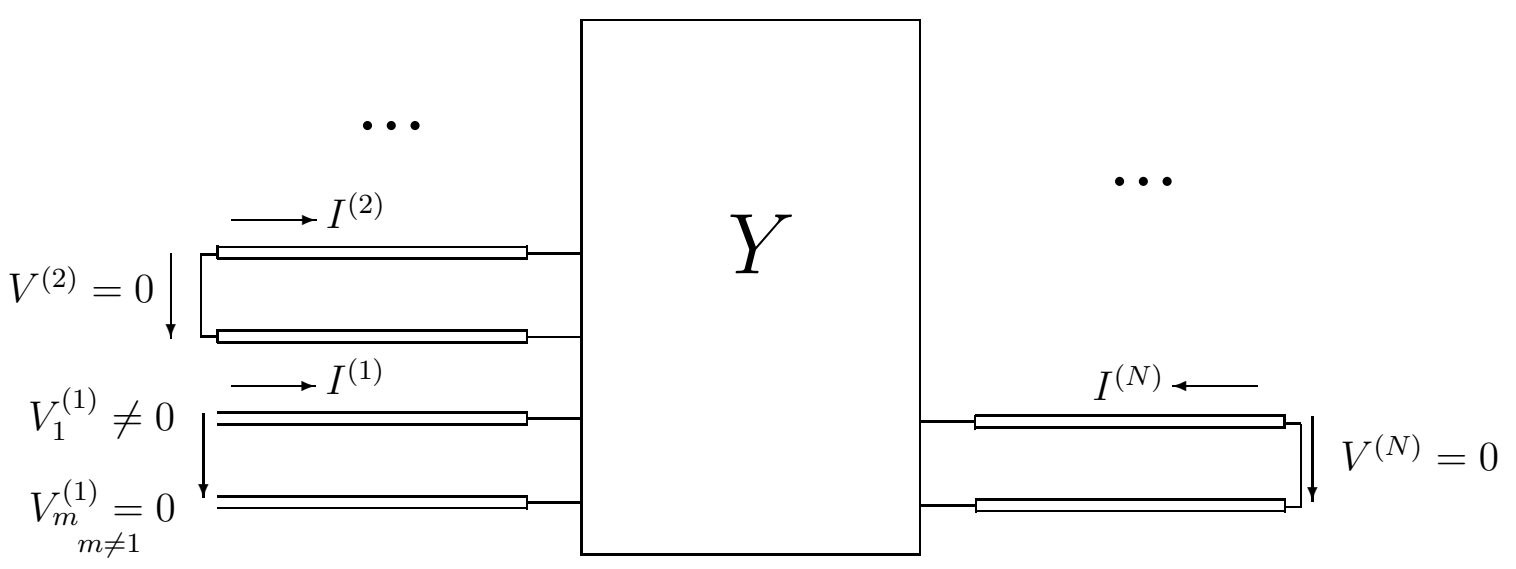

Figura 3.8: Configuración para el cálculo de los parámetros $Y_{m 1}^{(i, 1)}, i \in[1, \ldots, N], m \in$ $\left[1, \ldots, M_{i}\right]$

La ecuación anterior indica que se pueden calcular los parámetros $Y_{m n}^{(i, j)}$ de la red (con $i=$ $\left.[1, \ldots, N], m=\left[1, \ldots, M_{i}\right]\right)$, si se consigue que las tensiones se anulen en todos los puertos excepto en el puerto $j$ donde las tensiones han de ser nulas todas excepto la $n$-ésima. Esto se consigue colocando un cortocircuito en todos los puertos excepto en el $j\left(V_{p}^{(k)}=0, k \neq\right.$ $j, \forall p)$, y excitando el acceso $j$ sólo con el modo $n$-ésimo $\left(V_{p}^{(j)}=0, p \neq n\right)$. En la figura 3.8 se muestra la estructura que se debe analizar para calcular los parámetros $Y_{m 1}^{(i, 1)}$, con $i=$ $[1, \ldots, N], m=\left[1, \ldots, M_{i}\right]$. No obstante, la red de la figura 3.8 es un circuito equivalente. En la figura 3.9 se muestra la estructura real que se debe analizar mediante el método de análisis híbrido de problemas 2-D, suponiendo que la estructura guiada original sea la de la figura 3.2. Nótese que es necesario colocar cortocircuitos en todos los accesos menos en uno, y que además se debe conseguir que la excitación en ese acceso sea únicamente la del un determinado modo de dicho acceso.

Una vez construida una estructura como la de la figura 3.9, pero suponiendo que hay cortocircuitos en todos los accesos excepto en el acceso $j$, y que en el acceso $j$ tan sólo se excita el modo $T E_{n 0}^{(j)}$, podemos obtener los parámetros $Y_{m n}^{(i, j)}\left(i=[1, \ldots, N], m=\left[1, \ldots, M_{i}\right]\right) \mathrm{de}$ 


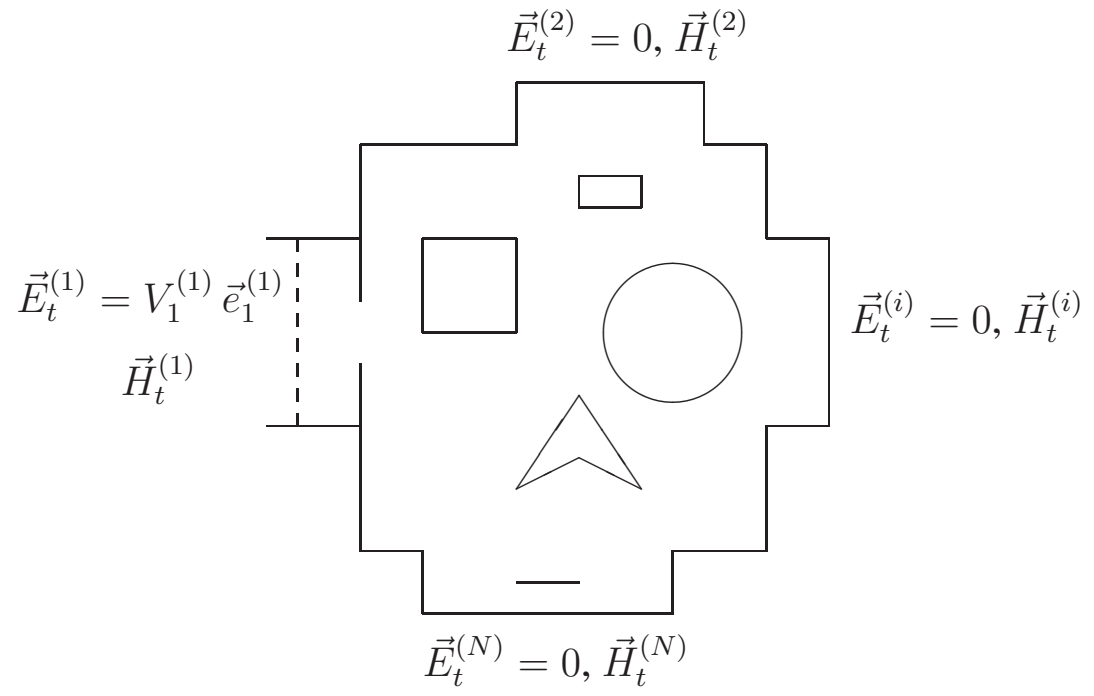

Figura 3.9: Estructura que debe analizarse para el cálculo de los parámetros $Y_{m 1}^{(i, 1)}$ de la red de la figura 3.2

la siguiente forma:

$$
Y_{m n}^{(i, j)}=\frac{I_{m}^{(i)}\left(z_{i}=d^{(i)}\right)}{V_{n}^{(j)}\left(z_{j}=d^{(j)}\right)}
$$

donde $d^{(i)}$ y $d^{(j)}$ son, respectivamente, las posiciones de los planos de referencia en los accesos $i$ y $j$. Utilizando (3.6) y (3.7):

$$
Y_{m n}^{(i, j)}=\frac{I_{m}^{(i)}\left(d^{(i)}\right)}{V_{n}^{(j)}\left(d^{(j)}\right)}=\frac{\iint_{S_{i}} \vec{H}_{t}^{(i)} \cdot \vec{h}_{m}^{(i)} \mathrm{d} S}{\iint_{S_{j}} \vec{E}_{t}^{(j)} \cdot \vec{e}_{n}^{(j)} \mathrm{d} S}
$$

donde $S_{i}$ y $S_{j}$ son las secciones transversales de las guías de ondas de los accesos $i$ y $j$. Como se desea analizar estructuras con guías de ondas rectangulares y geometría invariante en altura, todos los accesos serán guías con la misma altura $b$. Por tanto:

$$
\begin{aligned}
Y_{m n}^{(i, j)} & =\frac{\int_{y_{i}=0}^{b} \int_{x_{i}=0}^{a_{i}} \vec{H}_{t}^{(i)} \cdot \vec{h}_{m}^{(i)} \mathrm{d} x_{i} \mathrm{~d} y_{i}}{\int_{y_{j}=0}^{b} \int_{x_{j}=0}^{a_{j}} \vec{E}_{t}^{(j)} \cdot \vec{e}_{n}^{(j)} \mathrm{d} x_{j} \mathrm{~d} y_{j}}=\frac{b \int_{x_{i}=0}^{a_{i}} \vec{H}_{t}^{(i)} \cdot \vec{h}_{m}^{(i)} \mathrm{d} x_{i}}{b \int_{x_{j}=0}^{a_{j}} \vec{E}_{t}^{(j)} \cdot \vec{e}_{n}^{(j)} \mathrm{d} x_{j}} \\
= & \frac{\int_{x_{i}=0}^{a_{i}} \vec{H}_{t}^{(i)}\left(x_{i}, z_{i}=d^{(i)}\right) \cdot \vec{h}_{m}^{(i)}\left(x_{i}\right) \mathrm{d} x_{i}}{\int_{x_{j}=0}^{a_{j}} \vec{E}_{t}^{(j)}\left(x_{j}, z_{j}=d^{(j)}\right) \cdot \vec{e}_{n}^{(j)}\left(x_{j}\right) \mathrm{d} x_{j}}
\end{aligned}
$$


donde $\left(x_{i}, y_{i}, z_{i}\right)$ y $\left(x_{j}, y_{j}, z_{j}\right)$ son coordenadas locales de los accesos $i$ y $j$ definidas como se indica en la figura 3.1.

La expresión anterior permite concluir que para obtener los parámetros de la MAG el problema se reduce a calcular los campos eléctrico y magnético transversales en los planos de referencia de cada acceso en una estructura con cortocircuitos como la de la figura 3.9, y luego proyectar dichos campos sobre las funciones vectoriales modales correspondientes.

\subsubsection{Excitación del modo $T E_{n 0}^{(j)}$}

Se ha explicado anteriormente que resulta necesario excitar tan sólo un modo en el acceso sin cortocircuito para calcular correctamente los parámetros de admitancia. Para excitar el modo $T E_{n 0}^{(j)}$ en el acceso $j$ de la estructura de la figura 3.9, colocaremos una fuente puntual delante de dicho acceso, de forma que se excite en él el modo deseado.

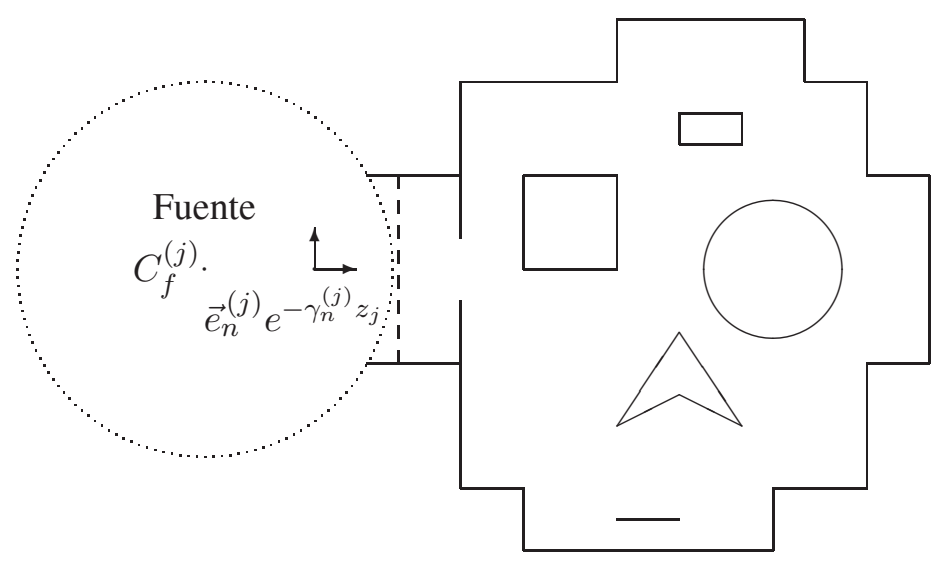

Figura 3.10: Excitación de la estructura de la figura 3.9 con el modo $T E_{n 0}^{(j)}$

En la figura 3.10 se muestra cómo se debe colocar una fuente puntual para excitar el modo $T E_{n 0}^{(j)}$ en el acceso $j$. El procedimiento es el siguiente:

1. Se analiza la estructura de la figura 3.9 con el método de análisis híbrido de estructuras 2-D arbitrarias, y se obtienen las matrices de dispersión de espectros cilíndricos de cada uno de los objetos de la estructura guiada (matrices $\underline{\underline{D_{i}}}$, con $i=\left[1, \ldots, N_{o}\right]$, siendo $N_{o}$ el número de objetos 2-D que conforman la estructura). Estas matrices ya tienen en cuenta el acoplo modal entre objetos.

2. En lugar de suponer un campo incidente con espectro cilíndrico incidente centrado en el centro de los objetos, se debe considerar la incidencia de una campo con un espectro cilíndrico emergente del punto $C_{f}^{(j)}$ (ver figura 3.10).

3. Se construye el espectro de campo emergente de $C_{f}^{(j)}$ de modo que el campo en el interior del acceso $j$ sea el correspondiente al modo $T E_{n 0}^{(j)}$. 
4. Se multiplica el espectro del campo de excitación por las matrices $\underline{\underline{D_{i}}}$ y se obtiene el espectro de campo dispersado por cada uno de los objetos 2-D de la estructura, ante la excitación del modo $T E_{n 0}^{(j)}$.

5. Se calcula el campo dispersado por todos los objetos en cada uno de los planos de referencia de los $N$ puertos de acceso a la estructura.

6. Se calcula el campo de excitación es esos planos de referencia.

7. Se suma el campo de excitación con el dispersado por los objetos, y ya se dispone del valor de los campos eléctrico y magnético total en los $N$ planos de referencia.

8. Se utilizan la expresión (3.34) para calcular los parámetros $Y_{m n}^{(i, j)}$, con $i=[1, \ldots, N]$, $m=\left[1, \ldots, M_{i}\right]$.

9. Se varía el valor de $n$ y se vuelve al punto 3. Se repite este procedimiento variando $n$ de 1 a $M_{i}$.

10. Se vuelve al punto 1, cambiando el acceso de excitación $i$. Se varía $i$ desde 1 hasta $N$.

Una vez terminado el proceso anterior ya se ha construido la MAG por completo.

A continuación se abordará el problema de cómo construir el espectro del campo excitación, para asegurar que en el acceso $j$ se excita el modo $T E_{n 0}^{(j)}$. La excitación debe ser tal que en el acceso $j$ la distribución de campo producido por dicha fuente sea la función modal correspondiente al $n$-ésimo modo con amplitud unidad, y con la fase correspondiente a una onda progresiva. Es decir, se debe excitar un campo en el acceso $j$ de la forma:

$$
\vec{E}_{t}^{(j)}=\vec{e}_{n}^{(j)} e^{-\gamma_{n}^{(j)} z_{j}}=-\hat{y}_{j} \frac{\sqrt{2}}{\sqrt{a_{j} b_{j}}} \sin \left(\frac{n \pi}{a_{j}} x_{j}\right) e^{-\gamma_{n}^{(j)} z_{j}}
$$

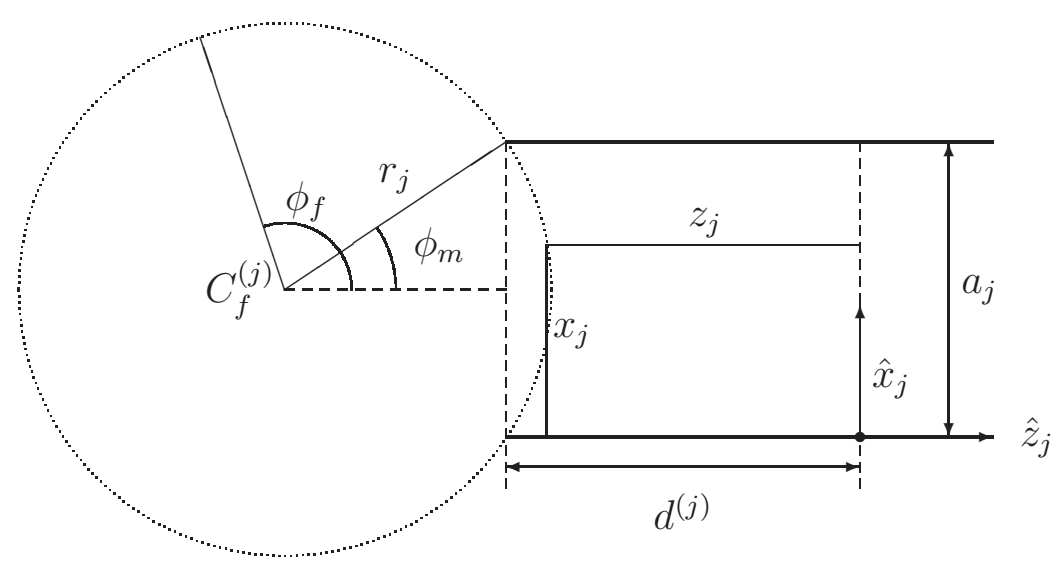

Figura 3.11: Detalle de la excitación del acceso $j$ con el modo $T E_{n 0}^{(j)}$ 
En la figura 3.11 se muestra un detalle de la excitación del modo $T E_{n 0}^{(j)}$ en el acceso $j$. En dicha figura se muestran las coordenadas locales $x_{j}$ y $z_{j}$ del acceso $j$, así como el punto $C_{f}^{(j)}$ donde está centrado el espectro del campo de excitación.

Por otro lado, el campo de excitación se puede poner en función de su espectro, que como ya se ha indicado será un espectro cilíndrico emergente del punto $C_{f}^{(j)}$ :

$$
\vec{E}_{t}^{(j)}=\sum_{q=-N_{f}}^{N_{f}} c_{q} H_{q}^{(2)}\left(k \vec{\rho}_{f}\right) e^{j q \phi_{f}} \hat{z}
$$

donde $\vec{\rho}_{f}$ es la distancia al punto de observación desde el punto $C_{f}$, y $\phi_{f}$ es el ángulo que se muestra en la figura 3.11.

Si forzamos que las expresiones (3.35) y (3.36) coincidan en la circunferencia de radio $r_{j}$ (ver figura 3.11), y teniendo en cuenta que $\hat{y}_{j}=\hat{z}$ (véase la figura 3.30):

$$
\sum_{q=-N_{f}}^{N_{f}} c_{q} H_{q}^{(2)}\left(k r_{j}\right) e^{j q \phi_{f}}=E\left(\phi_{f}\right)= \begin{cases}-\frac{\sqrt{2}}{\sqrt{a_{j} b_{j}}} \sin \left(\frac{n \pi}{a_{j}} x_{j}\right) e^{-\gamma_{n}^{(j)} z_{j}} & \left|\phi_{f}\right| \leq \phi_{m} \\ 0 & \left|\phi_{f}\right|>\phi_{m}\end{cases}
$$

donde las coordenadas $x_{j}$ y $z_{j}$ tienen la siguiente dependencia con $\phi_{f}$ :

$$
\begin{aligned}
x_{j}\left(\phi_{f}\right) & =\frac{a_{j}}{2}+r_{j} \sin \phi_{f} \\
z_{j}\left(\phi_{f}\right) & =-d^{(j)}-r_{j} \cos \phi_{m}+r_{j} \cos \phi_{f}
\end{aligned}
$$

donde:

$$
\phi_{m}=\arctan \left(\frac{a_{j}}{2 r_{j}}\right)
$$

Utilizando las conocidas expresiones de la transformada de Fourier de tiempo discreto (DTFT) y de la transformada inversa

$$
\begin{aligned}
W(\Omega)=X(-\Omega) & =\sum_{n=-\infty}^{\infty} x[n] e^{j n \Omega} \\
x[n] & =\frac{1}{2 \pi} \int_{-\pi}^{\pi} W(\Omega) e^{-j n \Omega} \mathrm{d} \Omega
\end{aligned}
$$

podemos escribir:

$$
\begin{aligned}
s_{q}=c_{q} H_{q}^{(2)}\left(k r_{j}\right) & =\frac{1}{2 \pi} \int_{-\pi}^{\pi} E\left(\phi_{f}\right) e^{-j q \phi_{f}} \mathrm{~d} \phi_{f} \\
& =\operatorname{DTFT}^{(-1)}\left\{E\left(-\phi_{f}\right)\right\}
\end{aligned}
$$


Teniendo en cuenta que los coeficientes $s_{q}$ se pueden considerar nulos para $|q|>N_{f}$, definimos la serie $\hat{s}_{q}$, igual a la serie $s_{q}$, pero repetida periódicamente cada $N=2 N_{f}$ posiciones (se considera también que $s_{N_{f}} \simeq s_{-N_{f}}$, ya que $\left|s_{N_{f}}\right| \ll 1$ ):

$$
\hat{s}[q]=s[q] * \delta\left[q-2 N_{f}\right]=s[q] * \delta[q-N]
$$

Y una serie temporal discreta periódica se puede obtener de forma sencilla a partir de su espectro utilizando la transformada rápida de Fourier (FFT) de la siguiente forma $[46,47]$ :

$$
\begin{gathered}
\hat{s}[q]=\mathrm{FFT}^{(-1)}\{S[k]\}=\frac{1}{N} \mathrm{FFT}^{(-1)}\left\{S\left(k \frac{2 \pi}{N}\right)\right\} \\
q, k=\left[-\frac{N}{2},-\frac{N}{2}+1, \ldots, \frac{N}{2}-1\right]
\end{gathered}
$$

donde $S[k]$ es la DTFS o FFT, y $S(\Omega)$ es la DTFT de $s[q]$.

Sustituyendo (3.42) en la expresión anterior:

$$
\begin{aligned}
s_{q}=c_{q} H_{q}^{(2)}\left(k r_{j}\right) \simeq \hat{s}[q] & =\frac{1}{N} \mathrm{FFT}^{(-1)}\left\{E\left(-k \frac{2 \pi}{N}\right)\right\} \\
& =\frac{1}{2 N_{f}} \mathrm{FFT}^{(-1)}\left\{E\left(-k \frac{\pi}{N_{f}}\right)\right\}
\end{aligned}
$$

$\operatorname{con} q, k=\left[-N_{f},-N_{f}+1, \ldots, N_{f}-1\right]$

Finalmente:

$$
c_{q}=\frac{1}{2 N_{f} H_{q}^{(2)}\left(k r_{j}\right)} \mathrm{FFT}^{(-1)}\left\{E\left(-k \frac{\pi}{N_{f}}\right)\right\}
$$

donde $E\left(-k \pi / N_{f}\right)$ es la función $E\left(\phi_{f}\right)$ definida en (3.37) y evaluada en los puntos $\phi_{f}=$ $-k \pi / N_{f}, \operatorname{con} k=\left[-N_{f},-N_{f}+1, \ldots, N_{f}-1\right]$.

\subsubsection{Cálculo de los campos transversales}

Para obtener los parámetros de admitancia, ya se ha explicado que resulta necesario calcular los campos eléctrico y magnético transversales en cada uno de los $N$ planos de referencia. En la figura 3.12 se ha representado un caso sencillo en el que se analiza una cavidad inductiva en guía rectangular con un poste circular metálico en el centro.

Para calcular los parámetros de admitancia $Y_{m n}^{(i, 1)}$, se debe analizar la estructura de la figura 3.12, en la que se ha colocado un cortocircuito en el acceso 2 (el de la derecha). A continuación se debe excitar el acceso 1 con el modo $T E_{n 0}^{(1)}$, y luego calcular los campos tangenciales en los planos de referencia de los accesos 1 y 2 (marcados como $T_{1}$ y $T_{2}$ en la figura). Para analizar la estructura, se segmenta en dispersores 2-D individuales tal y como 


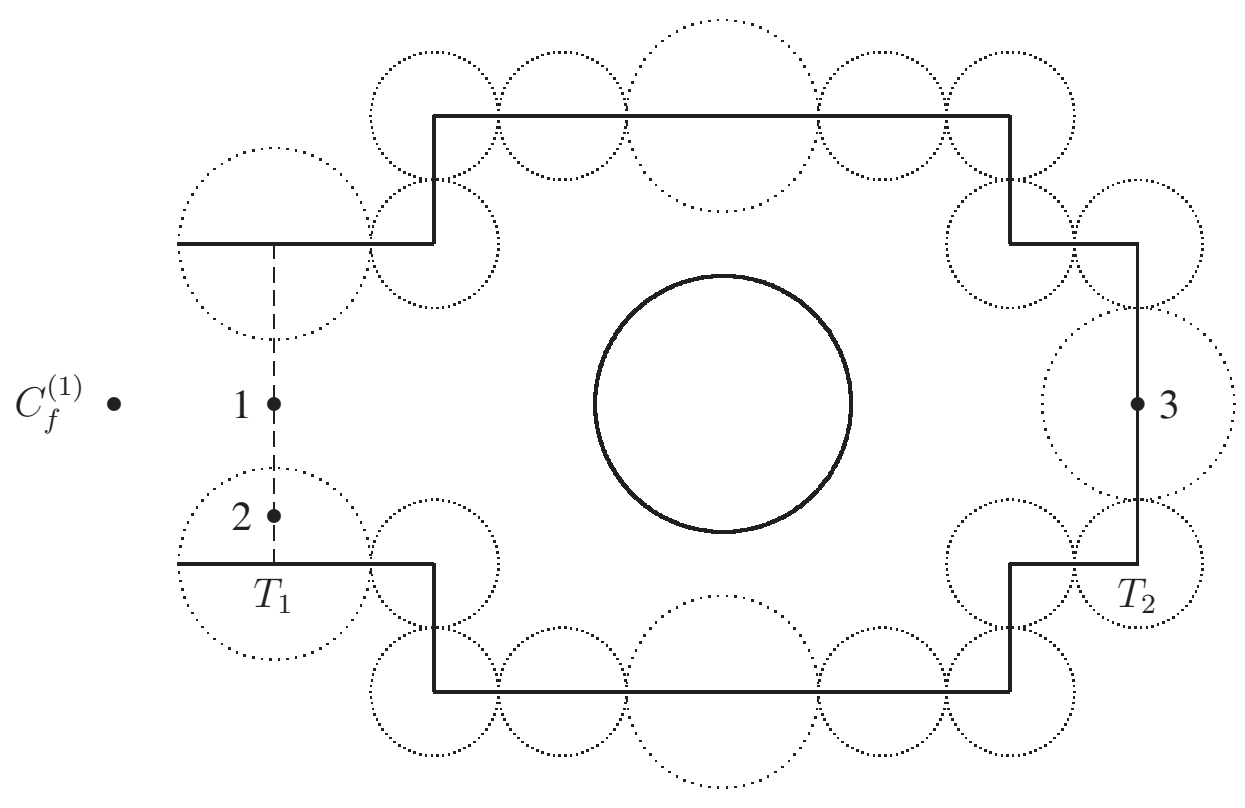

Figura 3.12: Diferentes puntos para el cálculo de los campos transversales durante la obtención de los parámetros de admitancia

se muestra en la figura, en la que se puede apreciar que existen tras segmentar 19 tiras 2-D y un cilindro metálico 2-D. Utilizando el método híbrido de análisis de problemas 2-D obtendremos $N_{o}=20$ matrices de dispersión conjuntas $\underline{\underline{D^{(i)}}}$, con $i=\left[1, \cdots, N_{o}\right]$. Cada matriz caracteriza el comportamiento de un objeto 2-D ante la incidencia de un campo cuyo espectro cilíndrico es emergente del punto $C_{f}^{(1)}$ teniendo ya en cuenta el acoplo electromagnético con el resto de objetos. $\mathrm{Si}\left[c_{p}\right]$ es el espectro del campo excitación, el espectro del campo eléctrico dispersado por cada objeto $i$ es:

$$
\left[c_{q}^{(i)}\right]=\underline{\underline{D^{(i)}}} \cdot\left[c_{p}\right], \quad i=\left[1, \ldots, N_{o}\right]
$$

A partir de la información de $\left[c_{q}^{(i)}\right]$ podremos calcular los campos eléctrico y magnético producidos por cada objeto dispersor, de modo que los campos transversales en el plano de referencia del puerto $j$, se calcularán de la siguiente forma:

$$
\begin{aligned}
\vec{E}_{t}^{(j)} & =\sum_{i=1}^{N_{o}} \vec{E}^{(i)} \cdot \hat{y}_{j}=\sum_{i=1}^{N_{o}} \vec{E}^{(i)} \cdot \hat{z} \\
\vec{H}_{t}^{(j)} & =\sum_{i=1}^{N_{o}} \vec{H}^{(i)} \cdot \hat{x}_{j}
\end{aligned}
$$

donde $\vec{E}^{(i)}$ y $\vec{H}^{(i)}$ son los campos eléctrico y magnético dispersados por el objeto $i$, y $\hat{x}_{j}$ e $\hat{y}_{j}$ son los vectores unitarios que definen el sistema de coordenadas en el puerto $j$. Como los 
campos eléctricos son $T M^{z}$, el campo eléctrico incidente y dispersado sólo tiene componente en $\hat{z}$. Además $\hat{z}=\hat{y}_{j}$, lo cual concuerda con el hecho de que los modos $T E_{m 0}^{(j)}$ tan sólo tienen componente según $\hat{y}_{j}$. Por eso, para obtener el campo eléctrico transversal tan sólo hay que multiplicar el campo escalarmente por $\hat{z}$. Y si el campo eléctrico sólo tiene componente en $\hat{z}=\hat{y}_{j}$, el campo magnético sólo tiene componente según $\hat{x}_{j}$ y $\hat{z}_{j}$. Y de esas dos componentes sólo interesa la transversal, es decir, la que va según $\hat{x}_{j}$.

Por tanto, para obtener los campos transversales en el plano de referencia del puerto $j$ es suficiente con calcular el campo eléctrico y magnético $\left(\vec{E}^{(i)}\right.$ y $\left.\vec{H}^{(i)}\right)$ dispersado por cada objeto $i$. No obstante, el espectro $\left[c_{q}^{(i)}\right]$ nos proporciona el campo producido por el objeto $i$ fuera de la circunferencia que lo contiene, pero no dentro. Dentro de dicha circunferencia se debe calcular el campo que produce la corriente inducida en la superficie del objeto 2-D. Teniendo esto presente, se concluye fácilmente que el cálculo del campo eléctrico y magnético producido por los $N_{o}$ objetos 2-D en cada uno de los planos de referencia $T_{1}$ y $T_{2}$ se debe realizar de forma diferente en cada punto según la posición de éste. En concreto, existen tres tipos diferentes de puntos, y en cada uno de ellos el cálculo del campo es diferente. Estos tres tipos de puntos, que se han representado en la figura 3.12, se describen a continuación:

Puntos externos a todos los objetos El punto 1 de la figura es un punto de este tipo. El campo dispersado por todos los objetos sobre este tipo de puntos se puede calcular a partir de los espectros cilíndricos de campo dispersado por cada objeto.

Puntos situados en el interior de algún objeto, pero no sobre su superficie El punto 2 de la figura es un punto de este tipo. En estos casos se puede utilizar el espectro de campo dispersado para todos los objetos excepto para el objeto en el interior del cual se encuentra el punto de observación. El campo dispersado por ese objeto sobre un punto situado dentro de la circunferencia que lo contiene se calculará integrando las contribuciones de campo de las corrientes inducidas en la superficie del dispersor.

Puntos situados sobre la superficie de algún objeto El punto 3 es un punto de este tipo. En este caso de nuevo podemos utilizar los espectros cilíndricos para todos los objetos excepto para aquel en cuya superficie se halla el punto de observación. Para calcular el campo eléctrico y magnético que produce un objeto 2-D sobre un punto situado en su superficie se deben hacer unos cálculos específicos.

A continuación se detalla el cálculo del campo dispersado por un objeto 2-D en las siguientes zonas:

- Fuera de la circunferencia que lo circunscribe

- Dentro de la circunferencia que lo circunscribe, pero no sobre su superficie.

- En la superficie del propio objeto 2-D. 


\section{Cálculo del campo fuera del objeto}

El campo eléctrico dispersado por un objeto fuera de la circunferencia que lo circunscribe se puede obtener a partir de su espectro $\left[c_{q}^{(i)}\right]$ :

$$
\vec{E}^{(i)}=\sum_{q=-N_{d}^{(i)}}^{N_{d}^{(i)}} c_{q}^{(i)} H_{q}^{(2)}\left(k \rho_{i}\right) e^{j q \phi_{i}} \hat{z}
$$

donde $\left(\rho_{i}, \phi_{i}\right)$ son las coordenadas locales del objeto $i$, tal y como se muestran en la figura 3.13, y $2 N_{d}^{(i)}+1$ es el número de modos cilíndricos del espectro de campo dispersado por el objeto $i$ que no se consideran nulos.

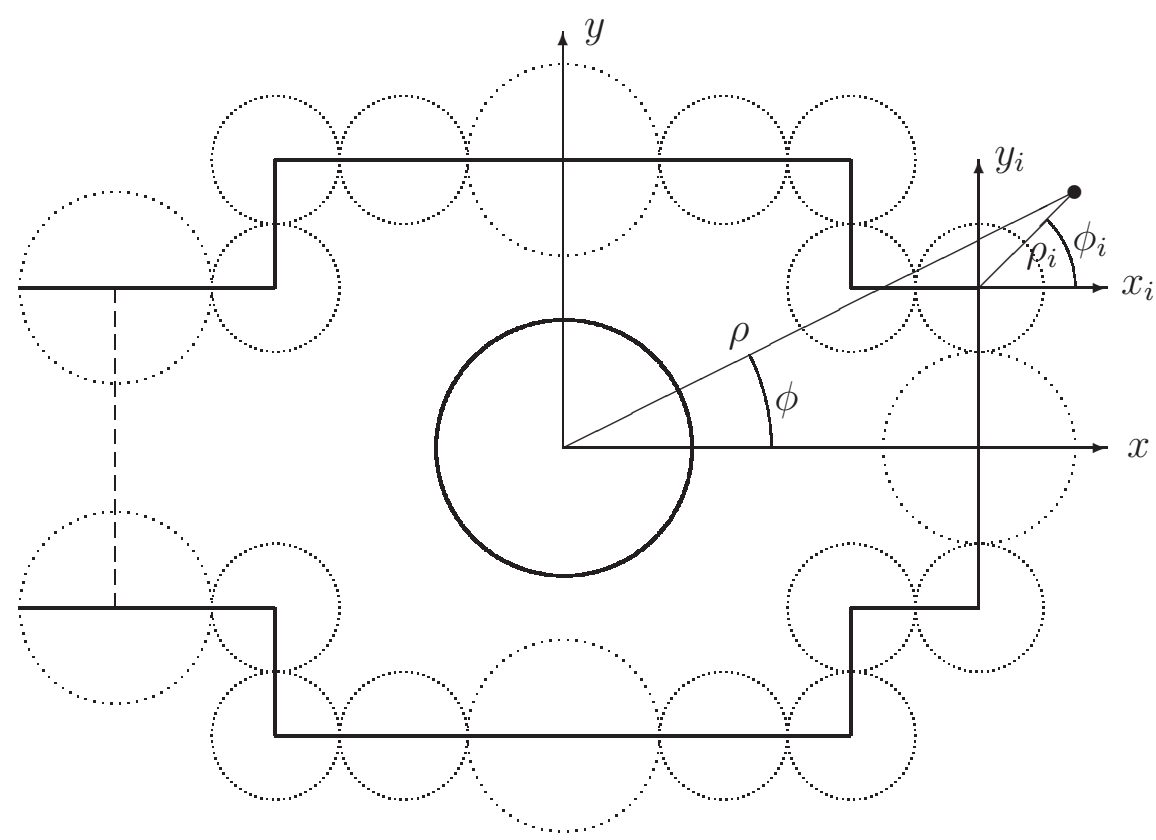

Figura 3.13: Sistemas de coordenadas global $(x, y)$ y local $\left(x_{i}, y_{i}\right)$ para cada objeto en un problema de dispersión múltiple 2-D

El campo magnético producido por el objeto $i$ será:

$$
\vec{H}^{(i)}=\frac{j}{\omega \mu} \nabla \times \vec{E}^{(i)}=\frac{j}{\omega \mu}\left(\hat{x} \frac{\partial E_{z}^{(i)}}{\partial y}-\hat{y} \frac{\partial E_{z}^{(i)}}{\partial x}\right)
$$

Como se puede apreciar en la figura 3.13, las derivadas con respecto a $x$ e $y$ son las mismas que las derivadas con respecto a las coordenadas locales $x_{i}$ e $y_{i}$. Por tanto:

$$
\vec{H}^{(i)}=\frac{j}{\omega \mu}\left(\hat{x} \frac{\partial E_{z}^{(i)}}{\partial y_{i}}-\hat{y} \frac{\partial E_{z}^{(i)}}{\partial x_{i}}\right)
$$


A continuación calcularemos las derivadas de $E_{z}^{(i)}$ :

$$
\begin{aligned}
& \frac{\partial E_{z}^{(i)}}{\partial y_{i}}=\sum_{q=-N_{d}^{(i)}}^{N_{d}^{(i)}} c_{q}^{(i)}\left[H_{q}^{(2)^{\prime}}\left(k \rho_{i}\right) \frac{\partial\left(k \rho_{i}\right)}{\partial y_{i}}+H_{q}^{(2)}\left(k \rho_{i}\right) j q \frac{\partial\left(\phi_{i}\right)}{\partial y_{i}}\right] e^{j q \phi_{i}} \\
& \frac{\partial E_{z}^{(i)}}{\partial x_{i}}=\sum_{q=-N_{d}^{(i)}}^{N_{d}^{(i)}} c_{q}^{(i)}\left[H_{q}^{(2)^{\prime}}\left(k \rho_{i}\right) \frac{\partial\left(k \rho_{i}\right)}{\partial x_{i}}+H_{q}^{(2)}\left(k \rho_{i}\right) j q \frac{\partial\left(\phi_{i}\right)}{\partial x_{i}}\right] e^{j q \phi_{i}}
\end{aligned}
$$

donde:

$$
H_{q}^{(2)^{\prime}}\left(k \rho_{i}\right)=\frac{\partial H_{q}^{(2)}\left(k \rho_{i}\right)}{\partial\left(k \rho_{i}\right)}
$$

Por otro lado:

$$
\begin{gathered}
\rho_{i}^{2}=x_{i}^{2}+y_{i}^{2} \\
2 \rho_{i} \frac{\partial \rho_{i}}{\partial x_{i}}=2 x_{i}=2 \rho_{i} \cos \phi_{i} \\
\frac{\partial \rho_{i}}{\partial x_{i}}=\cos \phi_{i}
\end{gathered}
$$

De igual forma:

$$
\begin{gathered}
\rho_{i}^{2}=x_{i}^{2}+y_{i}^{2} \\
2 \rho_{i} \frac{\partial \rho_{i}}{\partial y_{i}}=2 y_{i}=2 \rho_{i} \sin \phi_{i} \\
\frac{\partial \rho_{i}}{\partial x_{i}}=\sin \phi_{i}
\end{gathered}
$$

Para las derivadas de $\phi_{i}$ :

$$
\begin{gathered}
\tan \phi_{i}=\frac{y_{i}}{x_{i}} \\
\frac{1}{\cos ^{2} \phi_{i}} \frac{\partial \phi_{i}}{\partial x_{i}}=-\frac{y_{i}}{x_{i}^{2}}=-\frac{\rho_{i} \sin \phi_{i}}{\rho_{i}^{2} \cos ^{2} \phi_{i}}=-\frac{\sin \phi_{i}}{\rho_{i} \cos ^{2} \phi_{i}} \\
\frac{\partial \phi_{i}}{\partial x_{i}}=-\frac{\sin \phi_{i}}{\rho_{i}} \\
\frac{1}{\cos ^{2} \phi_{i}} \frac{\partial \phi_{i}}{\partial y_{i}}=\frac{1}{x_{i}}=\frac{1}{\rho_{i} \cos \phi_{i}} \\
\frac{\partial \phi_{i}}{\partial y_{i}}=\frac{\cos \phi_{i}}{\rho_{i}}
\end{gathered}
$$


Sustituyendo el valor de estas derivadas en (3.53) y (3.54):

$$
\begin{aligned}
\frac{\partial E_{z}^{(i)}}{\partial y_{i}} & =\sum_{q=-N_{d}^{(i)}}^{N_{d}^{(i)}} c_{q}^{(i)}\left[k \sin \phi_{i} H_{q}^{(2)^{\prime}}\left(k \rho_{i}\right)+j q \frac{\cos \phi_{i}}{\rho_{i}} H_{q}^{(2)}\left(k \rho_{i}\right)\right] e^{j q \phi_{i}} \\
\frac{\partial E_{z}^{(i)}}{\partial x_{i}} & =\sum_{q=-N_{d}^{(i)}}^{N_{d}^{(i)}} c_{q}^{(i)}\left[k \cos \phi_{i} H_{q}^{(2)^{\prime}}\left(k \rho_{i}\right)-j q \frac{\sin \phi_{i}}{\rho_{i}} H_{q}^{(2)}\left(k \rho_{i}\right)\right] e^{j q \phi_{i}}
\end{aligned}
$$

Finalmente podemos sustituir en (3.51) y obtener el campo magnético dispersado por cada objeto $i$ :

$$
\begin{aligned}
\vec{H}^{(i)}= & \hat{x} \sum_{q=-N_{d}^{(i)}}^{N_{d}^{(i)}} c_{q}^{(i)}\left[j \frac{\sin \phi_{i}}{\eta} H_{q}^{(2)^{\prime}}\left(k \rho_{i}\right)-q \frac{\cos \phi_{i}}{k \eta \rho_{i}} H_{q}^{(2)}\left(k \rho_{i}\right)\right] e^{j q \phi_{i}} \\
& -\hat{y} \sum_{q=-N_{d}^{(i)}}^{N_{d}^{(i)}} c_{q}^{(i)}\left[j \frac{\cos \phi_{i}}{\eta} H_{q}^{(2)^{\prime}}\left(k \rho_{i}\right)+q \frac{\sin \phi_{i}}{k \eta \rho_{i}} H_{q}^{(2)}\left(k \rho_{i}\right)\right] e^{j q \phi_{i}}
\end{aligned}
$$

\section{Cálculo del campo dentro del objeto pero no en su superficie}

Como ya se ha explicado con anterioridad, el campo producido por un objeto dispersor dentro de la circunferencia que lo contiene no se puede calcular a partir de su espectro cilíndrico de campo dispersado $\left[c_{q}^{(i)}\right]$, ya que la expresión (3.50) sólo es válida fuera de dicha circunferencia. En el interior de la citada circunferencia, el campo se debe calcular a partir de las corrientes inducidas en la superficie del objeto. El método híbrido de análisis de problemas 2-D no sólo proporciona las matrices de dispersión $\underline{\underline{D^{(i)}}}$, que relacionan espectro de campo dispersado con espectro de campo incidente, sino que también proporciona las matrices de corrientes $\underline{\underline{D I^{(i)}}}$, que relacionan la corriente excitada en la superficie del objeto con el espectro de campo incidente.

El método de análisis 2-D utiliza el método de los momentos para encontrar la corriente excitada en el objeto dispersor. En concreto, se utilizan funciones pulso como funciones base y deltas como funciones de test (Point-Matching) [7]. De modo que la densidad de corriente en la superficie del objeto se expande como una serie de funciones base (en este caso, funciones pulso), de la forma:

$$
\vec{J}_{s}\left(\vec{\rho}^{\prime}\right) \simeq \sum_{n=1}^{N_{s}} \alpha_{n} f_{n}\left(\vec{\rho}^{\prime}\right) \hat{z}
$$

donde $N_{s}$ es el número de funciones base y $f_{n}$ es la $n$-ésima función base. En el método de los momentos las incógnitas son los coeficientes $\alpha_{n}$. Una vez se obtienen dichos coeficientes, se conoce también la corriente en la superficie del objeto dispersor. 
Las matriz de corrientes $\underline{\underline{D I^{(i)}}}$ de cada objeto $i$ permite conocer las amplitudes $\left[\alpha_{n}\right]$ de la corriente inducida en la superficie del objeto a partir del espectro de campo incidente al objeto $\left[i_{p}^{(i)}\right]$ de la siguiente forma:

$$
\left[\alpha_{n} \Delta C_{n}\right]=\underline{\underline{D I^{(i)}}} \cdot\left[i_{p}^{(i)}\right]
$$

donde $\underline{\underline{D I^{(i)}}}$ es una matriz de tamaño $N_{s} \times\left(2 N_{i}^{(i)}+1\right)$, siendo $N_{i}^{(i)}$ el número de modos del espectro cilíndrico de campo incidente al objeto $i . \Delta C_{n}$ es la longitud de la parte del contorno del dispersor en la que la $n$-ésima función base pulso está definida. El vector $\left[\alpha_{n}\right]$ proporciona la corriente inducida teniendo ya en cuenta el acoplo electromagnético entre los múltiples objetos dispersores.

El campo dispersado por la corriente $\vec{J}_{s}\left(\vec{\rho}^{\prime}\right)$ será:

$$
\vec{E}^{(i)}(\vec{\rho})=-\frac{k \eta}{4} \int_{C} J_{s}\left(\vec{\rho}^{\prime}\right) H_{0}^{(2)}\left(k\left|\vec{\rho}-\vec{\rho}^{\prime}\right|\right) \mathrm{d} \rho^{\prime} \hat{z}
$$

donde $C$ es el contorno de la sección transversal del objeto 2-D.

Sustituyendo la corriente por su expansión en suma de funciones base pulso:

$$
\begin{aligned}
\vec{E}^{(i)}(\vec{\rho}) & =-\frac{k \eta}{4} \sum_{n=1}^{N_{s}} \alpha_{n} \int_{C} f_{n}\left(\vec{\rho}^{\prime}\right) H_{0}^{(2)}\left(k\left|\vec{\rho}-\vec{\rho}^{\prime}\right|\right) \mathrm{d} \rho^{\prime} \hat{z} \\
& =-\frac{k \eta}{4} \sum_{n=1}^{N_{s}} \alpha_{n} \int_{\Delta C_{n}} H_{0}^{(2)}\left(k\left|\vec{\rho}-\vec{\rho}^{\prime}\right|\right) \mathrm{d} \rho^{\prime} \hat{z}
\end{aligned}
$$

Puesto que los intervalos $\Delta C_{n}$ en los que está definida cada función base pulso son muy pequeños en términos eléctricos, ya que el número de funciones base $N_{s}$ es muy elevado, se puede aproximar el campo dispersado por cada función base como el campo producido por un hilo de corriente situado en el centro del intervalo $\Delta C_{n}$ (punto $\vec{\rho}_{n}$ ). Y la corriente que circula por dicho hilo de corriente será la densidad superficial de corriente multiplicada por la longitud $\Delta C_{n}$. Por tanto:

$$
\vec{E}^{(i)}(\vec{\rho}) \simeq-\frac{k \eta}{4} \sum_{n=1}^{N_{s}} \alpha_{n} \Delta C_{n} H_{0}^{(2)}\left(k\left|\vec{\rho}-\overrightarrow{\rho_{n}}\right|\right) \hat{z}
$$

Si definimos a continuación:

$$
\begin{aligned}
I_{n} & =\alpha_{n} \Delta C_{n} \\
d_{n} & =\left|\vec{\rho}-\overrightarrow{\rho_{n}}\right| \\
\vec{\rho}-\overrightarrow{\rho_{n}} & =x_{n} \hat{x}+y_{n} \hat{y}
\end{aligned}
$$

Se puede reescribir $\vec{E}^{(i)}$ de la siguiente forma:

$$
\vec{E}^{(i)}(\vec{\rho}) \simeq-\frac{k \eta}{4} \sum_{n=1}^{N_{s}} I_{n} H_{0}^{(2)}\left(k d_{n}\right) \hat{z}
$$


Y el correspondiente campo magnético será:

$$
\begin{aligned}
\vec{H}^{(i)}(\vec{\rho}) & =\frac{j}{\omega \mu}\left(\hat{x} \frac{\partial E_{z}^{(i)}}{\partial y}-\hat{y} \frac{\partial E_{z}^{(i)}}{\partial x}\right) \\
& =\frac{j}{\omega \mu}\left(\hat{x} \frac{\partial E_{z}^{(i)}}{\partial y_{n}}-\hat{y} \frac{\partial E_{z}^{(i)}}{\partial x_{n}}\right) \\
& =-\frac{j}{4}\left(\hat{x} \sum_{n=1}^{N_{s}} I_{n} k H_{0}^{(2)^{\prime}}\left(k d_{n}\right) \frac{y_{n}}{d_{n}}-\hat{y} \sum_{n=1}^{N_{s}} I_{n} k H_{0}^{(2)^{\prime}}\left(k d_{n}\right) \frac{x_{n}}{d_{n}}\right)
\end{aligned}
$$

\section{Cálculo del campo en la superficie del objeto}

En el caso de que se desee calcular el campo en un punto situado sobre la superficie de un objeto dispersor metálico, como es el caso de las tiras 2-D que modelan cortocircuitos en la figura 3.12, ya no se puede aproximar el campo dispersado por todos los pulsos de corriente como el campo dispersado por un hilo de corriente situado en el centro de cada pulso. Volviendo a la ecuación (3.62):

$$
\vec{E}^{(i)}(\vec{\rho})=-\frac{k \eta}{4} \sum_{n=1}^{N_{s}} \alpha_{n} \int_{\Delta C_{n}} H_{0}^{(2)}\left(k\left|\vec{\rho}-\vec{\rho}^{\prime}\right|\right) \mathrm{d} \rho^{\prime} \hat{z}
$$

Si el punto de observación $\vec{\rho}$ está dentro de la zona del contorno del objeto en la que el $k$-ésimo pulso de corriente está definido, entonces podremos aproximar el campo producido por todos los pulsos de corriente excepto el $k$-ésimo como el campo dispersado por un hilo de corriente situado en el centro del pulso $\left(\vec{\rho}_{n}\right)$ :

$$
\begin{aligned}
\vec{E}^{(i)}(\vec{\rho}) \simeq & -\frac{k \eta}{4} \sum_{n=1, n \neq k}^{N_{s}} \alpha_{n} \Delta C_{n} H_{0}^{(2)}\left(k\left|\vec{\rho}-\overrightarrow{\rho_{n}}\right|\right) \hat{z} \\
& -\frac{k \eta}{4} \alpha_{k} \int_{\Delta C_{k}} H_{0}^{(2)}\left(k\left|\vec{\rho}-\vec{\rho}^{\prime}\right|\right) \mathrm{d} \rho^{\prime}
\end{aligned}
$$

Para simplificar los cálculos, tomaremos el punto de observación en el centro del intervalo $\Delta C_{k}\left(\vec{\rho}=\vec{\rho}_{k}\right)$, de modo que siempre que deseemos calcular el campo en la superficie de un objeto metálico lo haremos en los puntos en los que se sitúan las funciones pulso utilizadas en el método de los momentos para caracterizar dicho objeto. Por lo tanto el campo que deseamos calcular es:

$$
\begin{aligned}
\vec{E}^{(i)}\left(\overrightarrow{\rho_{k}}\right) \simeq & -\frac{k \eta}{4} \sum_{n=1, n \neq k}^{N_{s}} \alpha_{n} \Delta C_{n} H_{0}^{(2)}\left(k\left|\overrightarrow{\rho_{k}}-\overrightarrow{\rho_{n}}\right|\right) \hat{z} \\
& -\frac{k \eta}{4} \alpha_{k} \int_{\Delta C_{k}} H_{0}^{(2)}\left(k\left|\overrightarrow{\rho_{k}}-\vec{\rho}^{\prime}\right|\right) \mathrm{d} \rho^{\prime}
\end{aligned}
$$


Como el intervalo $\Delta C_{k}$ del contorno del objeto es muy pequeño, puede aproximarse por una recta (además, el campo en la superficie de un objeto nos interesa para el caso de tiras 2-D, que son las que modelan los cortocircuitos, y en las tiras la superficie es siempre recta), con lo que $|l|=\left|\overrightarrow{\rho_{k}}-\vec{\rho}^{\prime}\right|$ será una variable que oscilará entre $-\Delta C_{k} / 2$ y $\Delta C_{k} / 2$. La integral se podrá entonces calcular de la siguiente forma:

$$
\begin{aligned}
I & =\int_{\Delta C_{k}} H_{0}^{(2)}\left(k\left|\vec{\rho}_{k}-\vec{\rho}^{\prime}\right|\right) \mathrm{d} \rho^{\prime} \simeq \int_{l=-\frac{\Delta C_{k}}{2}}^{l=\frac{\Delta C_{k}}{2}} H_{0}^{(2)}(k|l|) \mathrm{d} l \\
& =2 \int_{l=0}^{l=\frac{\Delta C_{k}}{2}} H_{0}^{(2)}(k l) \mathrm{d} l
\end{aligned}
$$

Para resolver la integral, utilizaremos la aproximación de argumento pequeño de la función de Hankel:

$$
H_{0}^{(2)}(x) \simeq 1-j \frac{2}{\pi} \ln \left(\frac{\gamma x}{2}\right)
$$

donde $\gamma=0,57721 \ldots$ es la constante de Euler, que se define como:

$$
\gamma=1+\frac{1}{2}+\frac{1}{2}+\cdots+\frac{1}{n}+\ln (n) \quad n \rightarrow \infty
$$

Utilizando (3.73):

$$
\begin{aligned}
I & \simeq 2 \int_{l=0}^{l=\frac{\Delta C_{k}}{2}}\left(1-j \frac{2}{\pi} \ln \left(\frac{\gamma k l}{2}\right)\right) \mathrm{d} l \\
& =2\left[\left.l\right|_{l=0} ^{\frac{\Delta C_{k}}{2}}-j \frac{2}{\pi} \int_{l=0}^{\frac{\Delta C_{k}}{2}} \ln \left(\frac{\gamma k l}{2}\right) \mathrm{d} l\right]
\end{aligned}
$$

La integral del logaritmo puede resolverse por partes de la siguiente forma:

$$
\begin{aligned}
\int \ln (C x) \mathrm{d} x & =\int u \mathrm{~d} v=u v-\int v \mathrm{~d} u \\
& =\left|\begin{array}{ll}
u=\ln (C x) & \rightarrow \mathrm{d} u=\frac{1}{x} \mathrm{~d} x \\
\mathrm{~d} v=\mathrm{d} x & \rightarrow v=x
\end{array}\right| \\
& =x \ln (C x)-x=x[\ln (C x)-1]
\end{aligned}
$$

Comparando la expresión anterior con la integral que se pretende resolver, la constante $C$ dentro del argumento del logaritmo es $\gamma k / 2$, y finalmente se puede obtener $I$ como:

$$
\begin{aligned}
I & =2\left[\frac{\Delta C_{k}}{2}-j \frac{2}{\pi} \frac{\Delta C_{k}}{2}\left[\ln \left(\frac{\gamma k \Delta C_{k}}{4}\right)-1\right]\right] \\
& =2\left[\frac{\Delta C_{k}}{2}-j \frac{2}{\pi} \frac{\Delta C_{k}}{2} \ln \left(\frac{\gamma k \Delta C_{k}}{4 e}\right)\right]
\end{aligned}
$$


Y, definitivamente:

$$
I=\Delta C_{k}\left[1-j \frac{2}{\pi} \ln \left(\frac{\gamma k \Delta C_{k}}{4 e}\right)\right]
$$

donde $e=2,718 \ldots$

Sustituyendo el resultado de esta integral en (3.71) se obtiene:

$$
\begin{aligned}
\vec{E}^{(i)}\left(\overrightarrow{\rho_{k}}\right) \simeq & -\frac{k \eta}{4} \sum_{n=1, n \neq k}^{N_{s}} \alpha_{n} \Delta C_{n} H_{0}^{(2)}\left(k\left|\overrightarrow{\rho_{k}}-\overrightarrow{\rho_{n}}\right|\right) \hat{z} \\
& -\frac{k \eta}{4} \alpha_{k} \Delta C_{k}\left[1-j \frac{2}{\pi} \ln \left(\frac{\gamma k \Delta C_{k}}{4 e}\right)\right]
\end{aligned}
$$

El correspondiente campo magnético será:

$$
\vec{H}^{(i)}\left(\vec{\rho}_{k}\right)=\left.\frac{j}{\omega \mu}\left(\hat{x} \frac{\partial E_{z}^{(i)}}{\partial y}-\hat{y} \frac{\partial E_{z}^{(i)}}{\partial x}\right)\right|_{\vec{\rho}=\vec{\rho}_{k}}
$$

Para calcular la derivada de $E_{z}^{(i)}$, es necesario partir de la expresión (3.70), en la que todavía no se ha particularizado el campo para ningún punto concreto, tal y como sucede en la expresión (3.79), donde se ha obtenido el campo en el punto $\overrightarrow{\rho_{k}}$.

Utilizando (3.66), podemos escribir (3.70) de la siguiente forma:

$$
\begin{aligned}
\vec{E}^{(i)}(\vec{\rho}) \simeq & -\frac{k \eta}{4} \sum_{n=1, n \neq k}^{N_{s}} I_{n} H_{0}^{(2)}\left(k d_{n}\right) \hat{z} \\
& -\frac{k \eta}{4} \frac{I_{k}}{\Delta C_{k}} \int_{\Delta C_{k}} H_{0}^{(2)}\left(k\left|\vec{\rho}-\vec{\rho}^{\prime}\right|\right) \mathrm{d} \rho^{\prime}
\end{aligned}
$$

Llamaremos:

$$
I(\vec{\rho})=\int_{\Delta C_{k}} H_{0}^{(2)}\left(k\left|\vec{\rho}-\vec{\rho}^{\prime}\right|\right) \mathrm{d} \rho^{\prime}
$$

Por tanto:

$$
\vec{E}^{(i)}(\vec{\rho}) \simeq-\frac{k \eta}{4} \sum_{n=1, n \neq k}^{N_{s}} I_{n} H_{0}^{(2)}\left(k d_{n}\right) \hat{z}-\frac{k \eta}{4} \frac{I_{k}}{\Delta C_{k}} I(\vec{\rho})
$$


Y el campo magnético se expresará como:

$$
\begin{aligned}
\vec{H}^{(i)}(\vec{\rho})= & \frac{j}{\omega \mu}\left(\hat{x} \frac{\partial E_{z}^{(i)}}{\partial y}-\hat{y} \frac{\partial E_{z}^{(i)}}{\partial x}\right) \\
= & \frac{j}{\omega \mu}\left(\hat{x} \frac{\partial E_{z}^{(i)}}{\partial y_{n}}-\hat{y} \frac{\partial E_{z}^{(i)}}{\partial x_{n}}\right) \\
= & -\frac{j}{4}\left(\sum_{n=1, n \neq k}^{N_{s}} I_{n} k H_{0}^{(2)^{\prime}}\left(k d_{n}\right) \frac{y_{n}}{d_{n}}+\frac{I_{k}}{\Delta C_{k}} \frac{\partial I(\vec{\rho})}{\partial y_{k}}\right) \hat{x} \\
& +\frac{j}{4}\left(\sum_{n=1, n \neq k}^{N_{s}} I_{n} k H_{0}^{(2)^{\prime}}\left(k d_{n}\right) \frac{x_{n}}{d_{n}}+\frac{I_{k}}{\Delta C_{k}} \frac{\partial I(\vec{\rho})}{\partial x_{k}}\right) \hat{y}
\end{aligned}
$$

En el punto $\vec{\rho}=\vec{\rho}_{k}$ :

$$
\begin{aligned}
\vec{H}^{(i)}\left(\vec{\rho}_{k}\right)= & -\frac{j}{4}\left(\sum_{n=1, n \neq k}^{N_{s}} I_{n} k H_{0}^{(2)^{\prime}}\left(k d_{k n}\right) \frac{y_{k n}}{d_{k n}}+\left.\frac{I_{k}}{\Delta C_{k}} \frac{\partial I(\vec{\rho})}{\partial y_{k}}\right|_{\vec{\rho}=\vec{\rho}_{k}}\right) \hat{x} \\
& +\frac{j}{4}\left(\sum_{n=1, n \neq k}^{N_{s}} I_{n} k H_{0}^{(2)^{\prime}}\left(k d_{k n}\right) \frac{x_{k n}}{d_{k n}}+\left.\frac{I_{k}}{\Delta C_{k}} \frac{\partial I(\vec{\rho})}{\partial x_{k}}\right|_{\vec{\rho}=\vec{\rho}_{k}}\right) \hat{y}
\end{aligned}
$$

donde:

$$
\begin{aligned}
d_{k n} & =\left|\vec{\rho}_{k}-\vec{\rho}_{n}\right| \\
\vec{\rho}_{k}-\vec{\rho}_{n} & =x_{k n} \hat{x}+y_{k n} \hat{y}
\end{aligned}
$$

Para completar la expresión del campo magnético tan sólo queda por determinar las derivadas de $I(\vec{\rho})$ y particularizarlas en el punto $\vec{\rho}_{k}$.

En primer lugar resolveremos la integral $I(\vec{\rho})$. Teniendo en cuenta que calculamos el campo sobre un cortocircuito, modelado por tiras 2-D, siempre se calculará el campo sobre una superficie recta. Por tanto la integral la podremos expresar de la siguiente forma:

$$
\begin{aligned}
I(\vec{\rho}) & =\int_{\Delta C_{k}} H_{0}^{(2)}\left(k\left|\vec{\rho}-\vec{\rho}^{\prime}\right|\right) \mathrm{d} \rho^{\prime} \\
& =\int_{l=-\frac{\Delta C_{k}}{2}}^{l=\frac{\Delta C_{k}}{2}} H_{0}^{(2)}\left(k\left|\vec{\rho}-\vec{\rho}^{\prime}\right|\right) \mathrm{d} l
\end{aligned}
$$

A continuación se definen las siguientes magnitudes escalares y vectoriales (ver figura 3.14) en el entorno del intervalo $k$-ésimo sobre la superficie del cortocircuito (modelado por una tira 2-D):

$$
\begin{aligned}
\vec{d}_{k} & =\vec{\rho}-\vec{\rho}_{k}=x_{k} \hat{x}+y_{k} \hat{y} \\
\vec{d} & =\vec{\rho}-\vec{\rho}^{\prime}=\vec{d}_{k}-l \hat{u}_{k} \\
d & =|\vec{d}|=\sqrt{\vec{d} \cdot \vec{d}}=\sqrt{d_{k}^{2}+l^{2}-2 l \vec{d}_{k} \cdot \hat{u}_{k}} \\
& =\sqrt{x_{k}^{2}+y_{k}^{2}+l^{2}-2 l\left(x_{k} u_{k_{x}}+y_{k} u_{k_{y}}\right)}
\end{aligned}
$$




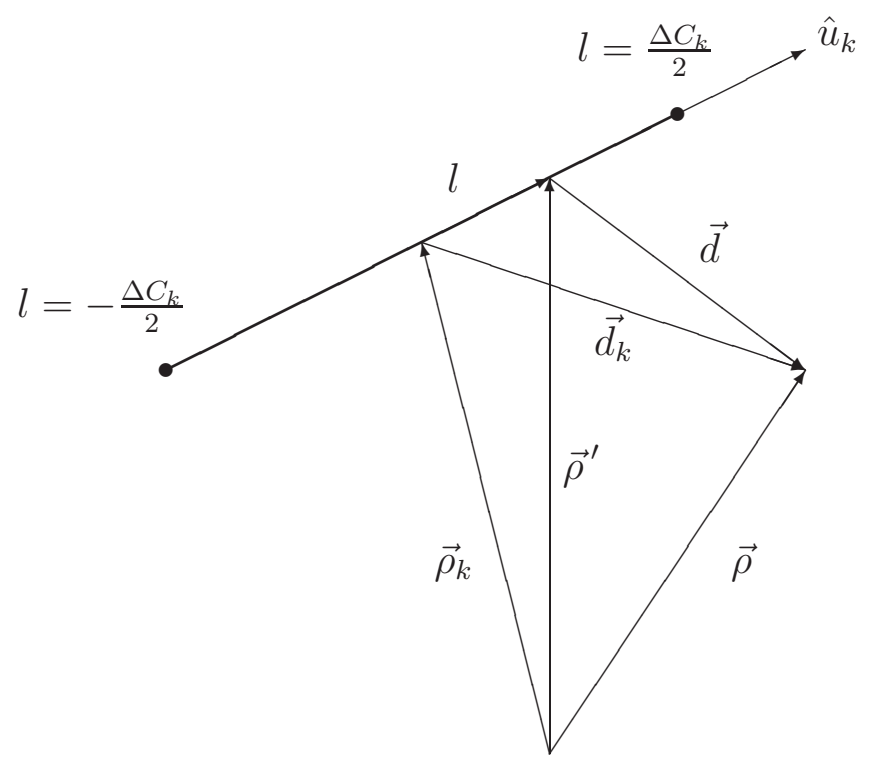

Figura 3.14: Sistema de coordenadas en el entorno del intervalo $\Delta C_{k}$ sobre la superficie de una tira 2-D

siendo $\hat{u}_{k}$ un vector unitario que indica la inclinación del $k$-ésimo intervalo sobre la superficie del objeto.

Utilizando las definiciones anteriores:

$$
I(\vec{\rho})=I\left(\vec{\rho}_{k}+\vec{d}_{k}\right)=I\left(x_{k}, y_{k}\right)=\int_{l=-\frac{\Delta C_{k}}{2}}^{l=\frac{\Delta C_{k}}{2}} H_{0}^{(2)}(k d(l)) \mathrm{d} l
$$

Utilizando la aproximación de Hankel para argumento pequeño:

$$
\begin{aligned}
I\left(x_{k}, y_{k}\right) & \simeq \int_{l=-\frac{\Delta C_{k}}{2}}^{l=\frac{\Delta C_{k}}{2}}\left(1-j \frac{2}{\pi} \ln \left(\frac{\gamma k d(l)}{2}\right)\right) \mathrm{d} l \\
& \int_{l=-\frac{\Delta C_{k}}{2}}^{l=\frac{\Delta C_{k}}{2}}\left(1-j \frac{2}{\pi} \ln \left(\frac{\gamma k \sqrt{x_{k}^{2}+y_{k}^{2}+l^{2}-2 l\left(x_{k} u_{k_{x}}+y_{k} u_{k_{y}}\right)}}{2}\right)\right) \mathrm{d} l
\end{aligned}
$$

Lo que se necesita son las derivadas direccionales de $I$ en las direcciones $x_{k}$ e $y_{k}$, y en el entorno del punto $\left(x_{k}, y_{k}\right)=(0,0)$. Es decir:

$$
\begin{aligned}
& \lim _{x_{k} \rightarrow 0} \frac{\partial I\left(x_{k}, y_{k}=0\right)}{\partial x_{k}} \\
& \lim _{y_{k} \rightarrow 0} \frac{\partial I\left(x_{k}=0, y_{k}\right)}{\partial y_{k}}
\end{aligned}
$$


Utilizando un software de cálculo simbólico comercial [48] se ha resuelto la integral anterior con el siguiente resultado:

$$
\begin{aligned}
& \lim _{x_{k} \rightarrow 0} \frac{\partial I\left(x_{k}, y_{k}=0\right)}{\partial x_{k}}= \begin{cases}0 & u_{k_{x}} \neq 0 \\
-2 j & u_{k_{x}}=0 \mathrm{y} x_{k} \rightarrow 0^{+} \\
2 j & u_{k_{x}}=0 \mathrm{y} x_{k} \rightarrow 0^{-}\end{cases} \\
& \lim _{y_{k} \rightarrow 0} \frac{\partial I\left(x_{k}=0, y_{k}\right)}{\partial y_{k}}= \begin{cases}0 & u_{k_{y}} \neq 0 \\
-2 j & u_{k_{y}}=0 \mathrm{y} y_{k} \rightarrow 0^{+} \\
2 j & u_{k_{y}}=0 \mathrm{y} y_{k} \rightarrow 0^{-}\end{cases}
\end{aligned}
$$

Se define:

$$
\begin{aligned}
& i_{k_{x}}= \begin{cases}0 & u_{k_{x}} \neq 0 \\
-2 j & u_{k_{x}}=0 \mathrm{y} x_{k} \rightarrow 0^{+} \\
2 j & u_{k_{x}}=0 \mathrm{y} x_{k} \rightarrow 0^{-}\end{cases} \\
& i_{k_{y}}= \begin{cases}0 & u_{k_{y}} \neq 0 \\
-2 j & u_{k_{y}}=0 \mathrm{y} y_{k} \rightarrow 0^{+} \\
2 j & u_{k_{y}}=0 \mathrm{y} y_{k} \rightarrow 0^{-}\end{cases}
\end{aligned}
$$

Nótese que el hecho de que los límites sean diferentes según nos acerquemos al punto $\vec{\rho}_{k}$ en un sentido u otro quiere decir que el campo magnético en el cortocircuito se habrá de calcular de manera diferente en una u otra cara del mismo. Para calcular los parámetros de admitancia se deberá seleccionar, de la la tira 2-D que modela el cortocircuito, la cara que da al interior del segmento que se está analizando (la cara de la izquierda en el punto 3 de la figura 3.12).

En el caso particular del punto 3 de la figura $3.12, i_{k_{x}}=2 j$ e $i_{k_{y}}=0$.

Finalmente, sustituyendo en (3.85) se tiene la siguiente expresión para el campo magnético:

$$
\begin{aligned}
\vec{H}^{(i)}\left(\vec{\rho}_{k}\right)= & -\frac{j}{4}\left(\sum_{n=1, n \neq k}^{N_{s}} I_{n} k H_{0}^{(2)^{\prime}}\left(k d_{k n}\right) \frac{y_{k n}}{d_{k n}}+\frac{I_{k}}{\Delta C_{k}} i_{k_{y}}\right) \hat{x} \\
& +\frac{j}{4}\left(\sum_{n=1, n \neq k}^{N_{s}} I_{n} k H_{0}^{(2)^{\prime}}\left(k d_{k n}\right) \frac{x_{k n}}{d_{k n}}+\frac{I_{k}}{\Delta C_{k}} i_{k_{x}}\right) \hat{y}
\end{aligned}
$$

\section{Resumen}

- Campo fuera de la circunferencia que contiene al objeto:

$$
\vec{E}^{(i)}=\sum_{q=-N_{d}^{(i)}}^{N_{d}^{(i)}} c_{q}^{(i)} H_{q}^{(2)}\left(k \rho_{i}\right) e^{j q \phi_{i}} \hat{z}
$$




$$
\begin{aligned}
\vec{H}^{(i)}= & \hat{x} \sum_{q=-N_{d}^{(i)}}^{N_{d}^{(i)}} c_{q}^{(i)}\left[j \frac{\sin \phi_{i}}{\eta} H_{q}^{(2)^{\prime}}\left(k \rho_{i}\right)-q \frac{\cos \phi_{i}}{k \eta \rho_{i}} H_{q}^{(2)}\left(k \rho_{i}\right)\right] e^{j q \phi_{i}} \\
& -\hat{y} \sum_{q=-N_{d}^{(i)}}^{N_{d}^{(i)}} c_{q}^{(i)}\left[j \frac{\cos \phi_{i}}{\eta} H_{q}^{(2)^{\prime}}\left(k \rho_{i}\right)+q \frac{\sin \phi_{i}}{k \eta \rho_{i}} H_{q}^{(2)}\left(k \rho_{i}\right)\right] e^{j q \phi_{i}}
\end{aligned}
$$

- Campo dentro de la circunferencia que contiene al objeto:

$$
\begin{gathered}
\vec{E}^{(i)}(\vec{\rho}) \simeq-\frac{k \eta}{4} \sum_{n=1}^{N_{s}} I_{n} H_{0}^{(2)}\left(k d_{n}\right) \hat{z} \\
\vec{H}^{(i)}(\vec{\rho})=-\frac{j}{4}\left(\hat{x} \sum_{n=1}^{N_{s}} I_{n} k H_{0}^{(2)^{\prime}}\left(k d_{n}\right) \frac{y_{n}}{d_{n}}-\hat{y} \sum_{n=1}^{N_{s}} I_{n} k H_{0}^{(2)^{\prime}}\left(k d_{n}\right) \frac{x_{n}}{d_{n}}\right)
\end{gathered}
$$

donde:

$$
\begin{aligned}
I_{n} & =\alpha_{n} \Delta C_{n} \\
d_{n} & =\left|\vec{\rho}-\overrightarrow{\rho_{n}}\right| \\
\vec{\rho}-\overrightarrow{\rho_{n}} & =x_{n} \hat{x}+y_{n} \hat{y}
\end{aligned}
$$

- Campo en la superficie del objeto (para una tira 2-D):

$$
\begin{aligned}
\vec{E}^{(i)}\left(\overrightarrow{\rho_{k}}\right) \simeq & -\frac{k \eta}{4} \sum_{n=1, n \neq k}^{N_{s}} \alpha_{n} \Delta C_{n} H_{0}^{(2)}\left(k d_{k n}\right) \hat{z} \\
& -\frac{k \eta}{4} \alpha_{k} \Delta C_{k}\left[1-j \frac{2}{\pi} \ln \left(\frac{\gamma k \Delta C_{k}}{4 e}\right)\right] \hat{z} \\
\vec{H}^{(i)}\left(\vec{\rho}_{k}\right)=- & \frac{j}{4}\left(\sum_{n=1, n \neq k}^{N_{s}} I_{n} k H_{0}^{(2)^{\prime}}\left(k d_{k n}\right) \frac{y_{k n}}{d_{k n}}+\frac{I_{k}}{\Delta C_{k}} i_{k_{y}}\right) \hat{x} \\
+ & \frac{j}{4}\left(\sum_{n=1, n \neq k}^{N_{s}} I_{n} k H_{0}^{(2)^{\prime}}\left(k d_{k n}\right) \frac{x_{k n}}{d_{k n}}+\frac{I_{k}}{\Delta C_{k}} i_{k_{x}}\right) \hat{y}
\end{aligned}
$$

con:

$$
\begin{aligned}
d_{k n} & =\left|\vec{\rho}_{k}-\vec{\rho}_{n}\right| \\
\vec{\rho}_{k}-\vec{\rho}_{n} & =x_{k n} \hat{x}+y_{k n} \hat{y}
\end{aligned}
$$




$$
\begin{aligned}
& i_{k_{x}}= \begin{cases}0 & u_{k_{x}} \neq 0 \\
-2 j & u_{k_{x}}=0 \mathrm{y} x_{k} \rightarrow 0^{+} \\
2 j & u_{k_{x}}=0 \mathrm{y} x_{k} \rightarrow 0^{-}\end{cases} \\
& i_{k_{y}}= \begin{cases}0 & u_{k_{y}} \neq 0 \\
-2 j & u_{k_{y}}=0 \mathrm{y} y_{k} \rightarrow 0^{+} \\
2 j & u_{k_{y}}=0 \mathrm{y} y_{k} \rightarrow 0^{-}\end{cases}
\end{aligned}
$$

\subsubsection{Resultados}

A continuación se muestran algunos resultados correspondientes al análisis de estructuras inductivas sencillas en guía de onda rectangular analizadas mediante la técnica de cálculo de matrices de admitancias que se acaba de explicar.

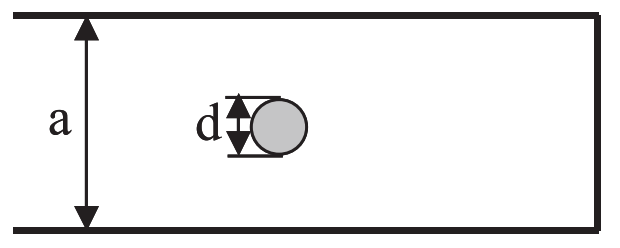

Figura 3.15: Esquema de una guía rectangular de anchura $a$ con un poste circular metálico inductivo de diámetro $d$

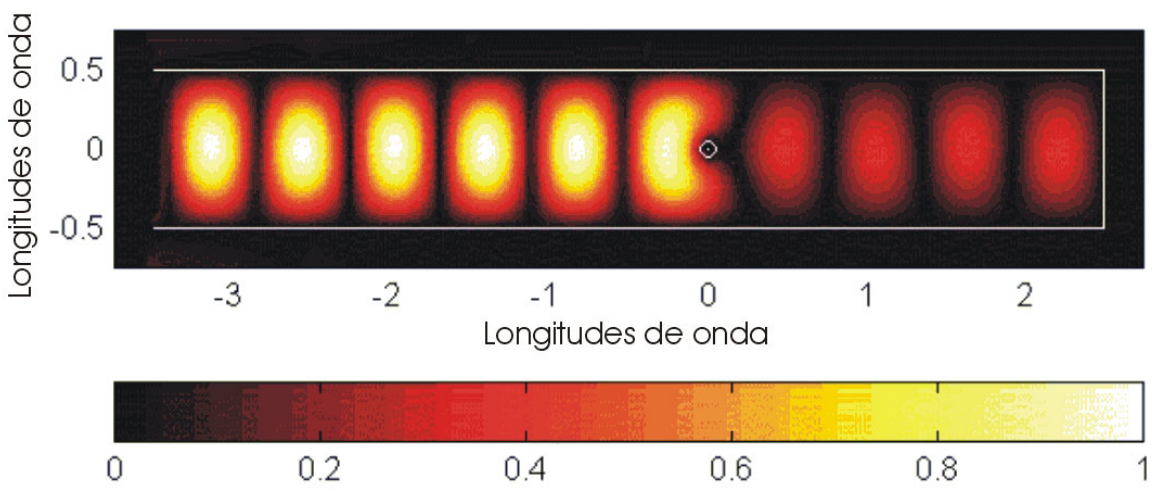

Figura 3.16: Amplitud del campo eléctrico $(\mathrm{dB})$ en el interior de una guía rectangular $(a=\lambda)$ con un poste metálico circular centrado (diámetro $d=0,2 \lambda$ ), bajo la excitación del modo fundamental en el acceso 1. El acceso 2 se halla cortocircuitado para calcular los parámetros de admitancia

El primer resultado que se muestra es para un poste circular metálico pasante situado en el centro de la guía. En la figura 3.15 se muestra el esquema de la estructura que se ha analizado. Se trata de una guía de anchura $a$ con un poste metálico circular de diámetro $d$.

Para poder obtener los parámetros de admitancia de la estructura, se ha simulado en primer lugar dicha estructura con un cortocircuito situado en el acceso 2 (el de la derecha) y 
con la excitación del modo fundamental $T E_{10}$. En la figura 3.16 se ha representado el campo que se obtiene en el interior de la citada estructura con cortocircuito, una vez que ésta se ha analizado con el método híbrido de análisis de estructuras 2-D. Para calcular el campo en todo el espacio, ha sido necesario utilizar las expresiones deducidas en el apartado anterior, y en cada región calcular el campo con la expresión adecuada.

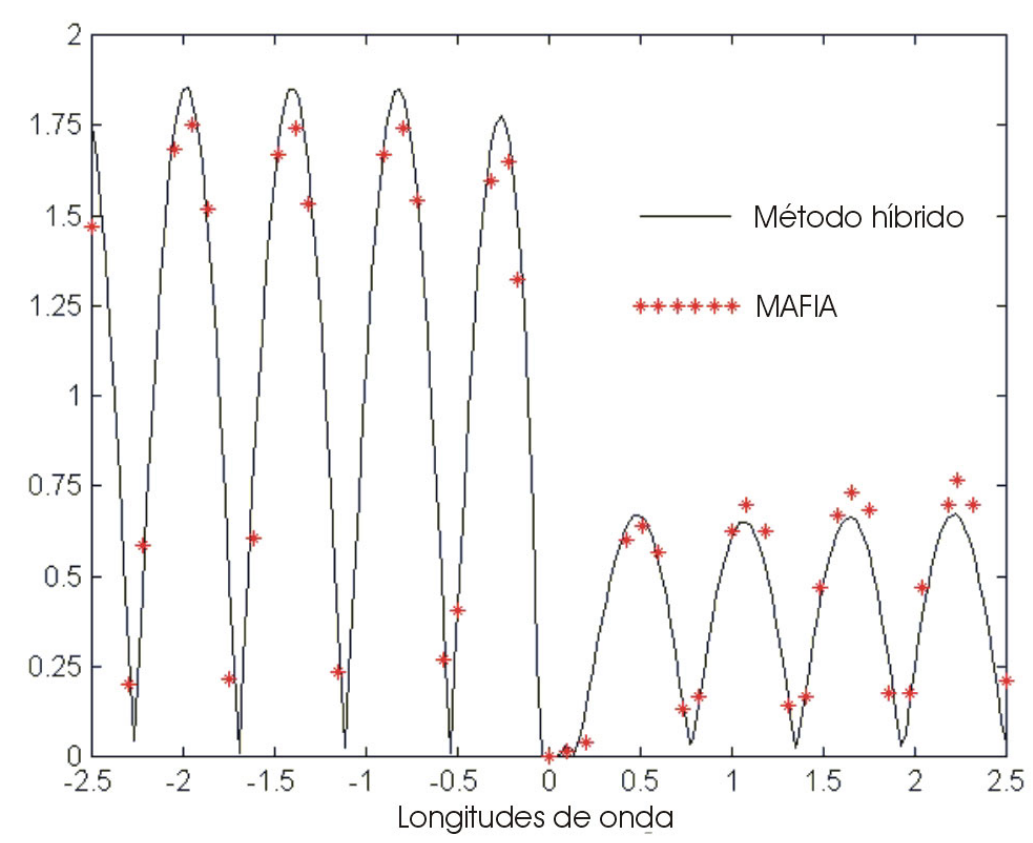

Figura 3.17: Amplitud del campo eléctrico a lo largo de una recta en el centro de una guía rectangular con un poste circular metálico en el centro y terminada en cortocircuito. Comparación con los mismos resultados proporcionados por el programa comercial MAFIA $\mathbb{R}$

En la figura 3.17 se muestra una comparación de los resultados del método de análisis híbrido 2-D y un programa de simulación electromagnética comercial basado en un algoritmo de integración finita (MAFIA(R): MAxwell Equations by the Finite Integration Algorithm, [55]). Se ha comparado la variación espacial de la amplitud del campo eléctrico a lo largo de una recta que recorre el eje central de la guía rectangular con la excitación del modo fundamental. Como se puede apreciar existe un buen nivel de coincidencia entre ambos resultados.

Una vez simulada la estructura con cortocircuito, se ha calculado el campo eléctrico y magnético en el primer acceso y en el segundo (sobre el cortocircuito), y utilizando las expresiones en (3.34), se han obtenido los parámetros $Y_{11}^{1,1}$ e $Y_{11}^{2,1}$. Como la estructura es simétrica, no es necesario calcular los parámetros $Y_{11}^{1,2}$ e $Y_{11}^{2,2}$, pues se cumple que $Y_{11}^{1,2}=Y_{11}^{2,1}$ e $Y_{11}^{2,2}=Y_{11}^{1,1}$. Con esto se completa el cálculo de la matriz de admitancias para el modo fundamental.

Con el fin de validar el resultado obtenido, se ha comparado con resultados de esta misma estructura publicados en la literatura técnica relacionada [12]. Concretamente, se han com- 


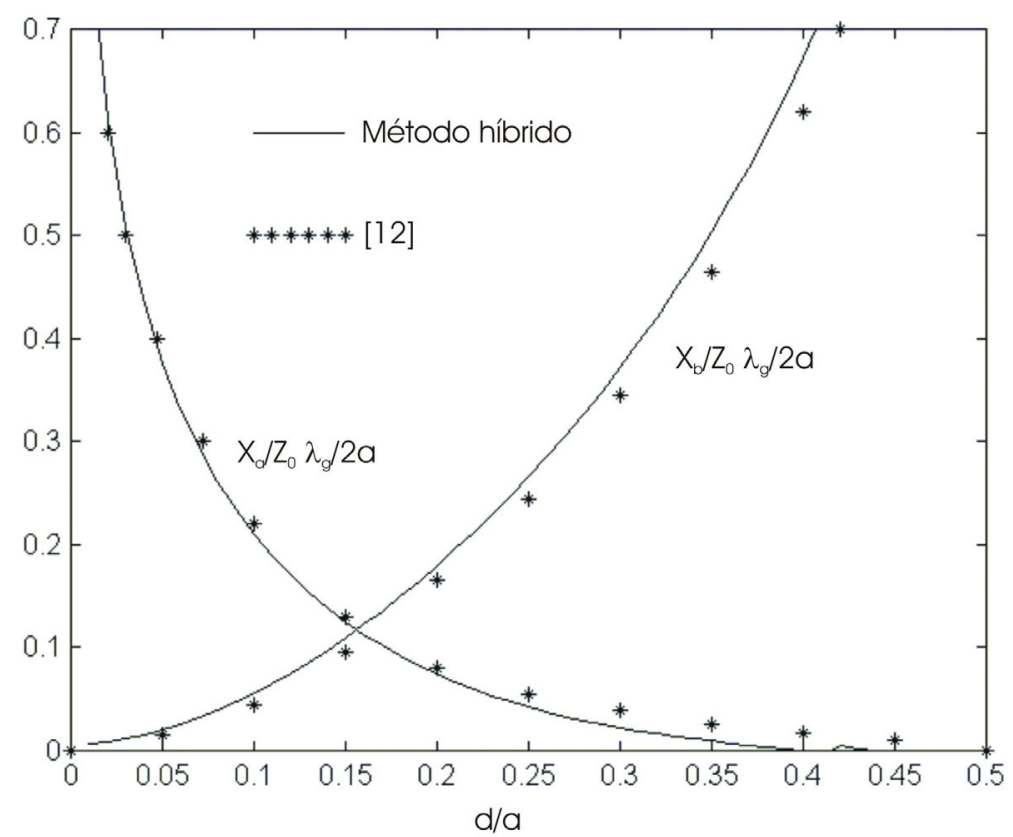

Figura 3.18: Parámetros circuitales normalizados de una guía rectangular con un poste metálico circular centrado. Variación con el tamaño del poste. $\lambda / a=1,01$

parado los parámetros circuitales de la estructura $X_{a}$ y $X_{b}$, definidos de la siguiente manera:

$$
\begin{aligned}
j \frac{X_{a}}{Z_{01}} & =\frac{2 S_{11}^{(2,1)}}{\left|1-S_{11}^{(1,1)}\right|^{2}-\left|S_{11}^{(2,1)}\right|^{2}} \\
-j \frac{X_{b}}{Z_{01}} & =\frac{1+S_{11}^{(1,1)}-S_{11}^{(2,1)}}{1-S_{11}^{(1,1)}+S_{11}^{(2,1)}}
\end{aligned}
$$

donde $Z_{01}$ es la impedancia característica del modo fundamental de los accesos 1 y 2 .

La matriz de dispersión para el modo fundamental, se puede obtener de forma sencilla a partir de la matriz de admitancias ya calculada mediante la siguiente expresión:

$$
\underline{\underline{S}}=(I-\underline{\underline{\underline{Y}}})(I+\underline{\underline{\underline{Y}}})^{-1}
$$

donde $\underline{\underline{Y}}$ es la matriz de admitancias del modo fundamental normalizada por la admitancia característica del modo fundamental $\left(Y_{01}=1 / Z_{01}\right)$.

En las figuras 3.18 y 3.19 se muestra la variación de los parámetros circuitales normalizados con el diámetro del poste para dos frecuencias diferentes. Estos resultados se comparan con los proporcionados por [12]. Como se puede observar, los resultados proporcionados por el método no son exactamente iguales a los de [12], si bien se aproximan bastante.

En la figura 3.20 se muestra el esquema de una estructura idéntica a la analizada anteriormente, pero con el poste descentrado. Concretamente, el poste se sitúa a una distancia $x_{0}$ 


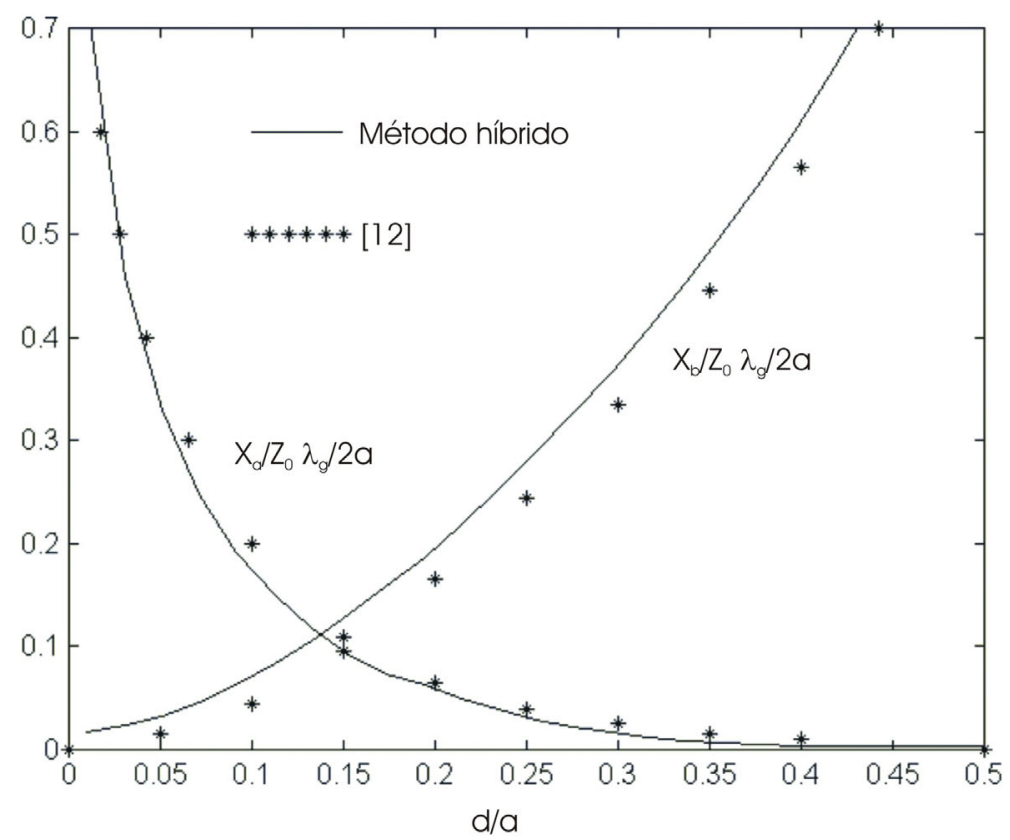

Figura 3.19: Parámetros circuitales normalizados de una guía rectangular con un poste metálico circular centrado. Variación con el tamaño del poste. $\lambda / a=1,2$

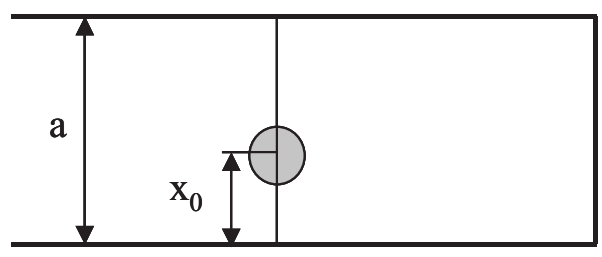

Figura 3.20: Esquema de una guía rectangular de anchura $a$ con un poste circular metálico inductivo descentrado

de una de las paredes de la guía. Los resultados se comparan de nuevo con [12] en la figura 3.21, y de nuevo se observa que aunque los resultados obtenidos son buenos, no se ajustan perfectamente a los de [12].

Para acabar de comprobar la eficacia del método, se han analizado dos nuevas estructuras. Éstas son un iris y una ventana inductiva. Un esquema de dichas estructuras se muestra en la figura 3.22, y en la figura 3.23 se ilustra la variación de los parámetros circuitales normalizados con el tamaño del obstáculo. De nuevo al comparar con resultados de la literatura técnica se observan ligeras diferencias.

Estas desviaciones de los resultados proporcionados por el método de obtención de parámetros de admitancia se debe a que la forma de obtener dichos parámetros no es lo suficientemente precisa. 


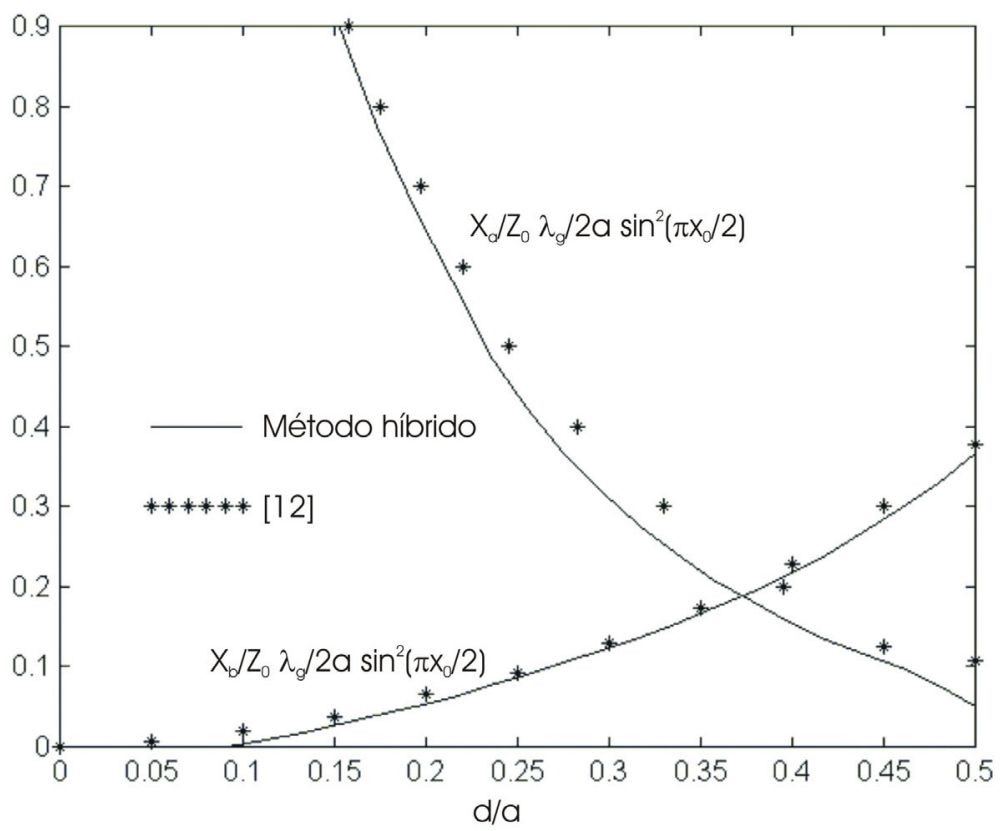

Figura 3.21: Parámetros circuitales normalizados de una guía rectangular con un poste metálico circular descentrado $\left(x_{0}=0,3 a, \lambda / a=1,01\right)$. Variación con el tamaño del poste

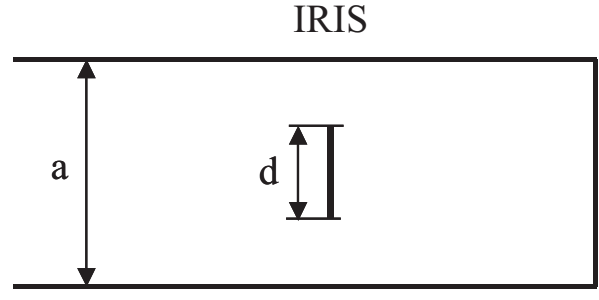

VENTANA

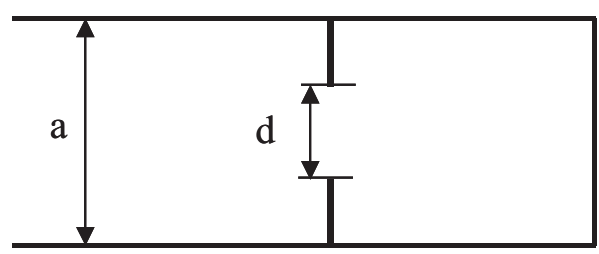

Figura 3.22: Dos esquemas de una guía rectangular de anchura $a$ con un iris y una ventana inductivos, respectivamente, ambos de anchura $d$ 


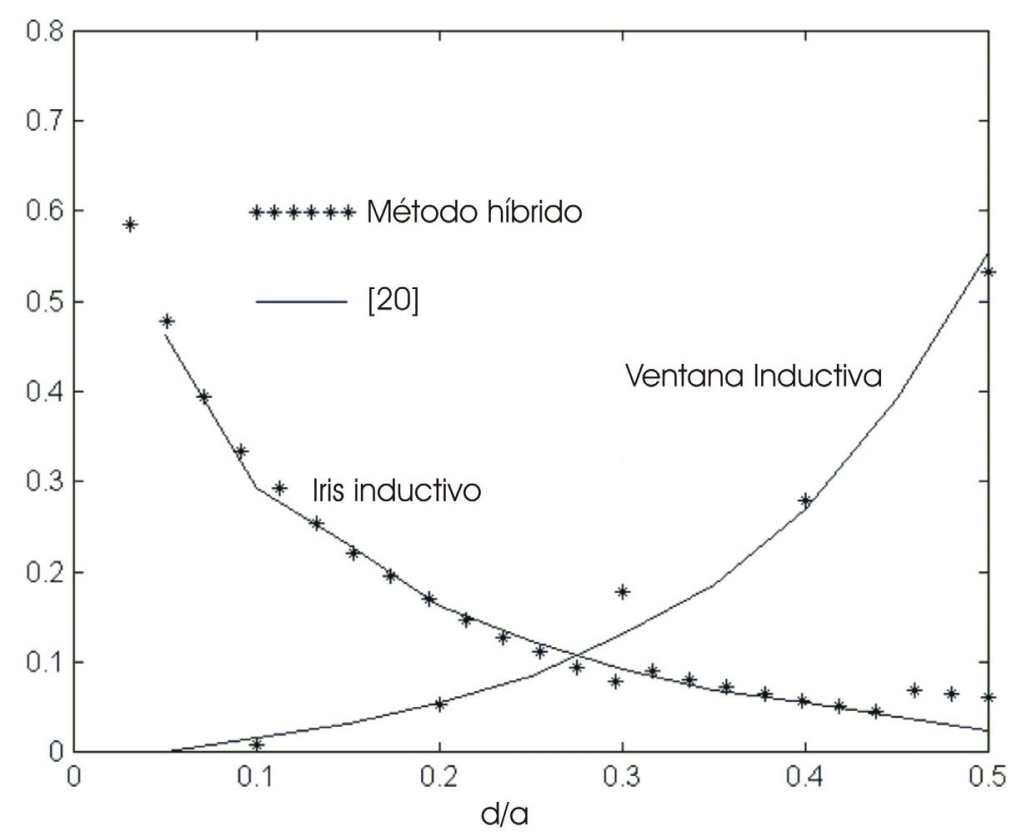

Figura 3.23: Parámetros circuitales normalizados de una guía rectangular con un iris y una ventana inductivos $\left(x_{0}=0,3 a, \lambda / a=1,46\right)$. Variación con la anchura del obstáculo

Por un lado la excitación no es exactamente la del modo fundamental, pues, como se ha explicado anteriormente, la excitación es la de una fuente puntual situada próxima a la boca de la guía. Se ha asegurado que el campo que dicha fuente produce es el adecuado a lo largo de una circunferencia tangente a la boca de la guía, pero eso no garantiza que se excite tan sólo el modo fundamental en el interior de la misma. De hecho, para obtener los resultados medianamente satisfactorios de las figuras 3.18 y 3.19 ha sido necesario situar los planos de referencia de los dos accesos muy lejos del poste, como se puede apreciar en la figura 3.16. Esto se debe a que si la fuente está muy cerca del poste, no hay espacio suficiente para que los modos de orden superior que también se excitan se atenúen antes de incidir sobre el obstáculo. Esto implica que se deben simular estructuras grandes en términos eléctricos, lo que disminuye en gran medida la eficiencia del método.

Por otro lado, para proyectar el campo sobre las funciones vectoriales normalizadas según (3.34), se toman una serie de puntos a lo largo de los dos accesos, se calcula el campo en dichos puntos, y se calcula la integral de (3.34) numéricamente, y no analíticamente.

Para mejorar los resultados, se tomó la decisión de cambiar la forma de calcular los parámetros circuitales de la estructura, ya que el método de análisis de problemas 2-D está suficientemente validado, y lo único que podía fallar es el paso de la matriz de dispersión de espectros cilíndricos $\underline{\underline{D}}$ a la matriz de parámetros circuitales (admitancias en este caso) $\underline{\underline{Y}}$. En la siguiente sección se detalla cómo se han obtenido resultados mucho mejores utilizando un método para obtener la matriz de dispersión $\underline{\underline{S}}$ en lugar de la de admitancias. 


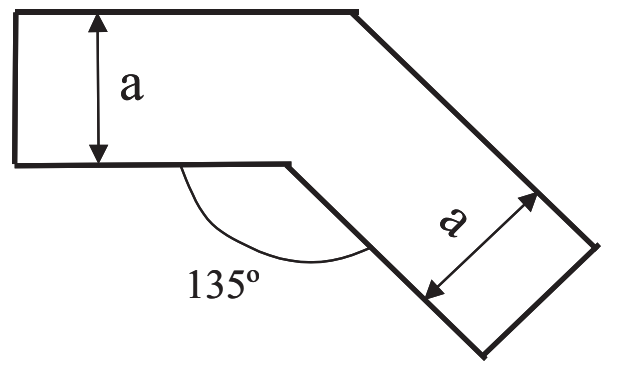

Figura 3.24: Esquema de un codo de $135^{\circ}$ en una guía rectangular de anchura $a$

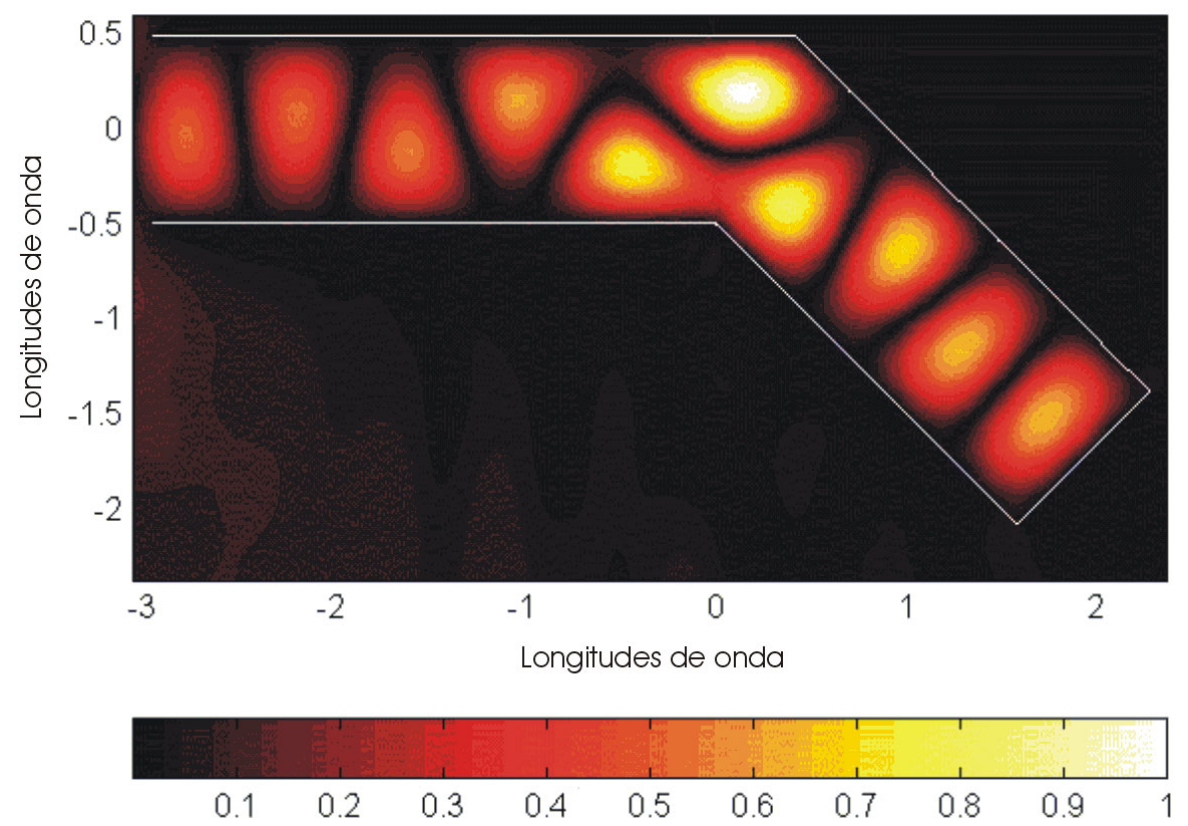

Figura 3.25: Amplitud del campo eléctrico $(\mathrm{dB})$ en el interior de una guía rectangular $(a=$ $0,99 \lambda$ ) con un codo inductivo de $135^{\circ}$, bajo la excitación del modo fundamental en el acceso 1. El acceso 2 se halla cortocircuitado para calcular los parámetros de admitancia

Para terminar la sección se muestran algunos elementos circuitales inductivos analizados mediante la técnica de matrices de admitancias. En la figura 3.24 se muestra el esquema de un codo de $135^{\circ}$ en una guía rectangular. Para obtener los parámetros de admitancia de la estructura se sitúa un cortocircuito en el acceso 2 y se analiza la estructura resultante con el método híbrido de análisis de estructuras 2-D. En la figura 3.25 se muestra la distribución de campo eléctrico resultante.

Finalmente, se muestra la distribución de campo obtenida en la estructura con cortocircuito analizada para obtener los parámetros de admitancias de un salto de sección inductivo (figuras 3.26 y 3.27), de un poste de sección cuadrada (figure 3.28.a) y de un poste de sección triangular (figura 3.28.b). 


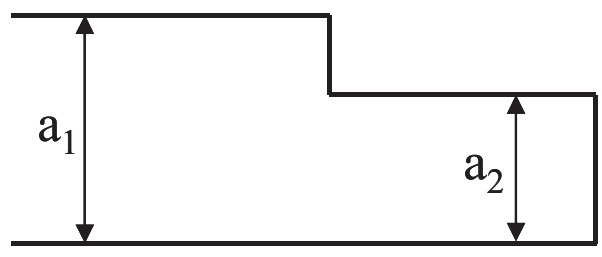

Figura 3.26: Esquema de un salto de sección inductivo en una guía rectangular de anchuras $a_{1}$ y $a_{2}$

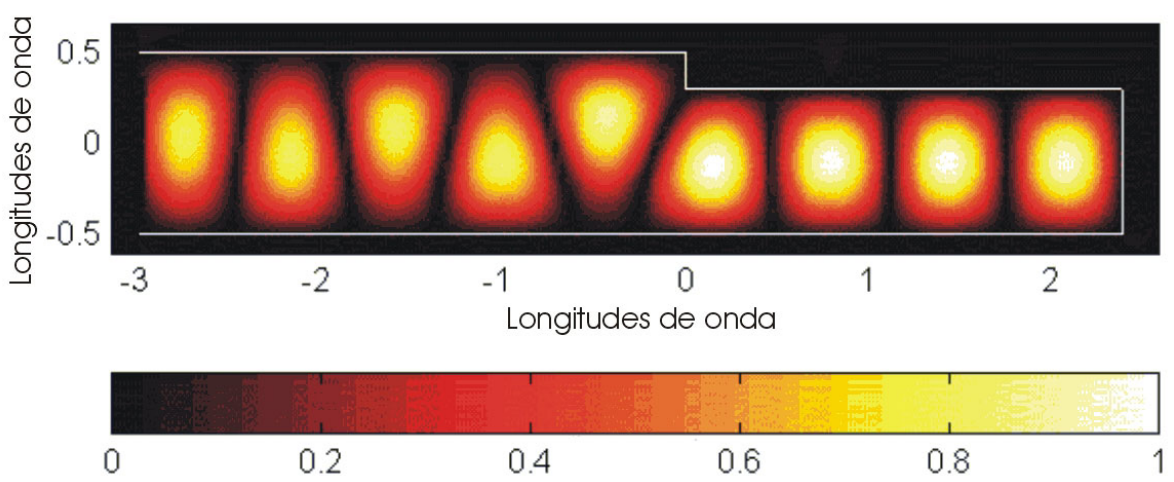

Figura 3.27: Amplitud del campo eléctrico $(\mathrm{dB})$ en el interior de una guía rectangular con un salto de sección inductivo $\left(a_{1}=0,99 \lambda, a_{2}=0,8 \lambda\right)$, bajo la excitación del modo fundamental en el acceso 1. El acceso 2 se halla cortocircuitado para calcular los parámetros de admitancia 

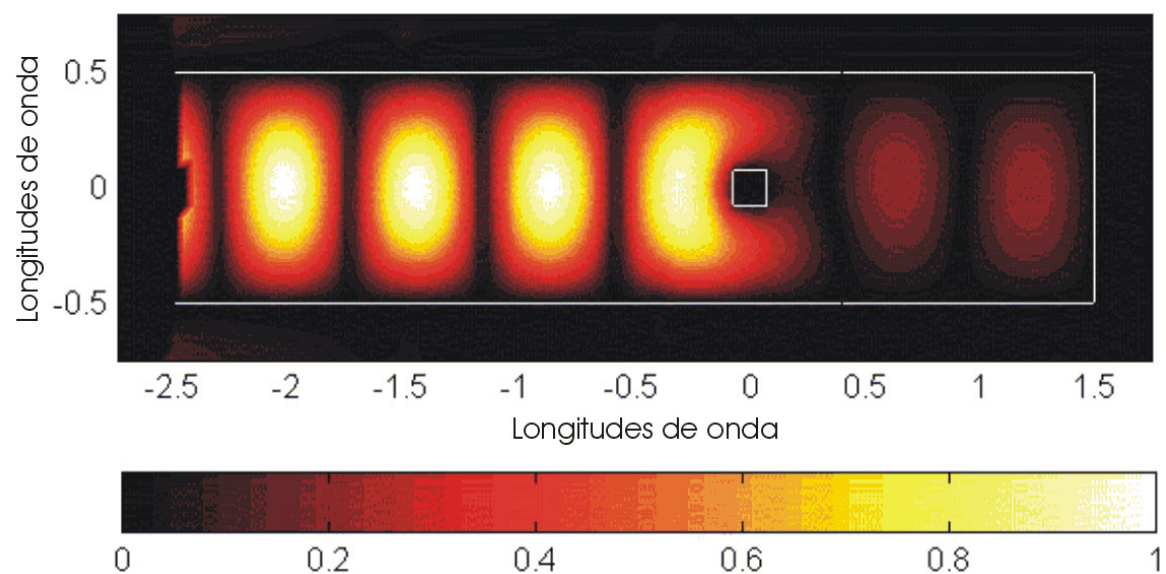

(a) Poste cuadrado

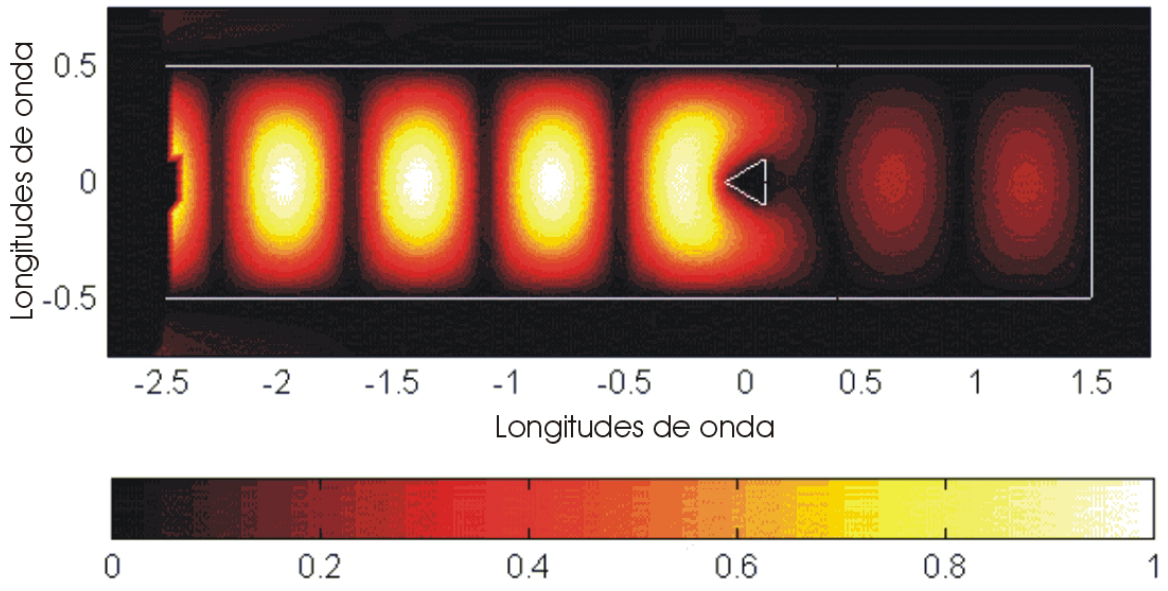

(b) Poste triangular

Figura 3.28: Amplitud del campo eléctrico $(\mathrm{dB})$ en el interior de una guía rectangular con postes de geometrías no canónicas (poste cuadrado y triangular), bajo la excitación del modo fundamental en el acceso 1. El acceso 2 se halla cortocircuitado para calcular los parámetros de admitancia 


\subsection{Obtención de la MDG mediante acoplo de modos}

\subsubsection{Introducción}

De acuerdo con la definición de los parámetros de dispersión dada por (3.26), se puede deducir la siguiente expresión para dichos parámetros:

$$
S_{m n}^{(i, j)}=\frac{b_{m}^{(i)}}{a_{n}^{(j)}}, \quad \operatorname{con} \begin{cases}a_{p}^{(k)}=0 & k \neq j, \quad \forall p \\ a_{p}^{(j)}=0 & p \neq n\end{cases}
$$

La ecuación anterior indica que se podrían calcular los parámetros $S_{m n}^{(i, j)}$ de la red (con $\left.i=[1, \ldots, N], m=\left[1, \ldots, M_{i}\right]\right)$, si se consiguiera que las ondas incidentes se anulasen todas excepto la $n$-ésima onda del acceso $j$-ésimo $\left(a_{n}^{(j)}\right)$. En un análisis unimodal, es decir, si tan sólo se puede propagar el modo fundamental en cada uno de los puertos de acceso, se pueden calcular los parámetros de dispersión colocando una impedancia de valor igual a la impedancia característica del modo fundamental en cada acceso excepto en el $j$-ésimo, y excitando dicho acceso con el modo fundamental. En la figura 3.29 se muestra la estructura que se debe analizar para calcular los parámetros $S_{11}^{(i, 1)}$, con $i=[1, \ldots, N]$.

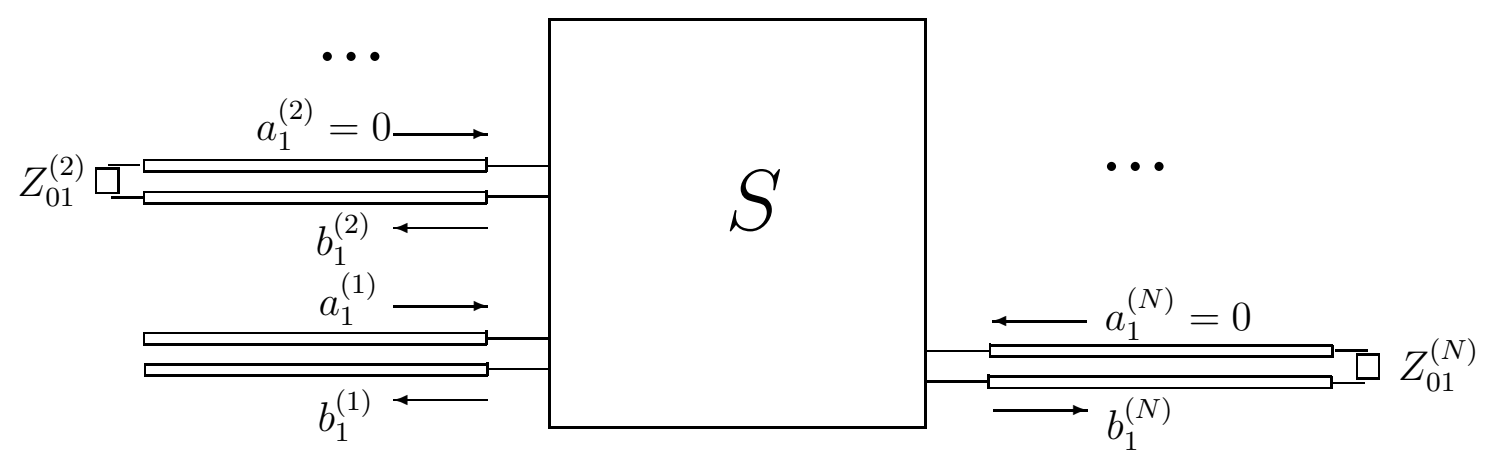

Figura 3.29: Configuración para el cálculo de los parámetros $S_{11}^{(i, 1)}, i \in[1, \ldots, N]$

En el caso de que se desee realizar un análisis multimodal, no se puede terminar cada acceso $j$ con una impedancia que consiga que todas las ondas modales $a_{n}^{(j)}$ se anulen. Si situamos una impedancia $Z_{01}^{(j)}$, conseguiremos que $a_{1}^{(j)}$ sea cero, pero no así $a_{2}^{(j)}, a_{3}^{(j)}$, etc. Por tanto, para obtener la matriz de dispersión generalizada será necesario seguir otro procedimiento. En la siguiente sección se detalla cómo se ha conseguido calcular la MDG mediante un proceso de acoplo de modos.

\subsubsection{Acoplo de modos}

El esquema general del problema que se desea analizar se muestra en la figura 3.30. Se tiene un segmento irregular de la guía rectangular, invariante en altura, al que acceden un número arbitrario $J$ de puertos. Denominaremos $\hat{x}_{i}, \hat{y}_{i}$ y $\hat{z}_{i}$, con $i \in[1, \ldots, J]$, a los vectores unitarios del sistema de coordenadas local al acceso $i$ definidos en la forma estándar 
para una guía rectangular, es decir, del mismo modo que en la figura 3.1. También definiremos un sistema de coordenadas global, válido para todo el segmento irregular de la guía, definido por los vectores $\hat{x}, \hat{y}$ y $\hat{z}$ (ver figura 3.30), de manera que la geometría de la guía es invariante a lo largo del eje $\hat{z}$, que está alineado con el eje $\hat{y}_{i}$ de cada acceso $i$. Como la geometría es invariante en una dimensión, a partir de ahora obviaremos dicha dimensión, y consideraremos únicamente las coordenadas $\left(x_{i}, z_{i}\right)$ para denotar la posición dentro del acceso $i$ en el sistema de coordenadas local a ese puerto; y las coordenadas $(x, y)$ para denotar la posición en cualquier punto con el sistema de coordenadas global (ver figura 3.30). Como caracterizaremos el segmento irregular utilizando modos cilíndricos, usaremos con frecuencia las coordenadas cilíndricas $(\rho, \phi)$ para denotar la posición en el sistema de coordenadas global, siendo:

$$
\begin{aligned}
\rho & =\sqrt{x^{2}+y^{2}} \\
\phi & =\arctan \frac{y}{x}
\end{aligned}
$$

Como se puede observar en la figura 3.30, pueden existir múltiples objetos metálicos o dieléctricos de geometría arbitraria, y cada acceso $i$ puede tener una anchura diferente $a_{i}$ $(i \in[1, \ldots, J])$. La posición y la inclinación de cada acceso viene definida por el punto $P_{i}$ y por los vectores unitarios $\hat{x}_{i}$ y $\hat{z}_{i}$. El segmento irregular de la guía está encerrado en una circunferencia de radio $R$, de manera que fuera de esa circunferencia sólo existen varias guías rectangulares (las que acceden al segmento) de geometría regular.

Como se ha comentado con anterioridad, por cada acceso inciden al segmento únicamente modos $T E_{m 0}$. Como el segmento es invariante en la dimensión $\hat{z}$, los modos que se excitan en él son sólo modos $T E_{m 0}$, también invariantes en $\hat{z}$. Por tanto, todos los campos eléctricos están polarizados linealmente según $\hat{z}$.

En consecuencia, el segmento puede caracterizarse utilizando alguno de los métodos de análisis de dispersión múltiple bidimensional del capítulo 2. Para utilizar estos métodos se debe emplear una formulación de espacio abierto, expresando los campos eléctricos incidente y dispersado (polarizados según $\hat{z}$ ) como sumas de modos cilíndricos:

$$
\begin{aligned}
& \vec{E}^{i n}(\rho, \phi)=\sum_{p=-\infty}^{\infty} i_{p} J_{p}(K \rho) e^{j p \phi} \hat{z} \quad \text { (Campo incidente) } \\
& \vec{E}^{s c}(\rho, \phi)=\sum_{q=-\infty}^{\infty} c_{q} H_{q}^{(2)}(K \rho) e^{j q \phi} \hat{z} \quad \text { (Campo dispersado) }
\end{aligned}
$$

donde $J_{p}(K \rho) e^{j p \phi}$ es el modo espectral cilíndrico incidente $p$-ésimo (finito en el origen de coordenadas), y $H_{q}^{(2)}(K \rho) e^{j q \phi}$ es el $q$-ésimo modo espectral cilíndrico emergente (singular en el origen de coordenadas). Los coeficientes $i_{p}$ constituyen el espectro cilíndrico de campo incidente, y los coeficientes $c_{q}$ el espectro cilíndrico de campo dispersado. Por razones computacionales, las sumas infinitas de modos incidentes y dispersados se deben truncar de la siguiente forma: 


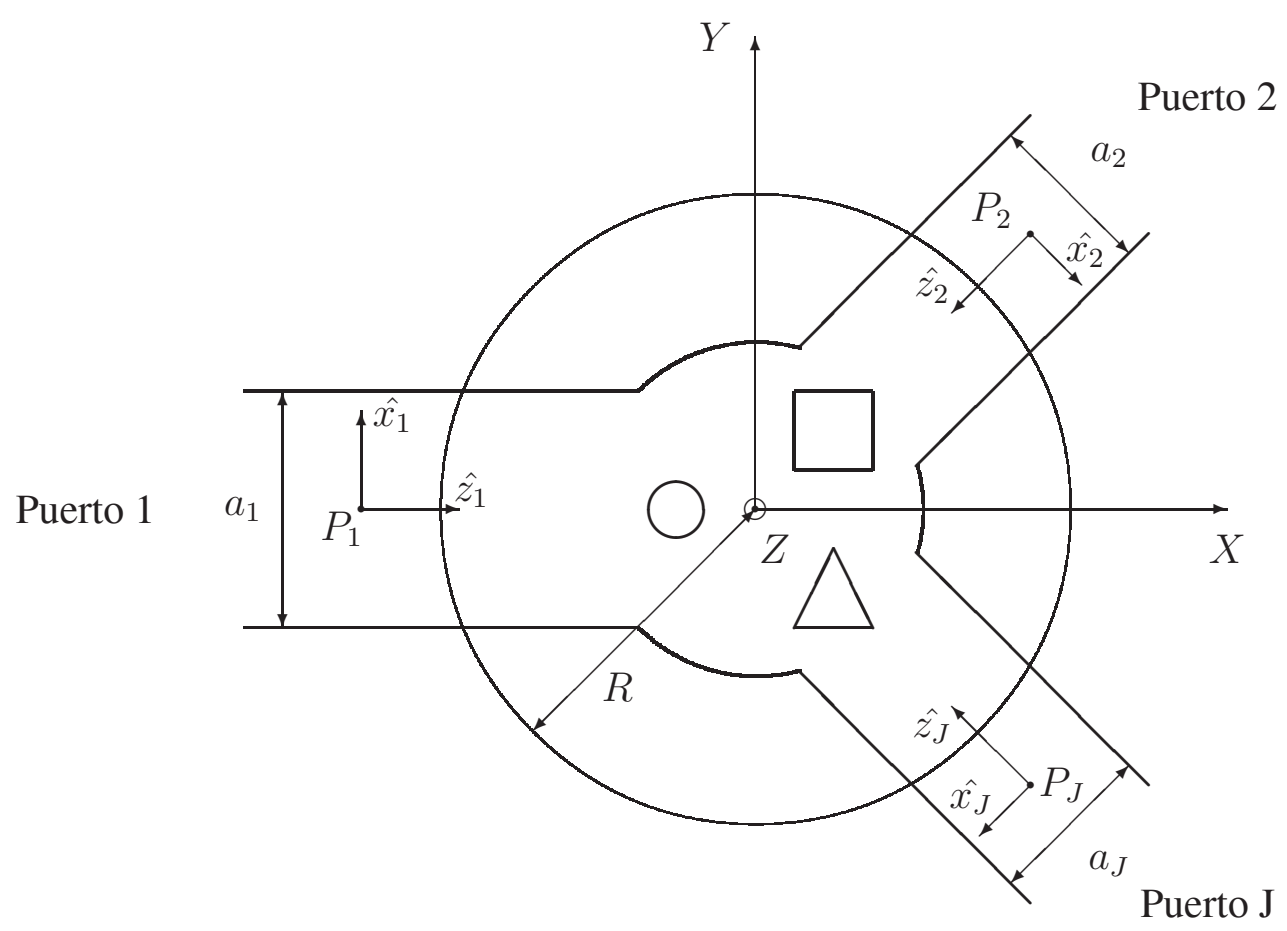

Figura 3.30: Esquema de un problema inductivo de geometría arbitraria en una guía rectangular

$$
\begin{aligned}
\vec{E}^{i n}(\rho, \phi) & =\sum_{p=-N_{i n}}^{N_{i n}} i_{p} J_{p}(K \rho) e^{j p \phi} \hat{z} \\
\vec{E}^{s c}(\rho, \phi) & =\sum_{q=-N_{s c}}^{N_{s c}} c_{q} H_{q}^{(2)}(K \rho) e^{j q \phi} \hat{z}
\end{aligned}
$$

donde $N_{s c}$ debe cumplir que $N_{s c}>K R$, siendo $R$ el radio de la circunferencia que contiene el segmento irregular de la guía. Por otro lado, $N_{\text {in }}$ debe ser lo suficientemente elevado como para que la suma de modos cilíndricos incidentes reconstruya correctamente el campo incidente dentro de la circunferencia que contiene al segmento irregular. Cuantos más modos guiados se tomen en consideración, mayor será la variación espacial del campo eléctrico, y por lo tanto mayor debe ser el número de modos cilíndricos $N_{i n}$ necesarios para reconstruir correctamente ese campo. En principio, para una reconstrucción totalmente correcta $N_{\text {in }}$ debiera ser infinito. Como esto es imposible desde el punto de vista computacional, se ha seguido el criterio de truncar la serie de modo que los términos que se desprecien no excedan nunca en amplitud de un determinado porcentaje $\left(1 / R_{A B}\right)$ del máximo coeficiente de dicha serie (ver anexo C). Aplicando ese criterio, se obtiene que el valor de $N_{i n}^{i}$ para reconstruir correctamente el campo dentro de la circunferencia de radio $R$ debido a la incidencia de 
modos guiados por el acceso $i$-ésimo es:

$$
N_{i n}^{i}=\operatorname{ceil}\left(\frac{M_{i} \pi R}{a_{i}}+n_{0}^{i}\right)
$$

donde $M_{i}$ es el número de modos guiados $T E_{m 0}$ en el acceso $i$-ésimo, ceil es la función que obtiene un número entero a partir de uno real redondeando hacia arriba, y $n_{0}^{i}$ se obtiene como:

$$
n_{0}^{i}=\operatorname{ceil}\left(\frac{20}{L} \frac{C(K, L)}{D(K, L)}\right)
$$

donde:

$$
\begin{aligned}
C(K, L)= & C_{0}\left(945 K^{5} L^{10}+3150 K^{4} L^{8}\left(12-L^{2}\right)\right. \\
& +315 K^{3} L^{6}\left(7 L^{4}-240 L^{2}+160 \cdot 4 !\right) \\
& -15 K^{2} L^{4}\left(17 L^{6}-1512 L^{4}+2 \cdot 8 ! L^{2}-48 \cdot 8 !\right) \\
& +K L^{2}\left(L^{8}-360 L^{6}+2 \cdot 8 ! L^{4}-240 \cdot 8 ! L^{2}+128 \cdot 10 !\right) \\
& +1024 \cdot 10 !) \\
D(K, L)= & D_{0}\left(945 K^{5} L^{10}+1050 K^{4} L^{8}\left(44-3 L^{2}\right)\right. \\
& +15 K^{3} L^{6}\left(147 L^{4}-6160 L^{2}+176 \cdot 6 !\right) \\
& -15 K^{2} L^{4}\left(17 L^{6}-1848 L^{4}+176 \cdot 6 ! L^{2}-528 \cdot 8 ! / 5\right) \\
& +K L^{2}\left(L^{8}-440 L^{6}+176 \cdot 6 ! L^{4}-528 \cdot 8 ! L^{2}+42240 \cdot 8 !\right) \\
& +11264 \cdot 10 !) \\
C_{0}= & \frac{L e^{-K}}{2048 \cdot 10 ! \pi} \\
D_{0}= & \frac{L e^{-K}}{22528 \cdot 10 ! \pi} \\
L= & 2 \arcsin \left(\frac{a_{i}}{2 R}\right) \\
K= & \gamma_{M_{i}}^{i} R
\end{aligned}
$$

Con el objetivo de simplificar la notación, es conveniente extender las series de (3.106) y (3.107) al mismo número de términos $N=\operatorname{máx}\left(N_{i n}, N_{s c}\right)$, donde:

$$
N_{i n}=\operatorname{máx}\left(N_{i n}^{1}, N_{i n}^{2}, \ldots, N_{i n}^{J}\right)
$$

Por lo tanto, el valor de $N$ que permite truncar las series de (3.106) y (3.107) sin pérdida significativa de precisión es:

$$
N \geq \max \left(\frac{M_{i} \pi R}{a_{i}}+n_{0}^{i}, K R\right) \quad i \in[1, \ldots, J]
$$

Utilizando un método de análisis de problemas de dispersión bidimensionales como los del capítulo 2, se obtiene una función de transferencia o matriz de dispersión $\underline{\underline{D}}$ de todos los 
objetos contenidos en la circunferencia de radio $R$. Esta matriz permite obtener el espectro de campo dispersado a partir del espectro de campo incidente de la siguiente forma:

$$
\left[c_{q}\right]=\underline{\underline{D}} \cdot\left[i_{p}\right] \rightarrow c_{q}=\sum_{p=-N_{i n}}^{N_{i n}} D_{q p} i_{p}
$$

Como se ha indicado anteriormente, el número de modos del espectro incidente y dispersado se ha extendido en ambos casos a $N=\operatorname{máx}\left(N_{i n}, N_{s c}\right)$. Por tanto, la matriz $\underline{\underline{D}}$ será cuadrada.

Una vez que la matriz $\underline{\underline{D}}$ ha sido calculada, el segmento irregular de la guía con todos los obstáculos y discontinuidades inductivas queda totalmente caracterizado usando formulación típica de problemas abiertos (modos cilíndricos). Con el objeto de caracterizar el segmento desde el punto de vista de los modos guiados, se debe realizar un acoplo de modos de una guía rectangular estándar a modos cilíndricos. Esto puede conseguirse forzando la continuidad de los campos eléctrico y magnético en la circunferencia de radio $R$ que encierra al segmento irregular. Los campos transversales totales en la circunferencia pueden expresarse como una suma de modos guiados de la siguiente forma:

$$
\begin{aligned}
\vec{E}_{t}^{g}(\rho=R, \phi) & =\sum_{i=1}^{J} \vec{E}_{t}^{(i)}(\rho=R, \phi) \\
\vec{H}_{t}^{g}(\rho=R, \phi) & =\sum_{i=1}^{J} \vec{H}_{t}^{(i)}(\rho=R, \phi)
\end{aligned}
$$

donde $\vec{E}_{t}^{(i)}$ y $\vec{H}_{t}^{(i)}$ son los campos eléctrico y magnético transversales en el acceso $i$, los cuales pueden expresarse como suma de modos incidentes y reflejados utilizando el sistema de coordenadas local al acceso $i$ tal y como ya se vio en (3.25):

$$
\begin{aligned}
\vec{E}_{t}^{(i)}(\rho, \phi) & =\sum_{m=1}^{M_{i}} a_{m}^{(i)} \vec{e}_{m}^{(i)^{\prime}}\left(x_{i}\right) e^{-\gamma_{m}^{(i)} z_{i}}+\sum_{m=1}^{M_{i}} b_{m}^{(i)} \vec{e}_{m}^{(i)^{\prime}}\left(x_{i}\right) e^{\gamma_{m}^{(i)} z_{i}} \\
\vec{H}_{t}^{(i)}(\rho, \phi) & =\sum_{m=1}^{M_{i}} a_{m}^{(i)} Y_{0 m}^{(i)} \vec{h}_{m}^{(i))^{\prime}}\left(x_{i}\right) e^{-\gamma_{m}^{(i)} z_{i}}-\sum_{m=1}^{M_{i}} b_{m}^{(i)} Y_{0 m}^{(i)} \vec{h}_{m}^{(i)^{\prime}}\left(x_{i}\right) e^{\gamma_{m}^{(i)} z_{i}}
\end{aligned}
$$


En el caso de guía de onda rectangular, y para modos $T E_{m 0}$ :

$$
\begin{aligned}
\vec{e}_{m}^{(i)^{\prime}}\left(x_{i}\right) & =-\hat{y}_{i} \sqrt{\frac{2 Z_{0 m}^{(i)}}{a_{i} b_{i}}} \sin \left(\frac{m \pi}{a_{i}} x_{i}\right) \\
\vec{h}_{m}^{(i)^{\prime}}\left(x_{i}\right) & =\hat{x}_{i} \sqrt{\frac{2 Z_{0 m}^{(i)}}{a_{i} b_{i}}} \sin \left(\frac{m \pi}{a_{i}} x_{i}\right) \\
\gamma_{m}^{(i)} & =\sqrt{\left(\frac{m \pi}{a_{i}}\right)^{2}-K^{2}} \\
Y_{m 0}^{(i)} & =\frac{1}{Z_{m 0}^{(i)}}=\frac{\gamma_{m}^{(i)}}{j \omega \mu}
\end{aligned}
$$

donde $a_{i}$ y $b_{i}$ son la anchura y altura del acceso $i, K$ es el número de onda ( $\left.K=\omega \sqrt{\mu \varepsilon}\right)$, y los vectores unitarios $\hat{x}_{i}$ e $\hat{y}_{i}$ son los definidos en la figura 3.1. De acuerdo con la figura 3.30, $\hat{y}_{i}=\hat{z}$. Por tanto:

$$
\begin{aligned}
\vec{E}_{t}^{(i)}(\rho, \phi)= & \sum_{m=1}^{M_{i}} a_{m}^{(i)} e_{m}^{(i)^{\prime \prime}}\left(x_{i}\right) e^{-\gamma_{m}^{(i)} z_{i}} \hat{z}+\sum_{m=1}^{M_{i}} b_{m}^{(i)} e_{m}^{(i)^{\prime \prime}}\left(x_{i}\right) e^{\gamma_{m}^{(i)} z_{i}} \hat{z} \\
\vec{H}_{t}^{(i)}(\rho, \phi)= & \sum_{m=1}^{M_{i}}-a_{m}^{(i)} Y_{0 m}^{(i)} e_{m}^{(i)^{\prime \prime}}\left(x_{i}\right) e^{-\gamma_{m}^{(i)} z_{i}} \hat{x}_{i} \\
& +\sum_{m=1}^{M_{i}} b_{m}^{(i)} Y_{0 m}^{(i)} e_{m}^{(i)^{\prime \prime}}\left(x_{i}\right) e^{\gamma_{m}^{(i)} z_{i}} \hat{x}_{i}
\end{aligned}
$$

donde $a_{m}^{i}$ y $b_{m}^{i}$ son las amplitudes de los modos guiados $T E_{m 0}$ incidentes y reflejados en el acceso $i$-ésimo, y:

$$
e_{m}^{(i)^{\prime \prime}}\left(x_{i}\right)= \begin{cases}-\sqrt{\frac{2 Z_{0 m}^{(i)}}{a_{i} b_{i}}} \sin \left(\frac{m \pi}{a_{i}} x_{i}\right) & \phi \in\left[\phi_{i m}, \phi_{i M}\right] \\ 0 & \text { otro }\end{cases}
$$

En estas expresiones $\phi_{i m}$ y $\phi_{i M}$ son los límites de la inserción del acceso $i$-ésimo en la circunferencia de radio $R$, tal y como se muestra en la figura 3.31. Los valores de $\phi_{i m}$ y $\phi_{i M}$ pueden determinarse fácilmente a partir de la figura 3.31.

Para forzar la continuidad de los campos eléctrico y magnético en la circunferencia de radio $R$ (los modos guiados que definen el campo fuera de la circunferencia deben ser continuos con los modos cilíndricos que definen el campo dentro), las coordenadas locales a cada acceso $i\left(x_{i}\right.$ y $\left.z_{i}\right)$, deben ser expresadas en relación con las coordenadas del sistema de coordenadas global $(\rho$ y $\phi)$. Esta relación se deduce en el apéndice $\mathbf{D}$, resultando ser la siguiente:

$$
\left[\begin{array}{l}
z_{i} \\
x_{i}
\end{array}\right]=\left[\begin{array}{ll}
z_{i_{x}} & x_{i_{x}} \\
z_{i_{y}} & x_{i_{y}}
\end{array}\right]^{(-1)} \cdot\left(R\left[\begin{array}{c}
\cos \phi \\
\sin \phi
\end{array}\right]-\left[\begin{array}{c}
P_{i_{x}} \\
P_{i_{y}}
\end{array}\right]+\frac{a_{i}}{2}\left[\begin{array}{l}
x_{i_{x}} \\
x_{i_{y}}
\end{array}\right]\right)
$$




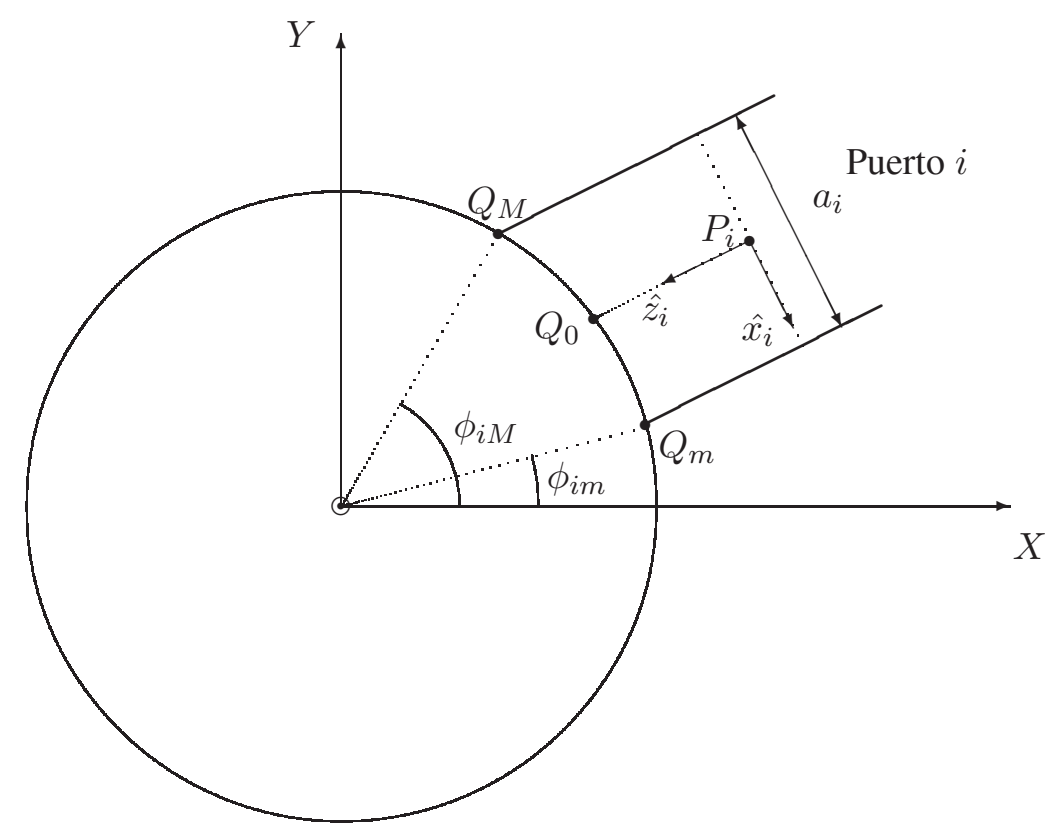

Figura 3.31: Sistema de coordenadas global y local al puerto $i$

donde el valor de $\phi$ está contenido en el intervalo $\left[\phi_{i m}, \phi_{i M}\right]$.

El plano de referencia para el puerto $i$ está dado por el punto $P_{i}$, el cual determina a qué altura se halla el valor cero de la coordenada $z_{i}$ del acceso $i$. Si $z_{i}$ alcanza valores muy elevados, podrían aparecer problemas de singularidad a la hora de resolver los sistemas matriciales que será necesario plantear para realizar el acoplo modal. Esto se debe al término $e^{\gamma_{m}^{(i)} z_{i}}$ en la expresión de los campos guiados (ver ecuación (3.123)), ya que la constante de propagación $\gamma_{m}^{(i)}$ es un número real elevado para los modos guiados de mayor orden, y si $z_{i}$ es también elevado, la exponencial será demasiado grande y eso puede dar lugar a problemas de singularidad. Con el objeto de evitar la aparición de estas posibles singularidades, el plano de referencia de cada acceso $i$ se mueve para asegurar que la coordenada $z_{i}$ alcance valores no muy elevados a lo largo de la circunferencia de radio $R$ entre los límites $\phi_{i m} \mathrm{y}$ $\phi_{i M}$ del acceso $i$ (ver figura 3.31). Concretamente, el nuevo plano de referencia en el acceso $i$ se sitúa a una distancia $d_{i}$ del plano de referencia original (punto $P_{i}$ ), donde $d_{i}$ se calcula de la siguiente forma:

$$
d_{i}=\frac{2 z_{i}\left(Q_{0}\right)+z_{i}\left(Q_{m}\right)+z_{i}\left(Q_{M}\right)}{4}
$$

Por lo tanto, el movimiento de los planos de referencia supone el uso de una nueva 
coordenada $z_{i}^{\prime}=z_{i}-d_{i}$ en cada acceso $i$.

Una vez definidas las expresiones de los campos en términos de modos cilíndricos y guiados, se está en disposición de forzar la continuidad de los campos eléctrico y magnético en la circunferencia de radio $R$. En primer lugar forzaremos la continuidad de campo eléctrico:

$$
\vec{E}^{i n}(\rho=R, \phi)+\vec{E}^{s c}(\rho=R, \phi)=\sum_{i=1}^{J} \vec{E}_{t}^{(i)}(\rho=R, \phi)
$$

donde:

$$
\begin{aligned}
\vec{E}^{i n}(R, \phi)+\vec{E}^{s c}(R, \phi) & =\sum_{p=-N}^{N} i_{p} J_{p}(K R) e^{j p \phi} \hat{z}+\sum_{q=-N}^{N} c_{q} H_{q}^{(2)}(K R) e^{j q \phi} \hat{z} \\
& =\sum_{p=-N}^{N}\left[i_{p} J_{p}(K R)+c_{p} H_{p}^{(2)}(K R)\right] e^{j p \phi} \hat{z}
\end{aligned}
$$

$\mathrm{y}$

$$
\begin{aligned}
\sum_{i=1}^{J} \vec{E}_{t}^{(i)}(R, \phi) & =\sum_{m=1}^{M} a_{m} e_{m}^{+}(\rho=R, \phi) \hat{z}+\sum_{m=1}^{M} b_{m} e_{m}^{-}(\rho=R, \phi) \hat{z} \\
& =\sum_{m=1}^{M}\left[a_{m} e_{m}^{+}(\phi)+b_{m} e_{m}^{-}(\phi)\right] \hat{z}
\end{aligned}
$$

con

$$
M=\sum_{i=1}^{J} M_{i}
$$

Los coeficientes $a_{m}$ y $b_{m}$ se definen de la siguiente forma:

$$
\begin{array}{r}
a_{m}=\left\{\begin{array}{cl}
a_{m}^{(1)} & m \in\left[1, M_{1}\right] \\
a_{m-M_{1}}^{(2)} & m \in\left[M_{1}+1, M_{1}+M_{2}\right] \\
\vdots & \\
b_{m}^{(J)}= & \left\{\left[\sum_{j=1}^{J-1} M_{j}+1, M\right]\right. \\
b_{m-\sum_{j=1}^{J-1} M_{j}}^{(1)} & m \in\left[1, M_{1}\right] \\
b_{m-M_{1}}^{(2)} & m \in\left[M_{1}+1, M_{1}+M_{2}\right] \\
\vdots & \\
b_{m-\sum_{j=1}^{(J)} M_{j}}^{J-1} & m \in\left[\sum_{j=1}^{J-1} M_{j}+1, M\right]
\end{array}\right.
\end{array}
$$


y los vectores modales incidente $\left(e_{m}^{+}\right)$y reflejado $\left(e_{m}^{-}\right)$se definen como:

$$
e_{m}^{ \pm}(\phi)=\left\{\begin{array}{r}
e_{m}^{(1)^{\prime \prime}}\left(x_{1}(R, \phi)\right) \cdot e^{\mp \gamma_{m}^{(1)} z_{1}^{\prime}(R, \phi)} \cdot \prod\left(\frac{\phi-\phi_{01}}{\phi_{d 1}}\right) \\
\text { si } m \in\left[1, M_{1}\right] \\
e_{m-M_{1}}^{(2)^{\prime \prime}}\left(x_{2}(R, \phi)\right) \cdot e^{\mp \gamma_{m-M_{1}}^{(2)} z_{2}^{\prime}(R, \phi)} \cdot \prod\left(\frac{\phi-\phi_{02}}{\phi_{d 2}}\right) \\
\text { si } m \in\left[M_{1}+1, M_{1}+M_{2}\right] \\
\vdots \\
e_{m-\sum_{j=1}^{J-1} M_{j}}^{(J)^{\prime \prime}}\left(x_{J}(R, \phi)\right) \cdot e^{\mp \gamma_{m-\sum_{j=1}^{J-1} M_{j}}^{z_{J}^{\prime}(R, \phi)} \cdot \prod\left(\frac{\phi-\phi_{0 J}}{\phi_{d J}}\right)} \\
\text { si } m \in\left[\sum_{j=1}^{J-1} M_{j}+1, M\right]
\end{array}\right.
$$

donde $\phi_{0 i}$ es el valor de la coordenada $\phi$ justo en el centro del acceso $i, \phi_{d i}$ es la anchura angular de dicho acceso $\left(\phi_{d i}=\phi_{i M}-\phi_{i m}\right)$, y $z_{i}^{\prime}$ es la nueva coordenada local en el acceso $i$ $\left(z_{i}^{\prime}=z_{i}-d_{i}\right)$.

Substituyendo (3.128) y (3.129) en (3.127), se obtiene

$$
\sum_{p=-N}^{N}\left[i_{p} J_{p}(K R)+c_{p} H_{p}^{(2)}(K R)\right] e^{j p \phi}=\sum_{m=1}^{M}\left[a_{m} e_{m}^{+}(\phi)+b_{m} e_{m}^{-}(\phi)\right]
$$

Utilizando las conocidas expresiones de la transformada de Fourier de tiempo discreto (DTFT) y de la transformada inversa

$$
\begin{aligned}
W(\Omega)=X(-\Omega) & =\sum_{n=-\infty}^{\infty} x[n] e^{j n \Omega} \\
x[n] & =\frac{1}{2 \pi} \int_{-\pi}^{\pi} W(\Omega) e^{-j n \Omega} \mathrm{d} \Omega
\end{aligned}
$$

Se puede deducir de la expresión (3.134) la siguiente relación:

$$
\begin{aligned}
i_{p} J_{p}(K R)+c_{p} H_{p}^{(2)}(K R) & =\frac{1}{2 \pi} \int_{-\pi}^{\pi} \sum_{m=1}^{M}\left[a_{m} e_{m}^{+}(\phi)+b_{m} e_{m}^{-}(\phi)\right] e^{-j p \phi} \mathrm{d} \phi \\
& =\sum_{m=1}^{M}\left[a_{m} E_{p m}^{+}+b_{m} E_{p m}^{-}\right]
\end{aligned}
$$

donde:

$$
E_{p m}^{ \pm}=\frac{1}{2 \pi} \int_{-\pi}^{\pi} e_{m}^{ \pm}(\phi) e^{-j p \phi} \mathrm{d} \phi
$$

Debemos recordar que el espectro de campo dispersado $c_{q}$ está relacionado con el espectro de campo incidente $i_{p}$ mediante la matriz $\underline{\underline{D}}$. Utilizando esta relación y escribiendo 
(3.137) en forma matricial, podemos relacionar el espectro de campo incidente $i_{p}$ con las amplitudes de los modos guiados $a_{m}$ y $b_{m}$ :

$$
\begin{aligned}
\underline{\underline{J}} \underline{i}+\underline{\underline{H}} \underline{c} & =\underline{\underline{J}} \underline{i}+\underline{\underline{H}} \underline{\underline{D}} \underline{i}=\underline{\underline{E}}^{+} \underline{a}+\underline{\underline{E}}^{-} \underline{b} \\
\underline{i} & =(\underline{\underline{J}}+\underline{\underline{H}} \underline{\underline{D}})^{-1}\left(\underline{\underline{E}}^{+} \underline{a}+\underline{\underline{E}}^{-} \underline{b}\right)
\end{aligned}
$$

donde:

$$
\begin{aligned}
& \underline{i}=\left[i_{-N}, i_{-N+1}, \cdots, i_{N}\right]^{T} \\
& \underline{c}=\left[c_{-N}, c_{-N+1}, \cdots, c_{N}\right]^{T} \\
& \underline{a}=\left[a_{1}, a_{2}, \cdots, a_{M}\right]^{T} \\
& \underline{b}=\left[b_{1}, b_{2}, \cdots, b_{M}\right]^{T} \\
& \underline{\underline{J}}=\left[\begin{array}{cccc}
J_{-N}(K R) & 0 & \cdots & 0 \\
0 & J_{-N+1}(K R) & \cdots & 0 \\
\vdots & \vdots & \ddots & \vdots \\
0 & 0 & \cdots & J_{N}(K R)
\end{array}\right]_{(2 N+1) \times(2 N+1)} \\
& \underline{\underline{H}}=\left[\begin{array}{cccc}
H_{-N}^{(2)}(K R) & 0 & \cdots & 0 \\
0 & H_{-N+1}^{(2)}(K R) & \cdots & 0 \\
\vdots & \vdots & \ddots & \vdots \\
0 & 0 & \cdots & H_{N}^{(2)}(K R)
\end{array}\right]_{(2 N+1) \times(2 N+1)} \\
& \underline{\underline{E}}^{ \pm}=\left[\begin{array}{cccc}
E_{-N, 1}^{ \pm} & E_{-N, 2}^{ \pm} & \cdots & E_{-N, M}^{ \pm} \\
E_{-N+1,1}^{ \pm} & E_{-N+1,2}^{ \pm} & \cdots & E_{-N+1, M}^{ \pm} \\
\vdots & \vdots & \ddots & \vdots \\
E_{N, 1}^{ \pm} & E_{N, 2}^{ \pm} & \cdots & E_{N, M}^{ \pm}
\end{array}\right]_{(2 N+1) \times M}
\end{aligned}
$$

Una vez relacionado el espectro $c_{q}$ con el espectro $i_{p}$, y relacionado $i_{p}$ con las amplitudes $a_{m}$ y $b_{m}$, resulta necesaria otra ecuación para poder eliminar la incógnita $i_{p}$ y despejar $b_{m}$ en función de $a_{m}$. Esta ecuación se puede obtener forzando la continuidad de campo magnético en la circunferencia de radio $R$ :

$$
\vec{H}^{i n}(\rho=R, \phi)+\vec{H}^{s c}(\rho=R, \phi)=\sum_{i=1}^{J}\left[\vec{H}_{t}^{(i)}(\rho=R, \phi)+\vec{H}_{z}^{(i)}(\rho=R, \phi)\right]
$$

donde $\vec{H}_{t}^{(i)}$ y $\vec{H}_{z}^{(i)}$ son, respectivamente, las componentes transversal y longitudinal de campo magnético en el acceso $i$-ésimo.

Como sólo se necesita una ecuación, forzaremos continuidad de una componente de campo magnético. En concreto, se forzará la continuidad de la componente de campo magnético 
transversal en cada acceso. Para ello proyectaremos la expresión (3.147) en una dirección $\hat{t}$ que nos defina la mencionada dirección transversal:

$$
\begin{aligned}
{\left[\vec{H}^{i n}(\rho=R, \phi)+\vec{H}^{s c}(\rho=R, \phi)\right] \cdot \hat{t}=} & \\
& \sum_{i=1}^{J}\left[\vec{H}_{t}^{(i)}(\rho=R, \phi) \cdot \hat{t}+\vec{H}_{z}^{(i)}(\rho=R, \phi) \cdot \hat{t}\right]= \\
& \sum_{i=1}^{J} H_{t}^{(i)}(\rho=R, \phi)
\end{aligned}
$$

donde $\hat{t}$ se define de la siguiente manera

$$
\hat{t}= \begin{cases}\hat{x}_{1} & \phi \in\left[\phi_{1 m}, \phi_{1 M}\right] \\ \hat{x}_{2} & \phi \in\left[\phi_{2 m}, \phi_{2 M}\right] \\ \vdots & \\ \hat{x}_{J} & \phi \in\left[\phi_{J m}, \phi_{J M}\right] \\ \hat{x}_{1} & \text { otro valor de } \phi\end{cases}
$$

Nótese que se ha tomado como dirección transversal en cada tramo de la circunferencia de radio $R$ a la componente transversal de campo dentro del acceso $i\left(\hat{x}_{i}\right)$ que se inserta en ese punto de la circunferencia. No obstante, pueden existir tramos a lo largo de la circunferencia de radio $R$ en los que no haya ningún acceso. En estos tramos se toma la dirección $\hat{x}_{1}$ como dirección transversal.

Los campos magnéticos incidente y dispersado se pueden obtener de manera sencilla a partir de los campos eléctricos de (3.106) y (3.107) mediante las ecuaciones de Maxwell:

$$
\begin{aligned}
& \vec{H}^{i n}(R, \phi)+\vec{H}^{s c}(R, \phi)= \\
& \quad-\frac{1}{K \eta R} \sum_{p=-N}^{N}\left[i_{p} J_{p}(K R)+c_{p} H_{p}^{(2)}(K R)\right] p e^{j p \phi} \hat{\rho} \\
&-\frac{j}{\eta} \sum_{p=-N}^{N}\left[i_{p} J_{p}^{\prime}(K R)+c_{p} H_{p}^{(2)^{\prime}}(K R)\right] e^{j p \phi} \hat{\phi}
\end{aligned}
$$

Proyectando sobre la dirección $\hat{t}$ :

$$
\begin{aligned}
{\left[\vec{H}_{t}^{i n}(R, \phi)+\right.} & \left.\vec{H}_{t}^{s c}(R, \phi)\right] \cdot \hat{t}= \\
& -\frac{1}{K \eta R} \sum_{p=-N}^{N}\left[i_{p} J_{p}(K R)+c_{p} H_{p}^{(2)}(K R)\right] p e^{j p \phi}(\hat{\rho} \cdot \hat{t}) \\
& -\frac{j}{\eta} \sum_{p=-N}^{N}\left[i_{p} J_{p}^{\prime}(K R)+c_{p} H_{p}^{(2)^{\prime}}(K R)\right] e^{j p \phi}(\hat{\phi} \cdot \hat{t})
\end{aligned}
$$


El campo magnético transversal en las guías puede expresarse de la siguiente manera:

$$
\begin{aligned}
\sum_{i=1}^{J} \vec{H}_{t}^{(i)}(R, \phi) & =\sum_{m=1}^{M}-a_{m} Y_{0 m} e_{m}^{+}(\phi) \hat{t}+\sum_{m=1}^{M} b_{m} Y_{0 m} e_{m}^{-}(\phi) \hat{t} \\
& =\sum_{m=1}^{M}\left[-a_{m} Y_{0 m} e_{m}^{+}(\phi)+b_{m} Y_{0 m} e_{m}^{-}(\phi)\right] \hat{t}
\end{aligned}
$$

donde

$$
Y_{0 m}=\left\{\begin{array}{cl}
Y_{0 m}^{(1)} & m \in\left[1, M_{1}\right] \\
Y_{0 m-M_{1}}^{(2)} & m \in\left[M_{1}+1, M_{1}+M_{2}\right] \\
\vdots & \\
Y_{0 m-\sum_{j=1}^{J-1} M_{j}}^{(J)} & m \in\left[\sum_{j=1}^{J-1} M_{j}+1, M\right]
\end{array}\right.
$$

Sustituyendo (3.151) y (3.152) en (3.148), obtenemos:

$$
\begin{aligned}
& \sum_{m=1}^{M}\left[-a_{m} Y_{0 m} e_{m}^{+}(\phi)+b_{m} Y_{0 m} e_{m}^{-}(\phi)\right] \\
= & -\frac{1}{K \eta R} \sum_{p=-N}^{N}\left[i_{p} J_{p}(K R)+c_{p} H_{p}^{(2)}(K R)\right] p e^{j p \phi}(\hat{\rho} \cdot \hat{t}) \\
- & \frac{j}{\eta} \sum_{p=-N}^{N}\left[i_{p} J_{p}^{\prime}(K R)+c_{p} H_{p}^{(2)^{\prime}}(K R)\right] e^{j p \phi}(\hat{\phi} \cdot \hat{t})
\end{aligned}
$$

donde los términos $\left[i_{p} J_{p}(K R)+c_{p} H_{p}^{(2)}(K R)\right]$ e $\left[i_{p} J_{p}^{\prime}(K R)+c_{p} H_{p}^{(2)^{\prime}}(K R)\right]$ deben relacionarse con los coeficientes de modos guiados $a_{m}$ y $b_{m}$, para conseguir que sólo $a_{m}$ y $b_{m}$ aparezcan en la expresión, y poder obtener así una relación de $b_{m}$ con $a_{m}$ que será la matriz de parámetros de dispersión generalizada (MDG) buscada. Al forzar continuidad de campo eléctrico, se llegó a la expresión (3.137), que relacionaba el término $\left[i_{p} J_{p}(K R)+c_{p} H_{p}^{(2)}(K R)\right]$ con $a_{m}$ y $b_{m}$. Para relacionar el término $\left[i_{p} J_{p}^{\prime}(K R)+c_{p} H_{p}^{(2)^{\prime}}(K R)\right]$ con $a_{m} \mathrm{y} b_{m}$, hacemos uso de la expresión matricial (3.139):

$$
\begin{aligned}
\underline{\underline{J^{\prime}}} \underline{i}+{\underline{\underline{H^{\prime}}}}^{\underline{c}} & =\underline{\underline{J^{\prime}}} \underline{i}+\underline{\underline{H^{\prime}}} \underline{\underline{D}} \underline{i}=\left(\underline{\underline{J^{\prime}}}+\underline{\underline{H^{\prime}}} \underline{\underline{D}}\right) \underline{i} \\
& =\left(\underline{\underline{J^{\prime}}}+\underline{\underline{H^{\prime}}} \underline{\underline{D}}\right)(\underline{\underline{J}}+\underline{\underline{H}} \underline{\underline{D}})^{-1}\left(\underline{\underline{E}}^{+} \underline{a}+\underline{\underline{E}}^{-} \underline{b}\right)
\end{aligned}
$$

donde $\underline{\underline{J^{\prime}}}$ y $\underline{\underline{H^{\prime}}}$ son matrices diagonales como $\underline{\underline{J}}$ y $\underline{\underline{H}}$, pero con la primera derivada de las funciones de Bessel y de Hankel en la diagonal principal. 
Si definimos la siguiente matriz $\underline{\underline{W}}$ :

$$
\underline{\underline{W}}=\left(\underline{\underline{J^{\prime}}}+\underline{\underline{H^{\prime}}} \underline{\underline{D}}\right)(\underline{\underline{J}}+\underline{\underline{H}} \underline{\underline{D}})^{-1}
$$

finalmente obtenemos la siguiente relación:

$$
\underline{\underline{J^{\prime}}} \underline{i}+\underline{\underline{H^{\prime}}} \underline{c}=\underline{\underline{W}}\left(\underline{\underline{E}}^{+} \underline{a}+\underline{\underline{E}}^{-} \underline{b}\right)
$$

La matriz $\underline{\underline{W}}$ permite relacionar $\left[i_{p} J_{p}^{\prime}(K R)+c_{p} H_{p}^{(2)^{\prime}}(K R)\right]$ con los coeficientes $a_{m}$ y $b_{m}$, tal y como deseábamos. Sin embargo, el cálculo de $\underline{\underline{W}}$ requiere de la inversión de la matriz $\underline{\underline{B}}=\underline{\underline{J}}+\underline{\underline{H}} \underline{\underline{D}}$, la cual normalmente es una matriz singular si se toma un elevado número de modos cilíndricos $N$. El motivo de esta singularidad es que la matriz $\underline{\underline{D}}$ está mal condicionada, ya que hemos obligado a que sea cuadrada eligiendo el mismo número de modos cilíndricos incidentes y dispersados. Sin embargo, la serie de modos dispersados decae mucho más rápidamente que la de modos incidentes, resultando en una matriz $\underline{\underline{D}}$ cuyos elementos significativos se concentran en las filas centrales, mientras que en las primeras y últimas filas los elementos son prácticamente nulos, tal y como se muestra en la siguiente expresión:

$$
\underline{\underline{D}}=\left[\begin{array}{c}
{[0]_{\left(N_{i n}-N_{s c}\right) \times\left(2 N_{i n}+1\right)}} \\
{\left[D_{s}\right]_{\left(2 N_{s c}+1\right) \times\left(2 N_{i n}+1\right)}} \\
{[0]_{\left(N_{i n}-N_{s c}\right) \times\left(2 N_{i n}+1\right)}}
\end{array}\right]_{\left(2 N_{i n}+1\right) \times\left(2 N_{i n}+1\right)}
$$

donde $N_{s c}$ es el número de modos cilíndricos del espectro de campo dispersado, que ya se vio que debe ser mayor que $K R$, siendo $R$ el radio de la circunferencia que contiene el segmento irregular de la guía. Concretamente, se tomará el siguiente valor para $N_{s c}$ :

$$
N_{s c}=C_{s c} K R
$$

siendo $C_{s c}>1$. Normalmente se toma $C_{s c}=6$, ya que si $C_{s c}$ fuera mayor que 6 se ha comprobado experimentalmente que podrían aparecer problemas de singularidades.

Como $\underline{\underline{J}}$ y $\underline{\underline{H}}$ son matrices diagonales, la matriz $\underline{\underline{B}}$ tendrá la siguiente estructura:

$$
\underline{\underline{B}}=\left[\begin{array}{c|c|c}
{\left[B_{1}\right]_{\left(N_{i n}-N_{s c}\right) \times\left(N_{i n}-N_{s c}\right)}} & {[0]} & {[0]} \\
\hline\left[B_{2}\right]_{\left(2 N_{s c}+1\right) \times\left(N_{i n}-N_{s c}\right)} & {\left[B_{3}\right]_{\left(2 N_{s c}+1\right) \times\left(2 N_{s c}+1\right)}} & {\left[B_{4}\right]_{\left(2 N_{s c}+1\right) \times\left(N_{i n}-N_{s c}\right)}} \\
\hline[0] & {[0]} & {\left[B_{5}\right]_{\left(N_{i n}-N_{s c}\right) \times\left(N_{i n}-N_{s c}\right)}}
\end{array}\right]
$$

donde $\underline{\underline{B_{1}}}$ y $\underline{\underline{B_{5}}}$ son matrices diagonales, y $\underline{\underline{B_{2}}}, \underline{\underline{B_{3}}}$ y $\underline{\underline{B_{4}}}$ son matrices llenas. La matriz $\underline{\underline{B}}$ es de dimensión $\left.2 N_{\text {in }}+1\right) \times\left(2 N_{\text {in }}+1\right)$.

La misma estructura es válida para la matriz $\underline{\underline{A}}=\underline{\underline{J^{\prime}}}+\underline{\underline{H^{\prime}}} \underline{\underline{D}}$ :

$$
\underline{\underline{A}}=\left[\begin{array}{c|c|c}
{\left[A_{1}\right]} & {[0]} & {[0]} \\
\hline\left[A_{2}\right] & {\left[A_{3}\right]} & {\left[A_{4}\right]} \\
\hline[0] & {[0]} & {\left[A_{5}\right]}
\end{array}\right]
$$


Teniendo en cuenta la estructura de las matrices $\underline{\underline{A}}$ y $\underline{\underline{B}}$, se puede calcular la matriz $\underline{\underline{W}}$ buscada del siguiente modo:

$$
\begin{aligned}
\underline{\underline{W}} & \underline{\underline{B}} \\
\underline{\underline{W}}\left[\begin{array}{c|c|c}
{\left[B_{1}\right]} & {[0]} & {[0]} \\
\hline\left[B_{2}\right] & {\left[B_{3}\right]} & {\left[B_{4}\right]} \\
\hline[0] & {[0]} & {\left[B_{5}\right]}
\end{array}\right] & =\left[\begin{array}{c|c|c}
{\left[A_{1}\right]} & {[0]} & {[0]} \\
\hline\left[A_{2}\right] & {\left[A_{3}\right]} & {\left[A_{4}\right]} \\
\hline[0] & {[0]} & {\left[A_{5}\right]}
\end{array}\right]
\end{aligned}
$$

$\underline{\underline{W}}=\left[\begin{array}{c|c|c}{\left[A_{1}\right]\left[B_{1}\right]^{-1}} & {[0]} & {[0]} \\ \hline\left(\left[A_{2}\right]-\left[A_{3}\right]\left[B_{3}\right]^{-1}\left[B_{2}\right]\right)\left[B_{1}\right]^{-1} & {\left[A_{3}\right]\left[B_{3}\right]^{-1}} & \left(\left[A_{4}\right]-\left[A_{3}\right]\left[B_{3}\right]^{-1}\left[B_{4}\right]\right)\left[B_{5}\right]^{-1} \\ \hline[0] & {[0]} & {\left[A_{5}\right]\left[B_{5}\right]^{-1}}\end{array}\right]$

Usando (3.162) se puede calcular $\underline{\underline{W}}$ evitando la inversión de toda la matriz $\underline{\underline{B}}$. Únicamente resulta necesario invertir las submatrices $\underline{\underline{B_{1}}}, \underline{\underline{B_{3}}} \mathrm{y} \underline{\underline{B_{5}}}$. Las submatrices $\underline{\underline{B_{1}}} \mathrm{y} \underline{\underline{B_{5}}}$ son diagonales, de modo que su inversión es sencilla. Únicamente se debe invertir la submatriz $\underline{\underline{B_{3}}}$, que es una matriz llena que por tanto no presenta problemas de singularidad.

Una vez que $\left[i_{p} J_{p}^{\prime}(K R)+c_{p} H_{p}^{(2)^{\prime}}(K R)\right]$ ha sido relacionado con $a_{m}$ y $b_{m}$ mediante la matriz $\underline{\underline{W}}$, la ecuación (3.154) puede simplificarse como sigue:

$$
\begin{aligned}
& \sum_{m=1}^{M}\left[-a_{m} Y_{0 m} e_{m}^{+}(\phi)+b_{m} Y_{0 m} e_{m}^{-}(\phi)\right] \\
= & -\frac{1}{K \eta R} \sum_{p=-N}^{N} \sum_{m=1}^{M}\left[a_{m} E_{p m}^{+}+b_{m} E_{p m}^{-}\right] p e^{j p \phi}(\hat{\rho} \cdot \hat{t}) \\
- & \frac{j}{\eta} \sum_{p=-N}^{N} \sum_{m=1}^{M}\left[a_{m} \sum_{q=-N}^{N} W_{p q} E_{q m}^{+}+b_{m} \sum_{q=-N}^{N} W_{p q} E_{q m}^{-}\right] e^{j p \phi}(\hat{\phi} \cdot \hat{t})
\end{aligned}
$$

Reordenando los sumandos se tiene que:

$$
\begin{aligned}
& \sum_{m=1}^{M}\left[-a_{m} Y_{0 m} e_{m}^{+}(\phi)+b_{m} Y_{0 m} e_{m}^{-}(\phi)\right]= \\
= & -\frac{1}{K \eta R} \sum_{m=1}^{M}\left[a_{m}\left(\sum_{p=-N}^{N} E_{p m}^{+} p e^{j p \phi}\right)+b_{m}\left(\sum_{p=-N}^{N} E_{p m}^{-} p e^{j p \phi}\right)\right](\hat{\rho} \cdot \hat{t}) \\
- & \frac{j}{\eta} \sum_{m=1}^{M}\left[a_{m} \sum_{q=-N}^{N}\left(\sum_{p=-N}^{N} W_{p q} e^{j p \phi}\right) E_{q m}^{+}+b_{m} \sum_{q=-N}^{N}\left(\sum_{p=-N}^{N} W_{p q} e^{j p \phi}\right) E_{q m}^{-}\right](\hat{\phi} \cdot \hat{t})
\end{aligned}
$$


Usando la propiedad de derivación de la DTFT:

$$
\begin{aligned}
& \sum_{m=1}^{M}\left[-a_{m} Y_{0 m} e_{m}^{+}(\phi)+b_{m} Y_{0 m} e_{m}^{-}(\phi)\right]= \\
= & \frac{j}{K \eta R} \sum_{m=1}^{M}\left[a_{m} \frac{\mathrm{d} e_{m}^{+}}{\mathrm{d} \phi}+b_{m} \frac{\mathrm{d} e_{m}^{-}}{\mathrm{d} \phi}\right](\hat{\rho} \cdot \hat{t}) \\
- & \frac{j}{\eta} \sum_{m=1}^{M}\left[a_{m} \sum_{q=-N}^{N} w_{q}(\phi) E_{q m}^{+}+b_{m} \sum_{q=-N}^{N} w_{q}(\phi) E_{q m}^{-}\right](\hat{\phi} \cdot \hat{t})
\end{aligned}
$$

Separando los términos con $a_{m} \mathrm{y}$ con $b_{m}$ :

$$
\begin{aligned}
& \sum_{m=1}^{M} b_{m}\left[Y_{0 m} e_{m}^{-}(\phi)-\frac{j}{K \eta R} \frac{\mathrm{d} e_{m}^{-}}{\mathrm{d} \phi}(\hat{\rho} \cdot \hat{t})+\frac{j}{\eta} \sum_{q=-N}^{N} w_{q}(\phi) E_{q m}^{-}(\hat{\phi} \cdot \hat{t})\right] \\
= & \sum_{m=1}^{M} a_{m}\left[Y_{0 m} e_{m}^{+}(\phi)+\frac{j}{K \eta R} \frac{\mathrm{d} e_{m}^{+}}{\mathrm{d} \phi}(\hat{\rho} \cdot \hat{t})-\frac{j}{\eta} \sum_{q=-N}^{N} w_{q}(\phi) E_{q m}^{+}(\hat{\phi} \cdot \hat{t})\right]
\end{aligned}
$$

A continuación se deben proyectar ambos términos de la expresión anterior sobre $e_{n}^{-}(\phi)$, obteniendo la siguiente expresión:

$$
\begin{aligned}
& \sum_{m=1}^{M} b_{m} \frac{1}{2 \pi} \int_{0}^{2 \pi}\left[Y_{0 m} e_{m}^{-}(\phi)-\frac{j}{K \eta R} \frac{\mathrm{d} e_{m}^{-}}{\mathrm{d} \phi}(\hat{\rho} \cdot \hat{t})\right. \\
+ & \left.\frac{j}{\eta} \sum_{q=-N}^{N} w_{q}(\phi) E_{q m}^{-}(\hat{\phi} \cdot \hat{t})\right] e_{n}^{-}(\phi)^{*} \mathrm{~d} \phi \\
= & \sum_{m=1}^{M} a_{m} \frac{1}{2 \pi} \int_{0}^{2 \pi}\left[Y_{0 m} e_{m}^{+}(\phi)+\frac{j}{K \eta R} \frac{\mathrm{d} e_{m}^{+}}{\mathrm{d} \phi}(\hat{\rho} \cdot \hat{t})\right. \\
& \left.-\frac{j}{\eta} \sum_{q=-N}^{N} w_{q}(\phi) E_{q m}^{+}(\hat{\phi} \cdot \hat{t})\right] e_{n}^{-}(\phi)^{*} \mathrm{~d} \phi
\end{aligned}
$$

Si repetimos este proceso con $n$ variando desde 1 hasta $M$, obtendremos $M$ ecuaciones lineales de $M$ incógnitas, que se pueden expresar de forma matricial de la siguiente manera:

$$
\triangleq \underline{\underline{b}}=\underline{\underline{Q}} \underline{a}
$$


donde $\triangleq \mathrm{y} \underline{\underline{Q}}$ son matrices de dimensión $M \times M$, cuyos elementos se definen como sigue:

$$
\begin{aligned}
\Delta_{m n}= & \frac{1}{2 \pi} \int_{0}^{2 \pi}\left[Y_{0 n} e_{n}^{-}(\phi)-\frac{j}{K \eta R} \frac{\mathrm{d} e_{n}^{-}}{\mathrm{d} \phi}(\hat{\rho} \cdot \hat{t})\right. \\
& \left.+\frac{j}{\eta} \sum_{q=-N}^{N} w_{q}(\phi) E_{q n}^{-}(\hat{\phi} \cdot \hat{t})\right] e_{m}^{-}(\phi)^{*} \mathrm{~d} \phi \\
Q_{m n}= & \frac{1}{2 \pi} \int_{0}^{2 \pi}\left[Y_{0 n} e_{n}^{+}(\phi)+\frac{j}{K \eta R} \frac{\mathrm{d} e_{n}^{+}}{\mathrm{d} \phi}(\hat{\rho} \cdot \hat{t})\right. \\
& \left.-\frac{j}{\eta} \sum_{q=-N}^{N} w_{q}(\phi) E_{q n}^{+}(\hat{\phi} \cdot \hat{t})\right] e_{m}^{-}(\phi)^{*} \mathrm{~d} \phi
\end{aligned}
$$

Finalmente, los vectores $\underline{b}$ y $\underline{a}$ pueden relacionarse a través de la matriz MDG que caracteriza al segmento irregular de la figura 3.30 en términos de modos guiados, obteniéndose la siguiente expresión:

$$
\underline{b}=\underline{\triangleq}^{-1} \underline{\underline{Q}} \underline{a}=\underline{\underline{S}} \underline{a}
$$

donde $\underline{\underline{S}}$ es finalmente la matriz MDG buscada.

El cálculo de los elementos $\Delta_{m n}$ and $Q_{m n}$ requiere, como se puede apreciar en las ecuaciones (3.168) y (3.169), de la solución de complejas integrales a lo largo de la circunferencia de radio $R$, donde el integrando es el producto de dos o tres funciones definidas en la variable $\phi$. Estas integrales no es necesario resolverlas de forma numérica, ya que pueden reducirse a simples series si hacemos uso de la DTFT. Así pues, la integración de un producto de dos funciones de $\phi$ a lo largo de la circunferencia de radio $R$ se puede simplificar si sustituimos cada función por su serie de Fourier discreta de la siguiente manera:

$$
\begin{aligned}
\frac{1}{2 \pi} \int_{0}^{2 \pi} f(\phi) g^{*}(\phi) \mathrm{d} \phi & =\frac{1}{2 \pi} \int_{0}^{2 \pi}\left(\sum_{p} F_{p} e^{j p \phi}\right)\left(\sum_{k} G_{k}^{*} e^{-j k \phi}\right) \mathrm{d} \phi \\
& =\frac{1}{2 \pi} \int_{0}^{2 \pi} \sum_{p} \sum_{k} F_{p} G_{k}^{*} e^{j(p-k) \phi} \mathrm{d} \phi \\
& =\sum_{p} \sum_{k} F_{p} G_{k}^{*}\left(\frac{1}{2 \pi} \int_{0}^{2 \pi} e^{j(p-k) \phi} \mathrm{d} \phi\right)
\end{aligned}
$$

Como,

$$
\frac{1}{2 \pi} \int_{0}^{2 \pi} e^{j(p-k) \phi} \mathrm{d} \phi= \begin{cases}1 & \text { si } p=k \\ 0 & \text { si } p \neq k\end{cases}
$$

finalmente se obtiene:

$$
\frac{1}{2 \pi} \int_{0}^{2 \pi} f(\phi) g^{*}(\phi) \mathrm{d} \phi=\sum_{p} F_{p} G_{p}^{*}
$$


De forma análoga, se puede simplificar la integral de un producto de tres funciones:

$$
\frac{1}{2 \pi} \int_{0}^{2 \pi} f(\phi) g(\phi) h^{*}(\phi) \mathrm{d} \phi=\sum_{p} \sum_{k} \sum_{r} F_{p} G_{k} H_{r}^{*}\left(\frac{1}{2 \pi} \int_{0}^{2 \pi} e^{j(p+k-r) \phi} \mathrm{d} \phi\right)
$$

donde:

$$
\frac{1}{2 \pi} \int_{0}^{2 \pi} e^{j(p+k-r) \phi} \mathrm{d} \phi= \begin{cases}1 & \text { si } p+k-r=0 \\ 0 & \text { si } p+k-r \neq 0\end{cases}
$$

Finalmente:

$$
\begin{aligned}
& \frac{1}{2 \pi} \quad \int_{0}^{2 \pi} f(\phi) g(\phi) h^{*}(\phi) \mathrm{d} \phi=\left.\sum_{p} \sum_{k} \sum_{r} F_{p} G_{k} H_{r}^{*}\right|_{p+k-r=0} \\
& \quad=\sum_{p} F_{p}\left(G_{-p} H_{0}^{*}+G_{-p+1} H_{1}^{*}+G_{-p+2} H_{2}^{*}+\cdots+G_{-p-1} H_{-1}^{*}+G_{-p-2} H_{-2}^{*}+\cdots\right) \\
& \frac{1}{2 \pi} \quad \int_{0}^{2 \pi} f(\phi) g(\phi) h^{*}(\phi) \mathrm{d} \phi=\sum_{p} F_{p} \sum_{k} G_{k-p} H_{k}^{*}
\end{aligned}
$$

Aplicando los resultados de (3.173) y (3.176) en las integrales definidas en (3.168) y (3.169), se obtienen las siguientes expresiones para los elementos $\Delta_{m n}$ y $Q_{m n}$ :

$$
\begin{aligned}
\Delta_{m n}= & Y_{0 n} \sum_{p=-\infty}^{\infty} E_{p n}^{-} E_{p m}^{-*}+\frac{1}{K \eta R} \sum_{p=-\infty}^{\infty} p E_{p n}^{-} \sum_{k=-\infty}^{\infty} \Gamma_{k-p} E_{k m}^{-*} \\
& +\frac{j}{\eta} \sum_{q=-N}^{N} E_{q n}^{-} \sum_{p=-\infty}^{\infty} W_{p q} \sum_{k=-\infty}^{\infty} \Phi_{k-p} E_{k m}^{-*} \\
Q_{m n}= & Y_{0 n} \sum_{p=-\infty}^{\infty} E_{p n}^{+} E_{p m}^{-*}-\frac{1}{K \eta R} \sum_{p=-\infty}^{\infty} p E_{p n}^{+} \sum_{k=-\infty}^{\infty} \Gamma_{k-p} E_{k m}^{-}{ }^{*} \\
& -\frac{j}{\eta} \sum_{q=-N}^{N} E_{q n}^{+} \sum_{p=-\infty}^{\infty} W_{p q} \sum_{k=-\infty}^{\infty} \Phi_{k-p} E_{k m}^{-*}
\end{aligned}
$$

donde:

$$
\begin{aligned}
\Gamma_{n} & =\frac{1}{2 \pi} \int_{-\pi}^{\pi}(\hat{\rho} \cdot \hat{t}) e^{-j n \phi} \mathrm{d} \phi \\
\Phi_{n} & =\frac{1}{2 \pi} \int_{-\pi}^{\pi}(\hat{\phi} \cdot \hat{t}) e^{-j n \phi} \mathrm{d} \phi
\end{aligned}
$$


Los valores de $\Gamma_{n}$ y $\Phi_{n}$ se pueden obtener de forma analítica:

$$
\begin{aligned}
\Gamma_{n} & =\frac{1}{2 \pi} \int_{-\pi}^{\pi}(\hat{\rho} \cdot \hat{t}) e^{-j n \phi} \mathrm{d} \phi \\
& =\frac{1}{2 \pi} \int_{-\pi}^{\pi}\left(\cos \phi t_{x}+\sin \phi t_{y}\right) e^{-j n \phi} \mathrm{d} \phi \\
\Gamma_{-n} & =\mathrm{DTFT}^{-1}\left\{\cos \phi t_{x}(\phi)+\sin \phi t_{y}(\phi)\right\} \\
& =\frac{\delta[n+1]+\delta[n-1]}{2} * T_{x}[-n]+\frac{\delta[n+1]-\delta[n-1]}{2 j} * T_{y}[-n] \\
\Gamma_{n} & =\frac{\delta[-n+1]+\delta[-n-1]}{2} * T_{x}[n]+\frac{\delta[-n+1]-\delta[-n-1]}{2 j} * T_{y}[n] \\
& =\frac{\delta[n-1]+\delta[n+1]}{2} * T_{x}[n]+\frac{\delta[n-1]-\delta[n+1]}{2 j} * T_{y}[n] \\
& =\frac{T_{x}[n-1]+T_{x}[n+1]}{2}+\frac{T_{y}[n-1]-T_{y}[n+1]}{2 j}
\end{aligned}
$$

Se puede deducir a partir de la geometría del problema que:

$$
\begin{aligned}
t_{x}(\phi) & =\sum_{i=1}^{J} x_{i_{x}} \prod\left(\frac{\phi-\phi_{0 i}}{\phi_{d i}}\right)+x_{1_{x}}\left(1-\sum_{i=1}^{J} \prod\left(\frac{\phi-\phi_{0 i}}{\phi_{d i}}\right)\right) \\
& =\sum_{i=2}^{J}\left(x_{i_{x}}-x_{1_{x}}\right) \prod\left(\frac{\phi-\phi_{0 i}}{\phi_{d i}}\right)+x_{1_{x}} \\
t_{y}(\phi) & =\sum_{i=1}^{J} x_{i_{y}} \prod\left(\frac{\phi-\phi_{0 i}}{\phi_{d i}}\right)+x_{1_{y}}\left(1-\sum_{i=1}^{J} \prod\left(\frac{\phi-\phi_{0 i}}{\phi_{d i}}\right)\right) \\
& =\sum_{i=2}^{J}\left(x_{i_{y}}-x_{1_{y}}\right) \prod\left(\frac{\phi-\phi_{0 i}}{\phi_{d i}}\right)+x_{1_{y}}
\end{aligned}
$$

donde $\phi_{0 i}$ y $\phi_{d i}$ son, respectivamente, la posición angular por la que entra el acceso $i$ a la circunferencia de radio $R$, y la anchura angular que ocupa dicho acceso. $x_{i_{x}}$ y $x_{i_{y}}$ son las componentes $\hat{x}$ e $\hat{y}$ del vector unitario $\hat{x}_{i}$ en el sistema de coordenadas global.

$$
\hat{x}_{i}=x_{i_{x}} \hat{x}+x_{i_{y}} \hat{y}
$$

La transformada de Fourier inversa de un pulso rectangular es la función sinc:

$$
\begin{aligned}
& T_{x}[-n]=\sum_{i=2}^{J}\left(x_{i_{x}}-x_{1_{x}}\right) \frac{\phi_{d i}}{2 \pi} \operatorname{sinc}\left(\frac{\phi_{d i}}{2 \pi} n\right) e^{j \phi_{0 i} n}+x_{1_{x}} \delta[n] \\
& T_{y}[-n]=\sum_{i=2}^{J}\left(x_{i_{y}}-x_{1_{y}}\right) \frac{\phi_{d i}}{2 \pi} \operatorname{sinc}\left(\frac{\phi_{d i}}{2 \pi} n\right) e^{j \phi_{0 i} n}+x_{1_{y}} \delta[n]
\end{aligned}
$$


Sustituyendo $n$ por $-n$ :

$$
\begin{aligned}
& T_{x}[n]=\sum_{i=2}^{J}\left(x_{i_{x}}-x_{1_{x}}\right) \frac{\phi_{d i}}{2 \pi} \operatorname{sinc}\left(\frac{\phi_{d i}}{2 \pi} n\right) e^{-j \phi_{0 i} n}+x_{1_{x}} \delta[n] \\
& T_{y}[n]=\sum_{i=2}^{J}\left(x_{i_{y}}-x_{1_{y}}\right) \frac{\phi_{d i}}{2 \pi} \operatorname{sinc}\left(\frac{\phi_{d i}}{2 \pi} n\right) e^{-j \phi_{0 i} n}+x_{1_{y}} \delta[n]
\end{aligned}
$$

Sustituyendo en (3.181):

$$
\begin{aligned}
\Gamma_{n}= & \sum_{i=2}^{J} \frac{e^{-j \phi_{0 i} n} \phi_{d i}}{4 \pi} \operatorname{sinc}\left(\frac{\phi_{d i}(n-1)}{2 \pi}\right) e^{j \phi_{0 i}}\left(\left(x_{i_{x}}-x_{1_{x}}\right)-j\left(x_{i_{y}}-x_{1_{y}}\right)\right) \\
& +\sum_{i=2}^{J} \frac{e^{-j \phi_{0 i} n} \phi_{d i}}{4 \pi} \operatorname{sinc}\left(\frac{\phi_{d i}(n+1)}{2 \pi}\right) e^{-j \phi_{0 i}}\left(\left(x_{i_{x}}-x_{1_{x}}\right)+j\left(x_{i_{y}}-x_{1_{y}}\right)\right) \\
& +\left(\frac{x_{1_{x}}-j x_{1_{y}}}{2}\right) \delta[n-1]+\left(\frac{x_{1_{x}}+j x_{1_{y}}}{2}\right) \delta[n+1]
\end{aligned}
$$

De manera análoga, se puede obtener que:

$$
\begin{aligned}
\Phi_{n}= & \sum_{i=2}^{J} \frac{e^{-j \phi_{0 i} n} \phi_{d i}}{4 \pi} \operatorname{sinc}\left(\frac{\phi_{d i}(n-1)}{2 \pi}\right) e^{j \phi_{0 i}}\left(\left(x_{i_{y}}-x_{1_{y}}\right)+j\left(x_{i_{x}}-x_{1_{x}}\right)\right) \\
& +\sum_{i=2}^{J} \frac{e^{-j \phi_{0 i} n} \phi_{d i}}{4 \pi} \operatorname{sinc}\left(\frac{\phi_{d i}(n+1)}{2 \pi}\right) e^{-j \phi_{0 i}}\left(\left(x_{i_{y}}-x_{1_{y}}\right)-j\left(x_{i_{x}}-x_{1_{x}}\right)\right) \\
& +\left(\frac{x_{1_{y}}+j x_{1_{x}}}{2}\right) \delta[n-1]+\left(\frac{x_{1_{y}}-j x_{1_{x}}}{2}\right) \delta[n+1]
\end{aligned}
$$

Truncando en (3.177) y (3.178) las sumas de los coeficientes espectrales a $2 N+1$ términos, se pueden escribir dichas expresiones en forma matricial:

$$
\begin{aligned}
& \triangleq=\underline{\underline{E}}^{-+}\left[\underline{\underline{E}}^{-} \underline{\underline{Y}}+\left(\frac{1}{K \eta R} \underline{\underline{\Gamma}}^{T} \underline{\underline{P}}+\frac{j}{\eta} \underline{\underline{\Phi}}^{T} \underline{\underline{W}}\right) \underline{\underline{E}}^{-}\right] \\
& \underline{\underline{Q}}=\underline{\underline{E}}^{-+}\left[\underline{\underline{E}}^{+} \underline{\underline{Y}}-\left(\frac{1}{K \eta R} \underline{\underline{\Gamma}}^{T} \underline{\underline{P}}+\frac{j}{\eta} \underline{\underline{\Phi}}^{T} \underline{\underline{W}}\right) \underline{\underline{E}}^{+}\right]
\end{aligned}
$$


donde:

$$
\begin{aligned}
\underline{\underline{W}}= & \left(\underline{\underline{J^{\prime}}}+\underline{\underline{H^{\prime}}} \underline{\underline{D}}\right)(\underline{\underline{J}}+\underline{\underline{H}} \underline{\underline{D}})^{-1} \\
\underline{\underline{Y}}= & {\left[\begin{array}{cccc}
Y_{01} & 0 & \cdots & 0 \\
0 & Y_{02} & \cdots & 0 \\
\vdots & \vdots & \ddots & \vdots \\
0 & 0 & \cdots & Y_{0 M}
\end{array}\right]_{M \times M} } \\
\underline{\underline{\Gamma}}= & {\left[\begin{array}{cccc}
\Gamma_{0} & \Gamma_{1} & \cdots & \Gamma_{2 N} \\
\Gamma_{-1} & \Gamma_{0} & \cdots & \Gamma_{2 N-1} \\
\vdots & \vdots & \ddots & \vdots \\
\Gamma_{-2 N} & \Gamma_{-2 n+1} & \cdots & \Gamma_{0}
\end{array}\right]_{(2 N+1) \times(2 N+1)} } \\
\underline{\underline{\Phi}}= & {\left[\begin{array}{cccc}
\Phi_{0} & \Phi_{1} & \cdots & \Phi_{2 N} \\
\Phi_{-1} & \Phi_{0} & \cdots & \Phi_{2 N-1} \\
\vdots & \vdots & \ddots & \vdots \\
\Phi_{-2 N} & \Phi_{-2 n+1} & \cdots & \Phi_{0}
\end{array}\right]_{(2 N+1) \times(2 N+1)} } \\
\underline{\underline{P}}= & {\left[\begin{array}{cccc}
-N & 0 & \cdots & 0 \\
0 & -N+1 & \cdots & 0 \\
\vdots & \vdots & \ddots & \vdots \\
0 & 0 & \cdots & N
\end{array}\right]_{(2 N+1) \times(2 N+1)} }
\end{aligned}
$$

Sea:

$$
\underline{\underline{L}}=\left(\frac{1}{K \eta R} \underline{\Gamma}^{T} \underline{\underline{P}}+\frac{j}{\eta} \underline{\underline{\Phi}}^{T} \underline{\underline{W}}\right)
$$

Sustituyendo (3.188) y (3.189) en (3.167):

$$
\underline{\underline{E}}^{-+}\left[\underline{\underline{E}}^{-} \underline{\underline{Y}}+\underline{\underline{L}} \underline{\underline{E}}^{-}\right] \underline{b}=\underline{\underline{E}}^{-+}\left[\underline{\underline{E}}^{+} \underline{\underline{Y}}-\underline{\underline{L}} \underline{\underline{E}}^{+}\right] \underline{a}
$$

Podemos finalmente obtener una expresión matricial para la matriz de dispersión generalizada $(\underline{b}=\underline{\underline{S}} \underline{a})$ :

$$
\underline{\underline{S}}=\left(\underline{\underline{E}}^{-+}\left[\underline{\underline{E}}^{-} \underline{\underline{Y}}+\underline{\underline{L}} \underline{\underline{E}}^{-}\right]\right)^{-1}\left(\underline{\underline{E}}^{-+}\left[\underline{\underline{E}}^{+} \underline{\underline{Y}}-\underline{\underline{L}}^{\underline{E}^{+}}\right]\right)
$$

Puesto que previamente se han desplazado los planos de referencia de cada acceso una distancia $d_{i}$, debemos ahora deshacer esos desplazamientos y obtener una matriz $\underline{\underline{S}}$ con los planos de referencia ubicados en su posición original. Esto se puede conseguir multiplicando a derecha e izquierda la matriz $\underline{\underline{S}}$ ya obtenida por una matriz de cambio de planos de referencia $\underline{\underline{P}}$ :

$$
\underline{\underline{S^{\prime}}}=\underline{\underline{P}} \cdot \underline{\underline{S}} \cdot \underline{\underline{P}}
$$


donde $\underline{\underline{S^{\prime}}}$ es la matriz de dispersión generalizada (MDG) con los planos de referencia en su posición original, y la matriz $\underline{\underline{P}}$ es una matriz diagonal de dimensión $M \times M$, cuyos elementos son:

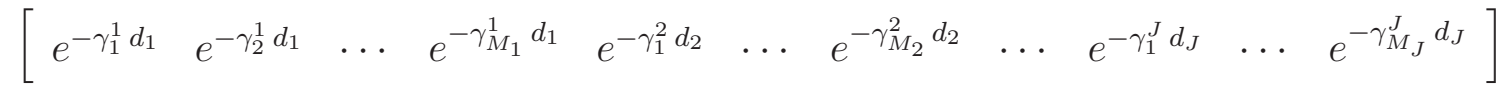

\subsection{Resultados}

En esta sección, el nuevo método híbrido de análisis de problemas inductivos se utiliza para resolver algunos problemas inductivos clásicos, con el objetivo de validar la eficiencia y eficacia de este método.

En primer lugar se analizan obstáculos inductivos de geometría arbitraria en una guía rectangular (postes circulares y cuadrados, metálicos o dieléctricos). A continuación, se comprueba la validez del método para el análisis de una gran variedad de discontinuidades inductivas (codos en ángulo recto, biselados y circulares, cambios de sección, y uniones T). Finalmente, se analizan dispositivos y componentes complejos en plano $\mathrm{H}$, tales como filtros de cavidades resonantes o filtros de múltiples postes acoplados.

Para cada problema analizado se incluye un esquema que muestra la sección longitudinal de la guía. En este esquema se puede apreciar la geometría de los obstáculos y/o discontinuidades objeto de análisis, la circunferencia de radio $R$ que delimita la sección irregular de la guía (que ha sido analizada como un problema de espacio abierto) y los puertos de geometría regular que acceden a dicho segmento irregular. Además del esquema, se presentan en una tabla las características de los objetos dispersores individuales en los que se ha segmentado el problema de espacio abierto. Las características que se presentan en la mencionada tabla para cada objeto, son:

Tipo Tipo de objeto dispersor en función de su geometría y constitución: tira metálica, cilindro metálico o dieléctrico, arco metálico, etc.

$\mathbf{C}_{\mathbf{i x}} \mathbf{y} \mathbf{C}_{\mathbf{i y}}$ Coordenadas $\hat{x}$ e $\hat{y}$ del centro del objeto $i$ en el sistema de coordenadas global.

$\mathbf{R}_{\mathbf{i}}$ Radio de la circunferencia que circunscribe al objeto. El campo dispersado por el objeto expresado como suma de modos cilíndricos sólo tiene validez fuera de la circunferencia de radio $R_{i}$.

$\mathbf{d N c}_{\mathbf{i}}$ Número de puntos de corrientes por cada longitud de onda a lo largo de la superficie del objeto $i$ cuando éste es metálico y es caracterizado utilizando el Método de los Momentos. A mayor densidad, mayor exactitud en la obtención del campo dispersado por dicho objeto, y mayor coste computacional.

$\mathrm{C}_{\mathrm{in}}^{\mathrm{i}} \mathbf{y} \mathrm{C}_{\mathrm{sc}}^{\mathrm{i}}$ Criterio para calcular el número de modos cilíndricos del espectro de campo incidente y dispersado, respectivamente, para el objeto $i$ (diferente del número de modos 
cilíndricos del conjunto total de objetos requerido por cada acceso guiado $i$, expresado en la ecuación (3.108)):

$$
\begin{aligned}
N_{i n}^{i} & =C_{i n}^{i} K R_{i} \\
N_{s c}^{i} & =C_{s c}^{i} K R_{i}
\end{aligned}
$$

A mayor número de modos, mayor exactitud, y mayor coste computacional.

Finalmente, se detallan en otra tabla los valores utilizados para los parámetros más relevantes del algoritmo de acoplo de modos en el análisis del problema inductivo correspondiente. Dichos parámetros son los siguientes:

R Radio de la circunferencia que delimita el segmento irregular de la guía. Los objetos dentro de dicha circunferencia se analizan como si de una problema de dispersión en espacio abierto se tratase.

$\mathbf{R}_{\mathrm{AB}}$ Factor que determina la cantidad de modos del espectro cilíndrico de campo incidente a todo el segmento irregular (ver anexo C).

$\mathbf{R}_{\mathrm{fft}}$ Indica el número de puntos utilizado al calcular la transformada discreta de Fourier. Cuanto mayor sea este número, menor será el error producido por el solapamiento temporal debido al uso de la transformada rápida de Fourier (FFT) sobre señales de duración infinita.

$\mathrm{C}_{\mathrm{sc}}$ Criterio para limitar el número de modos del espectro de campo dispersado por todo el segmento irregular de la guía, con el objeto de evitar posibles singularidades:

$$
N_{s c}=C_{s c} K R
$$

Modos Número de modos guiados considerados en cada puerto de acceso.

Tiempo Tiempo de computación en segundos de los parámetros de dispersión de la estructura por cada punto de frecuencia con un procesador Pentium III a $400 \mathrm{MHz}$.

Cuanto mayores sean $R_{A B}, R_{f f t}, C_{s c}$ y el número de modos guiados, más exacto será el resultado, pero también mayor será el coste computacional.

\subsubsection{Obstáculos inductivos}

A continuación se presentan los resultados del método híbrido para el análisis de estructuras guiadas con obstáculos inductivos de diferentes geometrías.

El primer problema que se va a analizar es el de un poste circular metálico inductivo como el que se muestra en la figura 3.32. Como se puede observar en la figura, el poste posee un diámetro $d$, y está situado a una distancia $x_{0}$ de una de las paredes de la guía. Cuando 


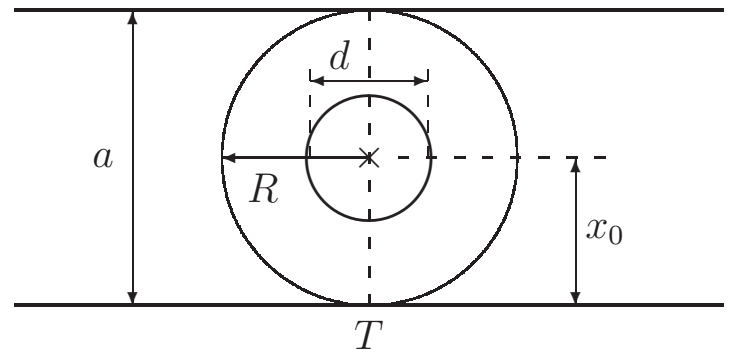

Figura 3.32: Poste metálico circular

$x_{0}=a / 2$, siendo $a$ la anchura de la guía, el poste está situado en el centro de la misma. El plano de referencia $T$ para el cálculo de los parámetros de dispersión se ha tomado para los dos puertos de acceso coincidiendo con el centro del poste.

Para analizar esta estructura con el método híbrido, se ha encerrado el obstáculo en una circunferencia de radio $R$, tal y como se muestra en la figura 3.32. Cuanto menor sea la circunferencia, menor será el problema de espacio abierto que se deba analizar, y menor será el coste computacional. En el caso del poste circular, con una circunferencia de radio $R=a / 2$ es suficiente, ya que todo lo que hay fuera de dicha circunferencia son los dos puertos de acceso con una geometría regular. De manera que fuera de la circunferencia de radio $R$ podemos suponer que los campos son una superposición de los modos propios de la guía rectangular. Sin embargo, la circunferencia no puede tener un radio inferior a $R=a / 2$, ya que en ese caso no podríamos realizar el acoplo modal de modos cilíndricos de espacio abierto a modos de la guía rectangular, al no forzar continuidad de contorno en todo el dominio de los modos guiados (desde $x_{i}=0$ a $x_{i}=a$ para cada acceso).

Por lo tanto, el proceso que se ha seguido es el de caracterizar lo que hay dentro de la circunferencia de radio $R$ como si de un problema de espacio abierto se tratara. Como dentro de esa circunferencia sólo existe un poste metálico circular bidimensional, éste se ha caracterizado mediante su función de transferencia utilizando la solución analítica descrita en [4]. Posteriormente, se ha resuelto el acoplo modal de modos cilíndricos a modos de la guía rectangular y se ha obtenido la matriz de dispersión generalizada (MDG).

Para poder comparar los resultados obtenidos con los resultados para el mismo problema de [12], se ha obtenido el circuito equivalente al poste inductivo [20], que es el que se muestra en la figura 3.33. Como se puede apreciar, el circuito equivalente es un circuito en $\mathrm{T}$, constituido por tres reactancias. El valor de $X_{a}$ y $X_{b}$ se puede obtener a partir de los parámetros de dispersión: 


$$
\begin{aligned}
& X_{a}=-j Z_{01}\left(\frac{2 S_{11}^{(2,1)}}{\left(1-S_{11}^{(1,1)}\right)^{2}-\left(S_{11}^{(2,1)}\right)^{2}}\right) \\
& X_{b}=j Z_{01}\left(\frac{1+S_{11}^{(1,1)}-S_{11}^{(2,1)}}{1-S_{11}^{(1,1)}+S_{11}^{(2,1)}}\right)
\end{aligned}
$$

donde $Z_{01}$ es la impedancia del modo fundamental $T E_{10}$ en la guía de anchura $a$.

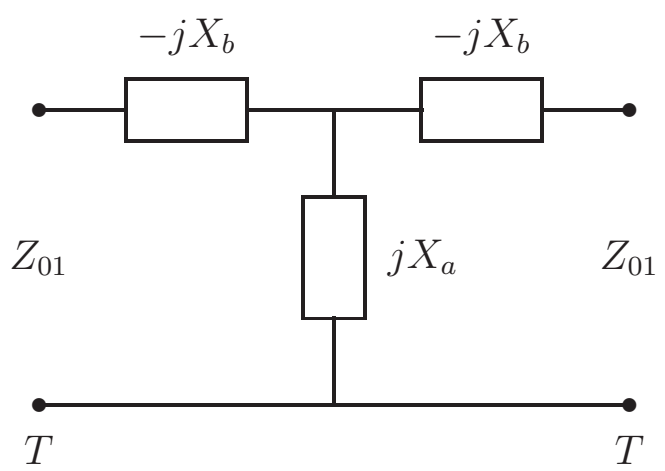

Figura 3.33: Circuito equivalente del poste inductivo

En la figura 3.34 se muestran los parámetros circuitales $X_{a}$ y $X_{b}$ normalizados para dos frecuencia distintas $\left(\frac{\lambda}{a}=1,01\right.$ y $\left.\frac{\lambda}{a}=1,2\right)$, y variando el tamaño del poste circular. En dicha figura se comparan los resultados proporcionados por el nuevo método híbrido con los de [12], observándose una buena coincidencia entre ambos resultados.

\begin{tabular}{|c|c|c|c|c|c|c|c|}
\cline { 2 - 7 } \multicolumn{1}{c|}{} & Tipo & $\mathrm{C}_{\mathrm{ix}}$ & $\mathrm{C}_{\mathrm{iy}}$ & $\mathrm{R}_{\mathrm{i}}$ & $\mathrm{dNc}_{\mathbf{i}}$ & $\mathrm{C}_{\mathrm{in}}^{\mathrm{i}}$ & $\mathrm{C}_{\mathrm{sc}}^{\mathrm{i}}$ \\
\hline Objeto 1 & Cilindro & 0 & 0 & $\frac{d}{2}$ & - & 3 & 1.5 \\
\hline
\end{tabular}

Tabla 3.1: Características de los dispersores individuales. Poste metálico circular

\begin{tabular}{|c|c|c|c|c|c|}
\hline $\mathbf{R}$ & $\mathbf{R}_{\mathrm{AB}}$ & $\mathbf{R}_{\mathrm{fft}}$ & $\mathbf{C}_{\mathrm{sc}}$ & Modos & Tiempo \\
\hline$\frac{a}{2}$ & 1 & 1 & 3 & 5 & $0.66 \mathrm{~s}$ \\
\hline
\end{tabular}

Tabla 3.2: Valor de los parámetros del acoplo de modos. Poste metálico circular

En la tabla 3.1 se muestran los valores utilizados para el análisis del poste circular. Obsérvese como el poste se ha situado en el centro de la circunferencia de radio $R\left(C_{i x}=\right.$ $C_{i y}=0$ ), su radio es de $\frac{d}{2}$, y como al caracterizarse de forma analítica no se ha utilizado el 


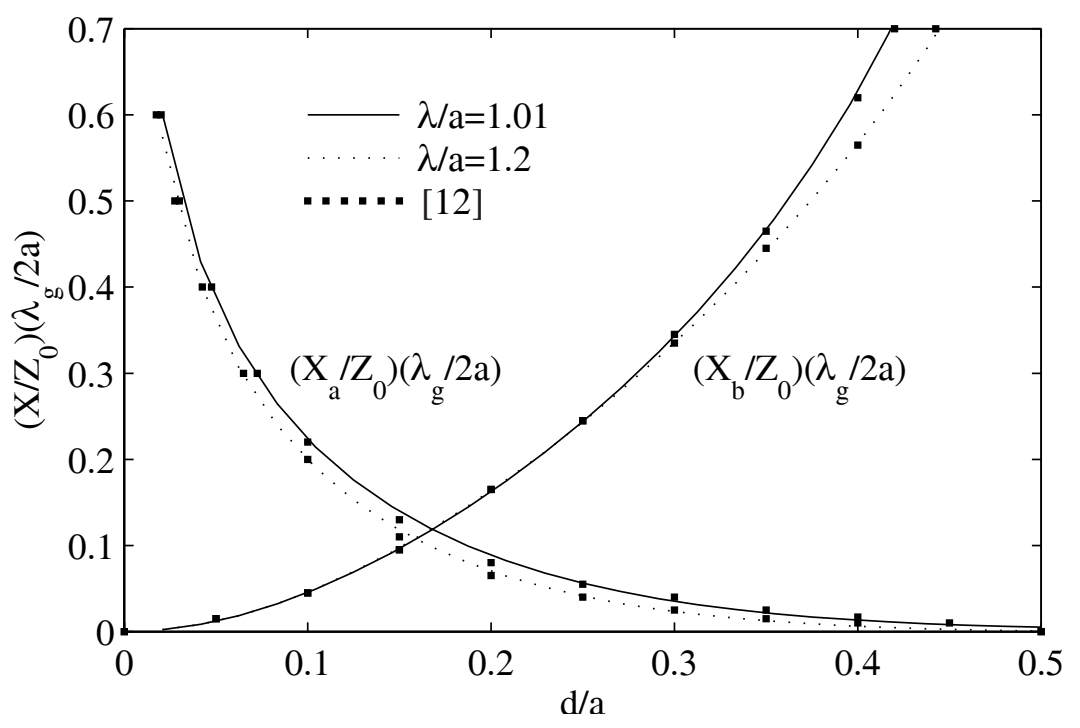

Figura 3.34: Parámetros circuitales de un poste metálico circular centrado

Método de los Momentos (por tanto el parámetro $d N c_{i}$ no se ha utilizado). Por otro lado, se han tomado $3 K \frac{d}{2}$ modos cilíndricos para el espectro de campo incidente y $1,5 K \frac{d}{2}$ para el espectro de campo dispersado por el cilindro.

En la tabla 3.2 se reflejan los valores de los parámetros de control del algoritmo de acoplo de modos. Se desprende de la tabla que para conseguir resultados satisfactorios han sido necesarios únicamente cinco modos guiados en cada puerto de acceso, que la circunferencia donde se ha forzado la continuidad de campo es de radio $R=\frac{a}{2}$, que para decidir el número de modos del espectro incidente a la circunferencia de radio $R$ se ha tomado $R_{A B}=1 \mathrm{y}$ para el espectro dispersado se han tomado el límite de $3 K R$ modos, que para realizar la transformada rápida de Fourier no se han tomado más puntos $\left(R_{f f t}=1\right)$, y que el tiempo de proceso necesario por cada punto de la gráfica ha sido de 0.43 segundos en un Pentium a $400 \mathrm{MHz}$.

La selección de los valores de los parámetros de control del método se ha realizado en base a unas pruebas de convergencia. Se ha realizado una prueba para cada uno de los parámetros de control, de modo que se fijan los valores de todos los parámetros menos uno, cuyo valor se va aumentando hasta que se observa que el valor del factor de reflexión se mantiene constante, señal de que el método ya ha alcanzado la convergencia. Una vez alcanzada la convergencia para un determinado parámetro, éste queda determinado y se siguen haciendo pruebas con el resto de parámetros hasta obtener el valor óptimo de todos ellos (mínimo coste computacional con la adecuada precisión).

Las figuras 3.35 a 3.40 muestran la evolución de la amplitud y la fase del factor de reflexión al aumentar el valor de cada uno de los parámetros de control. En cada figura se han realizado los cálculos para toda la curva variando un parámetro y manteniendo el resto de los parámetros constantes. Se comprueba que para los valores elegidos $\left(C_{i n}^{i}=3, C_{s c}^{i}=1.5\right.$, $R_{A B}=1, R_{f f t}=1, C_{s c}=3$, Modos=5) se ha alcanzado en todos los casos la convergencia. 

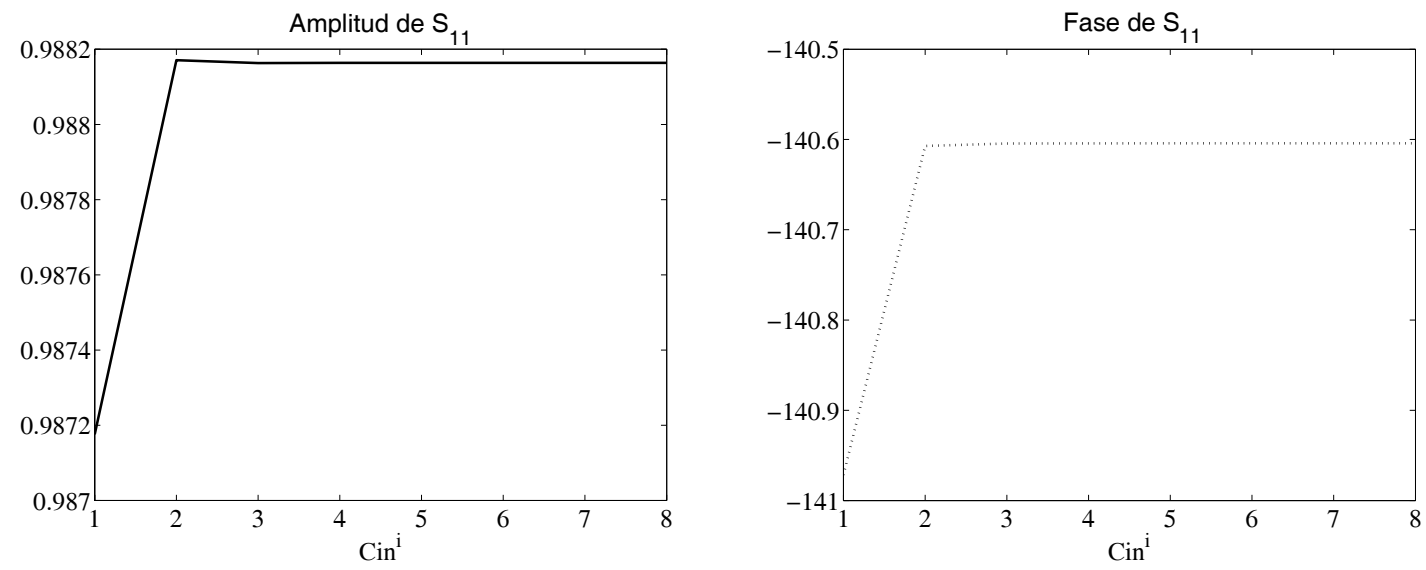

Figura 3.35: Variación de la amplitud y la fase de $S_{11}$ con el parámetro $C_{i n}^{i}$ en un poste metálico situado en el centro de una guía de ondas rectangular $\left(C_{s c}^{i}=3, R_{A B}=1, R_{f f t}=8\right.$, $C_{s c}=6, \operatorname{Modos}=9$ )
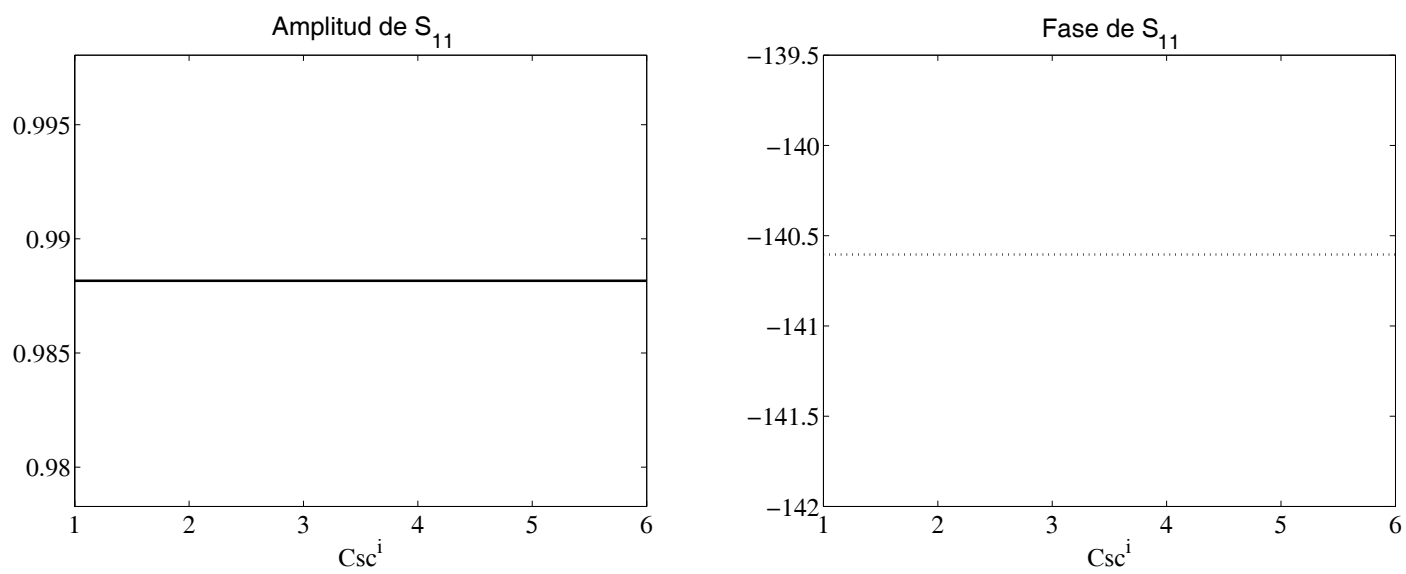

Figura 3.36: Variación de la amplitud y la fase de $S_{11}$ con el parámetro $C_{s c}^{i}$ en un poste metálico situado en el centro de una guía de ondas rectangular $\left(C_{i n}^{i}=6, R_{A B}=1, R_{f f t}=8\right.$, $C_{s c}=6, \operatorname{Modos}=9$ ) 

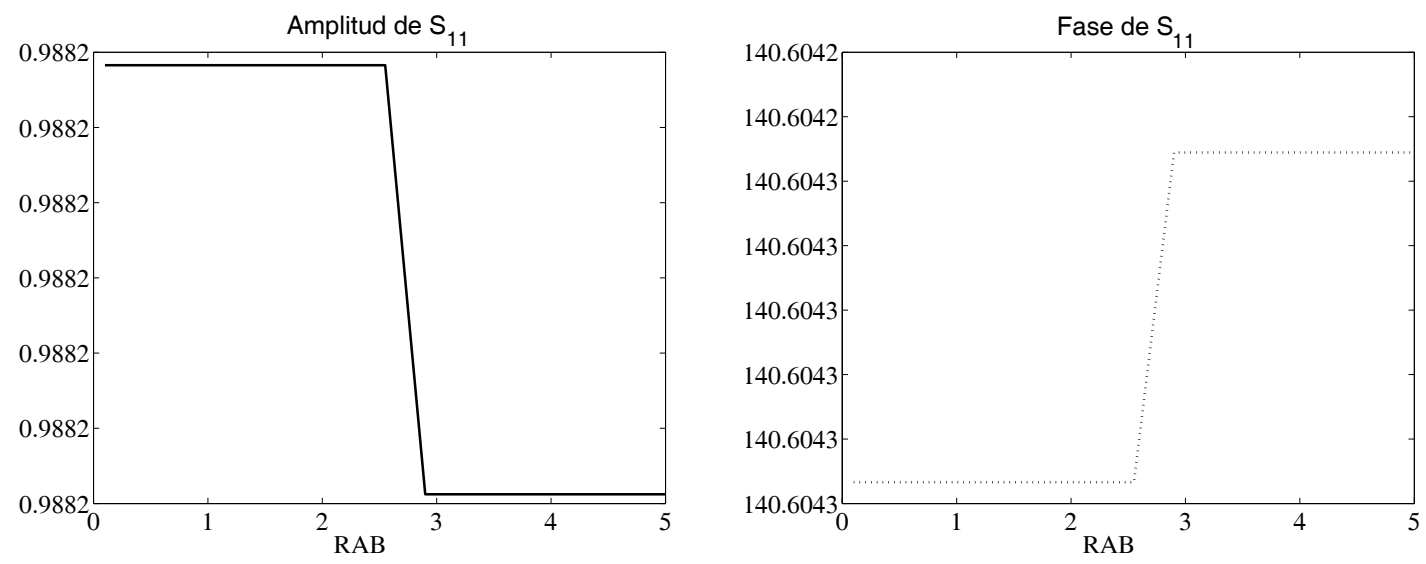

Figura 3.37: Variación de la amplitud y la fase de $S_{11}$ con el parámetro $R_{A B}$ en un poste metálico situado en el centro de una guía de ondas rectangular $\left(C_{i n}^{i}=6, C_{s c}^{i}=3, R_{f f t}=8, C_{s c}=6\right.$, Modos=9)
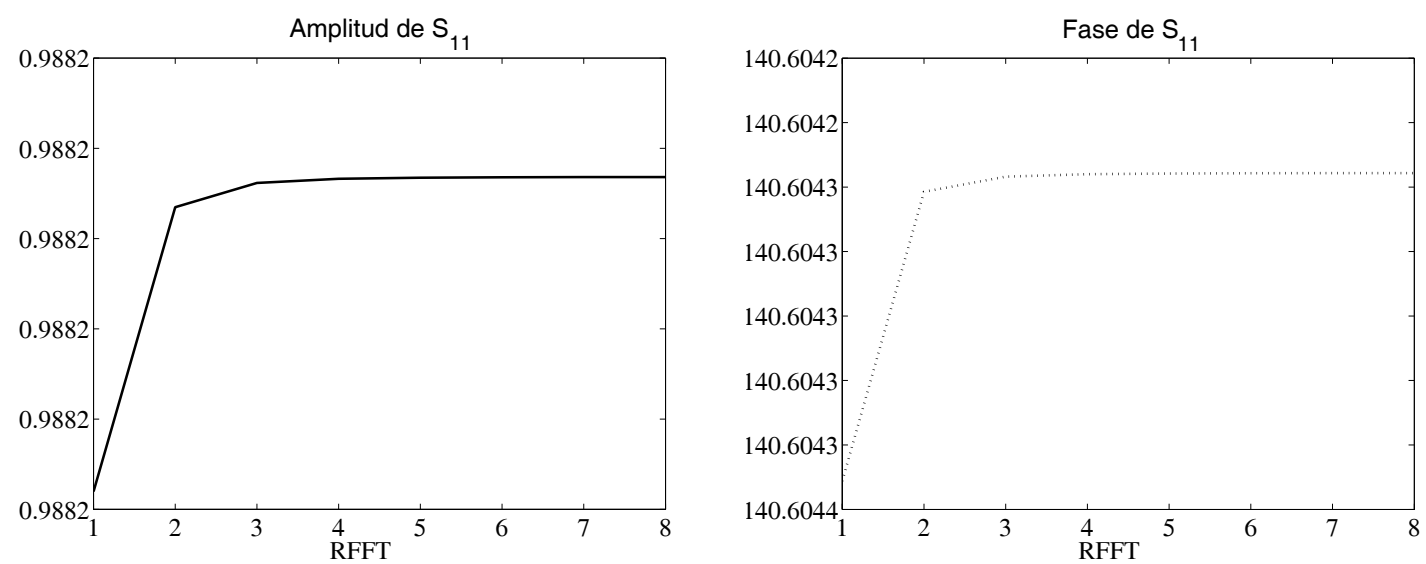

Figura 3.38: Variación de la amplitud y la fase de $S_{11}$ con el parámetro $R_{f f t}$ en un poste metálico situado en el centro de una guía de ondas rectangular $\left(C_{i n}^{i}=6, C_{s c}^{i}=3, R_{A B}=1, C_{s c}=6\right.$, Modos=9) 

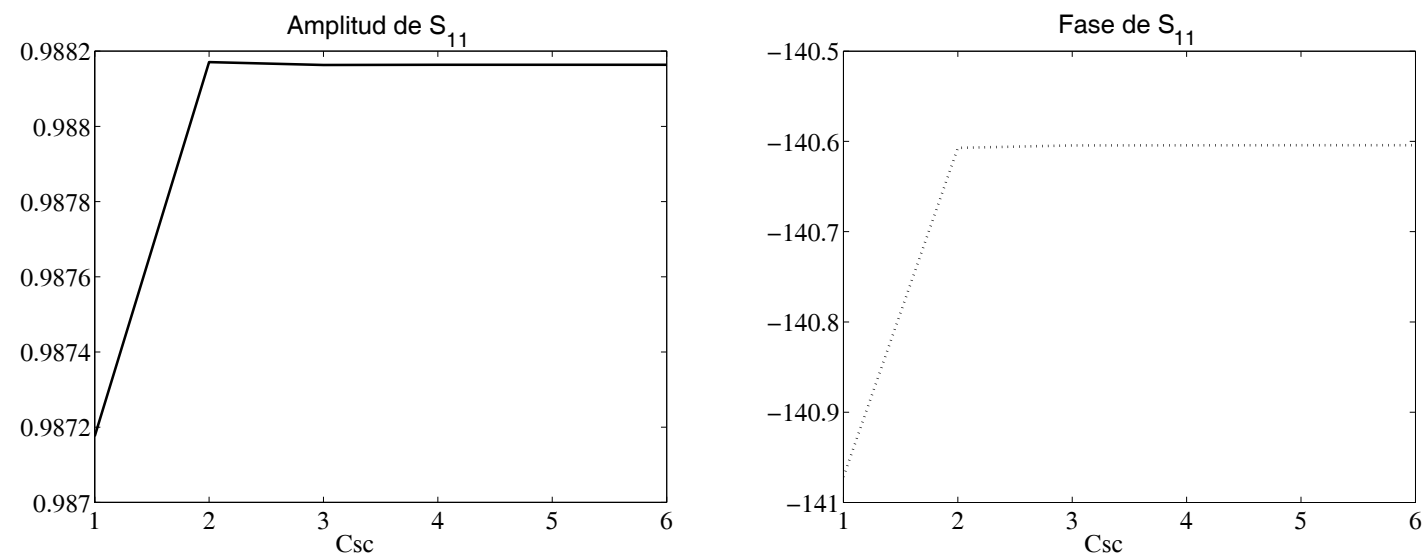

Figura 3.39: Variación de la amplitud y la fase de $S_{11}$ con el parámetro $C_{s c}$ en un poste metálico situado en el centro de una guía de ondas rectangular $\left(C_{i n}^{i}=6, C_{s c}^{i}=3, R_{A B}=1\right.$, $R_{f f t}=8$, Modos $=9$ )
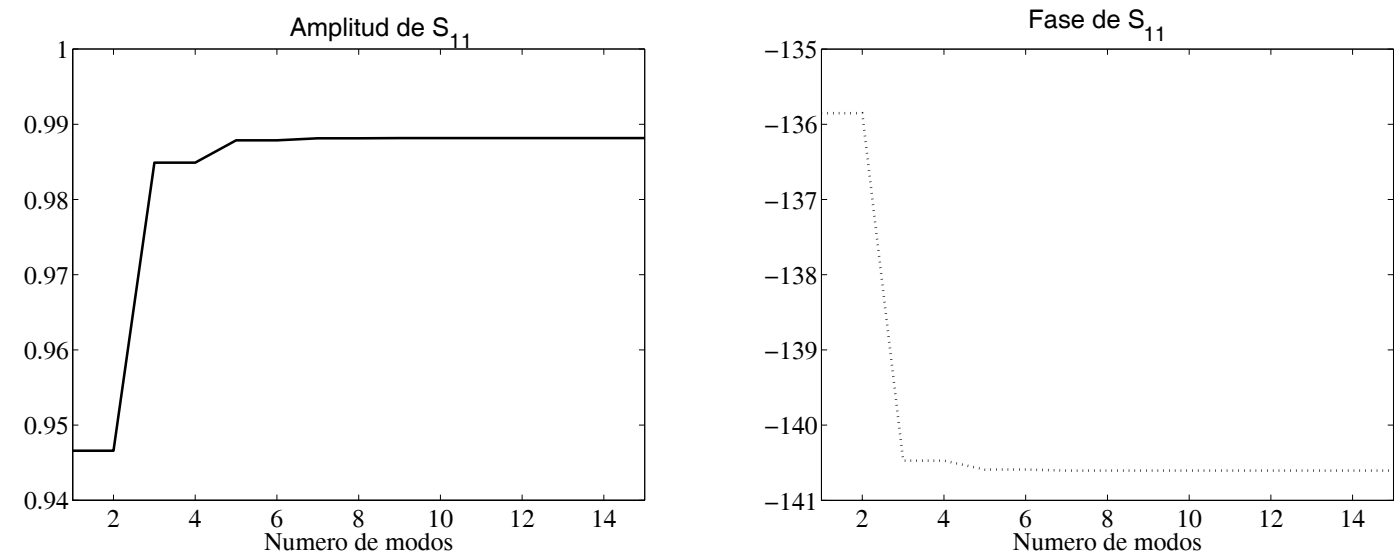

Figura 3.40: Variación de la amplitud y la fase de $S_{11}$ con el número de modos guiados en un poste metálico situado en el centro de una guía de ondas rectangular $\left(C_{i n}^{i}=6, C_{s c}^{i}=3\right.$, $\left.R_{A B}=1, R_{f f t}=8, C_{s c}=6\right)$ 

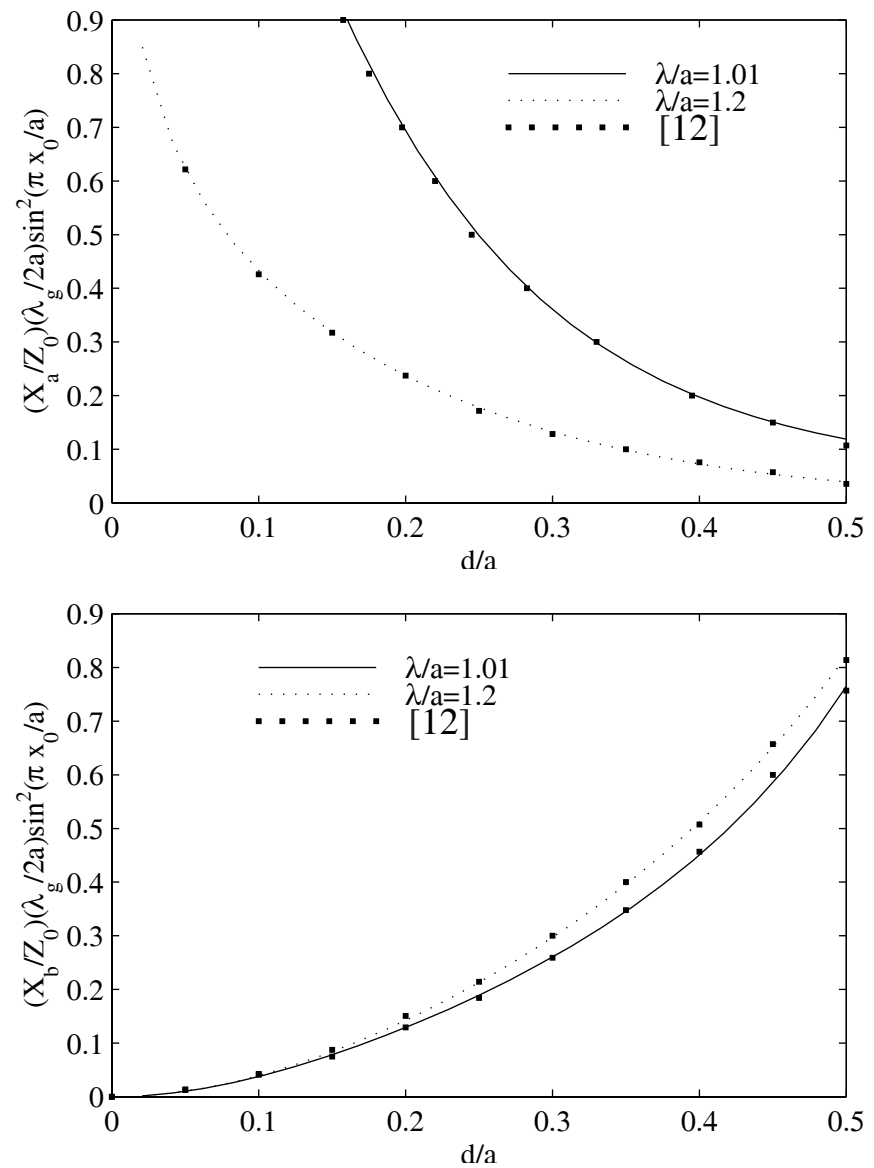

Figura 3.41: Parámetros circuitales de un poste metálico circular descentrado

En la figura 3.41 se muestran los parámetros circuitales de la guía con el poste circular descentrado $\left(x_{0}=0,3 a\right)$. De nuevo los resultados se comparan con [12], siendo los parámetros utilizados en la simulación los mismos de las tablas 3.1 y 3.2, a excepción de la posición del poste que ahora se halla situado en el punto $(0,-0,2 a)$.

Las figuras 3.42 y 3.43 muestran los parámetros circuitales de un iris inductivo y una apertura inductiva en una guía WR-90. En ambos casos (iris y apertura), $a=22,86 \mathrm{~mm}$ y $f=9 \mathrm{GHz}\left(\frac{\lambda}{a}=1,46\right)$. Se han utilizado los mismos valores de los parámetros de control del método de acoplo de modos que en el caso del poste circular metálico. No obstante, el tiempo de computación ha resultado ser algo superior. Esto es debido a que en el análisis del poste circular metálico (figura 3.41), el método de análisis es completamente analítico, ya que la caracterización del poste circular es analítica [4], y el acoplo de modos circulares a guiados también lo es. Sin embargo, la caracterización de las tiras metálicas que forman el iris o la apertura de la figura 3.42, ha sido realizada utilizando el método de los momentos, que es un método numérico. Por lo tanto, en el caso del iris y de la apertura, el método de análisis utilizado es un método híbrido numérico y analítico. En las figuras 3.42 y 3.41 se ha comparado la inductancia del circuito equivalente del iris y de la apertura con los resultados de [20], obteniéndose una coincidencia satisfactoria. 


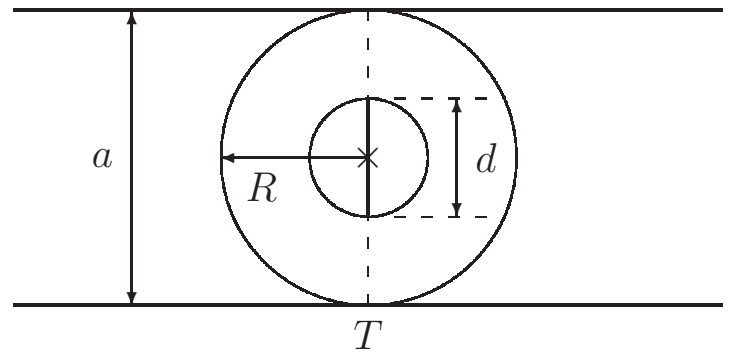

\begin{tabular}{|l|c|c|c|c|c|c|c|}
\cline { 2 - 7 } \multicolumn{1}{c|}{} & Tipo & $\mathrm{C}_{\mathrm{ix}}$ & $\mathrm{C}_{\mathrm{iy}}$ & $\mathbf{R}_{\mathbf{i}}$ & $\mathbf{d N c}_{\mathbf{i}}$ & $\mathrm{C}_{\mathrm{in}}^{\mathrm{i}}$ & $\mathrm{C}_{\mathbf{s c}}^{\mathrm{i}}$ \\
\hline Objeto 1 & Tira & 0 & 0 & $\frac{d}{2}$ & 75 & 3 & 2 \\
\hline
\end{tabular}

\begin{tabular}{|c|c|c|c|c|c|}
\hline $\mathbf{R}$ & $\mathbf{R}_{\mathrm{AB}}$ & $\mathbf{R}_{\mathrm{fft}}$ & $\mathbf{C}_{\mathrm{sc}}$ & Modos & Tiempo \\
\hline$\frac{a}{2}$ & 1 & 1 & 3 & 5 & $0.88 \mathrm{~s}$ \\
\hline
\end{tabular}

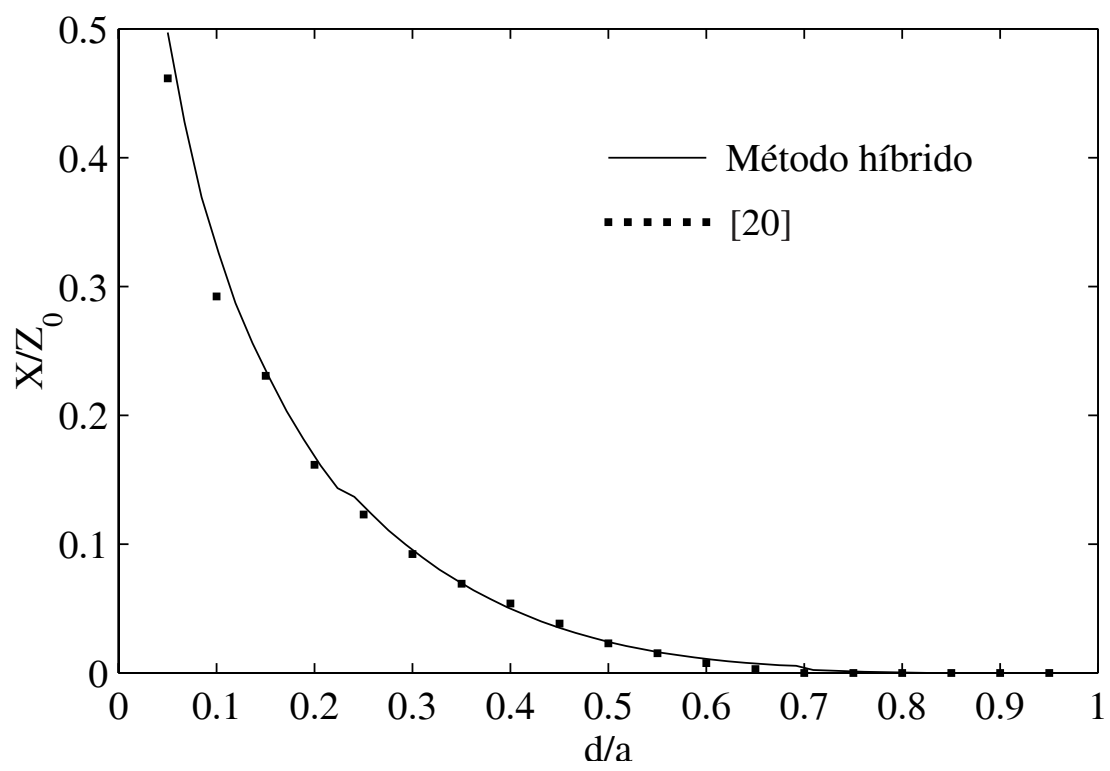

Figura 3.42: Iris inductivo. Esquema, valores de los parámetros de simulación y reactancia normalizada $\frac{X}{Z_{0}}$ para diferentes tamaños 


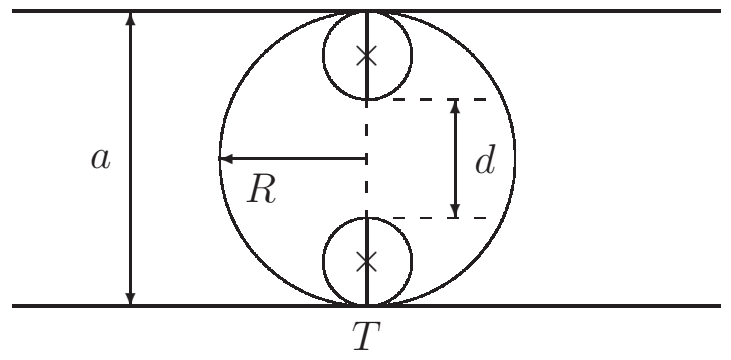

\begin{tabular}{|c|c|c|c|c|c|c|c|}
\cline { 2 - 8 } \multicolumn{1}{c|}{} & Tipo & $\mathbf{C}_{\mathbf{i x}}$ & $\mathbf{C}_{\mathbf{i y}}$ & $\mathbf{R}_{\mathbf{i}}$ & $\mathbf{d N c}_{\mathbf{i}}$ & $\mathbf{C}_{\mathbf{i n}}^{\mathbf{i}}$ & $\mathbf{C}_{\mathbf{s c}}^{\mathbf{i}}$ \\
\hline Objeto 1 & Tira & 0 & $a-\frac{a-d}{4}$ & $\frac{a-d}{4}$ & 75 & 6 & 3 \\
\hline Objeto 2 & Tira & 0 & $\frac{a-d}{4}-a$ & $\frac{a-d}{4}$ & 75 & 6 & 3 \\
\hline
\end{tabular}

\begin{tabular}{|c|c|c|c|c|c|}
\hline $\mathbf{R}$ & $\mathbf{R}_{\mathrm{AB}}$ & $\mathbf{R}_{\mathrm{fft}}$ & $\mathbf{C}_{\mathrm{sc}}$ & Modos & Tiempo \\
\hline$\frac{a}{2}$ & 1 & 1 & 3 & 5 & $0.76 \mathrm{~s}$ \\
\hline
\end{tabular}

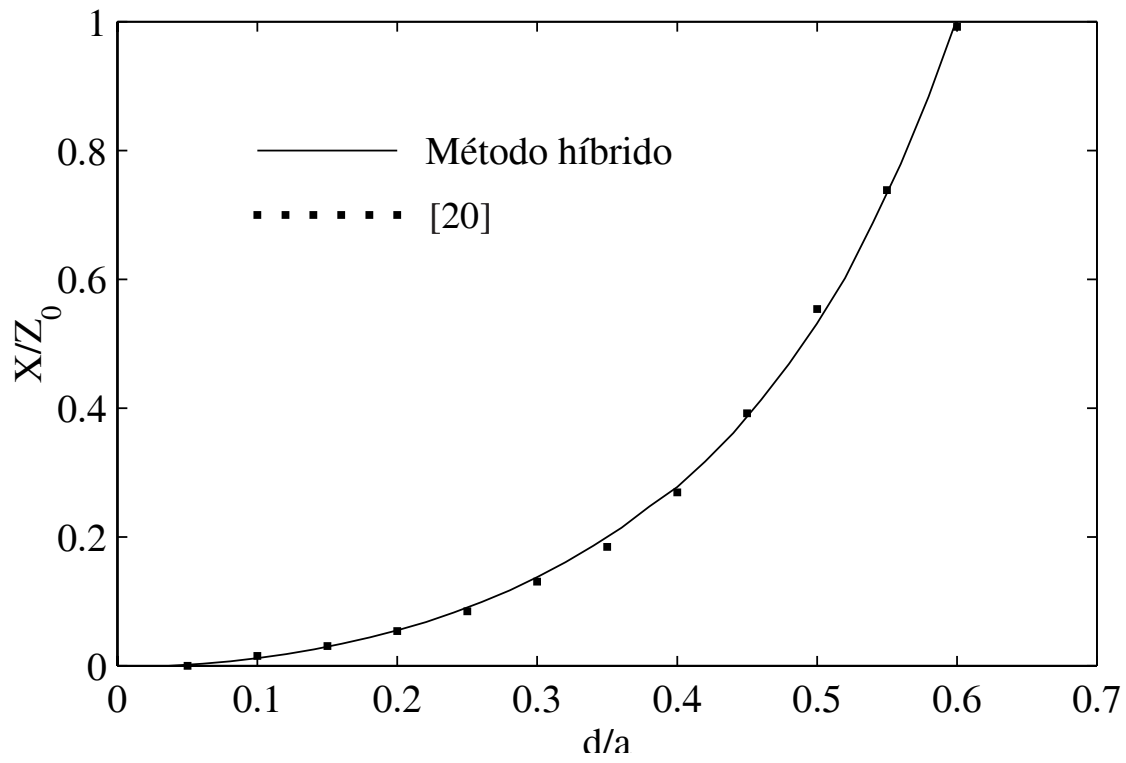

Figura 3.43: Ventana inductiva. Esquema, valores de los parámetros de simulación y reactancia normalizada $\frac{X}{Z_{0}}$ para diferentes tamaños 
En la figura 3.44 se analiza un poste circular dieléctrico sin pérdidas en el interior de una guía rectangular. El poste se sitúa en el centro de la guía, su diámetro es $d=0,1 / a$, y para su análisis se han utilizado los mismos valores de los parámetros de control que en los casos anteriores. Como el cilindro presenta solución analítica, el tiempo de cálculo por punto es inferior al del iris y la apertura, cuyo análisis se realizaba de forma numérica. En la figura se muestra la variación del factor de reflexión con la permitividad del poste, observándose un coincidencia total con los resultados de [43].

Un filtro de banda eliminada constituido por una guía rectangular con un poste dieléctrico con pérdidas (permitividad compleja) ha sido también analizado con el método híbrido, con el objetivo de validar el método para el análisis de obstáculos con pérdidas. Los resultados se presentan en la figura 3.45 para $a=22,86 \mathrm{~mm}, \varepsilon_{r}=38,5, \tan \delta=2 \times 10^{-4}$ y $d=0,03 a$. La respuesta del filtro se ha calculado para tres posiciones diferentes del poste dieléctrico ( $r=0,5 a, r=0,6 a$ and $r=0,7 a)$, y en todos los casos el resultado está en consonancia con el de Geshe y Lochel [11].

El método híbrido es capaz de analizar obstáculos inductivos de geometría arbitraria, ya sean metálicos o dieléctricos, tal y como se muestra en la figura 3.46, donde se muestra el factor de reflexión $\left(\left|S_{11}\right|\right)$ de una guía con un poste dieléctrico cuadrado. El factor de reflexión ha sido calculado para distintos valores de la constante dieléctrica del poste $(d / a=0,1$, $\lambda / a=1,4)$, y se puede observar que existe una muy buena coincidencia con los resultados previamente publicados en [16].

El método híbrido es capaz también de analizar problemas inductivos con múltiples obstáculos. Para demostrarlo, en la figura 3.47 se muestra el comportamiento de una guía con tres postes dieléctricos circulares $\left(\varepsilon_{r}=38,0\right)$. Se puede observar que los resultados no coinciden con los publicados para el mismo problema por Hsu y Auda [22]. Esta divergencia se puede justificar teniendo presente que el método utilizado por Hsu y Auda, tal y como se señala en [16], discretiza el contorno circular de los postes utilizando elementos radiantes infinitesimales de sección triangular. Esto puede introducir pequeños errores, que se pueden multiplicar en el caso de múltiples postes circulares. Además, los resultados de otra publicación [44] para el mismo problema sí coinciden con los resultados del método híbrido (ver figura 3.47), siendo también diferentes de los de Hsu y Auda.

En el análisis de los problemas con postes inductivos de esta sección, el tiempo de computación por cada punto de frecuencia ha sido algo inferior a 1 segundo con un ordenador con procesador Pentium III a $400 \mathrm{MHz}$, y utilizando 5 modos guiados en cada puerto de acceso. 


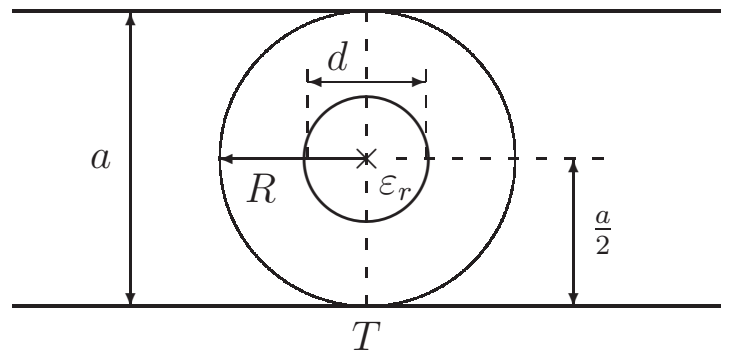

\begin{tabular}{|c|c|c|c|c|c|c|c|}
\cline { 2 - 7 } \multicolumn{1}{c|}{} & Tipo & $\mathrm{C}_{\mathbf{i x}}$ & $\mathrm{C}_{\mathrm{iy}}$ & $\mathbf{R}_{\mathbf{i}}$ & $\mathrm{dNc}_{\mathbf{i}}$ & $\mathrm{C}_{\mathrm{in}}^{\mathrm{i}}$ & $\mathrm{C}_{\mathbf{s c}}^{\mathrm{i}}$ \\
\hline Objeto 1 & $\begin{array}{c}\text { Cilindro } \\
\text { dieléctrico }\end{array}$ & 0 & 0 & 0.05 & - & 3 & 1.5 \\
\hline
\end{tabular}

\begin{tabular}{|c|c|c|c|c|c|}
\hline $\mathbf{R}$ & $\mathbf{R}_{\mathrm{AB}}$ & $\mathbf{R}_{\mathrm{fft}}$ & $\mathbf{C}_{\mathbf{s c}}$ & Modos & Tiempo \\
\hline$\frac{a}{2}$ & 1 & 1 & 3 & 5 & $0.7 \mathrm{~s}$ \\
\hline
\end{tabular}

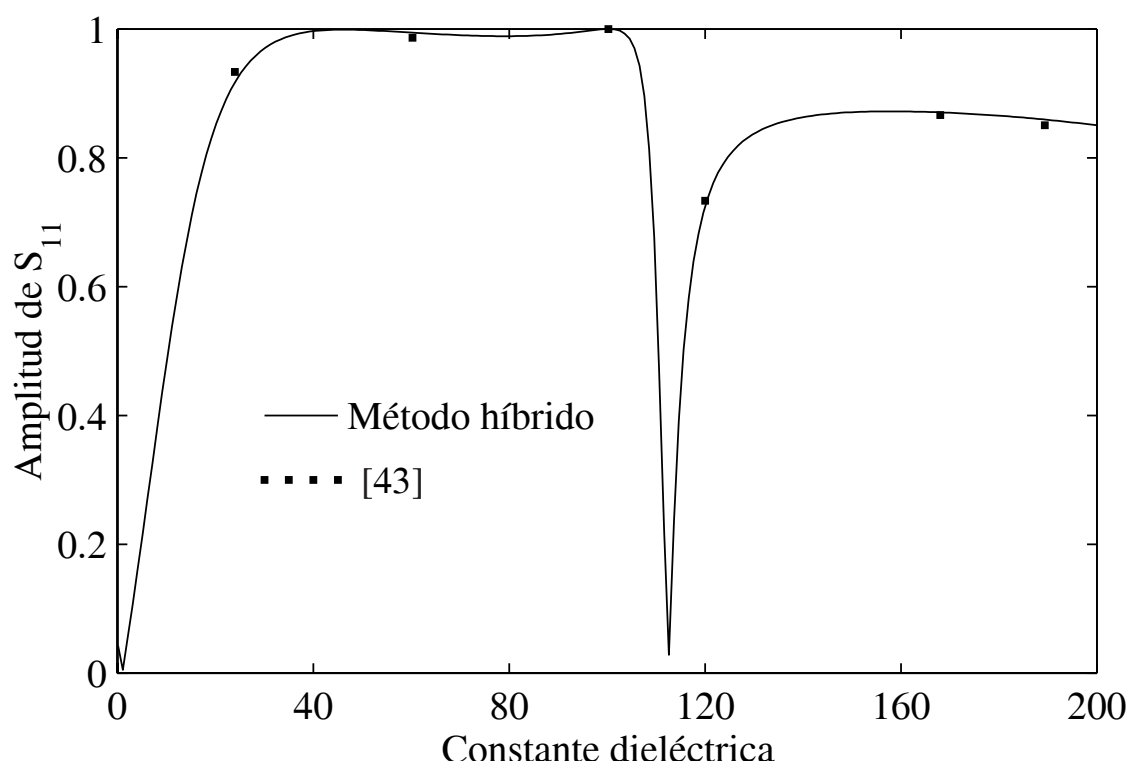

Figura 3.44: Poste dieléctrico circular. Esquema, valores de los parámetros de simulación y variación de $\left|S_{11}\right|$ con la constante dieléctrica $(d / a=0,1, \lambda / a=1,4)$ 


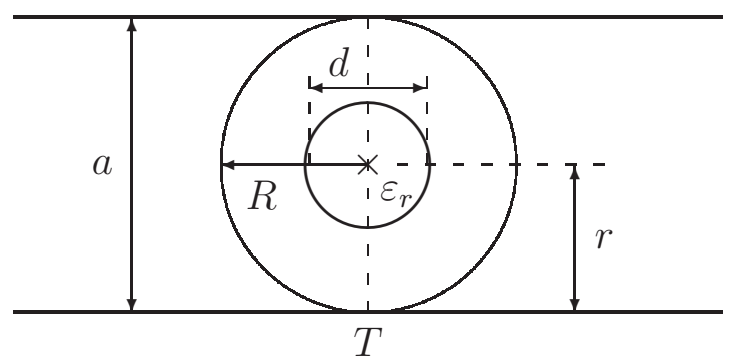

\begin{tabular}{|c|c|c|c|c|c|c|c|}
\cline { 2 - 7 } \multicolumn{1}{c|}{} & Tipo & $\mathrm{C}_{\mathrm{ix}}$ & $\mathrm{C}_{\mathrm{iy}}$ & $\mathbf{R}_{\mathbf{i}}$ & $\mathrm{dNc}_{\mathbf{i}}$ & $\mathrm{C}_{\mathrm{in}}^{\mathrm{i}}$ & $\mathrm{C}_{\mathrm{sc}}^{\mathrm{i}}$ \\
\hline Objeto 1 & $\begin{array}{c}\text { Cilindro } \\
\text { dieléctrico } \\
\text { con pérdidas }\end{array}$ & 0 & 0 & 0.015 & - & 3 & 1.5 \\
\hline
\end{tabular}

\begin{tabular}{|c|c|c|c|c|c|}
\hline $\mathbf{R}$ & $\mathbf{R}_{\mathrm{AB}}$ & $\mathbf{R}_{\mathrm{fft}}$ & $\mathbf{C}_{\mathrm{sc}}$ & Modos & Tiempo \\
\hline$\frac{a}{2}$ & 1 & 1 & 3 & 5 & $0.85 \mathrm{~s}$ \\
\hline
\end{tabular}

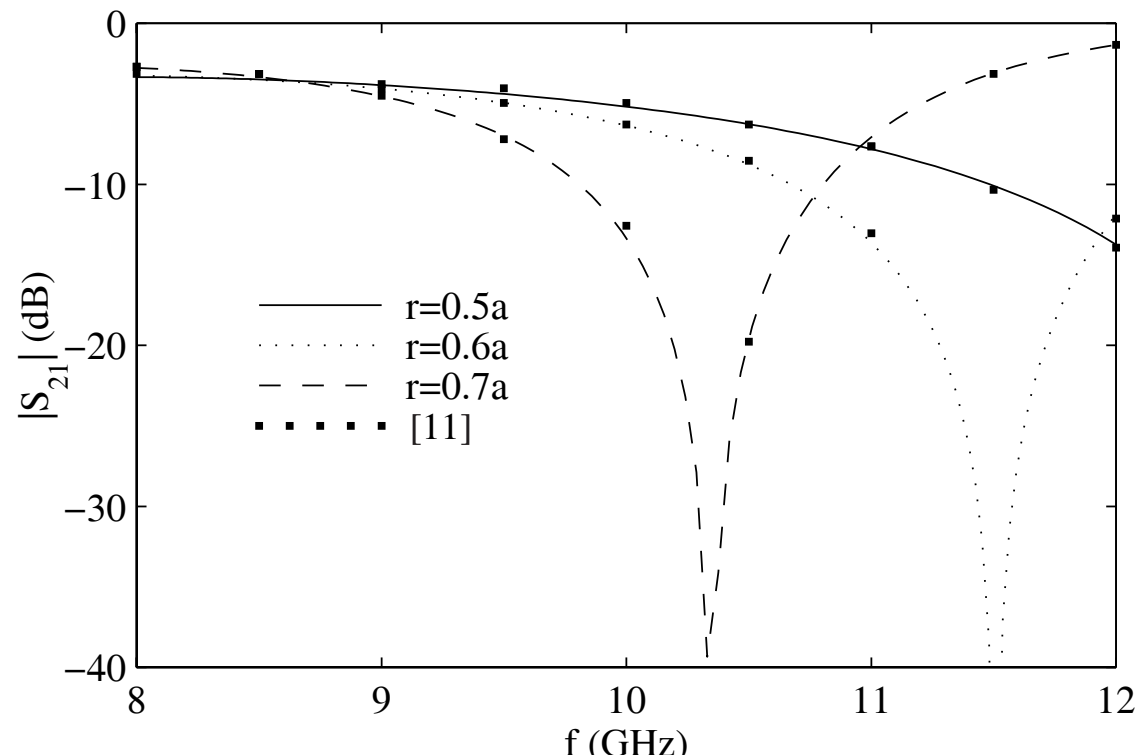

Figura 3.45: Poste dieléctrico circular con pérdidas. Esquema, valores de los parámetros de simulación y respuesta en frecuencia $\left(\left|S_{21}\right|\right) \cdot \varepsilon_{r}=38,5, \tan \delta=2 \times 10^{-4}, d=0,03 a$, $a=22,86 \mathrm{~mm}$ 


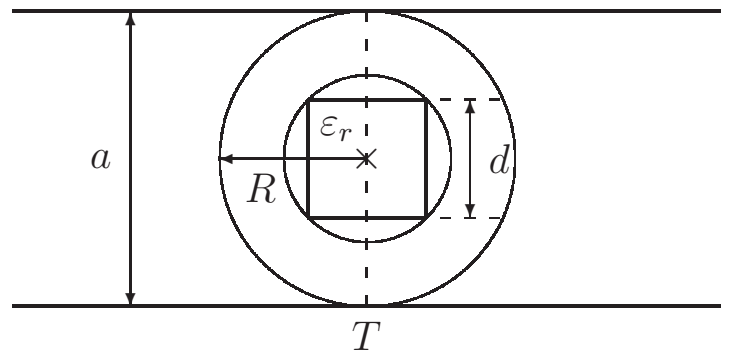

\begin{tabular}{|c|c|c|c|c|c|c|c|}
\cline { 2 - 7 } \multicolumn{1}{c|}{} & Tipo & $\mathbf{C}_{\mathbf{i x}}$ & $\mathbf{C}_{\mathbf{i y}}$ & $\mathbf{R}_{\mathbf{i}}$ & $\mathbf{d N c}_{\mathbf{i}}$ & $\mathbf{C}_{\mathbf{i n}}^{\mathbf{i}}$ & $\mathbf{C}_{\mathbf{s c}}^{\mathbf{i}}$ \\
\hline Objeto 1 & $\begin{array}{c}\text { Poste } \\
\text { cuadrado } \\
\text { dieléctrico }\end{array}$ & 0 & 0 & $0,05 \sqrt{2} a$ & 100 & 3 & 1.5 \\
\hline
\end{tabular}

\begin{tabular}{|c|c|c|c|c|c|}
\hline $\mathbf{R}$ & $\mathbf{R}_{\mathrm{AB}}$ & $\mathbf{R}_{\mathrm{fft}}$ & $\mathbf{C}_{\mathrm{sc}}$ & Modos & Tiempo \\
\hline$\frac{a}{2}$ & 1 & 1 & 3 & 5 & $\simeq 5 \mathrm{~s}$ \\
\hline
\end{tabular}

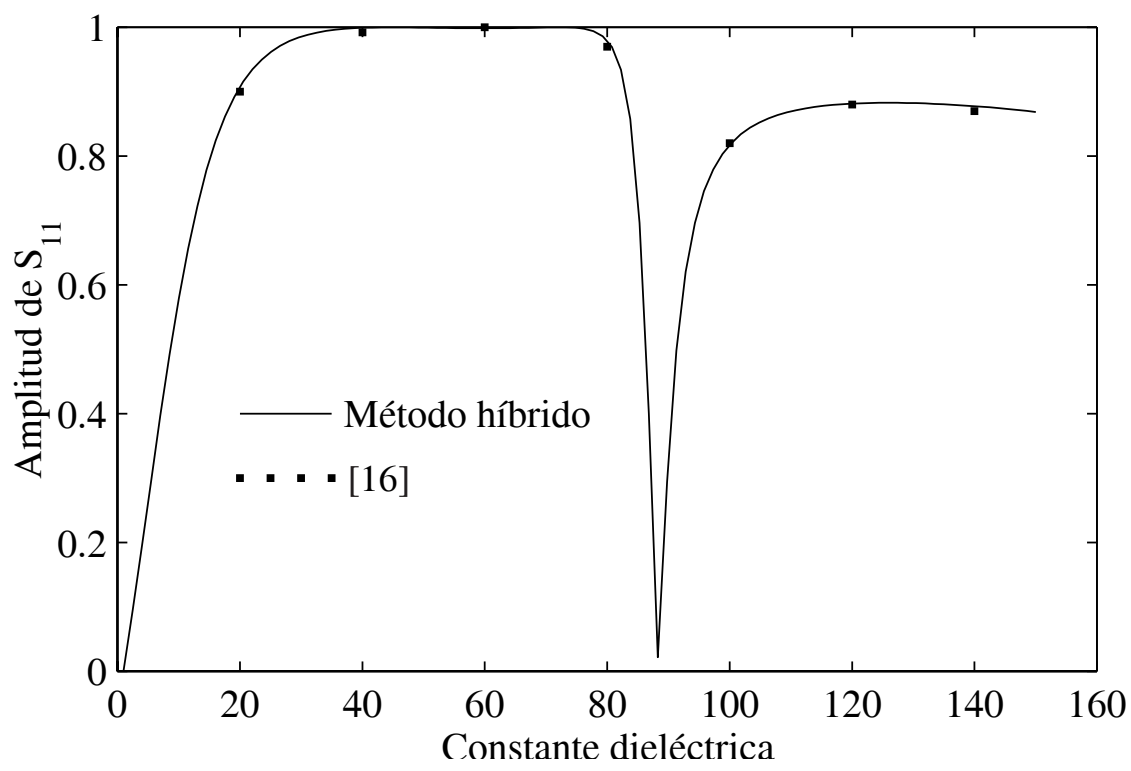

Figura 3.46: Poste dieléctrico cuadrado. Esquema, valores de los parámetros de simulación y variación de $\left|S_{11}\right|$ con la constante dieléctrica $(d / a=0,1, \lambda / a=1,4)$ 


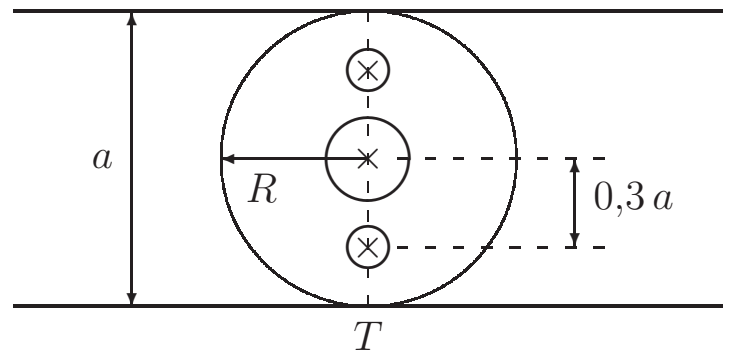

\begin{tabular}{|c|c|c|c|c|c|c|c|}
\cline { 2 - 7 } \multicolumn{1}{c|}{} & Tipo & $\mathrm{C}_{\mathbf{i x}}$ & $\mathrm{C}_{\mathbf{i y}}$ & $\mathbf{R}_{\mathbf{i}}$ & $\mathbf{d N c}_{\mathbf{i}}$ & $\mathrm{C}_{\mathrm{in}}^{\mathrm{i}}$ & $\mathbf{C}_{\mathbf{s c}}^{\mathrm{i}}$ \\
\hline Objeto 1 & $\begin{array}{c}\text { Cil. diel. } \\
\text { con pérdidas }\end{array}$ & 0 & 0 & 0.05 & - & 3 & 1.5 \\
\hline Objeto 2 & $\begin{array}{c}\text { Cil. diel. } \\
\text { con pérdidas }\end{array}$ & 0 & $0,03 a$ & 0.025 & - & 3 & 1.5 \\
\hline Objeto 3 & $\begin{array}{c}\text { Cil. diel. } \\
\text { con pérdidas }\end{array}$ & 0 & $-0,03 a$ & 0.025 & - & 3 & 1.5 \\
\hline
\end{tabular}

\begin{tabular}{|c|c|c|c|c|c|}
\hline $\mathbf{R}$ & $\mathbf{R}_{\mathrm{AB}}$ & $\mathbf{R}_{\mathrm{fft}}$ & $\mathbf{C}_{\mathrm{sc}}$ & Modos & Tiempo \\
\hline$\frac{a}{2}$ & 1 & 1 & 3 & 5 & $0.94 \mathrm{~s}$ \\
\hline
\end{tabular}

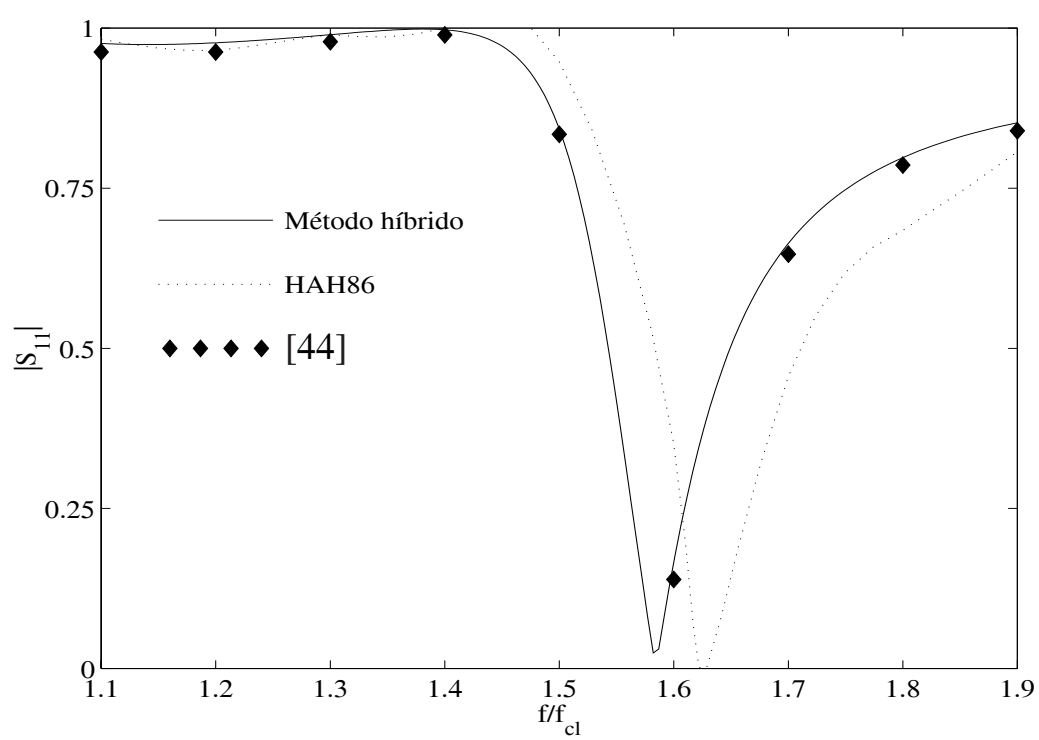

Figura 3.47: Tres postes dieléctricos circulares. Esquema, valores de los parámetros de simulación y variación de $\left|S_{11}\right|$ y $\left|S_{21}\right|$ con la frecuencia. $\varepsilon_{r}=38,0$ 


\subsubsection{Discontinuidades inductivas}

En esta apartado se verifica la capacidad de análisis de discontinuidades inductivas de geometría arbitraria por parte del método híbrido. En primer lugar se analizan en las figuras 3.49 y 3.50 un salto de sección en plano $\mathrm{H}$ centrado y lateral, respectivamente. En la figura 3.49 se muestran los valores de los parámetros del algoritmo de acoplo de modos que ha sido necesario utilizar para conseguir la convergencia de los resultados. También se muestra la amplitud y la fase del factor de reflexión comparando los resultados con los de [49] ( $f=$ $12 \mathrm{GHz}, a_{1}=19,05 \mathrm{~mm}$ ), comprobándose que existe una buena coincidencia. En la figura 3.50 se repite el análisis para el caso de un salto de sección lateral.

En la figura 3.51 se analizan un codo de $90^{\circ}$ y otro de $135^{\circ}$ en plano $\mathrm{H}$ en una guía WR62. Para realizar el análisis se ha fragmentado el segmento irregular de la guía (circunferencia de radio $R$ ) en tres objetos. Como se observa el la figura 3.51, se consiguen resultados satisfactorios al comparar el factor de reflexión con [14], con un coste computacional de $0.74 \mathrm{~s}$ por punto en frecuencia. En la figura 3.52 se repite al mismo análisis que en la figura anterior (codos de $90^{\circ}$ y $135 \circ$ en plano H), pero en esta ocasión el segmento irregular de la guía se ha caracterizado como un sólo objeto, utilizando el método de los momentos.

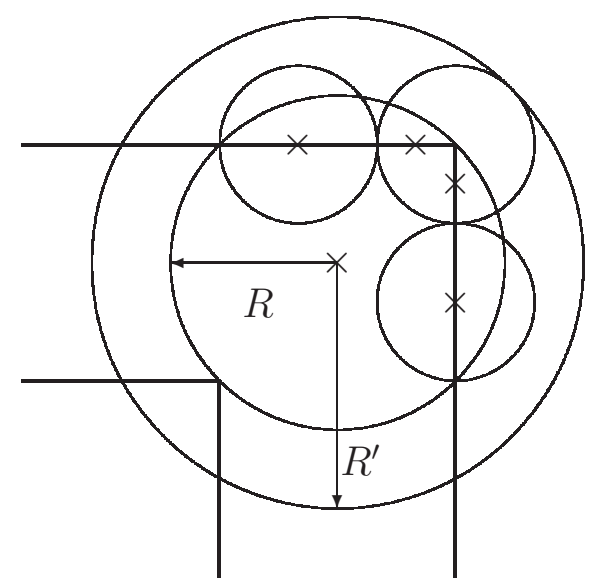

Figura 3.48: Esquema ilustrativo del problema existente al fragmentar el segmento irregular contenido en la circunferencia de radio $R$ en tres objetos en el caso de un codo de $90^{\circ}$

Se puede observar que los resultados son más precisos que cuando se fragmentaba el problema en tres objetos diferentes. Esto es debido a que al fragmentar en tres objetos y expresar el campo dispersado por los tres objetos como un único espectro de campo emergente, dicho espectro tan sólo reconstruye correctamente el campo fuera de la circunferencia de radio $R^{\prime}$ (ver figura 3.48). Si intentamos obtener el campo dispersado por los tres objetos a lo largo de la circunferencia de radio $R$ a partir de un único espectro de campo emergente, dicho espectro proporcionará un valor bastante exacto (pero no correcto totalmente) en aquellas zonas de la circunferencia de radio $R$ que queden fuera de las tres circunferencias que contienen a cada uno de los tres objetos. Este es el motivo de que los resultados de la figura 3.52 (donde no se comete ninguna irregularidad) sean mejores que los de la figura 3.51 
(donde el campo a lo largo de la circunferencia $R$ no se reconstruye correctamente). Hay que destacar, sin embargo, que el análisis de la figura 3.52 es más costoso computacionalmente, ya que se analiza toda la estructura de forma numérica, no como en la figura 3.51 donde se alterna el Método de los Momentos con el método de resolución de problemas múltiples 2-D.

Continuando con la validación del método para el análisis de codos en plano $\mathrm{H}$, en la figura 3.53 se muestra el factor de reflexión de un codo biselado en plano $\mathrm{H}$ en una guía WR62 para diferentes grados de biselado $\left(a_{\max }=a \sqrt{2}, a_{\min }=a / \sqrt{2}\right.$ and $\left.a_{\text {opt }}=0,68 a\right)$, y los resultados se comparan satisfactoriamente con los de [50].

Cualquier codo en plano $\mathrm{H}$ de geometría arbitraria en una guía rectangular puede ser analizados con el método híbrido. Un ejemplo de esto lo tenemos en las figuras 3.54 y 3.55 , donde se muestra, respectivamente, el factor de reflexión de dos codos circulares de $90^{\circ}$ ( $a=19,05 \mathrm{~mm}, L=21,6 \mathrm{~mm}$ ) y $60^{\circ}$ ( $a=22,9 \mathrm{~mm}, L=15,24 \mathrm{~mm}$ ). Los resultados se ha validado comparándolos con los mismos problemas publicados en [51] y [52].

En la figura 3.56 se analiza un codo en $\mathrm{U}$ en plano $\mathrm{H}$ formado por dos codos circulares de $90^{\circ}$ en WR62. Las matrices GSM de los dos codos han sido enlazadas siguiendo la formulación desarrollada en el apéndice F. Los resultados del método para la fase del factor de transmisión se han comparado satisfactoriamente con medidas de laboratorio.

La figura 3.57 muestra un ejemplo de análisis de una estructura multipuerto. La amplitud y la fase de los parámetros de dispersión de una unión T en plano H se comparan satisfactoriamente con resultados de [53]. También se pueden introducir obstáculos en la unión T y analizarlos con el método híbrido. Así, por ejemplo, en la figura 3.58 se presentan los resultados del análisis de una union $\mathrm{T}$ en plano $\mathrm{H}$ compensada con un poste circular metálico. El factor de reflexión de dicha estructura se muestra en la figura 3.58 y se compara exitosamente con resultados de [14].

El número de modos guiados (3 en todos los casos), y el tiempo computacional requerido para el análisis de los problemas de discontinuidades inductivas (algo menor a 1 segundo excepto an algún codo circular) fueron similares a los de los problemas de obstáculos inductivos. 


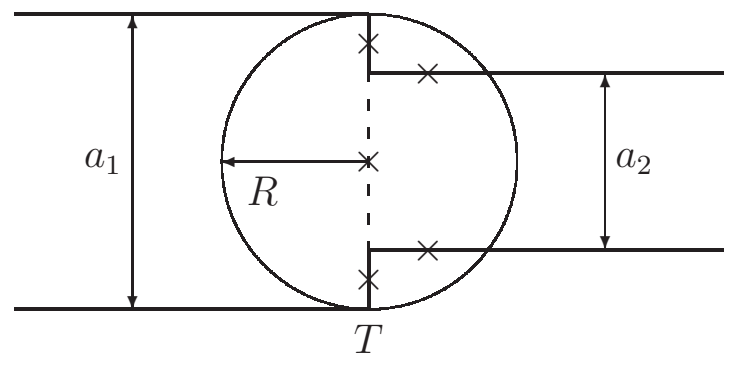

\begin{tabular}{|c|c|c|c|c|c|c|c|}
\cline { 2 - 7 } \multicolumn{1}{c|}{} & Tipo & $\mathrm{C}_{\mathrm{ix}}$ & $\mathrm{C}_{\mathrm{iy}}$ & $\mathbf{R}_{\mathbf{i}}$ & $\mathbf{d N c}_{\mathbf{i}}$ & $\mathrm{C}_{\mathrm{in}}^{\mathrm{i}}$ & $\mathrm{C}_{\mathrm{sc}}^{\mathrm{i}}$ \\
\hline Objeto 1 & $\begin{array}{c}\text { Compuesto de } \\
4 \text { tiras }\end{array}$ & 0 & 0 & $\frac{a_{1}}{2}$ & 75 & 6 & 2 \\
\hline
\end{tabular}

\begin{tabular}{|c|c|c|c|c|c|}
\hline $\mathbf{R}$ & $\mathbf{R}_{\mathrm{AB}}$ & $\mathbf{R}_{\mathrm{fft}}$ & $\mathbf{C}_{\mathrm{sc}}$ & Modos & Tiempo \\
\hline$\frac{a_{1}}{2}$ & 1 & 1 & 3 & 5 & $0.82 \mathrm{~s}$ \\
\hline
\end{tabular}

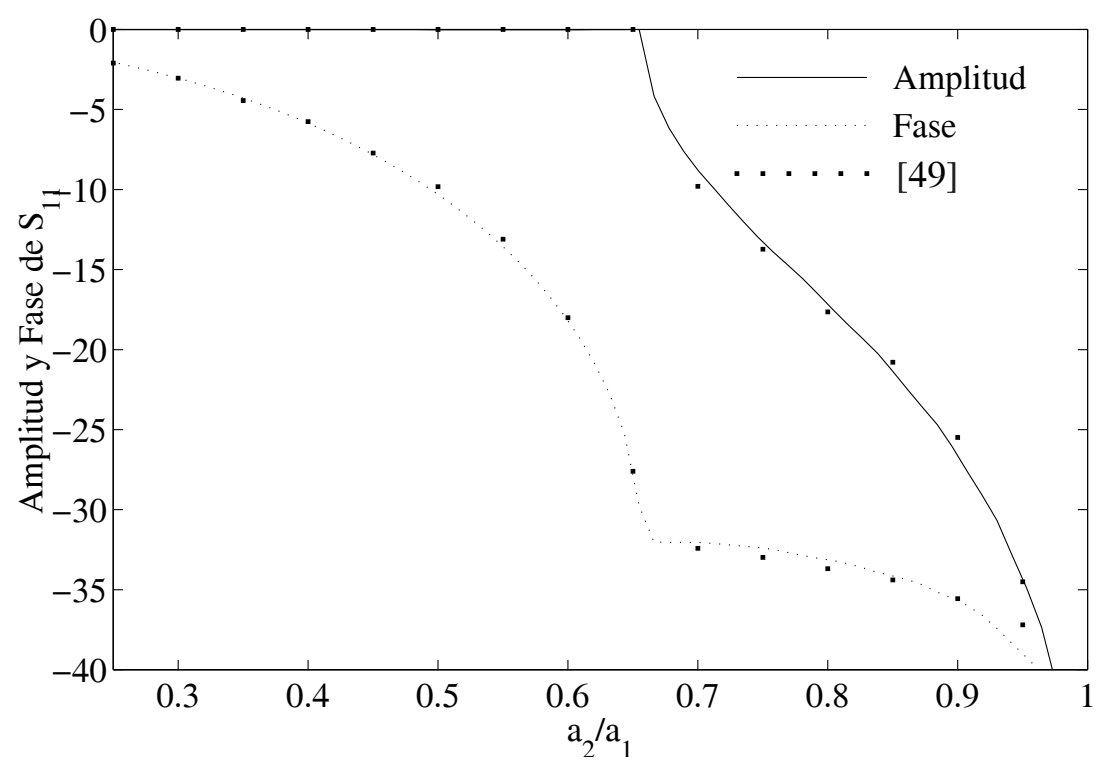

Figura 3.49: Salto de sección centrado. Esquema, valores de los parámetros de simulación y variación de $S_{11}$ (amplitud y fase) con la magnitud del cambio sección $\left(a_{2} / a_{1}\right) . f=12 \mathrm{GHz}$, $a_{1}=19,05 \mathrm{~mm}$ 


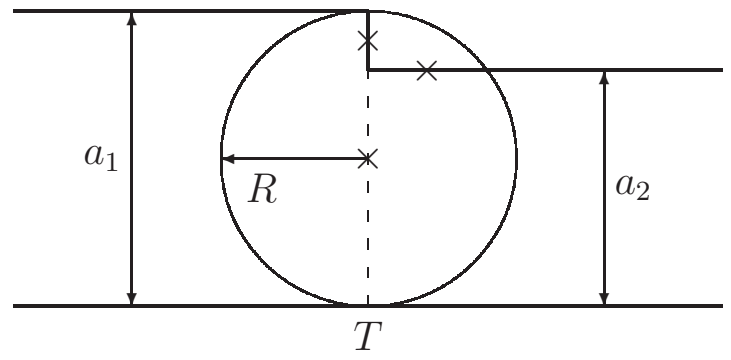

\begin{tabular}{|c|c|c|c|c|c|c|c|}
\cline { 2 - 7 } \multicolumn{1}{c|}{} & Tipo & $\mathbf{C}_{\mathbf{i x}}$ & $\mathbf{C}_{\mathrm{iy}}$ & $\mathbf{R}_{\mathbf{i}}$ & $\mathbf{d N c}_{\mathbf{i}}$ & $\mathrm{C}_{\mathrm{in}}^{\mathrm{i}}$ & $\mathrm{C}_{\mathbf{s c}}^{\mathrm{i}}$ \\
\hline Objeto 1 & $\begin{array}{c}\text { Compuesto de } \\
2 \text { tiras }\end{array}$ & 0 & 0 & $\frac{a_{1}}{2}$ & 75 & 6 & 2 \\
\hline
\end{tabular}

\begin{tabular}{|c|c|c|c|c|c|}
\hline $\mathbf{R}$ & $\mathbf{R}_{\mathrm{AB}}$ & $\mathbf{R}_{\mathrm{fft}}$ & $\mathbf{C}_{\mathbf{s c}}$ & Modos & Tiempo \\
\hline$\frac{a_{1}}{2}$ & 1 & 1 & 3 & 5 & $0.82 \mathrm{~s}$ \\
\hline
\end{tabular}

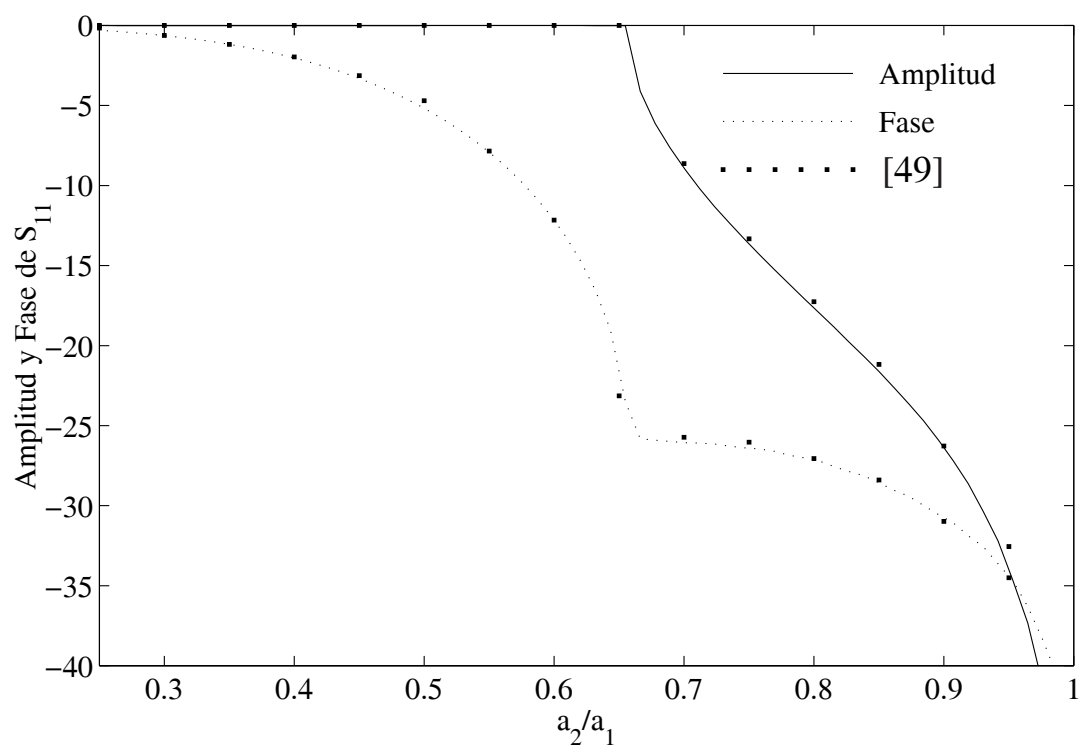

Figura 3.50: Salto de sección lateral. Esquema, valores de los parámetros de simulación y variación de $S_{11}$ (amplitud y fase) con la magnitud del cambio sección $\left(a_{2} / a_{1}\right) . f=12 \mathrm{GHz}$, $a_{1}=19,05 \mathrm{~mm}$ 


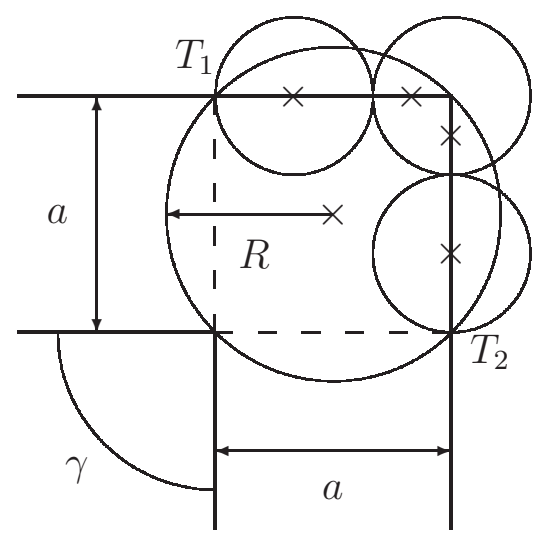

\begin{tabular}{|c|c|c|c|c|c|c|c|}
\hline$\gamma=90^{\circ}$ & Tipo & $\mathbf{C}_{\mathbf{i x}}$ & $\mathbf{C}_{\mathbf{i y}}$ & $\mathbf{R}_{\mathbf{i}}$ & $\mathbf{d N c}_{\mathbf{i}}$ & $\mathbf{C}_{\mathbf{i n}}^{\mathbf{i}}$ & $\mathbf{C}_{\mathbf{s c}}^{\mathbf{i}}$ \\
\hline Objeto 1 & Tira & $-\frac{a}{6}$ & $\frac{a}{2}$ & $\frac{a}{3}$ & 25 & 3 & 1.2 \\
\hline Objeto 2 & $\begin{array}{c}\text { Compuesto de } \\
2 \text { tiras }\end{array}$ & $\frac{a}{2}$ & $\frac{a}{2}$ & $\frac{a}{3}$ & 25 & 3 & 1.2 \\
\hline Objeto 3 & Tira & $\frac{a}{2}$ & $-\frac{a}{6}$ & $\frac{a}{3}$ & 25 & 3 & 1.2 \\
\hline
\end{tabular}

\begin{tabular}{|c|c|c|c|c|c|}
\hline $\mathbf{R}$ & $\mathbf{R}_{\mathrm{AB}}$ & $\mathbf{R}_{\mathrm{fft}}$ & $\mathbf{C}_{\mathrm{sc}}$ & Modos & Tiempo \\
\hline$\frac{a}{2} \sqrt{2}$ & 3 & 8 & 3 & 5 & $0.74 \mathrm{seg}$. \\
\hline
\end{tabular}

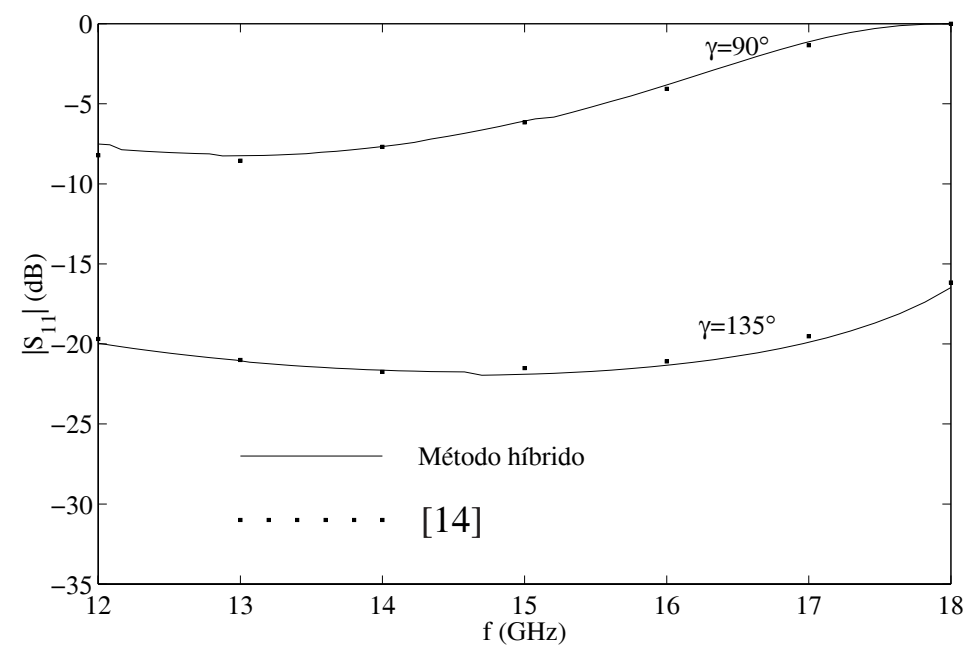

Figura 3.51: Codo en plano H. Esquema, valores de los parámetros de simulación $\left(\gamma=90^{\circ}\right)$ y variación de $S_{11}$ con la frecuencia para dos inclinaciones distintas $\left(\gamma=90^{\circ}\right.$ y $\left.\gamma=135^{\circ}\right)$ 


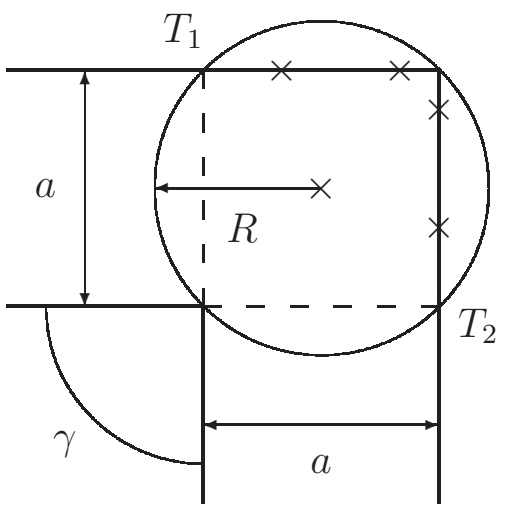

\begin{tabular}{|c|c|c|c|c|c|c|c|}
\hline$\gamma=90^{\circ}$ & Tipo & $\mathbf{C}_{\mathbf{i x}}$ & $\mathbf{C}_{\mathbf{i y}}$ & $\mathbf{R}_{\mathbf{i}}$ & $\mathbf{d N c}_{\mathbf{i}}$ & $\mathbf{C}_{\mathbf{i n}}^{\mathbf{i}}$ & $\mathbf{C}_{\mathbf{s c}}^{\mathbf{i}}$ \\
\hline Objeto 1 & $\begin{array}{c}\text { Compuesto de } \\
4 \text { tiras }\end{array}$ & 0 & 0 & $\frac{a}{2} \sqrt{2}$ & 25 & 6 & 3 \\
\hline
\end{tabular}

\begin{tabular}{|c|c|c|c|c|c|}
\hline $\mathbf{R}$ & $\mathbf{R}_{\mathrm{AB}}$ & $\mathbf{R}_{\mathrm{fft}}$ & $\mathbf{C}_{\mathbf{s c}}$ & Modos & Tiempo \\
\hline$\frac{a}{2} \sqrt{2}$ & 3 & 1 & 3 & 5 & $0.85 \mathrm{seg}$. \\
\hline
\end{tabular}

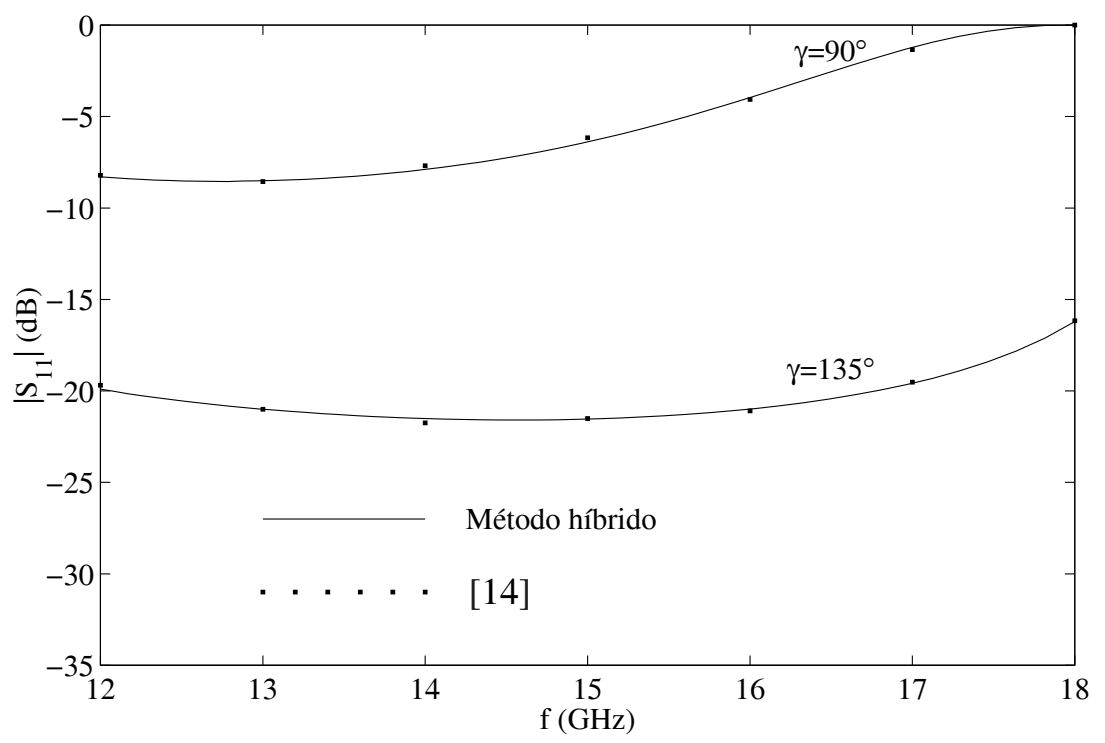

Figura 3.52: Codo en plano H. Esquema, valores de los parámetros de simulación $\left(\gamma=90^{\circ}\right)$ y variación de $S_{11}$ con la frecuencia para dos inclinaciones distintas $\left(\gamma=90^{\circ}\right.$ y $\left.\gamma=135^{\circ}\right)$ 


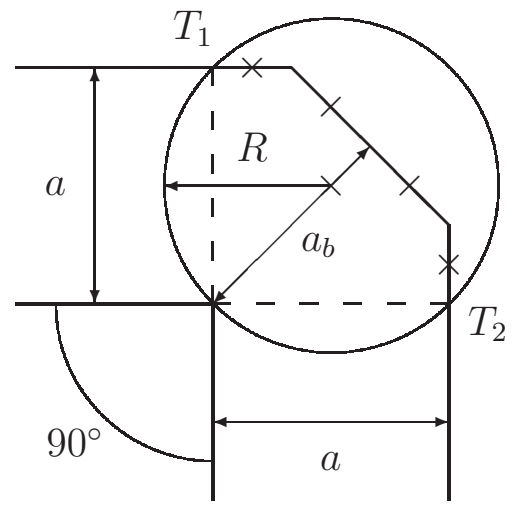

\begin{tabular}{|c|c|c|c|c|c|c|c|}
\cline { 2 - 7 } \multicolumn{1}{c|}{} & Tipo & $\mathrm{C}_{\mathbf{i x}}$ & $\mathrm{C}_{\mathrm{iy}}$ & $\mathbf{R}_{\mathbf{i}}$ & $\mathrm{dNc}_{\mathbf{i}}$ & $\mathrm{C}_{\mathrm{in}}^{\mathrm{i}}$ & $\mathrm{C}_{\mathrm{sc}}^{\mathrm{i}}$ \\
\hline Objeto 1 & $\begin{array}{c}\text { Compuesto de } \\
4 \text { tiras }\end{array}$ & 0 & 0 & $\frac{a}{2} \sqrt{2}$ & 25 & 1.5 & 1 \\
\hline
\end{tabular}

\begin{tabular}{|c|c|c|c|c|c|}
\hline $\mathbf{R}$ & $\mathbf{R}_{\mathrm{AB}}$ & $\mathbf{R}_{\mathrm{fft}}$ & $\mathbf{C}_{\mathbf{s c}}$ & Modos & Tiempo \\
\hline$\frac{a}{2} \sqrt{2}$ & 3 & 2 & 3 & 5 & $0.69 \mathrm{seg}$. \\
\hline
\end{tabular}

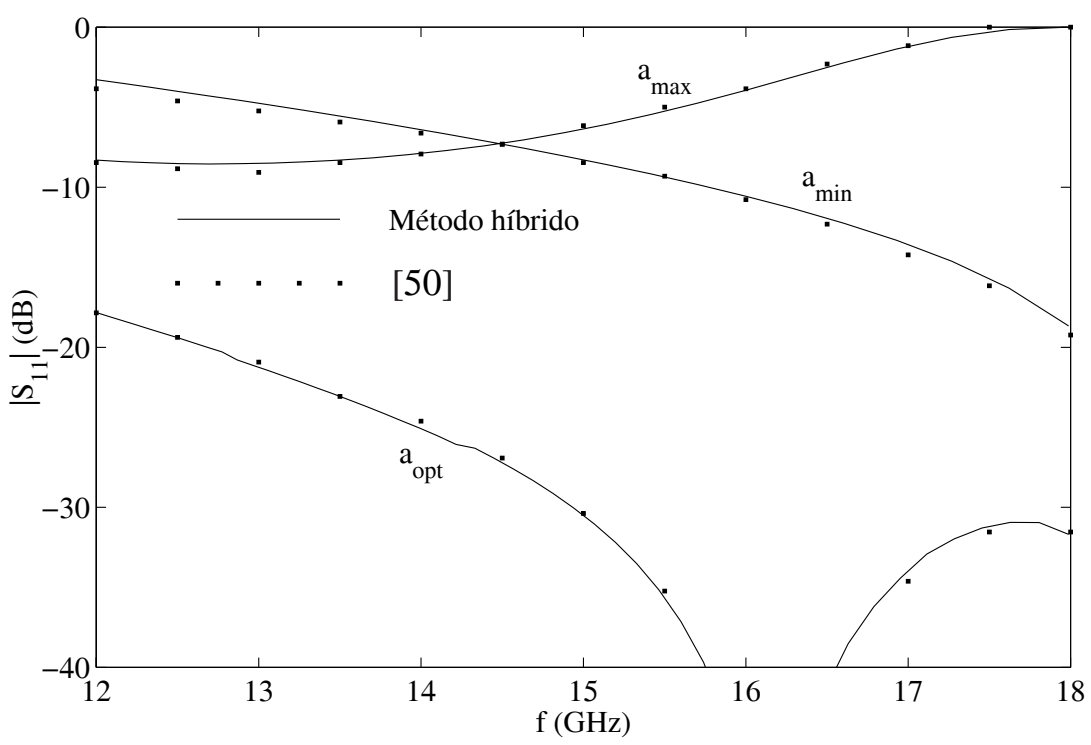

Figura 3.53: Codo biselado en plano $H$. Esquema, valores de los parámetros de simulación $\left(a_{b}=0,68 a\right)$ y variación de $\left|S_{11}\right|$ con la frecuencia para tres niveles de biselado $\left(a_{b}=a_{\max }=\right.$ $a \sqrt{2}, a_{b}=a_{\text {min }}=a / \sqrt{2}$ y $\left.a_{b}=a_{\text {opt }}=0,68 a, a=15,799 \mathrm{~mm}\right)$ 


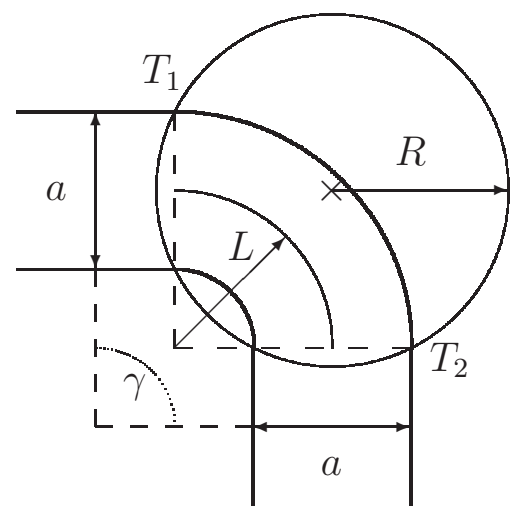

\begin{tabular}{|c|c|c|c|c|c|c|c|}
\cline { 2 - 7 } \multicolumn{1}{c|}{} & Tipo & $\mathbf{C}_{\mathbf{i x}}$ & $\mathbf{C}_{\mathbf{i y}}$ & $\mathbf{R}_{\mathbf{i}}$ & $\mathbf{d N c}_{\mathbf{i}}$ & $\mathbf{C}_{\mathbf{i n}}^{\mathbf{i}}$ & $\mathbf{C}_{\mathbf{s c}}^{\mathbf{i}}$ \\
\hline Objeto 1 & $\begin{array}{c}\text { Compuesto de } \\
2 \text { tiras curvas }\end{array}$ & 0 & 0 & $\sqrt{\left(\frac{a}{2}\right)^{2}+L^{2}}$ & 75 & 12 & 5 \\
\hline
\end{tabular}

\begin{tabular}{|c|c|c|c|c|l|}
\hline $\mathbf{R}$ & $\mathbf{R}_{\mathrm{AB}}$ & $\mathbf{R}_{\mathrm{fft}}$ & $\mathbf{C}_{\mathbf{s c}}$ & Modos & Tiempo \\
\hline$\sqrt{\left(\frac{a}{2}\right)^{2}+L^{2}}$ & 3 & 2 & 3 & 5 & $1.96 \mathrm{seg}$. \\
\hline
\end{tabular}

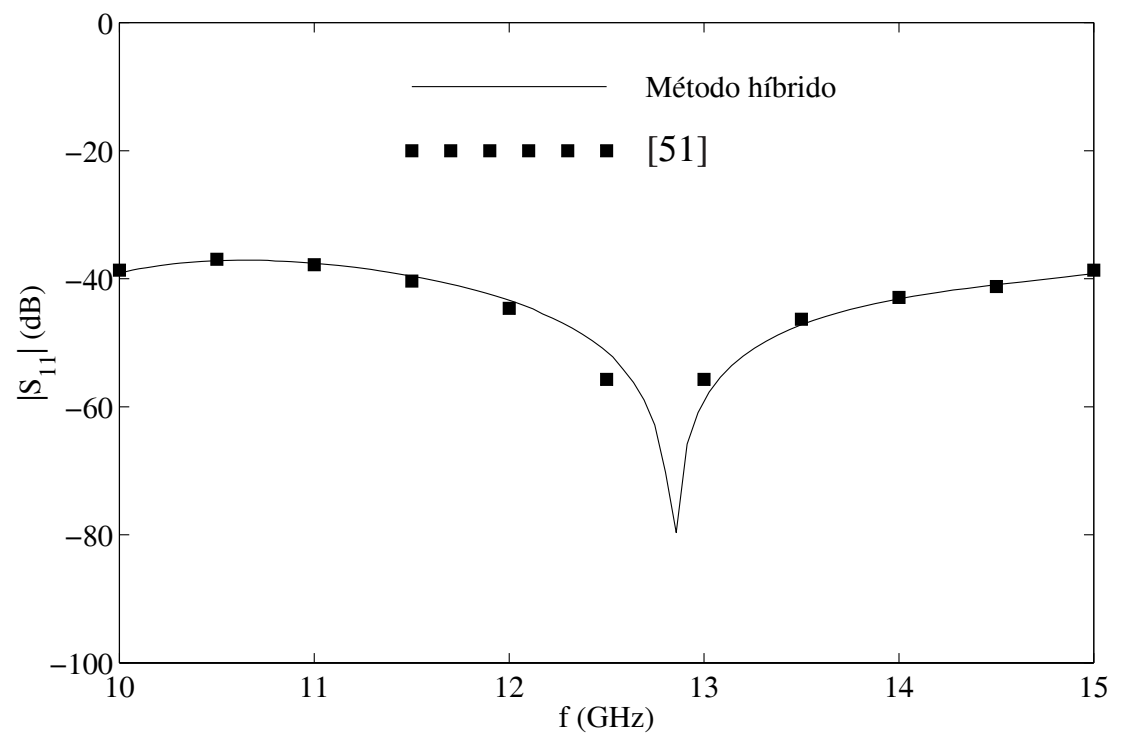

Figura 3.54: Codo circular en plano $H$. Esquema, valores de los parámetros de simulación y variación de $\left|S_{11}\right|$ con la frecuencia $\left(a=19,05 \mathrm{~mm}, \gamma=90^{\circ}, L=21,6 \mathrm{~mm}\right)$ 


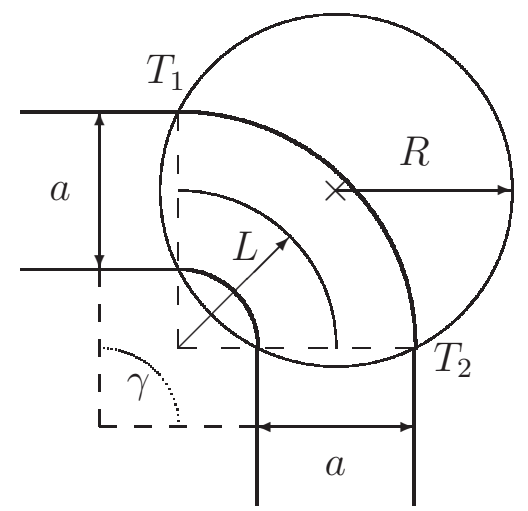

\begin{tabular}{|c|c|c|c|c|c|c|c|}
\cline { 2 - 7 } \multicolumn{1}{c|}{} & Tipo & $\mathbf{C}_{\mathbf{i x}}$ & $\mathbf{C}_{\mathbf{i y}}$ & $\mathbf{R}_{\mathbf{i}}$ & $\mathbf{d N c}_{\mathbf{i}}$ & $\mathbf{C}_{\mathrm{in}}^{\mathbf{i}}$ & $\mathbf{C}_{\mathbf{s c}}^{\mathbf{i}}$ \\
\hline Objeto 1 & $\begin{array}{c}\text { Compuesto de } \\
2 \text { tiras curvas }\end{array}$ & 0 & 0 & $\sqrt{\left(\frac{a}{2}\right)^{2}+L^{2}}$ & 25 & 6 & 4 \\
\hline
\end{tabular}

\begin{tabular}{|c|c|c|c|c|c|}
\hline $\mathbf{R}$ & $\mathbf{R}_{\mathrm{AB}}$ & $\mathbf{R}_{\mathrm{fft}}$ & $\mathbf{C}_{\mathbf{s c}}$ & Modos & Tiempo \\
\hline$\sqrt{\left(\frac{a}{2}\right)^{2}+L^{2}}$ & 3 & 2 & 3 & 5 & $2.06 \mathrm{~s}$ \\
\hline
\end{tabular}

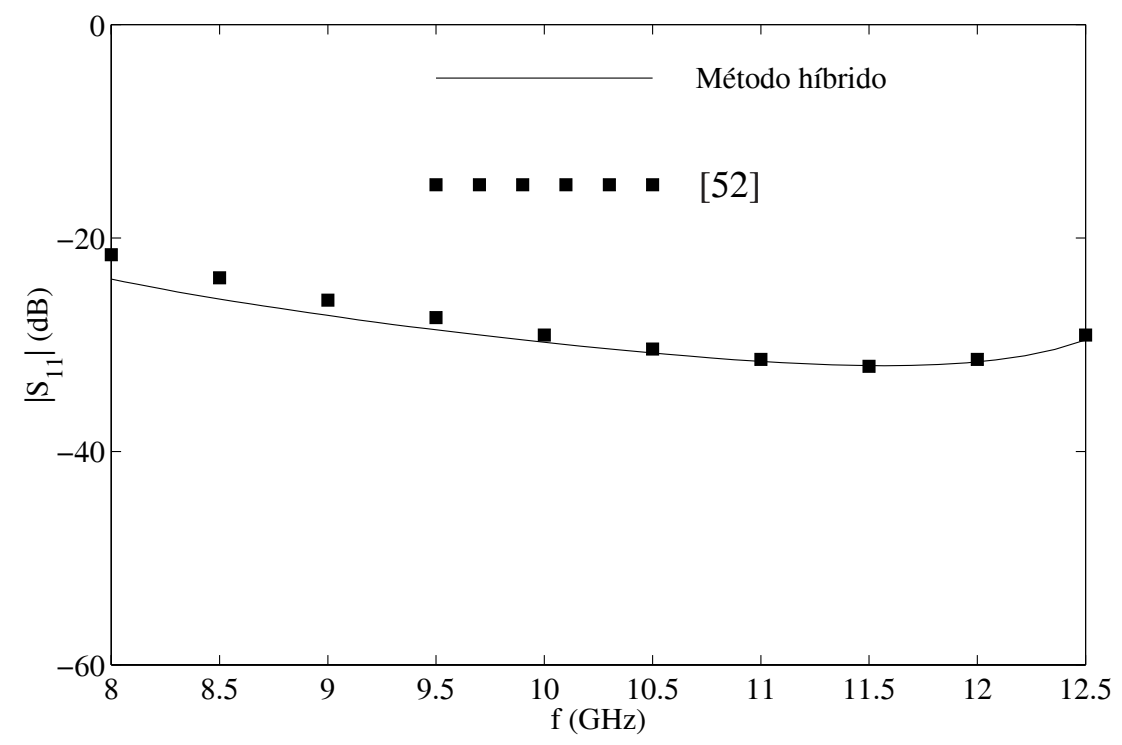

Figura 3.55: Codo circular en plano H. Esquema, valores de los parámetros de simulación y variación de $\left|S_{11}\right|$ con la frecuencia $\left(a=22,9 \mathrm{~mm}, \gamma=60^{\circ}, L=15,24 \mathrm{~mm}\right)$ 


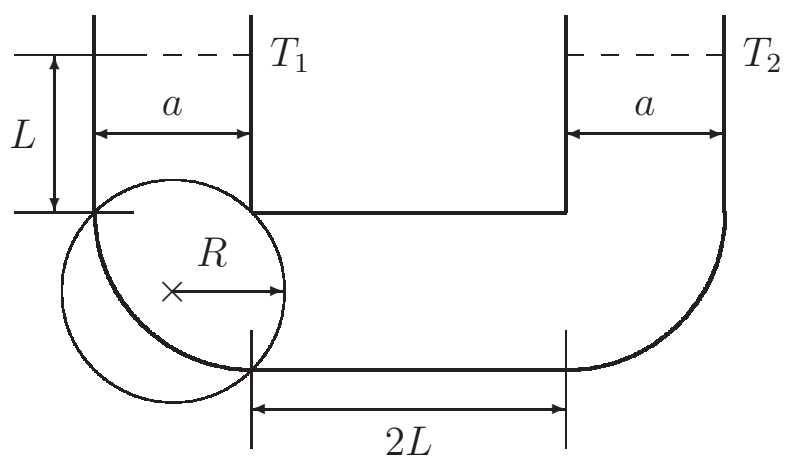

\begin{tabular}{|l|c|c|c|c|c|c|c|}
\cline { 2 - 8 } \multicolumn{1}{c|}{} & Tipo & $\mathbf{C}_{\mathrm{ix}}$ & $\mathrm{C}_{\mathrm{iy}}$ & $\mathbf{R}_{\mathbf{i}}$ & $\mathbf{d N c}_{\mathbf{i}}$ & $\mathrm{C}_{\mathrm{in}}^{\mathrm{i}}$ & $\mathrm{C}_{\mathbf{s c}}^{\mathbf{i}}$ \\
\hline Objeto 1 & 1 tira curva & 0 & 0 & $\sqrt{2} \frac{a}{2}$ & 50 & 6 & 2 \\
\hline
\end{tabular}

\begin{tabular}{|c|c|c|c|c|c|}
\hline $\mathbf{R}$ & $\mathbf{R}_{\mathrm{AB}}$ & $\mathbf{R}_{\mathrm{fft}}$ & $\mathbf{C}_{\mathbf{s c}}$ & Modos & Tiempo \\
\hline$\sqrt{2} \frac{a}{2}$ & 3 & 2 & 3 & 5 & $0.97 \mathrm{seg}$. \\
\hline
\end{tabular}

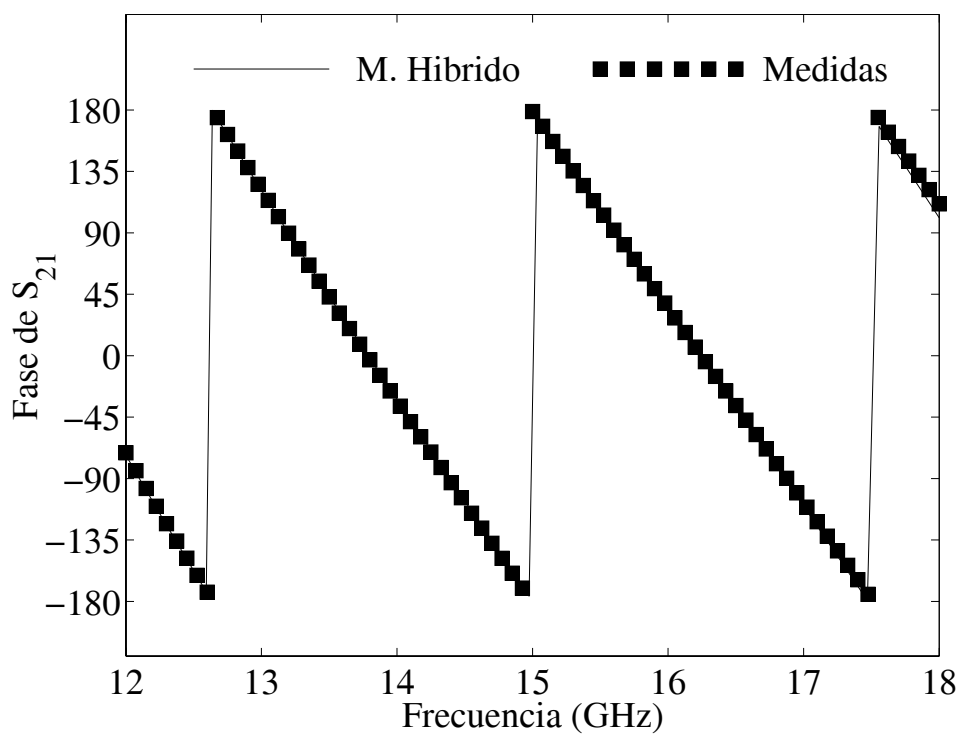

Figura 3.56: Codo circular en U en plano $\mathrm{H}$. Esquema, valores de los parámetros de simulación y variación de la fase de $\left|S_{21}\right|$ con la frecuencia ( $a=15,799 \mathrm{~mm}, L=16,6 \mathrm{~mm}$ ) 


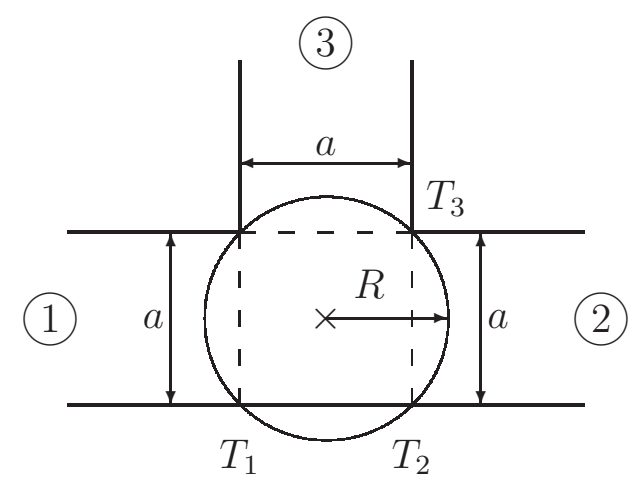

\begin{tabular}{|c|c|c|c|c|c|c|c|}
\cline { 2 - 7 } \multicolumn{1}{c|}{} & Tipo & $\mathbf{C}_{\mathbf{i x}}$ & $\mathbf{C}_{\mathbf{i y}}$ & $\mathbf{R}_{\mathbf{i}}$ & $\mathbf{d N c}_{\mathbf{i}}$ & $\mathbf{C}_{\mathbf{i n}}^{\mathrm{i}}$ & $\mathbf{C}_{\mathbf{s c}}^{\mathbf{i}}$ \\
\hline Objeto 1 & 1 tira & 0 & 0 & $\sqrt{2} \frac{a}{2}$ & 25 & 6 & 2 \\
\hline
\end{tabular}

\begin{tabular}{|c|c|c|c|c|c|}
\hline $\mathbf{R}$ & $\mathbf{R}_{\mathrm{AB}}$ & $\mathbf{R}_{\mathrm{fft}}$ & $\mathbf{C}_{\mathrm{sc}}$ & Modos & Tiempo \\
\hline$\sqrt{2} \frac{a}{2}$ & 1 & 1 & 3 & 5 & $0.75 \mathrm{seg}$. \\
\hline
\end{tabular}
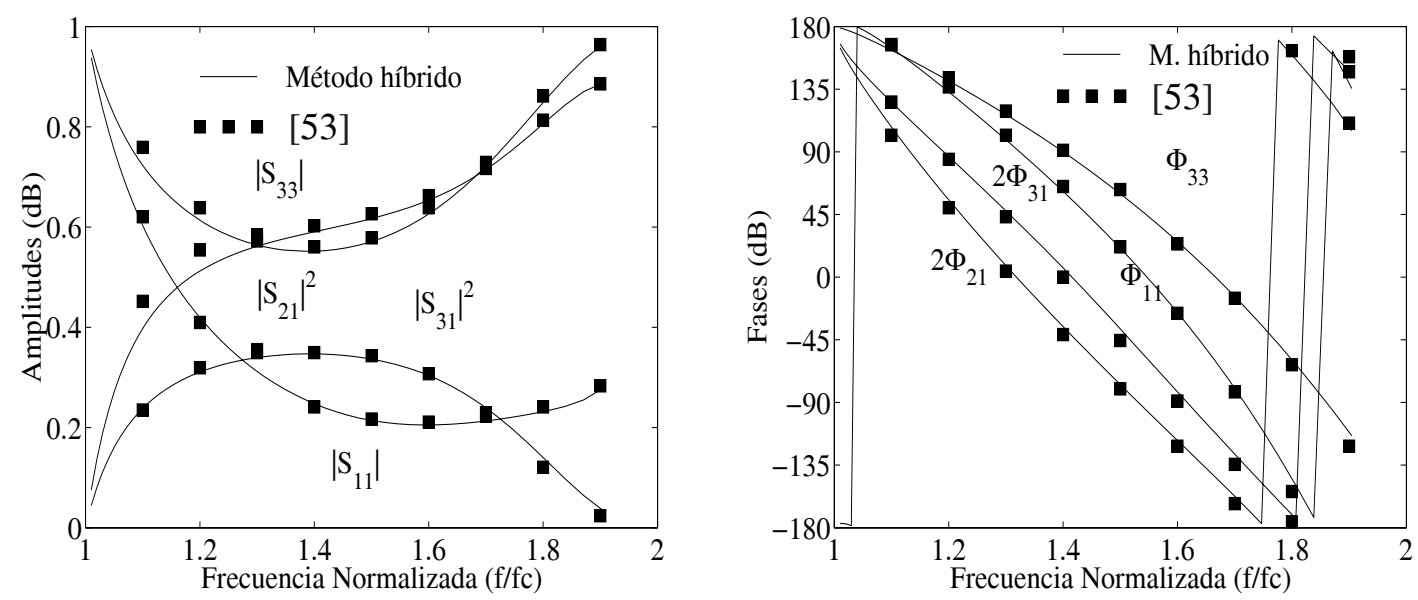

Figura 3.57: Unión $T$ en plano $H$. Esquema, valores de los parámetros de simulación y variación de la amplitud y fase de los parámetros de dispersión con la frecuencia 


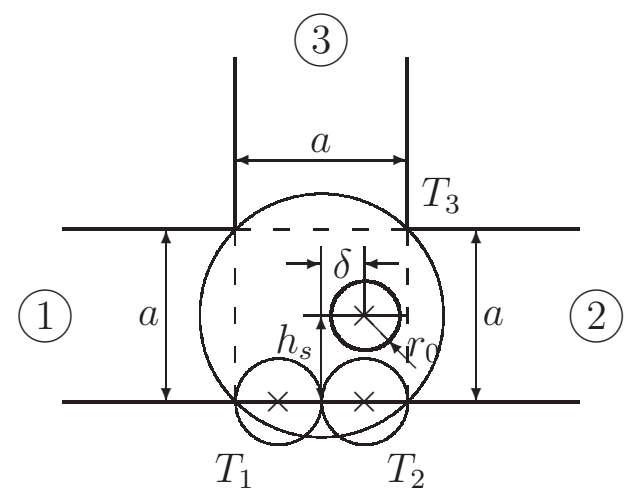

\begin{tabular}{|c|c|c|c|c|c|c|c|}
\cline { 2 - 8 } \multicolumn{1}{c|}{} & Tipo & $\mathbf{C}_{\mathbf{i x}}$ & $\mathbf{C}_{\mathbf{i y}}$ & $\mathbf{R}_{\mathbf{i}}$ & $\mathbf{d N c}_{\mathbf{i}}$ & $\mathbf{C}_{\mathbf{i n}}^{\mathbf{i}}$ & $\mathbf{C}_{\mathbf{s c}}^{\mathbf{i}}$ \\
\hline Objeto 1 & $\begin{array}{c}\text { Cilindro } \\
\text { metálico }\end{array}$ & $\delta$ & $-\frac{a}{2}+h_{s}$ & $r_{0}$ & - & 6 & 3 \\
\hline Objeto 2 & Tira & $\frac{a}{4}$ & $-\frac{a}{2}$ & $\frac{a}{4}$ & 50 & 6 & 3 \\
\hline Objeto 3 & Tira & $-\frac{a}{4}$ & $-\frac{a}{2}$ & $\frac{a}{4}$ & 50 & 6 & 3 \\
\hline
\end{tabular}

\begin{tabular}{|c|c|c|c|c|l|}
\hline $\mathbf{R}$ & $\mathbf{R}_{\mathrm{AB}}$ & $\mathbf{R}_{\mathrm{fft}}$ & $\mathbf{C}_{\mathbf{s c}}$ & Modos & Tiempo \\
\hline$\sqrt{2} \frac{a}{2}$ & 3 & 2 & 3 & 5 & $0.78 \mathrm{seg}$. \\
\hline
\end{tabular}

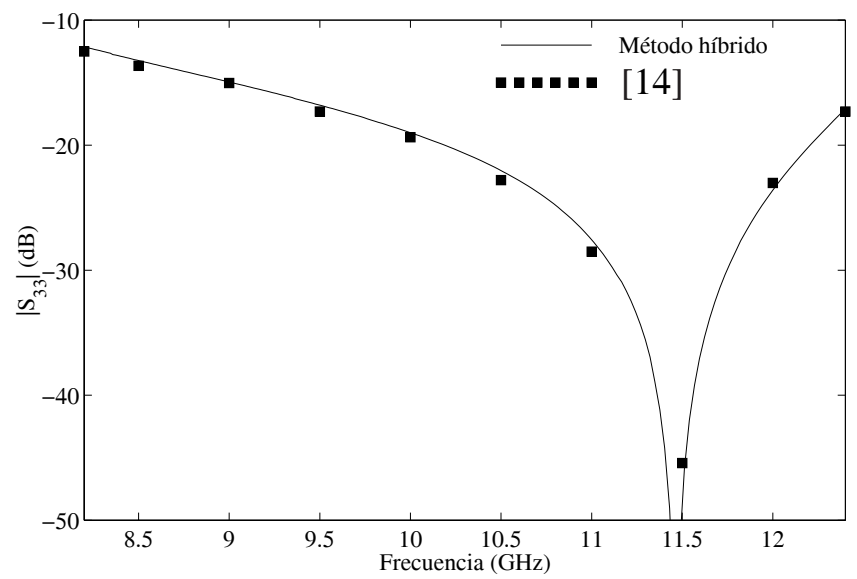

Figura 3.58: Unión $T$ en plano $H$ con un poste circular metálico. Esquema, valores de los parámetros de simulación y variación de la amplitud del factor de reflexión en el acceso 3 con la frecuencia ( $a=22,9 \mathrm{~mm}, h_{s}=8,8 \mathrm{~mm}, \delta=10^{-4} \mathrm{~mm}, r_{0}=7,510^{-4} \mathrm{~mm}$ ) 


\subsubsection{Componentes y dispositivos en plano $H$}

En este apartado se presentan dos resultados finales que permiten demostrar la validez del método híbrido en el análisis de estructuras inductivas complejas. En la figura 3.59 se muestra la respuesta en frecuencia del factor de transmisión de una filtro paso banda con un pequeño poste circular dieléctrico y otro grande metálico. El poste dieléctrico es de un material cerámico con permitividad compleja $\left(\varepsilon_{r}=38,5\right.$ y $\left.\tan \delta=2 \times 10^{-4}\right)$ y radio $r_{1}=$ $0,03 a$, donde $a$ es la anchura de la guía rectangular. El poste metálico es de mayor tamaño, de radio $r_{2}=0,3 a$, y situado de manera que toca una de las paredes de la guía $\left(b_{2}=\right.$ $0,3 a=r_{2}$ ). El factor de transmisión (amplitud y fase) se muestra en la figura 3.59 para varios posiciones del poste dieléctrico $\left(b_{1}=0,85 a, b_{1}=0,9 a\right.$ y $\left.b_{1}=0,95 a\right)$. Los resultados han sido validados con los publicados en [13].

En la figura 3.60 se analiza una cavidad en plano $\mathrm{H}$ con un poste circular dieléctrico en el centro. Se muestra un esquema de la estructura analizada $\left(a_{1}=17 \mathrm{~mm}, a_{2}=22,86 \mathrm{~mm}\right.$, $\left.r=5 \mathrm{~mm}, l=30 \mathrm{~mm}, \varepsilon_{r}=2,17\right)$, donde se aprecia que en el análisis se ha considerado la existencia de tres redes diferentes: un salto de sección en plano $\mathrm{H}$ de una guía de anchura $a_{1}$ a otra de anchura $a_{2}$, un poste circular dieléctrico en el interior de una guía rectangular de anchura $a_{2}$, y un salto de sección de una guía de anchura $a_{2}$ a otra de anchura $a_{1}$. Para obtener los parámetros de dispersión de esta estructura, se ha analizado cada red por separado. Una vez obtenida la MDG de cada red, se ha calculado la MDG de la estructura completa utilizando las expresiones de conexión de redes en cascada del apéndice F. En la figura 3.60 se muestran los valores de los parámetros del método utilizados en la simulación para cada red, y se compara la amplitud y fase de $\left|S_{21}\right|$ con los resultados de [54] obteniendo una buena coincidencia.

Finalmente, la figura 3.61 muestra los factores de reflexión y transmisión de un filtro de cavidades inductivas resonantes en una guía rectangular WR15 $(a=3,7591 \mathrm{~mm})$. Las dimensiones del filtro son: $l_{1}=l_{4}=2,73 \mathrm{~mm}, l_{2}=l_{3}=3,02 \mathrm{~mm}, w_{1}=w_{5}=1,88 \mathrm{~mm}$, $w_{2}=w_{4}=w_{3}=1,18 \mathrm{~mm}, d_{1}=d_{5}=0,56 \mathrm{~mm}, d_{2}=d_{4}=0,52 \mathrm{~mm}$, y $d_{3}=0,63 \mathrm{~mm}$. Para obtener los parámetros de dispersión de esta estructura, se ha analizado cada estrechamiento inductivo por separado (la zona dentro del círculo en la figura 3.61 es lo que se entiende en este texto por estrechamiento inductivo), posteriormente se ha calculado la MDG de la estructura completa utilizando las expresiones de conexión de redes en cascada del apéndice F. Los resultados están en concordancia con los proporcionados en [51] para el mismo filtro. Se necesitaron 13 modos guiados en cada acceso para analizar correctamente la estructura, y alrededor de 6 segundos fue el tiempo de computación para cada estrechamiento y por cada punto de frecuencia con un Pentium a $400 \mathrm{MHz}$. 


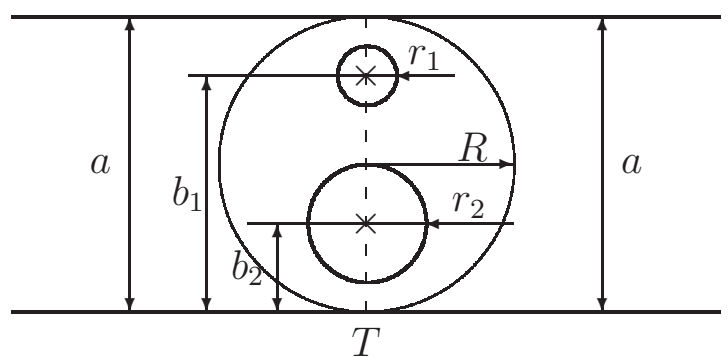

\begin{tabular}{|c|c|c|c|c|c|c|c|}
\cline { 2 - 8 } \multicolumn{1}{c|}{} & Tipo & $\mathbf{C}_{\mathbf{i x}}$ & $\mathbf{C}_{\mathbf{i y}}$ & $\mathbf{R}_{\mathbf{i}}$ & $\mathbf{d N c}_{\mathbf{i}}$ & $\mathbf{C}_{\mathbf{i n}}^{\mathbf{i}}$ & $\mathbf{C}_{\mathbf{s c}}^{\mathbf{i}}$ \\
\hline Objeto 1 & $\begin{array}{c}\text { Cilindro } \\
\text { dieléctrico }\end{array}$ & 0 & $-\frac{a}{2}+b_{1}$ & $r_{1}$ & - & 6 & 2 \\
\hline Objeto 2 & $\begin{array}{c}\text { Cilindro } \\
\text { metálico }\end{array}$ & 0 & $-\frac{a}{2}+b_{2}$ & $r_{2}$ & - & 6 & 2 \\
\hline
\end{tabular}

\begin{tabular}{|c|c|c|c|c|c|}
\hline $\mathbf{R}$ & $\mathbf{R}_{\mathrm{AB}}$ & $\mathbf{R}_{\mathrm{fft}}$ & $\mathbf{C}_{\mathbf{s c}}$ & Modos & Tiempo \\
\hline$\sqrt{2} \frac{a}{2}$ & 3 & 2 & 3 & 5 & $0.68 \mathrm{seg}$. \\
\hline
\end{tabular}
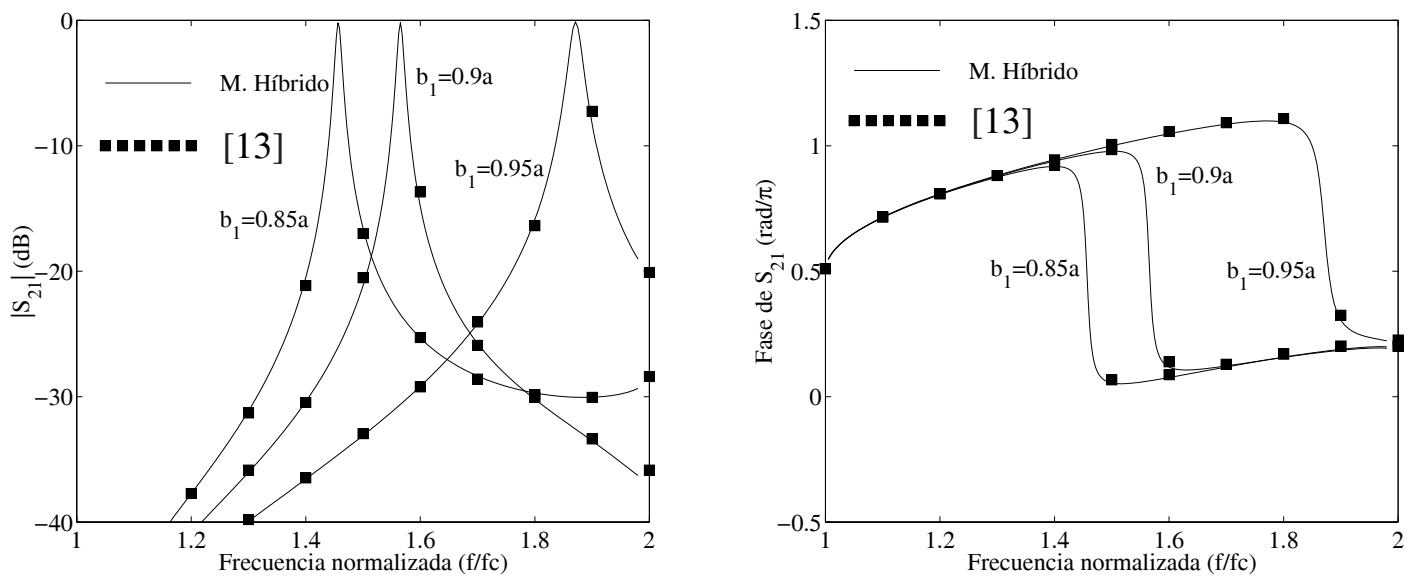

Figura 3.59: Filtro paso banda formado por dos postes circulares, uno metálico (grande) y otro dieléctrico (pequeño). Esquema, valores de los parámetros de simulación y variación de la amplitud y fase de $\left|S_{21}\right|$ con la frecuencia normalizada $\left(\varepsilon_{r}=38,5, \tan \delta=2 \times 10^{-4}\right.$, $\left.r_{1}=0,03 a, r_{2}=0,3 a, b_{2}=r_{2}, b_{1}=0,85 a, 0,9 a, \mathrm{y} 0,95 a\right)$ 


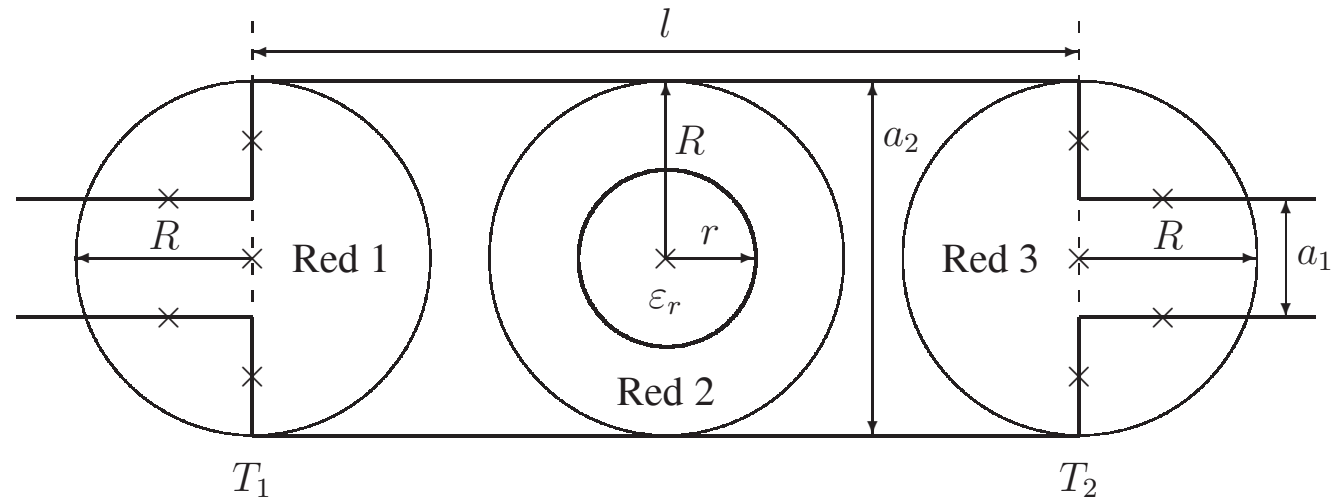

\begin{tabular}{|c|c|c|c|c|c|c|c|}
\cline { 2 - 7 } \multicolumn{1}{c|}{} & Tipo & $\mathbf{C}_{\mathbf{i x}}$ & $\mathbf{C}_{\mathbf{i y}}$ & $\mathbf{R}_{\mathbf{i}}$ & $\mathbf{d N \mathbf { c } _ { \mathbf { i } }}$ & $\mathbf{C}_{\mathbf{i n}}^{\mathbf{i}}$ & $\mathbf{C}_{\mathbf{s c}}^{\mathbf{i}}$ \\
\hline $\begin{array}{c}\text { Red 1 } \\
\text { objeto 1 }\end{array}$ & $\begin{array}{c}\text { Compuesto } \\
\text { de 4 tiras }\end{array}$ & 0 & 0 & $a_{2} / 2$ & 25 & 6 & 3 \\
\hline $\begin{array}{c}\text { Red 2 } \\
\text { objeto 1 }\end{array}$ & $\begin{array}{c}\text { Poste } \\
\text { circular }\end{array}$ & 0 & 0 & $a_{2} / 2$ & - & 3 & 2 \\
\hline $\begin{array}{c}\text { Red 3 } \\
\text { objeto 1 }\end{array}$ & $\begin{array}{c}\text { Compuesto } \\
\text { de 4 tiras }\end{array}$ & 0 & 0 & $a_{2} / 2$ & 25 & 6 & 3 \\
\hline
\end{tabular}

\begin{tabular}{|c|c|c|c|c|c|}
\hline Red & $\mathbf{R}_{\mathrm{AB}}$ & $\mathbf{R}_{\mathrm{fft}}$ & $\mathrm{C}_{\mathbf{s c}}$ & $\begin{array}{c}\text { Modos } \\
\text { entr./sal. }\end{array}$ & Tiempo \\
\hline 1 & 1 & 1 & 6 & $3 / 5$ & $0.84 \mathrm{seg}$. \\
\hline 2 & 3 & 1 & 6 & $5 / 5$ & $0.5 \mathrm{seg}$. \\
\hline 3 & 1 & 1 & 6 & $5 / 3$ & $0.84 \mathrm{seg}$. \\
\hline
\end{tabular}
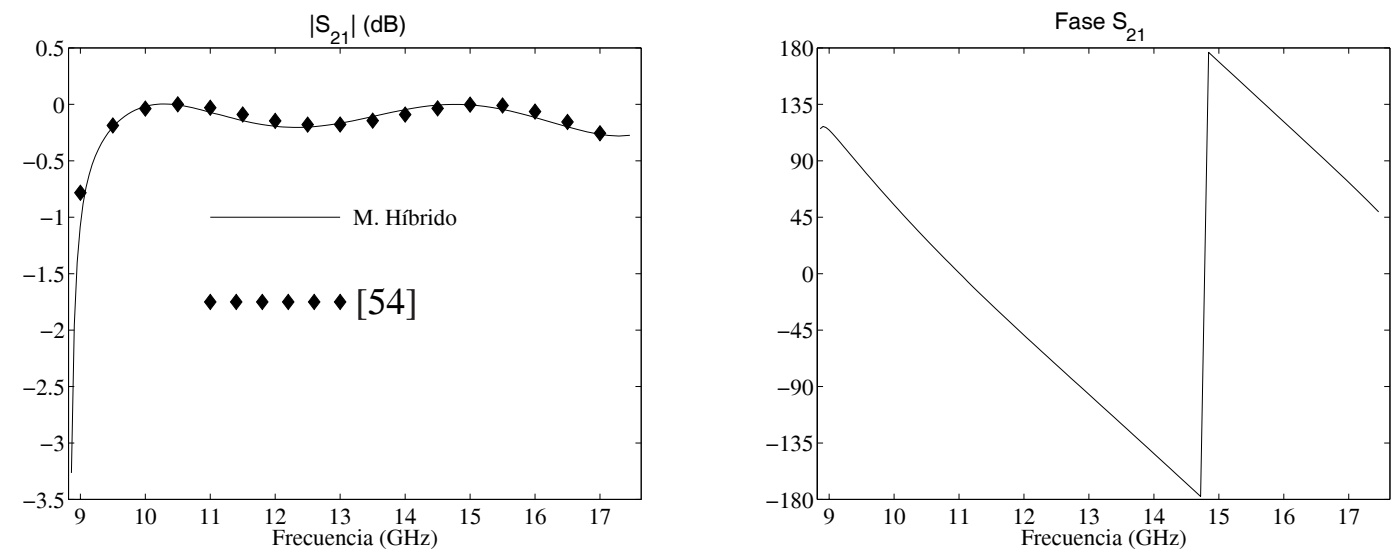

Figura 3.60: Cavidad en plano $H$ en una guía rectangular con poste circular dieléctrico. Esquema, valores de los parámetros de simulación y variación de la amplitud y fase de $\left|S_{21}\right|$ con la frecuencia $\left(a_{1}=17 \mathrm{~mm}, a_{2}=22,86 \mathrm{~mm}, r=5 \mathrm{~mm}, l=30 \mathrm{~mm}, \varepsilon_{r}=2,17\right)$ 


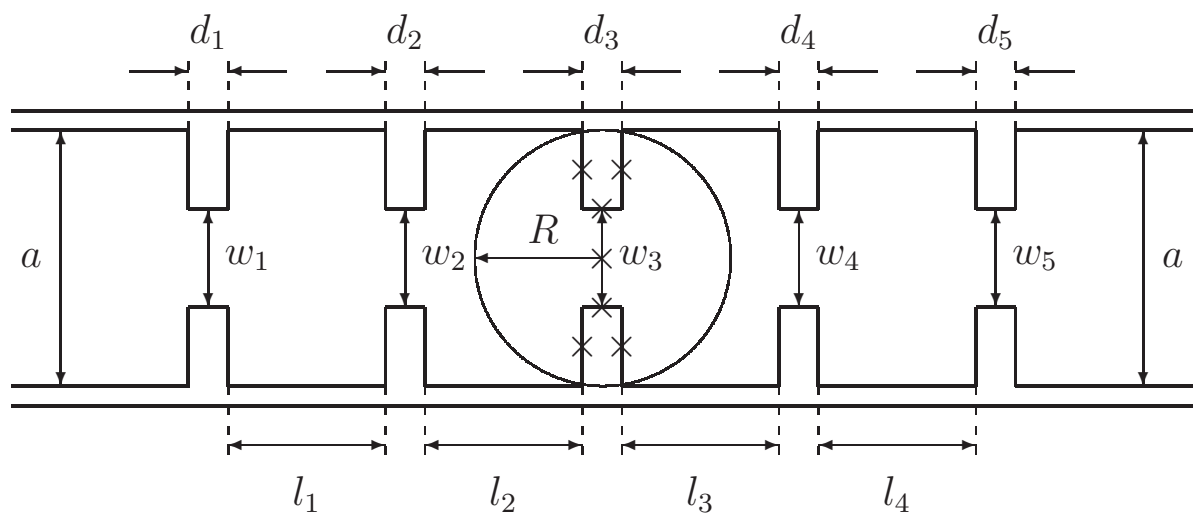

\begin{tabular}{|c|c|c|c|c|c|c|c|c|}
\hline & Tipo & $\mathrm{C}_{\mathrm{ix}}$ & $\mathrm{C}_{\mathrm{iy}}$ & \multicolumn{2}{|c|}{$\mathbf{R}_{\mathrm{i}}$} & $\mathrm{dNc}_{\mathrm{i}}$ & $\mathrm{C}_{\mathrm{in}}^{\mathrm{i}}$ & $\mathrm{C}_{\mathrm{sc}}^{\mathrm{i}}$ \\
\hline Objeto 1 & $\begin{array}{c}\text { Compuesto de } \\
6 \text { tiras }\end{array}$ & 0 & 0 & \multicolumn{2}{|c|}{$\left.\sqrt{(}\left(\frac{a}{2}\right)^{2}+\left(\frac{d_{i}}{2}\right)^{2}\right)$} & 200 & 12 & 6 \\
\hline & $\mathbf{R}$ & $\mathbf{R}_{\mathrm{AB}}$ & $\mathbf{R}_{\mathrm{fft}}$ & $\mathrm{C}_{\mathrm{sc}}$ & Modos & \multicolumn{2}{|c|}{ Tiempo } & \\
\hline \multicolumn{2}{|c|}{$\left.\sqrt{(}\left(\frac{a}{2}\right)^{2}+\left(\frac{d_{i}}{2}\right)^{2}\right)$} & 5 & 8 & 6 & 13 & \multicolumn{2}{|c|}{$\begin{array}{c}6.3 \mathrm{~s} \mathrm{c} / \mathrm{u} \\
18.9 \mathrm{~s} \text { total }\end{array}$} & \\
\hline
\end{tabular}
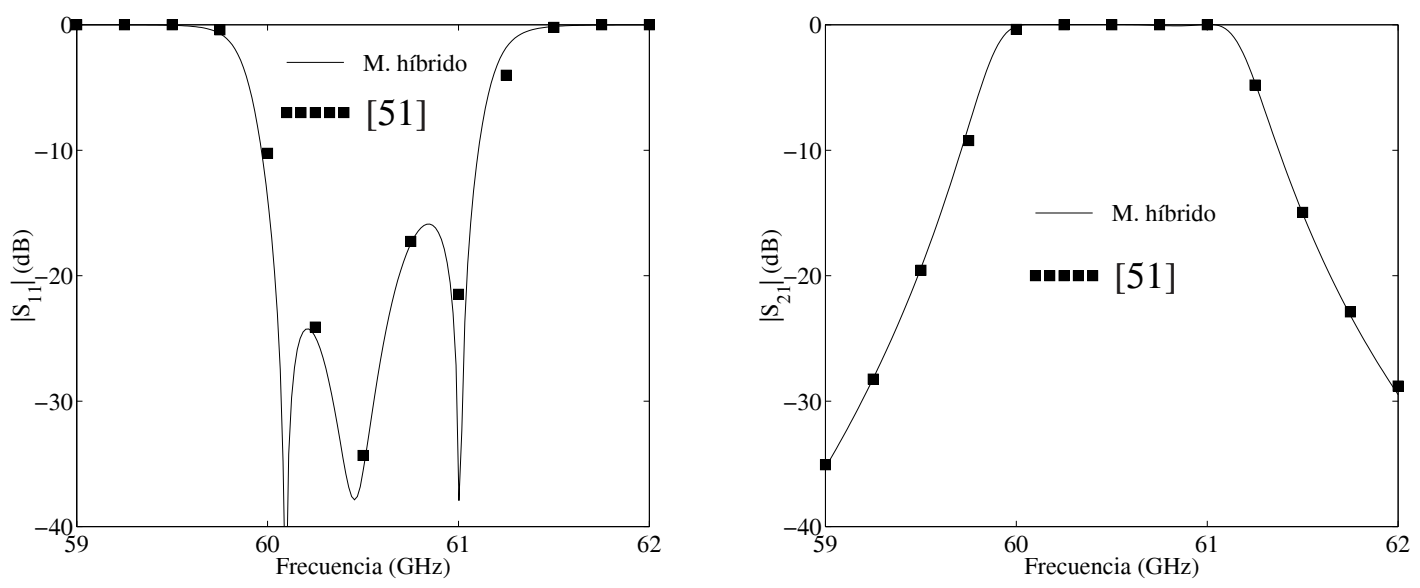

Figura 3.61: Filtro paso banda formado por 5 cavidades inductivas. Esquema, valores de los parámetros de simulación y variación de los factores de reflexión y transmisión con la frecuencia ( $a=3,7591 \mathrm{~mm}, l_{1}=l_{4}=2,73 \mathrm{~mm}, l_{2}=l_{3}=3,02 \mathrm{~mm}, w_{1}=w_{5}=1,88 \mathrm{~mm}$, $w_{2}=w_{4}=w_{3}=1,18 \mathrm{~mm}, d_{1}=d_{5}=0,56 \mathrm{~mm}, d_{2}=d_{4}=0,52 \mathrm{~mm}$, y $\left.d_{3}=0,63 \mathrm{~mm}\right)$ 
Las figuras 3.62, 3.63 y 3.64 muestran la convergencia del método al variar el valor de $d N c_{i}, C_{s c}$ y el número de modos, respectivamente.

En la figura 3.62 se puede apreciar que la convergencia con él número de funciones base del método de los momentos $\left(d N c_{i}\right)$ es bastante lenta, y es necesaria una densidad de funciones base de por lo menos 200 por cada longitud de onda. Esto se debe probablemente a la lenta convergencia del método de los momentos al utilizar Point-Matching, destacada en numerosas publicaciones técnicas [56].

En las figuras 3.63 y 3.64 se comprueba que con $C_{s c}=6$ y 13 modos guiados el método ha alcanzado ya la convergencia.
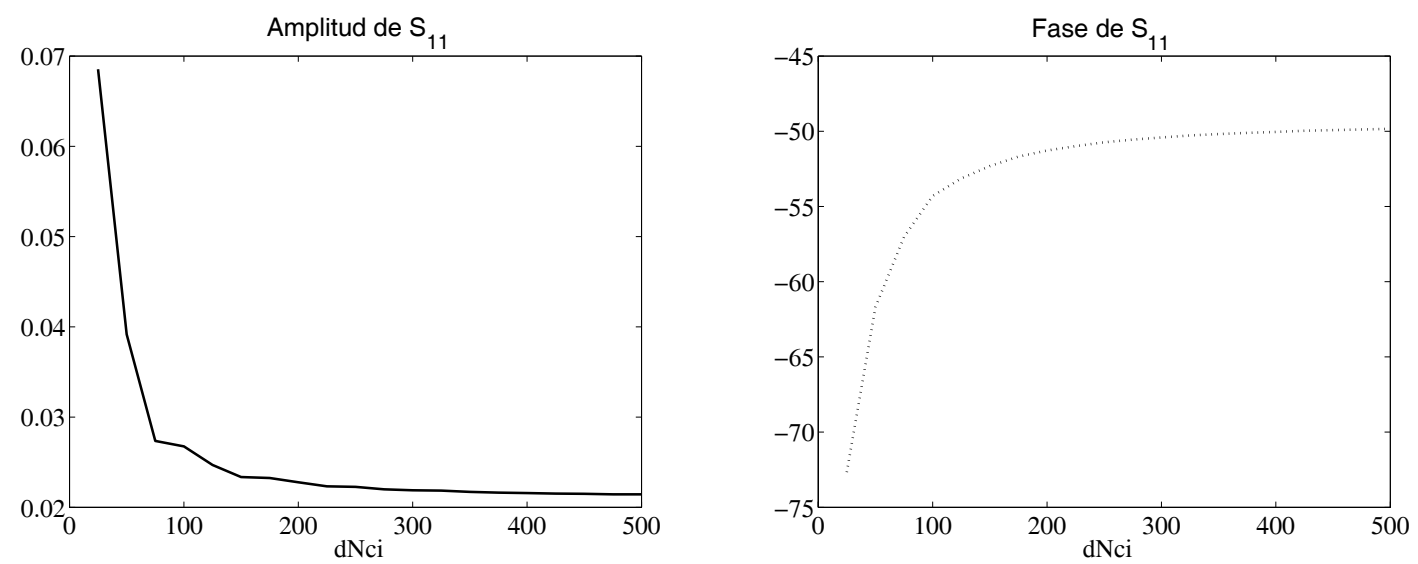

Figura 3.62: Variación de la amplitud y la fase de $S_{11}$ con el parámetro $d N c_{i}$ del filtro de cavidades inductivas $\left(C_{s c}^{i}=6, R_{A B}=3, R_{f f t}=8\right.$, Modos $=13$ )
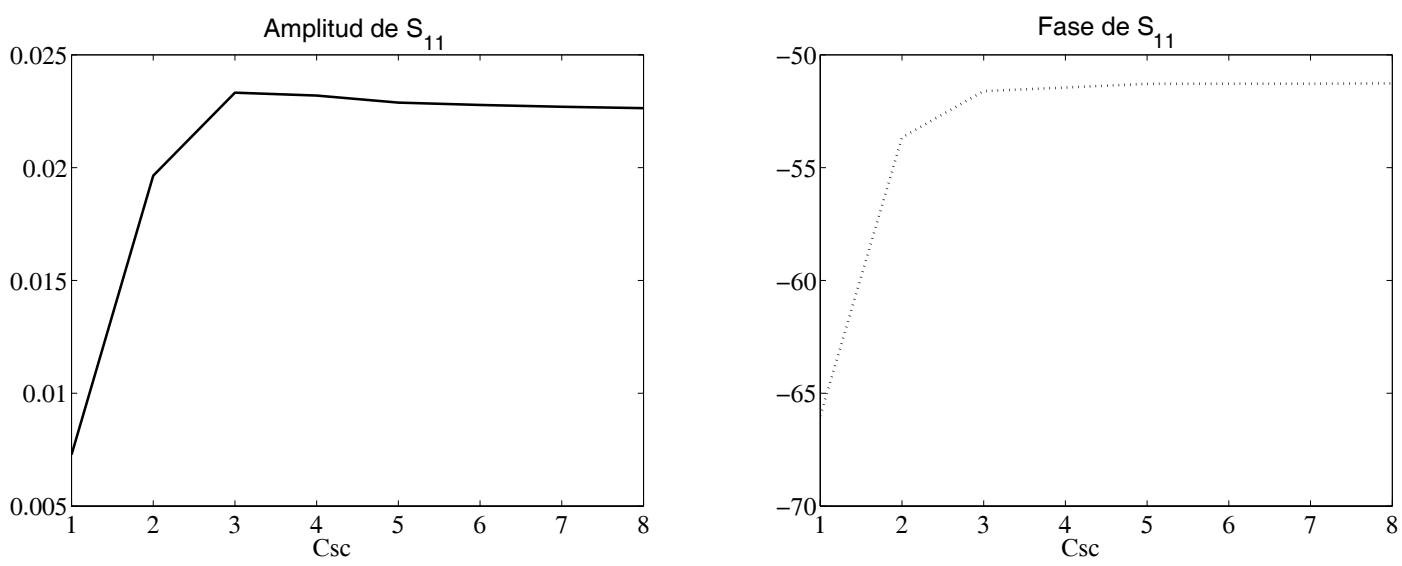

Figura 3.63: Variación de la amplitud y la fase de $S_{11}$ con el parámetro $C_{s c}$ del filtro de cavidades inductivas $\left(d N c_{i}=200, R_{A B}=3, R_{f f t}=8\right.$, Modos $=13$ ) 

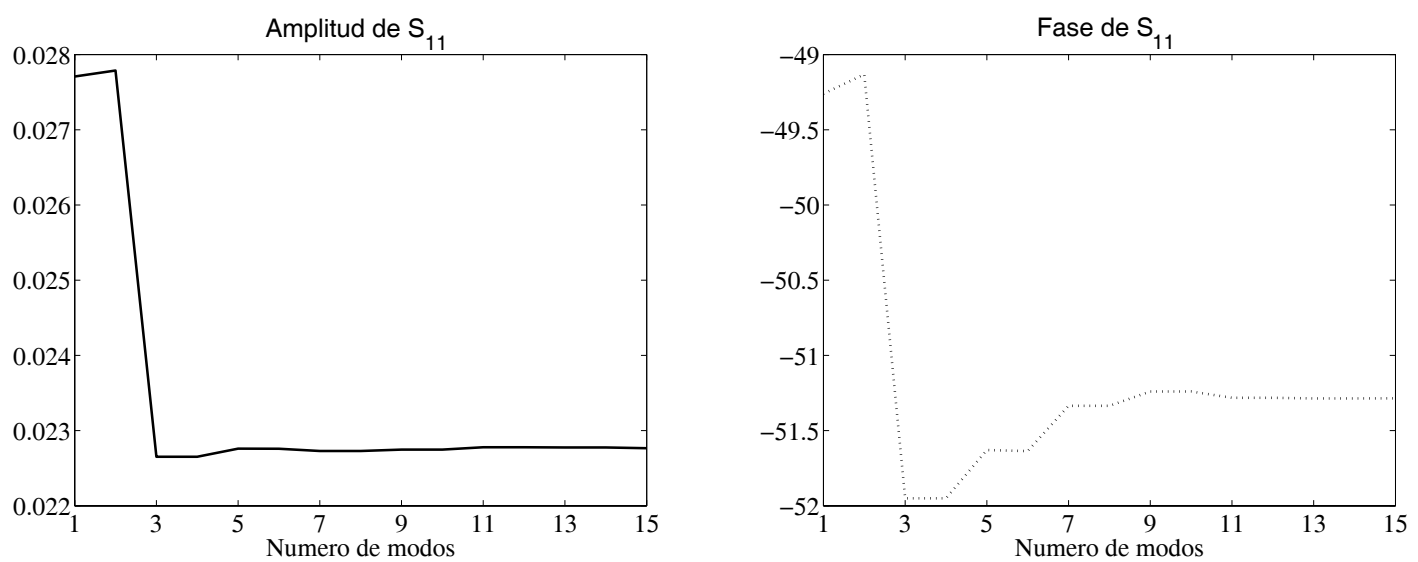

Figura 3.64: Variación de la amplitud y la fase de $S_{11}$ con el número de modos guiados del filtro de cavidades inductivas $\left(d N c_{i}=200, C_{s c}^{i}=6, R_{A B}=3, R_{f f t}=8\right)$

\subsection{Conclusiones}

En este capítulo se ha aplicado el método híbrido de análisis de problemas de dispersión múltiple 2-D al análisis de problemas inductivos de geometría arbitraria en una guía rectangular: múltiples obstáculos metálicos o dieléctricos de geometría arbitraria, saltos de sección, codos, estructuras multipuerto, etc. El método de análisis 2-D es híbrido porque utiliza el método de los momentos (método numérico) para el análisis de dispersores individuales, y a continuación usa un método espectral para resolver el acoplo electromagnético entre los múltiples dispersores. Una vez caracterizado el problema mediante espectros cilíndricos, se han utilizado dos técnicas diferentes para obtener los parámetros circuitales de la estructura guiada. En primer lugar se ha utilizado una técnica basada en la colocación de cortocircuitos en la estructura guiada para calcular la Matriz de Admitancias Generalizada (MAG). Los resultados obtenidos no han sido muy precisos, y además requieren de la simulación de estructuras de grandes dimensiones eléctricas, lo que lo convierte en un método poco eficiente, además de poco eficaz. A continuación se ha aplicado un nuevo método de acoplo modal que ha permitido obtener la Matriz de Dispersión Generalizada (MDG) de forma sencilla. Los resultados obtenidos son altamente satisfactorios, tanto por la precisión obtenida en los resultados (validados con resultados de publicaciones científicas para múltiples dispositivos de microondas), como por la eficiencia del método. Los tiempos de computación se mantienen razonablemente bajos para un método de propósito general como el propuesto, que permite analizar estructuras con geometrías muy diversas. 


\section{Capítulo 4}

\section{Aplicaciones}

La mejor característica del método de análisis de problemas inductivos en guía rectangulares desarrollado en la tesis, y descrito en el capítulo 3, es su flexibilidad y capacidad para analizar problemas complejos. Así, como ya se ha demostrado, se puede analizar con exactitud con dicho método cualquier problema inductivo, sea cual sea su geometría. Por este motivo el método resulta especialmente útil para aquellas aplicaciones en las que se requiera de un análisis exacto pero veloz de estructuras complejas. En este campo el método resulta mucho más adecuado que por ejemplo los métodos de elementos finitos, que también analizan problemas complejos con exactitud, pero no de manera veloz.

En este capítulo se describe el resultado de aplicar el método a la solución de algunos problemas en los que se requiere del análisis eficiente de problemas inductivos complejos. Concretamente, se ha aplicado el método a los siguientes problemas:

- Análisis de filtros con esquinas redondeadas, que modelan defectos de fabricación en filtros de cavidades inductivas.

- Determinación de las propiedades dieléctricas de los materiales, especialmente de líquidos por su mayor complejidad.

- Diseño de filtros inductivos de geometría compleja.

- Estudio de problemas de propagación en guías rectangulares con postes y obstáculos inductivos de distintas geometrías, con fines educativos.

Estas son las aplicaciones que se le han dado al método en la presente tesis, aunque como es lógico las posibles aplicaciones son mucho más extensas.

A continuación se describen cada una de la aplicaciones que se le han dado al método. 


\subsection{Determinación de las propiedades dieléctricas de ma- teriales}

\subsubsection{Introducción}

El conocimiento preciso de las propiedades dieléctricas de los materiales es de gran interés en muchas aplicaciones de microondas, como se destaca en [57] y en [58]. En consecuencia, la determinación de las propiedades dieléctricas de los materiales ha recibido una considerable atención en las publicaciones técnicas relacionadas en los últimos años. De entre las técnicas que se han descrito en dichas publicaciones, las técnicas de cavidad son normalmente las que proporcionan resultados más precisos, aunque presentan el problema de que se hallan limitadas al análisis de únicamente muestras de pequeño tamaño y pocas pérdidas. Además, estas técnicas proporcionan resultados válidos únicamente a una frecuencia (ver [59]). Otro tipo de técnicas también muy extendidas son las que se basan en líneas de transmisión. En dichas técnicas la muestra dieléctrica ha de ser introducida completamente dentro de la sección transversal de una guía de ondas o de una línea coaxial (ver [60]). Esta configuración de la muestra dieléctrica es difícil de llevar a la práctica en muchos casos debido a la aparición de pequeños huecos entre la muestra y las paredes de la guía de ondas. Además, el ancho de banda en el que son válidos los resultados de dichas técnicas (frecuencia máxima) viene limitado por la posible excitación de modos de propagación de orden superior, cuya frecuencia de corte depende de las propiedades dieléctricas del material que queremos caracterizar, y que a priori desconocemos, por lo que no podemos saber de manera general el ancho de banda en el que serán válidas las medidas.

Con el objetivo de superar las limitaciones que se han expuesto tanto de los métodos de cavidad como los basados en líneas de transmisión, se ha desarrollado un nuevo método para la determinación precisa de las propiedades dieléctricas de los materiales, haciendo uso del método híbrido de análisis de problemas inductivos complejos. Este nuevo método consiste en situar un poste de material dieléctrico a lo largo del eje $y$ de una guía de ondas rectangular, y posteriormente medir los parámetros de dispersión de dicha estructura con un analizador de redes automático (ANA: Automatic Network Analyzer). A continuación, un algoritmo de optimización toma las medidas realizadas por el analizador de redes como referencia, y utiliza el método híbrido del capítulo 3 para estimar la permitividad eléctrica compleja del material dieléctrico.

Dado que el método de análisis híbrido permite el análisis de postes dieléctricos multicapa dentro de la guía rectangular, se puede también caracterizar mediante este método materiales líquidos. En este caso, el material líquido se puede introducir en una pipeta y posteriormente insertar la pipeta con el líquido dentro de la guía, para de esta forma medir con el analizador de redes los parámetros de dispersión. Seguidamente se compararan dichas medidas con los resultados del simulador mediante un algoritmo de optimización hasta que la estima de la permitividad sea adecuada y las curvas medidas y simuladas coincidan.

Es precisamente la caracterización de materiales líquidos aquélla que más nos interesa del método que se ha desarrollado, ya que los líquidos son materiales cuya caracterización es especialmente difícil con los métodos existentes. Por ello en esta sección se presentarán 
resultados de la caracterización de un material líquido (etanol).

\subsubsection{Medida de los parámetros de dispersión}

El objetivo de esta aplicación será la caracterización de la permitividad compleja de un material líquido, concretamente el etanol. Para realizar las medidas de la dispersión de este material, se utiliza una guía rectangular WR340, y se inserta en en ella una pipeta circular completamente llena de la muestra de material dieléctrico, tal y como se muestra en la figura 4.1. En dicha figura $r_{1}$ y $r_{2}$ son, respectivamente, los radios externo e interno de la pipeta. $\mathrm{Y}$ $\varepsilon_{r_{1}} \mathrm{y} \varepsilon_{r_{2}}$ son, respectivamente, las permitividades de la pipeta y del material líquido.

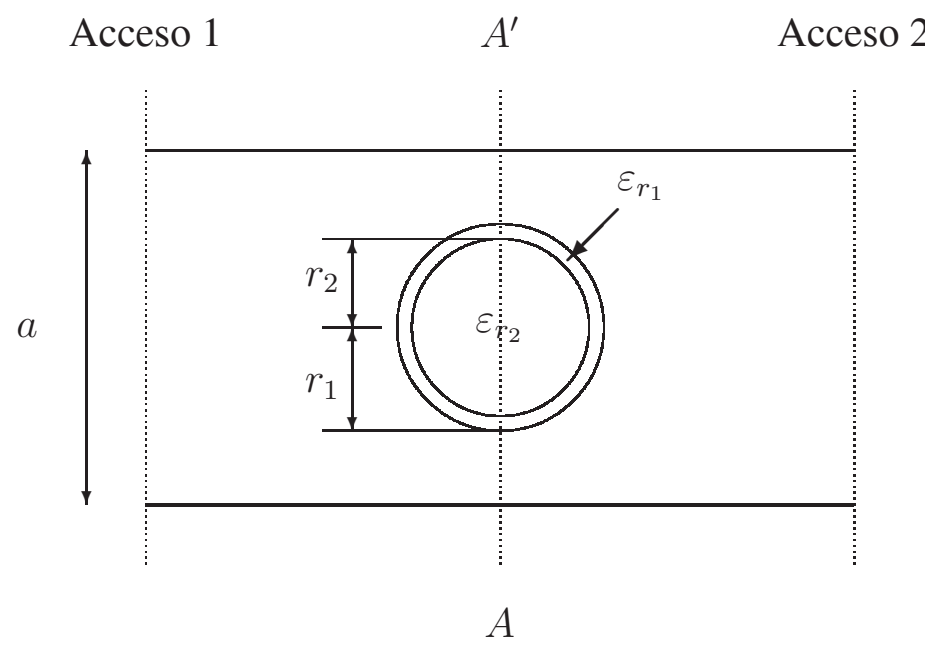

Sección longitudinal

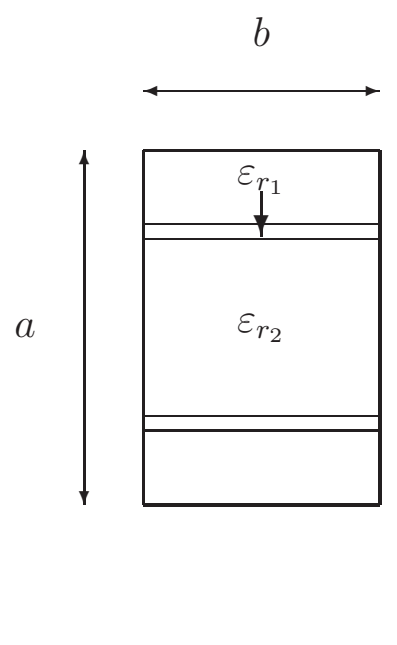

Sección transversal $A-A^{\prime}$

Figura 4.1: Material dieléctrico bajo estudio situado dentro de una pipeta circular situada en una guía rectangular

La muestra de material dieléctrico (la pipeta rellena de líquido, ver figura 4.2) se debe situar dentro de la guía de ondas para poder medir los parámetros de dispersión de la estructura. Como nos interesa que la dispersión de la guía dependa en la mayor medida posible de la permitividad del dieléctrico, que es lo que queremos estimar, debemos situar la pipeta en el lugar donde más interfiera con las ondas que se propagan por la guía. Por tanto, teniendo en cuenta que la mayor parte de la energía del modo fundamental viaja por el centro de la misma, es ahí donde se debe poner la pipeta.

Con el objetivo de colocar la pipeta en el centro de la guía, se perfora la guía en el centro de su pared superior tal y como se muestra en la figura 4.3. Para conseguir que la pipeta esté firme dentro de la guía justo en el lugar que le corresponde, se produce un ligero rebaje 

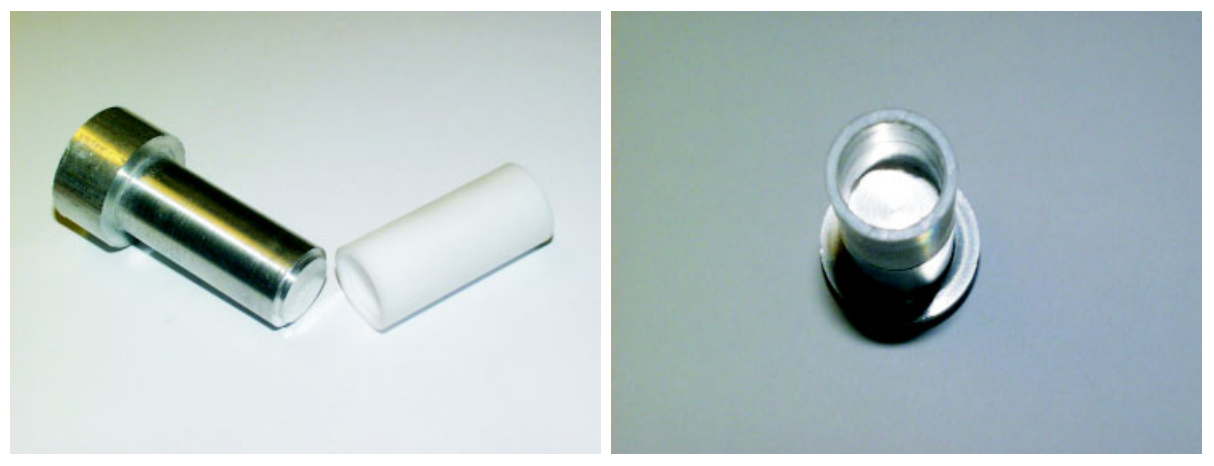

Figura 4.2: Detalles de la pipeta circular preparada para introducirle un líquido dieléctrico y ser insertada en una guía circular

en la pared inferior de la guía, justo donde la pipeta se apoya, de modo que ésta encaja en el rebaje que se ha producido cuando se mete por el agujero superior y ya no se mueve. Una vez la pipeta está firmemente sujeta y colocada en el centro de la guía, se rellena del material líquido bajo prueba. Finalmente, para evitar la pérdida de potencia que se produciría si se dejara el agujero superior de la guía al descubierto, se tapa la parte superior de la pipeta con un pistón metálico (ver figura 4.2). La altura de la pipeta coincide exactamente con la altura de la guía, de modo que una vez situado el pistón metálico, su cara inferior está justo a la misma altura que la pared metálica superior de la guía, evitando cualquier posible pérdida por radiación. De este modo conseguimos situar un poste circular multicapa (dieléctrico más pipeta) totalmente inductivo dentro de la guía circular. Esta estructura es posible analizarla con el método híbrido de análisis de estructuras inductivas arbitrarias.
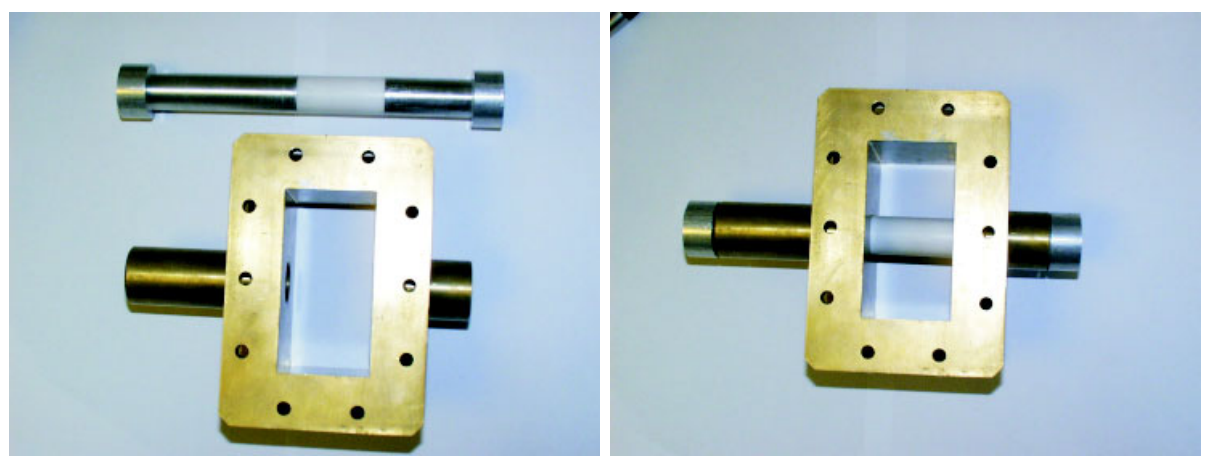

Figura 4.3: Vistas de la guía rectangular WR340 antes y después de introducirle la pipeta circular

Para poder estimar la permitividad del líquido, es preciso realizar medidas de la guía con la pipeta vacía y de la guía con la pipeta llena de líquido. Estas medidas de los parámetros de dispersión se han realizado en la banda $\mathrm{S}$ a las frecuencias en las que sólo se propaga el modo fundamental en la guía WR340. Para realizar las medidas se ha utilizado un analizador 
de redes HP-8720B calibrado siguiendo el procedimiento de calibración estándar denominado full two-port. Aunque el método de análisis permite obtener la respuesta teórica de la estructura también para frecuencias en las que se propagan varios modos, se utilizarán frecuencias monomodo debido a que las técnicas estándar actuales de calibración tan sólo permiten realizar medidas en ese rango de frecuencias.

\subsubsection{Proceso de optimización}

El proceso de optimización es el que se encargará de realizar, de forma iterativa, la estimación de la permitividad del material dieléctrico.

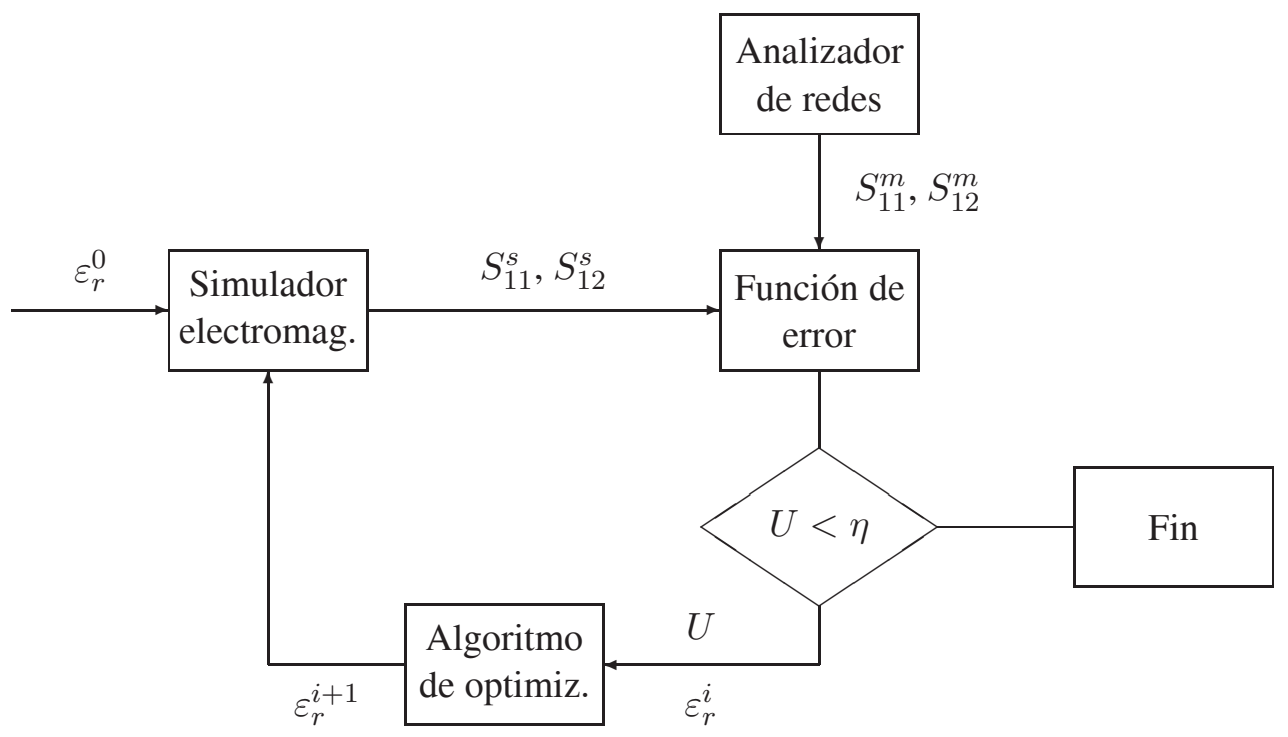

Figura 4.4: Diagrama del proceso seguido en la optimización

En la figura 4.4 se muestra un esquema de los pasos que se siguen en el proceso de optimización, y que a continuación se describen:

- Se parte de una estimación inicial $\left(\varepsilon_{r}^{0}\right)$ de la permitividad del material dieléctrico que se ha insertado en la guía. Esta estimación puede obtenerse con algún método rápido pero de poca precisión, o simplemente ser una estimación basada en la experiencia anterior con el material que se desea caracterizar. El hecho de que esta estimación inicial no esté muy lejos del valor real de la permitividad es crucial para evitar que el proceso de optimización sea muy largo, o, peor aún, que caiga en algún mínimo local y no llegue nunca a converger.

- A continuación se utiliza el simulador electromagnético para calcular los parámetros de dispersión de la guía rectangular con el poste de material dieléctrico dentro ( $S_{11}^{s}$ y $S_{12}^{s}$ ), tomando como permitividad del poste la estimación inicial. 
- En el siguiente paso, se extrae un indicador $(U)$ de la diferencia entre las curvas medidas por el analizador de redes $\left(S_{11}^{m}\right.$ y $\left.S_{12}^{m}\right)$ y las proporcionadas por el simulador $\left(S_{11}^{s}\right.$ y $S_{12}^{s}$ ) mediante alguna función de error. Si el indicador de error $U$ fuera nulo (en realidad, menor que una cantidad muy pequeña $\eta$ ), eso querría decir que las curvas medidas y simuladas son prácticamente iguales, y que por tanto la estimación inicial de la permitividad coincide con la permitividad real del material.

- Si el error entre las curvas medidas y simuladas es mayor que $\eta$, un algoritmo de optimización genera un nuevo valor de la permitividad del material dieléctrico $\left(\varepsilon_{r}^{i+1}\right)$, que sirve para que el simulador electromagnético calcule la respuesta de la estructura con el nuevo valor de la permitividad.

- De nuevo estos resultados de la simulación se compararan con los valores medidos y se obtiene el valor de la función de error $U$, repitiéndose este mismo proceso (simulación, comparación con medidas, cálculo de un nuevo valor de permitividad) hasta que la diferencia entre la simulación y las medidas es menor que un cierto umbral $\eta$.

- El algoritmo de optimización genera una nueva estimación de la permitividad según una determinada estrategia, cuyo objetivo es encontrar el valor de la permitividad que minimiza la función de error $U$. Existen diferentes algoritmos de optimización de funciones multidimensionales, y cada uno sigue una estrategia diferente para conseguir su objetivo.

\subsubsection{Función de error}

Para estimar la permitividad de la muestra dieléctrica es necesario establecer una función de error que nos proporcione, para cada valor de la permitividad, la diferencia entre las medidas y la simulación. Esta función de error $U$ será una función bidimensional, ya que nos da una medida de la diferencia entre las medidas y la simulación para cada posible valor de la parte real $\left(\varepsilon_{r}^{\prime}\right)$ e imaginaria $\left(\varepsilon_{r}^{\prime \prime}\right)$ de la permitividad del material:

$$
U=U\left(\varepsilon_{r}\right)=U\left(\varepsilon_{r}^{\prime}, \varepsilon_{r}^{\prime \prime}\right)
$$

En el proceso de optimización, un algoritmo de optimización buscará los valores de $\varepsilon_{r}^{\prime}$ y $\varepsilon_{r}^{\prime \prime}$ que minimizan la función de error $U$. Según cómo se defina la función de error, ésta puede ser una superficie más o menos adecuada para la búsqueda de su valor mínimo. Si, por ejemplo, la función de error presenta muchos mínimos locales, será fácil que el algoritmo de optimización se quede atrapado en un mínimo local y que no llegue al mínimo absoluto. En cambio, si la función de error fuera una función con un único mínimo y además la pendiente cerca de ese mínimo fuera suave, entonces el algoritmo llegaría muy rápidamente al mínimo desde cualquier punto de partida.

Resulta conveniente, por tanto, investigar en cada aplicación concreta cuál es la mejor función de error. En el caso concreto de la estimación de la permitividad de un dieléctrico insertado en una guía rectangular, la función de error se debe construir comparando el módulo y la fase (o la parte real e imaginaria) de los parámetros de dispersión medidos y los 
proporcionados por el simulador a una determinada frecuencia. La comparación se puede realizar utilizando norma 1, 2, 4, o incluso norma infinito. Además la diferencia puede ser normalizada o sin normalizar. Resulta evidente, por tanto, que existen muchas posibilidades a la hora de construir la función de error, y con el fin de encontrar una función de error adecuada, se ha probado con una gran cantidad de ellas, y se ha seleccionado la que mejores resultados proporcionaba en términos de eficiencia y robustez en la búsqueda del mínimo.

A continuación se enumeran las distintas funciones de error con las que se ha trabajado:

1. Diferencia de parte real e imaginaria normalizada

$$
\begin{aligned}
U= & \frac{\left|\operatorname{Re}\left\{S_{11}^{m}\right\}-\operatorname{Re}\left\{S_{11}^{s}\right\}\right|}{\left|S_{11}^{m}\right|}+\frac{\left|\operatorname{Im}\left\{S_{11}^{m}\right\}-\operatorname{Im}\left\{S_{11}^{s}\right\}\right|}{\left|S_{11}^{m}\right|} \\
& +\frac{\left|\operatorname{Re}\left\{S_{21}^{m}\right\}-\operatorname{Re}\left\{S_{21}^{s}\right\}\right|}{\left|S_{21}^{m}\right|}+\frac{\left|\operatorname{Im}\left\{S_{21}^{m}\right\}-\operatorname{Im}\left\{S_{21}^{s}\right\}\right|}{\left|S_{21}^{m}\right|}
\end{aligned}
$$

2. Diferencia de módulo normalizada

$$
U=\frac{|| S_{11}^{m}|-| S_{11}^{s}||}{\left|S_{11}^{m}\right|}+\frac{|| S_{21}^{m}|-| S_{21}^{s}||}{\left|S_{21}^{m}\right|}
$$

3. Máximo de la diferencia de parte real e imaginaria normalizada

$$
\begin{aligned}
U= & \max \left\{\frac{\left|\operatorname{Re}\left\{S_{11}^{m}\right\}-\operatorname{Re}\left\{S_{11}^{s}\right\}\right|}{\left|S_{11}^{m}\right|}, \frac{\left|\operatorname{Im}\left\{S_{11}^{m}\right\}-\operatorname{Im}\left\{S_{11}^{s}\right\}\right|}{\left|S_{11}^{m}\right|},\right. \\
& \left.\frac{\left|\operatorname{Re}\left\{S_{21}^{m}\right\}-\operatorname{Re}\left\{S_{21}^{s}\right\}\right|}{\left|S_{21}^{m}\right|}, \frac{\left|\operatorname{Im}\left\{S_{21}^{m}\right\}-\operatorname{Im}\left\{S_{21}^{s}\right\}\right|}{\left|S_{21}^{m}\right|}\right\}
\end{aligned}
$$

4. Diferencia de parte real e imaginaria normalizada (norma 2)

$$
\begin{aligned}
U= & \frac{\left|\operatorname{Re}\left\{S_{11}^{m}\right\}-\operatorname{Re}\left\{S_{11}^{s}\right\}\right|^{2}}{\left|S_{11}^{m}\right|}+\frac{\left|\operatorname{Im}\left\{S_{11}^{m}\right\}-\operatorname{Im}\left\{S_{11}^{s}\right\}\right|^{2}}{\left|S_{11}^{m}\right|} \\
& +\frac{\left|\operatorname{Re}\left\{S_{21}^{m}\right\}-\operatorname{Re}\left\{S_{21}^{s}\right\}\right|^{2}}{\left|S_{21}^{m}\right|}+\frac{\left|\operatorname{Im}\left\{S_{21}^{m}\right\}-\operatorname{Im}\left\{S_{21}^{s}\right\}\right|^{2}}{\left|S_{21}^{m}\right|}
\end{aligned}
$$

5. Diferencia de parte real e imaginaria normalizada (norma 4)

$$
\begin{aligned}
U= & \frac{\left|\operatorname{Re}\left\{S_{11}^{m}\right\}-\operatorname{Re}\left\{S_{11}^{s}\right\}\right|^{4}}{\left|S_{11}^{m}\right|}+\frac{\left|\operatorname{Im}\left\{S_{11}^{m}\right\}-\operatorname{Im}\left\{S_{11}^{s}\right\}\right|^{4}}{\left|S_{11}^{m}\right|} \\
& +\frac{\left|\operatorname{Re}\left\{S_{21}^{m}\right\}-\operatorname{Re}\left\{S_{21}^{s}\right\}\right|^{4}}{\left|S_{21}^{m}\right|}+\frac{\left|\operatorname{Im}\left\{S_{21}^{m}\right\}-\operatorname{Im}\left\{S_{21}^{s}\right\}\right|^{4}}{\left|S_{21}\right|^{m}}
\end{aligned}
$$


6. Diferencia de parte real e imaginaria normalizada con pesos distintos para $S_{11}$ y $S_{22}$

$$
\begin{aligned}
U= & 0,55\left\{\frac{\left|\operatorname{Re}\left\{S_{11}^{m}\right\}-\operatorname{Re}\left\{S_{11}^{s}\right\}\right|}{\left|S_{11}^{m}\right|}+\frac{\left|\operatorname{Im}\left\{S_{11}^{m}\right\}-\operatorname{Im}\left\{S_{11}^{s}\right\}\right|}{\left|S_{11}^{m}\right|}\right\} \\
& +0,45\left\{\frac{\left|\operatorname{Re}\left\{S_{21}^{m}\right\}-\operatorname{Re}\left\{S_{21}^{s}\right\}\right|}{\left|S_{21}^{m}\right|}+\frac{\left|\operatorname{Im}\left\{S_{21}^{m}\right\}-\operatorname{Im}\left\{S_{21}^{s}\right\}\right|}{\left|S_{21}^{m}\right|}\right\}
\end{aligned}
$$

7. Diferencia de parte real e imaginaria sin normalizar

$$
\begin{aligned}
U= & \left|\operatorname{Re}\left\{S_{11}^{m}\right\}-\operatorname{Re}\left\{S_{11}^{s}\right\}\right|+\left|\operatorname{Im}\left\{S_{11}^{m}\right\}-\operatorname{Im}\left\{S_{11}^{s}\right\}\right| \\
& +\left|\operatorname{Re}\left\{S_{21}^{m}\right\}-\operatorname{Re}\left\{S_{21}^{s}\right\}\right|+\left|\operatorname{Im}\left\{S_{21}^{m}\right\}-\operatorname{Im}\left\{S_{21}^{s}\right\}\right|
\end{aligned}
$$

8. Diferencia de módulo y fase

$$
\begin{aligned}
U= & || S_{11}^{m}|-| S_{11}^{s}||+|| S_{21}^{m}|-| S_{21}^{s}|| \\
& +\frac{\angle S_{11}^{m}-\angle S_{11}^{s}}{2 \pi}+\frac{\angle S_{21}^{m}-\angle S_{21}^{s}}{2 \pi}
\end{aligned}
$$

9. Diferencia de módulo normalizada (norma 2)

$$
U=\frac{\sqrt{\left.|| S_{11}^{m}|-| S_{11}^{s}\right|^{2}}}{\left|S_{11}^{m}\right|}+\frac{\sqrt{|| S_{21}^{m}|-| S_{21}^{s} \|^{2}}}{\left|S_{21}^{m}\right|}
$$

10. Diferencia de módulo y fase normalizada

$$
\begin{aligned}
U= & \frac{|| S_{11}^{m}|-| S_{11}^{s}||}{\left|S_{11}^{m}\right|}+\frac{|| S_{21}^{m}|-| S_{21}^{s}||}{\left|S_{21}^{m}\right|} \\
& +\frac{\angle S_{11}^{m}-\angle S_{11}^{s}}{2 \pi}+\frac{\angle S_{21}^{m}-\angle S_{21}^{s}}{2 \pi}
\end{aligned}
$$

11. Diferencia de módulo y fase con diferentes pesos

$$
\begin{aligned}
U= & || S_{11}^{m}|-| S_{11}^{s}||+|| S_{21}^{m}|-| S_{21}^{s}|| \\
& +2 \frac{\angle S_{11}^{m}-\angle S_{11}^{s}}{2 \pi}+2 \frac{\angle S_{21}^{m}-\angle S_{21}^{s}}{2 \pi}
\end{aligned}
$$


12. Diferencia de parte real e imaginaria normalizada con pesos distintos para $S_{11}$ y $S_{22}$

$$
\begin{aligned}
U= & 0,45\left\{\frac{\left|\operatorname{Re}\left\{S_{11}^{m}\right\}-\operatorname{Re}\left\{S_{11}^{s}\right\}\right|}{\left|S_{11}^{m}\right|}+\frac{\left|\operatorname{Im}\left\{S_{11}^{m}\right\}-\operatorname{Im}\left\{S_{11}^{s}\right\}\right|}{\left|S_{11}^{m}\right|}\right\} \\
& +0,55\left\{\frac{\left|\operatorname{Re}\left\{S_{21}^{m}\right\}-\operatorname{Re}\left\{S_{21}^{s}\right\}\right|}{\left|S_{21}^{m}\right|}+\frac{\left|\operatorname{Im}\left\{S_{21}^{m}\right\}-\operatorname{Im}\left\{S_{21}^{s}\right\}\right|}{\left|S_{21}^{m}\right|}\right\}
\end{aligned}
$$

13. Diferencia de logaritmo de parte real e imaginaria normalizada

$$
\begin{aligned}
U= & \frac{\left|\log _{10} \operatorname{Re}\left\{S_{11}^{m}\right\}-\log _{10} \operatorname{Re}\left\{S_{11}^{s}\right\}\right|}{\log _{10} \operatorname{Re}\left\{S_{11}^{m}\right\}}+\frac{\left|\log _{10} \operatorname{Im}\left\{S_{11}^{m}\right\}-\log _{10} \operatorname{Im}\left\{S_{11}^{s}\right\}\right|}{\log _{10} \operatorname{Im}\left\{S_{11}^{m}\right\}} \\
& +\frac{\left|\log _{10} \operatorname{Re}\left\{S_{21}^{m}\right\}-\log _{10} \operatorname{Re}\left\{S_{21}^{s}\right\}\right|}{\log _{10} \operatorname{Re}\left\{S_{21}^{m}\right\}}+\frac{\left|\log _{10} \operatorname{Im}\left\{S_{21}^{m}\right\}-\log _{10} \operatorname{Im}\left\{S_{21}^{s}\right\}\right|}{\log _{10} \operatorname{Im}\left\{S_{21}^{m}\right\}}
\end{aligned}
$$

14. Diferencia de logaritmo de módulo y fase

$$
\begin{aligned}
U= & \left|\log _{10}\right| S_{11}^{m}\left|-\log _{10}\right| S_{11}^{s}||+\left|\log _{10}\right| S_{21}^{m}\left|-\log _{10}\right| S_{21}^{s}|| \\
& +2 \frac{\angle S_{11}^{m}-\angle S_{11}^{s}}{2 \pi}+2 \frac{\angle S_{21}^{m}-\angle S_{21}^{s}}{2 \pi}
\end{aligned}
$$

15. Diferencia de logaritmo de módulo normalizada y fase con diferentes pesos

$$
\begin{aligned}
U= & \frac{\left|\log _{10}\right| S_{11}^{m}\left|-\log _{10}\right| S_{11}^{s}||}{\left|\log _{10}\right| S_{11}^{m}||}+\frac{\left|\log _{10}\right| S_{21}^{m}\left|-\log _{10}\right| S_{21}^{s}||}{\left|\log _{10}\right| S_{21}^{m}||} \\
& +2 \frac{\angle S_{11}^{m}-\angle S_{11}^{s}}{2 \pi}+2 \frac{\angle S_{21}^{m}-\angle S_{21}^{s}}{2 \pi}
\end{aligned}
$$

De entre todas las funciones de error se comprobó experimentalmente que las mejores (las que proporcionaban mejores estimaciones de la permitividad del dieléctrico) eran las que comparaban módulo y fase, en lugar de comparar parte real e imaginaria. Ambos tipos de funciones de error proporcionaban estimaciones de $\varepsilon_{r}^{\prime}$ bastante exactas, pero las que comparaban módulo y fase daban valores de $\varepsilon_{r}^{\prime \prime}$ más exactos que las que comparaban parte real e imaginaria. Esto se debe probablemente a que el hecho de que el dieléctrico presente mayores o menores pérdidas (mayor o menor valor de $\varepsilon_{r}^{\prime \prime}$ ) influye directamente en el módulo (mayor o menor absorción) de $S_{11}$ y $S_{21}$, más que influir en la parte real o imaginaria de forma aislada. También influye el valor de la permitividad (parte real e imaginaria) en el desfase que sufre la señal al atravesar el dieléctrico (fase del parámetro de transmisión $S_{21}$ ). De manera que comparar módulo y fase en esta aplicación es comparar directamente aquellas magnitudes que tienen la información de cómo es el poste dieléctrico. En cambio, comparar la parte 
real e imaginaria de los parámetros de reflexión y transmisión es comparar magnitudes que contiene la información de manera indirecta.

Una vez se dedujo que era más conveniente comparar módulo y fase, se probó con diferentes maneras de comparar módulo y fase (norma 1 o norma 2, normalizando o sin normalizar, con igual peso o distinto para módulo y fase). Y finalmente se llegó a la conclusión de que para esta aplicación era mejor comparar el módulo y la fase con norma 1 (módulo de la diferencia), y dando más peso a la información de la fase, pues es más sensible que el módulo a pequeños cambios en la permitividad.

Finalmente, la función de error seleccionada fue la siguiente:

$$
\begin{aligned}
U= & 0,25|| S_{11}^{m}|-| S_{11}^{s}||+0,25|| S_{21}^{m}|-| S_{21}^{s}|| \\
& +0,75\left|\frac{\angle S_{11}^{m}-\angle S_{11}^{s}}{2 \pi}\right|+0,75\left|\frac{\angle S_{21}^{m}-\angle S_{21}^{s}}{2 \pi}\right|
\end{aligned}
$$

Nótese que el hecho de dividir la fase por $2 \pi$ es para que tanto el módulo como la fase dividida por $2 \pi$ sean magnitudes que oscilen entre 0 y 1 . Además de esta normalización de la fase, se comprobó que dando más peso a la fase se conseguían mejores resultados, como ya se ha comentado. Concretamente, el $75 \%$ del error se corresponde a error de fase, y el otro $25 \%$ a error de módulo.

\subsubsection{Algoritmo de optimización}

Existen diferentes algoritmos de optimización para encontrar el máximo o el mínimo de una función multidimensional. En [63] se detallan los algoritmos de optimización más comunes. Se trata de algoritmos que ya han sido ampliamente utilizados y probados, y cuyas prestaciones son ya bien conocidas. A continuación se enumeran y detallan las características de las principales familias de algoritmos para la optimización de funciones multidimensionales, para posteriormente justificar el porqué de la elección del método de descenso del simplex.

Métodos de gradiente conjugado (Conjugate gradient methods) Los métodos de esta familia, tales como el algoritmo de Fletcher-Reeves o el de Polak-Ribiere, son métodos que calculan a cada paso las derivadas parciales de la función que se desea optimizar, con el fin de decidir a partir de la información de las derivadas en qué dirección avanzar. Una vez decidido en qué dirección avanzar, utilizan un sub-algoritmo de minimización unidimensional para decidir cuánto han de avanzar en dicha dirección. Estos métodos requieren una capacidad de almacenamiento de memoria del orden de $N$ posiciones, siendo $N$ el número de dimensiones de la función que se está optimizando.

Métodos quasi-Newton Los métodos de esta familia, tales como el algoritmo de DavidonFletcher-Powell (DFP) o el algoritmo de Broyden-Fletcher-Goldfrab-Shanno (BFGS), son también métodos que calculan las derivadas parciales de la función de error (o función objetivo) que se desea optimizar, y también requieren de un sub-algoritmo de 
minimización unidimensional. Estos métodos requieren del orden de $N^{2}$ posiciones de memoria. No se puede afirmar que los métodos de esta familia sean ni mejores ni peores que los de gradiente conjugado, sino que se deben ver como una alternativa.

Métodos direccionales (Direction-set methods) Estos métodos, como por ejemplo el método de Powell, no requieren del cálculo de las derivadas parciales, aunque sí utilizan un sub-algoritmo de minimización unidimensional. Requieren del orden de $N^{2}$ posiciones de memoria.

Método de descenso del simplex (Downhill simplex method) Este método se desplaza cuesta abajo a lo largo de la función de error de una forma directa sin hacer ningún tipo de asunción sobre las características de la función que se está minimizando, a diferencia de los métodos que utilizan derivadas parciales y que presuponen que la dirección de máxima pendiente nos llevará al mínimo de la función. Este método puede resultar lento, pero es extremadamente robusto. Además se trata de un método muy sencillo que se puede programar en pocas líneas de código totalmente autocontenido ( $\sin$ llamadas a otras rutinas). Los requerimientos de memoria son del orden de $N$ posiciones.

Método de enfriamiento progresivo (simulated annealing method) El método de enfriamiento progresivo es una técnica efectiva para la búsqueda de mínimos (o máximos) globales de funciones con una forma compleja que poseen varios mínimos (o máximos) locales. La base del método es análoga a la termodinámica, en particular con el modo en que los líquidos se congelan y cristalizan. A altas temperaturas, las moléculas de un líquido se mueven libremente. Si el líquido se enfría, pierden esa movilidad. En algunas ocasiones los átomos son capaces de alinearse y formar un cristal puro. Este cristal, completamente ordenado, se encuentra en estado de mínima energía. La naturaleza permite alcanzar este estado en el caso de un enfriamiento lento. De hecho, si un metal líquido es enfriado rápidamente, adopta un estado policristalino o amorfo con un nivel de energía superior.

La esencia del proceso es el enfriamiento progresivo. Dando tiempo a la redistribución de los átomos antes de que pierdan su movilidad, se asegura un estado de baja energía. Con el método de enfriamiento progresivo se permite un cierto desvío aleatorio, admitiendo no sólo los puntos que nos hacen ir rápidamente cuesta abajo en la función, sino también otros que aparentemente nos desvían del mínimo buscado. Se evita así que la precipitación nos haga caer en un mínimo local.

Una posible implementación del método de enfriamiento progresivo se puede realizar a partir del método de descenso del simplex. Consiste en utilizar el método de descenso del simplex tal cual, con la única diferencia de que se utiliza una variable aleatoria dependiente de una temperatura ficticia que hace que se puedan admitir con cierta probabilidad movimientos en direcciones diferentes de las de máximo descenso. Cada cierto número de iteraciones se disminuye la variable que representa la temperatura, de modo que cada vez es menos probable un movimiento en direcciones que no sean las de máximo descenso. 
De todos los métodos que se han descrito, se debe seleccionar el más adecuado para la aplicación de la estimación de la permitividad de materiales dieléctricos. En esta aplicación concreta el tiempo no es un factor crítico, de modo que no importa demasiado si el método de optimización es un poco lento. Por otra parte, el punto de partida de la optimización (estimación inicial de la permitividad) puede quedar bastante lejos del punto óptimo (permitividad real del material). Esta última característica nos hace descartar cualquier método de gradiente, de los que calculan las derivadas de la función de error, ya que estos métodos funcionan bien cuando las derivadas de la función nos conducen al mínimo, cosa que suele suceder al encontrarnos cerca del mismo, pero no así si el punto de partida está alejado del óptimo. En esta aplicación, por tanto, se necesita un método robusto aunque pueda resultar lento. Tanto el método de descenso del simplex como el de enfriamiento progresivo cumplen con estos requisitos, y se ha seleccionado el método de descenso del simplex por su mayor sencillez, ya que la función de error que resulta de comparar los parámetros de dispersión medidos y simulados de la guía con dieléctrico no presenta apenas mínimos locales, por lo que el uso de un sofisticado método de enfriamiento progresivo no se justifica.

En el apéndice E se detalla el funcionamiento del método de descenso del simplex.

Para acelerar el proceso de optimización, es conveniente seleccionar un punto de partida lo más cercano posible al mínimo de la función de error. En este caso, la estimación inicial de la permitividad del material dieléctrico se ha obtenido utilizando la ya conocida teoría perturbacional descrita en [59]. Como se desea estimar la permitividad del material en un amplio rango de frecuencias, se realiza una estima inicial para el primer punto de frecuencia, y el resultado de la optimización para ese punto se utiliza como punto de partida para el siguiente punto de frecuencia. Para el resto de frecuencias se utiliza como punto de partida el resultado de la optimización anterior.

\subsubsection{Resultados}

Con el objeto de contrastar la validez del método propuesto para la determinación de las propiedades dieléctricas de los materiales, se ha realizado una estimación de la permitividad de una muestra de etanol, que es un material líquido. Como ya se ha explicado con anterioridad, el etanol se ha introducido en una pipeta de teflón, y a su vez la pipeta se ha introducido en el interior de una guía rectangular WR340 (ver figuras 4.2 y 4.3). Los radios interior y exterior de la pipeta de teflón son, respectivamente, 16 y $20 \mathrm{~mm}$. Se han realizado medidas de los parámetros de dispersión de la guía con la muestra dieléctrica entre 2 y $3 \mathrm{GHz}$, que se corresponden con frecuencias en las que se propaga tan sólo el modo fundamental. Como ya se ha explicado, esta técnica permite caracterizar el dieléctrico en frecuencias multimodo, pero los programas de calibración del equipo de medida no permiten calibrar a dichas frecuencias.

Aunque el objetivo es la estimación de la permitividad del etanol ( $\varepsilon_{r_{2}}$ en la figura 4.1), resulta necesario como paso previo conocer la permitividad del material que forma la pipeta ( $\varepsilon_{r_{1}}$ en la figura 4.1), pues el simulador electromagnético necesita conocer dicha permitividad para poder generar los parámetros de dispersión de la estructura. Para estimar la permitividad de la pipeta, ésta se inserta vacía en el interior de la guía de ondas, y se miden sus parámetros 
de dispersión con el analizador de redes entre 2 y $3 \mathrm{GHz}$ a temperatura ambiente.

Con el objetivo de reducir la potencia del ruido existente en las medidas, éstas han sido filtradas utilizando una ventana de Hamming. Los coeficientes de una ventana de Hamming de tamaño $M$ muestras son [47]:

$$
h[n]= \begin{cases}0,54-0,46 \cos (2 \pi n / M), & 0 \leq n \leq M \\ 0, & \text { otherwise }\end{cases}
$$

El efecto de filtrar las medidas con una ventana de Hamming de tamaño $M$ es el de realizar un promediado en cada punto de medida con los $(M-1) / 2$ puntos anteriores y los $(M-1) / 2$ posteriores, dando más importancia a los puntos más próximos al actual.

En nuestra aplicación se tomaron 801 puntos de medida en el rango entre 2 y $3 \mathrm{GHz}$, y se ha probado con varios valores de $M$ para el tamaño de la ventana, llegando a la conclusión de que con una ventana de tamaño $M=11$ se reduce satisfactoriamente el rizado en las medidas (debido al ruido) sin que se deforme la envolvente (debida a la respuesta del sistema). En la figura 4.5 se representa el aspecto de una ventana de Hamming de tamaño $M=11$.

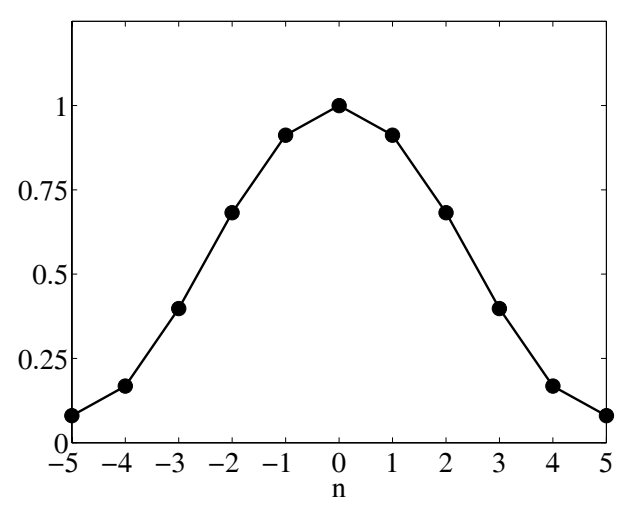

Figura 4.5: Ventana de Hamming de tamaño $M=11$

Como las medidas son complejas, por un lado se filtra la parte real de cada parámetro de dispersión, y por otro lado se filtra la parte imaginaria. Como los parámetros de dispersión no varían de forma brusca en frecuencia, este filtrado reduce el ruido satisfactoriamente, como se muestra en la figura 4.6, donde se representan el módulo y la fase del parámetro $S_{11}$ antes y después de filtrar.

Una vez que las medidas has sido realizadas y procesadas, se sigue un proceso de optimización, en el que se utiliza el algoritmo de descenso del simplex. El proceso de optimización busca los valores de la permitividad de la pipeta que minimizan la diferencia entre las medidas y los resultados de la simulación.

Tan sólo se ha optimizado la parte real de la permitividad, debido a que la parte imaginaria es muy pequeña, pues se trata de un material con muy bajas pérdidas. Si se intenta, a pesar de todo, optimizar tanto la parte real como la imaginaria, nos encontramos con que la función de error resultante de comparar las medidas con los resultados de la simulación 
presenta un valle muy estrecho a lo largo del eje correspondiente a la parte imaginaria de la permitividad $\left(\varepsilon_{r}^{\prime \prime}\right)$.

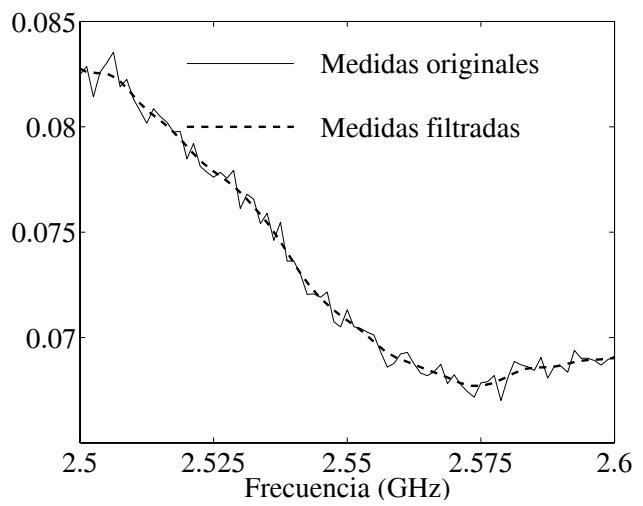

(a)

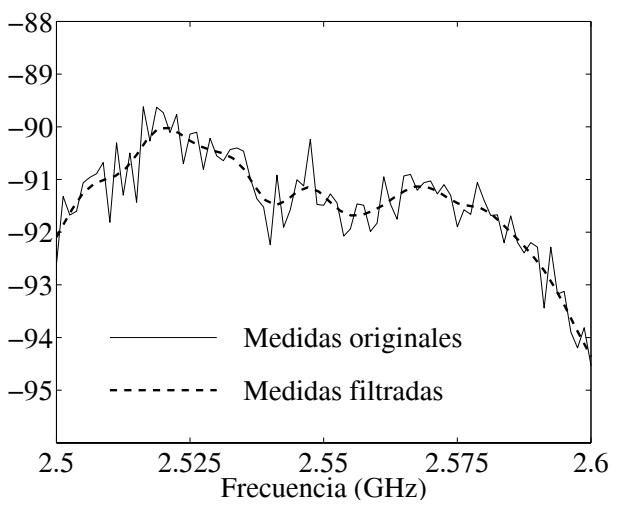

(b)

Figura 4.6: Parámetro de reflexión de la guía con un poste dieléctrico.Comparación de las medias sin procesar y filtradas. a) Módulo. b) Fase

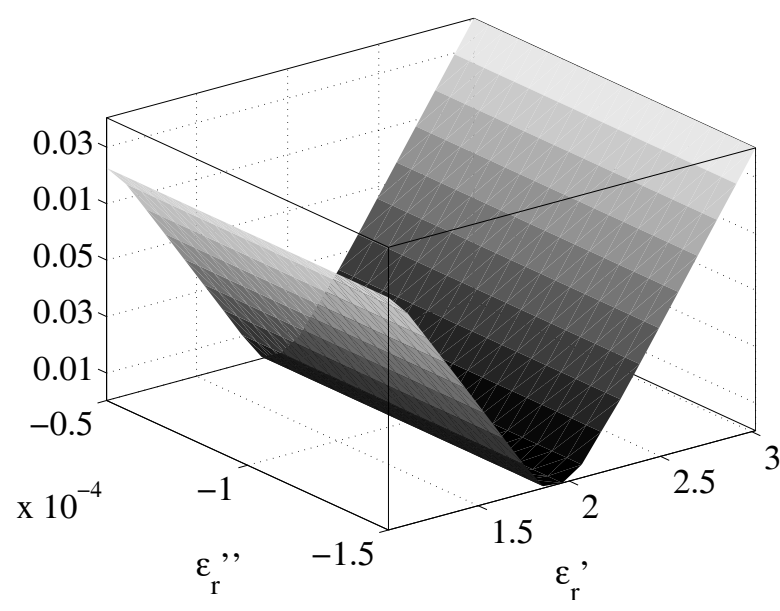

(a)

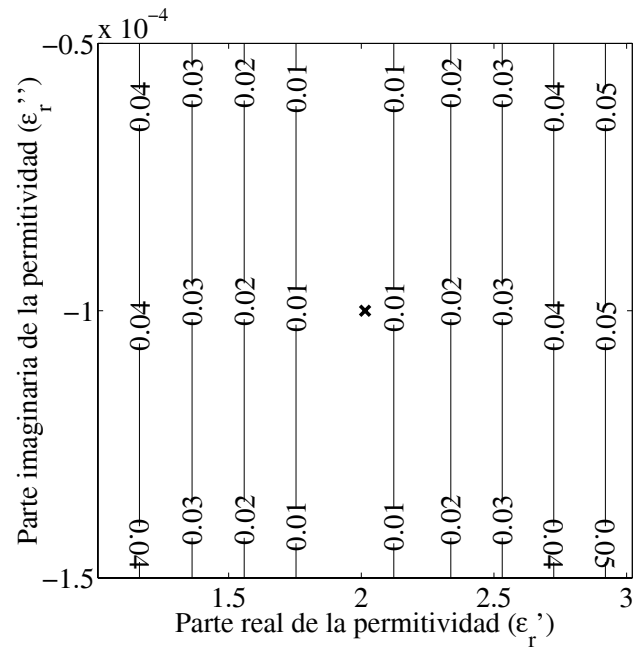

(b)

Figura 4.7: Función de error en la optimización de la permitividad de la pipeta. a) Vista tridimensional. b) Curvas de nivel

En la figura 4.7 se representa la función de error calculada según (4.2) en el entorno del punto $\varepsilon_{r}=2-j 10^{-4}$ y a una frecuencia de $2 \mathrm{GHz}$. Se puede observar que una vez el algoritmo de optimización llega al fondo del valle, es difícil decidir en qué dirección moverse 
a lo largo del mismo porque la pendiente es prácticamente nula. Por otro lado, como las pérdidas son tan bajas, se puede afirmar que las medidas proporcionadas por el analizador de redes presentan un grado de incertidumbre demasiado elevado como para poder determinar el nivel de dichas pérdidas $[60,64]$. Por tanto se ha fijado $\varepsilon_{r}^{\prime \prime}=10^{-4}$, y se ha optimizado tan sólo la parte real de la permitividad $\varepsilon_{r}^{\prime}$.

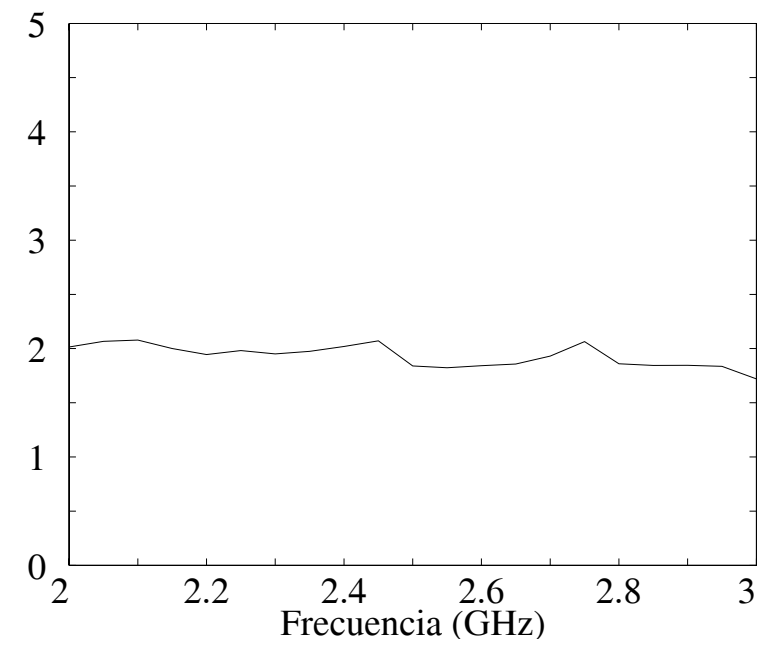

Figura 4.8: Variación con la frecuencia de la permitividad de la pipeta (teflón) a temperatura ambiente

En la figura 4.8 se muestran los valores obtenidos tras la optimización para estimar la permitividad de la pipeta. Se ha utilizado el algoritmo de descenso del simplex, y en término medio el valor final de la función de error ha resultado de 0.001 , y el número medio de evaluaciones de la función de error necesarias para converger en cada punto en frecuencia es de 36. Se entiende por convergencia del algoritmo que entre una iteración y la siguiente el tamaño del simplex sea menor que un umbral $\left(10^{-4}\right.$ en este caso), y que la variación de la función de error entre una iteración y la siguiente sea menor que otro umbral $\left(10^{-4}\right)$.

Una vez se ha estimado la permitividad de la pipeta, se puede pasar a determinar la permitividad del etanol, que es nuestro objetivo. Para ello se introduce el etanol en la pipeta, y la pipeta en la guía. Se miden los parámetros de dispersión de la estructura, y de nuevo las medidas son filtradas con una ventana de Hamming para reducir la potencia de ruido. En un nuevo proceso de optimización, y utilizando los valores de la permitividad de la pipeta obtenidos previamente para simular la respuesta de la estructura, se buscan los valores real e imaginario de la permitividad del etanol que minimizan el error entre las medidas y la simulación calculado según 4.2. En este caso, y utilizando de nuevo el algoritmo de descenso del simplex, se ha conseguido la convergencia en todos los puntos de frecuencia. Para alcanzar el mínimo de la función de error se ha necesitado de una media de 104 evaluaciones de la función, y se ha conseguido en media reducir el error hasta un valor de 0.007.

A modo de ejemplo, se muestra en la figura 4.9 el aspecto de la función de error que se 
debe minimizar para la frecuencia de $2 \mathrm{GHz}$. Superpuestas a las curvas de nivel se encuentran pequeñas flechas que indican la dirección del gradiente en cada punto. Una cruz indica la posición del mínimo encontrado por el algoritmo de optimización, y un círculo indica el punto de partida del algoritmo. Para completar la optimización a esta frecuencia $(2 \mathrm{GHz}), \mathrm{se}$ han necesitado 103 evaluaciones de la función de error.

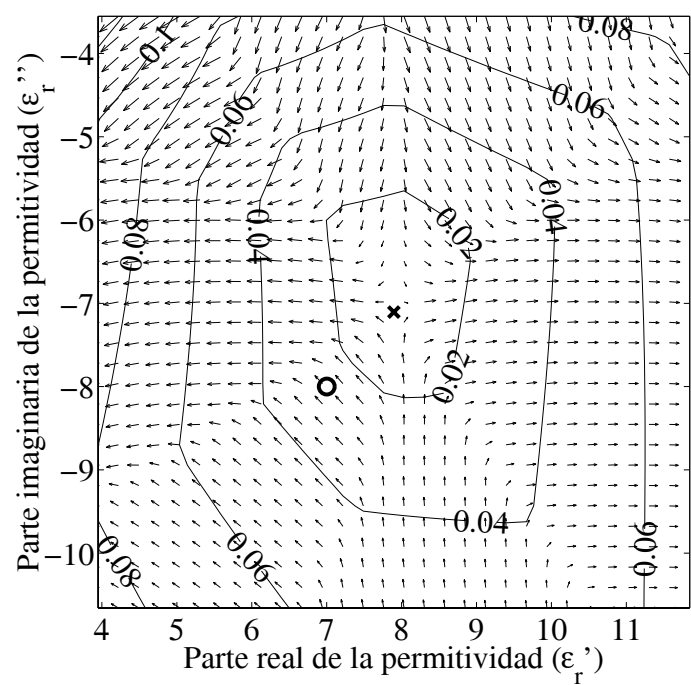

(a)

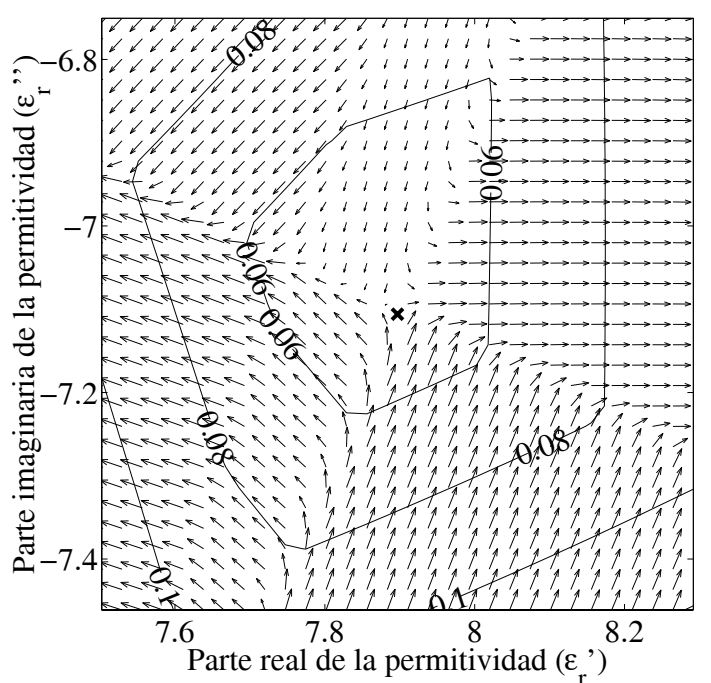

(b)

Figura 4.9: Función de error de (4.2) para el caso de una pipeta rellena de etanol en una guía WR340 a $2 \mathrm{GHz}$. Con una cruz se marca la posición del mínimo encontrado tras la optimización. a) Entorno amplio. b) Entorno más reducido próximo al mínimo

En la figura 4.10 se muestra la permitividad compleja (parte real e imaginaria) del etanol resultante del proceso de optimización. Estos resultados se comparan con estimaciones de la permitividad del etanol realizadas utilizando otras técnicas de medida ([61, 62]), y se observa que existe una buena concordancia en todo el rango de frecuencia entre dichos resultados y los de la técnica presentada en este trabajo. También se ha representado en la figura 4.10 la permitividad del etanol obtenida tras un único proceso de optimización en el que se ha obviado la presencia de la pipeta que soporta el etanol. Este resultado se ha incluido porque es frecuente a la hora de estimar la permitividad de líquidos el despreciar la presencia del soporte. En la figura 4.10 se observa cómo la inclusión en el análisis del soporte de teflón (cuya permitividad ha sido estimada tras un proceso de optimización), mejora en gran medida los resultados. 


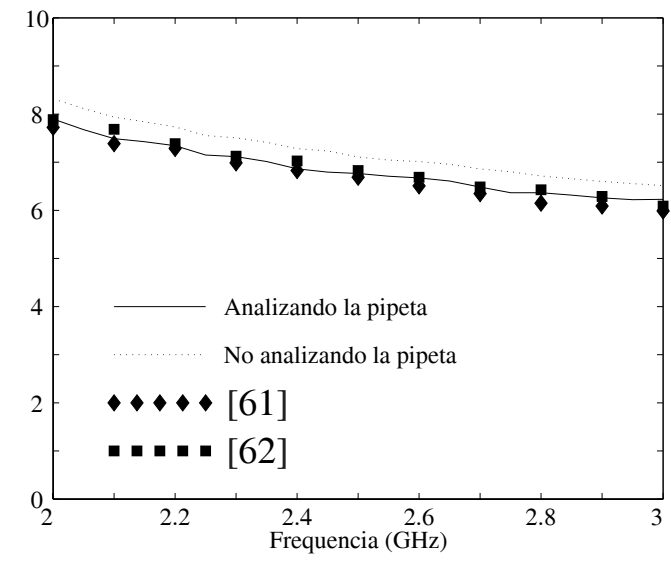

(a)

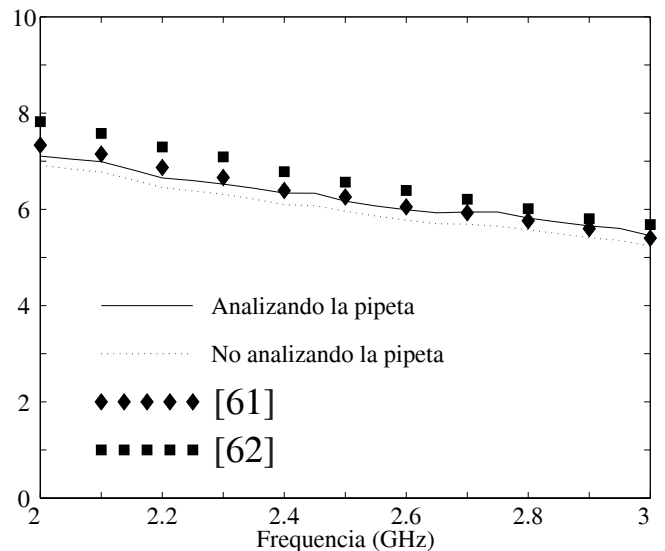

(b)

Figura 4.10: Permitividad compleja del etanol a temperatura ambiente. (a) Parte real. (b) Parte imaginaria

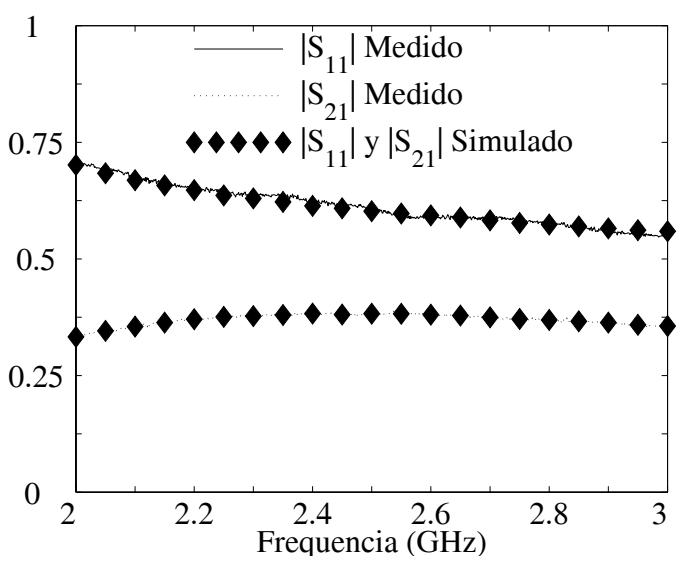

(a)

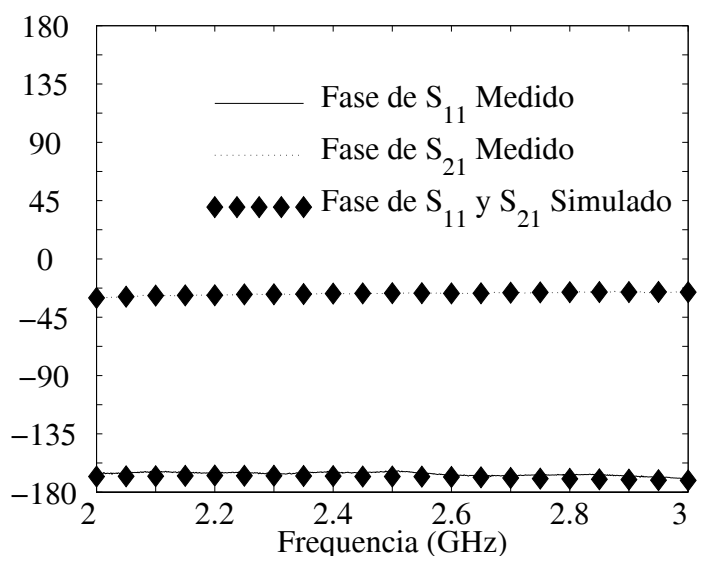

(b)

Figura 4.11: Comparación entre medidas y simulación de los parámetros de dispersión de una guía WR340 con una pipeta rellena de etanol. a) Amplitud. b) Fase

Con el objeto de comprobar que el proceso de optimización se realizó correctamente, y que se minimizó la diferencia entre las medidas y los resultados de la simulación, en la figura 4.11 se representa la amplitud y la fase de los factores de reflexión y transmisión de la guía WR340 con la pipeta llena de etanol. Se puede constatar la poca diferencia entre la amplitud y la fase de las medidas y de los resultados de la simulación (utilizando para la simulación los valores de la permitividad proporcionados por el proceso de optimización). 


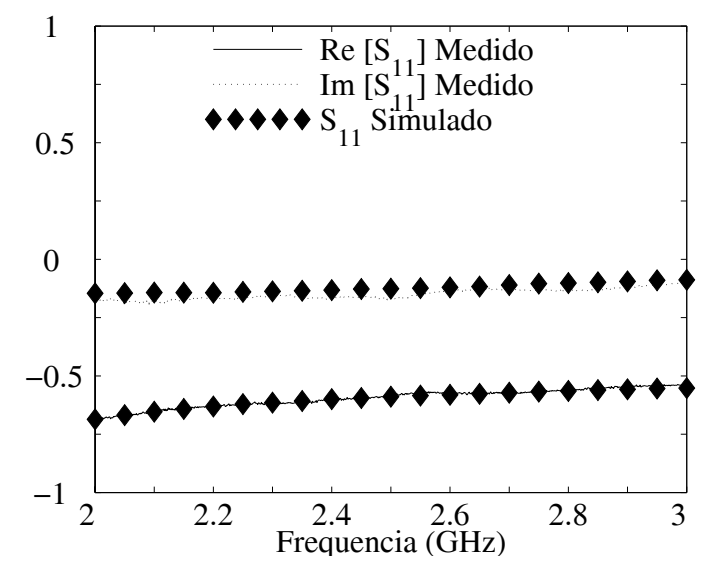

(a)

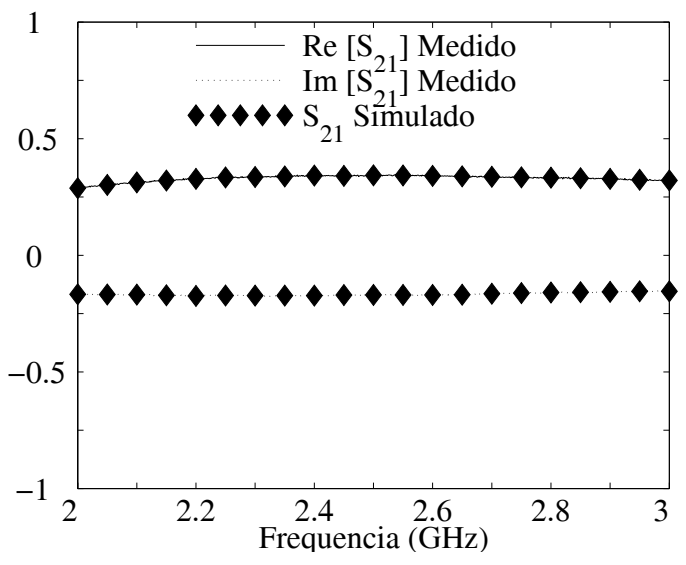

(b)

Figura 4.12: Comparación entre medidas y simulación de la parte real e imaginaria de los parámetros de dispersión de una guía WR340 con una pipeta rellena de etanol. a) factor de reflexión. b) factor de transmisión

Puesto que la función de error se ha definido en (4.2) como la diferencia entre la amplitud y la fase de $S_{11}$ y $S_{21}$, resulta lógico que la diferencia entre la amplitud y la fase de las medidas y de la simulación sea mínima. Podemos pensar que si se compara la parte real y la parte imaginaria de los parámetros de dispersión esa diferencia no debe ser muy grande, pero tampoco tiene porqué ser tan pequeña. En la figura 4.12 se has representado las partes real e imaginaria de los parámetros de dispersión, y se puede observar que, efectivamente, la diferencia entre las medidas y la simulación es pequeña, pero no tanto como en el caso de la amplitud y la fase. Si lo que se desea es ajustar bien las partes real e imaginaria, se debería haber utilizado otra función de error, como por ejemplo la descrita en la página 145 (diferencia de parte real e imaginaria normalizada, apartado 1). No obstante, tal y como se comentó con anterioridad, en esta aplicación la amplitud y la fase aportan mayor información sobre la permitividad del etanol, y por ello se eligió definir la función de error como se detalla en (4.2). 


\subsection{Análisis de filtros inductivos con esquinas redondeadas}

\subsubsection{Introducción}

Las actuales técnicas de fabricación de dispositivos en guía rectangular ${ }^{1}$ introducen internamente esquinas redondeadas cuando estos procesos son de bajo coste, debido a que en la práctica resulta complicado, y por tanto costoso económicamente, realizar ángulos rectos [65]. Si este efecto de mecanizado pudiera considerarse de forma rigurosa en los programas de análisis de estructuras guiadas de las herramientas de diseño asistido por ordenador (Computer Aided Design, CAD), la fabricación de tales dispositivos podría mejorarse enormemente en términos de precisión, costes de producción y tiempos de desarrollo.

A continuación se describen los principales métodos de fabricación de filtros inductivos, y se describen las imperfecciones que suelen producir cada uno de estos método de fabricación.

\subsubsection{Principales métodos de fabricación}

De los métodos de bajo coste que se emplean actualmente para la fabricación de filtros inductivos en guía rectangular destacan principalmente dos de ellos, a los que denominaremos "corte en plano H" y "corte en plano E". La filosofía es en ambas técnicas la misma: dividir la pieza en dos partes y así poder erosionar y dar forma al interior del filtro con la mayor comodidad posible. Si la producción es de bajo coste, la forma interior del filtro se suele moldear utilizando una fresa. Esto supone la aparición de esquinas redondeadas cuyo tamaño depende del diámetro de la fresa [66]. A continuación se describirán las características, ventajas e inconvenientes de cada uno de los dos métodos de fabricación mencionados. También se describirá una novedosa técnica de fabricación, aún en fase de prueba, en la que se utiliza un molde para fabricar los filtros inductivos, y a la que denominaremos "fabricación con molde".

\section{Corte en plano $\mathbf{H}$}

Este tipo de proceso de fabricación consiste en cortar la pieza en dos bloques: una lámina superior y otra pieza en la que se eliminará mediante la fresa el material necesario para obtener el filtro inductivo con las dimensiones deseadas. Posteriormente se atornillarán ambas piezas. En la figura 4.13 puede verse la estructura de las dos piezas y cómo se unen finalmente para dar lugar al filtro. La principal ventaja de este proceso de fabricación es la facilidad del modelado del interior del filtro, así como la posterior unión de las dos piezas resultantes.

Como puede observarse en la figura 4.13, esta técnica de fabricación introduce esquinas redondeadas en el plano $\mathrm{H}$ del filtro. Se ha comprobado que en este caso el radio de curvatura $(R)$ de las esquinas redondeadas tiene una fuerte influencia en la respuesta del dispositivo.

\footnotetext{
${ }^{1}$ Fresado controlado por ordenador (=computer controlled milling), electroerosión (=spark-eroding), electroformado(=electro-forming) o troquelado(=die casting).
} 


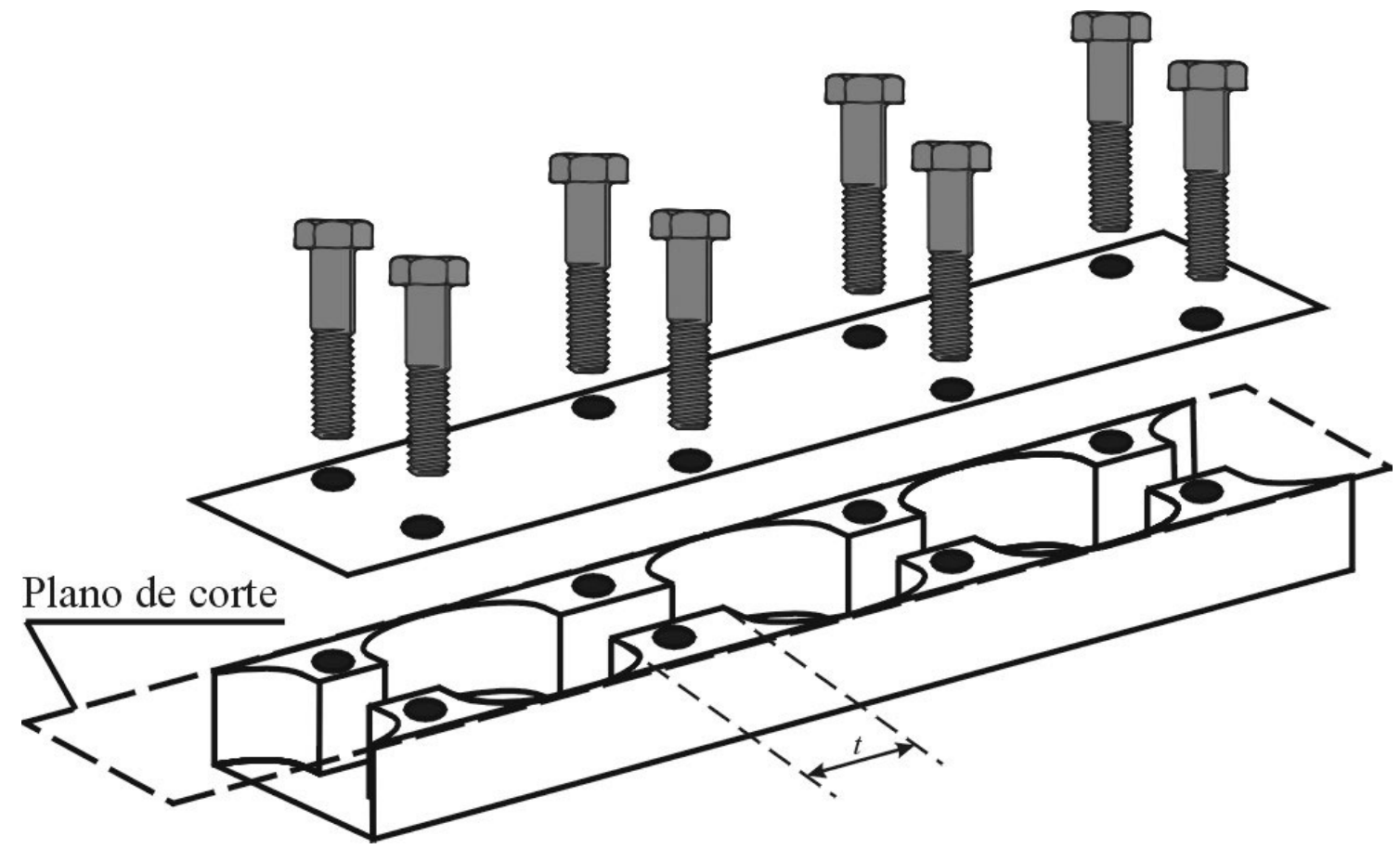

(a) Piezas separadas

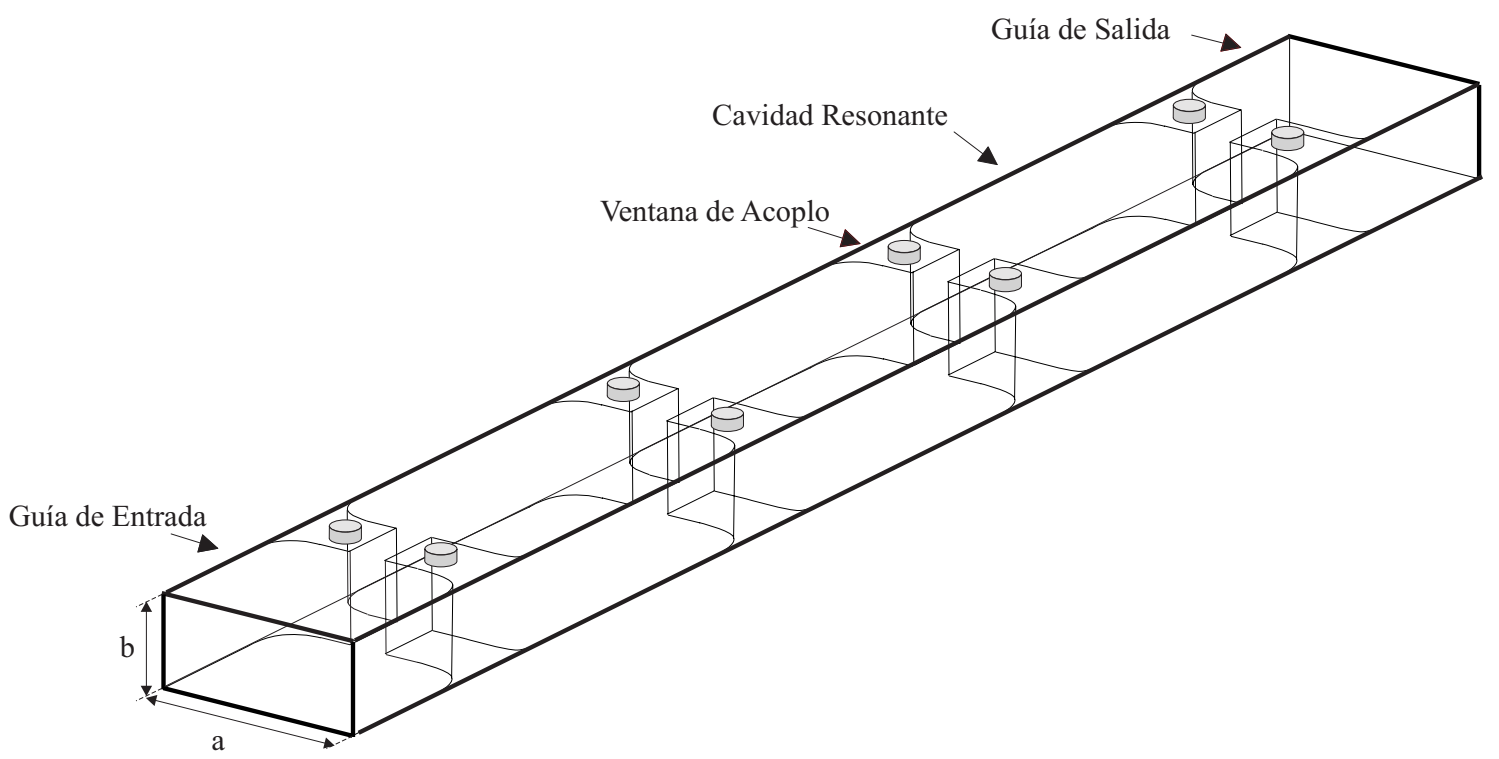

(b) Piezas atornilladas

Figura 4.13: Filtro inductivo en guía rectangular fabricado con la técnica de corte en plano $\mathrm{H}$ 
Otro problema de este método de fabricación está asociado a las altas pérdidas que normalmente presenta la estructura. Dichas pérdidas se deben a que el plano de corte interrumpe completamente la continuidad de la corriente eléctrica presente en la pared superior del filtro. Para minimizar este efecto la tapa superior debe atornillarse lo mejor posible al cuerpo del filtro.

Finalmente, la longitud de las ventanas de acoplo $t$ señaladas en la figura 4.13 se aprovechan para colocar los tornillos que se encargan de sujetar la tapa al resto del filtro. Para que dicha sujeción sea lo suficientemente efectiva y no haya muchas pérdidas, los tornillos de ajuste deben tener un diámetro igual o superior a $3 \mathrm{~mm}$. Sin embargo, a altas frecuencias (por ejemplo en banda X: $8-12 \mathrm{GHz}$ ), las ventanas de acoplo obtenidas en el proceso de diseño son de una longitud menor (típicamente $2 \mathrm{~mm}$ ) y ello impide colocar con facilidad los tornillos aprovechando ese espacio. Este problema se acrecienta a medida que se trabaja a frecuencias más elevadas, tendencia presente en la mayoría de aplicaciones prácticas (por ejemplo sector espacial) donde se emplea esta tecnología.

\section{Corte en plano $\mathrm{E}$}

En la figura 4.14 se muestra este segundo método de fabricación. En este caso se secciona la pieza inicial en dos piezas simétricas, y ambas se vacían de forma adecuada para que al unirse se forme el filtro inductivo con las dimensiones adecuadas, tal y como se muestra en la figura 4.14 .

La principal ventaja sobre el método anterior reside en que las dos piezas son simétricas y el plano de corte no produce efectos tan perniciosos en cuanto a pérdidas y respuesta en frecuencia del filtro. Por otro lado, el análisis de esta estructura requiere de métodos complejos tales como elementos finitos que necesitan de un gran esfuerzo computacional, por lo que el diseño mediante herramientas CAD de filtros fabricados con esta técnica no resulta factible con las capacidades de computación existentes en la actualidad.

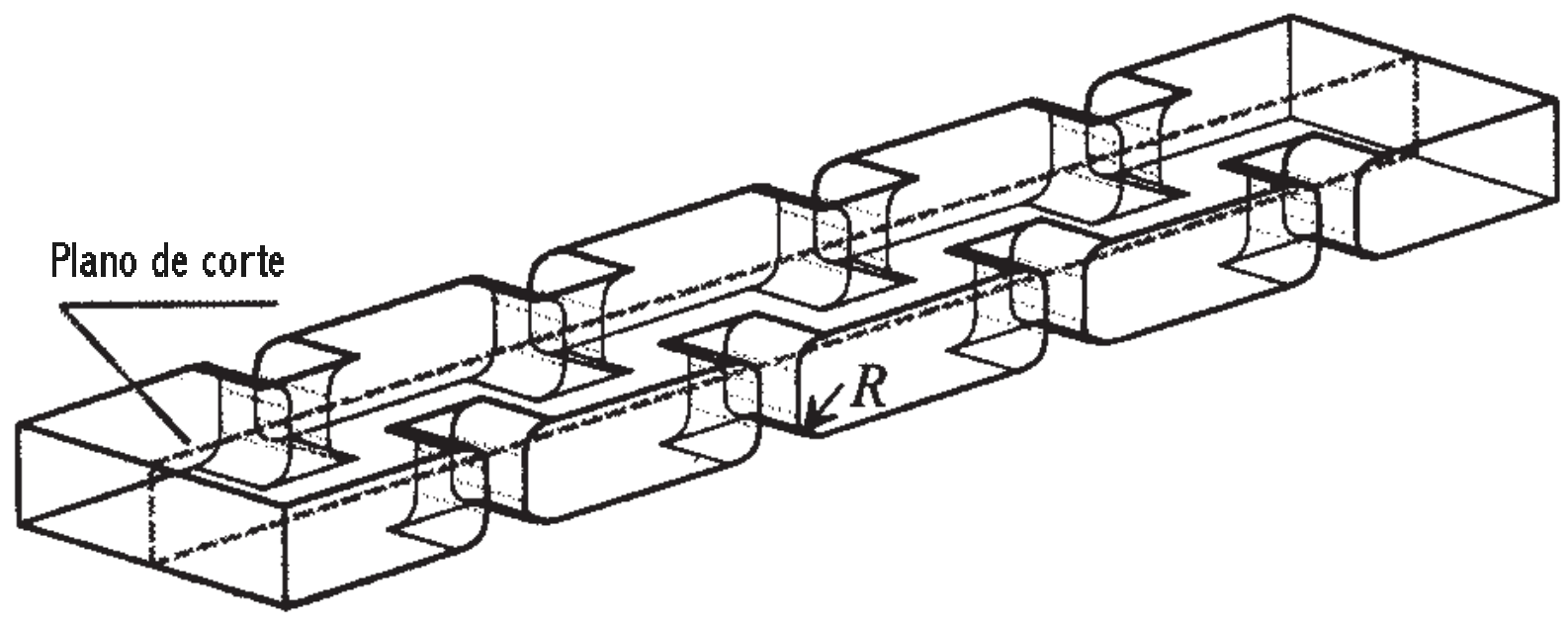

Figura 4.14: Técnica de corte en plano E 


\section{Fabricación con molde}

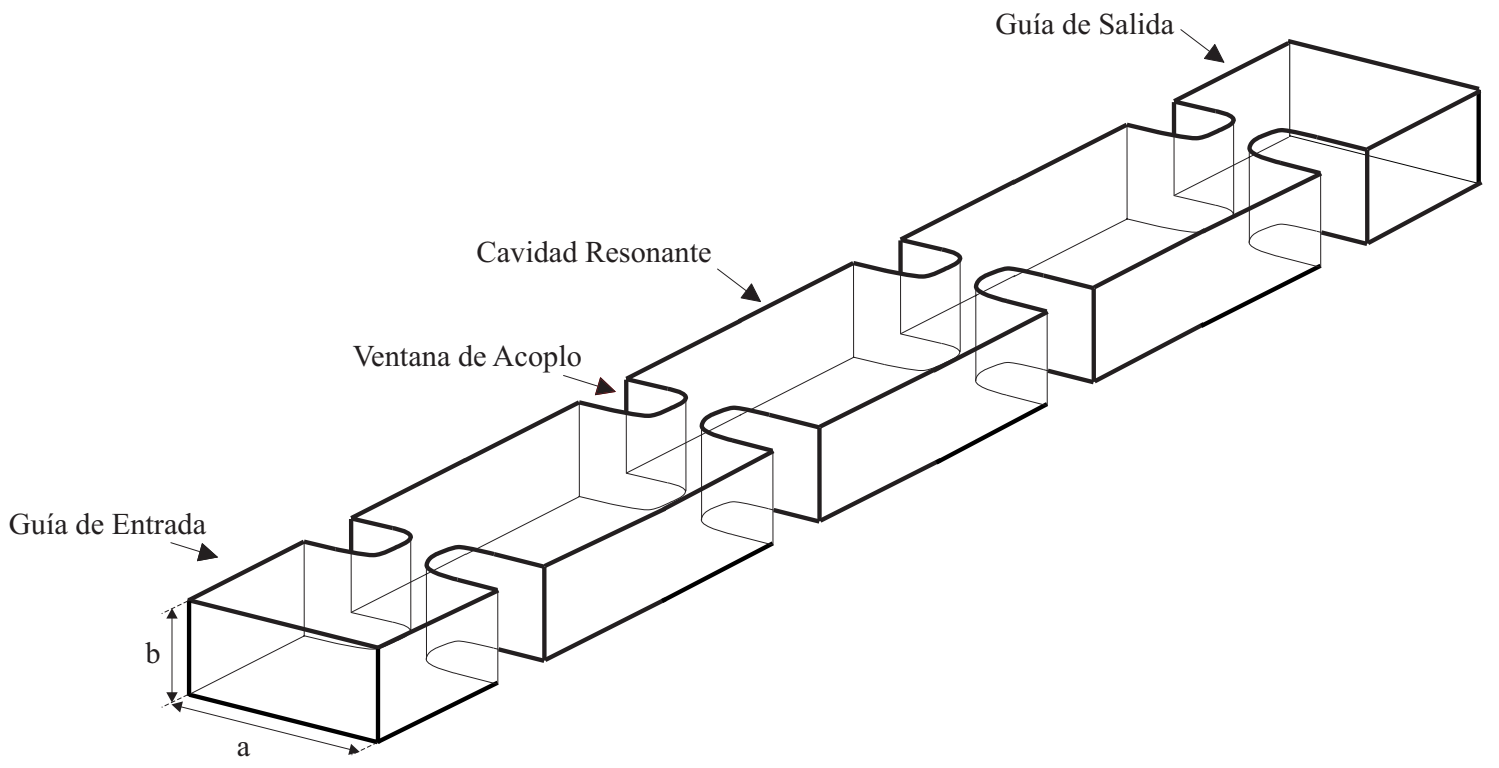

(a) Molde

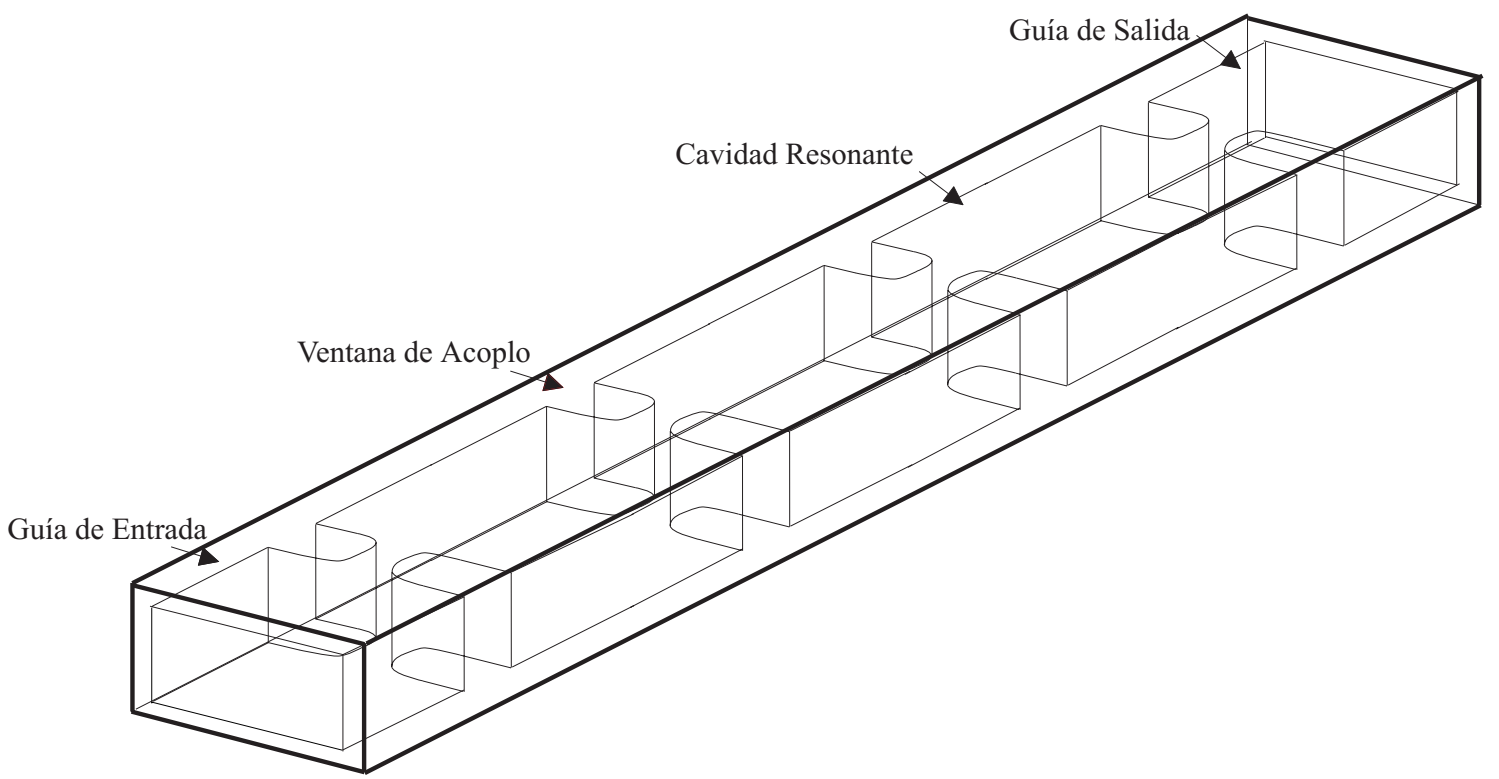

(b) Molde recubierto de metal

Figura 4.15: Técnica de fabricado con molde

Esta técnica consiste en fabricar un molde como el mostrado en la figura 4.15.a. Posteriormente el mode se cubre de metal, y el molde se elimina con un líquido abrasivo no daña 
a la estructura metálica. Tras finalizar el proceso de fabricación, se obtiene un filtro inductivo con esquinas redondeadas en las ventanas de acoplo. Las esquinas redondeadas están situadas en plano $\mathrm{H}$ como en el caso de fabricación por corte en plano $\mathrm{H}$, pero se sitúan en posiciones diferentes. La ventaja de esta técnica de fabricación frente a la de corte en plano $\mathrm{H}$ o en plano E es que en este caso se fabrica el filtro de una sola pieza sin interrumpir de ningún modo la corriente, lo que supone unas pérdidas menores.

\subsubsection{Análisis de filtros inductivos con efectos de mecanizado en pla- no $\mathbf{H}$}

La presencia de esquinas redondeadas en filtros de guía rectangular ha sido un tema ampliamente estudiado en la literatura siguiendo diferentes técnicas de análisis. Por ejemplo, en [66] se propone una primera aproximación con una técnica de acoplo modal (modematching) que emplea un modelo escalonado para simular el efecto de la curvatura. Para mejorar la precisión de tal aproximación se pueden emplear métodos híbridos que combinan técnicas numéricas (discretización espacial) y técnicas modales. Como ejemplo de este tipo de técnicas se puede citar el método riguroso que combina adaptación modal y elementos de contorno (Boundary Contour Mode-Matching, BCMM) descrito en [14] o el método de integral de contorno y expansión modal resonante (Boundary Integral-Resonant Mode Expansion, BI-RME) formulado inicialmente en [67] para cavidades arbitrarias 3D y, tras algunas mejoras, particularizado en [68] al caso de filtros con redondeces en el plano E.

En esta sección se aplicará la técnica híbrida de análisis de problemas 2-D en conjunción con la técnica de acoplo modal para obtener los parámetros de dispersión de filtros inductivos con errores de mecanizado. De los tres tipos de fabricación de filtros inductivos, con la técnica expuesta en los capítulos anteriores tan sólo se puede analizar estructuras en plano $\mathrm{H}$ (inductivas). Por tanto, se pueden analizar aquellos filtros fabricados con la técnica de corte en plano $\mathrm{H}$ y con la técnica de molde. En ambos casos las esquinas redondeadas tienen una geometría invariante a lo largo del eje $\hat{y}$, y se puede aplicar por tanto la técnica de análisis de problemas 2-D.

El análisis preciso y eficiente de este tipo de estructuras mediante el método híbrido permitirá en un futuro integrar este método de análisis en una herramienta de CAD (diseño asistido por ordenador) de diseño de filtros inductivos en la que se proporcionarán las dimensiones que debe tener el filtro para cumplir con unas determinadas especificaciones. El diseño resultará mucho más preciso al tenerse en cuenta los efectos de redondeo en la esquinas por imperfecciones en la fabricación.

\section{Análisis de errores de mecanizado por la técnica de corte en plano $\mathbf{H}$}

Para analizar la influencia de los errores de mecanizado en filtros inductivos fabricados con la técnica de corte en plano $\mathrm{H}$, se ha analizado un filtro inductivo de 8 polos ( 8 cavidades inductivas). En la figura 4.16 se muestra la amplitud del factor de reflexión para la banda de paso del filtro. Junto a la respuesta en frecuencia del filtro con esquinas rectas ( $R=0 \mathrm{~mm})$, se ha representado la respuesta en frecuencia del mismo filtro pero considerando una esquinas 


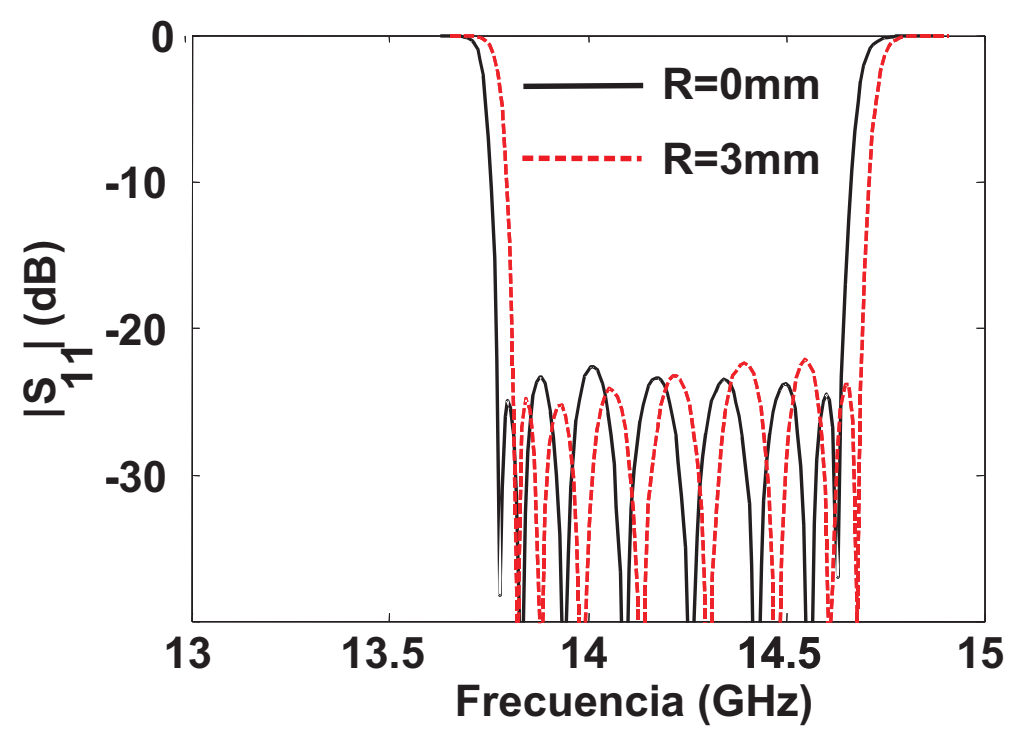

Figura 4.16: Amplitud del factor de reflexión en un filtro de 8 cavidades inductivas con esquinas redondeadas producidas por la técnica de fabricación por corte en plano $\mathrm{H}$

redondeadas de radio $3 \mathrm{~mm}$, La posición de las esquinas redondeadas es la que se indica en la figura 4.13. Se puede apreciar que el efecto del redondeo es importante, especialmente por el desplazamiento en frecuencia de la respuesta. Por tanto, si dicho efecto no se tiene en cuenta en los simuladores que se utilicen en el proceso de diseño se obtendrán unas dimensiones erróneas para el filtro. Tradicionalmente, una vez fabricados los filtros se ajustan de forma manual con unos tornillos de sintonía que corrigen errores de diseño y/o fabricación hasta conseguir sintonizar la respuesta del filtro de forma adecuada. Para realizar dichos ajustes es necesaria una gran destreza y experiencia. Utilizando un simulador que permita analizar esquinas redondeadas se consigue que el diseño sea más preciso, y por tanto que la respuesta del filtro tras su fabricación (sin ajustes con tornillos) se parezca mucho más a las especificaciones. En consecuencia, los ajustes a posteriori habrán de ser mucho menores y más sencillos.

El tiempo de computación, tanto para el filtro con esquinas rectas como para el filtro con esquinas redondeadas es de aproximadamente de 8 segundos por punto en frecuencia en un PC con procesador Pentium II a $300 \mathrm{MHz}$. Si bien existen técnicas de análisis muy eficientes para filtros inductivos con esquinas rectas, dichas técnicas no pueden simular esquinas redondeadas. Y aquellas técnicas que sí pueden analizar esquinas redondeadas (por ejemplo el software comercial HFSS $囚[69]$ ) requieren de mucho más de 8 segundos por punto en frecuencia, al utilizar en el análisis de esta estructura un método numérico como el de los elementos finitos.

\section{Análisis de errores de mecanizado por fabricación con molde}

Los errores de mecanizado producidos por la novedosa técnica de fabricación por molde también pueden analizarse con el método híbrido de análisis de problemas 2-D. En la figura 
4.17 se muestra la respuesta en frecuencia (factor de reflexión) de un filtro inductivo de cuatro polos. Se han representado dicha respuesta en frecuencia para el caso de esquinas rectas $(R=0 \mathrm{~mm})$ y para esquinas redondeadas con radio pequeño $(R=0.1 \mathrm{~mm})$ y con radio grande $(R=0.5 \mathrm{~mm})$. Podemos observar que en este caso los errores de mecanizado son mucho más importantes que en la fabricación por corte en plano $\mathrm{H}$. Esto se debe a que en la fabricación con molde las esquinas redondeadas quedan más cerca del centro de la guía, donde la intensidad de campo es mayor para el modo fundamental. En cambio, en la fabricación por corte en plano $\mathrm{H}$ las esquinas redondeadas quedan al lado de las paredes laterales de la guía, donde la intensidad de campo es mucho menor. Por esta razón, con radios de las esquinas más pequeños, la fabricación con molde produce una distorsión mucho mayor que la anterior técnica de mecanizado. No obstante, si en el proceso de diseño se tienen en cuenta las esquinas redondeadas, se pueden conseguir dimensiones óptimas del filtro que satisfagan las especificaciones.

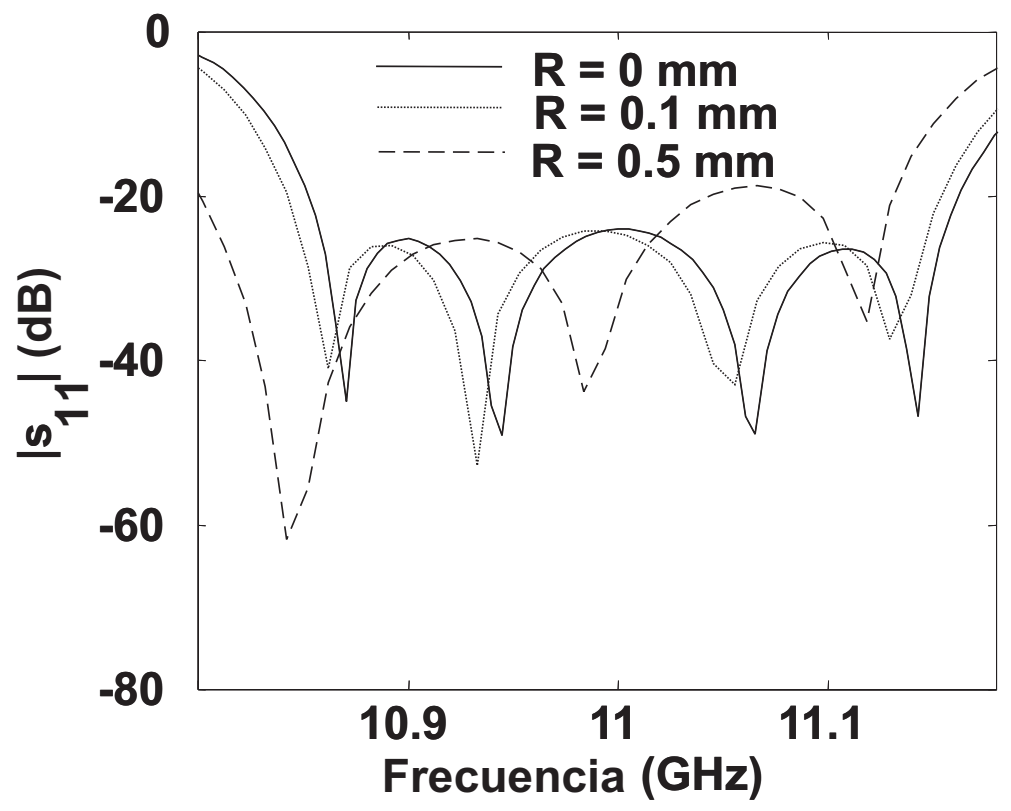

Figura 4.17: Amplitud del factor de reflexión de un filtro de 4 cavidades inductivas con esquinas redondeadas producidas por la técnica de fabricación con molde

Para validar el análisis de filtros con esquinas redondeadas se ha recurrido a un software comercial de simulación electromagnética (HFSS, ver [69]). Tal y como se ha comentado anteriormente, dicho software comercial utiliza el método numérico de los elementos finitos (FEM). Se ha comparado en la figura 4.18 la respuesta en frecuencia del filtro para un radio de redondeo grande $(R=0.5 \mathrm{~mm}$ ) obtenida con el método híbrido con la respuesta en frecuencia proporcionada por HFSS. Como se puede apreciar la coincidencia es total, si bien el tiempo de computación total con el método híbrido fue de aproximadamente 15 minutos (200 puntos a 5 segundos por punto con un Pentium II a $400 \mathrm{MHz}$ ), mientras que el tiempo de computación con HFSS fue de varias horas. 


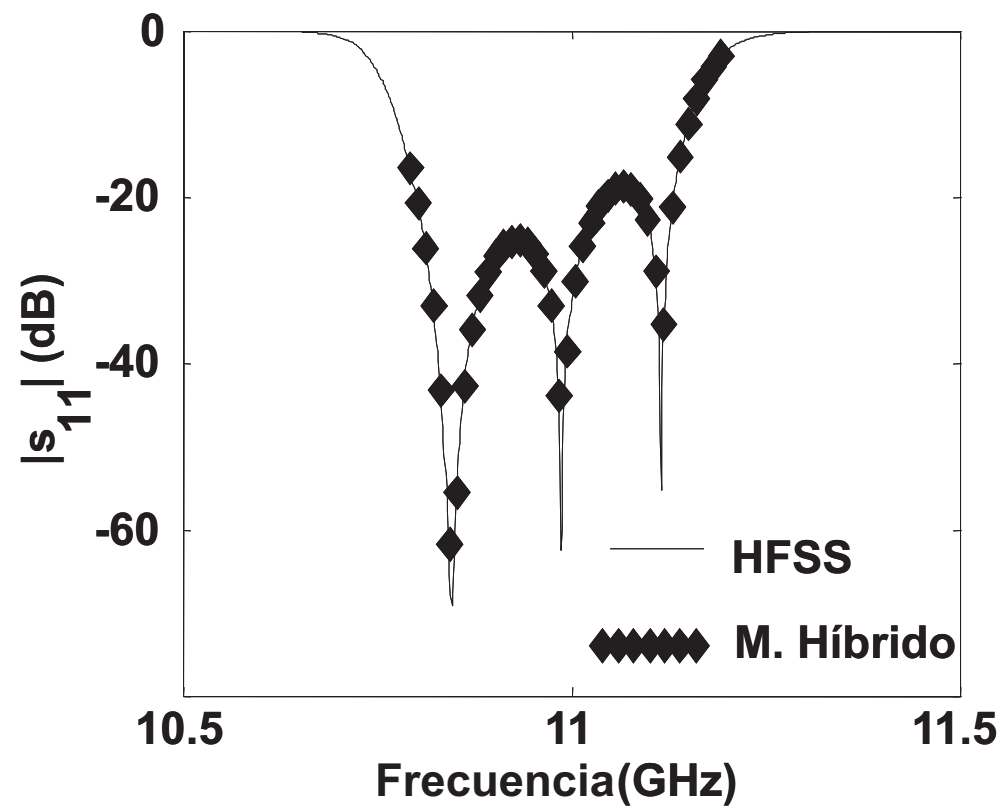

Figura 4.18: Respuesta en frecuencia de un filtro de 4 cavidades inductivas con esquinas redondeadas producidas por la técnica de fabricación con molde. Comparación del método híbrido con HFSS 


\subsection{Análisis de diplexores}

\subsubsection{Introducción}

Un diplexor es un tipo particular de multiplexor, que se define como un dispositivo que permite combinar múltiples entradas en una señal completa para transportarla mediante un canal de transmisión simple. La multiplexación es una técnica inventada en primer lugar para los sistemas telefónicos basados en hilos de cobre como una manera de enviar más mensajes por una línea de capacidad limitada. Si un multiplexor combina tan sólo dos señales, recibe el nombre de diplexor. En los últimos tiempos los multiplexores han recibido considerable atención por parte de la comunidad científica, ya sea en tecnología microelectrónica para diplexores en banda K [70], como en tecnología guiada donde aparecen un gran número de referencias al respecto (por ejemplo $[71,72,73,74,75,76,77,78,79,80]$ ). En las citadas referencias se enfatiza tanto la geometría de los dispositivos (con sus pros y sus contras) como el proceso de diseño optimizado. Las razones de este interés son bastante obvias:

- Proliferación de estos dispositivos en sistemas de telecomunicación tales como satélites y estaciones terrenas en el sector espacio, así como estaciones base de comunicaciones móviles. Estos sistemas requieren frecuentemente utilizar diversas bandas de frecuencia simultáneamente, y los multiplexores brindan dicha posibilidad.

- Permiten duplexar la señal y utilizar la misma antena en transmisión y en recepción ahorrando espacio en el diseño (útil sobretodo en satélites donde el espacio reservado a la carga útil es un bien escaso).

- Dificultad de diseño empleando dispositivos pasivos recíprocos. Los diplexores/multiplexores usan como divisores de potencia las uniones T [81] ó Y [80] (que se diseñan para repartir la potencia por cada brazo del diplexor donde están situados los filtros de alta selectividad). Estos multiplexores/diplexores requieren un gran esfuerzo de diseño, ya que al montar los filtros al divisor de potencia el acoplo entre las diferentes partes de la estructura altera la respuesta total del dispositivo, y se requiere en general un alto nivel de precisión para obtener los parámetros de scattering deseados. Este diseño se simplifica si se utilizan circuladores ${ }^{2}$ con filtros muy selectivos. Este tipo de multiplexores se utilizan como multiplexores de entrada en satélites (ver figura 4.19) donde las pérdidas no son un parámetro de diseño crítico. Los inconvenientes de esta técnica de fabricación de multiplexores son varios [82]:

1. Pérdidas en los circuladores.

2. Aislamiento no perfecto entre los accesos.

3. Los circuladores son voluminosos y de masa relativamente grande.

\footnotetext{
${ }^{2}$ Un circulador es una red de tres accesos (también existen de cuatro pero su uso es menos frecuente) no recíproca. Su matriz de parámetros $\mathrm{S}$ es no simétrica y unitaria. Se fabrica con ferritas (materiales cerámicos no conductores pero con propiedades magnéticas muy intensas) sometidas a la acción de un campo magnético estático.
} 
4. Posibilidad de manejo de poca potencia.

5. Problemas en el diseño de los filtros selectivos.

- Pueden emplearse para aplicaciones espaciales (sobre todo en estaciones terrenas) multiplexores en etapas como los anteriormente expuestos formados por circuladores y filtros, pero construidos con dispositivos recíprocos. Utilizando filtros y acopladores direccionales se consigue un comportamiento de la estructura muy parecido [83]. Las ventajas en cuanto al diseño son las mismas que con circuladores: fácil realización modular y fácil extensión adicional para nuevos servicios. Las mejoras también son importantes: menores pérdidas de inserción y capacidad de manejo de mayor potencia. Los inconvenientes a remarcar se derivan del esfuerzo de fabricación de los módulos. Cada módulo requiere dos acopladores direccionales y dos filtros. Esto conlleva una dificultad nada despreciable de sintonización del módulo así como una gran masa y tamaño, que hacen impracticable estos diseños para aplicaciones espaciales embarcadas en satélites ${ }^{3}$.

- Los multiplexores formados por estructuras en guía se utilizan como multiplexores de señal de salida en satélites [84], debido a que las pérdidas en esta etapa son críticas, pues suponen una reducción en el nivel de potencia de la señal radiada. Se utilizan filtros montados en una guía común o colector (en inglés manifold) cuyo extremo está cortocircuitado (ver figura 4.20). Se realiza una división entre canales pares e impares dejando una banda de guarda entre cada canal de cada grupo igual a un canal de ancho de banda. Esto involucra restricciones menos severas en las especificaciones de los multiplexores. El diseño y optimización de este tipo de multiplexores es particularmente complicado, especialmente si se requiere una banda de guarda estrecha entre cada canal, lo cual es característico en este tipo de aplicaciones. Además, se requiere siempre insertar elementos de sintonía en el diseño para poder regular la respuesta final, ya que sin dichos elementos no suele conseguirse la respuesta deseada tras todo el proceso de diseño y fabricación del dispositivo. Incluso se ha estudiado la posibilidad de montar en satélites este tipo de dispositivos con motores accionados por telecomando para alterar la frecuencia de cada canal del multiplexor [85].

Por todas estas razones recién expuestas, el estudio de multiplexores en guía es, hoy en día, un tema de enorme interés que está generando mucha literatura al respecto. Parte de esta literatura está referida a las estrategias de diseño de estos dispositivos, que suelen requerir de programas de optimización para resolver un problema de esta envergadura.

El proceso de diseño de un multiplexor/diplexor suele ser bastante automatizado. Se parte de las especificaciones eléctricas y se diseñan los filtros (diseño de banda estrecha); seguidamente se hace una aproximación de la parte del diseño referida a la banda ancha (diseño del colector). Finalmente, se analiza el multiplexor completo y se comprueba si éste cumple con las especificaciones. Si no es así, se inicia un algoritmo de optimización que a cada paso

\footnotetext{
${ }^{3}$ Esta es la razón de que se utilice este tipo de multiplexores en equipamiento de alta potencia en estaciones terrenas donde el tamaño y la masa no son parámetros críticos.
} 
analiza el multiplexor completo y comprueba el cumplimiento de las especificaciones. El proceso de optimización continúa hasta que se cumplen las especificaciones requeridas.
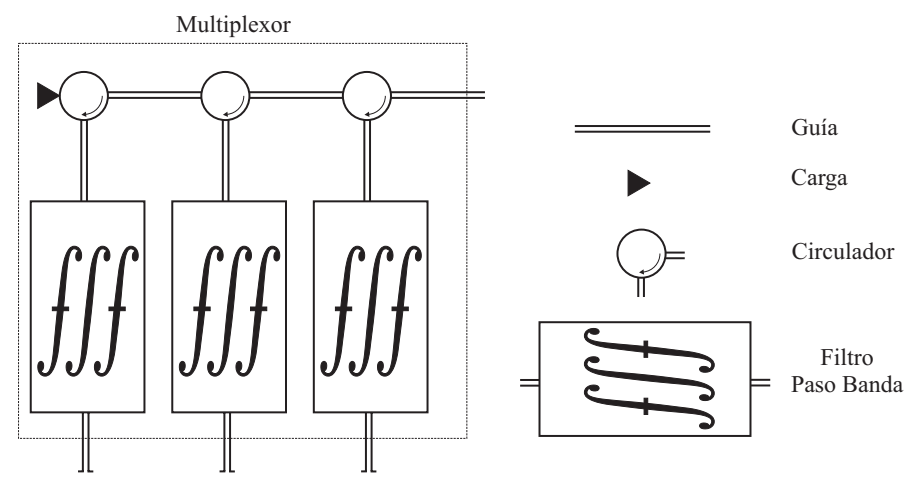

Figura 4.19: Multiplexor no recíproco utilizado como multiplexor de entrada en satélites. Este multiplexor tiene una entrada y tres canales de salida.

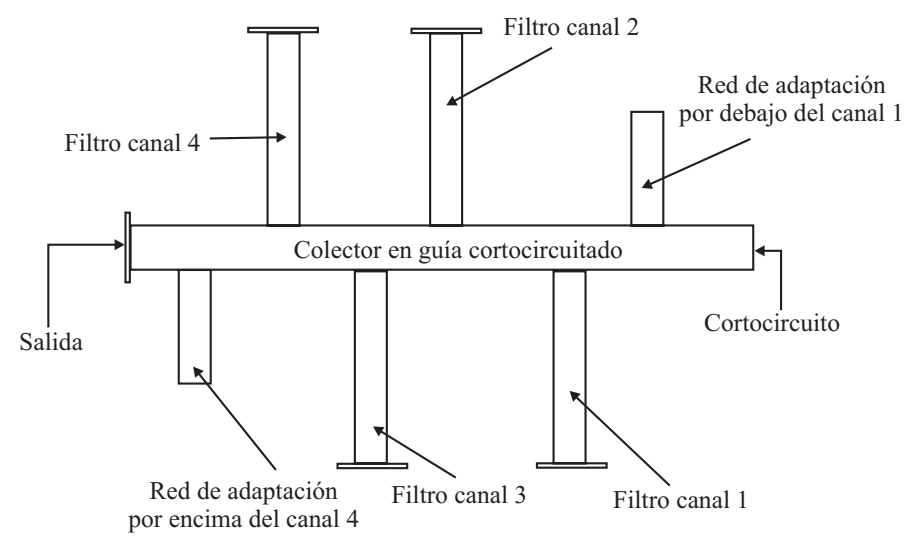

Figura 4.20: Multiplexor en guía con colector común utilizado como multiplexor de salida en satélites. La salida del multiplexor se conecta a la antena. Este multiplexor tiene 4 canales de entrada y una salida (aunque es recíproco). Tiene dos redes de adaptación para eliminar las frecuencias por debajo del canal inferior (canal 1) y por encima del canal superior (canal 4).

\subsubsection{Diferentes geometrías de diplexores}

Las diferentes geometrías existentes en la actualidad vienen dadas por las necesidades de espacio del sistema en el que se integran estos diplexores. Existen, por ejemplo, diplexores cuyo divisor de potencia es una bifurcación en plano $\mathrm{E}$ [80] a la que se añaden los filtros paso alto y paso bajo. La forma de la bifurcación es la que suele forzar gran parte del diseño de estos dispositivos. La forma de bifurcación más extendida es la que se utiliza en los diplexores en colector común, donde el colector puede simularse como un conjunto de uniones 
$\mathrm{T}$ en plano $\mathrm{H}$ conectadas en cascada. En los accesos laterales del colector se emplazan los diferentes filtros.

De todas las geometrías posibles de un diplexor se considerarán aquellas con una estructura en plano $\mathrm{H}$ y con uniones $\mathrm{T}$ en ese mismo plano, ya que el método híbrido tan sólo puede analizar estructuras con discontinuidades y saltos en plano $\mathrm{H}$. Pueden verse diferentes configuraciones posibles de diplexores en plano $\mathrm{H}$ en la figura 4.21.

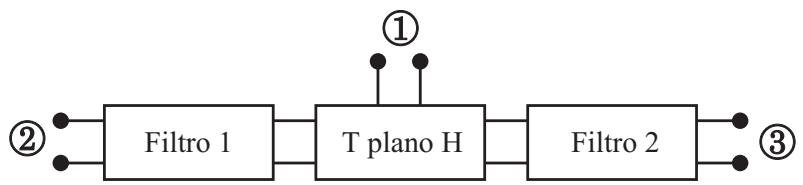

(a)

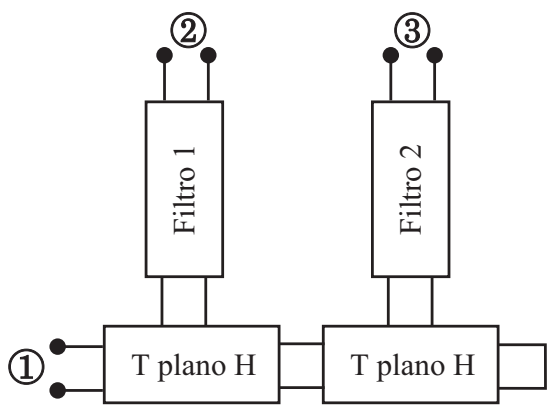

(b)

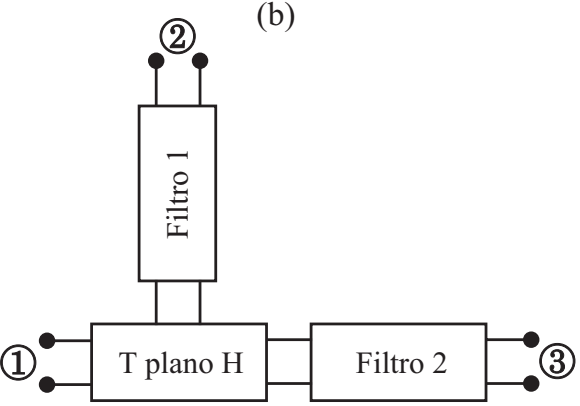

(c)

Figura 4.21: Diferentes configuraciones para diplexores con geometría en plano H.

Según [86] la unión T estándar tiene unas pobres características en cuanto a división de potencia, que hace que el diseño de tales diplexores presente problemas. En la citada referencia se propone una unión $\mathrm{T}$ con un reentrante lateral en la cavidad de la unión para conseguir un mayor ancho de banda en las respuestas de los filtros del diplexor, y relajando a su vez las tolerancias de fabricación. Esto también se puede conseguir con transiciones entre la unión T y los filtros. 


\subsubsection{Análisis de un diplexor del satélite Hispasat 1-C (Alcatel Espacio)}

En esta sección se va a utilizar el método híbrido de análisis de problemas 2-D para analizar un diplexor real utilizado a bordo del satélite Hispasat 1-C, cedido por la empresa Alcatel Espacio S.A.

\section{Características del diplexor}

Este diplexor presenta la configuración (a) de la figura 4.21. Como se observa consta de dos filtros y una unión en T. Para evitar los problemas de la T estándar mencionados en [86], se utiliza una unión T simétrica pero que se conecta a los filtros a través de unas transiciones asimétricas para adaptar las impedancias de dichos filtros y de la unión $\mathrm{T}$ de forma adecuada (las dimensiones $d_{2}, t_{2}$ y $a_{3}^{\prime}$ de un brazo son diferentes a las dimensiones $d_{3}, t_{3}$ y $a_{3}$ del otro). La geometría de la unión T puede verse en la figura 4.22.

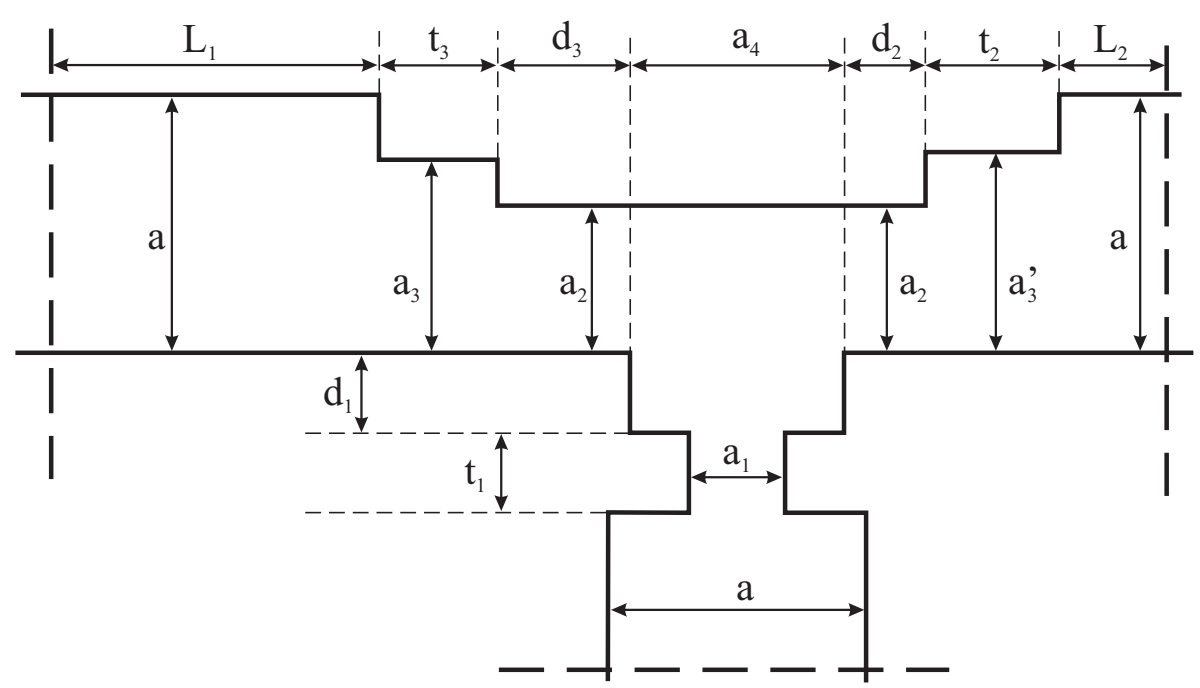

Figura 4.22: Geometría de la unión T asimétrica en plano H con adaptadores de impedancia en los accesos laterales. Los filtros se conectan a los adaptadores de los lados de la unión T. Las entradas/salidas son guías estándar WR-75 ( $a=19,05 \mathrm{~mm}, b=9,525 \mathrm{~mm})$. La dimensión $b$ es constante en todo el diplexor.

Los filtros que se conectan a ambos lados de la unión T son filtros inductivos de 6 cavidades, cuya geometría se recoge en la figura 4.23.

El diplexor en su totalidad se ha construido en guía rectangular estándar WR-75 ( $a=$ $19,05 \mathrm{~mm}, b=9,525 \mathrm{~mm})$. Al ser totalmente inductivo, la dimensión $b$ se mantiene constante en todo el dispositivo.

Este dispositivo se analizará en primer lugar considerando que todos los tramos de línea que lo componen son guías rectangulares estándar. Posteriormente, se analizará el mismo dispositivo teniendo en cuenta las esquinas redondeadas que aparecen a causa de la fabricación con la técnica de corte en plano $\mathrm{H}$, tal y como se ha explicado en la sección anterior (ver figura 4.13). 


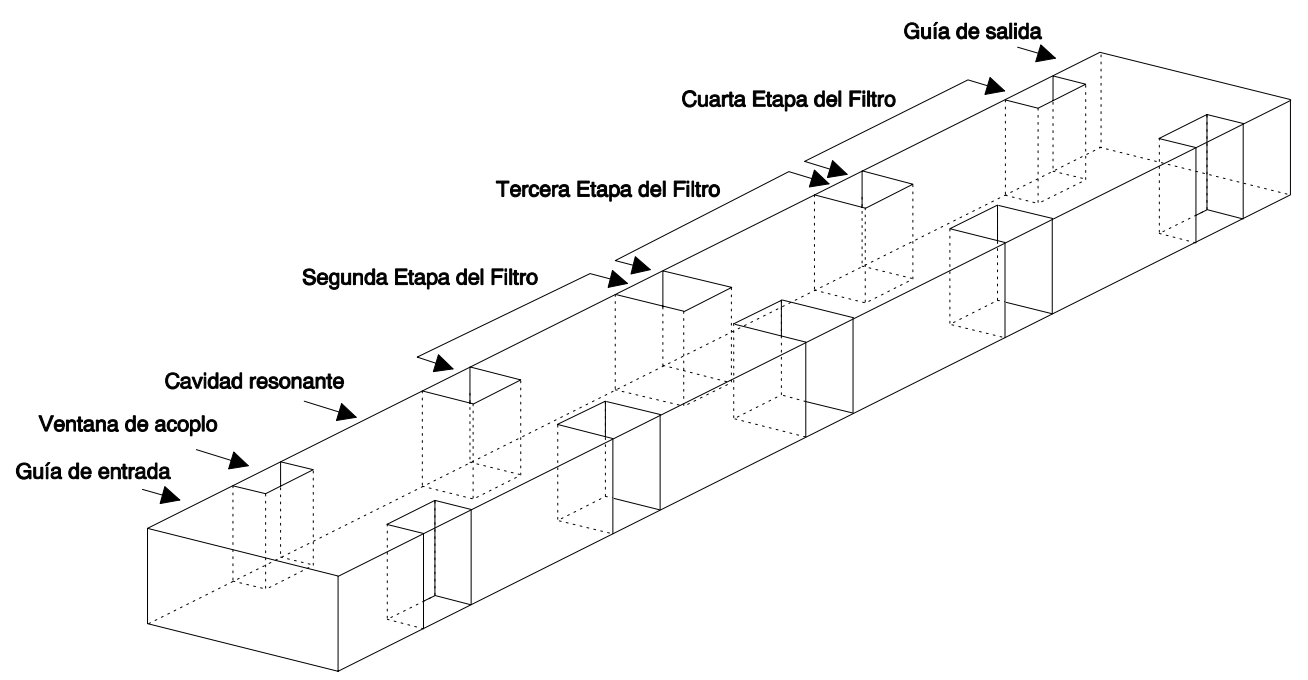

Figura 4.23: Geometría de los filtros inductivos que se conectarán a la unión T asimétrica. Aquí se ha representado un filtro de 4 cavidades que producirá una respuesta con 4 polos. En realidad, los filtros utilizados en el diplexor son de 6 cavidades.

Por cortesía de la empresa Alcatel Espacio, se ha dispuesto de una maqueta de este diplexor, cuyo aspecto puede observarse en las figuras 4.24 a 4.27. Como se ha comentado con anterioridad, los dos filtros inductivos del diplexor presentan tornillos de sintonía. Estos tornillos son necesarios porque aunque se fabrique el dispositivo conforme a las dimensiones proporcionadas por el diseño, pequeños errores en el diseño y/o en el proceso de fabricación hacen que el dispositivo real no presente exactamente la respuesta esperada. Para corregir estas desviaciones de la respuesta real con respecto al comportamiento ideal de la estructura se colocan los mencionados tornillos. Como se aprecia en las figuras 4.24 a 4.27, los tornillos se sitúan en la parte superior de los filtros. En concreto, hay un tornillo en cada cavidad y en cada ventana de acoplo de cada uno de los filtros.

Las características para el diseño del diplexor se resumen a continuación:

Banda de paso del Filtro 1 (F1): $13,00-13,25 \mathrm{GHz}$

Banda de paso del Filtro 2 (F2:) $13,75-14,00 \mathrm{GHz}$

Pérdidas de retorno: $>23 \mathrm{~dB}$ en ambas bandas

Rechazo fuera de banda F1: $>45 \mathrm{~dB} \forall f<12,75 \mathrm{GHz}$ y $\forall f>13,50 \mathrm{GHz}$

Rechazo fuera de banda F2: $>45 \mathrm{~dB} \forall f<13,50 \mathrm{GHz}$ y $\forall f>14,25 \mathrm{GHz}$

Las especificaciones anteriores han supuesto tener que utilizar filtros paso banda de Chebyshev de 6 polos. 


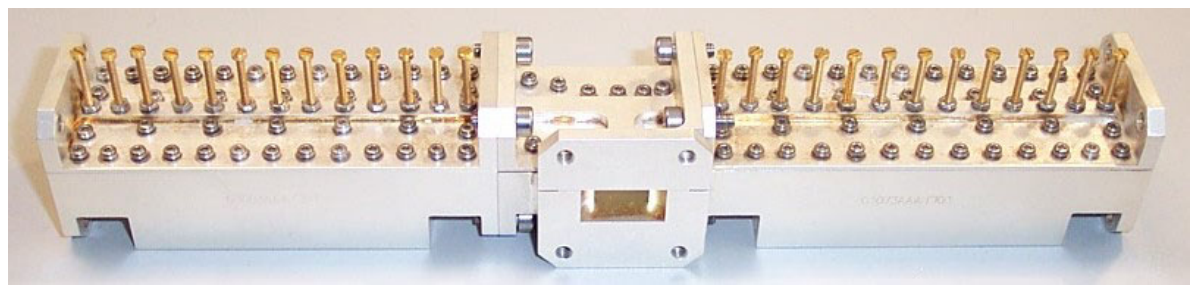

Figura 4.24: Foto del diplexor analizado. Se aprecian los dos filtros a ambos lados de la unión en T. En la parte superior de los filtros se aprecian tornillos que han sido colocados para sintonizar adecuadamente la respuesta del dispositivo.

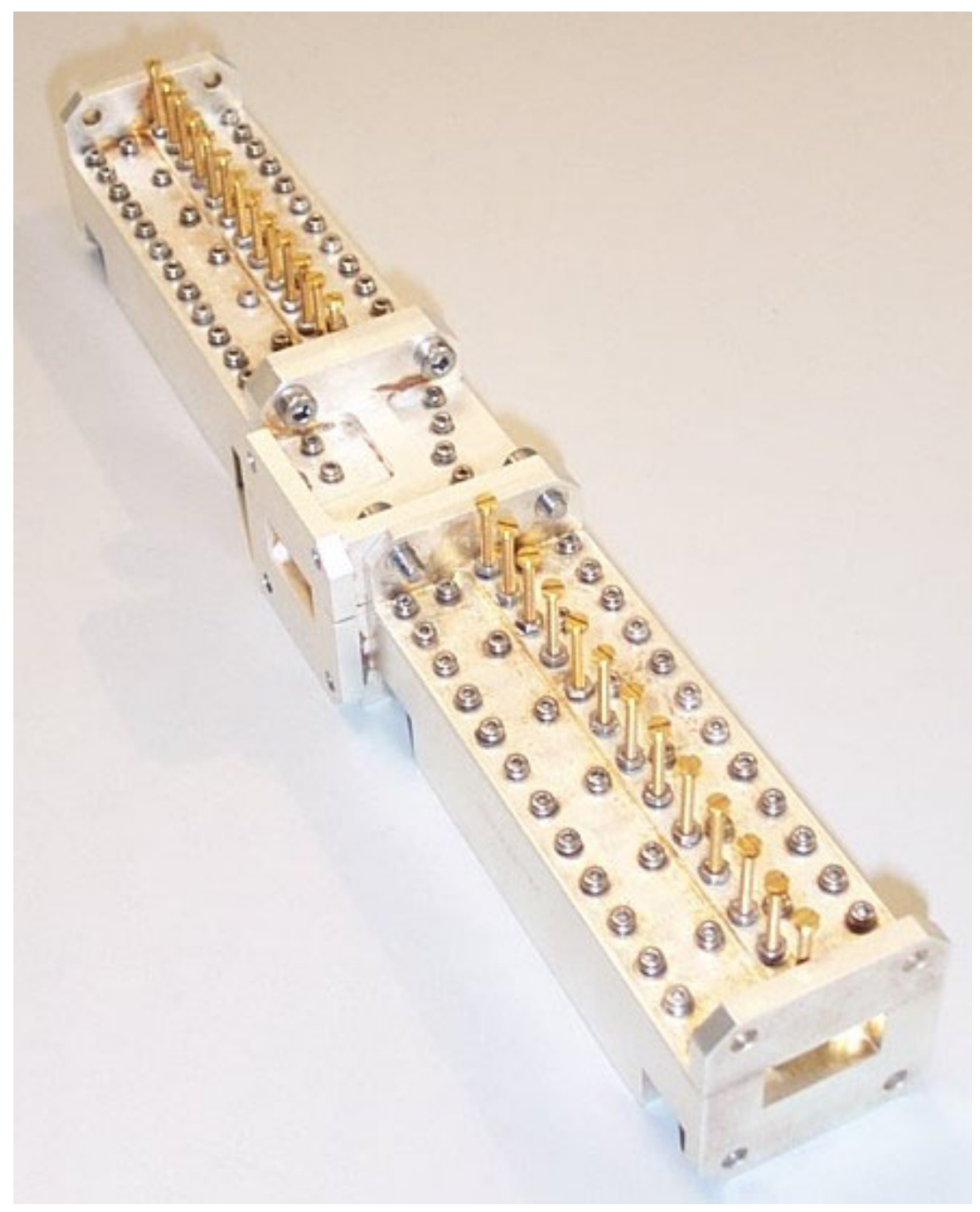

Figura 4.25: Foto del diplexor analizado. Se aprecian los accesos laterales así como los tornillos superiores para la sintonización del dispositivo. 


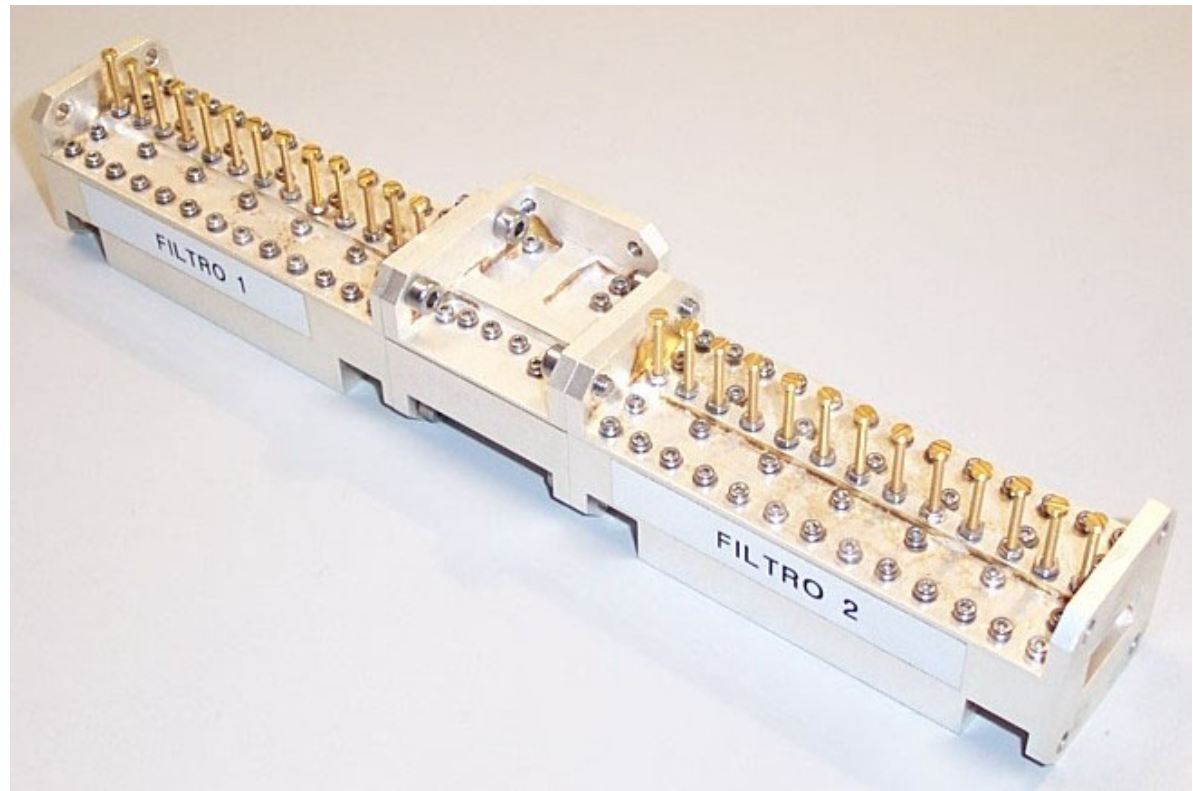

Figura 4.26: Foto del diplexor analizado. En la parte trasera del mismo se ha puesto una etiqueta de referencia para identificar los filtros.

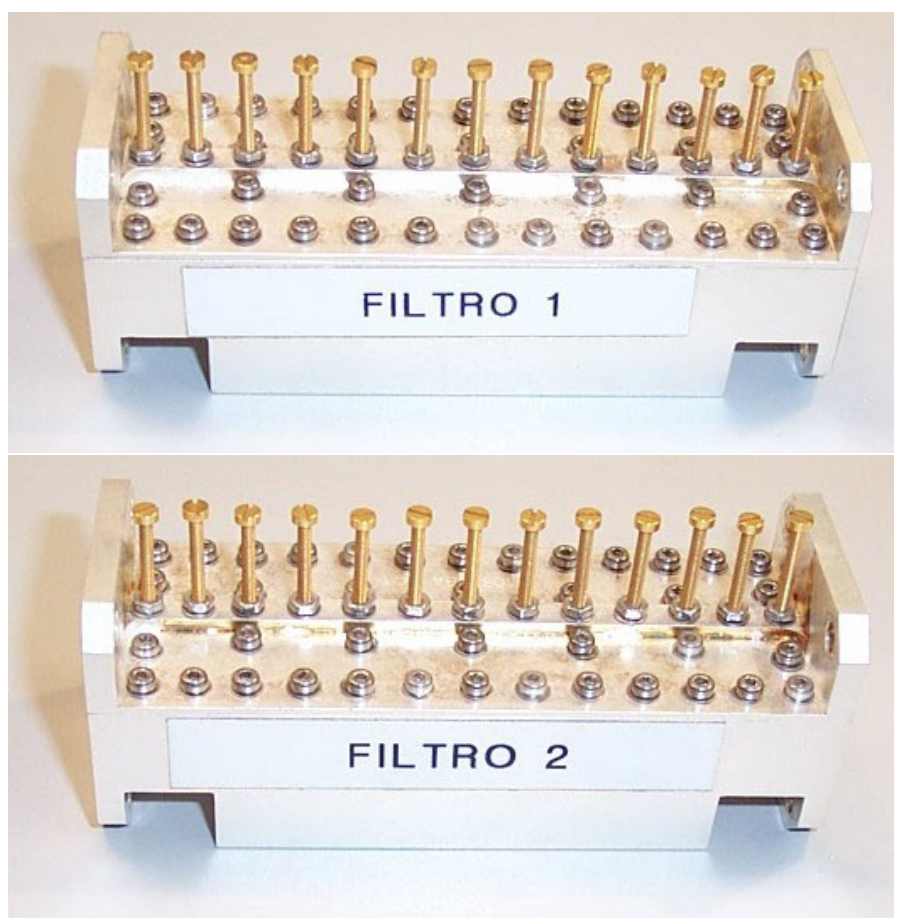

Figura 4.27: Detalle de cada uno de los filtros del diplexor. 


\section{Procedimiento de medida}

Con el objetivo de validar los resultados que proporcione el método híbrido tras analizar una estructura tan compleja como es el diplexor, se han realizado medidas de los parámetros de dispersión de la maqueta mostrada en las figuras 4.24 a 4.27. Dichas medidas se han realizado en el laboratorio de investigación de Radiocomunicaciones del Departamento de Comunicaciones de la Universidad Politécnica de Valencia. En dicho laboratorio se dispone recientemente de un analizador de redes con capacidad de medida hasta $20 \mathrm{GHz}$. El equipo de medida se muestra en la figura 4.28.

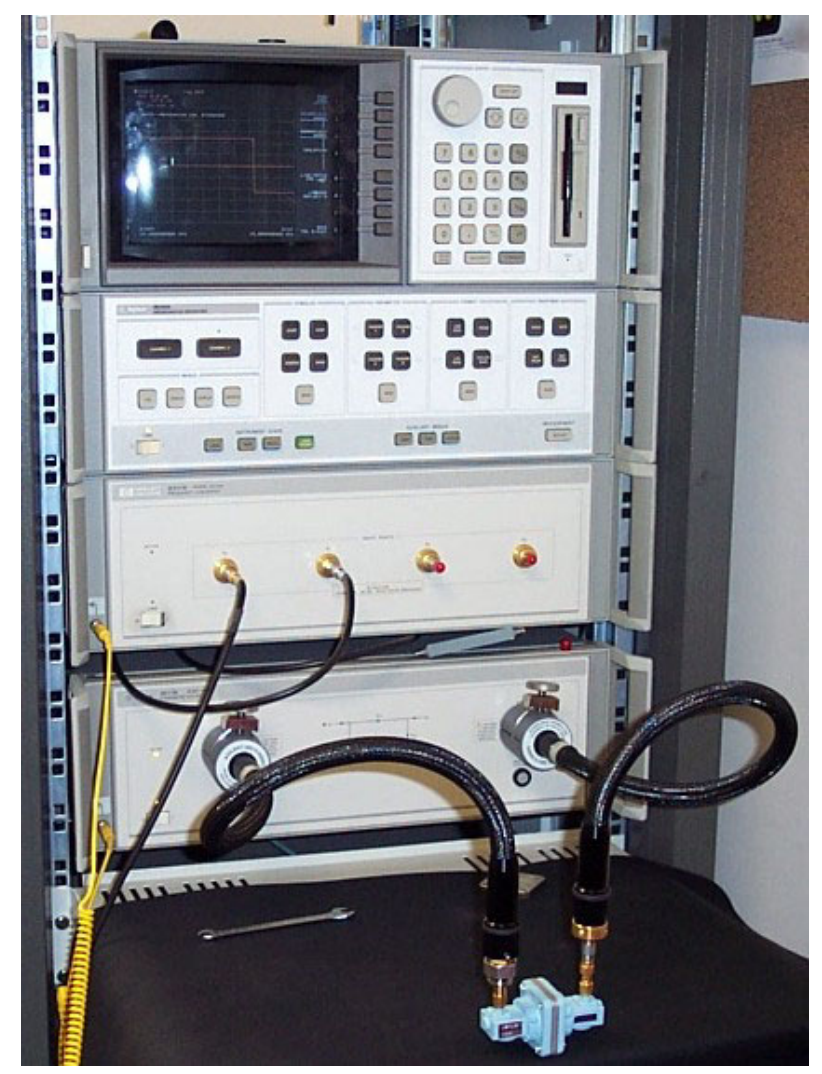

Figura 4.28: Analizador de redes

Para realizar las medidas, el primer paso es realizar una calibración. En este caso se realizó una calibración del tipo TRL (ver figura 4.29).

Para identificar los accesos y poder determinar de forma unívoca los parámetros de dispersión, se han numerado los puertos de acceso del diplexor siguiendo el criterio mostrado en el esquema de la figura 4.30. Como se puede apreciar en el esquema el acceso común (la entrada a la T) es el primer acceso, la salida del filtro 1 es el segundo acceso, y la salida del filtro 2 es el tercer acceso. Con esta numeración, el parámetro $S_{11}^{(2,1)}$ nos proporciona la transmisión de señal desde el puerto común (modo fundamental) a la salida del filtro 1 (modo fundamental). 


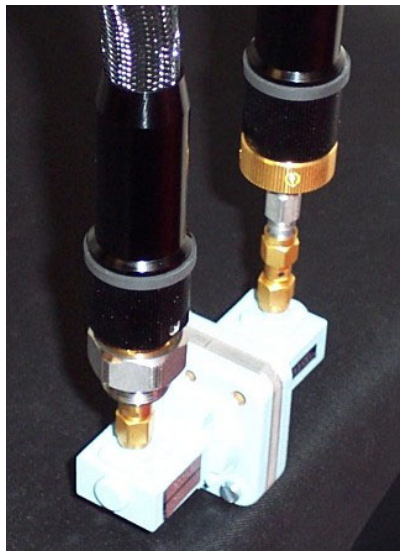

Figura 4.29: Montaje para realizar la calibración del analizador de redes HP8510B

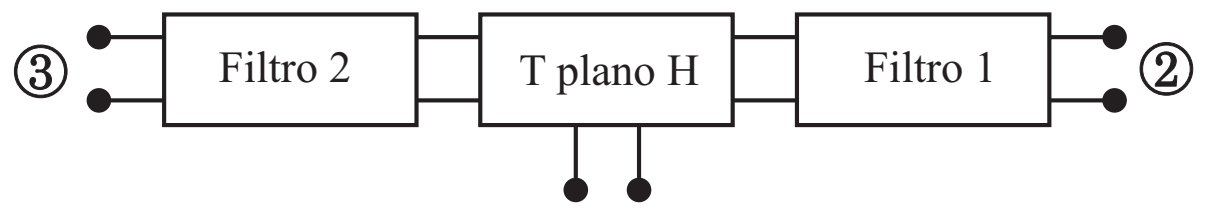

(1)

Figura 4.30: Numeración de los accesos en el diplexor. El primer acceso es el acceso común (entrada a la T). La salida del filtro 1 es el segundo acceso, y la salida del filtro 2 es el tercer acceso

El procedimiento de medida es el siguiente:

- En primer lugar se termina con una carga adaptada el filtro 2 (acceso 3) y se coloca la primera sonda de medida en el puerto común (acceso 1) y la segunda a la salida del filtro 1 (acceso 2). Esta configuración se muestra en la figura 4.31.a. Con esta configuración, se han medido los parámetros relativos a los puertos 1 y 2 del diplexor, es decir, los parámetros $S_{11}^{(1,1)}, S_{11}^{(2,1)}, S_{11}^{(1,2)}$ y $S_{11}^{(2,2)}$.

- A continuación se termina con una carga adaptada el filtro 1 (acceso 2) y se coloca la primera sonda en el puerto común (acceso 1) y la segunda sonda a la salida del filtro 2 (acceso 3), tal y como se muestra en la figura 4.31.b. De esta forma se han medido los parámetros $S_{11}^{(1,1)}, S_{11}^{(3,1)}, S_{11}^{(1,3)}$ y $S_{11}^{(3,3)}$.

- Finalmente, se coloca la carga adaptada en el puerto común, y la primera sonda de medida se conecta a la salida del filtro 1 (acceso 2) y la segunda sonda a la salida del filtro 2 (acceso 3). Con esta configuración al analizador nos proporcionará los parámetros $S_{11}^{(2,2)}, S_{11}^{(3,2)}, S_{11}^{(2,3)}$ y $S_{11}^{(3,3)}$. 


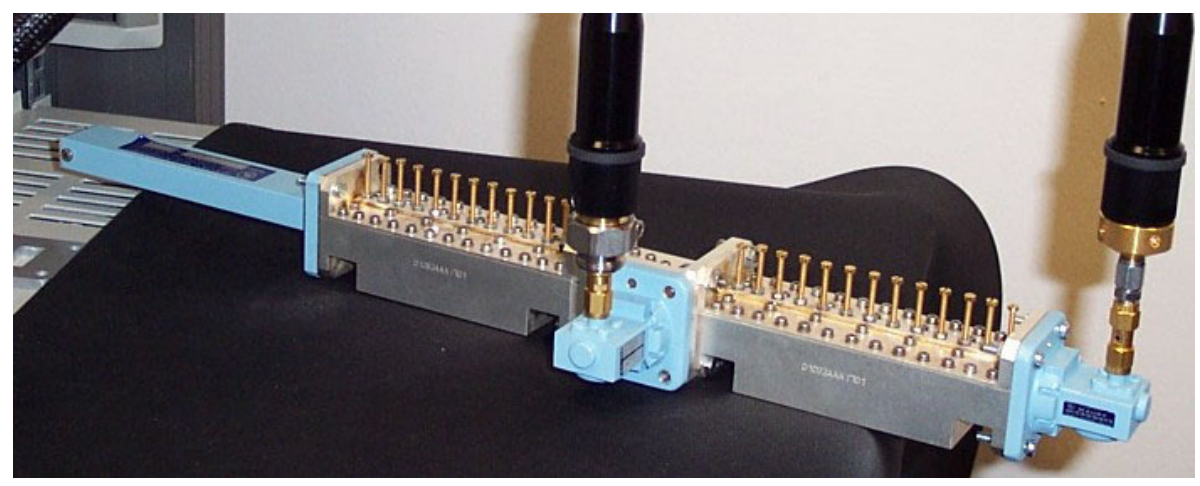

(a) Filtro 2 terminado con carga adaptada

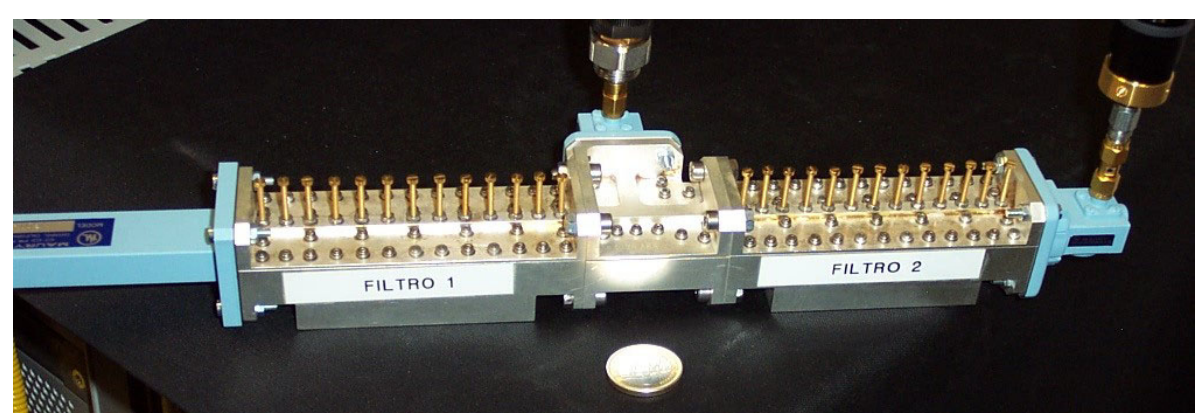

(b) Filtro 1 terminado con carga adaptada (se incluye una moneda de 1e para apreciar el tamaño)

Figura 4.31: Montaje para realizar las medidas de los parámetros de dispersión del diplexor del satélite Hispasat 1-C

\subsubsection{Simulación}

El análisis del diplexor se ha realizado con el método híbrido 2-D propuesto en el capítulo anterior, para lo cual el diplexor se ha descompuesto en los siguientes bloques (ver figura 4.32):

1. Unión $\mathrm{T}$ en plano $\mathrm{H}$ junto con dos saltos de sección también en plano H.

2. Estrechamiento inductivo a la entrada del puerto común.

3. Salto de sección en plano $\mathrm{H}$ a la entrada del filtro 1.

4. Filtro de cavidades inductivas número 1.

5. Salto de sección en plano $\mathrm{H}$ a la entrada del filtro 2.

6. Filtro de cavidades inductivas número 2. 


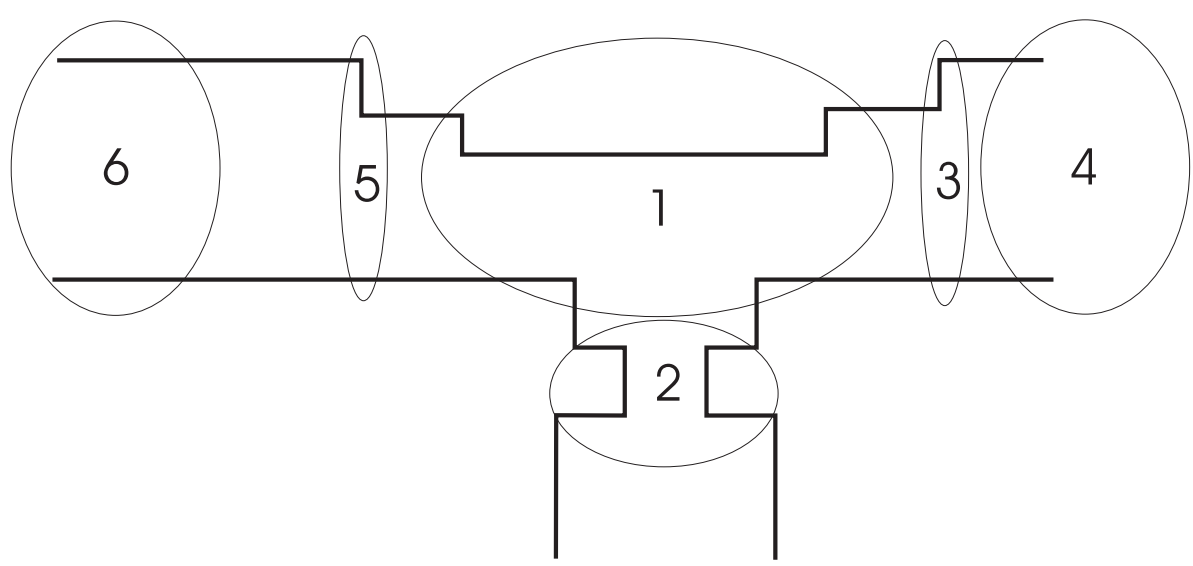

Figura 4.32: Esquema de los bloques en los que se ha dividido el diplexor para su análisis. (1) Unión T y dos saltos en plano H. (2) Estrechamiento en plano H. (3) Salto de sección a la entra del filtro 1. (4) Filtro 1. 5) Salto de sección a la entrada del filtro 2. (5) Filtro 2

Una vez segmentado el diplexor en varios bloques, cada uno de ellos ha sido analizado por separado con el método híbrido 2-D, y se han obtenido las MDGs de cada uno de los bloques. Finalmente, y de acuerdo con las expresiones que se detallan en el apéndice F, se han enlazado las MDGs de las redes por pasos hasta obtener la MDG total del diplexor. Primero se enlazan las MDGs de la unión T y la del estrechamiento, obteniendo una MDG conjunta de las dos redes. A continuación se enlaza la MDG resultante con el salto a la entrada del filtro 1, y luego con la MDG del filtro 1. Y finalmente con la MDG del salto a la entrada del filtro 2, y se termina enlazando con la MDG del filtro 2, tras lo cual se dispone de la MDG completa del diplexor.

Para analizar cada uno de los bloques se han utilizado los siguientes parámetros de control a la hora de realizar el acoplo modal de modos de abiertos a modos guiados (el significado de cada parámetros ha sido explicado con detalle en la sección 3.4):

- Unión T en plano H junto con dos saltos de sección también en plano H:

\begin{tabular}{|c|c|c|c|c|c|l|}
\hline $\mathrm{dNc}$ & $\mathbf{R}$ & $\mathbf{R}_{\mathrm{AB}}$ & $\mathbf{R}_{\mathrm{fft}}$ & $\mathbf{C}_{\mathrm{sc}}$ & Modos & Tiempo \\
\hline 150 & $13.5 \mathrm{~mm}$ & 5 & 8 & 6 & $13 / 13 / 13$ & $5.44 \mathrm{seg}$. \\
\hline
\end{tabular}

En la casilla de modos se ha apuntado el número de modos $T E_{m 0}$ utilizados en cada uno de los tres puertos de accesos de la red.

- Estrechamiento inductivo a la entrada del puerto común:

\begin{tabular}{|c|c|c|c|c|c|l|}
\hline $\mathrm{dNc}$ & $\mathbf{R}$ & $\mathbf{R}_{\mathrm{AB}}$ & $\mathbf{R}_{\mathrm{fft}}$ & $\mathbf{C}_{\mathrm{sc}}$ & Modos & Tiempo \\
\hline 150 & $19.06 \mathrm{~mm}$ & 5 & 8 & 6 & $13 / 13$ & $1.99 \mathrm{seg}$. \\
\hline
\end{tabular}


- Salto de sección en plano H a la entrada del filtro 1:

\begin{tabular}{|c|c|c|c|c|c|l|}
\hline $\mathbf{d N c}$ & $\mathbf{R}$ & $\mathbf{R}_{\mathrm{AB}}$ & $\mathbf{R}_{\mathrm{fft}}$ & $\mathbf{C}_{\mathrm{sc}}$ & Modos & Tiempo \\
\hline 150 & $19.05 \mathrm{~mm}$ & 5 & 8 & 6 & $13 / 17$ & $2.85 \mathrm{seg}$. \\
\hline
\end{tabular}

- Filtro de cavidades inductivas número 1:

\begin{tabular}{|c|c|c|c|c|c|c|}
\hline $\mathrm{dNc}$ & $\mathbf{R}$ & $\mathbf{R}_{\mathrm{AB}}$ & $\mathbf{R}_{\mathrm{fft}}$ & $\mathbf{C}_{\mathrm{sc}}$ & Modos & Tiempo \\
\hline 150 & - & 5 & 8 & 6 & $17 / 17$ & $12.43 \mathrm{seg}$. \\
\hline
\end{tabular}

En la casilla $R$ correspondiente al radio de la circunferencia que contiene a los objetos 2-D no se ha puesto nada porque el filtro inductivo se ha descompuesto en varias subredes (cada uno de los estrechamientos que separan dos cavidades) y cada una de ellas ha sido analizada por separado. Posteriormente se han enlazado las MDGs de todos los estrechamientos para obtener la MDG final de todo el filtro.

- Salto de sección en plano H a la entrada del filtro 2:

\begin{tabular}{|c|c|c|c|c|c|l|}
\hline $\mathrm{dNc}$ & $\mathbf{R}$ & $\mathbf{R}_{\mathrm{AB}}$ & $\mathbf{R}_{\mathrm{fft}}$ & $\mathbf{C}_{\mathrm{sc}}$ & Modos & Tiempo \\
\hline 150 & $19.05 \mathrm{~mm}$ & 5 & 8 & 6 & $17 / 13$ & $2.85 \mathrm{seg}$. \\
\hline
\end{tabular}

- Filtro de cavidades inductivas número 2:

\begin{tabular}{|c|c|c|c|c|c|l|}
\hline $\mathrm{dNc}$ & $\mathbf{R}$ & $\mathbf{R}_{\mathrm{AB}}$ & $\mathbf{R}_{\mathrm{fft}}$ & $\mathbf{C}_{\mathrm{sc}}$ & Modos & Tiempo \\
\hline 150 & - & 5 & 8 & 6 & $17 / 17$ & $10.44 \mathrm{seg}$. \\
\hline
\end{tabular}

Como se puede observar en las tablas anteriores, el número de modos guiados utilizado es muy elevado comparado con el número de modos empleado para obtener los resultados de problemas sencillos de la sección 3.4. También se ha aumentado el parámetros $R_{A B}$ que determina el número de modos del espectro cilíndrico que se consideran. Esto ha hecho que el tiempo de computación también aumente. La razón por la cual ha sido necesario aumentar tanto el número de modos guiados y cilíndricos es porque las MDGs de cada uno de los dispositivos han de ser muy exactas (y con muchos modos guiados) para que al enlazar las MDGs de dos redes consecutivas se analice correctamente las interacciones entre las citadas redes. En cambio, cuando tan sólo se desean los parámetros de dispersión de una red aislada para el modo fundamental, como es el caso de los resultados de la sección 3.4, con muchos menos modos se obtienen ya resultados satisfactorios, que se superponen con éxito 
al compararlos con los parámetros de dispersión del modo fundamental de publicaciones científicas.

Sumando los tiempos de computación de cada elemento por separado, se obtiene un tiempo de computación total del diplexor de 36 segundos por punto en frecuencia. Lo que supone un esfuerzo computacional de alrededor de una hora para una simulación con 100 puntos. Es obvio que existen métodos de análisis específicos para este tipo de dispositivos mucho más eficientes, pero que son incapaces de analizar geometrías no canónicas como por ejemplo diplexores con esquinas redondeadas producidas por la técnica de fabricación. En cambio, con el método híbrido, el diplexor con esquinas rectas o con esquinas redondeadas supone idéntico esfuerzo computacional, tal y como se mostrará más adelante.

\section{Resultados}

En la figura 4.33 se muestra el factor de reflexión (parámetro $S_{11}^{(1,1)}$ ) en la puerta común del diplexor. En la gráfica se comparan los resultados proporcionados por el método híbrido 2-D y las medidas realizadas con el analizador de redes. Las medidas se han realizado sobre el diplexor con tornillos de sintonía (ver figura 4.24).

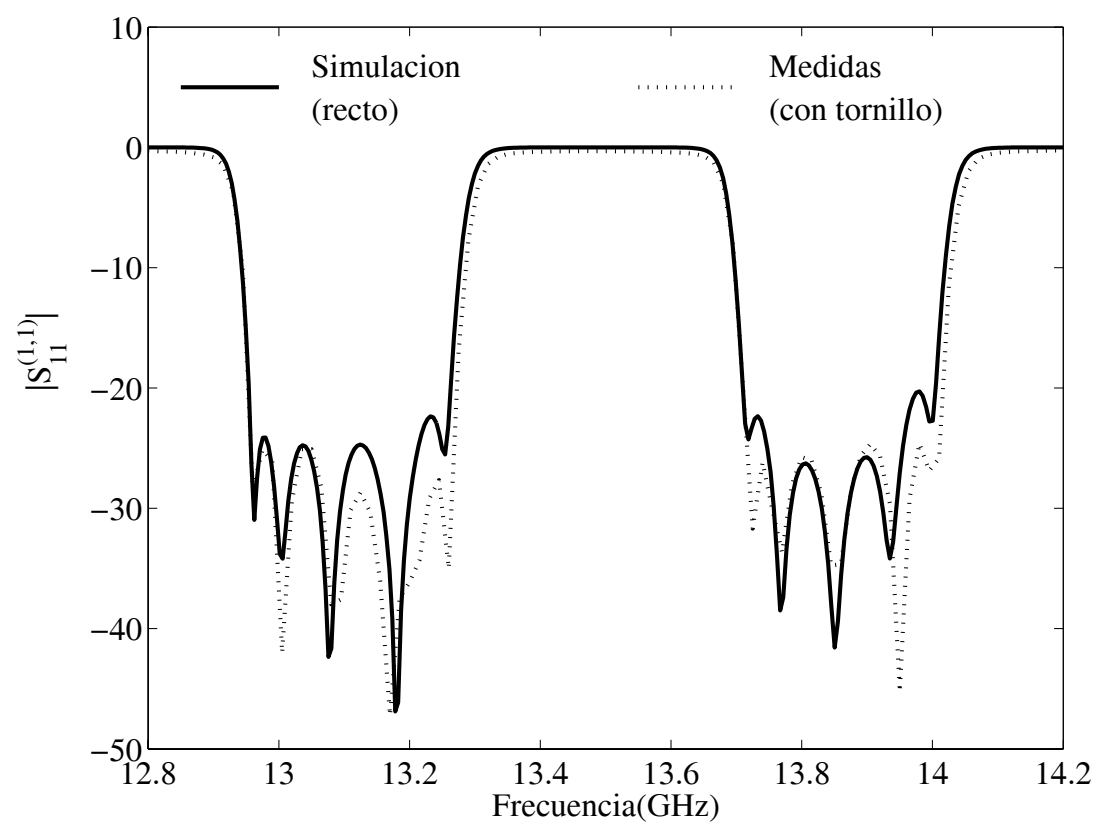

Figura 4.33: Factor de reflexión en la puerta común del diplexor con tornillos de sintonía. Comparación entre medidas y simulación (esquinas rectas)

Se observa en la figura que existe una buena concordancia entre las medidas del diplexor con tornillos y la simulación del método híbrido, a pesar de que en el método híbrido no se ha tenido en cuenta la presencia de los tornillos de sintonía. Esta concordancia se debe a que, como ya se ha comentado con anterioridad, los tornillos de sintonía se utilizan para 
corregir las desviaciones que sufre la respuesta del diplexor una vez fabricado con respecto a la respuesta ideal que predice el simulador electromagnético tras la etapa de diseño. De modo que los tornillos, ajustados de forma adecuada por personal experto de la empresa Alcatel Espacio, consiguen que la respuesta real del diplexor con tornillos sea muy parecida a la que proporciona su simulador electromagnético, y que por tanto se aproxima mucho también a la simulación del método híbrido 2-D.

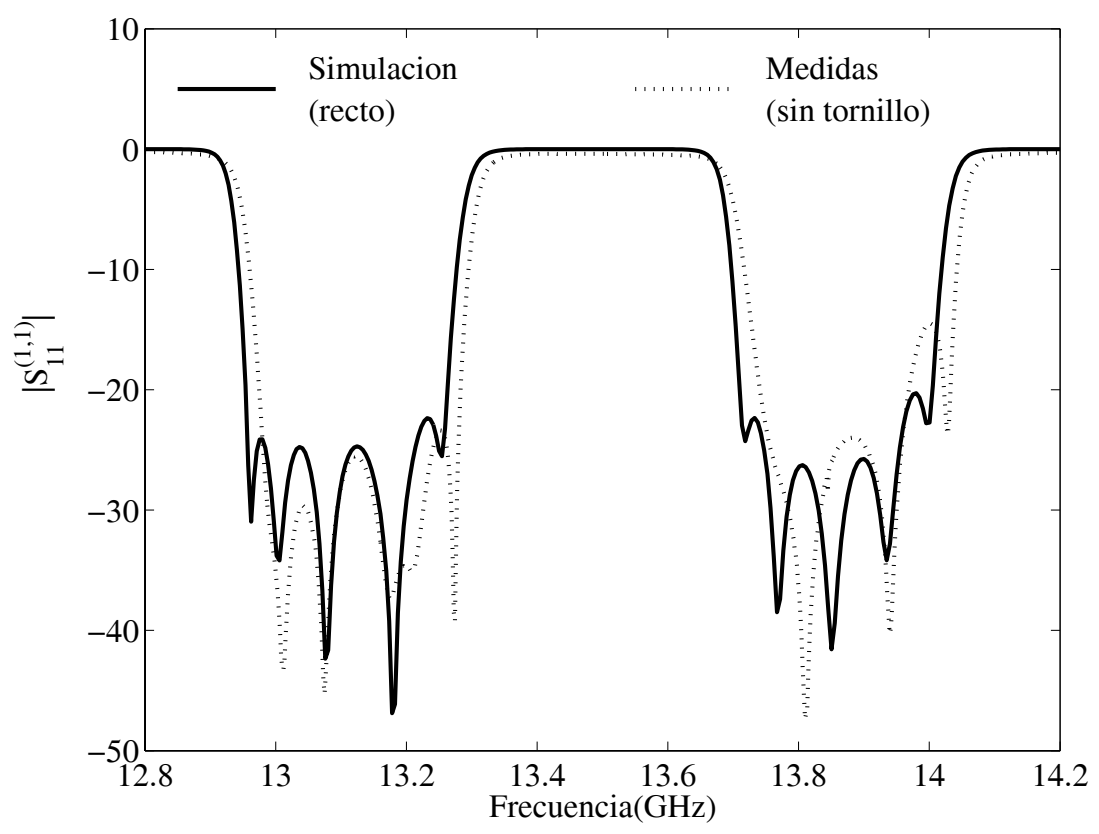

Figura 4.34: Factor de reflexión en la puerta común del diplexor sin tornillos de sintonía. Comparación entre medidas y simulación (esquinas rectas)

En la figura 4.34 se muestra de nuevo el factor de reflexión en la puerta común del diplexor, pero en este caso las medidas se han tomado sobre el diplexor sin tornillos de sintonía. Más exactamente, los tornillos de sintonía siguen presentes, pero se ha modificado la posición de todos ellos para que su penetración dentro del diplexor sea nula. Se observa en este caso la diferencia entre la respuesta que predice un simulador electromagnético y la respuesta real del mismo una vez fabricado.

Esta diferencia se debe fundamentalmente a los errores de mecanizado producidos por la técnica de fabricación. En este caso la técnica utilizada es el corte en plano $\mathrm{H}$, que como se ha comentado anteriormente produce esquinas redondeadas como las mostradas en el esquema de la figura 4.13. En la figura 4.35 se muestra un detalle de la unión T del diplexor en la que se puede apreciar la existencia de esquinas redondeadas como las mostradas en la figura 4.13. Se ha medido el radio de curvatura de las esquinas redondeadas del diplexor, y éste ha resultado ser de aproximadamente $3 \mathrm{~mm}$. 


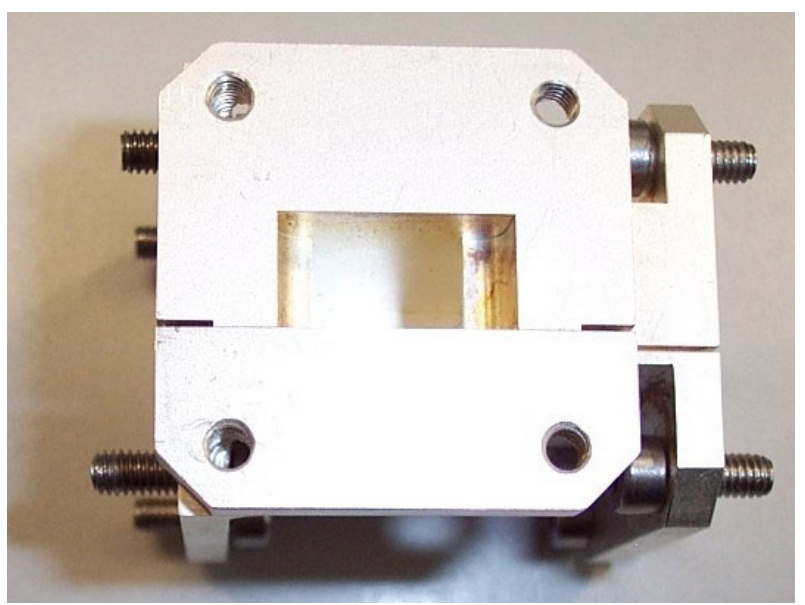

Figura 4.35: Detalle de las esquinas redondeadas existentes en la union $\mathrm{T}$ de la maqueta del diplexor a bordo del satélite Hispasat 1-C

Como el método híbrido 2-D permite analizar cualquier estructura en plano H en guía rectangular, se ha simulado la respuesta del diplexor con esquinas redondeadas con radio de curvatura $3 \mathrm{~mm}$. La simulación se ha realizado con el mismo valor de los parámetros de control que ya se han expuesto para el caso del diplexor con esquinas rectas, y el tiempo de computación consumido por la simulación ha resultado de 55 segundos por punto en frecuencia, ligeramente superior al del diplexor con esquinas rectas debido a la presencia de esquinas redondeadas que implica mayores dimensiones eléctricas de las subestructuras (estrechamientos entre cavidades) que se han de analizar.

En la figura 4.36 se muestra de nuevo el factor de reflexión en la puerta común del diplexor. Esta vez se comparan las medidas sobre el diplexor sin tornillos de sintonía con la respuesta proporcionada por el método híbrido considerando esquinas redondeadas.

Como se puede apreciar en la figura, al tener en cuenta las esquinas redondeadas el método híbrido es capaz de predecir correctamente la posición en frecuencia de la respuesta del diplexor. No obstante, en las dos bandas de paso, el número de polos, su posición, y la altura de los lóbulos no es coincidente entre las medidas y la simulación.

Estas diferencias pueden ser debidas a varias causas. En primer lugar, el simulador no considera la existencia de tornillos de sintonía, y sin embargo en el diplexor sí existen tornillos. Aunque se ha ajustado manualmente la penetración de los tornillos para que queden a ras de la pared superior de los filtros, es difícil realizar estos ajustes a ojo, y las penetraciones pueden no ser adecuadas (pueden penetrar un poco o retirarse hacia atrás ligeramente). Además la terminación de los tornillos no es completamente plana. Todas estas imperfecciones pueden afectar en gran medida a los resultados, ya que los tornillos se hallan en el centro de la guía, donde el flujo de potencia electromagnética es máximo. Por otro lado, la medida del radio de curvatura de las esquinas redondeadas del diplexor no pudo realizarse con exactitud, ya que para ello hubiera sido necesario retirar la tapa superior del diplexor, y no se disponía del equipo necesario para volver a colocar de nuevo la tapa correctamente. 


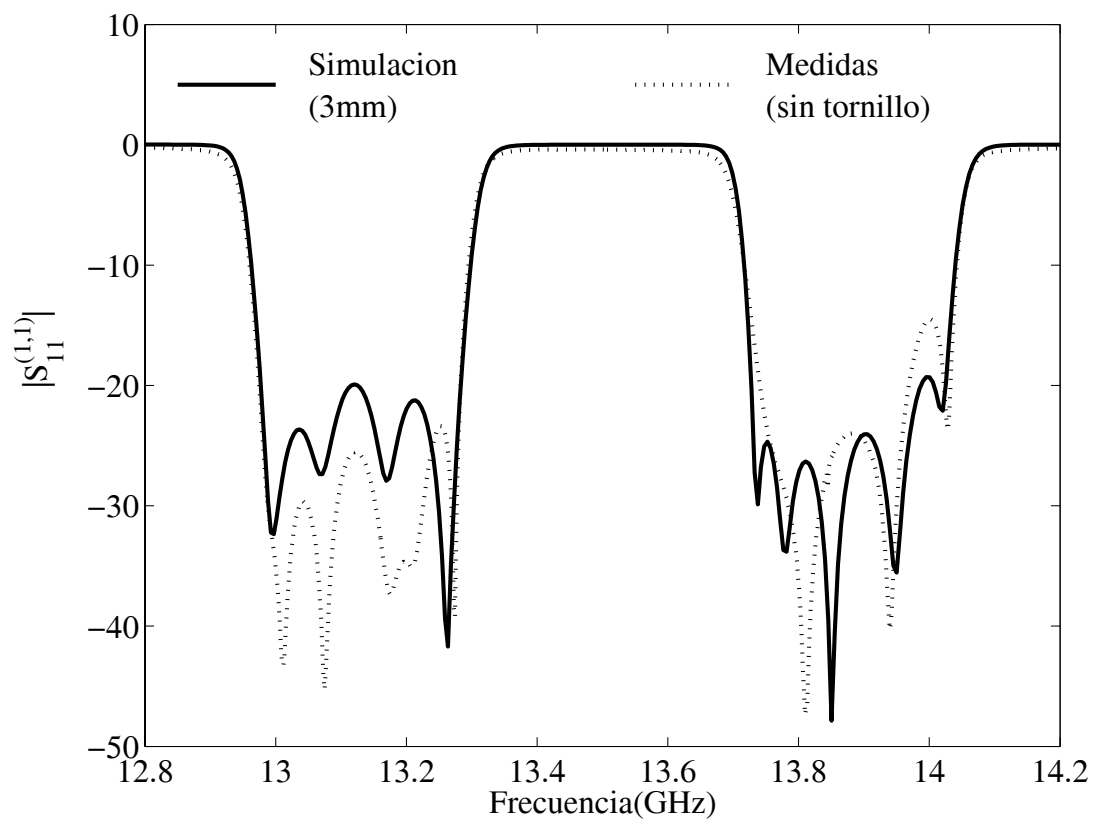

Figura 4.36: Factor de reflexión en la puerta común del diplexor sin tornillos de sintonía. Comparación entre medidas y simulación con esquinas redondeadas ( $R=3 \mathrm{~mm})$

En las figuras 4.37, 4.38 y 4.39 se muestran el resto de los parámetros de dispersión del diplexor. En la figura 4.37 se muestran $S_{11}^{(2,1)}$, y $S_{11}^{(3,1)}$. Se trata de la transmisión desde el puerto común a las salidas de los filtros 1 y 2 .

En la figura 4.38 se representan los parámetros $S_{11}^{(2,2)}, S_{11}^{(1,2)}$, y $S_{11}^{(3,2)}$. Se trata, respectivamente, del factor de reflexión en el puerto 2 (la salida del filtro 1), y la transmisión desde el filtro 1 a las salidas del puerto común y del filtro 2 .

Finalmente, en la figura 4.39 se representan los parámetros $S_{11}^{(3,3)}, S_{11}^{(1,3)}$, y $S_{11}^{(2,3)}$. Se trata, respectivamente, del factor de reflexión en el puerto 3 (la salida del filtro 2), y la transmisión desde el filtro 2 a las salidas del puerto común y del filtro 1 .

Los comentarios respecto a la comparación de las medidas y los resultados de la simulación con esquinas redondeadas para los parámetros de las figuras 4.37, 4.38 y 4.39 son los mismos que ya se han hecho para los parámetros de la figura 4.36, añadiendo que en el caso de los parámetros $S_{11}^{(2,3)}$ y $S_{11}^{(3,2)}$ el nivel de los mismos se encuentra por debajo del fondo de ruido del analizador y que por tanto las medidas no son significativas, no pudiéndose establecer pues una comparación con las simulaciones. 
En conclusión, si en el proceso de diseño de un diplexor se utilizase el método híbrido 2-D en lugar de un simulador que no considera la existencia de esquinas redondeadas, posiblemente no sería necesaria la inclusión de tornillos de sintonía en el diplexor. De esta forma se reduciría la complejidad en la fabricación del mismo, y no sería necesaria la intervención manual de un operario experto que ajuste la posición de los tornillos. Y en caso de que los tornillos siguieran siendo necesarios, el ajuste que se habría de conseguir con los tornillos sería de menor magnitud.
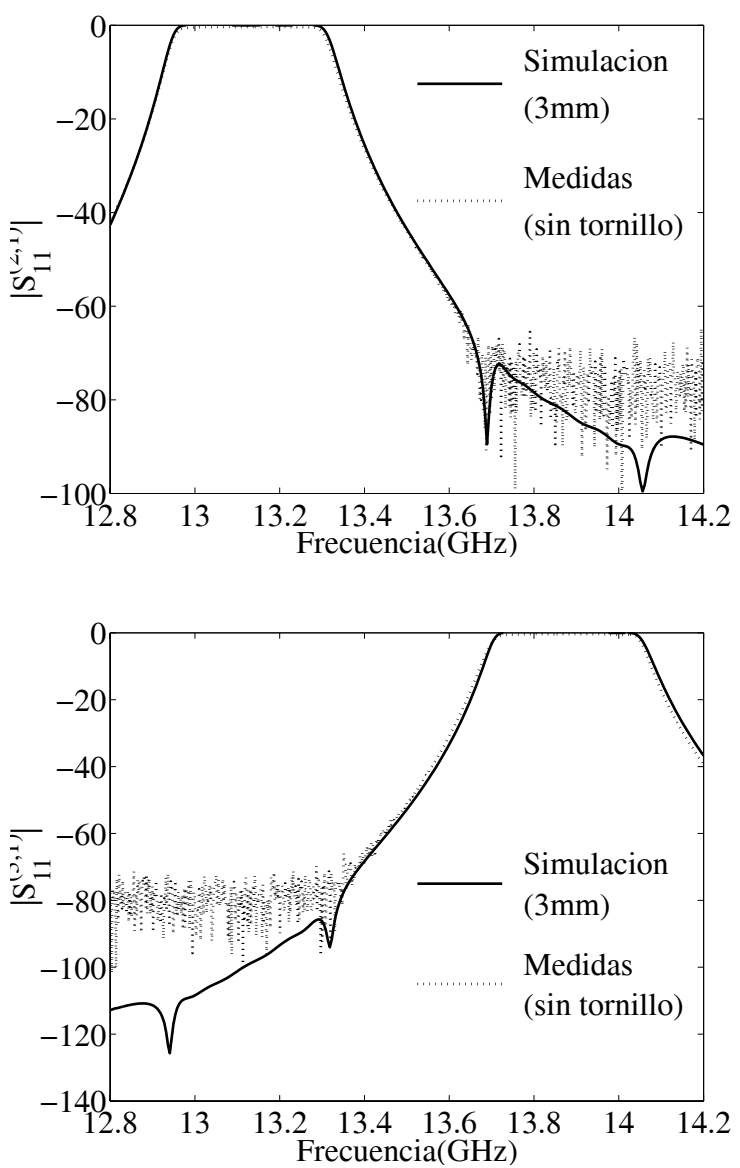

Figura 4.37: Parámetros de dispersión del diplexor a bordo del Hispasat 1-C. $S_{11}^{(2,1)}$, y $S_{11}^{(3,1)}$ 

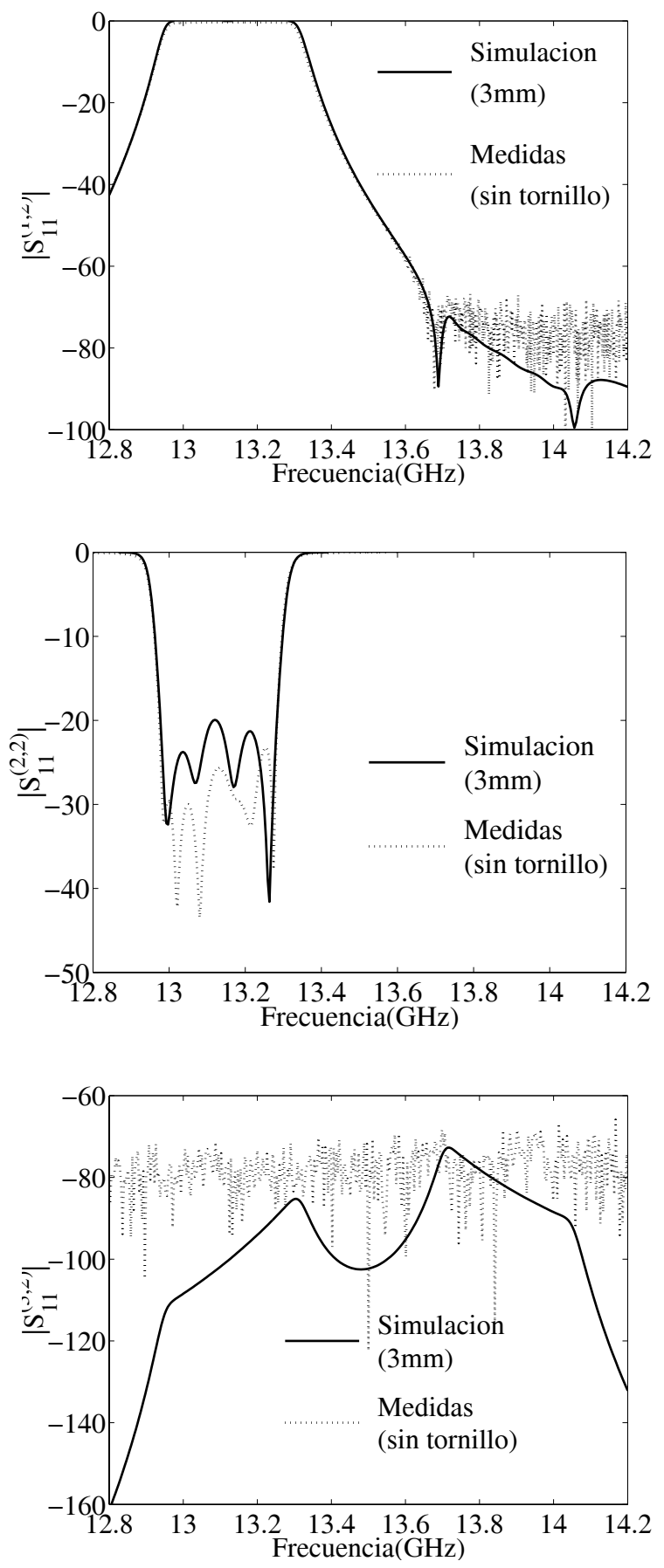

Figura 4.38: Parámetros de dispersión del diplexor a bordo del Hispasat 1-C. $S_{11}^{(1,2)}, S_{11}^{(2,2)}$, y $S_{11}^{(3,2)}$ 

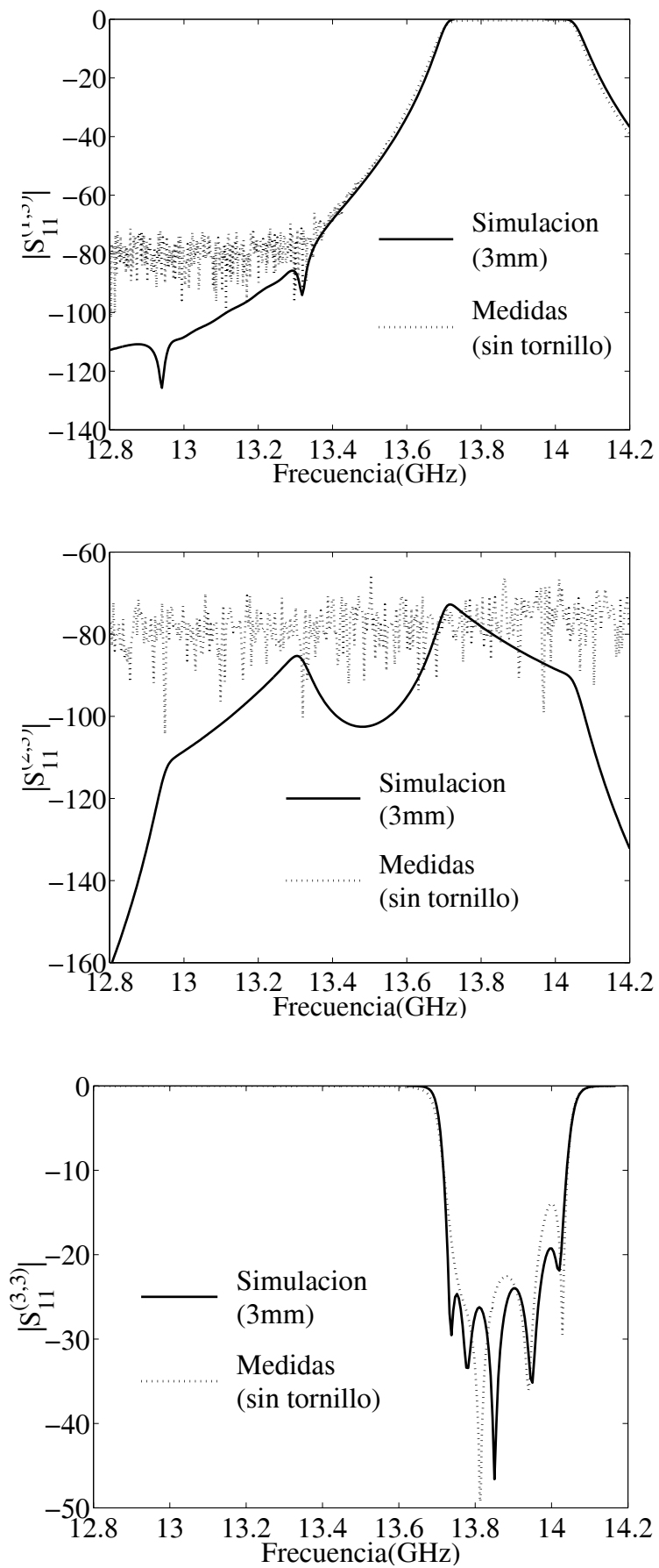

Figura 4.39: Parámetros de dispersión del diplexor a bordo del Hispasat 1-C. $S_{11}^{(1,3)}, S_{11}^{(2,3)}$, y $S_{11}^{(3,3)}$ 


\subsection{Conclusiones}

En este capítulo se ha aplicado el método híbrido de análisis 2-D junto con la técnica de acoplo modal a la determinación de los parámetros circuitales de varios problemas de gran interés práctico.

Utilizando el método para obtener la respuesta en frecuencia de una poste dieléctrico dentro de una guía rectangular, en combinación con medidas realizadas con un analizador de redes y tras un proceso de optimización, se ha conseguido desarrollar un método muy preciso y eficiente para la determinación de la propiedades dieléctricas de materiales. La nueva técnica de medida supera las limitaciones de otras técnicas de medidas anteriores. El nuevo método puede determinar las propiedades dieléctricas de materiales sólidos y también de materiales líquidos de una forma muy precisa, ya que en el caso de los líquidos se los introduce en una pipeta y la presencia de la pipeta también se ha tenido en cuenta. Otra ventaja del método de medida es la sencillez de la forma cilíndrica que debe tener la muestra de material a analizar, aunque se podría utilizar otra forma (por ejemplo, un poste cuadrado) y se obtendrían los mismos resultados. La forma de introducir la muestra evita el inconveniente de otros métodos en los que el material dieléctrico tiene que llenar gran parte de la guía, existiendo pues el peligro de que queden huecos de aire entre el dieléctrico y la guía que deterioren las medidas. Esta nueva técnica propuesta se ha aplicado a la medida de la permitividad eléctrica del etanol, obteniendo unos resultados muy precisos.

También se ha utilizado el método híbrido para analizar filtros inductivos de cavidades inductivas con defectos de fabricación. Estos filtros son muy utilizados en el sector de las comunicaciones espaciales. El hecho de poder analizar las esquinas redondeadas que aparecen cuando la técnica de fabricación no es muy precisa permite obtener las dimensiones óptimas del filtro aún con estos defectos, de modo que se pueden obtener las mismas prestaciones con un menor coste de fabricación. Se ha simulado la respuesta de varios filtros con esquinas redondeadas producidas por dos técnicas de fabricación distintas, y se ha comprobado el efecto que dichas esquinas producen en la respuesta de los filtros. Se ha validado la precisión de los resultados comparando con resultados proporcionados por un programa comercial de análisis electromagnético.

Finalmente, se ha comprobado la validez del método híbrido para analizar un dispositivo de comunicaciones tan complejo como es un diplexor en guía rectangular con filtros de cavidades inductivas. Los resultados del método han sido validados con medidas realizadas en el laboratorio sobre una maqueta de un diplexor actualmente a bordo del satélite Hispasat 1-C. Se ha analizado el diplexor considerando la presencia de esquinas redondeadas debidas a la técnica de fabricación por corte en plano $\mathrm{H}$, y se ha comprobado que los resultados se ajustan bastante bien a las medidas del diplexor sin tornillos de sintonía. Esto hace pensar que en caso de realizar el diseño del diplexor con el método híbrido, teniendo en cuenta los errores de mecanizado en la simulación, los tornillos de sintonía podrían no ser necesarios.

Se ha comprobado que el nuevo método de análisis de problemas inductivos con geometría arbitraria en guía rectangular posee múltiples aplicaciones en el campo de las comunicaciones, y que resulta preciso y razonablemente eficiente para el análisis de estructuras de gran complejidad. 


\section{Capítulo 5}

\section{Conclusiones}

El primer trabajo realizado en la tesis ha sido el de mejorar las técnicas de análisis de problemas de dispersión en espacio abierto que ya se habían desarrollado con anterioridad en el Departamento de Comunicaciones de la Universidad Politécnica de Valencia. Con relación a las capacidades de análisis de objetos dispersores de que se disponía [7] (tiras y cilindros metálicos), se han implementado técnicas que permiten analizar además otros objetos como son cilindros multicapa con pérdidas eléctricas y magnéticas (solución analítica), objetos dieléctricos inhomogéneos de sección arbitraria, arcos circulares metálicos y objetos cuya geometría se puede expresar como combinaciones de objetos metálicos sencillos (tiras y cilindros). Además se ha mejorado el cálculo de los elementos de la diagonal principal de la matriz de coeficiente resultante de aplicar el método de los momentos al cálculo de la corriente inducida en la superficie de un objeto metálico 2-D. Esta mejora ha supuesto una reducción del coste computacional a la cuarta parte sin pérdida de precisión en los resultados.

A continuación se ha desarrollado un nuevo método de análisis del acoplo electromagnético entre múltiples dispersores con el objeto de superar las limitaciones del método de que se disponía al comenzar la tesis [3], que hacían imposible el análisis de estructuras muy cerradas, como las que son necesarias analizar para obtener los parámetros circuitales de muchos dispositivos en guía de onda rectangular. Con el nuevo método es posible analizar prácticamente cualquier problema de dispersión múltiple 2-D independientemente de la posición de los objetos, incluidos muchos dispositivos reales inductivos en guía rectangular.

Una vez conseguido un método de análisis de problemas múltiples 2-D eficiente y capaz de analizar prácticamente cualquier estructura, se ha aplicado al análisis de dispositivos inductivos en guía rectangular. Para ello ha sido necesario extraer los parámetros circuitales de la estructura guiada a partir de la matriz de dispersión de modos cilíndricos del problema 2-D equivalente. En primer lugar se ha desarrollado una técnica para la obtención de la matriz de admitancias generalizadas (MAG). Los resultados obtenidos son satisfactorios si se analiza un único dispositivo, pero la precisión de esta técnica resulta insuficiente si se desea caracterizar varios dispositivos en guía y enlazar sus matrices de parámetros circuitales para obtener la respuesta global de la red formada por la unión en cascada de varios dispositivos. Para superar esta limitación se ha desarrollado otra técnica que permite construir la matriz de dispersión generalizada (MDG). Para desarrollar esta otra técnica ha sido necesario realizar 
un estudio exhaustivo de la cantidad de modos cilíndricos que son necesarios para reconstruir correctamente los modos de una guía rectangular estándar a lo largo de una circunferencia. Los resultados obtenidos con esta técnica son excelentes y se ha analizado con éxito una gran variedad de dispositivos en guía rectangular, y en todos los casos los resultados se han validado con otros publicados en la bibliografía técnica relacionada.

Esta técnica también ha permitido el análisis con éxito de filtros y diplexores inductivos. Ambos dispositivos están formados por la unión en cascada de varias subredes cada una de las cuales ha sido caracterizada de forma independiente. Con la formulación que se ha desarrollado en el apéndice $\mathrm{F}$ ha sido posible unir las matrices de dispersión generalizadas de las subredes con éxito. Además se ha analizado el efecto que produce la presencia de esquinas redondeadas debidas a defectos en la fabricación en dichos dispositivos, y se ha validado con éxito la precisión del método comparando sus resultados con medidas de la respuesta de un diplexor real con esquinas redondeadas debidas a defectos de fabricación. Hay que destacar que esta última geometría resulta muy costosa computacionalmente utilizando técnicas clásicas de análisis de problemas de geometría arbitraria. En el caso de la nueva técnica el coste computacional ha resultado ser más de 10 veces inferior al coste computacional de un software comercial de análisis electromagnético de propósito general. El análisis eficiente de los defectos de mecanizado en dispositivos de comunicaciones en guía rectangular es una muestra de la la gran aplicabilidad práctica de la herramienta desarrollada en la tesis, ya que permitirá en un futuro el diseño automatizado de estos dispositivos teniendo en cuenta los defectos de mecanizado. Por un lado esto garantiza un mejor diseño, en el que las dimensiones óptimas del dispositivo se calculan teniendo ya en cuenta que se van a producir defectos al fabricar. Y por otro lado permite relajar las tolerancias de fabricación, pues aunque los defectos de fabricación sean grandes de igual forma seremos capaces de encontrar unas dimensiones óptimas de la estructura que satisfagan las especificaciones.

Además de la aplicación al análisis de filtros y diplexores con esquinas redondeadas, la nueva herramienta de análisis ha sido aplicada con éxito a la determinación precisa de las propiedades dieléctricas de materiales, aunque éstos sean líquidos. Y con ello se han conseguido superar algunas limitaciones (poder analizar sólo materiales con bajas pérdidas y en un margen de frecuencia estrecho) de otros métodos que se han utilizado recientemente para el mismo propósito. Para esta aplicación ha sido necesario implementar un algoritmo de optimización de funciones $N$-dimensionales (método de descenso del simplex).

Como valoración final del trabajo realizado en la tesis se puede afirmar que se ha conseguido un método eficaz y muy eficiente de análisis de problemas arbitrarios 2-D en espacio abierto y de problemas inductivos de geometría arbitraria en guía rectangular. Las primeras aplicaciones de esta nueva herramienta han resultado de un gran interés práctico, y son muchas las aplicaciones que aún quedan por explotar, como por ejemplo el análisis de cristales fotónicos que cada vez está recibiendo más interés por parte de la comunidad científica, la combinación con métodos de optimización para diseñar dispositivos de comunicaciones teniendo en cuenta defectos de fabricación, el diseño de nuevos dispositivos tales como filtros con postes dieléctricos, etc.

El interés científico de la tesis queda justificado por las publicaciones de tipo científico a que ha dado lugar (ver apéndice $G$ ): 2 artículos publicados y uno en revisión en revistas 
de ámbito internacional, 4 ponencias en congresos nacionales, y 14 ponencias en congresos internacionales.

Entre las muchas líneas futuras de investigación relacionadas con esta nueva herramienta cabe destacar la extensión del método, primero al análisis de problemas capacitivos (p. ej. saltos de sección capacitivos, y estructuras en plano E en general), y posteriormente al análisis de estructuras guiadas completamente tridimensionales, tales como tornillos de sintonía, iris elípticos, o excitación mediante cable coaxial, etc. Para conseguir este objetivo resulta necesario extender el método de análisis de problemas de dispersión múltiple en espacio abierto a tres dimensiones. En caso de lograr una técnica capaz de resolver problemas de acoplo 3-D, se podría aplicar también al análisis de antenas o a la predicción de la respuesta electromagnética de cubiertas vegetales o de otro tipo de blancos, también de gran interés práctico. Otra posible ampliación de la herramienta de análisis desarrollada en la tesis es el análisis de transiciones entre una estructura guiada (alimentador) y una estructura radiante (antena), que permitiría en un futuro el diseño integrado de sistemas reales de comunicación considerando a la vez circuitos de alimentación y elementos radiantes. 


\section{Apéndice A}

\section{Espectros Cilíndrico y Plano de campos electromagnéticos. Transformaciones de espectros}

En este apéndice se introduce el concepto de espectro de campo electromagnético, y se describen dos espectros distintos, el cilíndrico y el de ondas planas. Finalmente, se explica cómo transformar un espectro cilíndrico en otro de ondas planas.

\section{A.1. Espectro cilíndrico}

La solución de la ecuación de onda en coordenadas cilíndricas son un conjunto infinito de modos o funciones cilíndricas, como se desarrolla en [29], de forma que los campos eléctrico y magnético pueden expresarse como una suma infinita de dichas funciones o modos, cada uno ponderado por un peso. Estos pesos constituyen el llamado espectro cilíndrico de campo. Los modos cilíndricos representan ondas cuyo frente de fase es un cilindro con centro en el origen de coordenadas.

Los modos cilíndricos son:

$$
H_{n}^{(1)}\left(k_{\rho} \rho\right) e^{j n \phi} e^{ \pm j k_{z} z} \quad \text { y } \quad H_{n}^{(2)}\left(k_{\rho} \rho\right) e^{j n \phi} e^{ \pm j k_{z} z}
$$

A partir de dichos modos, el campo eléctrico se puede expresar como:

$$
E_{z}(\rho, \phi)=\sum_{i=1}^{2} \sum_{n=-\infty}^{\infty} \int_{k_{\rho}} c_{i n}\left(k_{\rho}\right) H_{n}^{(i)}\left(k_{\rho} \rho\right) e^{j n \phi} e^{ \pm j k_{z} z} \mathrm{~d} k_{\rho}
$$

donde los coeficientes $c_{i n}\left(k_{\rho}\right)$ constituyen el espectro escalar cilíndrico del campo eléctrico.

En problemas bidimensionales estos modos son constantes para cualquier valor de $z$, de modo que el número de onda no depende de $z$, y $k_{\rho}=k=2 \pi / \lambda$. Por lo tanto, los modos cilíndricos con invarianza en $z$ son:

$$
H_{n}^{(1)}(k \rho) e^{j n \phi} \quad \text { y } \quad H_{n}^{(2)}(k \rho) e^{j n \phi}
$$


Utilizando los modos anteriores, el campo eléctrico en problemas invariantes en $z$ se puede expresar como:

$$
E_{z}(\rho, \phi)=\sum_{i=1}^{2} \sum_{n=-\infty}^{\infty} c_{i n} H_{n}^{(i)}(k \rho) e^{j n \phi}
$$

donde los coeficientes $c_{i n}$ constituyen el espectro cilíndrico escalar de campo para problemas bidimensionales, siendo independientes de $k_{\rho}$.

La función de Hankel de segunda especie, $H_{n}^{(2)}(k \rho)$ es una función emergente desde el origen de coordenadas hacia el infinito, mientras que la de primera especie $H_{n}^{(1)}(k \rho)$ representa una onda que avanza desde el infinito hasta el origen. La parte imaginaria de ambas funciones es singular en el origen.

Consecuentemente, el campo dispersado producido por unas fuentes situadas en el origen de coordenadas presenta un espectro cilíndrico cuyos coeficientes $c_{1 n}$ serán nulos, y por tanto la expresión del campo se reduce a la siguiente expresión:

$$
E_{z}(\rho, \phi)=\sum_{n=-\infty}^{\infty} c_{2 n} H_{n}^{(2)}(k \rho) e^{j n \phi}
$$

Si en el origen de coordenadas el campo es no singular (porque las fuentes que lo producen están lejos del origen), como es el caso del campo incidente a un objeto dispersor, los coeficientes $c_{1 n}$ y $c_{2 n}$ son iguales necesariamente para que las partes imaginarias de las funciones de Hankel de primera y segunda especie se anulen y el campo quede acotado en el origen. La expresión del campo eléctrico es en este caso:

$$
E_{z}(\rho, \phi)=\sum_{n=-\infty}^{\infty} i_{n} J_{n}(k \rho) e^{j n \phi}
$$

donde $i_{n}=2 c_{1 n}=2 c_{2 n}$, y $J_{n}(k \rho)=\left(H_{n}^{(1)}(k \rho)+H_{n}^{(2)}(k \rho)\right) / 2$ es la función de Bessel, que representa una onda que se propaga hacia el origen pero que no es singular en él.

Por lo tanto se puede hablar de un espectro cilíndrico para el campo dispersado por un objeto dispersor (coeficientes $c_{n}=c_{2 n}$ cuando $c_{1 n}=0$ ), y un espectro cilíndrico para el campo incidente sobre un objeto situado en el origen de coordenadas pero generado en otro punto, y que viene dado por los coeficientes $i_{n}$.

\section{A.2. Espectro de ondas planas}

Resolviendo la ecuación de onda en coordenadas cartesianas se obtienen unas soluciones que son ondas planas que se propagan cada una con una dirección diferente, como se explica en [29]. Una onda plana es una onda con un frente de fase plano y que se propaga con una dirección dada. Estos modos u ondas planas son de la forma:

$$
e^{-j k_{x} x} e^{-j k_{y} y} e^{ \pm j k_{z} z}
$$


donde:

$$
\begin{aligned}
k & =\frac{2 \pi}{\lambda} \\
k_{x} & =k \cos (\beta) \sin (\alpha) \\
k_{y} & =k \sin (\beta) \sin (\alpha) \\
k_{z} & =k \cos (\alpha) \\
k_{\rho} & =k \sin (\alpha)=\sqrt{k_{x}^{2}+k_{y}^{2}} \\
x & =\rho \cos (\phi) \sin (\theta) \\
y & =\rho \sin (\phi) \sin (\theta) \\
z & =\rho \cos (\theta)
\end{aligned}
$$

Los ángulos $\beta$ y $\alpha$ indican la dirección de avance del frente de fase, tal y como se muestra en la figura A.1, mientras que $\rho, \theta$ y $\phi$ son las coordenadas cilíndricas del punto donde se evalúa el campo.

El campo eléctrico se puede expresar como una suma infinita (una integral) de ondas planas, cada una con una dirección $(\alpha, \beta)$, es decir, con unos valores distintos de $k_{x}$ y $k_{y}$. Y cada onda plana vendrá ponderada por un peso distinto. La función continua que contiene los pesos para cada onda es el espectro de ondas planas del campo, que se representa como $p\left(k_{x}, k_{y}\right)$, o como $p\left(k_{\rho}, \beta\right)$. Y el campo se expresa en función de dicho espectro como:

$$
E_{z}(x, y, z)=\int_{k_{x}} \int_{k_{y}} p\left(k_{x}, k_{y}\right) e^{-j k_{x} x} e^{-j k_{y} y} e^{ \pm j k_{z} z} \mathrm{~d} k_{x} \mathrm{~d} k_{y}
$$

Si se desea utilizar coordenadas cilíndricas, se deben tener en cuenta las siguientes equivalencias:

$$
\begin{aligned}
k_{x} x+k_{y} y & =k_{\rho} \rho \sin (\theta) \cos (\phi-\beta) \\
\mathrm{d} k_{x} \mathrm{~d} k_{y} & =\left|\begin{array}{cc}
\frac{\partial k_{x}}{\partial k_{\rho}} & \frac{\partial k_{x}}{\partial \beta} \\
\frac{\partial k_{y}}{\partial k_{\rho}} & \frac{\partial k_{y}}{\partial \beta}
\end{array}\right| \mathrm{d} k_{\rho} \mathrm{d} \beta=k_{\rho} \mathrm{d} k_{\rho} \mathrm{d} \beta
\end{aligned}
$$

Las relaciones anteriores permiten particularizar la expresión en A.6 del campo eléctrico en función de su espectro de ondas planas para el caso de utilizar coordenadas cilíndricas, obteniéndose la siguiente expresión:

$$
E_{z}(\rho, \theta, \phi)=\int_{k_{\rho}} \int_{\beta} p\left(k_{\rho}, \beta\right) e^{-j k_{\rho} \rho \sin (\theta) \cos (\phi-\beta)} e^{ \pm j k_{z} z} k_{\rho} \mathrm{d} k_{\rho} \mathrm{d} \beta
$$




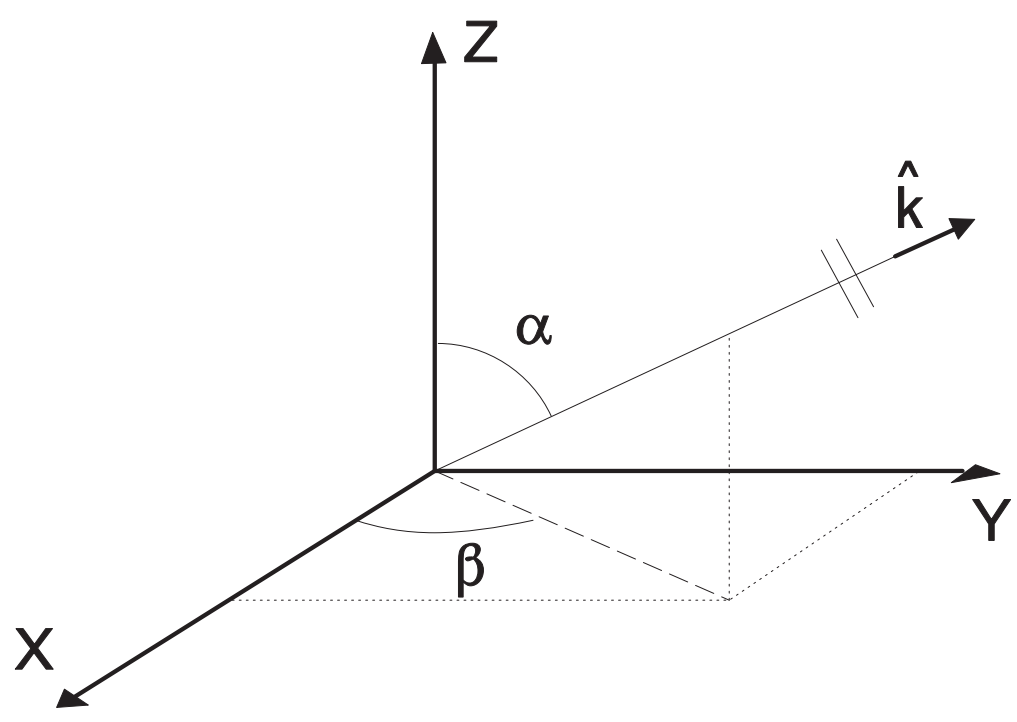

Figura A.1: Diagrama de avance de una onda plana

En el caso de problemas invariantes en $z$, bidimensionales, la dirección de estas ondas planas estará contenida en el plano $X Y$, de modo que $\alpha=90^{\circ}$ y $\theta=90^{\circ}$, y $k_{\rho}=k=$ $2 \pi / \lambda$. Por lo tanto en dos dimensiones las expresiones en A.5 se simplifican, quedando de la siguiente forma:

$$
\begin{aligned}
k_{x} & =k \cos (\beta) \\
k_{y} & =k \sin (\beta) \\
k_{z} & =0 \\
k_{\rho} & =k \\
x & =\rho \cos (\phi) \\
y & =\rho \sin (\phi)
\end{aligned}
$$

Y la onda plana en dos dimensiones queda como:

$$
e^{-j k_{x} x} e^{-j k_{y} y}=e^{-j k \rho \cos (\phi-\beta)}
$$

Finalmente, el campo eléctrico en problemas invariantes en $z$ se reconstruye a partir de su espectro bidimensional $p(\beta)$ de ondas planas de acuerdo con la siguiente expresión:

$$
E_{z}(\rho, \phi)=k \int_{\beta} p(\beta) e^{-j k \rho \cos (\phi-\beta)} \mathrm{d} \beta
$$




\section{A.3. Equivalencia entre espectro plano y cilíndrico}

La equivalencia de espectros plano y cilíndrico se obtiene a partir de las siguientes relaciones [29]:

$$
\begin{aligned}
c_{i n}(k \rho) & =j^{-n} k_{\rho} \frac{1}{2} \int_{\beta=0}^{2 \pi} p\left(k_{\rho}, \beta\right) e^{-j n \beta} \mathrm{d} \beta \\
p\left(k_{\rho}, \beta\right) & =\frac{1}{\pi k_{\rho}} \sum_{n=-\infty}^{\infty} j^{n} c_{i n}\left(k_{\rho}\right) e^{j n \beta}
\end{aligned}
$$

Para el caso bidimensional estas equivalencias se reducen a:

$$
\begin{aligned}
c_{i n} & =j^{-n} \frac{k}{2} \int_{\beta=0}^{2 \pi} p(\beta) e^{-j n \beta} \mathrm{d} \beta \\
p(\beta) & =\frac{1}{\pi k} \sum_{n=-\infty}^{\infty} j^{n} c_{i n} e^{j n \beta}
\end{aligned}
$$

\section{A.4. Espectro cilíndrico de una onda incidente plana}

Las relaciones de A.3 servirán para obtener el espectro cilíndrico del campo incidente a un objeto dispersor cuando el campo incidente es una onda plana. Con ello se obtiene el campo dispersado como el producto de la función de transferencia del objeto por el espectro cilíndrico de campo incidente.

Una onda plana que se propague en el plano $X Y$ con una dirección de avance $\phi=\beta_{0}$ es de la forma:

$$
E_{z}(\rho, \phi)=e^{-j k \rho \cos \left(\phi-\beta_{0}\right)}
$$

El espectro de ondas planas de esta onda es:

$$
\begin{aligned}
p(\beta) & =\frac{1}{k} \frac{1}{2 \pi} \int_{\phi=0}^{2 \pi} E_{z}(\rho, \phi) e^{j k \rho \cos (\phi-\beta)} \mathrm{d} \phi \\
& = \begin{cases}\frac{1}{k} & \text { si } \beta=\beta_{0} \\
0 & \text { si } \beta \neq \beta_{0}\end{cases} \\
& =\frac{1}{k} \delta\left(\beta-\beta_{0}\right)
\end{aligned}
$$


Por lo tanto, y usando (A.11), el espectro cilíndrico de una onda plana es:

$$
\begin{aligned}
c_{i n} & =j^{-n} \frac{k}{2} \int_{\beta=0}^{2 \pi} \frac{1}{k} \delta\left(\beta-\beta_{0}\right) e^{-j n \beta} \mathrm{d} \beta \\
& =j^{-n} \frac{e^{-j n \beta_{0}}}{2}
\end{aligned}
$$

Puesto que $c_{1 n}=c_{2 n}$, se puede expresar el campo en función de su espectro cilíndrico incidente (coeficientes $i_{n}$ ), de modo que $i_{n}=2 c_{1 n}=2 c_{2 n}=\left(j e^{j \beta_{0}}\right)^{-n}$.

En resumen:

$$
\begin{aligned}
E_{z}(\rho, \phi) & =e^{-j k \rho \cos \left(\phi-\beta_{0}\right)} \\
& =\sum_{n=-\infty}^{\infty}\left(j e^{j \beta_{0}}\right)^{-n} J_{n}(k \rho) e^{j n \phi}
\end{aligned}
$$




\section{Apéndice B}

\section{Matrices de Traslación de espectros}

\section{B.1. Introducción}

En la resolución de problemas electromagnéticos con múltiples objetos dispersores es necesario obtener el espectro de un campo electromagnético referido a un origen a partir del espectro de dicho campo referido a otro origen. En este apéndice se obtienen unas matrices llamadas matrices de traslación de espectros $[29,6,8]$ que permiten obtener el espectro de un campo referido a otros puntos a partir del espectro de dicho campo referido a un punto concreto.

Un tipo de matrices de traslación proporciona el espectro de campo incidente centrado en un origen a partir del espectro de dicho campo emergente desde otro origen. A estas matrices se las denomina matrices de traslación de espectro emergente a incidente.

Otro tipo de matrices de traslación proporciona el espectro de campo incidente centrado en un origen a partir del espectro de dicho campo incidente centrado en otro origen. Estas son las matrices de traslación de espectro incidente a incidente.

\section{B.2. Traslación de espectro emergente a incidente}

Supongamos la situación de la figura B.1, en la que hay dos objetos dispersores $O_{1}$ y $O_{2}$. El objeto $O_{1}$ dispersa un campo:

$$
E_{z}\left(\overrightarrow{\rho_{1}}\right)=\sum_{q=-N_{d_{1}}}^{N_{d_{1}}} c_{q} H_{q}^{(2)}\left(K \rho_{1}\right) e^{j q \phi_{1}}
$$

donde $\overrightarrow{\rho_{1}}$ está referido a un sistema de coordenadas local a $O_{1}\left(x_{1}\right.$ e $y_{1}$ en la figura B.1).

El campo $E_{z}$ dispersado por $O_{1}$ se propaga e incide sobre $O_{2}$. Para conocer la respuesta de $\mathrm{O}_{2}$ ante dicha incidencia nos interesa expresar el campo $E_{z}$ en el sistema local de coorde- 


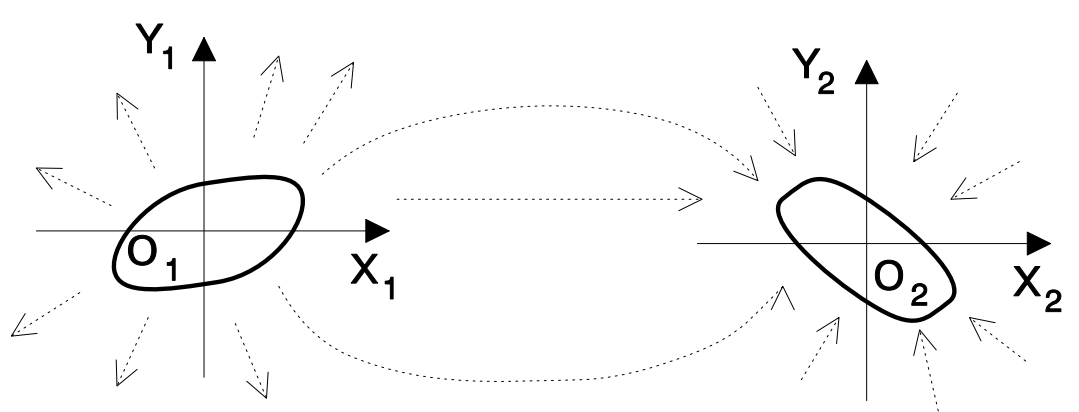

Figura B.1: Ubicación de los objetos $O_{1}$ y $O_{2}$. El campo dispersado por $O_{1}$ incide en $O_{2}$

nadas a $\mathrm{O}_{2}$ como:

$$
E_{z}\left(\overrightarrow{\rho_{2}}\right)=\sum_{p=-N_{i_{2}}}^{N_{i_{2}}} i_{p} J_{p}\left(K \rho_{2}\right) e^{j p \phi_{2}}
$$

donde $\overrightarrow{\rho_{2}}$ está referido al sistema de coordenadas local a $O_{2}\left(x_{2}\right.$ e $y_{2}$ en la figura B.1).

Para poder realizar esta traslación de un espectro emergente a otro incidente, debemos expresar los modos cilíndricos $H_{q}^{(2)}\left(k \rho_{1}\right) e^{j q \phi_{1}}$ emergentes de $O_{1}$ como sumatorio de modos cilíndricos $J_{p}\left(k \rho_{2}\right) e^{j p \phi_{2}}$ incidentes a $O_{2}$. Esta relación viene recogida en $[8,87,88]$ y se expresa de la siguiente manera:

$$
H_{q}^{(2)}\left(k \rho_{1}\right) e^{j q \phi_{1}}=\sum_{p=-\infty}^{\infty} H_{p-q}^{(2)}\left(k d_{21}\right) e^{-j(p-q) \phi_{21}} J_{p}\left(k \rho_{2}\right) e^{j p \phi_{2}} \rho_{2} \leq d_{21}
$$

donde $d_{21}$ es la distancia entre los centros de los sistemas de coordenadas de $O_{1}$ y $O_{2}$, y $\phi_{21}$ es la coordenada $\phi$ del origen del sistema de coordenadas de $O_{1}$ referido al sistema de coordenadas de $\mathrm{O}_{2}$. La expresión anterior es válida en la circunferencia centrada en el objeto $\mathrm{O}_{2}$ y de radio $d_{21}$. Los parámetros $d_{21}$ y $\phi_{21}$ se ilustran en la figura B.2, y se pueden obtener como:

$$
\begin{aligned}
d_{21} & =\sqrt{\left(x_{c_{1}}-x_{c_{2}}\right)^{2}+\left(y_{c_{1}}-y_{c_{2}}\right)^{2}} \\
\tan \phi_{21} & =\frac{y_{c_{1}}-y_{c_{2}}}{x_{c_{1}}-x_{c_{2}}}
\end{aligned}
$$

donde $\overrightarrow{\rho_{c_{1}}}=\left(x_{c_{1}}, y_{c_{1}}\right)$ son las coordenadas del origen del sistema de coordenadas local a $O_{1}$ en un sistema de coordenadas global a $O_{1}$ y a $O_{2}$, como se ilustra en la figura B.2. Y consecuentemente $\overrightarrow{\rho_{c_{2}}}=\left(x_{c_{2}}, y_{c_{2}}\right)$ son las coordenadas del origen del sistema local a $\mathrm{O}_{2}$.

La relación entre el espectro $\left[c_{q}\right]$ dispersado por $O_{1}$ y el incidente $\left[i_{p}\right]$ a $O_{2}$ se puede establecer utilizando las expresiones anteriores: 


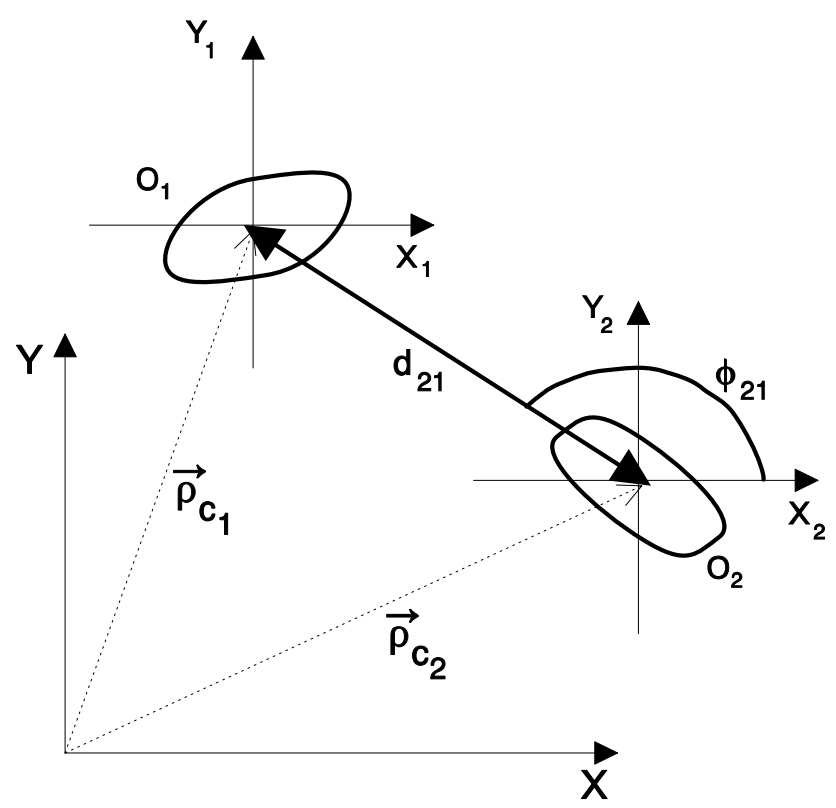

Figura B.2: Diagrama explicativo de los parámetros $d_{21}$ y $\phi_{21}$

$$
\begin{aligned}
E_{z} & =\sum_{q=-N_{d_{1}}}^{N_{d_{1}}} c_{q} H_{q}^{(2)}\left(k \rho_{1}\right) e^{j q \phi_{1}} \\
& =\sum_{q=-N_{d_{1}}}^{N_{d_{1}}} c_{q} \sum_{p=-\infty}^{\infty} H_{p-q}^{(2)}\left(k d_{21}\right) e^{-j(p-q) \phi_{21}} J_{p}\left(k \rho_{2}\right) e^{j p \phi_{2}} \\
& =\sum_{p=-\infty}^{\infty}\left(\sum_{q=-N_{d_{1}}}^{N_{d_{1}}} c_{q} H_{p-q}^{(2)}\left(k d_{21}\right) e^{-j(p-q) \phi_{21}}\right) J_{p}\left(k \rho_{2}\right) e^{j p \phi_{2}} \\
& =\sum_{p=-\infty}^{\infty} i_{p} J_{p}\left(k \rho_{2}\right) e^{j p \phi_{2}} \\
& \simeq \sum_{p=-N_{i_{2}}}^{N_{i_{2}}} i_{p} J_{p}\left(k \rho_{2}\right) e^{j p \phi_{2}}
\end{aligned}
$$

A partir de estas expresiones se puede construir ya la matriz $T_{21}$ de traslación de espec- 
tro emergente desde $O_{1}$ a incidente sobre $O_{2}$ :

$$
\left[\begin{array}{c}
i_{-N_{i_{2}}} \\
i_{-N_{i_{2}}+1} \\
\vdots \\
i_{N_{i_{2}}}
\end{array}\right]=\begin{gathered}
-N_{i_{2}} \\
p \\
\downarrow \\
N_{i_{2}}
\end{gathered} \overbrace{\left[\begin{array}{l}
H_{p-q}^{(2)}\left(k d_{21}\right) e^{-j(p-q) \phi_{21}} \\
\end{array}\right]}^{-N_{d_{1}} \longrightarrow q \longrightarrow N_{d_{1}}} \cdot\left[\begin{array}{c}
c_{-N_{d_{1}}} \\
c_{-N_{d_{1}}+1} \\
\vdots \\
c_{N_{d_{1}}}
\end{array}\right]
$$

La expresión del campo dispersado por el objeto $O_{1}$ tan sólo es válida en la región libre de fuentes, es decir, fuera de la circunferencia que circunscribe al objeto $O_{1}$. Por lo tanto, la expresión del campo $E_{z}$ a partir de modos cilíndricos emergentes de $O_{1}$

$$
\sum_{q=-N_{d_{1}}}^{N_{d_{1}}} c_{q} H_{q}^{(2)}\left(k \rho_{1}\right) e^{j q \phi_{1}}
$$

es tan sólo válida en el exterior de la circunferencia que contiene al objeto $O_{1}$. La expresión del campo incidente a $\mathrm{O}_{2}$ con infinitos modos

$$
\sum_{p=-\infty}^{\infty}\left(\sum_{q=-N_{d_{1}}}^{N_{d_{1}}} c_{q} H_{p-q}^{(2)}\left(k d_{21}\right) e^{-j(p-q) \phi_{21}}\right) J_{p}\left(k \rho_{2}\right) e^{j p \phi_{2}}
$$

es válida dentro de la circunferencia centrada en el objeto $O_{2}$ y de radio $d_{21}$, pero fuera de la circunferencia que circunscribe a $O_{1}$.

$\mathrm{Si}$ en lugar de tomar infinitos modos cilíndricos incidentes a $\mathrm{O}_{2}$, ( $p$ variando desde $-\infty$ a $\infty$ ), tomamos $2 N_{i_{2}}+1$ modos, la expresión

$$
\sum_{p=-N_{i_{2}}}^{N_{i_{2}}}\left(\sum_{q=-N_{d_{1}}}^{N_{d_{1}}} c_{q} H_{p-q}^{(2)}\left(k d_{21}\right) e^{-j(p-q) \phi_{21}}\right) J_{p}\left(k \rho_{2}\right) e^{j p \phi_{2}}
$$

es válida en la región externa a la circunferencia que contiene a $O_{1}$ e interna a la circunferencia de radio $N_{i_{2}} / k$ centrada en $O_{2}$, como se ilustra en la figura B.3.

En las figuras B.4, B.5 y B.6 se ilustra un ejemplo de traslación de espectro emergente a incidente. La figura B.4 presenta la amplitud y la fase del campo dispersado por un cilindro metálico de radio $2 \lambda$ ante la incidencia de una onda plana que avanza paralela al eje $X$, hacia valores crecientes de la coordenada $x$ (es decir, $\beta=0$ ). El cilindro se halla centrado en el punto $(-4,0)$.

El campo dispersado por el cilindro y en general todos los campos serán representados en una rejilla bidimensional para apreciar las zonas donde la reconstrucción de los campos es correcta. En el caso del campo dispersado por el cilindro, éste se reconstruye correctamente a partir de su espectro emergente $\left[c_{q}\right]$ en la zona externa a la circunferencia que lo circunscribe. El campo que proporciona dicho espectro dentro de la circunferencia que circuncribe al 


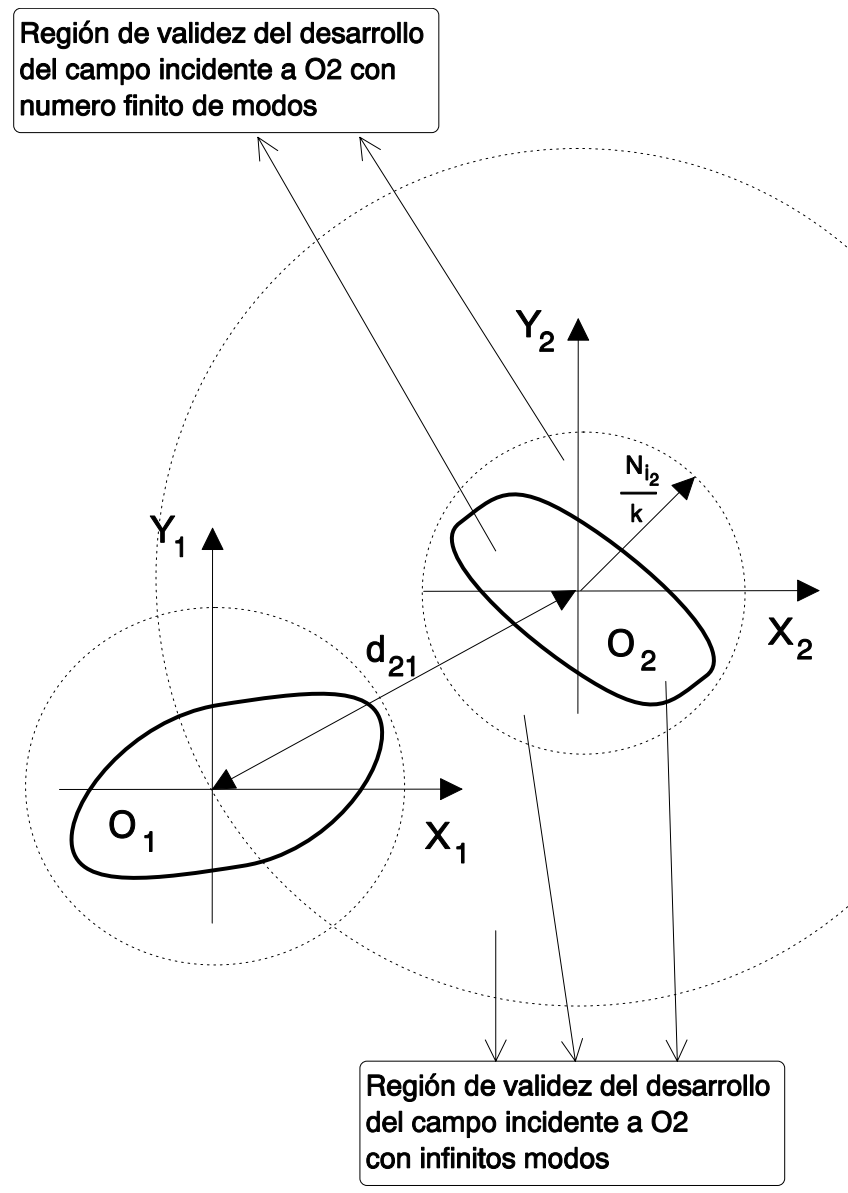

Figura B.3: Regiones de validez de los distintos desarrollos modales en la traslación de espectro emergente a incidente

objeto no ha sido representado ya que éste es singular, y carece de interés. El campo real dentro del cilindro es nulo, y este es el valor que se ha tomado para dicha zona.

Se ha dibujado una circunferencia con línea discontinua cuyo centro es el punto $(3,0)$ y con radio $4 \lambda$. Dicha circunferencia representa la zona donde queremos reconstruir el campo dispersado con el cilindro a partir de un espectro incidente centrado en el punto $(3,0)$. Utilizando la matriz de traslación de coordenadas de espectro emergente de $(-4,0)$ a espectro incidente en el punto $(3,0)$, se obtiene el espectro de campo incidente centrado en dicho punto.

La amplitud y la fase de la reconstrucción del campo dispersado por el cilindro a partir del espectro incidente centrado en $(3,0)$ se representa en la figura B.5. Para reconstruir el campo se han utilizado $2 N_{i_{2}}+1$ modos, donde $N_{i_{2}}=k 4 \lambda$.

Se observa en la reconstrucción del campo a partir del espectro incidente que tanto la amplitud como la fase coinciden con las del campo proporcionado por el espectro emergente dentro de la circunferencia de trazo discontinuo. Dicha circunferencia corresponde con la zona donde se reconstruye correctamente el campo incidente con $2 N_{i_{2}}+1$ modos para $N_{i_{2}}=$ 

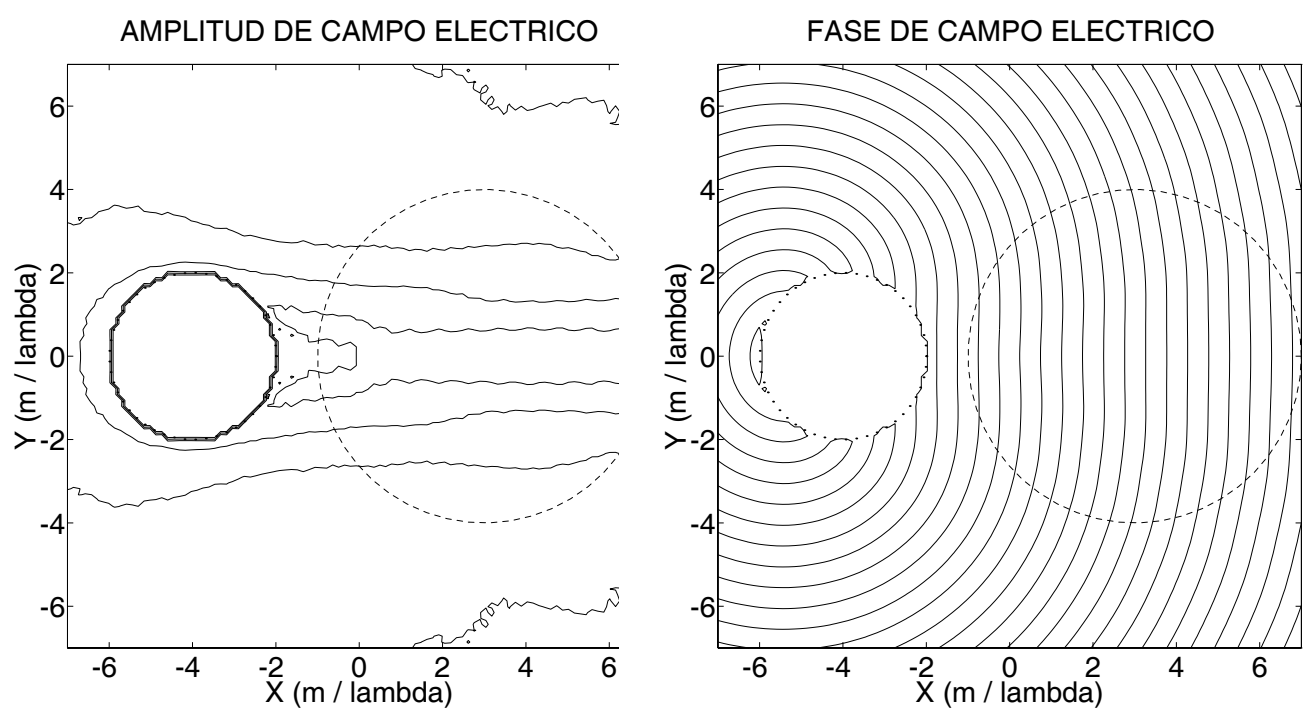

Figura B.4: Amplitud y fase del campo dispersado por un cilindro infinito de radio $2 \lambda$ centrado en $(-4,0)$ ante una onda plana $(\beta=0)$. Reconstrucción a partir del espectro emergente de $(-4,0)$
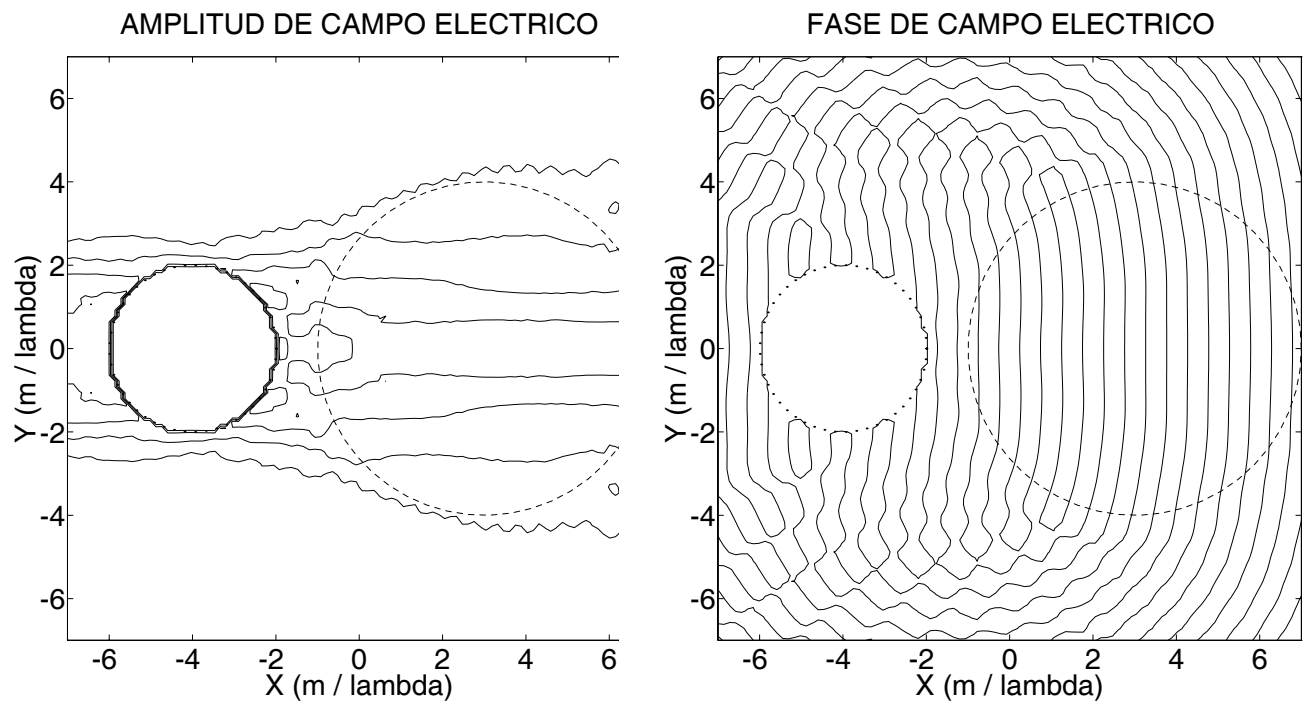

Figura B.5: Amplitud y fase del campo dispersado por un cilindro infinito de radio $2 \lambda$ centrado en $(-4,0)$ ante una onda plana $(\beta=0)$. Reconstrucción a partir del espectro incidente a $(3,0) \operatorname{con} N_{i_{2}}=k 4 \lambda$

$k 4 \lambda$. Para reconstruir el campo correctamente en una zona más amplia se debería tomar un valor de $N_{i_{2}}$ mayor.

En la figura B.6 se representa la amplitud y la fase del campo dispersado por el cilindro a lo largo del eje $X$. Se ha representado la reconstrucción del campo a partir del espectro emergente (reconstrucción total) y a partir del incidente (reconstrucción parcial). Se observa cómo el espectro incidente proporciona un campo dispersado que se ajusta bastante bien, 
especialmente la fase, en la zona interna a la circunferencia de radio $4 \lambda$ centrada en $(3,0)$, pero que difiere totalmente fuera de dicha zona.
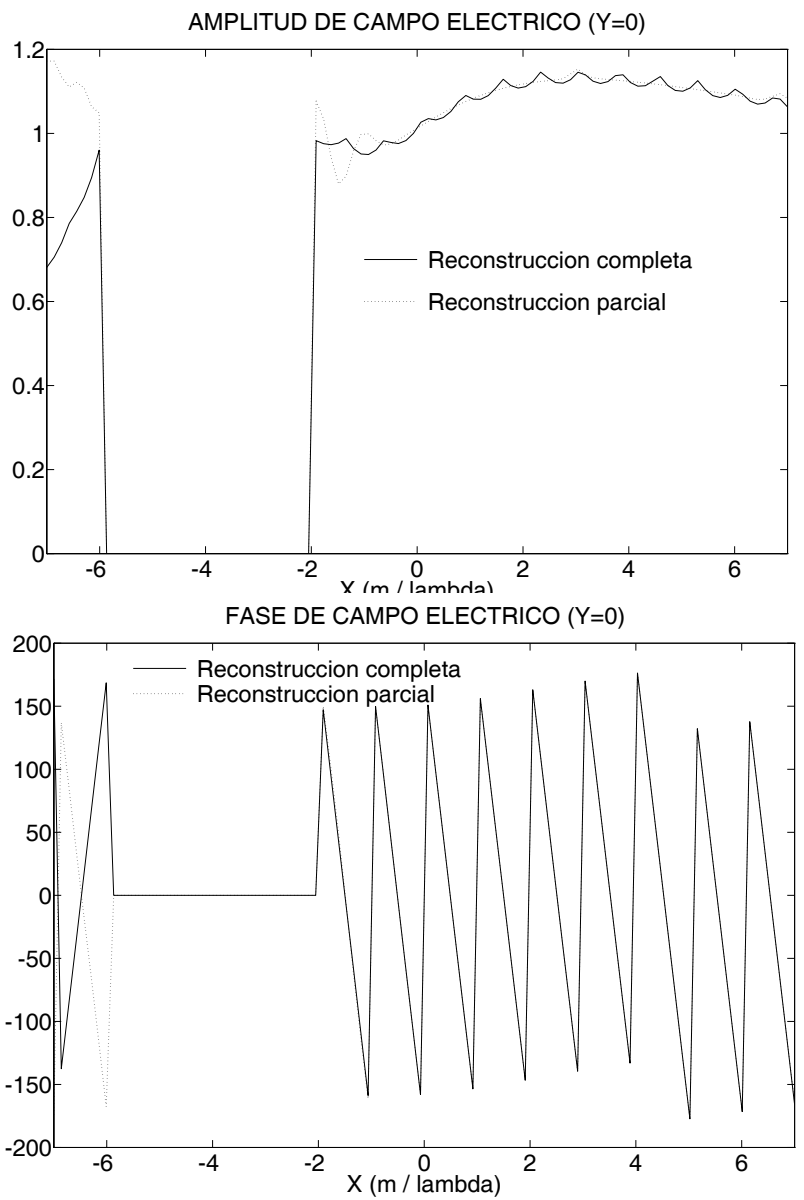

Figura B.6: Amplitud y fase del campo dispersado por un cilindro infinito de radio $2 \lambda$ centrado en $(-4,0)$ ante una onda plana $(\beta=0)$. Reconstrucciones total y parcial $\left(N_{i_{2}}=k 4 \lambda\right)$.

La reconstrucción del campo dispersado por el cilindro como campo incidente centrado en $(3,0)$ con un número mayor de modos se representa en la figura B.7. En este caso se ha escogido un valor de $N_{i_{2}}=k 8 \lambda$. Dicho valor de $N_{i_{2}}$ supone que, en principio, el campo se debería reconstruir correctamente en un radio de $8 \lambda$ alrededor del punto $(3,0)$. No obstante, la reconstrucción del campo a partir del espectro incidente obtenido por medio de la matriz de traslación de espectro emergente a incidente es tan sólo válida dentro de la circunferencia centrada en $(3,0)$ y de radio $d_{21}$. Dicho parámetro es la distancia entre los centros de los espectros emergente e incidente, y en este caso concreto dicha distancia es de valor $d_{21}=7 \lambda$. Por lo tanto, según el criterio $N_{i_{2}}=k a$, el número de modos tomado para recontruir el campo incidente permitiría la reconstrucción correcta en un radio de $8 \lambda$, pero como dicho radio excede el valor de $d_{21}$, el campo, como se aprecia en la amplitud y la fase de la figura B.7, se reconstruye correctamente en un radio de valor $d_{21}=7 \lambda$, que es la zona más amplia en la que podemos reconstruir el campo a partir de un espectro incidente centrado en $(3,0)$. 

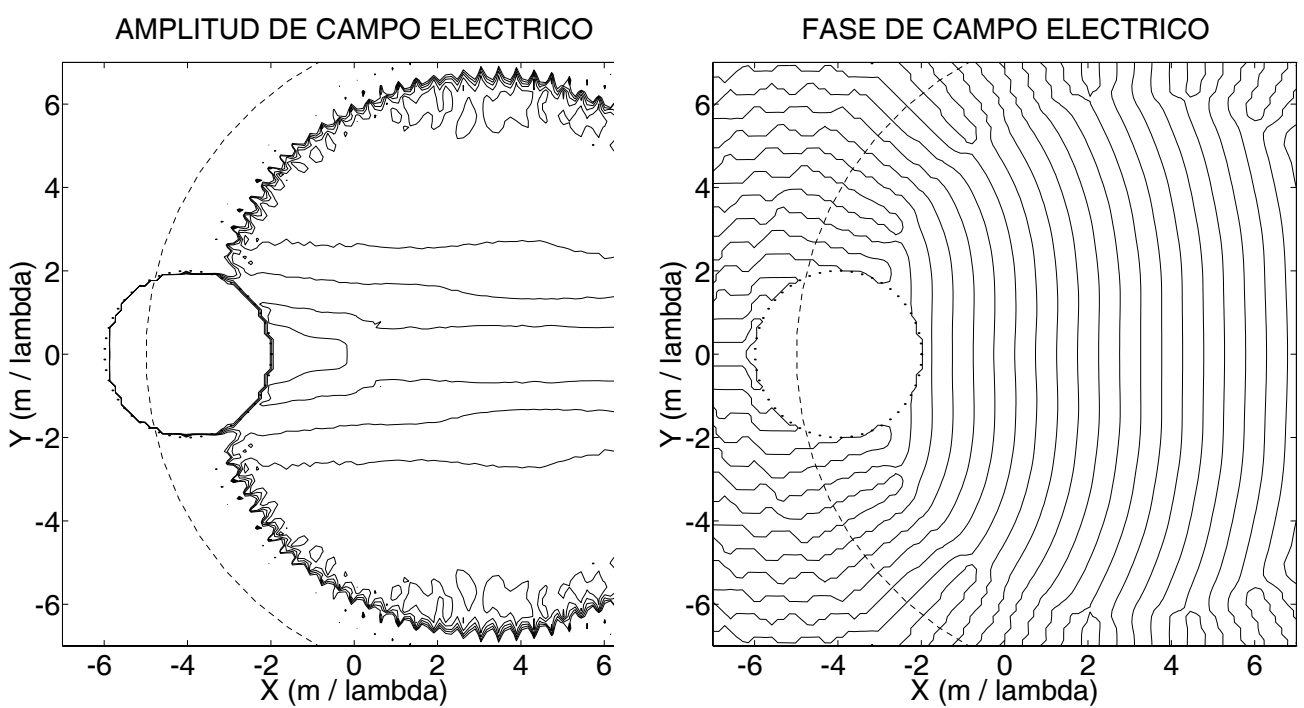

Figura B.7: Amplitud y fase del campo dispersado por un cilindro infinito de radio $2 \lambda$ centrado en $(-4,0)$ ante una onda plana $(\beta=0)$. Reconstrucción a partir del espectro incidente a $(3,0) \operatorname{con} N_{i_{2}}=k 8 \lambda$

De la circunferencia centrada en $(3,0)$ y de radio $d_{21}$ se debe excluir lógicamente la parte de la circunferencia que circunscribe al cilindro y que entra dentro de aquélla. En dicha zona ni siquiera el espectro emergente de $(-4,0)$ proporciona una reconstrucción correcta del campo, y por tanto tampoco lo hace el espectro incidente obtenido a partir del emergente.

En la figura B.8 se comparan la amplitud y la fase del campo dispersado por el cilindro a lo largo del eje $X$, reconstruyéndolo a partir del espectro emergente (reconstrucción total) y a partir del incidente (reconstrucción parcial) con $N_{i_{2}}=k 8 \lambda$. Como se aprecia en las gráficas la reconstrucción es buena en un círculo centrado en $(3,0)$ de radio aproximadamente $7 \lambda$. Fuera de dicha zona la reconstrucción del campo a partir del espectro incidente proporciona un campo cuya amplitud es muy elevada, mucho más que en la reconstrucción parcial de la figura B.6 con un número menor de modos.

El hecho de que al reconstruir el campo incidente con mayor número de modos aumenten considerablemente los valores de campo fuera de la zona de interés viene acompañado por el fenómeno de que algunos de los elementos de la matriz de traslación de espectro emergente a incidente son cada vez de mayor tamaño. Dicho fenómeno no afecta a la reconstrucción del campo en la zona de interés, pues en dicha zona el campo incidente se reconstruye correctamente. Sin embargo, en los problemas de acoplo electromagnético o realimentación entre varios dispersores, es preciso resolver sistemas matriciales. En estos casos la utilización de matrices de traslación con elementos muy elevados puede provocar que los sistemas matriciales que se deben resolver estén mal condicionados y no se pueda obtener una solución.

La tabla B.1 muestra la evolución de la matriz de traslación de espectro emergente a incidente conforme reconstruimos el campo incidente con mayor número de modos. En dicha tabla se muestra el radio de la zona donde supuestamente se reconstruiría correctamente el campo $N_{i_{2}} / k$. En otra columna se ha incluido el margen entre dicho radio y el valor del 

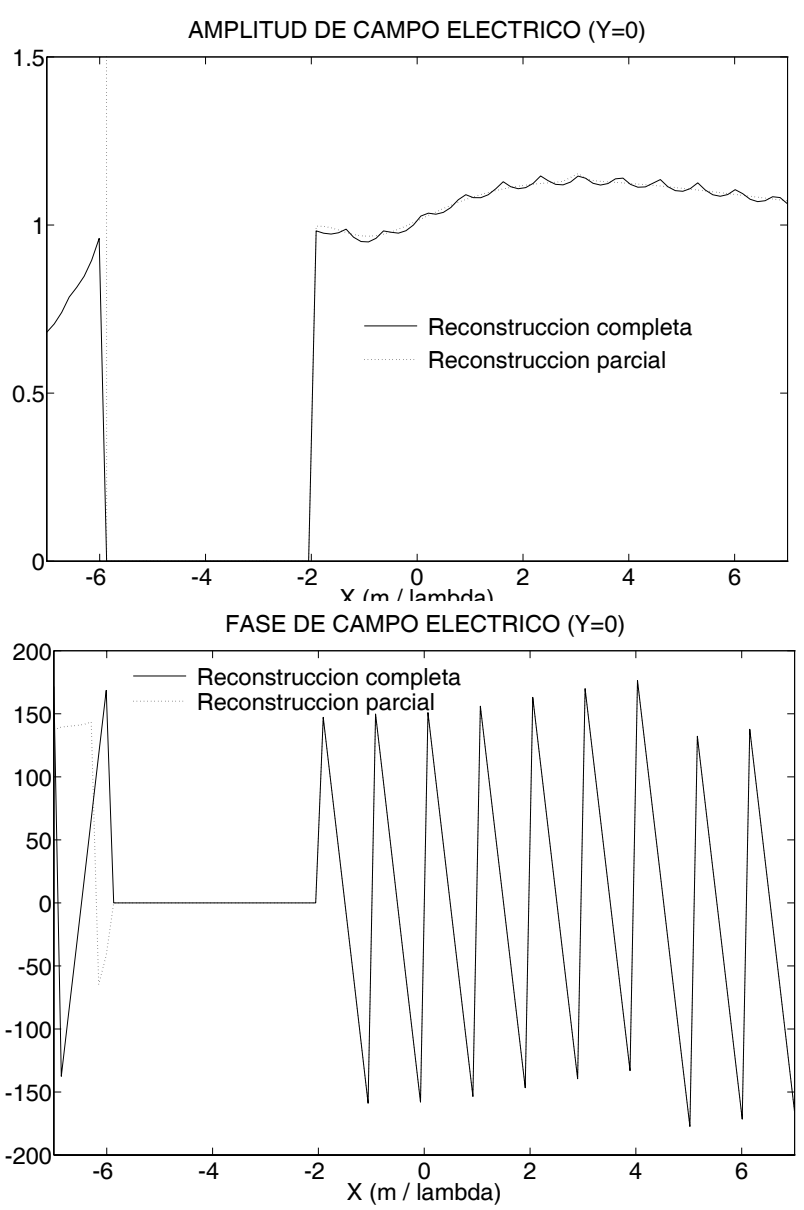

Figura B.8: Amplitud y fase del campo dispersado por un cilindro infinito de radio $2 \lambda$ centrado en $(-4,0)$ ante una onda plana $(\beta=0)$. Reconstrucciones total y parcial $\left(N_{i_{2}}=k 8 \lambda\right)$.

parámetro $d_{21}$, que en este ejemplo vale $7 \lambda$. Y en la tercera columna se representa la amplitud del elemento de la matriz de traslación que mayor amplitud presenta. Se aprecia un aumento de la amplitud del mayor elemento de la matriz de traslación conforme el margen con $d_{21}$ disminuye. Mientras dicho margen es positivo la matriz de traslación no presenta elementos muy singulares, pero valores negativos para el margen con $d_{21}$ supone que queremos reconstruir el campo dispersado por el cilindro en una zona que incluye el punto interior al cilindro donde está centrado el espectro emergente. En dicho punto el espectro emergente es singular, y por tanto la pretensión de reconstruir el campo incluso en ese punto es lo que provoca la aparición de elementos en la matriz de traslación que son cada vez más singulares cuanto más negativo es el margen respecto a $d_{21}$.

La conclusión de esta discusión es que no debe tomarse un margen sobre $d_{21}$ negativo, ya que eso puede provocar problemas de singularidades en la resolución de sistemas de ecuaciones en problemas de acoplo electromagnético, y además esta decisión se refuerza por el hecho de que escoger un número de modos $N_{i_{2}}$ tan elevado que haga que el margen con $d_{21}$ sea negativo no amplía la zona donde se reconstruye correctamente el campo a partir del 
espectro incidente, como ya se ha comentado anteriormente.

\begin{tabular}{|c|c|c|}
\hline \hline Radio $\left(N_{i_{2}} / k\right)$ & Margen con $d_{21}$ & Elemento de mayor amplitud \\
\hline 2 & 5 & 0.1451 \\
\hline 2.5 & 4.5 & 0.1543 \\
\hline 3 & 4 & 0.1685 \\
\hline 3.5 & 3.5 & 0.1941 \\
\hline 4 & 3 & 0.2941 \\
\hline 4.5 & 2.5 & 0.6190 \\
\hline 5 & 2 & 2.2402 \\
\hline 5.5 & 1.5 & 12.5295 \\
\hline 6 & 1 & 96.8612 \\
\hline 6.5 & 0.5 & 977.2143 \\
\hline 7 & 0 & $1,242810^{4}$ \\
\hline 7.5 & -0.5 & $5,073810^{5}$ \\
\hline 8 & -1 & $1,016810^{7}$ \\
\hline 8.5 & -1.5 & $2,413310^{8}$ \\
\hline \hline
\end{tabular}

Tabla B.1: Evolución del elemento más singular de la matriz de traslación de espectro emergente a incidente al disminuir el margen con $d_{21}$

\section{B.3. Traslación de espectro incidente a incidente}

Supongamos la situación de la figura B.9, en la que un campo $E_{z}$ incidente está expresado como una suma de modos cilíndricos centrados en el punto $\left(x_{c_{1}}, y_{c_{1}}\right)$, origen del sistema de coordenadas formado por los ejes $X_{1}, Y_{1}$ :

$$
E_{z}\left(\overrightarrow{\rho_{1}}\right)=\sum_{p=-N_{i_{1}}}^{N_{i_{1}}} i_{p} J_{p}\left(k \rho_{1}\right) e^{j p \phi_{1}}
$$

donde $\overrightarrow{\rho_{1}}$ está referido al sistema de coordenadas definido por los ejes $X_{1}, Y_{1}$.

Se desea expresar el campo $E_{z}$ como una suma de modos cilíndricos centrados en el punto $\left(x_{c_{2}}, y_{c_{2}}\right)$, origen del sistema de coordenadas formado por los ejes $X_{2}, Y_{2}$ :

$$
E_{z}\left(\overrightarrow{\rho_{2}}\right)=\sum_{r=-N_{i_{2}}}^{N_{i_{2}}} i_{r}^{\prime} J_{r}\left(k \rho_{2}\right) e^{j r \phi_{2}}
$$

donde $\overrightarrow{\rho_{2}}$ está referido al sistema de coordenadas definido por los ejes $X_{2}, Y_{2}$. 


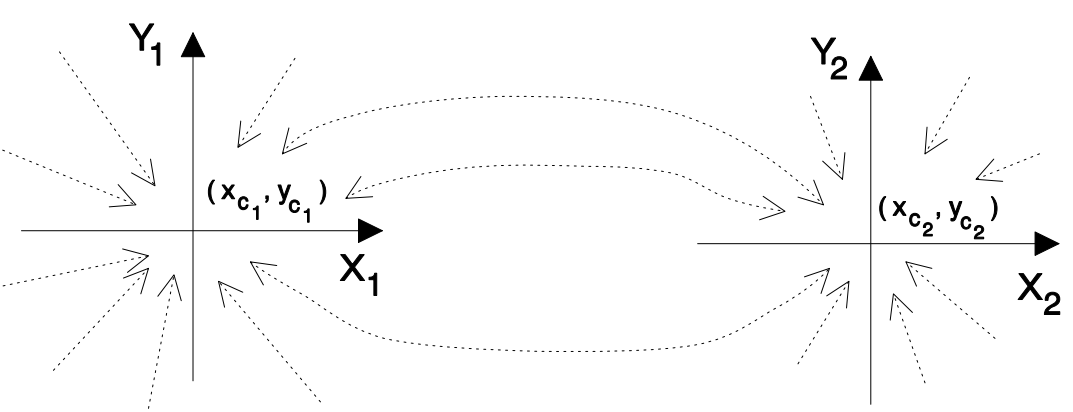

Figura B.9: Posición de los ejes $X_{1}, Y_{1}$ y $X_{2}, Y_{2}$.

Para poder realizar esta traslación de un espectro incidente centrado en $\left(x_{c_{1}}, y_{c_{1}}\right)$ a otro incidente centrado en $\left(x_{c_{2}}, y_{c_{2}}\right)$, debemos expresar los modos cilíndricos $J_{p}\left(k \rho_{1}\right) e^{j p \phi_{1}}$ centrados en $\left(x_{c_{1}}, y_{c_{1}}\right)$ como sumatorio de modos cilíndricos $J_{r}\left(k \rho_{2}\right) e^{j r \phi_{2}}$ centrados en $\left(x_{c_{2}}, y_{c_{2}}\right)$. Esta relación la proporciona la siguiente expresión [8]:

$$
J_{p}\left(k \rho_{1}\right) e^{j p \phi_{1}}=\sum_{r=-\infty}^{\infty} J_{r-p}\left(k d_{21}\right) e^{-j(r-p) \phi_{21}} J_{r}\left(k \rho_{2}\right) e^{j r \phi_{2}}
$$

donde $d_{21}$ es la distancia entre los puntos $\left(x_{c_{1}}, y_{c_{1}}\right)$ y $\left(x_{c_{2}}, y_{c_{2}}\right)$ y $\phi_{21}$ es la coordenada $\phi$ del punto $\left(x_{c_{1}}, y_{c_{1}}\right)$ en el sistema de coordenadas definido por los ejes $X_{2}, Y_{2}$. Ambos parámetros $d_{21}$ y $\phi_{21}$ se ilustran en la figura B.2, y pueden obtenerse como:

$$
\begin{aligned}
d_{21} & =\sqrt{\left(x_{c_{1}}-x_{c_{2}}\right)^{2}+\left(y_{c_{1}}-y_{c_{2}}\right)^{2}} \\
\tan \phi_{21} & =\frac{y_{c_{1}}-y_{c_{2}}}{x_{c_{1}}-x_{c_{2}}}
\end{aligned}
$$

La expresión anterior permite obtener la relación entre el espectro $\left[i_{p}\right]$ centrado en $\left(x_{c_{1}}, y_{c_{1}}\right)$ y el espectro $\left[i_{r}^{\prime}\right]$ centrado en $\left(x_{c_{2}}, y_{c_{2}}\right)$ : 


$$
\begin{aligned}
E_{z} & =\sum_{p=-N_{i_{1}}}^{N_{i_{1}}} i_{p} J_{p}\left(k \rho_{1}\right) e^{j p \phi_{1}} \\
& =\sum_{p=-N_{i_{1}}}^{N_{i_{1}}} i_{p} \sum_{r=-\infty}^{\infty} J_{r-p}\left(k d_{21}\right) e^{-j(r-p) \phi_{21}} J_{r}\left(k \rho_{2}\right) e^{j r \phi_{2}} \\
& =\sum_{r=-\infty}^{\infty}\left(\sum_{p=-N_{i_{1}}}^{N_{i_{1}}} i_{p} J_{r-p}\left(k d_{21}\right) e^{-j(r-p) \phi_{21}}\right) J_{r}\left(k \rho_{2}\right) e^{j r \phi_{2}} \\
& =\sum_{r=-\infty}^{\infty} i_{r}^{\prime} J_{r}\left(k \rho_{2}\right) e^{j r \phi_{2}} \\
& \simeq \sum_{r=-N_{i_{2}}}^{N_{i_{2}}} i_{r}^{\prime} J_{r}\left(k \rho_{2}\right) e^{j r \phi_{2}}
\end{aligned}
$$

A partir de estas expresiones se puede construir ya la matriz $T J_{21}$ de traslación de espectro incidente centrado en $\left(x_{c_{1}}, y_{c_{1}}\right)$ a incidente centrado en $\left(x_{c_{2}}, y_{c_{2}}\right)$ :

$$
\left[\begin{array}{c}
i_{-N_{i_{2}}} \\
i_{-N_{i_{2}}+1} \\
\vdots \\
i_{N_{i_{2}}}
\end{array}\right]=\begin{gathered}
-N_{i_{2}} \\
\downarrow \\
r \\
\downarrow \\
N_{i_{2}}
\end{gathered} \overbrace{\left[\begin{array}{l}
J_{r-p}\left(k d_{21}\right) e^{-j(r-p) \phi_{21}} \\
\end{array}\right]}^{-N_{i_{1}} \longrightarrow p \longrightarrow N_{i_{1}}}\left[\begin{array}{c}
c_{-N_{i_{1}}} \\
c_{-N_{i_{1}}+1} \\
\vdots \\
c_{N_{i_{1}}}
\end{array}\right]
$$

La siguiente expresión del campo reconstruido a partir de su espectro centrado en el punto $\left(x_{c_{1}}, y_{c_{1}}\right)$ con $2 N_{i_{1}}+1$ modos

$$
\sum_{p=-N_{i_{1}}}^{N_{i_{1}}} i_{p} J_{p}\left(k \rho_{1}\right) e^{j p \phi_{1}}
$$

tan sólo es válida dentro de la circunferencia de radio $N_{i_{1}} / k$ y centrada en el punto $\left(x_{c_{1}}, y_{c_{1}}\right)$, como se muestra en la figura B.10.

La reconstrucción del campo a partir de su espectro centrado en el punto $\left(x_{c_{2}}, y_{c_{2}}\right)$ utilizando infinitos modos

$$
\sum_{r=-\infty}^{\infty}\left(\sum_{p=-N_{i_{1}}}^{N_{i_{1}}} i_{p} J_{r-p}\left(k d_{21}\right) e^{-j(r-p) \phi_{21}}\right) J_{r}\left(k \rho_{2}\right) e^{j r \phi_{2}}
$$




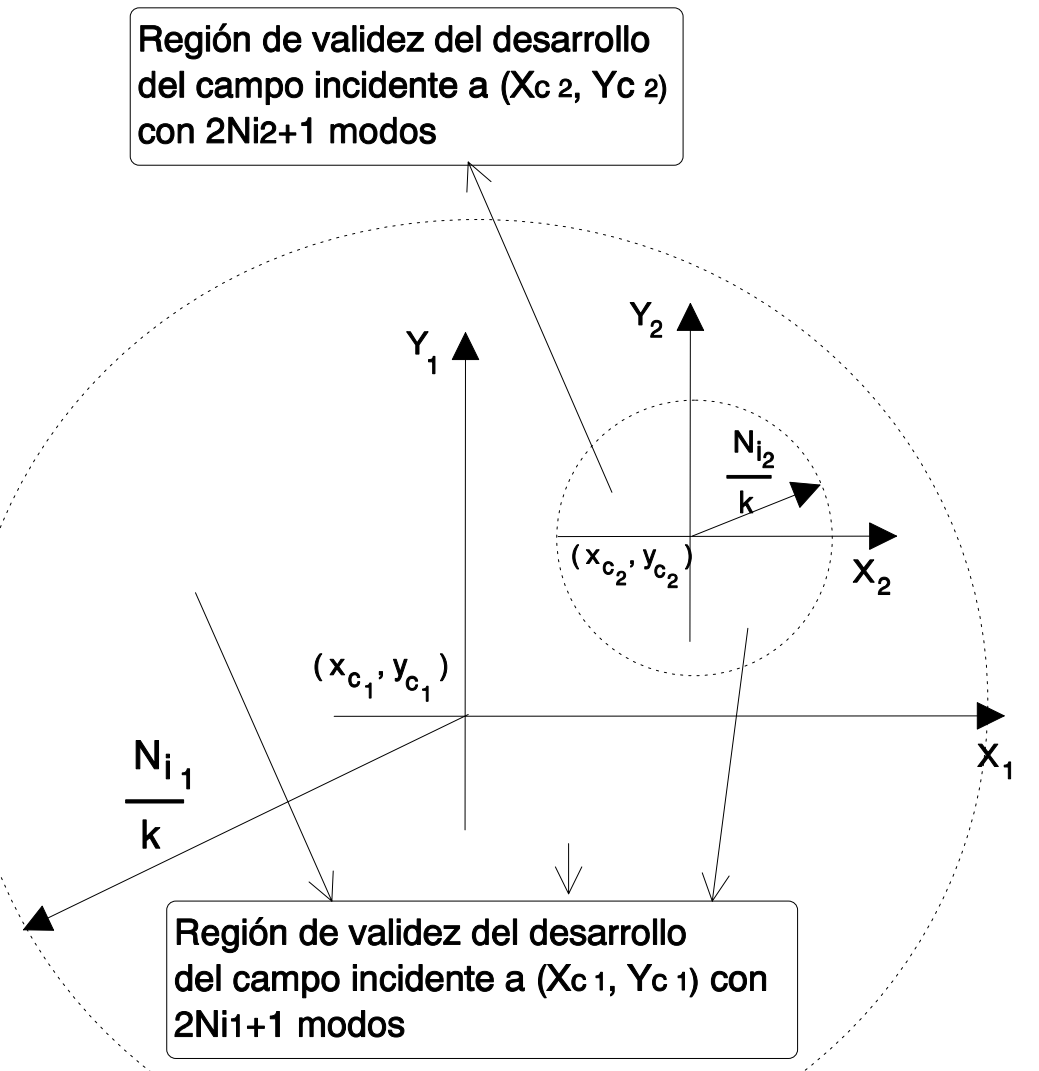

Figura B.10: Regiones de validez de los distintos desarrollos modales en la traslación de espectro incidente a incidente

es también sólo válida en la misma región.

Si en lugar de tomar infinitos modos cilíndricos centrados en $\left(x_{c_{2}}, y_{c_{2}}\right),(r$ variando desde $-\infty$ a $\infty$ ), tomamos $2 N_{i_{2}}+1$ modos, la reconstrucción del campo mediante la siguiente expresión:

$$
\sum_{r=-N_{i_{2}}}^{N_{i_{2}}}\left(\sum_{p=-N_{i_{1}}}^{N_{i_{1}}} i_{p} J_{r-p}\left(k d_{21}\right) e^{-j(r-p) \phi_{21}}\right) J_{r}\left(k \rho_{2}\right) e^{j r \phi_{2}}
$$

es válida en la región interna a la circunferencia de radio $N_{i_{1}} / k \mathbf{y}$ centrada en el punto $\left(x_{c_{1}}, y_{c_{1}}\right)$, e interna a la circunferencia de radio $N_{i_{2}} / k$ y centrada en el punto $\left(x_{c_{2}}, y_{c_{2}}\right)$, como se ilustra en la figura B.10.

En las figuras B.11, B.12 y B.13 se ilustra un ejemplo de traslación de espectro incidente centrado en un punto a incidente centrado en otro. La figura B.11 presenta la amplitud y la fase de una onda plana que avanza paralela al eje $X$, hacia valores crecientes de la coordenada $x$ (es decir, $\beta=0$ ). Dicha onda plana ha sido reconstruida a partir de su espectro cilíndrico 

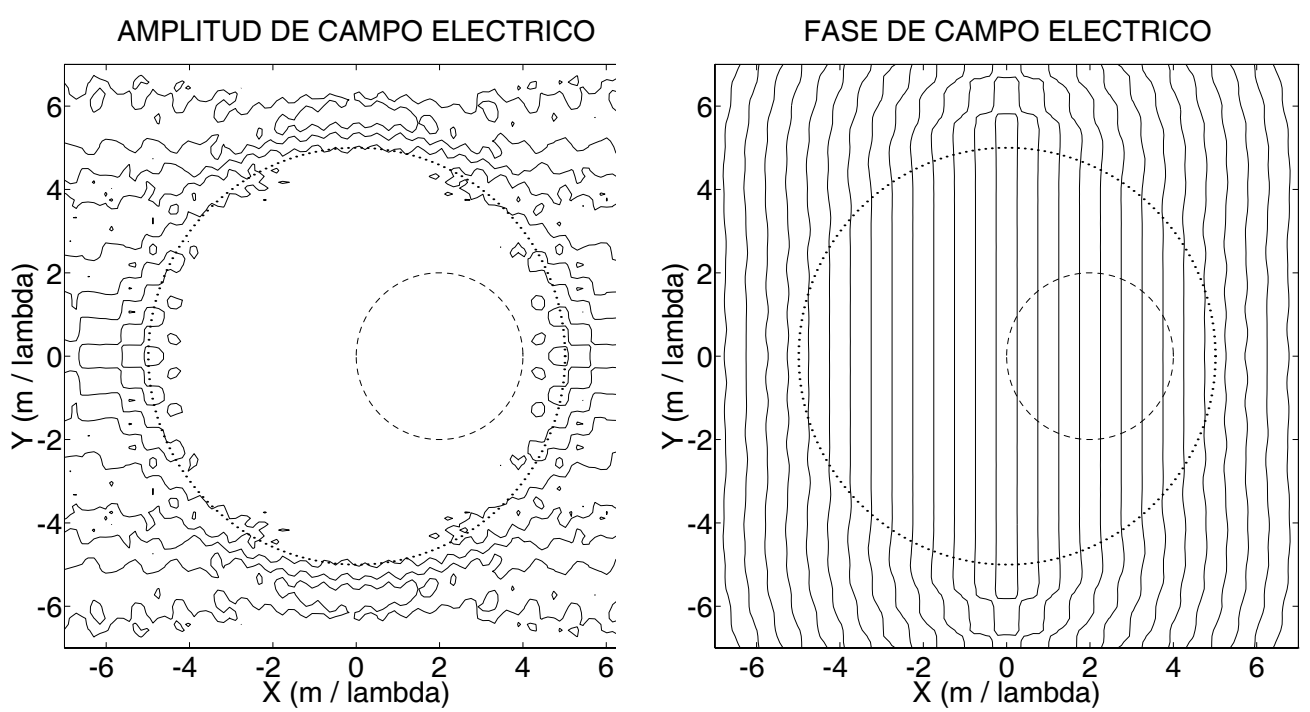

Figura B.11: Amplitud y fase una onda plana $(\beta=0)$. Reconstrucción a partir del espectro cilíndrico centrado en $(0,0)\left(N_{i_{1}}=k 5 \lambda\right)$
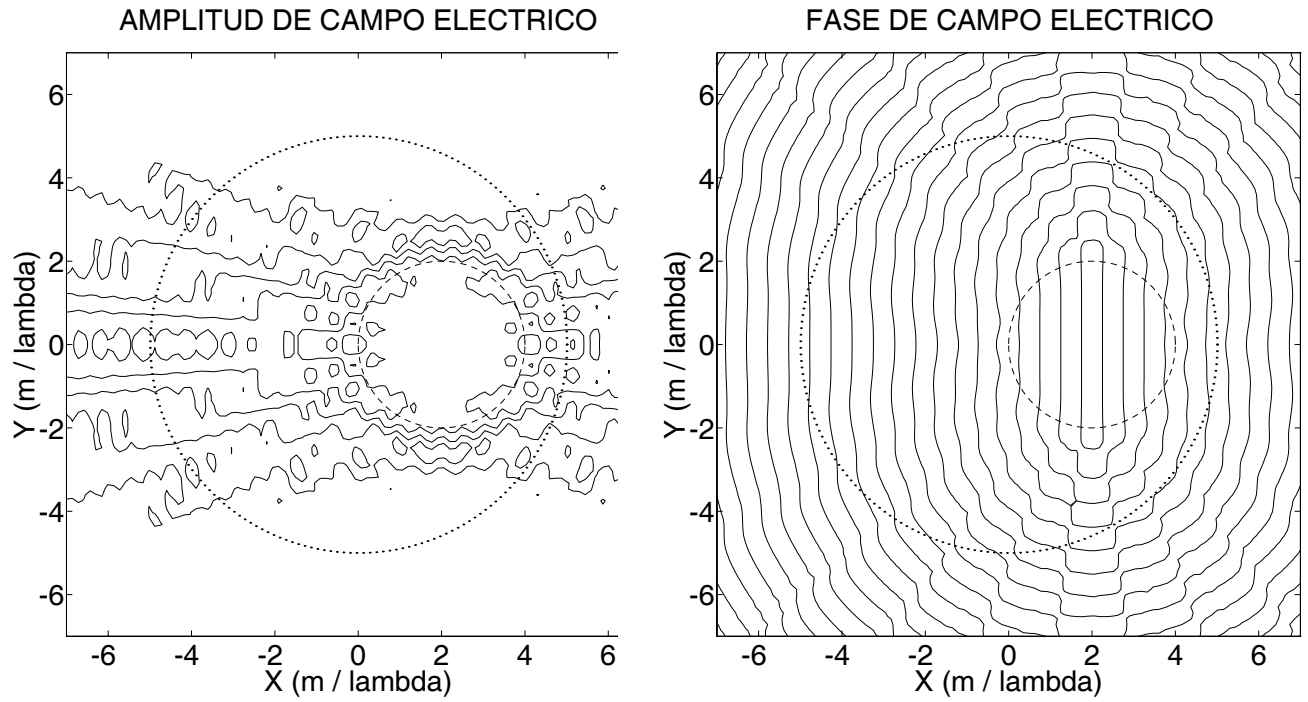

Figura B.12: Amplitud y fase una onda plana $(\beta=0)$. Reconstrucción a partir del espectro cilíndrico centrado en $(2,0)\left(N_{i_{2}}=k 2 \lambda\right)$

(ver apéndice A) con $2 N_{i_{1}}+1$ modos centrados en el punto $(0,0)$, siendo $N_{i_{1}}=k 5 \lambda$.

Se aprecia como la amplitud de la reconstrucción de la onda plana es constante en amplitud y por tanto correcta en la circunferencia centrada en $(0,0)$ y de radio $5 \lambda$. La fase se corresponde correctamente a la de una onda plana en la misma zona. Esta región es la zona donde el campo se reconstruye correctamente para el número de modos utilizados $\left(N_{i_{1}}=k 5 \lambda\right)$.

Se ha dibujado una circunferencia con línea discontinua con centro en el punto $(2,0)$ y radio $2 \lambda$. Dicha circunferencia representa la zona donde queremos reconstruir la onda plana a partir de un espectro incidente centrado en el punto $(2,0)$. Utilizando la matriz de traslación 

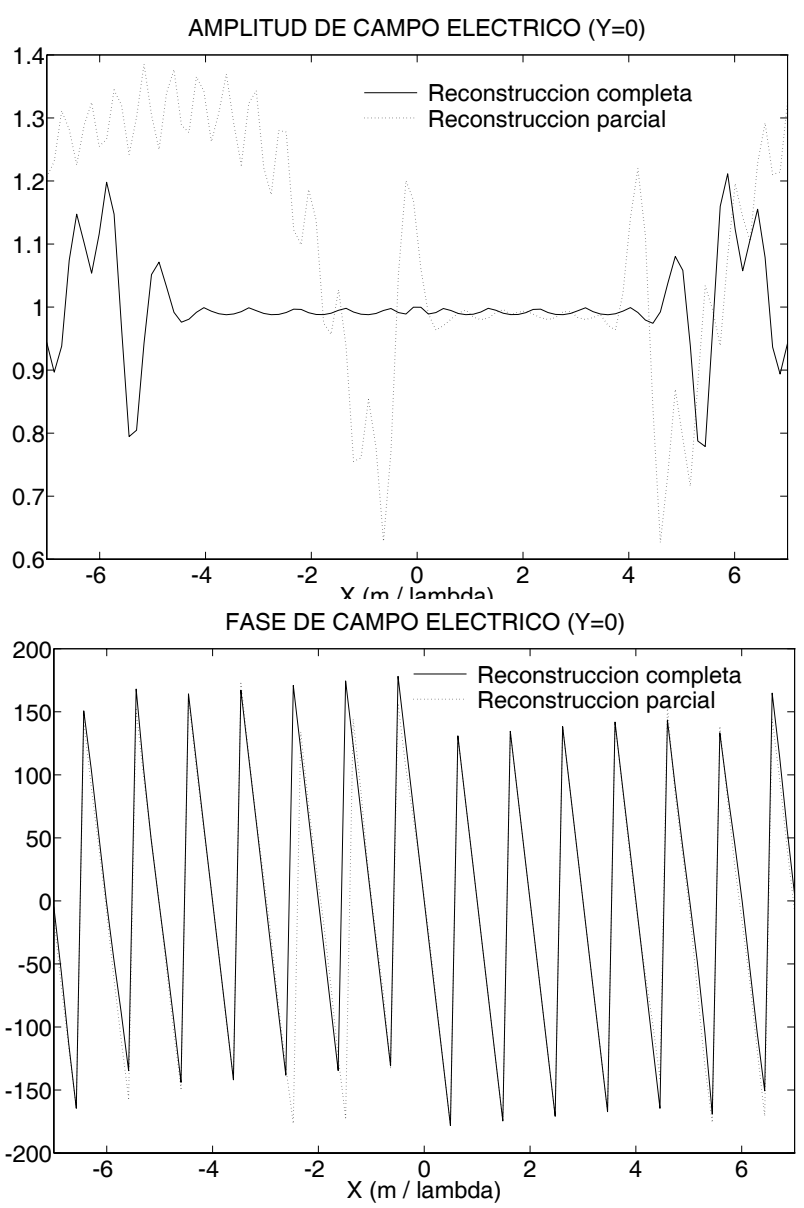

Figura B.13: Amplitud y fase una onda plana $(\beta=0)$. Reconstrucciones total $\left(N_{i_{1}}=k 5 \lambda\right)$ y parcial $\left(N_{i_{2}}=k 2 \lambda\right)$.

de coordenadas de espectro incidente centrado en $(0,0)$ a espectro incidente centrado en el punto $(2,0)$, se obtiene el espectro de campo incidente centrado en el nuevo origen $(2,0)$.

La amplitud y la fase de la reconstrucción de la onda plana a partir del espectro incidente centrado en $(2,0)$ se representa en la figura B.12. Para reconstruir el campo se han utilizado $2 N_{i_{2}}+1$ modos, donde $N_{i_{2}}=k 2 \lambda$.

La reconstrucción de la onda plana con $2 N_{i_{2}}+1$ modos centrados en $(2,0)$ coincide como se esperaba con la de la figura B.11 dentro de la circunferencia de línea discontinua, que es una circunferencia de radio $2 \lambda$, correspondiente a la zona teórica de reconstrucción correcta para el número de modos utilizados. Para reconstruir el campo correctamente en una zona más amplia se debe escoger un valor de $N_{i_{2}}$ mayor.

En la figura B.13 se representan la amplitud y la fase de la misma onda plana a lo largo del eje $X$. Se ha representado la reconstrucción del campo a partir del espectro centrado en $(0,0)$ (reconstrucción total) y a partir del espectro centrado en $(2,0)$ (reconstrucción parcial). Ambas reconstrucciones son muy semejantes dentro del intervalo $x \in[0,4]$, de modo que el espectro centrado en $(2,0)$ obtenido mediante la matriz de traslación permite reconstruir un 

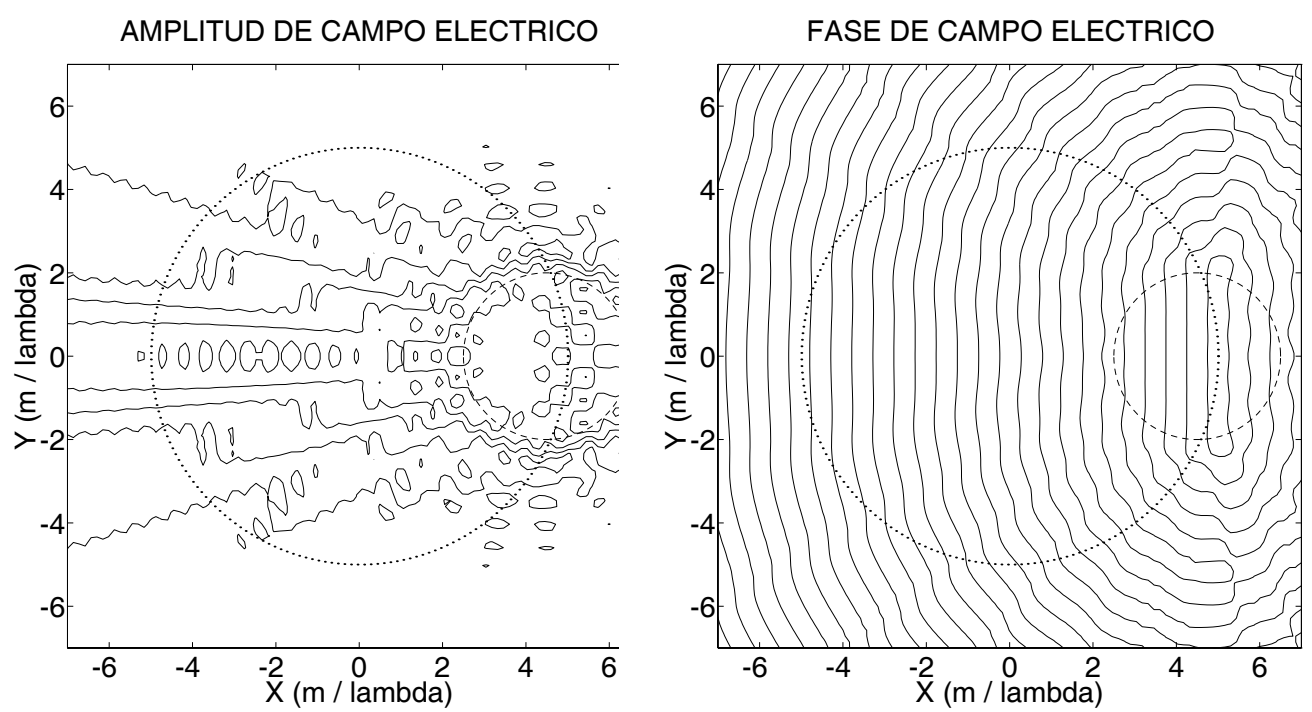

Figura B.14: Amplitud y fase una onda plana $(\beta=0)$. Reconstrucción a partir del espectro cilíndrico centrado en $(4,5,0)\left(N_{i_{2}}=k 2 \lambda\right)$

campo que se ajusta bien en esa zona al campo original centrado en $(0,0)$.

En la figura B.14 se presenta la amplitud y la fase de la onda plana reconstruida con un espectro centrado en $(4,5,0)$ con $N_{i_{2}}=k 2 \lambda$. Se ha tomado el mismo número de modos que antes, pero el espectro se ha centrado en $(4,5,0)$ y no en $(2,0)$. Esto nos permite comprobar cómo la nueva reconstrucción se ajusta bien a la original en toda la circunferencia centrada en $(4,5,0)$ y de radio $2 \lambda$. Sin embargo la reconstrucción original, con $N_{i_{1}}=k 5 \lambda$, proporciona una amplitud y fase correspondientes a una onda plana tan sólo en un radio de $5 \lambda$ desde $(0,0)$. Por este motivo el espectro centrado en $(4,5,0)$ no reconstruye una onda plana correctamente dentro de la toda la circunferencia de radio $2 \lambda$ centrada en $(4,5,0)$, sino tan sólo en la parte de dicha circunferencia contenida dentro de la circunferencia de radio $5 \lambda$ centrada en $(0,0)$.

En la figura B.15 se presenta el mismo resultado que en la figura B.14 pero a lo largo del eje $X$. Se aprecia con mayor precisión en esta figura cómo se ajusta la reconstrucción proporcionada por el espectro centrado en $(4,5,0)$ al original en el intervalo $x \in[2,5,6,5]$. Sin embargo, tan sólo se reconstruye correctamente una onda plana en el intervalo $x \in$ $[2,5,5]$. 

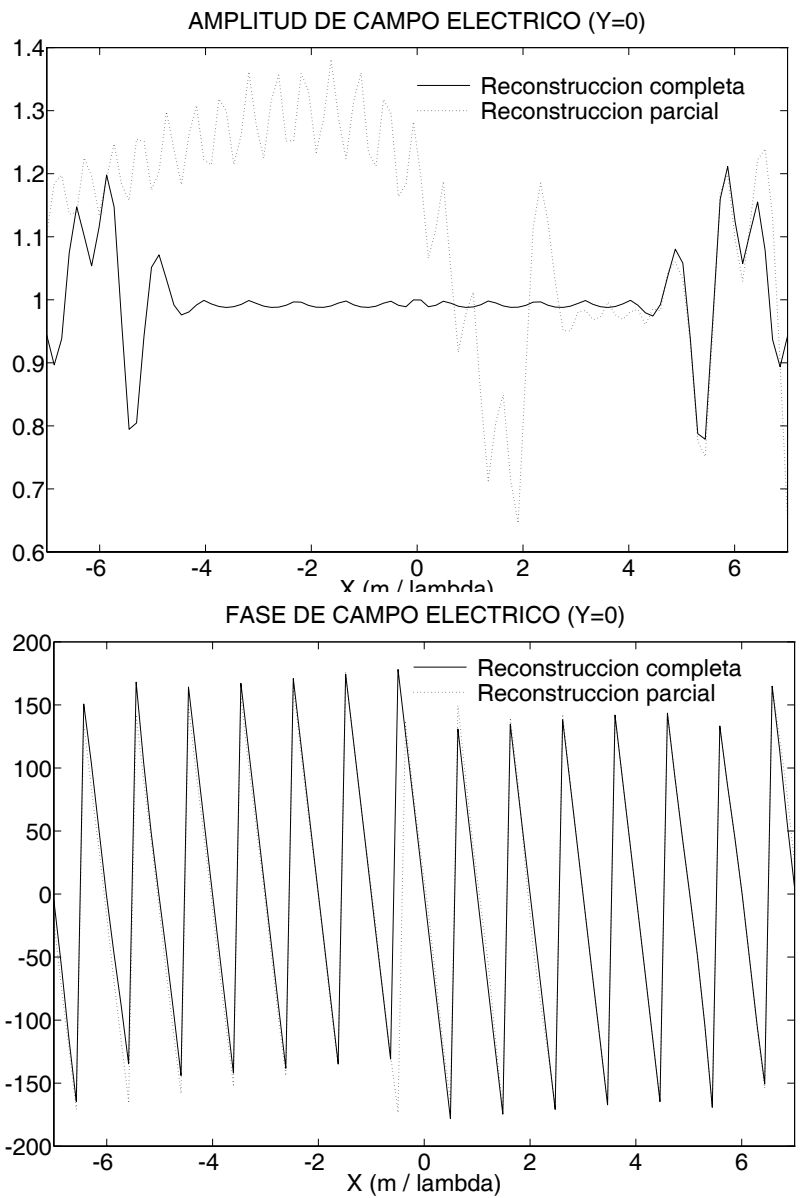

Figura B.15: Amplitud y fase una onda plana $(\beta=0)$. Reconstrucciones total $\left(N_{i_{1}}=k 5 \lambda\right)$ y parcial $\left(N_{i_{2}}=k 2 \lambda\right)$. 


\section{Apéndice C}

\section{Número de modos cilíndricos para el acoplo con modos guiados}

Al forzar la igualdad de campos transversales expresados como suma de los modos guiados y de modos cilíndricos, resulta necesario realizar la DTFT inversa sobre las expresiones de modos guiados. Esta operación nos proporcionará los coeficientes de la serie en modos cilíndricos que corresponde a los campos guiados. En realidad lo que estamos haciendo es expresar cada modo guiado como suma de modos cilíndricos, para así poder comparar las expresiones de los campos transversales guiados con las de espacio abierto. El resultado de dicha DTFT inversa se trunca de modo que tan sólo se toman $2 N+1$ términos. Pero para asegurarnos de que al truncar no estamos perdiendo información es necesario realizar un estudio previo que determine el mínimo valor de $N$ que permite truncar la serie de modos cilíndricos sin perder información.

La expresión del campo eléctrico en la circunferencia de radio $R$ como suma de modos guiados es:

$$
\vec{E}_{t}^{g}(\rho=R, \phi)=\sum_{i=1}^{J} \vec{E}_{t}^{(i)}(R, \phi)
$$

donde $\vec{E}_{t}^{(i)}$ es el campo eléctrico transversal en el acceso $i$, el cual se pueden expresar como suma de modos incidentes y reflejados en dicho acceso de la siguiente manera:

$$
\vec{E}_{t}^{(i)}(R, \phi)=\sum_{m=1}^{M_{i}} a_{m}^{(i)} e_{m}^{(i)^{\prime \prime}}\left(x_{i}\right) e^{-\gamma_{m}^{(i)} z_{i}} \hat{z}+\sum_{m=1}^{M_{i}} b_{m}^{(i)} e_{m}^{(i)^{\prime \prime}}\left(x_{i}\right) e^{\gamma_{m}^{(i)} z_{i}} \hat{z}
$$

De todos los modos en el acceso $i$, el más crítico (el que más variaciones tiene entre $x_{i}=0$ y $x_{i}=a_{i}$ ), y por tanto mayor número de modos cilíndricos requiere para reconstruirlo correctamente es el modo de mayor orden, el $M_{i}$, tanto si se trata del modo progresivo como del regresivo. Llamaremos $F^{+}(\phi)$ y $F^{-}(\phi)$ a las distribuciones de campo de estos modos 
a lo largo de la circunferencia de radio $R$, y estudiaremos el número de modos cilíndricos necesario para reconstruirlos correctamente.

$$
\begin{aligned}
& F^{+}(\phi)=a_{M_{i}}^{(i)} e_{M_{i}}^{(i)^{\prime \prime}}\left(x_{i}\right) e^{-\gamma_{M_{i}}^{(i)} z_{i}} \\
& F^{-}(\phi)=b_{M_{i}}^{(i)} e_{M_{i}}^{(i)^{\prime \prime}}\left(x_{i}\right) e^{\gamma_{M_{i}}^{(i)} z_{i}}
\end{aligned}
$$

Teniendo en cuenta que

$$
\begin{aligned}
e_{M_{i}}^{(i)^{\prime \prime}}\left(x_{i}\right) & = \begin{cases}-\sqrt{\frac{2 Z_{0 m}^{(i)}}{a_{i} b_{i}}} \sin \left(\frac{M_{i} \pi}{a_{i}} x_{i}\right) & \phi \in\left[\phi_{i m}, \phi_{i M}\right] \\
0 & \text { resto }\end{cases} \\
& =-\sqrt{\frac{2 Z_{0 m}^{(i)}}{a_{i} b_{i}}} \sin \left(\frac{M_{i} \pi}{a_{i}} x_{i}\right) \prod\left(\frac{\phi-\phi_{0 i}}{\phi_{d i}}\right)
\end{aligned}
$$

donde:

$$
\begin{aligned}
\phi_{0 i} & =\frac{\phi_{i M}+\phi_{i m}}{2} \\
\phi_{d i} & =\phi_{i M}-\phi_{i m}
\end{aligned}
$$

Las expresiones anteriores quedan pues de la siguiente manera:

$$
\begin{aligned}
F^{+}(\phi) & =-a_{M_{i}}^{(i)} \sqrt{\frac{2 Z_{0 m}^{(i)}}{a_{i} b_{i}}} \sin \left(\frac{M_{i} \pi}{a_{i}} x_{i}\right) \prod\left(\frac{\phi-\phi_{0 i}}{\phi_{d i}}\right) e^{-\gamma_{M_{i}}^{(i)} z_{i}} \\
& =C_{+} \sin \left(\frac{M_{i} \pi}{a_{i}} x_{i}\right) \prod\left(\frac{\phi-\phi_{0 i}}{\phi_{d i}}\right) e^{-\gamma_{M_{i}}^{(i)} z_{i}} \\
F^{-}(\phi) & =-b_{M_{i}}^{(i)} \sqrt{\frac{2 Z_{0 m}^{(i)}}{a_{i} b_{i}}} \sin \left(\frac{M_{i} \pi}{a_{i}} x_{i}\right) \prod\left(\frac{\phi-\phi_{0 i}}{\phi_{d i}}\right) e^{\gamma_{M_{i}}^{(i)} z_{i}} \\
& =C_{-} \sin \left(\frac{M_{i} \pi}{a_{i}} x_{i}\right) \prod\left(\frac{\phi-\phi_{0 i}}{\phi_{d i}}\right) e^{\gamma_{M_{i}}^{(i)} z_{i}}
\end{aligned}
$$

Para simplificar el cálculo del número de modos cilíndricos necesario para reconstruir $F^{ \pm}(\phi)$, y sin pérdida de generalidad, consideraremos un caso sencillo en el que el acceso $i$ esté situado paralelo al eje $x$ y acceda a la circunferencia de radio $R$ por $\phi=0$ (ver figura C.1). En este caso: 


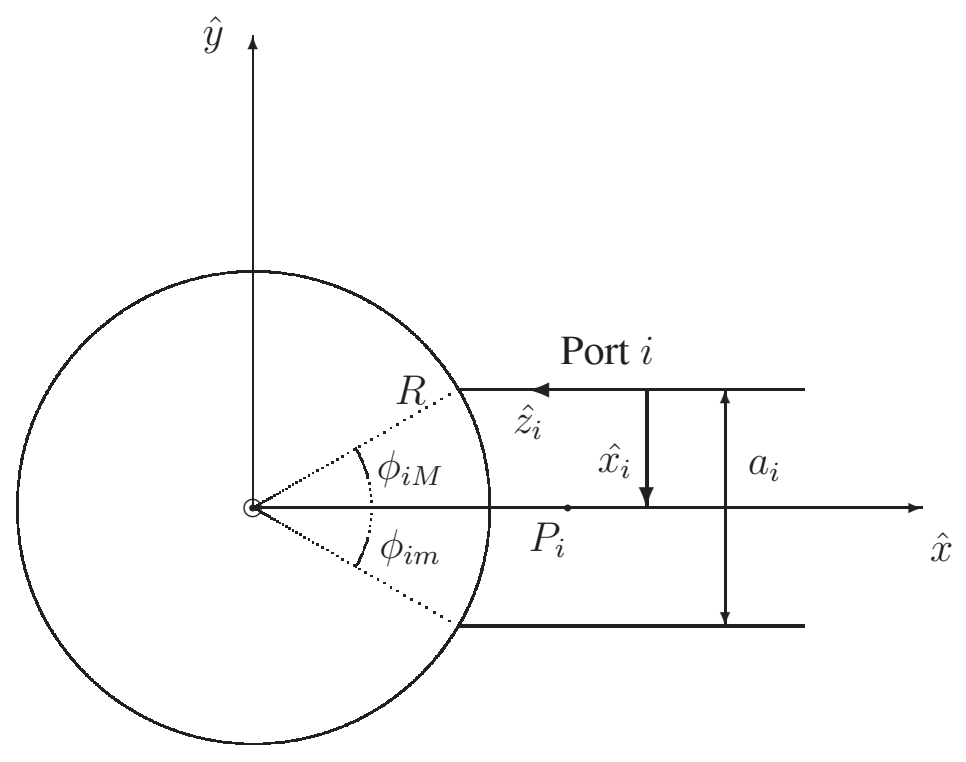

Figura C.1: Caso sencillo de acceso situado en $\phi=0$

$$
\begin{aligned}
\hat{x}_{i} & =-\hat{y} \\
\hat{z}_{i} & =-\hat{x} \\
x_{i} & =-R \sin (\phi)+\frac{a_{i}}{2} \\
z_{i} & =-R \cos (\phi)+\left\|P_{i}\right\| \\
\phi_{i m} & =-\arcsin \left(\frac{a_{i}}{2 R}\right) \\
\phi_{i M} & =\arcsin \left(\frac{a_{i}}{2 R}\right) \\
\phi_{0 i} & =0 \\
\phi_{d i} & =2 \arcsin \left(\frac{a_{i}}{2 R}\right)
\end{aligned}
$$

Podemos expresar $F^{ \pm}(\phi)$ como el producto de dos funciones diferentes:

$$
F^{ \pm}(\phi)=C_{ \pm} F_{1}(\phi) F_{2}(\phi)
$$


donde

$$
\begin{aligned}
& F_{1}(\phi)=\sin \left(\frac{M_{i} \pi}{a_{i}}\left(-R \sin (\phi)+\frac{a_{i}}{2}\right)\right) \\
& F_{2}(\phi)=\prod\left(\frac{\phi}{2 \arcsin \left(\frac{a_{i}}{2 R}\right)}\right) e^{ \pm \gamma_{M_{i}}^{(i)} R \cos (\phi)} e^{\mp \gamma_{M_{i}}^{(i)}\left\|P_{i}\right\|}
\end{aligned}
$$

Los coeficientes de la serie de modos cilíndricos que reconstruye $F^{ \pm}(\phi)$ se obtienen a partir de la DTFT inversa de la siguiente manera:

$$
\begin{gathered}
F^{ \pm}(\phi)=\sum_{n=-\infty}^{\infty}\left[f^{\prime}[n] J_{n}(K R)\right] e^{j n \phi}=\sum_{n=-\infty}^{\infty} f^{ \pm}[n] e^{j n \phi} \simeq \sum_{n=-N}^{N} f^{ \pm}[n] e^{j n \phi} \\
f^{ \pm}[n]=\frac{1}{2 \pi} \int_{-\pi}^{\pi} F^{ \pm}(\phi) e^{-j n \phi}
\end{gathered}
$$

O lo que es lo mismo:

$$
f^{ \pm}[-n]=\operatorname{DTFT}^{(-1)}\left\{F^{ \pm}(\phi)\right\}
$$

Por tanto el valor de $N$ (número mínimo de modos cilíndricos de dicha serie) nos lo proporcionará la duración de dicha DTFT inversa $f^{ \pm}[-n]$. Como hemos expresado $F^{ \pm}(\phi)$ como el producto de dos funciones, la DTFT inversa será la convolución de la DTFT inversa de esas dos funciones:

$$
f^{ \pm}[-n]=C_{ \pm} f_{1}[-n] * f_{2}[-n]
$$

Y la duración será:

$$
\operatorname{Dur}\left\{f^{ \pm}[-n]\right\}=\operatorname{Dur}\left\{f_{1}[-n]\right\}+\operatorname{Dur}\left\{f_{2}[-n]\right\}-1
$$

Para calcular la DTFT inversa de $F_{1}(\phi)$, aproximaremos la función $\sin (\phi)$ por su serie de Taylor en torno a $\phi=0$. Esta aproximación será bastante exacta ya que, como se puede apreciar en la figura C.1, el valor de $\phi$ estará próximo a 0 dentro de la guía, y en el peor de los casos llegará a valer $\pi / 2$, pero no más. Por tanto:

$$
\sin (\phi) \simeq \sin (0)+\cos (0) \phi-\sin (0) \frac{\phi^{2}}{2 !}+\cdots \simeq \phi
$$

Sustituyendo la aproximación del seno en (C.19), se obtiene

$$
F_{1}(\phi) \simeq \sin \left(\frac{M_{i} \pi}{a_{i}}\left(-R \phi+\frac{a_{i}}{2}\right)\right)
$$


Ahora la transformada inversa de $F_{1}(\phi)$ es inmediata:

$$
f_{1}[-n] \simeq \frac{1}{2 j}\left(e^{j M_{i} \frac{\pi}{2}} \delta\left[n-\frac{M_{i} \pi R}{a_{i}}\right]-e^{-j M_{i} \frac{\pi}{2}} \delta\left[n+\frac{M_{i} \pi R}{a_{i}}\right]\right)
$$

Vemos que la señal $f_{1}[-n]$ es aproximadamente un par de deltas centradas en $n=\frac{M_{i} \pi R}{a_{i}}$ y $n=-\frac{M_{i} \pi R}{a_{i}}$. Por lo tanto su duración será:

$$
\operatorname{Dur}\left\{f_{1}[-n]\right\}=2\left[\frac{M_{i} \pi R}{a_{i}}\right]+1
$$

Para hallar la duración de $f_{2}[-n]$, debemos primero hallar la transformada inversa de $F_{2}(\phi)$. De forma analítica no es posible hallar $f_{2}[-n]$, ya que el pulso de $F_{2}(\phi)$ sí tiene transforma inversa analítica, pero no así la exponencial del coseno. Así que deberemos utilizar algún tipo de aproximación para la exponencial del coseno. No sirve de nada aproximar el coseno por su serie de Taylor, porque nos quedarían exponenciales reales de potencias de $\phi$, y eso tampoco tiene transformada inversa analítica. Una posible solución es plantear la ecuación de la transformada inversa y aproximar el integrando por su serie de Taylor en el entorno de $\phi=0$.

Por tanto, comenzaremos por plantear la ecuación de la transformada inversa:

$$
\begin{aligned}
f_{2}[-n] & =\frac{1}{2 \pi} \int_{-\pi}^{\pi} F_{2}(\phi) e^{j n \phi} \mathrm{d} \phi \\
& =\frac{1}{2 \pi} \int_{-\pi}^{\pi} \prod\left(\frac{\phi}{2 \arcsin \left(\frac{a_{i}}{2 R}\right)}\right) e^{ \pm \gamma_{M_{i}}^{(i)} R \cos (\phi)} e^{\mp \gamma_{M_{i}}^{(i)}\left\|P_{i}\right\|} e^{j n \phi} \mathrm{d} \phi \\
& =\frac{1}{2 \pi} \int_{-\arcsin \left(\frac{a_{i}}{2 R}\right)}^{\arcsin \left(\frac{a_{i}}{2 R}\right)} e^{\mp \gamma_{M_{i}}^{(i)}\left\|P_{i}\right\|} e^{ \pm \gamma_{M_{i}}^{(i)} R \cos (\phi)} e^{j n \phi} \mathrm{d} \phi
\end{aligned}
$$

Por simplicidad, utilizaremos la siguiente notación:

$$
\begin{aligned}
L & =2 \arcsin \left(\frac{a_{i}}{2 R}\right) \\
K & =\gamma_{M_{i}}^{(i)} R \\
P & =e^{\mp \gamma_{M_{i}}^{(i)}\left\|P_{i}\right\|}
\end{aligned}
$$

Sustituyendo en (C.29) obtenemos:

$$
f_{2}[-n]=\frac{1}{2 \pi} \int_{-\frac{L}{2}}^{\frac{L}{2}} P e^{ \pm K \cos (\phi)} e^{j n \phi} \mathrm{d} \phi
$$


Vemos que la exponencial real puede tener argumento positivo o negativo. El caso peor, puesto que el coseno es una función decreciente en el entorno de $\phi=0$ es cuando la exponencial es negativa, y por tanto creciente. En ese caso la duración de $f_{2}[-n]$ será mayor. Por tanto, analizaremos ese caso:

$$
f_{2}[-n]=\frac{1}{2 \pi} \int_{-\frac{L}{2}}^{\frac{L}{2}} P e^{-K \cos (\phi)} e^{j n \phi} \mathrm{d} \phi
$$

Esta integral no tiene solución analítica. Lo que haremos, como se ha sugerido con anterioridad, será aproximar la exponencial real por su serie de Taylor en el entorno de $\phi=0$, tomando hasta la décima potencia, ya que deseamos que la aproximación sea buena en todo el intervalo $\phi=\left[-\frac{\pi}{2}, \frac{\pi}{2}\right]$, pues el valor máximo de $L$ es $\pi$. Además, interesa tomar muchas potencias dado que la señal, para valores grandes de $K$, variará de forma rápida y se necesitará de un polinomio de grado elevado para seguir esa variación. Por otro lado, como la exponencial considerada es una función par, sus derivadas impares en el origen serán nulas, de modo que la serie de Taylor hasta la décima potencia se reduce a lo siguiente:

$$
\begin{aligned}
G(\phi)= & e^{-K \cos (\phi)} \\
\simeq & G(0)+G^{\prime \prime}(0) \frac{\phi^{2}}{2 !}+G^{(4)}(0) \frac{\phi^{4}}{4 !} \\
& +G^{(6)}(0) \frac{\phi^{6}}{6 !}+G^{(8)}(0) \frac{\phi^{8}}{8 !}+G^{(10)}(0) \frac{\phi^{10}}{10 !}
\end{aligned}
$$

A continuación resolveremos analíticamente la integral de (C.33) sustituyendo el integrando por su desarrollo en serie de Taylor hasta la décima potencia:

$$
\begin{aligned}
f_{2}[-n] & =\frac{1}{2 \pi} \int_{-\frac{L}{2}}^{\frac{L}{2}} G(\phi) e^{j n \phi} \mathrm{d} \phi \\
& \simeq \frac{A_{1}(K, L, n)}{A_{2}(K, L, n)} \cos \left(\frac{L n}{2}\right)+\frac{B_{1}(K, L, n)}{B_{2}(K, L, n)} \operatorname{sinc}\left(\frac{L n}{2 \pi}\right)
\end{aligned}
$$

donde $A_{2}, B_{1}$ y $B_{2}$ son polinomios en $n$ de orden 10 , y $A_{1}$ es un polinomio de orden 8 . Los coeficientes de los polinomios son función de las constantes $K$ y $L$. Las expresiones de $A_{1}$, $A_{2}, B_{1}$ y $B_{2}$ no se reproducen por su elevada longitud.

Como se puede apreciar en (C.35), la duración de $f_{2}[-n]$ es infinita, lo cual indica que deberíamos utilizar un número infinito de modos cilíndricos para reconstruir de forma totalmente correcta el campo a partir de su espectro. Como esto no es posible, debemos truncar por algún sitio y quedarnos con un número finito de modos cilíndricos. En este caso eso se podrá hacer sin gran pérdida de precisión, puesto que la señal $f_{2}[-n]$, aunque es de duración infinita, es decreciente, y por tanto podemos despreciar las colas a partir de un cierto momento. La solución que se ha adoptado es la de tomar como duración de la señal $f_{2}[-n]$ desde su 
valor máximo (en $n=0$ ) hasta el momento (en $n=n_{0}$ ) en que su amplitud se reduzca a su valor inicial dividido por un factor al que llamaremos $R_{A B}$ :

$$
\operatorname{Dur}\left\{f_{2}[-n]\right\} \simeq 2 n_{0}+1
$$

donde $n_{0}$ es un valor de $n$ que cumple lo siguiente:

$$
\left|f_{2}[-n]\right|<\frac{1}{R_{A B}} f_{2}[0] \quad \forall n,|n| \geq n_{0}
$$

y $f_{2}[0]$ se obtiene resolviendo la expresión (C.33) para $n=0$ :

$$
f_{2}[0]=\frac{1}{2 \pi} \int_{-\frac{L}{2}}^{\frac{L}{2}} e^{-K \cos (\phi)} \mathrm{d} \phi=D(K, L)
$$

Cuanto mayor sea $R_{A B}$ mayor será la duración estimada de $f_{2}[-n]$, y por tanto con más exactitud reconstruiremos el campo a partir del espectro de modos cilíndricos. Sin embargo, cuanto mayor sea $R_{A B}$, mayor será el esfuerzo computacional requerido. Se deberá buscar una solución de compromiso en la que la exactitud con que se reconstruya el campo sea suficiente y el esfuerzo computacional no sea excesivo. Para la mayoría de problemas en guía rectangular analizados se ha comprobado que ese valor de compromiso es aproximadamente $R_{A B}=3$.

Por otro lado, aún nos queda por determinar el valor de $n_{0}$. Para ello, hemos de determinar a partir de qué valor de $n$ la señal ya no supera nunca el valor máximo multiplicado por el factor $R_{A B}$. Para simplificar este cálculo, podemos utilizar el hecho de que el polinomio $A_{1}$ es de orden 8, mientras que $A_{2}$ es de orden 10. Eso quiere decir que para valores grandes de $n$, el cociente de polinomios $A_{1} / A_{2}$ tiende a cero, y podemos despreciar el coseno de la expresión (C.35). Además, como $B_{1}$ y $B_{2}$ son polinomios del mismo orden, el cociente $B_{1} / B_{2}$ tiende a una constante cuando $n$ aumenta.

$$
\begin{aligned}
& \lim _{n \rightarrow \infty} \frac{A_{1}(K, L, n)}{A_{2}(K, L, n)}=0 \\
& \lim _{n \rightarrow \infty} \frac{B_{1}(K, L, n)}{B_{2}(K, L, n)}=C(K, L)
\end{aligned}
$$

Por tanto, para valores grandes de $n$ podremos afirmar lo siguiente:

$$
f_{2}[-n] \simeq C(K, L) \operatorname{sinc}\left(\frac{L n}{2 \pi}\right) \quad, n \gg 0
$$

Se observa que para valores grandes de $n$, la señal $f_{2}[-n]$ se comporta como una sinc, tal y como se muestra en la figura C.2.

La envolvente de la sinc es $C(K, L) \frac{2}{L|n|}$, como se muestra en la figura C.2. De manera que lo que debemos asegurar es que a partir de cierto valor $n_{0}$ la envolvente sea menor que el $10 \%$ de $f_{2}[0]$ :

$$
C(K, L) \frac{2}{L|n|}<\frac{f_{2}[0]}{10}=\frac{D(K, L)}{10} \quad \forall n, n \geq n_{0}
$$




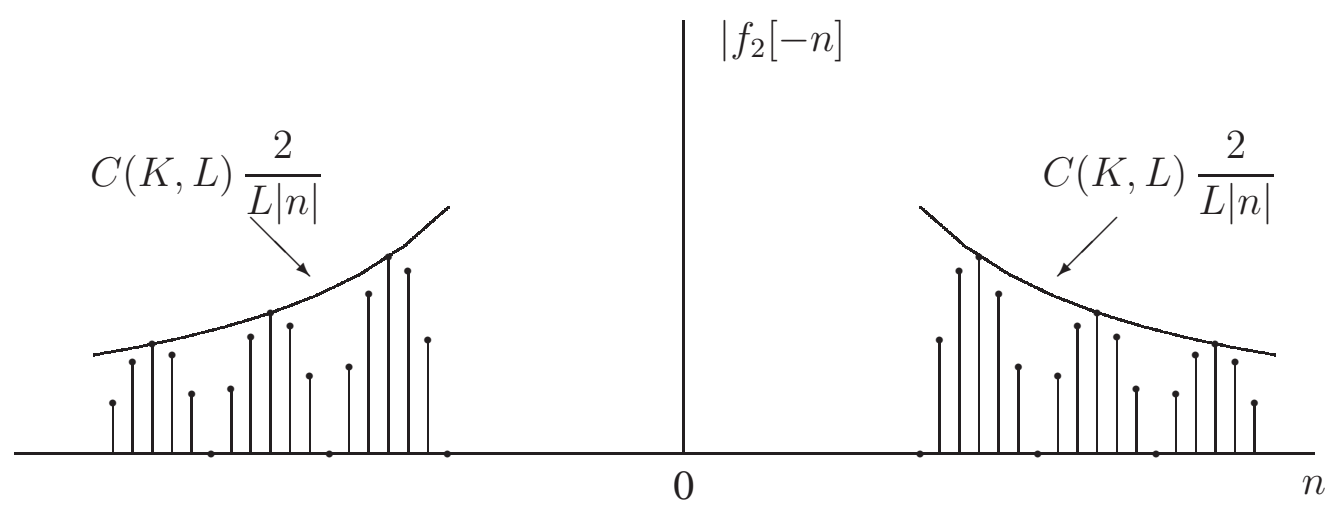

Figura C.2: Aspecto de la señal $f_{2}[-n]$ para $n \gg 0$

Despejando se obtiene:

$$
|n|>\frac{C(K, L)}{D(K, L)} \frac{20}{L} \quad \forall n, n \geq n_{0}
$$

Por tanto $n_{0}$ es el primer número entero por encima de $\frac{C(K, L)}{D(K, L)} \frac{20}{L}$. Es decir:

$$
n_{0}=\operatorname{ceil}\left(\frac{20}{L} \frac{C(K, L)}{D(K, L)}\right)
$$

siendo ceil la función que obtiene un número entero a partir de uno real redondeando hacia arriba, y donde las expresiones de $C(K, L)$ y $D(K, L)$, son:

$$
\begin{aligned}
C(K, L)= & C_{0}\left(945 K^{5} L^{10}+3150 K^{4} L^{8}\left(12-L^{2}\right)\right. \\
& +315 K^{3} L^{6}\left(7 L^{4}-240 L^{2}+160 \cdot 4 !\right) \\
& -15 K^{2} L^{4}\left(17 L^{6}-1512 L^{4}+2 \cdot 8 ! L^{2}-48 \cdot 8 !\right) \\
& +K L^{2}\left(L^{8}-360 L^{6}+2 \cdot 8 ! L^{4}-240 \cdot 8 ! L^{2}+128 \cdot 10 !\right) \\
& +1024 \cdot 10 !) \\
D(K, L)= & D_{0}\left(945 K^{5} L^{10}+1050 K^{4} L^{8}\left(44-3 L^{2}\right)\right. \\
+ & 15 K^{3} L^{6}\left(147 L^{4}-6160 L^{2}+176 \cdot 6 !\right) \\
- & 15 K^{2} L^{4}\left(17 L^{6}-1848 L^{4}+176 \cdot 6 ! L^{2}-528 \cdot 8 ! / 5\right) \\
+ & K L^{2}\left(L^{8}-440 L^{6}+176 \cdot 6 ! L^{4}-528 \cdot 8 ! L^{2}+42240 \cdot 8 !\right) \\
+ & 11264 \cdot 10 !)
\end{aligned}
$$

con:

$$
\begin{aligned}
C_{0} & =\frac{L e^{-K}}{2048 \cdot 10 ! \pi} \\
D_{0} & =\frac{L e^{-K}}{22528 \cdot 10 ! \pi}
\end{aligned}
$$


Finalmente, la duración de la señal $f^{ \pm}[-n]$ será:

$$
\begin{aligned}
\operatorname{Dur}\left\{f^{ \pm}[-n]\right\} & =\operatorname{Dur}\left\{f_{1}[-n]\right\}+\operatorname{Dur}\left\{f_{2}[-n]\right\}-1 \\
& =2\left[\frac{M_{i} \pi R}{a_{i}}\right]+1+2 n_{0}+1-1 \\
& =2\left[\frac{M_{i} \pi R}{a_{i}}+n_{0}\right]+1
\end{aligned}
$$

Como la duración de $f^{ \pm}[-n]$ es de $n=-N$ a $n=N$ :

$$
\operatorname{Dur}\left\{f^{ \pm}[-n]\right\}=2 N+1 \simeq 2\left[\frac{M_{i} \pi R}{a_{i}}+n_{0}\right]+1
$$

de donde se despeja finalmente que el valor de $N$ :

$$
N \simeq \frac{M_{i} \pi R}{a_{i}}+n_{0}
$$

Tomaremos este valor de forma aproximada como el valor mínimo que le podemos asignar a $N$ para truncar la serie con garantías de no estar perdiendo información. Y como $N$ debe ser un número entero:

$$
N=\operatorname{ceil}\left(\frac{M_{i} \pi R}{a_{i}}+n_{0}\right)
$$

Como se ha visto, para reconstruir las distribuciones de campo $F^{+}(\phi)$ y $F^{-}(\phi)$ de forma totalmente correcta a partir de suma de modos cilíndricos necesitaríamos un número infinito de términos. El valor que hemos obtenido para $N$ nos permite truncar la serie con garantías de cometer un error muy pequeño, prácticamente despreciable, ya que casi toda la energía de la señal $f^{ \pm}[-n]$ está contenida entre $-N$ y $N$. No obstante, siguen existiendo colas que no consideramos por ser pequeñas, pero que pueden traer problemas si aproximamos la DTFT inversa mediante la $f f t$ de tamaño $2 N+1$. Esto se debe a que al realizar la $f f t$, calculamos $f^{ \pm}[-n]$ tan sólo a partir de la información de $2 N+1$ muestras de $F^{ \pm}(\phi)$. Y este procedimiento tan sólo proporciona un resultado correcto en caso de que la duración de $f^{ \pm}[-n]$ sea $2 N+1$ o inferior, cosa que no sucede en este caso, pues su duración es infinita. Si realizamos la fft de tamaño $2 N+1$, el resultado será que se repetirá la señal $f^{ \pm}[-n]$ cada $2 N+1$ posiciones, y como su duración es infinita, se producirán solapamientos.

Este fenómeno se puede minimizar si realizamos una fft tomando muchas más muestras, aunque luego nos quedemos sólo con las $2 N+1$ centrales. De este modo se seguirá produciendo solapamiento, pero como se repetirá la señal con mucha más distancia, y las colas de la señal son decrecientes, al haber más distancia entre las réplicas el solapamiento será mucho menor. En nuestro caso, todas las fft se han realizado de tamaño $R_{f f t}(2 N+1)$. Es decir, se han tomado $R_{f f t}$ veces más muestras con lo que las réplicas están $R_{f f t}$ veces más separadas, minimizando en gran medida el solapamiento temporal. El valor adecuado del parámetro $R_{f f t}$ depende de lo rápidamente que decrezca la señal, lo cual varía según el 
problema que se desee analizar. Por ello se deja que sea el usuario quien determine el valor que desea que tome $R_{f f t}$. 


\section{Apéndice D}

\section{Sistemas de coordenadas local y global en una guía rectangular}

Para poder forzar la continuidad de los campos transversales en la circunferencia de radio $R$ entre las expresiones de campos guiados y en espacio abierto, es necesario relacionar los sistemas de coordenadas en que están expresados estos campos. Las expresiones de los campos como sumas de modos cilíndricos en espacio abierto se definen ambas en coordenadas $(x, y)$. No obstante, las expresiones del campo como suma de modos guiados utilizan un sistema de coordenadas $\left(x_{i}, y_{i}\right)$ en cada acceso $i$. En la figura D.1 se muestra la disposición del sistema de coordenadas global utilizado para los modos cilíndricos, y del sistema de coordenadas de un acceso cualquiera $i$-ésimo.

Las características del acceso $i$ vienen definidas por el punto $P_{i}$, el vector $\hat{z}_{i}$ y la anchura $a_{i}$. Como se puede apreciar en la figura D.1, los vectores unitarios en el sistema de coordenadas del acceso $i$ se relacionan con los vectores unitarios del sistema de coordenadas "global" de la siguiente manera:

$$
\begin{aligned}
& \hat{y}_{i}=\hat{z} \\
& \hat{x}_{i}=\hat{y}_{i} \times \hat{z}_{i}=\hat{z} \times \hat{z}_{i}
\end{aligned}
$$

Por otro lado, las coordenadas $\left(x_{i}, z_{i}\right)$ de un punto $P$ cualquiera de la circunferencia de radio $R$ se pueden hallar obteniendo el punto $Q$, resultado de la intersección de las rectas $r_{1}$ y $r_{2}$ :

$$
\begin{aligned}
r_{1} & \equiv Q=P_{i}-\frac{a_{i}}{2} \hat{x}_{i}+z_{i} \hat{z}_{i} \\
r_{2} & \equiv Q=P-x_{i} \hat{x}_{i}
\end{aligned}
$$

Igualando la expresión de las dos rectas se obtiene que:

$$
\begin{aligned}
P_{i}-\frac{a_{i}}{2} \hat{x}_{i}+z_{i} \hat{z}_{i} & =P-x_{i} \hat{x}_{i} \\
z_{i} \hat{z}_{i}+x_{i} \hat{x}_{i} & =\left(P-P_{i}+\frac{a_{i}}{2} \hat{x}_{i}\right)
\end{aligned}
$$




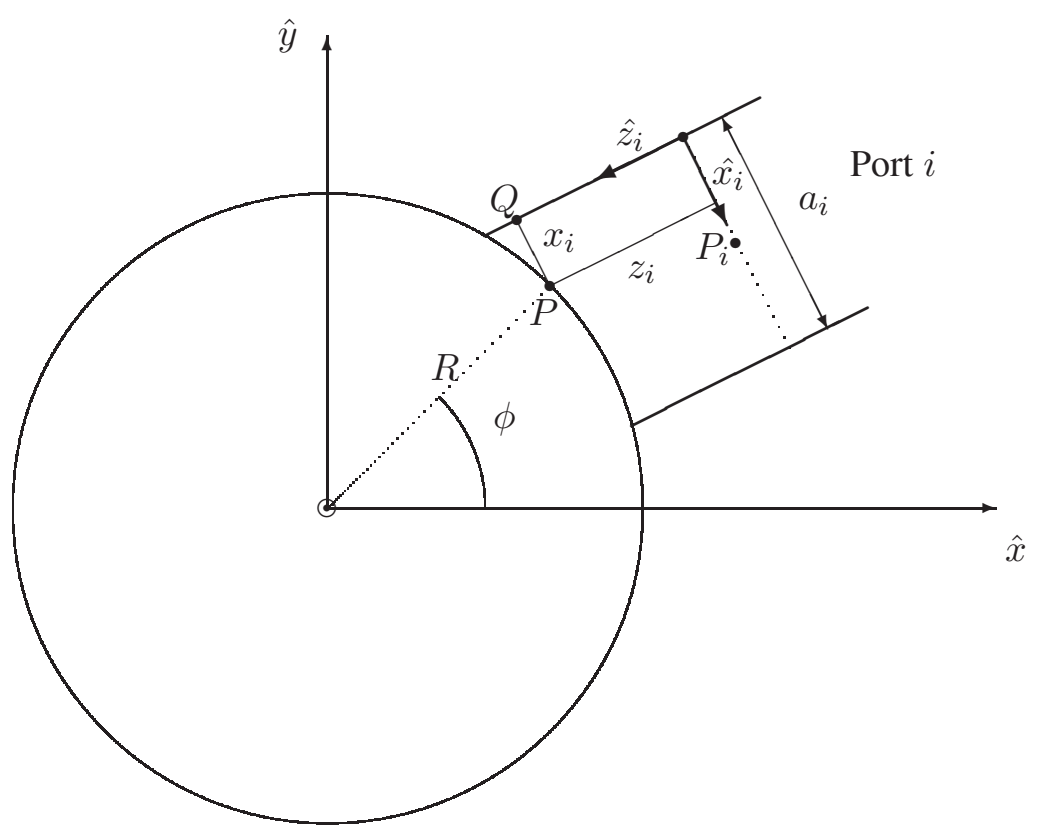

Figura D.1: Sistemas de coordenadas local al acceso y global

Para poder despejar los valores de $x_{i}$ y $z_{i}$, descompondremos los vectores $\hat{x}_{i}$ y $\hat{z}_{i}$ en sus componentes $(x, y)$ del sistema de referencia global:

$$
\begin{aligned}
& \hat{x_{i}}=x_{i_{x}} \hat{x}+x_{i_{y}} \hat{y}=\left[\begin{array}{l}
x_{i_{x}} \\
x_{i_{y}}
\end{array}\right] \\
& \hat{z}_{i}=z_{i_{x}} \hat{x}+z_{i_{y}} \hat{y}=\left[\begin{array}{l}
z_{i_{x}} \\
z_{i_{y}}
\end{array}\right]
\end{aligned}
$$

Utilizando la descomposición de los vectores unitarios $\hat{x}_{i}$ y $\hat{z}_{i}$, la expresión D.3 se puede reescribir de la siguiente forma:

$$
\left[\begin{array}{cc}
z_{i_{x}} & x_{i_{x}} \\
z_{i_{y}} & x_{i_{y}}
\end{array}\right] \cdot\left[\begin{array}{c}
z_{i} \\
x_{i}
\end{array}\right]=\left(\left[\begin{array}{c}
P_{x} \\
P_{y}
\end{array}\right]-\left[\begin{array}{c}
P_{i_{x}} \\
P_{i_{y}}
\end{array}\right]+\frac{a_{i}}{2}\left[\begin{array}{c}
x_{i_{x}} \\
x_{i_{y}}
\end{array}\right]\right)
$$

Despejando, y teniendo en cuenta que $P$ es un punto de la circunferencia de radio $R$ $\left(P=R[\cos \phi \sin \phi]^{T}\right)$, obtenemos la relación entre las coordenadas locales $\left(x_{i}, z_{i}\right)$ y las 
coordenadas globales $(\rho=R, \phi)$.

$$
\left[\begin{array}{c}
z_{i} \\
x_{i}
\end{array}\right]=\left[\begin{array}{ll}
z_{i_{x}} & x_{i_{x}} \\
z_{i_{y}} & x_{i_{y}}
\end{array}\right]^{(-1)} \cdot\left(R\left[\begin{array}{c}
\cos \phi \\
\sin \phi
\end{array}\right]-\left[\begin{array}{c}
P_{i_{x}} \\
P_{i_{y}}
\end{array}\right]+\frac{a_{i}}{2}\left[\begin{array}{c}
x_{i_{x}} \\
x_{i_{y}}
\end{array}\right]\right)
$$

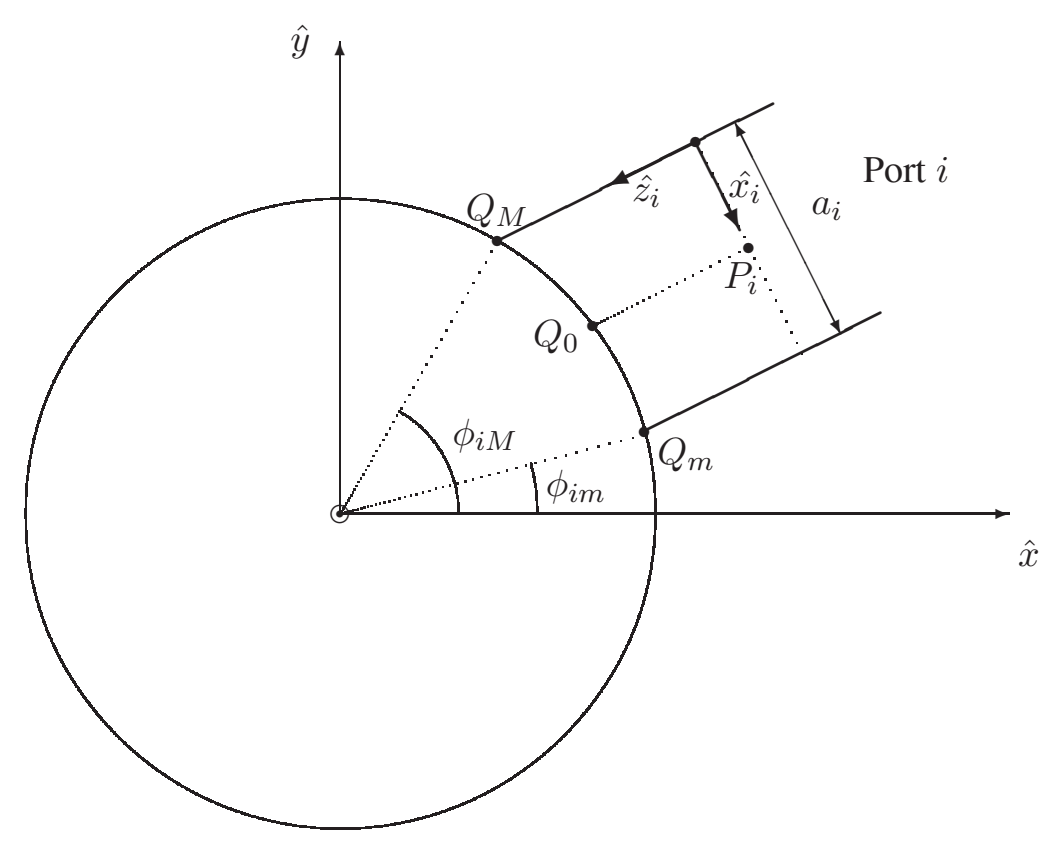

Figura D.2: Límites inferior y superior de la inserción del acceso $i$ en la circunferencia de radio $R$

Por otro lado, nos resultará necesario conocer entre qué valores de $\phi$, dentro de la circunferencia de radio $R$, se inserta el acceso $i$. A estos límites se les ha llamado, respectivamente, $\phi_{i m} \mathrm{y} \phi_{i M}$ (ver figura D.2). El valor de $\phi_{i m}$ y $\phi_{i M}$ lo obtendremos hallando la intersección de las rectas $s_{1}$ y $s_{2}$ con la circunferencia de radio $R$ (puntos $Q_{m}$ y $Q_{M}$ ):

$$
\begin{aligned}
s_{1} & \equiv Q_{M}=P_{i}-\frac{a_{i}}{2} \hat{x}_{i}+\lambda_{M} \hat{z}_{i}=R\left[\begin{array}{c}
\cos \phi_{i M} \\
\sin \phi_{i M}
\end{array}\right] \\
s_{2} & \equiv Q_{m}=P_{i}+\frac{a_{i}}{2} \hat{x}_{i}+\lambda_{m} \hat{z}_{i}=R\left[\begin{array}{c}
\cos \phi_{i m} \\
\sin \phi_{i m}
\end{array}\right]
\end{aligned}
$$

Para poder despejar de las ecuaciones anteriores el valor de $\lambda_{M}$, expresaremos el punto $P_{i}$ y los vectores unitarios $\hat{x}_{i}$ y $\hat{z}_{i}$ como vectores de dos componentes $(x, y)$ en el sistema de 
coordenadas global:

$$
\left[\begin{array}{c}
P_{i_{x}} \\
P_{i_{y}}
\end{array}\right]-\frac{a_{i}}{2}\left[\begin{array}{l}
x_{i_{x}} \\
x_{i_{y}}
\end{array}\right]+\lambda_{M}\left[\begin{array}{c}
z_{i_{x}} \\
z_{i_{y}}
\end{array}\right]=R\left[\begin{array}{c}
\cos \phi_{i M} \\
\sin \phi_{i M}
\end{array}\right]
$$

Igualando las componentes en $x$ y en $y$ en ambos lados de la expresión anterior, se obtiene:

$$
\begin{aligned}
R \cos \phi_{i M} & =P_{i_{x}}-\frac{a_{i}}{2} x_{i_{x}}+\lambda_{M} z_{i_{x}} \\
R \sin \phi_{i M} & =P_{i_{y}}-\frac{a_{i}}{2} x_{i_{y}}+\lambda_{M} z_{i_{y}}
\end{aligned}
$$

Operando con las dos ecuaciones anteriores:

$$
\begin{aligned}
R^{2}= & \left(R \cos \phi_{i M}\right)^{2}+\left(R \sin \phi_{i M}\right)^{2} \\
= & \left(P_{i_{x}}-\frac{a_{i}}{2} x_{i_{x}}+\lambda_{M} z_{i_{x}}\right)^{2}+\left(P_{i_{y}}-\frac{a_{i}}{2} x_{i_{y}}+\lambda_{M} z_{i_{y}}\right)^{2} \\
= & \left(P_{i_{x}}-\frac{a_{i}}{2} x_{i_{x}}\right)^{2}+\lambda_{M}^{2} z_{i_{x}}^{2}+2\left(P_{i_{x}}-\frac{a_{i}}{2} x_{i_{x}}\right) \lambda_{M} z_{i_{x}} \\
& +\left(P_{i_{y}}-\frac{a_{i}}{2} x_{i_{y}}\right)^{2}+\lambda_{M}^{2} z_{i_{y}}^{2}+2\left(P_{i_{y}}-\frac{a_{i}}{2} x_{i_{y}}\right) \lambda_{M} z_{i_{y}} \\
= & \left(z_{i_{x}}^{2}+z_{i_{y}}^{2}\right) \lambda_{M}^{2}+2\left[\left(P_{i_{x}}-\frac{a_{i}}{2} x_{i_{x}}\right) z_{i_{x}}+\left(P_{i_{y}}-\frac{a_{i}}{2} x_{i_{y}}\right) z_{i_{y}}\right] \lambda_{M} \\
& +\left[\left(P_{i_{x}}-\frac{a_{i}}{2} x_{i_{x}}\right)^{2}+\left(P_{i_{y}}-\frac{a_{i}}{2} x_{i_{y}}\right)^{2}\right]^{2} \\
= & \left|\hat{z}_{i}\right|^{2} \lambda_{M}^{2}+2\left(P_{i}-\frac{a_{i}}{2} \hat{x}_{i}\right)^{\mathrm{T}} \cdot \hat{z}_{i} \lambda_{M}+\left|P_{i}-\frac{a_{i}}{2} \hat{x}_{i}\right|^{2}
\end{aligned}
$$

A la vista de este resultado, $\lambda_{m}$ y $\lambda_{M}$ son la solución de las siguientes dos ecuaciones de segundo orden:

$$
\begin{gathered}
\left|\hat{z}_{i}\right|^{2} \lambda_{M}^{2}+2\left(P_{i}-\frac{a_{i}}{2} \hat{x}_{i}\right)^{\mathrm{T}} \cdot \hat{z}_{i} \lambda_{M}+\left|P_{i}-\frac{a_{i}}{2} \hat{x}_{i}\right|^{2}-R^{2}=0 \\
\left|\hat{z}_{i}\right|^{2} \lambda_{m}^{2}+2\left(P_{i}+\frac{a_{i}}{2} \hat{x}_{i}\right)^{\mathrm{T}} \cdot \hat{z}_{i} \lambda_{m}+\left|P_{i}+\frac{a_{i}}{2} \hat{x}_{i}\right|^{2}-R^{2}=0
\end{gathered}
$$

Como $\hat{z}_{i}$ es un vector unitario, $\left|\hat{z}_{i}\right|=1$. Cada una de las ecuaciones anteriores tiene dos soluciones, ya que las rectas $s_{1}$ y $s_{2}$ cortan a la circunferencia en dos puntos (al entrar y al 
salir). Esas soluciones son las siguientes:

$$
\begin{aligned}
\lambda_{M}= & \frac{-2\left(P_{i}-\frac{a_{i}}{2} \hat{x}_{i}\right)^{\mathrm{T}} \cdot \hat{z}_{i}}{2} \\
& \pm \frac{\sqrt{\left(2\left(P_{i}-\frac{a_{i}}{2} \hat{x}_{i}\right)^{\mathrm{T}} \cdot \hat{z}_{i}\right)^{2}-4\left(\left|P_{i}-\frac{a_{i}}{2} \hat{x}_{i}\right|^{2}-R^{2}\right)}}{2} \\
\lambda_{m}= & \frac{-2\left(P_{i}+\frac{a_{i}}{2} \hat{x}_{i}\right)^{\mathrm{T}} \cdot \hat{z}_{i}}{2} \\
& \pm \frac{\sqrt{\left(2\left(P_{i}+\frac{a_{i}}{2} \hat{x}_{i}\right)^{\mathrm{T}} \cdot \hat{z}_{i}\right)^{2}-4\left(\left|P_{i}+\frac{a_{i}}{2} \hat{x}_{i}\right|^{2}-R^{2}\right)}}{2}
\end{aligned}
$$

A nosotros nos interesa el punto por donde las rectas entran a la circunferencia, y por tanto de las dos soluciones tomaremos la menor tanto para $\lambda_{m}$ como para $\lambda_{M}$.

$$
\begin{aligned}
\lambda_{M}= & \frac{-2\left(P_{i}-\frac{a_{i}}{2} \hat{x}_{i}\right)^{\mathrm{T}} \cdot \hat{z}_{i}}{2} \\
& -\frac{\sqrt{\left(2\left(P_{i}-\frac{a_{i}}{2} \hat{x}_{i}\right)^{\mathrm{T}} \cdot \hat{z}_{i}\right)^{2}-4\left(\left|P_{i}-\frac{a_{i}}{2} \hat{x}_{i}\right|^{2}-R^{2}\right)}}{2} \\
\lambda_{m}= & \frac{-2\left(P_{i}+\frac{a_{i}}{2} \hat{x}_{i}\right)^{\mathrm{T}} \cdot \hat{z}_{i}}{2} \\
& -\frac{\sqrt{\left(2\left(P_{i}+\frac{a_{i}}{2} \hat{x}_{i}\right)^{\mathrm{T}} \cdot \hat{z}_{i}\right)^{2}-4\left(\left|P_{i}+\frac{a_{i}}{2} \hat{x}_{i}\right|^{2}-R^{2}\right)}}{2}
\end{aligned}
$$

Una vez obtenidas $\lambda_{m}$ y $\lambda_{M}$, los puntos $Q_{m}$ y $Q_{M}$ son:

$$
\begin{aligned}
Q_{M} & =P_{i}-\frac{a_{i}}{2} \hat{x}_{i}+\lambda_{M} \hat{z}_{i} \\
Q_{m} & =P_{i}+\frac{a_{i}}{2} \hat{x}_{i}+\lambda_{m} \hat{z}_{i}
\end{aligned}
$$

Y, finalmente, los ángulos $\phi_{i m}$ y $\phi_{i M}$ en los que empieza y acaba la inserción del acceso $i$ son:

$$
\begin{aligned}
\phi_{i m} & =\arctan \left(\frac{Q_{m_{y}}}{Q_{m_{x}}}\right) \\
\phi_{i M} & =\arctan \left(\frac{Q_{M_{y}}}{Q_{M_{x}}}\right)
\end{aligned}
$$


También nos interesará conocer las coordenadas del punto $Q_{0}$ (ver figura D.2). Ese punto es la intersección de la recta $s$ con la circunferencia de radio $R$ :

$$
s \equiv Q_{0}=P_{i}+\lambda_{0} \hat{z}_{i}=R\left[\begin{array}{c}
\cos \phi_{0} \\
\sin \phi_{0}
\end{array}\right]
$$

siendo $\phi_{0}$ la posición angular en la que la recta $s$ intersecta con la circunferencia de radio $R$. Despejando:

$$
\lambda_{0}=\frac{-2 P_{i}^{\mathrm{T}} \cdot \hat{z}_{i}-\sqrt{\left(2 P_{i}^{\mathrm{T}} \cdot \hat{z}_{i}\right)^{2}-4\left(\left|P_{i}\right|^{2}-R^{2}\right)}}{2}
$$

Una vez obtenida $\lambda_{0}, Q_{0}$ se obtiene fácilmente como:

$$
Q_{0}=P_{i}+\lambda_{0} \hat{z}_{i}
$$




\section{Apéndice E}

\section{Método de descenso del simplex}

El método de descenso del simplex está basado en un algoritmo desarrollado por Nelder y Mead [89]. Un simplex es una figura geométrica $\mathrm{N}$-dimensional, donde $\mathrm{N}$ es el número de variables independientes. El simplex consta de $\mathrm{N}+1$ vértices, no necesariamente equidistantes, con sus correspondientes caras y aristas. Por ejemplo, si la función depende de 2 variables, tendremos una figura de 2 dimensiones y 3 vértices, es decir, un triángulo; si son 3 las variables, estaremos en 3 dimensiones y el simplex consistirá en un tetraedro.

Para comenzar, el algoritmo necesita de un simplex inicial. En el método de Nelder y Mead, para obtener el simplex inicial, se parte de un vértice que se toma como punto de origen $\left(P_{0}\right)$. A partir del vértice inicial, los $N$ restantes vértices del simplex se obtienen desplazándose una longitud $\lambda$ en cada una de las $N$ direcciones del espacio (ver figura E.1):

$$
P_{i}=P_{0}+\lambda \hat{e_{i}} \quad \forall i \in[1, N]
$$

donde

- $P_{0}$ : Vértice inicial

- $P_{i}$ : Resto de vértices del simplex

- $\lambda$ : Longitud de las aristas

- $\hat{e}_{i}$ : Vectores unitarios ortogonales que definen una base en el espacio $N$-dimensional

Esta forma de calcular el simplex inicial no tiene en cuenta las magnitudes de las variables de la función a optimizar. De modo que el tamaño del simplex inicial es siempre el mismo tanto si los valores iniciales de las variables (parte real e imaginaria de la permitividad en nuestro caso) están alrededor de 1 como si están alrededor de 100.

Para subsanar esta circunstancia, en nuestro caso también se ha obtenido el simplex inicial a partir de un vértice inicial $P_{0}$, pero el resto de vértices se obtienen en cada dimensión sumando una cantidad proporcional al valor de $P_{0}$ en esa dimensión. Esta estrategia falla en aquellas dimensiones en las que el valor de $P_{0}$ sea nulo. En esos casos, no queda más remedio que desplazarnos una cantidad siempre fija para crear la nueva arista del simplex en esa dirección: 


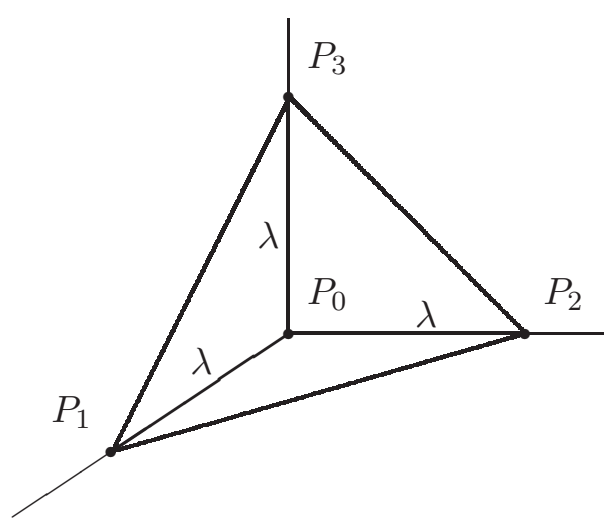

Figura E.1: Simplex inicial

$$
P_{i}=\left\{\begin{array}{lll}
P_{0}+\lambda_{0} \hat{e}_{i} & \text { si } & P_{0}(i)=0 \\
P_{0}+\lambda_{N} P_{0}(i) \hat{e}_{i} & \text { si } & P_{0}(i) \neq 0
\end{array} \quad, \quad \forall i \in[1, N]\right.
$$

donde $\lambda_{0}$ y $\lambda_{N}$ son parámetros de control que suelen tomar el valor de 0.00025 y 0.05 respectivamente. Y $P_{0}(i)$ es el valor de la coordenada $i$-ésima del punto inicial $P_{0}$, o, lo que es lo mismo, el valor inicial de la variable $i$-ésima de la función de error (en nuestro caso parte real o imaginaria de la permitividad).

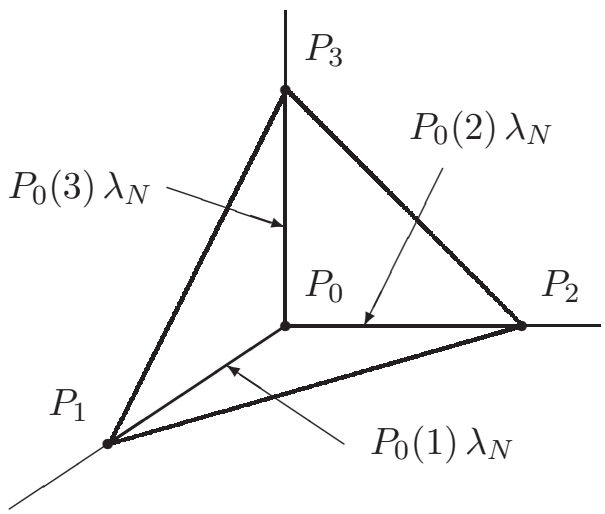

Figura E.2: Simplex inicial. Cálculo mejorado

Una vez obtenido el simplex inicial, se evalúa la función en los N+1 vértices, y éstos se ordenan y nombran de menor a mayor según el valor de la función en cada vértice. Tras la ordenación, el valor de la función en $P_{0}\left(F\left(P_{0}\right)\right)$ será menor que el valor de la función en cualquier otro vértice. Y $F\left(P_{N}\right)$ será mayor que el valor de la función en cualquier otro vértice.

A continuación se procede a efectuar movimientos en el simplex de forma iterativa, de manera que a cada iteración se elimina un vértice y se sustituye por otro. Los movimientos 
se realizan con la intención de que poco a poco el simplex se desplace hasta alcanzar el mínimo de la función. Como se busca el valor mínimo de la función, se desecha el vértice que se corresponda con el valor mayor, y se sustituye por otro en el que el valor de la función sea menor. Nótese que con este procedimiento no se pretende llegar demasiado rápido al mínimo, pues en lugar de intentar mover el simplex en la dirección del vértice con un valor menor de la función, lo que se hace es eliminar el peor vértice. De esta forma se evita quedar atrapados en mínimos locales, y el método resulta más robusto, con mayores garantías de convergencia hacia el mínimo absoluto de la función.

Los posibles movimientos del simplex consisten básicamente en sustituir el peor vértice $\left(P_{N}\right)$ por otro punto. Los movimientos posibles, en función del punto que sustituya a $P_{N}$, son:

- Reflexión

- Expansión

- Contracción externa

- Contracción interna

- Contracción multidimensional

A continuación se describe el procedimiento por el que se decide qué tipo de movimiento se va a realizar.

El primer paso consiste en buscar el nuevo punto que sustituya a $P_{N}$ en la recta que une el peor punto con el punto medio $\left(P_{m}\right)$ del resto de puntos del simplex (ver figura E.3.a):

$$
P_{m}=\frac{\sum_{i=0}^{N-1} P_{i}}{N}
$$

El motivo de buscar el nuevo punto en la recta que une $P_{N}$ con $P_{m}$ es que deseamos que el simplex se aleje del peor punto, de forma que al final se llegará al mínimo de la función. Como ya se ha dicho, no se pretende que el simplex se acerque de la forma más rápida posible al mínimo, lo que se haría buscando el nuevo punto en la dirección marcada por $P_{0}$.

Una vez hemos decidido buscar el nuevo punto a lo largo de la recta que une $P_{N}$ con $P_{m}$, el primer intento será el que llamaremos punto reflejado o $P_{r}$. Este punto se llama así porque es el punto reflejado de $P_{N}$ con respecto a la figura formada por todos los vértices del simplex excepto el $P_{N}$ (ver figura E.3.a):

$$
P_{r}=P_{m}+\rho \overrightarrow{P_{N} P_{m}}
$$

Obsérvese que si la distancia de $P_{N}$ a $P_{m}$ es $d=\overrightarrow{P_{N} P_{m}}$, el punto reflejado se halla a una distancia $\rho d$ del punto $P_{m}$ (ver figura E.3.a). Si $\rho=1, P_{N}$ y el punto reflejado se hallan a la misma distancia de $P_{m}$. $\rho$ es un parámetro de control del algoritmo, que puede ajustarse en cada aplicación. 


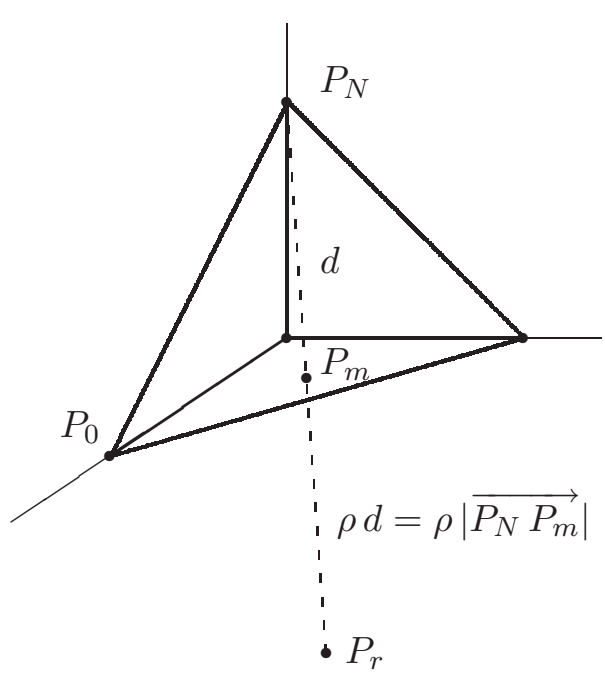

(a) Reflexión

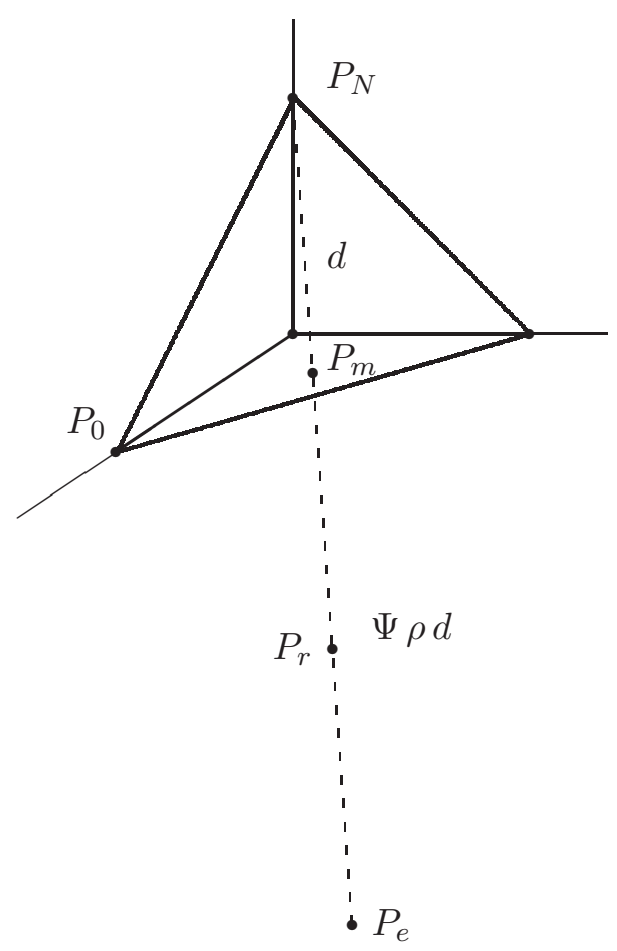

(b) Expansión

Figura E.3: Movimientos de reflexión y de expansión

Una vez calculado el punto reflejado, se obtiene el valor de la función en dicho punto $F\left(P_{r}\right)$. Si el punto es mejor que todos los que hay en el simplex (si $F\left(P_{r}\right)<F\left(P_{0}\right)$ ), eso quiere decir que estamos avanzando en una dirección y un sentido correctos. Por tanto lo que se hace a continuación es comprobar si avanzando más aún en esa dirección seguimos mejorando. Para comprobar esto se obtiene el que llamaremos punto de expansión, que es un punto que se halla a $\Psi$ veces más distancia de $P_{m}$ que el punto reflejado (ver figura E.3.b):

$$
P_{e}=P_{m}+\Psi \rho \overrightarrow{P_{N} P_{m}}
$$

donde $\Psi$ es un número mayor que 1.

Si el punto de expansión es mejor que el de reflexión $\left(F\left(P_{e}\right)<F\left(P_{r}\right)\right)$, se sustituye el punto $P_{N}$ por el punto $P_{e}$ y se consuma un movimiento de expansión del simplex. En cambio, si el punto de expansión es peor que el reflejado, es el punto reflejado el que se incorpora al simplex en el lugar de $P_{N}$, consumando un movimiento de reflexión.

En el caso de que el punto reflejado no sea mejor que todos los vértices del simplex $\left(F\left(P_{r}\right)>F\left(P_{0}\right)\right)$, se compara con el penúltimo mejor punto $\left(P_{N-1}\right)$. Si $F\left(P_{r}\right)<F\left(P_{N-1}\right)$, se acepta el punto reflejado y se completa el movimiento de reflexión. En caso contrario, es decir, si el punto reflejado es peor que el penúltimo punto del simplex, debemos suponer 
que nos hemos ido demasiado lejos a lo largo de la línea que une $P_{N}$ con $P_{m}$. Por tanto, el punto que buscamos que sustituya a $P_{N}$ debe estar entre $P_{N}$ y $P_{r}$. Comparando $F\left(P_{N}\right)$ y $F\left(P_{r}\right)$ decidiremos si el nuevo punto lo buscamos más cerca de $P_{N}$ o de $P_{r}$. Si $F\left(P_{r}\right)<$ $F\left(P_{N}\right)$, buscaremos el nuevo punto más cerca de $P_{r}$. A este punto le llamaremos punto de contracción externa (ver figura E.4.a):

$$
P_{c_{e}}=P_{m}+\phi \rho \overrightarrow{P_{N} P_{m}}
$$

donde $\phi$ es un número menor que 1 .

Si el punto de contracción externa es mejor o igual que $P_{r}\left(F\left(P_{c_{e}}\right) \leq F\left(P_{r}\right)\right)$, aceptamos el punto y realizamos un movimiento de contracción externa. En caso contrario, parece ya improbable que encontremos un punto a lo largo de la recta que une $P_{N}$ con $P_{m}$ que sea mejor que $P_{r}$. Y aunque $P_{r}$ es mejor que $P_{N}$, al no ser mejor que $P_{N-1}$ aceptar un movimiento de reflexión no supone una gran mejora. De modo que llegados a este punto consideraremos que no hemos podido encontrar un nuevo punto porque estamos ya muy cerca del mínimo, y lo que haremos será un movimiento de contracción multidimensional en torno al mejor punto del simplex $P_{0}$. Este movimiento se explicará más adelante.

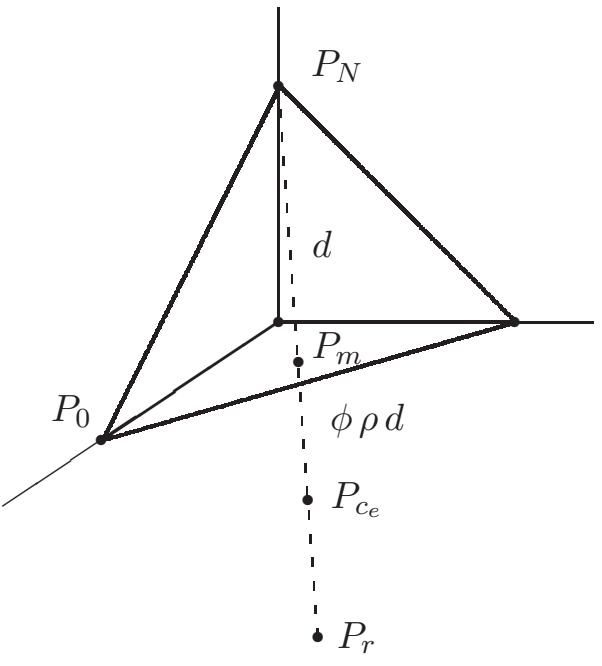

(a) Contracción externa

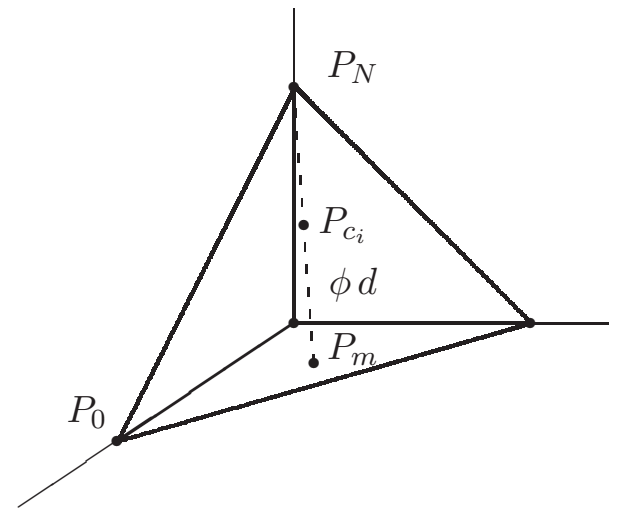

(b) Contracción interna

Figura E.4: Movimientos de contracción externa e interna

Si $F\left(P_{r}\right)>F\left(P_{N}\right)$, buscaremos el nuevo punto más cerca de $P_{N}$. A este punto le llamaremos punto de contracción interna (ver figura E.4.b):

$$
P_{c_{i}}=P_{m}-\phi \overrightarrow{P_{N} P_{m}}
$$

donde $\phi$ es un número menor que 1 . 


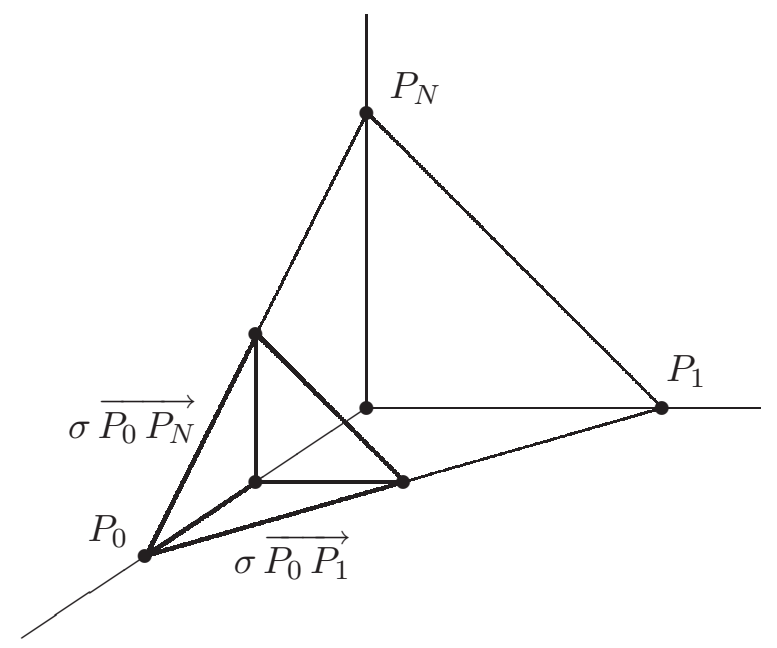

Figura E.5: Movimiento de contracción multidimensional

Si el punto de contracción interna es mejor que $P_{N}\left(F\left(P_{c_{i}}\right)<F\left(P_{N}\right)\right)$, aceptamos el punto y realizamos un movimiento de contracción interna. En caso contrario, ya parece poco probable que podamos encontrar un punto a lo largo de la línea de $P_{N}$ a $P_{m}$ que sea mejor que $P_{N}$. De modo que realizaremos una contracción del simplex en torno al punto mejor del simplex $P_{0}$. Realizaremos un movimiento de contracción multidimensional, en el que todos los vértices del simplex excepto $P_{0}$ se modifican de la siguiente manera (ver figura E.5):

$$
P_{i}=P_{0}+\sigma \overrightarrow{P_{0} P_{i}} \quad i \in[1, \ldots, N]
$$

En la figura E.6 se muestra un diagrama de flujo donde se esquematizan los pasos a seguir para completar un movimiento del simplex.

Como ya se ha explicado con anterioridad, una vez realizado un movimiento, los vértices del simplex se renombran de menor a mayor según el valor de la función en cada vértice. A continuación se comprueba si el simplex es de un tamaño muy reducido (menor que un cierto umbral), y si es así interpretamos que ya hemos alcanzado el mínimo de la función y que por tanto la función tiene su mínimo en el punto $P_{0}$, que es el mejor del simplex que hemos obtenido al final de la optimización. Si por el contrario el tamaño del simplex aún es grande, se continúa realizando más movimientos hasta que se consiga la convergencia o hasta que se exceda un determinado número de evaluaciones de la función o un determinado número de movimientos del simplex. Si terminamos por alguna de estas dos últimas razones, no podemos asegurar que el algoritmo haya convergido correctamente al mínimo de la función. 


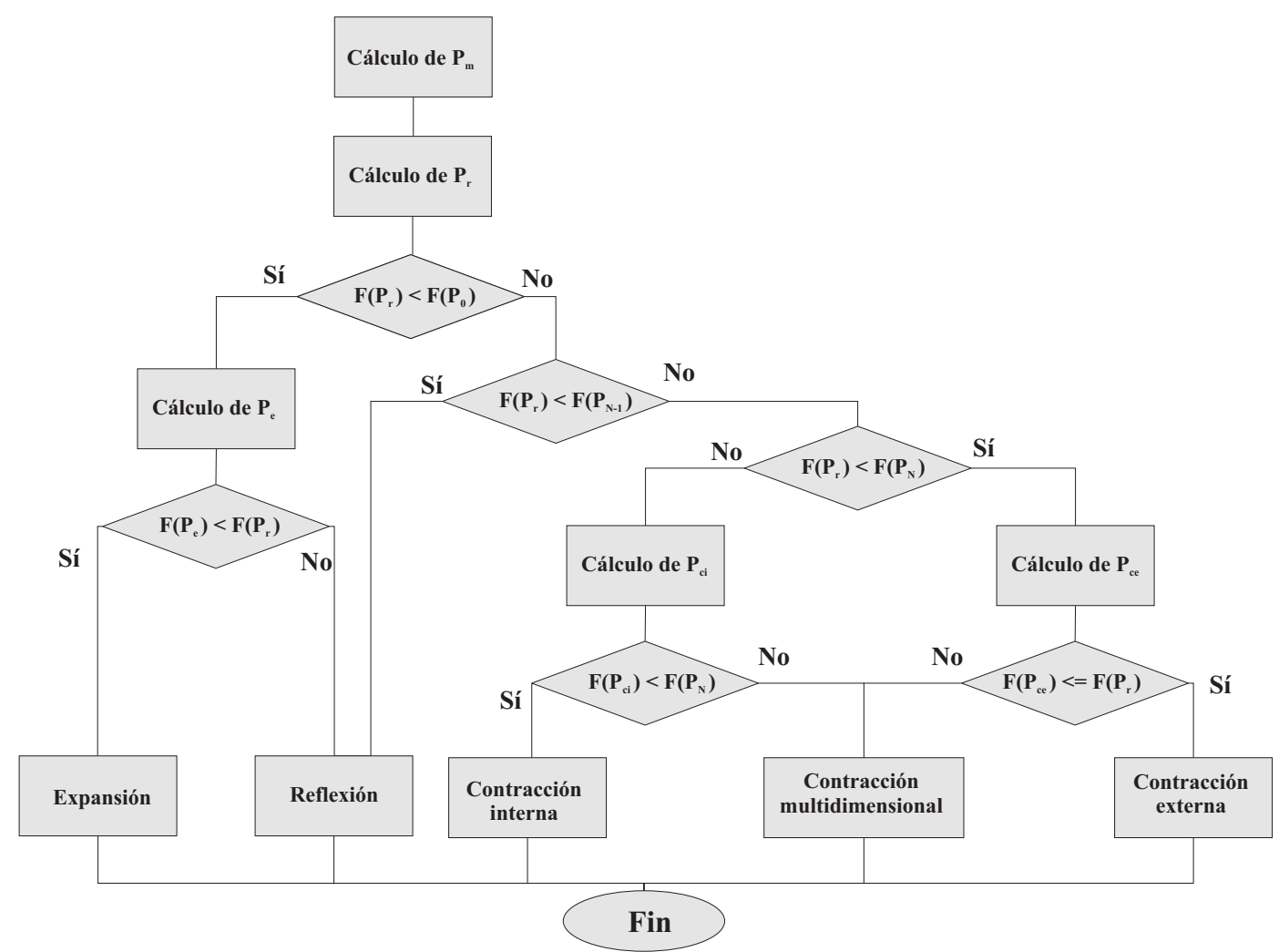

Figura E.6: Diagrama de flujo del algoritmo de descenso del simplex 


\section{Apéndice F}

\section{MDG conjunta de una conexión de dos redes}

\section{F.1. Conexión de dos accesos cualesquiera de dos redes}

En esta sección se aborda el problema de conectar dos accesos cualesquiera de dos redes. En la figura F.1 se muestra la conexión del acceso $n$-ésimo de la primera red con el acceso $m$-ésimo de la segunda red.

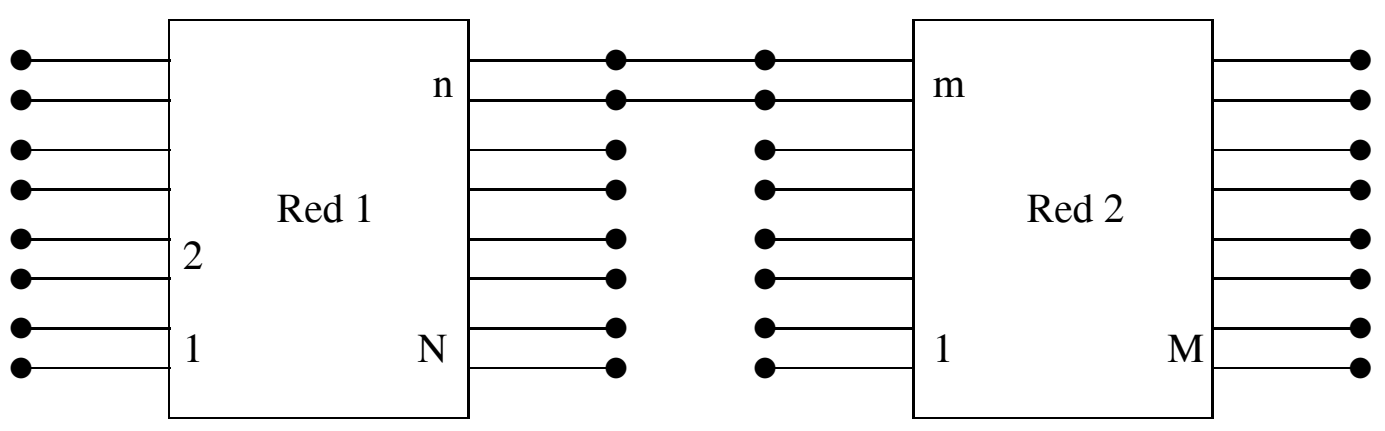

Figura F.1: Unión de dos redes en cascada

Al conectar las dos redes se obtiene una única red de $Q=N+M-2$ accesos, tal y como se muestra en la figura F.2. Los accesos de la red conjunta se numerarán siguiendo la siguiente convención:

- El acceso $i$ de la red global se corresponde con el acceso $i$ de la primera red para $i \in$ $[1, \ldots, n-1]$.

- El acceso $i$ de la red global se corresponde con el acceso $i+1$ de la primera red para $i$ $\in[n, \ldots, N-1]$.

- El acceso $i$ de la red global se corresponde con el acceso $i-N+1$ de la segunda red para $i \in[N, \ldots, N+m-2]$. 
- El acceso $i$ de la red global se corresponde con el acceso $i-N+2$ de la segunda red para $i \in[N+m-1, \ldots, Q]$.

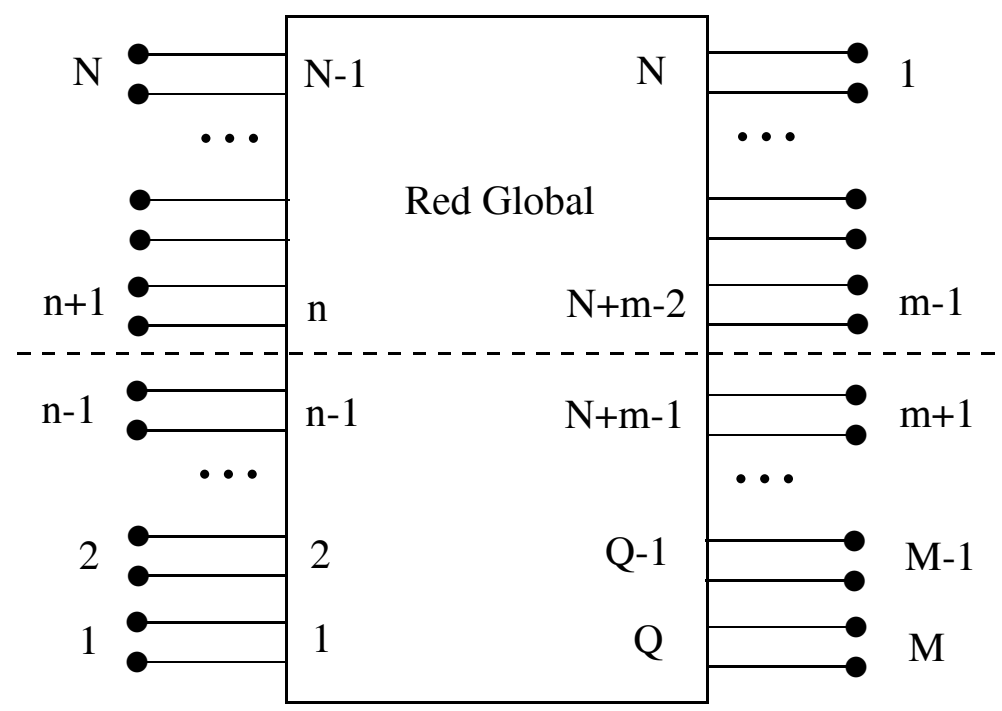

Figura F.2: Red global. Correspondencia con los accesos de la primera (izquierda) y segunda red (derecha)

En la figura F.2 se ha representado dentro de la red global la numeración de los accesos en dicha red, y fuera de la misma se ha representado la correspondencia con los accesos de la primera red (izquierda) y la segunda (derecha).

Para conseguir el objetivo de obtener la matriz de dispersión generalizada (MDG) de la red global (a la que llamaremos $S$ ), es necesario relacionar las ondas de tensión emergentes de los $Q$ accesos de la red global $\left(b_{i}, i \in[1, \ldots, Q]\right)$ con las ondas de tensión incidentes $\left(a_{i}\right.$, $i \in[1, \ldots, Q])$. Dicha relación se puede expresar matricialmente de la siguiente forma:

$$
\left[\begin{array}{c}
b_{1} \\
b_{2} \\
\vdots \\
b_{Q}
\end{array}\right]=\left[\begin{array}{cccc}
S_{11} & S_{12} & \cdots & S_{1 Q} \\
S_{21} & S_{22} & \cdots & S_{2 Q} \\
\vdots & \vdots & \ddots & \vdots \\
S_{Q 1} & S_{Q 2} & \cdots & S_{Q Q}
\end{array}\right] \cdot\left[\begin{array}{c}
a_{1} \\
a_{2} \\
\vdots \\
a_{Q}
\end{array}\right]
$$

De acuerdo con la definición de la MDG de la expresión anterior, podemos expresar cada onda de tensión emergente del acceso $i$ como una combinación lineal de las ondas incidentes a los $Q$ accesos:

$$
b_{i}=\sum_{j=1}^{Q} S_{i j} a_{j}, i \in[1, \ldots, Q]
$$

Para obtener los valores de los elementos $S_{i j}$, debemos hacer uso del conocimiento de las MDGs de las dos subredes. Si denominamos $S^{1}$ a la MDG de la primera red, y $S^{2}$ a la 
MDG de la segunda red, la relación entre ondas de tensión incidentes y dispersadas en los accesos de las dos redes será:

$$
\begin{aligned}
b_{i}^{1} & =\sum_{j=1}^{N} S_{i j}^{1} a_{j}^{1}, i \in[1, \ldots, N] \\
b_{i}^{2} & =\sum_{j=1}^{M} S_{i j}^{2} a_{j}^{2}, i \in[1, \ldots, M]
\end{aligned}
$$

donde $S_{i j}^{1}$ y $S_{i j}^{2}$ son conocidos.

Teniendo en cuenta cómo se han numerado los accesos de la red global (ver figura F.2), y su correspondencia con los accesos de las dos subredes, podemos establecer las siguientes relaciones entre ondas de tensión de la red global y de las subredes:

$$
\begin{gathered}
b_{i}= \begin{cases}b_{i}^{1} & i \in[1, \ldots, n-1] \\
b_{i+1}^{1} & i \in[n, \ldots, N-1] \\
b_{i-N+1}^{2} & i \in[N, \ldots, N+m-2] \\
b_{i-N+2}^{2} & i \in[N+m-1, \ldots, Q]\end{cases} \\
a_{i}= \begin{cases}a_{i}^{1} & i \in[1, \ldots, n-1] \\
a_{i+1}^{1} & i \in[n, \ldots, N-1] \\
a_{i-N+1}^{2} & i \in[N, \ldots, N+m-2] \\
a_{i-N+2}^{2} & i \in[N+m-1, \ldots, Q]\end{cases}
\end{gathered}
$$

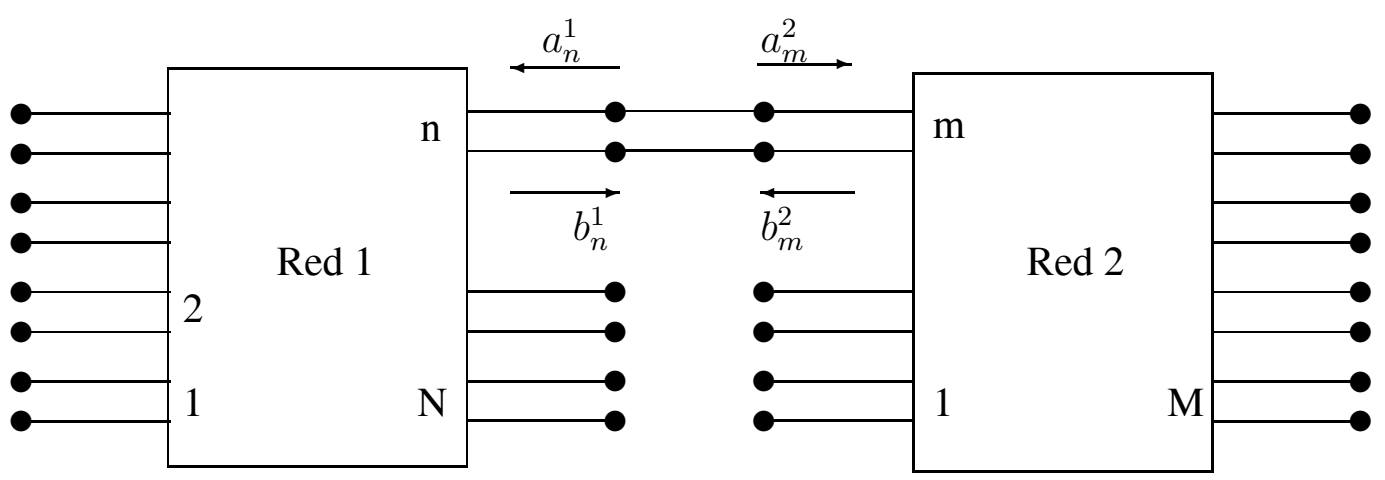

Figura F.3: Relación entre las ondas de tensión de los accesos conectados 
Además, existen las siguientes relaciones entre las ondas de tensión de los accesos de las subredes que se han conectado (ver figura F.3):

$$
\begin{aligned}
& a_{n}^{1}=b_{m}^{2} \\
& b_{n}^{1}=a_{m}^{2}
\end{aligned}
$$

Con el objetivo de relacionar las ondas de tensión emergentes e incidentes de la red global, utilizaremos las expresiones (F.3) - (F.6). Para las ondas de tensión reflejadas en los $n-1$ primeros accesos podemos escribir:

$$
\begin{aligned}
b_{i} & =b_{i}^{1}=\sum_{j=1}^{N} S_{i j}^{1} a_{j}^{1} \\
& =\sum_{j=1}^{n-1} S_{i j}^{1} a_{j}^{1}+S_{i n}^{1} a_{n}^{1}+\sum_{j=n+1}^{N} S_{i j}^{1} a_{j}^{1} \quad, i \in[1, \ldots, n-1] \\
& =\sum_{j=1}^{n-1} S_{i j}^{1} a_{j}+\sum_{j=n+1}^{N} S_{i j}^{1} a_{j-1}+S_{i n}^{1} a_{n}^{1}
\end{aligned}
$$

Para el resto de accesos procedentes de la red primera (del acceso $n$ hasta el $N-1$ ):

$$
\begin{aligned}
b_{i} & =b_{i+1}^{1}=\sum_{j=1}^{N} S_{i+1, j}^{1} a_{j}^{1} \\
& =\sum_{j=1}^{n-1} S_{i+1, j}^{1} a_{j}^{1}+S_{i+1, n}^{1} a_{n}^{1}+\sum_{j=n+1}^{N} S_{i+1, j}^{1} a_{j}^{1} \quad, i \in[n, \ldots, N-1] \\
& =\sum_{j=1}^{n-1} S_{i+1, j}^{1} a_{j}+\sum_{j=n+1}^{N} S_{i+1, j}^{1} a_{j-1}+S_{i+1, n}^{1} a_{n}^{1}
\end{aligned}
$$

Para los accesos desde el $N$ hasta el $N+m-2$ (primeros $m-1$ accesos de la red 2):

$$
\begin{aligned}
b_{i}= & b_{i-N+1}^{2}=\sum_{j=1}^{M} S_{i-N+1, j}^{2} a_{j}^{2} \\
= & \sum_{j=1}^{m-1} S_{i-N+1, j}^{2} a_{j}^{2}+S_{i-N+1, m}^{2} a_{m}^{2}+\sum_{j=m+1}^{M} S_{i-N+1, j}^{2} a_{j}^{2}, i \in[N, \ldots, N+m-2] \\
= & \sum_{j=1}^{m-1} S_{i-N+1, j}^{2} a_{j+N-1}+\sum_{j=m+1}^{M} S_{i-N+1, j}^{2} a_{j+N-2} \\
& +S_{i-N+1, m}^{2} a_{m}^{2}
\end{aligned}
$$


Finalmente, las ondas de tensión emergentes de los últimos accesos, desde el $N+m-1$ hasta el $Q$ (accesos del $m+1$ al $M$ de la red 2):

$$
\begin{aligned}
b_{i}= & b_{i-N+2}^{2}=\sum_{j=1}^{M} S_{i-N+2, j}^{2} a_{j}^{2} \\
= & \sum_{j=1}^{m-1} S_{i-N+2, j}^{2} a_{j}^{2}+S_{i-N+2, m}^{2} a_{m}^{2}+\sum_{j=m+1}^{M} S_{i-N+2, j}^{2} a_{j}^{2}, i \in[N+m-1, \ldots, Q] \\
= & \sum_{j=1}^{m-1} S_{i-N+2, j}^{2} a_{j+N-1}+\sum_{j=m+1}^{M} S_{i-N+2, j}^{2} a_{j+N-2} \\
& +S_{i-N+2, m}^{2} a_{m}^{2}
\end{aligned}
$$

Las expresiones (F.9) - (F.12) expresan las ondas de tensión emergentes en los accesos de la red global en función de las ondas de tensión incidentes, con la excepción de los términos $a_{n}^{1}$ y $a_{m}^{2}$. Necesitamos por tanto expresar $a_{n}^{1}$ y $a_{m}^{2}$ en función de las ondas de tensión incidentes $a_{i}$ de la red global para cumplir con nuestro objetivo. Para establecer esta relación nos valdremos de (F.3) y (F.4):

$$
\begin{aligned}
b_{n}^{1} & =\sum_{j=1}^{N} S_{n j}^{1} a_{j}^{1}=\sum_{j=1}^{n-1} S_{n j}^{1} a_{j}^{1}+S_{n n}^{1} a_{n}^{1}+\sum_{j=n+1}^{N} S_{n j}^{1} a_{j}^{1} \\
& =\sum_{j=1}^{n-1} S_{n j}^{1} a_{j}+\sum_{j=n+1}^{N} S_{n j}^{1} a_{j-1}+S_{n n}^{1} a_{n}^{1} \\
b_{m}^{2} & =\sum_{j=1}^{M} S_{m j}^{2} a_{j}^{2}=\sum_{j=1}^{m-1} S_{m j}^{2} a_{j}^{2}+S_{m m}^{2} a_{m}^{2}+\sum_{j=m+1}^{M} S_{m j}^{2} a_{j}^{2} \\
& =\sum_{j=1}^{m-1} S_{m j}^{2} a_{j+N-1}+\sum_{j=m+1}^{M} S_{m j}^{2} a_{j+N-2}+S_{m m}^{2} a_{m}^{2}
\end{aligned}
$$

Teniendo en cuenta que $b_{n}^{1}=a_{m}^{2}$ y $b_{m}^{2}=a_{n}^{1}$,

$$
\begin{aligned}
a_{m}^{2} & =\sum_{j=1}^{n-1} S_{n j}^{1} a_{j}+\sum_{j=n+1}^{N} S_{n j}^{1} a_{j-1}+S_{n n}^{1} a_{n}^{1} \\
a_{n}^{1} & =\sum_{j=1}^{m-1} S_{m j}^{2} a_{j+N-1}+\sum_{j=m+1}^{M} S_{m j}^{2} a_{j+N-2}+S_{m m}^{2} a_{m}^{2}
\end{aligned}
$$


Obtenemos un sistema de 2 ecuaciones con 2 incógnitas. Para resolverlo despejaremos primero $a_{m}^{2}$ :

$$
\begin{gathered}
a_{m}^{2}=\sum_{j=1}^{n-1} S_{n j}^{1} a_{j}+\sum_{j=n+1}^{N} S_{n j}^{1} a_{j-1} \\
+S_{n n}^{1}\left(\sum_{j=1}^{m-1} S_{m j}^{2} a_{j+N-1}+\sum_{j=m+1}^{M} S_{m j}^{2} a_{j+N-2}+S_{m m}^{2} a_{m}^{2}\right) \\
\left(I-S_{n n}^{1} S_{m m}^{2}\right) a_{m}^{2}= \\
\quad \sum_{j=1}^{n-1} S_{n j}^{1} a_{j}+\sum_{j=n+1}^{N} S_{n j}^{1} a_{j-1} \\
\quad+\sum_{j=1}^{m-1} S_{n n}^{1} S_{m j}^{2} a_{j+N-1}+\sum_{j=m+1}^{M} S_{n n}^{1} S_{m j}^{2} a_{j+N-2}
\end{gathered}
$$

Finalmente,

$$
\begin{aligned}
a_{m}^{2}= & \sum_{j=1}^{n-1} E S_{n j}^{1} a_{j}+\sum_{j=n+1}^{N} E S_{n j}^{1} a_{j-1} \\
& +\sum_{j=1}^{m-1} E S_{n n}^{1} S_{m j}^{2} a_{j+N-1}+\sum_{j=m+1}^{M} E S_{n n}^{1} S_{m j}^{2} a_{j+N-2}
\end{aligned}
$$

donde,

$$
E=\left(I-S_{n n}^{1} S_{m m}^{2}\right)^{-1}
$$

Sustituyendo (F.19) en (F.16) obtendremos ahora $a_{n}^{1}$ :

$$
\begin{aligned}
a_{n}^{1}= & \sum_{j=1}^{m-1} S_{m j}^{2} a_{j+N-1}+\sum_{j=m+1}^{M} S_{m j}^{2} a_{j+N-2} \\
& +\sum_{j=1}^{n-1} S_{m m}^{2} E S_{n j}^{1} a_{j}+\sum_{j=n+1}^{N} S_{m m}^{2} E S_{n j}^{1} a_{j-1} \\
& +\sum_{j=1}^{m-1} S_{m m}^{2} E S_{n n}^{1} S_{m j}^{2} a_{j+N-1}+\sum_{j=m+1}^{M} S_{m m}^{2} E S_{n n}^{1} S_{m j}^{2} a_{j+N-2}
\end{aligned}
$$


Agrupando términos:

$$
\begin{aligned}
a_{n}^{1}= & \sum_{j=1}^{n-1} S_{m m}^{2} E S_{n j}^{1} a_{j}+\sum_{j=n+1}^{N} S_{m m}^{2} E S_{n j}^{1} a_{j-1} \\
& +\sum_{j=1}^{m-1}\left(I+S_{m m}^{2} E S_{n n}^{1}\right) S_{m j}^{2} a_{j+N-1} \\
& +\sum_{j=m+1}^{M}\left(I+S_{m m}^{2} E S_{n n}^{1}\right) S_{m j}^{2} a_{j+N-2}
\end{aligned}
$$

Una vez determinados $a_{n}^{1}$ y $a_{m}^{2}$, podemos sustituir en (F.9):

$$
\begin{aligned}
b_{i}= & \sum_{j=1}^{n-1} S_{i j}^{1} a_{j}+\sum_{j=n+1}^{N} S_{i j}^{1} a_{j-1} \\
& +\sum_{j=1}^{n-1} S_{i n}^{1} S_{m m}^{2} E S_{n j}^{1} a_{j}+\sum_{j=n+1}^{N} S_{i n}^{1} S_{m m}^{2} E S_{n j}^{1} a_{j-1} \\
& +\sum_{j=1}^{m-1} S_{i n}^{1}\left(I+S_{m m}^{2} E S_{n n}^{1}\right) S_{m j}^{2} a_{j+N-1} \\
& +\sum_{j=m+1}^{M} S_{i n}^{1}\left(I+S_{m m}^{2} E S_{n n}^{1}\right) S_{m j}^{2} a_{j+N-2}
\end{aligned}
$$

Agrupando los sumatorios:

$$
\begin{aligned}
b_{i}= & \sum_{j=1}^{n-1}\left(S_{i j}^{1}+S_{i n}^{1} S_{m m}^{2} E S_{n j}^{1}\right) a_{j} \\
& +\sum_{j=n+1}^{N}\left(S_{i j}^{1}+S_{i n}^{1} S_{m m}^{2} E S_{n j}^{1}\right) a_{j-1} \\
& +\sum_{j=1}^{m-1} S_{i n}^{1}\left(I+S_{m m}^{2} E S_{n n}^{1}\right) S_{m j}^{2} a_{j+N-1} \\
& +\sum_{j=m+1}^{M} S_{i n}^{1}\left(I+S_{m m}^{2} E S_{n n}^{1}\right) S_{m j}^{2} a_{j+N-2}
\end{aligned}
$$

Realizando cambios en los índices de los sumatorios de manera que éstos recorran los accesos según la numeración de la red global, y no la numeración local de las dos redes (ver 
figura F.2):

$$
\begin{aligned}
b_{i}= & \sum_{j=1}^{n-1}\left(S_{i j}^{1}+S_{i n}^{1} S_{m m}^{2} E S_{n j}^{1}\right) a_{j} \\
& +\sum_{j=n}^{N-1}\left(S_{i, j+1}^{1}+S_{i n}^{1} S_{m m}^{2} E S_{n, j+1}^{1}\right) a_{j} \\
& +\sum_{j=N}^{N+m-2} S_{i n}^{1}\left(I+S_{m m}^{2} E S_{n n}^{1}\right) S_{m, j-N+1}^{2} a_{j} \\
& +\sum_{j=N+m-1}^{Q} S_{i n}^{1}\left(I+S_{m m}^{2} E S_{n n}^{1}\right) S_{m, j-N+2}^{2} a_{j}
\end{aligned}
$$

Sustituyendo (F.22) en (F.10), agrupando términos y cambiando los índices de los sumatorios, obtenemos para $i \in[n, \ldots, N-1]$ :

$$
\begin{aligned}
b_{i}= & \sum_{j=1}^{n-1}\left(S_{i+1, j}^{1}+S_{i+1, n}^{1} S_{m m}^{2} E S_{n j}^{1}\right) a_{j} \\
& +\sum_{j=n}^{N-1}\left(S_{i+1, j+1}^{1}+S_{i+1, n}^{1} S_{m m}^{2} E S_{n, j+1}^{1}\right) a_{j} \quad, i \in[n, \ldots, N-1] \\
& +\sum_{j=N}^{N+m-2} S_{i+1, n}^{1}\left(I+S_{m m}^{2} E S_{n n}^{1}\right) S_{m, j-N+1}^{2} a_{j} \\
& +\sum_{j=N+m-1}^{Q} S_{i+1, n}^{1}\left(I+S_{m m}^{2} E S_{n n}^{1}\right) S_{m, j-N+2}^{2} a_{j}
\end{aligned}
$$

Sustituyendo (F.19) en (F.11), agrupando términos y cambiando los índices de los suma- 
torios, obtenemos para $i \in[N, \ldots, N+m-2]$ :

$$
\begin{aligned}
b_{i}= & \sum_{j=1}^{n-1} S_{i-N+1, m}^{2} E S_{n j}^{1} a_{j} \\
& +\sum_{j=n}^{N-1} S_{i-N+1, m}^{2} E S_{n, j+1}^{1} a_{j} \\
& +\sum_{j=N}^{N+m-2}\left(S_{i-N+1, j-N+1}^{2}+S_{i-N+1, m}^{2} E S_{n n}^{1} S_{m, j-N+1}^{2}\right) a_{j} \quad, i \in[N, \ldots, N+m-2] \\
& +\sum_{j=N+m-1}^{Q}\left(S_{i-N+1, j-N+2}^{2}+S_{i-N+1, m}^{2} E S_{n n}^{1} S_{m, j-N+2}^{2}\right) a_{j}
\end{aligned}
$$

Sustituyendo (F.19) en (F.12), agrupando términos y cambiando los índices de los sumatorios, obtenemos para $i \in[N+m-1, \ldots, Q]$ :

$$
\begin{aligned}
b_{i}= & \sum_{j=1}^{n-1} S_{i-N+2, m}^{2} E S_{n j}^{1} a_{j} \\
& +\sum_{j=n}^{N-1} S_{i-N+2, m}^{2} E S_{n, j+1}^{1} a_{j} \\
& +\sum_{j=N}^{N+m-2}\left(S_{i-N+2, j-N+1}^{2}+S_{i-N+2, m}^{2} E S_{n n}^{1} S_{m, j-N+1}^{2}\right) a_{j} \quad, i \in[N+m-1, \ldots, Q] \\
& +\sum_{j=N+m-1}^{Q}\left(S_{i-N+2, j-N+2}^{2}+S_{i-N+2, m}^{2} E S_{n n}^{1} S_{m, j-N+2}^{2}\right) a_{j}
\end{aligned}
$$

Las expresiones (F.25), (F.26), (F.27) y (F.28) nos permiten relacionar las ondas de tensión emergentes de cada uno de los accesos de la red global con las ondas de tensión incidentes. Por tanto podemos ya construir la matriz de dispersión generalizada (MDG) de la red conjunta, fruto de la conexión de las dos subredes. Comparando (F.2) con (F.25), (F.26), (F.27) y (F.28), podemos identificar directamente los elementos $S_{i j}$ de la matriz global. 
Para $i \in[1, \ldots, n-1]$ :

$$
S_{i j}= \begin{cases}S_{i j}^{1}+S_{i n}^{1} S_{m m}^{2} E S_{n j}^{1} & j \leq n-1 \\ S_{i, j+1}^{1}+S_{i n}^{1} S_{m m}^{2} E S_{n, j+1}^{1} & n \leq j \leq N-1 \\ S_{i n}^{1}\left(I+S_{m m}^{2} E S_{n n}^{1}\right) S_{m, j-N+1}^{2} & N \leq j \leq N+m-2 \\ S_{i n}^{1}\left(I+S_{m m}^{2} E S_{n n}^{1}\right) S_{m, j-N+2}^{2} & N+m-1 \leq j \leq Q\end{cases}
$$

Para $i \in[n, \ldots, N-1]$ :

$$
S_{i j}= \begin{cases}S_{i+1, j}^{1}+S_{i+1, n}^{1} S_{m m}^{2} E S_{n j}^{1} & j \leq n-1 \\ S_{i+1, j+1}^{1}+S_{i+1, n}^{1} S_{m m}^{2} E S_{n, j+1}^{1} & n \leq j \leq N-1 \\ S_{i+1, n}^{1}\left(I+S_{m m}^{2} E S_{n n}^{1}\right) S_{m, j-N+1}^{2} & N \leq j \leq N+m-2 \\ S_{i+1, n}^{1}\left(I+S_{m m}^{2} E S_{n n}^{1}\right) S_{m, j-N+2}^{2} & N+m-1 \leq j \leq Q\end{cases}
$$

Para $i \in[N, \ldots, N+m-2]$ :

$$
S_{i j}= \begin{cases}S_{i-N+1, m}^{2} E S_{n j}^{1} & j \leq n-1 \\ S_{i-N+1, m}^{2} E S_{n, j+1}^{1} & n \leq j \leq N-1 \\ S_{i-N+1, j-N+1}^{2} & N \leq j \leq N+m-2 \\ \quad+S_{i-N+1, m}^{2} E S_{n n}^{1} S_{m, j-N+1}^{2} & \\ S_{i-N+1, j-N+2}^{2} & N+m-1 \leq j \leq Q \\ +S_{i-N+1, m}^{2} E S_{n n}^{1} S_{m, j-N+2}^{2} & \end{cases}
$$

Para $i \in[N+m-1, \ldots, Q]$ :

$$
S_{i j}= \begin{cases}S_{i-N+2, m}^{2} E S_{n j}^{1} & j \leq n-1 \\ S_{i-N+2, m}^{2} E S_{n, j+1}^{1} & n \leq j \leq N-1 \\ S_{i-N+2, j-N+1}^{2} & N \leq j \leq N+m-2 \\ \quad+S_{i-N+2, m}^{2} E S_{n n}^{1} S_{m, j-N+1}^{2} & \\ S_{i-N+2, j-N+2}^{2} & N+m-1 \leq j \leq Q \\ +S_{i-N+2, m}^{2} E S_{n n}^{1} S_{m, j-N+2}^{2} & \end{cases}
$$

Cada elemento $S_{i j}$ es una submatriz de tamaño $M_{i} \times M_{j}$, donde $M_{i}$ y $M_{j}$ son el número de modos guiados que se han considerado para el acceso $i$ y $j$, respectivamente. 


\begin{tabular}{|c||c|c|}
\hline Sub-bloque & Filas & Columnas \\
\hline \hline$G_{11}^{1}$ & $n-1$ & $n-1$ \\
\hline$G_{12}^{1}$ & $n-1$ & $N-n$ \\
\hline$G_{21}^{1}$ & $N-n$ & $n-1$ \\
\hline$G_{22}^{1}$ & $N-n$ & $N-n$ \\
\hline$C_{1}^{1}$ & $n-1$ & 1 \\
\hline$C_{2}^{1}$ & $N-n$ & 1 \\
\hline$F_{1}^{1}$ & 1 & $n-1$ \\
\hline$F_{2}^{1}$ & 1 & $N-n$ \\
\hline$G_{11}^{2}$ & $m-1$ & $m-1$ \\
\hline$G_{12}^{2}$ & $m-1$ & $M-m$ \\
\hline$G_{21}^{2}$ & $M-m$ & $m-1$ \\
\hline$G_{22}^{2}$ & $M-m$ & $M-m$ \\
\hline$C_{1}^{2}$ & $m-1$ & 1 \\
\hline$C_{2}^{2}$ & $M-m$ & 1 \\
\hline$F_{1}^{2}$ & 1 & $m-1$ \\
\hline$F_{2}^{2}$ & 1 & $M-m$ \\
\hline \multicolumn{2}{|c}{}
\end{tabular}

El cálculo de la matriz $S$ se puede simplificar si descomponemos las matrices MDG de las dos redes $\left(S^{1}\right.$ y $\left.S^{2}\right)$ en los siguientes sub-bloques:

$$
\begin{aligned}
S^{1} & =\left[\begin{array}{c|c|c}
G_{11}^{1} & C_{1}^{1} & G_{12}^{1} \\
\hline F_{1}^{1} & S_{n n}^{1} & F_{2}^{1} \\
\hline G_{21}^{1} & C_{2}^{1} & G_{22}^{1}
\end{array}\right]_{N \times N} \\
S^{2} & =\left[\begin{array}{c|c|c}
G_{11}^{2} & C_{1}^{2} & G_{12}^{2} \\
\hline F_{1}^{2} & S_{m m}^{2} & F_{2}^{2} \\
\hline G_{21}^{2} & C_{2}^{2} & G_{22}^{2}
\end{array}\right]_{M \times M}
\end{aligned}
$$

A continuación se detalla en una tabla el tamaño de cada uno de los sub-bloques en los que se han descompuesto las matrices $S^{1}$ y $S^{2}$.

Como ya se ha indicado con anterioridad, cada uno de los sub-bloques $A$ son matrices con elementos $A_{i j}$ que son a su vez matrices de tamaño $M_{i} \times M_{j}$, siendo $M_{i}$ y $M_{j}$ el número de modos guiados en el acceso $i$ y $j$, respectivamente. 
A continuación definimos las siguientes matrices:

$$
\begin{aligned}
& G^{1}=\left[\begin{array}{ll}
G_{11}^{1} & G_{12}^{1} \\
G_{21}^{1} & G_{22}^{1}
\end{array}\right] \\
& C^{1}=\left[\begin{array}{l}
C_{1}^{1} \\
C_{2}^{1}
\end{array}\right] \\
& F^{1}=\left[\begin{array}{ll}
F_{1}^{1} & F_{2}^{1}
\end{array}\right] \\
& G^{2}=\left[\begin{array}{ll}
G_{11}^{2} & G_{12}^{2} \\
G_{21}^{2} & G_{22}^{2}
\end{array}\right] \\
& C^{2}=\left[\begin{array}{ll}
C_{1}^{2} \\
C_{2}^{2}
\end{array}\right] \\
& F^{2}=\left[\begin{array}{ll}
F_{1}^{2} & F_{2}^{2}
\end{array}\right]
\end{aligned}
$$

Utilizando las matrices que acabamos de definir, la MDG de la red total se puede calcular de la siguiente forma:

$$
S=\left[\begin{array}{c|c}
A_{11} & A_{12} \\
\hline A_{21} & A_{22}
\end{array}\right]=\left[\begin{array}{c|c}
G^{1}+C^{1} S_{m m}^{2} E F^{1} & C^{1}\left(I+S_{m m}^{2} E S_{n n}^{1}\right) F^{2} \\
\hline C^{2} E F^{1} & G^{2}+C^{2} E S_{n n}^{1} F^{2}
\end{array}\right]
$$

donde $A_{11}$ es un sub-bloque de $(N-1) \times(N-1)$ elementos, $A_{12}$ de $(N-1) \times(M-1)$ elementos, $A_{21}$ de $(M-1) \times(N-1)$ elementos, y $A_{22}$ de $(M-1) \times(M-1)$ elementos.

\section{F.2. Cambio de accesos}

Una vez se han enlazado dos redes y se ha obtenido la MDG conjunta, la numeración de los accesos de la red queda tal y como se muestra en la figura F.2. Es posible que que se desee que la numeración de los accesos sea distinta. Para ello es preciso intercambiar unos o más accesos.

En esta sección se aborda el problema de intercambiar dos accesos cualesquiera en una red, tal y como se muestra en la figura F.4, donde se ha denominado $S$ a la red original y $S^{\prime}$ a la red resultante de intercambiar los accesos $i$-ésimo y $j$-ésimo de la red $\mathrm{S}$. 


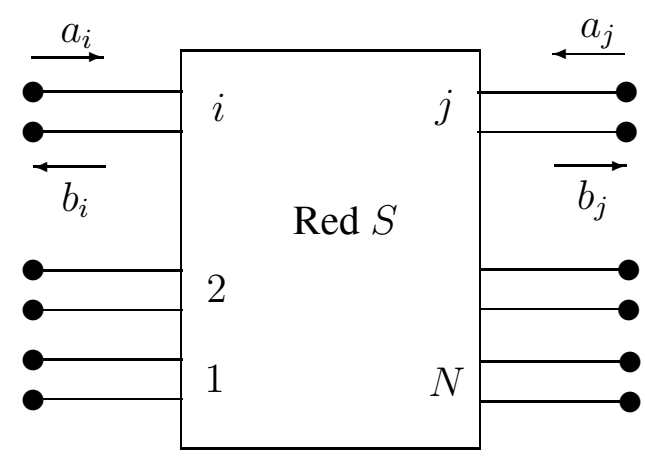

(a) Antes del cambio

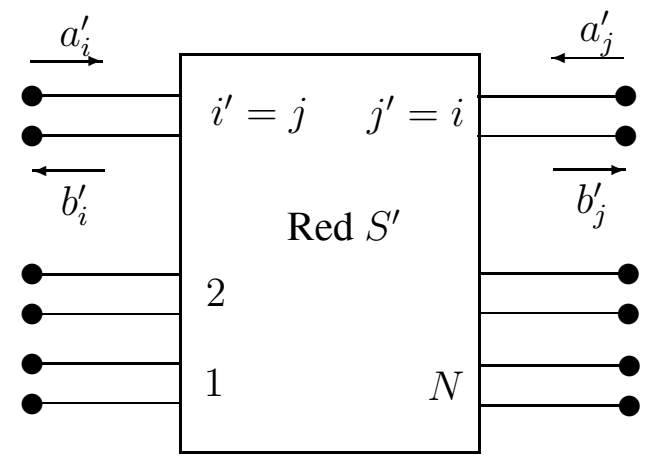

(b) Después del cambio

Figura F.4: Cambio del acceso $i$ por el acceso $j$ en una red de $N$ accesos

La relación entre las ondas de tensión incidentes y reflejadas en cada uno de los $N$ accesos de la red $S$ viene dada por su matriz de dispersión generalizada $S$ de la siguiente forma:

$$
\left[\begin{array}{c}
b_{1} \\
\vdots \\
b_{i} \\
\vdots \\
b_{j} \\
\vdots \\
b_{N}
\end{array}\right]=S \cdot\left[\begin{array}{c}
a_{1} \\
\vdots \\
a_{i} \\
\vdots \\
a_{j} \\
\vdots \\
a_{N}
\end{array}\right]
$$

donde los elementos $b_{n}$ y $a_{n}$ son a su vez vectores, de $M_{n}$ elementos, que contienen las amplitudes de los $M_{n}$ modos incidentes y reflejados en el acceso $n$.

Expandiendo la matriz $S$ en submatrices $S_{m n}$ de tamaño $M_{m} \times M_{n}$, con $m, n \in[1, \ldots, N]$ :

$$
\left[\begin{array}{c}
b_{1} \\
\vdots \\
b_{i} \\
\vdots \\
b_{j} \\
\vdots \\
b_{N}
\end{array}\right]=\left[\begin{array}{ccccccc}
S_{11} & \cdots & S_{1 i} & \cdots & S_{1 j} & \cdots & S_{1 N} \\
\vdots & \vdots & \vdots & \vdots & \vdots & \vdots & \vdots \\
S_{i 1} & \cdots & S_{i i} & \cdots & S_{i j} & \cdots & S_{i N} \\
\vdots & \vdots & \vdots & \vdots & \vdots & \vdots & \vdots \\
S_{j 1} & \cdots & S_{j i} & \cdots & S_{j j} & \cdots & S_{j N} \\
\vdots & \vdots & \vdots & \vdots & \vdots & \vdots & \vdots \\
S_{N 1} & \cdots & S_{N i} & \cdots & S_{N j} & \cdots & S_{N N}
\end{array}\right] \cdot\left[\begin{array}{c}
a_{1} \\
\vdots \\
a_{i} \\
\vdots \\
a_{j} \\
\vdots \\
a_{N}
\end{array}\right]
$$

En el caso de la red $S^{\prime}$, también se pueden relacionar las amplitudes de las ondas de 
tensión normalizadas $a_{n}^{\prime}$ y $b_{n}^{\prime}$ mediante la matriz de dispersión generalizada de la red:

$$
\left[\begin{array}{c}
b_{1}^{\prime} \\
\vdots \\
b_{i}^{\prime} \\
\vdots \\
b_{j}^{\prime} \\
\vdots \\
b_{N}^{\prime}
\end{array}\right]=\left[\begin{array}{ccccccc}
S_{11}^{\prime} & \cdots & S_{1 i}^{\prime} & \cdots & S_{1 j}^{\prime} & \cdots & S_{1 N}^{\prime} \\
\vdots & \vdots & \vdots & \vdots & \vdots & \vdots & \vdots \\
S_{i 1}^{\prime} & \cdots & S_{i i}^{\prime} & \cdots & S_{i j}^{\prime} & \cdots & S_{i N}^{\prime} \\
\vdots & \vdots & \vdots & \vdots & \vdots & \vdots & \vdots \\
S_{j 1}^{\prime} & \cdots & S_{j i}^{\prime} & \cdots & S_{j j}^{\prime} & \cdots & S_{j N}^{\prime} \\
\vdots & \vdots & \vdots & \vdots & \vdots & \vdots & \vdots \\
S_{N 1}^{\prime} & \cdots & S_{N i}^{\prime} & \cdots & S_{N j}^{\prime} & \cdots & S_{N N}^{\prime}
\end{array}\right] \cdot\left[\begin{array}{c}
a_{1}^{\prime} \\
\vdots \\
a_{i}^{\prime} \\
\vdots \\
a_{j}^{\prime} \\
\vdots \\
a_{N}^{\prime}
\end{array}\right]
$$

Si cambiamos los accesos $i$ y $j$ el uno por el otro, se cumplirán las siguientes relaciones:

$$
\begin{aligned}
a_{i}^{\prime} & =a_{j} \\
b_{i}^{\prime} & =b_{j} \\
a_{j}^{\prime} & =a_{i} \\
b_{j}^{\prime} & =b_{i} \\
a_{n}^{\prime} & =a_{n} n \neq i, j \\
b_{n}^{\prime} & =b_{n} n \neq i, j
\end{aligned}
$$

Utilizando las expresiones anteriores y sustituyendo en (F.43) podemos establecer una relación entre las ondas reflejadas $b_{n}^{\prime}$ y las incidentes $a_{n}^{\prime}$ en los accesos de la red $S^{\prime}$, en función de la matriz $S$ de la red original:

$$
\left[\begin{array}{c}
b_{1}^{\prime} \\
\vdots \\
b_{i}^{\prime} \\
\vdots \\
b_{j}^{\prime} \\
\vdots \\
b_{N}^{\prime}
\end{array}\right]=\left[\begin{array}{ccccccc}
S_{11} & \cdots & S_{1 j} & \cdots & S_{1 i} & \cdots & S_{1 N} \\
\vdots & \vdots & \vdots & \vdots & \vdots & \vdots & \vdots \\
S_{j 1} & \cdots & S_{j j} & \cdots & S_{j i} & \cdots & S_{j N} \\
\vdots & \vdots & \vdots & \vdots & \vdots & \vdots & \vdots \\
S_{i 1} & \cdots & S_{i j} & \cdots & S_{i i} & \cdots & S_{i N} \\
\vdots & \vdots & \vdots & \vdots & \vdots & \vdots & \vdots \\
S_{N 1} & \cdots & S_{N j} & \cdots & S_{N i} & \cdots & S_{N N}
\end{array}\right] \cdot\left[\begin{array}{c}
a_{1}^{\prime} \\
\vdots \\
a_{i}^{\prime} \\
\vdots \\
a_{j}^{\prime} \\
\vdots \\
a_{N}^{\prime}
\end{array}\right]
$$

De las expresiones (F.43) y (F.46) se desprende que la matriz $S^{\prime}$ de la red con los accesos cambiados es la misma que la matriz $S$ de la red original, con la salvedad de que se han intercambiado las posiciones de las filas $i$ y $j$, y posteriormente las de las columnas $i$ y $j$. Este intercambio de posiciones en las filas y las columnas se puede conseguir pre y post multiplicando $S$ por unas matrices adecuadas:

$$
S^{\prime}=F \cdot S \cdot C
$$

donde $F$ y $C$ son matrices de $N \times N$ elementos, cada uno de tamaño $M_{m} \times M_{n}(m, n \in$ $[1, \ldots, N])$. 
Para obtener $F$ y $C$, partiremos de una matriz diagonal $A$ de $N \times N$ elementos.

$$
A=\left[\begin{array}{ccccccc}
I_{1} & \cdots & 0 & \cdots & 0 & \cdots & 0 \\
\vdots & \vdots & \vdots & \vdots & \vdots & \vdots & \vdots \\
0 & \cdots & I_{i} & \cdots & 0 & \cdots & 0 \\
\vdots & \vdots & \vdots & \vdots & \vdots & \vdots & \vdots \\
0 & \cdots & 0 & \cdots & I_{j} & \cdots & 0 \\
\vdots & \vdots & \vdots & \vdots & \vdots & \vdots & \vdots \\
0 & \cdots & 0 & \cdots & 0 & \cdots & I_{N}
\end{array}\right]
$$

donde $I_{n}$ es la una matriz identidad de tamaño $M_{n} \times M_{n}(n \in[1, \ldots, N])$.

La matriz $F$ se obtiene cambiando las filas $i$ y $j$ de la matriz $A$ :

$$
F=\left[\begin{array}{ccccccc}
I_{1} & \cdots & 0 & \cdots & 0 & \cdots & 0 \\
\vdots & \vdots & \vdots & \vdots & \vdots & \vdots & \vdots \\
0 & \cdots & 0 & \cdots & I_{j} & \cdots & 0 \\
\vdots & \vdots & \vdots & \vdots & \vdots & \vdots & \vdots \\
0 & \cdots & I_{i} & \cdots & 0 & \cdots & 0 \\
\vdots & \vdots & \vdots & \vdots & \vdots & \vdots & \vdots \\
0 & \cdots & 0 & \cdots & 0 & \cdots & I_{N}
\end{array}\right]
$$

Y la matriz $C$ se obtiene cambiando las columnas $i$ y $j$ de $A$ :

$$
C=\left[\begin{array}{ccccccc}
I_{1} & \cdots & 0 & \cdots & 0 & \cdots & 0 \\
\vdots & \vdots & \vdots & \vdots & \vdots & \vdots & \vdots \\
0 & \cdots & 0 & \cdots & I_{i} & \cdots & 0 \\
\vdots & \vdots & \vdots & \vdots & \vdots & \vdots & \vdots \\
0 & \cdots & I_{j} & \cdots & 0 & \cdots & 0 \\
\vdots & \vdots & \vdots & \vdots & \vdots & \vdots & \vdots \\
0 & \cdots & 0 & \cdots & 0 & \cdots & I_{N}
\end{array}\right]
$$




\section{Apéndice G}

\section{Publicaciones}

En este apéndice se proporciona una relación de las publicaciones científicas realizadas como fruto del trabajo de la tesis. Se trata de 2 artículos publicados y uno en revisión en revistas de ámbito internacional, 4 ponencias en congresos nacionales, y 14 ponencias en congresos internacionales.

\section{G.1. Artículos en revistas de ámbito internacional}

\begin{tabular}{|c|l|l|l|}
\hline Autores & \multicolumn{4}{|l|}{ H. Esteban, J.M. Catalá, S. Cogollos, y V.E. Boria } \\
\hline Título & \multicolumn{4}{|l|}{$\begin{array}{l}\text { Characterization of complex permittivity properties of materials } \\
\text { in rectangular waveguides using a hybrid iterative method }\end{array}$} \\
\hline Revista & \multicolumn{4}{|l|}{ IEEE Microwave and Guided Wave Letters } \\
\hline Volumen & 10 & Número & 5 \\
\hline Fecha & Mayo 2000 & Páginas & $186-188$ \\
\hline ISSN & $1051-8207$ & \multicolumn{3}{|l}{} \\
\hline
\end{tabular}

\begin{tabular}{|c|l|c|l|}
\hline Autores & H. Esteban, S. Cogollos, V.E. Boria, A. San Blas, y M. Ferrando \\
\hline Título & \multicolumn{2}{|l|}{$\begin{array}{l}\text { A new Hybrid/Mode-Matching method for the analysis of arbi- } \\
\text { trarily shaped inductive obstacles and discontinuities in rectangu- } \\
\text { lar waveguides }\end{array}$} \\
\hline Revista & \multicolumn{4}{|l|}{ IEEE Transactions on Microwave Theory and Techniques } \\
\hline Volumen & 50 & Número & 4 \\
\hline Fecha & Abril 2002 & Páginas & $1219-1224$ \\
\hline ISBN & $0018-9480 / 02$ & \multicolumn{3}{l}{} \\
\hline
\end{tabular}




\begin{tabular}{|c|l|c|l|}
\hline Autores & C. Bachiller, H. Esteban, A.A. San Blas, A. Vidal, V.E. Boria \\
\hline Título & $\begin{array}{l}\text { Teaching of basic and advanced wave propagation phenomena us- } \\
\text { ing MATLAB GUIs }\end{array}$ \\
\hline Revista & IEEE Transactions on Microwave Theory and Techniques \\
\hline Volumen & - & Número & - \\
\hline Fecha & En revisión & Páginas & 8 \\
\hline ISBN & $0018-9480$ & \multicolumn{3}{l}{} \\
\hline
\end{tabular}

\section{G.2. Congresos}

\section{G.2.1. Congresos nacionales}

\begin{tabular}{|c|l|l|l|}
\hline Autores & \multicolumn{4}{|l|}{ H. Esteban, S. Cogollos, V.E. Boria y M. Ferrando } \\
\hline Título & \multicolumn{4}{|l|}{ Análisis de problemas de dispersión mediante técnicas espectrales } \\
\hline Congreso & $\begin{array}{l}\text { XIII Simposium Nacional de la Unión Científica Internacional de } \\
\text { Radio (URSI'98) }\end{array}$ \\
\hline Publicación & Libro de actas de la URSI '98 \\
\hline ISBN & - & Lugar & Pamplona \\
\hline Fecha & $\begin{array}{l}16-18 \text { de Septiembre de } \\
1998\end{array}$ & Páginas & $199-200$ \\
\hline
\end{tabular}

\begin{tabular}{|c|l|c|l|}
\hline Autores & Vicente E. Boria, S. Cogollos, H. Esteban, y B. Gimeno \\
\hline Título & $\begin{array}{l}\text { Desarrollo de Nuevos Componentes en las bandas de Microondas } \\
\text { y Milimétricas para Futuros Sistemas de Radiocomunicaciones }\end{array}$ \\
\hline Congreso & X Jornadas de Telecom I+D \\
\hline Publicación & Libro de actas & Lugar & Madrid \\
\hline ISBN & - & Páginas & - \\
\hline Fecha & Noviembre, 2000 &
\end{tabular}




\begin{tabular}{|c|c|c|c|}
\hline Autores & \multicolumn{3}{|c|}{ H. Esteban, A. San Blas, C. Bachiller, A. Vidal y V. E. Boria } \\
\hline Título & \multicolumn{3}{|c|}{$\begin{array}{l}\text { Análisis y diseño de dispositivos en guía rectangular con ge- } \\
\text { ometría arbitraria en el plano } \mathrm{H}\end{array}$} \\
\hline Congreso & \multicolumn{3}{|c|}{ II Encuentro de Electromagnetismo Computacional } \\
\hline Publicación & \multicolumn{3}{|l|}{ Libro de actas } \\
\hline ISBN & - & Lugar & Aracena \\
\hline Fecha & 1 de diciembre de 2001 & Páginas & - \\
\hline
\end{tabular}

\begin{tabular}{|c|l|c|l|}
\hline Autores & \multicolumn{2}{|l|}{$\begin{array}{l}\text { V.E. Boria, S. Cogollos, H. Esteban, P. Soto, A.A. San Blas, B. } \\
\text { Gimeno }\end{array}$} \\
\hline Título & \multicolumn{2}{|l|}{$\begin{array}{l}\text { Desarrollo de dispositivos pasivos de microondas y milimétricas } \\
\text { en guía de onda considerando efectos de mecanizado }\end{array}$} \\
\hline Congreso & \multicolumn{4}{|l|}{ XII Jornadas TELECOM I+D 2002 } \\
\hline Publicación & Actas del Congreso & Lugar & Madrid \\
\hline ISBN & - & Páginas & 4 \\
\hline Fecha & noviembre de 2002 & \multicolumn{3}{|l}{} \\
\hline
\end{tabular}

\section{G.2.2. Congresos internacionales}

\begin{tabular}{|c|l|l|l|}
\hline Autores & H. Esteban, S.Cogollos, V.E.Boria y M.Ferrando \\
\hline Título & \multicolumn{2}{|l|}{$\begin{array}{l}\text { Analysis of Discontinuities in a Rectangular Waveguide using } \\
\text { Hybrid Numerical and Spectral Techniques }\end{array}$} \\
\hline Congreso & \multicolumn{4}{|l|}{ Progress in Electromagnetics Research Symposium (PIERS'98) } \\
\hline Publicación & Proceedings of PIERS'98. Vol. 2 & Lugar & Nantes (Francia) \\
\hline ISBN & 2-909 805-10-7 & Páginas & 893 \\
\hline Fecha & $13-17$ de julio de 1998 &
\end{tabular}




\begin{tabular}{|c|l|l|l|}
\hline Autores & H. Esteban, J.M. Catalá, S.Cogollos, y V.E.Boria \\
\hline Título & \multicolumn{2}{|l|}{$\begin{array}{l}\text { Characterization of complex dielectric properties of materials us- } \\
\text { ing a hybrid iterative method }\end{array}$} \\
\hline Congreso & \multicolumn{2}{|l|}{$\begin{array}{l}\text { 1999 IEEE AP-S International Symposium and USNC/URSI Na- } \\
\text { tional Radio Science Meeting }\end{array}$} \\
\hline Publicación & Proceedings of the 1999 IEEE AP-S International, Vol. 3 \\
\hline ISBN & $0-7803-5639-X$ & Lugar & $\begin{array}{l}\text { Orlando, Florida, } \\
\text { Estados Unidos }\end{array}$ \\
\hline Fecha & Julio de 1999 & Páginas & $1946-1949$ \\
\hline
\end{tabular}

\begin{tabular}{|c|l|l|l|}
\hline Autores & H. Esteban, S. Cogollos, A. Vidal, V.E.Boria, y M.Ferrando \\
\hline Título & \multicolumn{2}{|l|}{$\begin{array}{l}\text { A new hybrid mode-matching method for the analysis of inductive } \\
\text { obstacles and discontinuities }\end{array}$} \\
\hline Congreso & \multicolumn{2}{|l|}{$\begin{array}{l}\text { 1999 IEEE AP-S International Symposium and USNC/URSI Na- } \\
\text { tional Radio Science Meeting }\end{array}$} \\
\hline Publicación & \multicolumn{1}{|l|}{ Proceedings of the 1999 IEEE AP-S International, Vol. 2 } \\
\hline ISBN & $0-7803-5639-X$ & Lugar & $\begin{array}{l}\text { Orlando, Florida, } \\
\text { Estados Unidos }\end{array}$ \\
\hline Fecha & Julio de 1999 & Páginas & 966-969 \\
\hline
\end{tabular}

\begin{tabular}{|c|l|c|l|}
\hline Autores & \multicolumn{3}{|l|}{ H. Esteban, S. Cogollos, V.E. Boria, y M. Ferrando } \\
\hline Título & \multicolumn{2}{|l|}{$\begin{array}{l}\text { Analysis of arbitrarily shaped inductive problems in rectangular } \\
\text { waveguides using a hybrid mode-matching method }\end{array}$} \\
\hline Congreso & \multicolumn{3}{|l|}{$\begin{array}{l}\text { 7th International Symposium on Recent Advances in Microwave } \\
\text { Technologies ISRAMT'99 }\end{array}$} \\
\hline Publicación & $\begin{array}{l}\text { Proceedings of 7th International Symposium on Recent Advances } \\
\text { in Microwave Technology }\end{array}$ \\
\hline ISBN & 84-7785-349-5 & Lugar & Málaga, España \\
\hline Fecha & 13-17 Diciembre, 1999 & Páginas & 533-536 \\
\hline
\end{tabular}




\begin{tabular}{|c|c|c|c|}
\hline Autores & \multicolumn{3}{|c|}{ M. Ferrando, V.E. Boria, y H. Esteban } \\
\hline Título & \multicolumn{3}{|c|}{$\begin{array}{l}\text { Efficient analysis of waveguide systems and open-space problems } \\
\text { using modal and hybrid techniques }\end{array}$} \\
\hline Congreso & \multicolumn{3}{|c|}{$\begin{array}{l}\text { 7th International Symposium on Recent Advances in Microwave } \\
\text { Technologies ISRAMT'99 }\end{array}$} \\
\hline Publicación & \multicolumn{3}{|c|}{$\begin{array}{l}\text { Proceedings of } 7 \text { th International Symposium on Recent Advances } \\
\text { in Microwave Technology }\end{array}$} \\
\hline ISBN & $84-7785-349-5$ & Lugar & Málaga, España \\
\hline Fecha & 13-17 Diciembre, 1999 & Páginas & $761-764$ \\
\hline
\end{tabular}

\begin{tabular}{|c|c|c|c|}
\hline Autores & \multicolumn{3}{|c|}{ H. Esteban, S. Cogollos, V.E. Boria, R. Chismol, y A. Vidal } \\
\hline Título & \multicolumn{3}{|c|}{$\begin{array}{l}\text { Efficient analysis of arbitrarily shaped H-plane bends using a hy- } \\
\text { brid mode-matching method }\end{array}$} \\
\hline Congreso & \multicolumn{3}{|c|}{$\begin{array}{l}\text { NUMELEC'2000, 3rd European Conference on Numerical Meth- } \\
\text { ods in Electromagnetism }\end{array}$} \\
\hline Publicación & \multicolumn{3}{|c|}{$\begin{array}{l}\text { Proceedings of 3rd European Conference on Numerical Methods } \\
\text { in Electromagnetism }\end{array}$} \\
\hline ISBN & - & Lugar & Poitiers, Francia \\
\hline Fecha & 20-22 Marzo, 2000 & Páginas & $172-173$ \\
\hline
\end{tabular}

\begin{tabular}{|c|l|l|l|}
\hline Autores & \multicolumn{4}{|l|}{ H. Esteban, J.M. Catalá, S. Cogollos, y V.E. Boria } \\
\hline Título & \multicolumn{2}{|l|}{$\begin{array}{l}\text { Automated determination of dielectric properties of materials } \\
\text { with arbitrary section using a hybrid iterative method }\end{array}$} \\
\hline Congreso & $\begin{array}{l}\text { 2nd World Congress on Microwave \& Radio Frequency Process- } \\
\text { ing }\end{array}$ \\
\hline Publicación & $\begin{array}{l}\text { Proceedings of 2nd World Congress on Microwave \& Radio Fre- } \\
\text { quency Processing }\end{array}$ \\
\hline ISBN & - & Lugar & $\begin{array}{l}\text { Orlando, } \\
\text { USA }\end{array}$ \\
\hline Fecha & 2-6 Abril, 2000 & Páginas & 41 \\
\hline
\end{tabular}




\begin{tabular}{|c|l|c|l|}
\hline Autores & H. Esteban, V.E. Boria, S. Cogollos, y M. Ferrando \\
\hline Título & \multicolumn{4}{|l|}{$\begin{array}{l}\text { Analysis of 2-D scattering problems in open-space and waveguide } \\
\text { systems using hybrid numerical-modal techniques }\end{array}$} \\
\hline Congreso & AP2000, Millenium Conference on Antennas and Propagation \\
\hline Publicación & Abstracts of Millenium Conference on Antennas and Propagation \\
\hline ISBN & $92-9092-776-3$ & Lugar & Davos, Suiza \\
\hline Fecha & $9-14$ Abril, 2000 & Páginas & 625 \\
\hline
\end{tabular}

\begin{tabular}{|c|c|c|c|}
\hline Autores & \multicolumn{3}{|c|}{ P. Soto, A. Bergner, J.L. Gómez, V.E. Boria, y H. Esteban } \\
\hline Título & \multicolumn{3}{|c|}{$\begin{array}{l}\text { Automated design of inductively coupled rectangular waveguide } \\
\text { filters using space mapping optimisation }\end{array}$} \\
\hline Congreso & \multicolumn{3}{|c|}{$\begin{array}{l}\text { ECCOMAS 2000, European Congress on Computational Meth- } \\
\text { ods in Applied Sciences and Engineering }\end{array}$} \\
\hline Publicación & \multicolumn{3}{|c|}{$\begin{array}{l}\text { Proceedings of European Congress on Computational Methods in } \\
\text { Applied Sciences and Engineering (ECCOMAS 2000) }\end{array}$} \\
\hline ISBN & $92-9092-776-3$ & Lugar & Barcelona, España \\
\hline Fecha & 11-14 Septiembre, 2000 & Páginas & 19 \\
\hline
\end{tabular}

\begin{tabular}{|c|l|c|l|}
\hline Autores & \multicolumn{2}{|l|}{$\begin{array}{l}\text { H. Esteban, S. Cogollos, C. Bachiller, A. A. San Blas y V. E. } \\
\text { Boria }\end{array}$} \\
\hline Título & $\begin{array}{l}\text { A new analytical method for the analysis of multiple scattering } \\
\text { problems using spectral techniques }\end{array}$ \\
\hline Congreso & $\begin{array}{l}\text { IEEE Antennas and Propagation Society International Sympo- } \\
\text { sium } 2002\end{array}$ \\
\hline Publicación & 2002 Digest. Volumen II & \multicolumn{2}{|l|}{} \\
\hline ISBN & $0-7803-7330-8$ & Lugar & $\begin{array}{l}\text { San Antonio (Texas, } \\
\text { Estados Unidos) }\end{array}$ \\
\hline Fecha & $16-21$ de Junio de 2002 & Páginas & $82-85$ \\
\hline
\end{tabular}




\begin{tabular}{|c|l|c|l|}
\hline Autores & \multicolumn{1}{|l|}{ A. Belenguer, A. Vidal, H. Esteban, C. Bachiller y V. E. Boria } \\
\hline Título & $\begin{array}{l}\text { Highly efficient MoM analysis of conducting 2-D scatterers using } \\
\text { wavelet basis functions }\end{array}$ \\
\hline Congreso & $\begin{array}{l}\text { IEEE Antennas and Propagation Society International Sympo- } \\
\text { sium 2002 }\end{array}$ \\
\hline Publicación & 2002 Digest. Volumen II & \multicolumn{2}{|l|}{} \\
\hline ISBN & $0-7803-7330-8$ & Lugar & $\begin{array}{l}\text { San Antonio (Texas, } \\
\text { Estados Unidos) }\end{array}$ \\
\hline Fecha & $16-21$ de Junio de 2002 & Páginas & $246-249$ \\
\hline
\end{tabular}

\begin{tabular}{|c|l|c|l|}
\hline Autores & \multicolumn{2}{|l|}{$\begin{array}{l}\text { J. V. Morro, H. Esteban, P. Soto, A. A. San Blas, V. E. Boria y B. } \\
\text { Gimeno }\end{array}$} \\
\hline Título & $\begin{array}{l}\text { Optimization methods for the automated design of inductively } \\
\text { coupled rectangular waveguide filters }\end{array}$ \\
\hline Congreso & $\begin{array}{l}\text { IEEE Antennas and Propagation Society International Sympo- } \\
\text { sium 2002 }\end{array}$ \\
\hline Publicación & 2002 Digest. Volumen II & \multicolumn{2}{|l}{} \\
\hline ISBN & $0-7803-7330-8$ & Lugar & $\begin{array}{l}\text { San Antonio (Texas, } \\
\text { Estados Unidos) }\end{array}$ \\
\hline Fecha & $16-21$ de Junio de 2002 & Páginas & $350-353$ \\
\hline
\end{tabular}

\begin{tabular}{|c|l|l|l|}
\hline Autores & \multicolumn{1}{|l|}{ V. E. Boria, S. Cogollos, H. Esteban y A. A. San Blas } \\
\hline Título & \multicolumn{2}{|l|}{$\begin{array}{l}\text { Efficient techniques for the accurate analysis and design of pas- } \\
\text { sive waveguide components with arbitrary geometry }\end{array}$} \\
\hline Congreso & $\begin{array}{l}\text { Progress in Electromagnetics Research Symposium (PIERS) } \\
2002\end{array}$ \\
\hline Publicación & Proceedings of PIERS'2002 \\
\hline ISBN & $0-9679674-2-2$ & Lugar & $\begin{array}{l}\text { Boston, } \\
\text { Unidos }\end{array}$ \\
\hline Fecha & $1-5$ de Julio de 2002 & Páginas & 615 \\
\hline
\end{tabular}




\begin{tabular}{|c|l|l|l|}
\hline Autores & \multicolumn{2}{|l|}{$\begin{array}{l}\text { J. V. Morro, H. Esteban, P. Soto, A. A. San Blas, V. E. Boria y B, } \\
\text { Gimeno }\end{array}$} \\
\hline Título & $\begin{array}{l}\text { DESAFIO 1.0: A novel CAD tools for the automated design of } \\
\text { inductively coupled rectangular waveguide filters }\end{array}$ \\
\hline Congreso & $\begin{array}{l}\text { Progress in Electromagnetics Research Symposium (PIERS) } \\
2002\end{array}$ \\
\hline Publicación & Proceedings of PIERS'2002 \\
\hline ISBN & $0-9679674-2-2$ & Lugar & $\begin{array}{l}\text { Boston, } \\
\text { Unidos }\end{array}$ \\
\hline Fecha & $1-5$ de Julio de 2002 & Páginas & 882 \\
\hline
\end{tabular}




\section{Bibliografía}

[1] V.E. Boria, M. Baquero, and M. Ferrando. Analysis of multireflector antenna clusters by spectral methods. In Proceedings of the 24th European Microwave Conference, volume 1, pages 870-875, Cannes, 5-8 Sept. 1994.

[2] V. Boria. Análisis de problemas electromagnéticos mediante métodos modales y matrices generalizadas, y aplicaciones. PhD thesis, Universitat Politècnica de València, València, 1997.

[3] H. Esteban, V.E. Boria, M. Baquero, and M. Ferrando. Generalised iterative method for solving 2D multiscattering problems using spectral techniques. IEE Proceedings on Microwave, Antennas and Propagation, 144(2):73-80, April 1997.

[4] C.A. Balanis. Advanced Engineering Electromagnetics. John Wiley \& Sons, 1989.

[5] A.F. Peterson, S. Ray, and R. Mittra. Computational Methods for Electromagnetics. IEEE Press, 1998.

[6] J.C. Cruellas. Análisis de la difracción de objetos dieléctricos mediante elementos finitos y realimentación modal. PhD thesis, U. Politècnica de Catalunya, Barcelona, 1989.

[7] H. Esteban, J.M. López, V.E. Boria, M. Baquero, and M. Ferrando. A generalized method for characterizing two-dimensional scattering problems with spectral techniques. Microwave and Optical Technology Letters, 14(1):6-9, January 1997.

[8] A.Z. Elsherbeni, M. Hamid, and G. Tian. Iterative scattering of a gaussian beam by an array of circular conducting and dielectric cylinders. Journal of Electromagnetic Waves and Applications, 7(10):1323-1342, 1993.

[9] F. Arndt, R. Beyer, J. Reiter, T. Sieverding, and T. Wolf. Automated design of waveguide components using hybrid mode-matching/numerical em building-blocks in optimization-oriented cad frame-works - state-of-the-art and recent advances. IEEE Trans. Microwave Theory Tech., 45(5):747-760, May 1997.

[10] R. H. MacPhie and Ke-Li Wu. A full wave modal analysis of arbitrarily shaped waveguide discontinuities using the finite plane-wave series expansion. IEEE Trans. Microwave Theory Tech., 47(2):232-237, February 1999. 
[11] Roland Geshe and Norbert Lochel. Scattering by a lossy dielectric cylinder in a rectangular waveguide. IEEE Trans. Microwave Theory Tech., 36(1):137-144, January 1988.

[12] Y. Leviatan., P.G. Li, A.T. Adams, and J. Perini. Single-post inductive obstacle in rectangular waveguide. IEEE Trans. Microwave Theory Tech., 31(10):806-811, October 1983.

[13] R. Geshe and N. Lochel. Two cylindrical obstacles in a rectangular waveguide - resonances and filter applications. IEEE Trans. Microwave Theory Tech., 37(6):962-968, June 1989.

[14] J.M. Reiter and F. Arndt. Rigorous analysis of arbitrarily shaped H- and E-plane discontinuities in rectangular waveguides by a full-wave boundary contour mode-matching method. IEEE Trans. Microwave Theory Tech., 43(4):796-801, April 1995.

[15] F. Alessandri, G. Bartolucci, and R. Sorrentino. Admittance matrix formulation of waveguide discontinuity problems: Computer-aided design of branch guide directional couplers. IEEE Trans. Microwave Theory Tech., 36(2):394-403, February 1988.

[16] J. Abdulnour and L. Marchildon. Boundary elements and analytic expansions applied to H-plane waveguide junctions. IEEE Trans. Microwave Theory Tech., 42(6):10381045, June 1994.

[17] M. O. Kolawole. Scattering from dielectric cilinders having radially layered permitivity. Journal of Electromagnetic Waves and Applications, 6(2):235-239, 1992.

[18] J. H. Richmond. Scattering by a dielectric cylinder of arbitrary cross-section shape. IEEE Trans. Antennas Propagat., 13(3):334-341, May 1965.

[19] M. Guglielmi and G. Gheri. Multimode network representation of multiple inductive and capacitive obstacles in parallel plate waveguides. IEEE Trans. Microwave Theory Tech., 42(6):1046-1051, June 1994.

[20] N. Marcuvitz. Waveguide Handbook. IEE Electromagnetic Wave Series 21, London, 1986.

[21] G.S. Sheaffer and Y. Leviatan. Composite inductive posts in waveguide - a multifilament analysis. IEEE Trans. Microwave Theory Tech., 36(4):779-783, April 1988.

[22] C.-I. Hsu and A. Auda. Multiple dielectric posts in a rectangular waveguide. IEEE Trans. Microwave Theory Tech., 34(8):883-891, August 1986.

[23] F. Alessandri, M. Mongiardo, and R. Sorrentino. Rigorous mode matching analysis of mitered e-plane bends in rectangular waveguide. IEEE Microwave and Guided Wave Letters, 4(12):408-410, December 1994. 
[24] M. Mongiardo, A. Morini, and T. Rozzi. Analysis and design of full-band matched waveguide bends. IEEE Trans. Microwave Theory Tech., 43(12):2965-2971, December 1995.

[25] H. Esteban, J. M. López, M. Baquero, J. Fortuny, G.Ñesti, and A. Sieber. An electromagnetic scattering model for multiple tree trunks over a tilted rough ground plane. IEEE Trans. Geosci. Remote Sensing, 37(2):659-667, March 1999.

[26] C.A. Balanis. Antenna Theory, Analysis and Design. Harper \& Row, New York, 1982.

[27] J. T. Londergan, J. P. Carini, and D. P. Murdock. Binding and Scattering in TwoDimensional Systems: Application to Quantum Wires, Waveguides and Photonic Crystals. Springer-Verlag, New York, 1999.

[28] A.B. Carlson. Communication Systems. An Introduction to Signals and Noise in Electrical Communication. McGraw-Hill Book Company, New York, 1986.

[29] M. Baquero. Transformaciones espectrales y aplicaciones a sintesis de ondas, medida de antenas y difracción. $\mathrm{PhD}$ thesis, Universitat Politècnica de València, València, 1994.

[30] R.F. Harrington. Field Computation by Moment Methods. The Mac Millan Company, New York, 1968.

[31] Héctor Esteban. Análisis de dispersores bidimensionales mediante técnicas espectrales. Proyecto de fin de carrera, Universidad Politécnica de Valencia, 1996.

[32] A. Belenguer. Mejora de la eficiencia en la resolución de problemas de dispersión 2-d mediante técnicas espectrales. Proyecto de fin de carrera, Universidad Politécnica de Valencia, 2000.

[33] H. Esteban, S. Cogollos, V.E. Boria, and M. Ferrando. Analysis of discontinuities in a rectangular waveguide using hybrid numerical and spectral techniques. In Progress in Electromagnetics Research Symposium PIERS, volume 2, page 893, Nantes, 13-14 July 1998.

[34] P. G. Li, A. T. Adams, Y. Leviatan, and J. Perini. Multiple post inductive obstacle in rectangular waveguide. IEEE Trans. Microwave Theory Tech., 32:365-373, April 1984.

[35] M. Koshiba and M. Suzuki. Application of the boundary-element method to waveguide discontinuities. IEEE Trans. Microwave Theory Tech., 34:301-307, February 1986.

[36] A. M. Khilla and I. Wolff. Field theory treatment of H-plane waveguide junction with triangular ferrite post. IEEE Trans. Microwave Theory Tech., 26:279-287, April 1978.

[37] M. Guglielmi. Simple CAD procedure for microwave filters and multiplexers. IEEE Trans. Microwave Theory Tech., 42(7):1347-1352, July 1994. 
[38] V.E. Boria, M. Guglielmi, and P. Arcioni. Computer-aided design of inductively coupled rectangular waveguide filters including tuning elements. International Journal of RF and Microwaves Computer-Aided Engineering, 8(3):226-236, May 1998.

[39] A. Alvarez, G. Connor, and M. Guglielmi. New simple procedure for the computation of the multimode admittance or impedance matrix of planar waveguide junctions. IEEE Trans. Microwave Theory Tech., 44(3):413-418, March 1996.

[40] H. Patzelt and F. Arndt. Double-plane steps in rectangular waveguides and their application for transformers, irises, and filters. IEEE Trans. Microwave Theory Tech., 30(5):771-776, May 1982.

[41] J. P. Webb and S. Porihar. Finite element analysis of H-plane rectangular waveguide problems. Proceedings of the Institute of Electrical Engineering, 133(2):91-94, April 1986.

[42] A. Taflove. Computational Electromagnetics: The Finite-Difference Time-Domain Method. Artech House, 1995.

[43] Y. Leviatan and G.S. Sheaffer. Analysis of inductive dielectric posts in rectangular waveguide. IEEE Trans. Microwave Theory Tech., 35(1):48-59, January 1987.

[44] A. Valero and M. Ferrando. Full-wave equivalent network representation for multiple arbitrarily shaped posts in H-plane waveguide. IEEE Trans. Microwave Theory Tech., 47(10):1997-2002, October 1999.

[45] A. Boetteger, Th. Sieverding, P. Krauss, and F. Arndt. Fast boundary contour modematching method for the cad of circular post coupled resonator filters. In Proceedings of the 28th European Microwave Conference, pages 712-715, October 1998.

[46] S. Haykin and B. Van Veen. Signals and Systems. John Wiley \& Sons, 1999.

[47] A. V. Oppenheim and R. W. Schafer. Discrete-Time Signal Processing. Prentice-Hall International, Inc., 1999.

[48] Texas Instruments. DERIVEß: The mathematical assistant for your pc. http://www.derive.com/.

[49] M. Guglielmi, G. Gheri, M. Calamia, and G. Pelosi. Rigurous mutimode network numerical representation of inductive step. IEEE Trans. Microwave Theory Tech., 42(2):317-325, February 1994.

[50] J.M. Reiter and F. Arndt. A full-wave boundary contour mode-matching method BCMM for the rigorous CAD of single and cascaded optimized H-plane and E-plane bends. IEEE MTT-S Digest, pages 1021-1024, 1994.

[51] R. Pregla. Concatenations of waveguide sections. IEE Proceedings on Microwave Antennas and Propagation, 114:119-125, April 1997. 
[52] A. Weisshaar, S.M. Goodnick, and V.K. Tripathi. A rigorous and efficient method of moments solution for curved waveguide bends. IEEE Trans. Microwave Theory Tech., 40:2200-2206, December 1992.

[53] X.-P. Liang, K. A. Zaki, and A. E. Atia. A rigorous three plane mode-matching technique for characterizing waveguide T-junctions, and its application in multiplexer design. IEEE Trans. Microwave Theory Tech., 39(12):2138-2147, 1991.

[54] D. Camilleri, B. Gimeno, A. Coves, M. V. Andrés, A. A. San Blas, H. Esteban, and V. E. Boria. Efficient technique for the analysis of cylindrical dielectric resonators in rectangular waveguide cavities. In Progress in Electromagnetic Research Symposium PIERS, editor, Proceedings of PIERS 2002, page 617, 2002.

[55] Inc. Advanced Electronics Technology. MAFIA®: MAxwell equations by the finite integration algorithm. http://aetassociates.com/.

[56] R. A. Shore and A. D. Yaghjian. Solving the electric field integral equation using the method of moments with pulse-basis functions and point-matching. In Progress in Electromagnetic Research Symposium PIERS, editor, Proceedings of PIERS 2002, page 578, 2002.

[57] G.T. Voelker, G.W. Lei, and B.K. Gilbert. Determination of complex permittivity of low-loss dielectrics. IEEE Trans. Microwave Theory Tech., 45(10):1955-1960, October 1997.

[58] B. Oswald, D. Erni., H.R. Benedickter, and W. Batchtold. Dielectric properties of natural materials. IEEE AP-S International Symposium, 4:2002-2005, June 1998.

[59] A. Parkash, J.K Vaid, and A. Mansingh. Measurement of dielectric parameters at microwave frequencies by cavity perturbation technique. IEEE Trans. Microwave Theory Tech., 27(9):791-795, September 1979.

[60] A. H. Boughriet, C. Legrand, and A. Chapton. Noniterative stable Transmission/Reflection method for low-loss material complex permitivity determination. IEEE Trans. Microwave Theory Tech., 45(1):52-57, January 1997.

[61] S. Jenkins, T.E. Hodgetts, R.N. Clarke, and A. W. Preece. Dielectric measurements on reference liquids using automatic network analysers and calculable geometries. Measurements Science \& Technology, 1:691-702, February 1990.

[62] S. Roberts and A. Von Hippel. A new method for measuring dielectric constant and loss in the range of centimeter waves. Journal of Applied Physics, 17:610-616, 1946.

[63] W. H. Press, S. A. Teukolsky, W. T. Vetterling, and B. P. Flannery. Numerical Recipes in C. Cambridge University Press, 1992. 
[64] J. Barker-Jarvis, E. Vanzura, and W. Kissick. Improved technique for determining complex permittivity with the transmission reflection method. IEEE Trans. Microwave Theory Tech., 38(8):571-577, August 1990.

[65] F. Arndt, R. Beyer, J.M. Reiter, T. Sieverding, and T. Wolf. Automated design of waveguide components using hybrid mode-matching/numerical EM building-blocks in optimization-oriented cad frameworks-state-of-the-art and recent advances. IEEE Trans. Microwave Theory Tech., 45(5):747-760, May 1997.

[66] J. E. Page. The effect of the machining method on the performances of rectangular waveguide devices. Proceedings of ESA workshop on advanced CAD for microwave filters and passive devices, pages 329-336, 1995.

[67] M Bozzi, M. Bressan, and L. Perregrini. Generalized Y-matrix of arbitrary 3D waveguide junctions by the BI-RME method. IEEE MTT-S International Microwave Symposium Digest, 3:1269-1272, June 1999.

[68] M. Bressan, L. Perregrini, and E. Regini. BI-RME modelling of 3D waveguide components enhanced by the Ewald technique. IEEE MTT International Symposium Digest, 2:1097-1100, 2000.

[69] Ansoft Corporation. HFSS®: 3d high-frequency electromagnetic simulation. http://www.ansoft.com/products/hf/hfss/index.cfm.

[70] A. R. Brown and G. M. Rebeiz. A high-performance integrated k-band diplexer. IEEE Trans. Microwave Theory Tech., 47(8):1477-1481, August 1999.

[71] M. Guglielmi. Simple CAD procedure for microwave filters and multiplexers. IEEE Trans. Microwave Theory Tech., 42(7):1347-1352, July 1994.

[72] R. Levy. Analytical design of contiguous multiplexers. IEEE MTT-S International Microwave Symposium Digest, 3:899-902, June 1999.

[73] J. Bornemann, S. Amari, and R. Vahldieck. A combined mode-matching and coupledintegral-equations technique for the design of narrow-band h-plane waveguide diplexers. IEEE AP-S International Symposium Digest, 2:950-953, July 1999.

[74] L. Accatino. CAD of a ku-band antena diplexer. Proceedings of the 23th European Microwave Conference, pages 544-546, 1993.

[75] F. Arndt, J. Dittloff, U. Papziner, D. Fasold, N.Ñathrath, and H. Wolf. Rigorous field theory design of compact and lightweight broadband diplexers for satellite systems. Proceedings of the 19th European Microwave Conference, pages 1214-1219, 1989.

[76] J. Dittloff and F. Arndt. Computer aided design of slit-coupled h-plane t-junctions diplexers with e-plane metal-insert filters. IEEE Trans. Microwave Theory Tech., 36(12):1833-1839, December 1988. 
[77] A. Morini, T. Rozzi, and M. Mongiardo. Efficient CAD of wideband contiguous channel multiplexers. IEEE MTT-S Int. Microwave Symp. Digest, pages 1651-1654, 1996.

[78] A. Morini and T. Rozzi. Design of optimum three port symmetrical junctions for diplexer applications. IEEE MTT-S Int. Microwave Symp. Digest, pages 739-742, 1994.

[79] J. Esteban and J. M. Rebollar. CAD for broadband antenna diplexer. ESA Workshop on Advanced CAD for microwave Filters and Passive Devices, pages 139-149, November 1995.

[80] J. R. Montejo-Garai and J. M. Rebollar. CAD of a ku band e-plane output diplexer for satellite applications. 7th Intenational Symposium on Recent Advances in Microwave Technology Proceedings, pages 769-772, December 1999.

[81] J. M. Rebollar and J. R. Montejo-Garai. Diseño de diplexor con t-plano h asimétrica en banda ku para aplicaciones espaciales. Libro de Actas del XIV Simposium Nacional de la Unión Científica Internacional De Radio, pages 388-389, Septiembre 1999.

[82] J. Bará. Circuitos de Microondas con Líneas de Transmisión. Edicions UPC, Barcelona, 1994.

[83] J. Uher, J. Bornemann, and U. Rosenberg. Waveguide Components for Antena Feed Systems: Theory and CAD. Artech House, Boston, 1993.

[84] G. Maral and M. Bousquet. Satellite Communications Systems. Wiley Series in Communication and Distributed Systems. John Wiley \& Sons, Chichester, third edition, 1998.

[85] U. Rosenberg, P. Rosowsky, W. Rümmer, and D. Wolk. Tunable manifold multiplexers: A new possibility for satellite redundancy philosophy. 18th European Microwave Conference, pages 870-875, September 1988.

[86] Y. Rong, H. Yao, K. A. Zaki, and T. Dolan. Millimeter wave ka-band h-plane diplexers and multiplexers. IEEE Trans. Microwave Theory Tech., 47(12):2325-2330, December 1999.

[87] J.A. Stratton. Electromagnetic Theory. Mc Graw-Hill Book Company, Inc., New York, 1941.

[88] R.F. Harrington. Time-Harmonic Electromagnetic Fields. McGraw-Hill Book Company, New York, 1961.

[89] J.A. Nelder and R. Mead. A simplex method for function minimization. Computer Journal, 7:308-313, January 1965. 